\title{
Marktorientierung in Business-to-Business-Märkten
}

Inaugural-Dissertation zur Erlangung des akademischen Grades eines Doktors der Wirtschaftswissenschaft des

Fachbereichs Wirtschaftswissenschaft der Freien Universität Berlin

vorgelegt von

Dipl.-Ing. Matthias Claßen

Berlin, 2015 
Erstgutachter: $\quad$ Prof. Dr. Dr. h.c. Michael Kleinaltenkamp

Zweitgutachter: $\quad$ Prof. Dr. Martin Gersch

Datum der Disputation: 11.02 .2015 


\section{Vorwort}

Die vorliegende Arbeit entstand während meiner Tätigkeit als Wissenschaftlicher Mitarbeiter und wurde im Januar 2015 als Dissertation vom Fachbereich Wirtschaftswissenschaft der Freien Universität Berlin angenommen.

An dieser Stelle möchte ich einer Reihe von Personen danken, die maßgeblich zum Gelingen dieser Arbeit beigetragen haben.

Zunächst gebührt besonderer Dank meinem Doktorvater Herrn Prof. Dr. Dr. h.c. Michael Kleinaltenkamp, der mir seit meinem ersten Tag an der Freien Universität Berlin das nötige Vertrauen entgegengebracht und das Thema dieser Arbeit angeregt hat. Neben dem eingeräumten Freiraum hat er mich durch seine Diskussionsbereitschaft und wertvolle Hinweise bei der Erstellung dieser Arbeit und auch darüber hinaus fachlich und persönlich unterstützt.

Ebenfalls gebührt großer Dank Herrn Prof. Dr. Martin Gersch, der die zügige Zweitbegutachtung dieser Arbeit übernahm und in kritisch-konstruktiver Weise bis zum erfolgreichen Abschluss begleitete.

Herrn Prof. Dr. Sascha Raithel möchte ich ebenfalls meinen Dank für die kurzfristige Bereitschaft, in meiner Promotionskommission tätig zu werden, und für die Inkaufnahme aller damit verbundenen Unwägbarkeiten aussprechen.

Prof. Dr. Henning Kreis und Alexander Mafael gebührt gleichfalls großer Dank. Als Mitglieder der Promotionskommission sorgten sie nicht nur für die reibungslose Durchführung des Promotionsverfahrens sondern ließen mir auch während meiner gesamten Zeit an der Freien Universität in fachlicher und persönlicher Hinsicht jede Unterstützung zukommen, um diese Arbeit erfolgreich zu Ende zu bringen.

Neben den namentlich Genannten habe ich während meiner Tätigkeit am Marketing-Department und beim Executive Master of Business Marketing eine Reihe von Kollegen und Freunden kennen und schätzen gelernt. Ohne nun einige davon in besonderer Weise hervorzuheben, sei gesagt, dass ohne die fortwährende Mo- 
tivation aus dem Kreise der Kollegen und Vorgesetzten die Anfertigung der Dissertation sich wesentlich schwieriger gestaltet hätte. Vielen Dank dafür!

Besonders große Anerkennung gebührt zudem meiner Familie. Meinen Eltern Marion und Günter danke ich für ihre bedingungslose, langjährige Unterstützung in jeglicher Weise sowie den notwendigen Ansporn zur Fertigstellung dieser Arbeit. Meiner lieben Frau Smita bin ich zu äußerstem Dank verpflichtet, da sie sich neben ihrer eigenen Promotion liebevoll und geduldig um unsere Kinder gekümmert und mir so den erforderlichen zeitlichen Freiraum zur Forschungsarbeit eröffnet hat. 


\section{Inhaltsverzeichnis}

Tabellenverzeichnis .................................................................................IX

Abkürzungsverzeichnis.................................................................... XII

$1 \quad$ Einleitung ................................................................................................. 1

1.1 Marktorientierung: Relevanz und Problemstellung .............................. 1

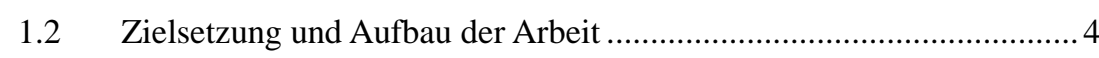

2 Grundlagen der Marktorientierung in mehrstufigen Märkten.. 9

2.1 Begriff und Merkmal der Marktorientierung ....................................... 9

2.1.1 Verhaltensorientierte Sichtweise von Marktorientierung.............. 12

2.1.2 Kulturbasierte Sichtweise von Marktorientierung .......................... 16

2.1.3 Gemeinsamkeiten der Sichtweisen .............................................. 19

2.2 Begriff und Merkmale von mehrstufigen Märkten und Mehrstufigem

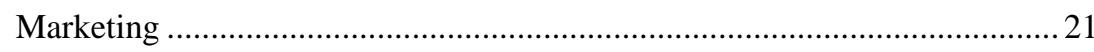

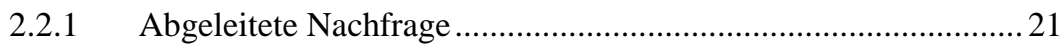

2.2.2 Grundlagen des Mehrstufigen Marketings.................................... 23

2.2.3 Möglichkeit der praktischen Ausgestaltung der einzelnen

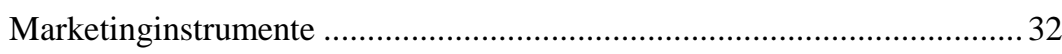

3 Diskussion der theoretischen Grundlage ....................................... 37

3.1 Bestimmung der theoretischen Grundlage .......................................... 37

3.1.1 Resource-Based View und Competence-Based View .................... 38

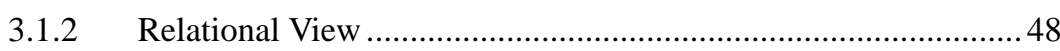

3.2 Eignung der theoretischen Grundlagen zur Erklärung des Konstrukts

der Marktorientierung und relevanter Konsequenzen....................................... 53

4 Bestimmung und Zusammenhang der untersuchten Konstrukte67

4.1 Allgemeines zur Konzeptualisierung und Operationalisierung von

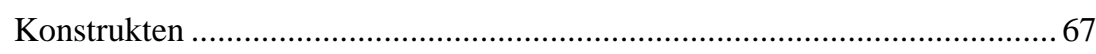

4.2 Zur Messung des Konstrukts Marktorientierung................................... 73 
4.3 Zur Konzeptualisierung des Konstrukts einer umfassenden

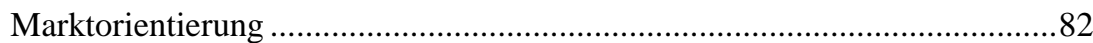

4.3.1 Theoretische Erwägungen..........................................................82

4.3.2 Messtechnische Erwägungen ....................................................86

4.3.3 Berücksichtigung B2B-relevanter Aspekte der gängigen Marktorientierungskonzepte ...................................................................8 87

4.3.4 Kundenorientierung ........................................................... 100

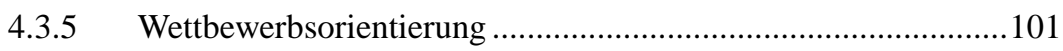

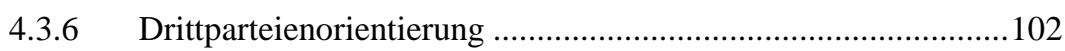

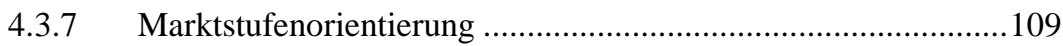

4.3.8 Abteilungsübergreifende Koordination....................................112

4.4 Konsequenzen einer umfassenden Marktorientierung und

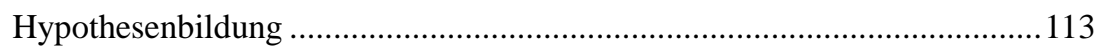

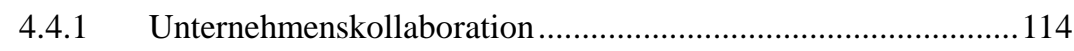

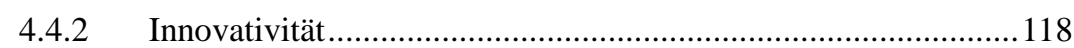

4.4.3 Anpassung der Marketingstrategien..........................................122

4.4.4 Neuprodukterfolg..............................................................126

4.5 Zusammenfassung der Hypothesen und das theoretische Modell ....132

4.6 Operationalisierung der Konstrukte ...............................................133

4.6.1 Zur Operationalisierung des Konstrukts Marktorientierung ......134

4.6.2 Operationalisierung der abhängigen Konstrukte.........................144

5 Empirische Analyse des theoretischen Modells ..........................151

5.1 Methodische Grundlagen zu Strukturgleichungsmodellen ................151

5.2 Der Varianz-analytische Ansatz zur Schätzung des

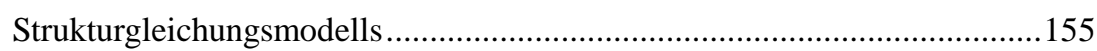

5.2.1 Grundlagen der Gütebeurteilung.............................................158

5.2.2 Gütekriterien reflektiver Messmodelle ......................................160

5.2.3 Gütekriterien formativer Messmodelle .......................................162

5.2.4 Gütekriterien des Strukturmodells ...............................................165

5.2.5 Gütebeurteilung von Mediationseffekten..................................166

5.2.6 Gütebeurteilung von Messmodellen zweiter Ordnung................169 
5.3 Datenerhebung und Datenbasis.................................................... 170

5.3.1 Wahl der Erhebungsmethode und Fragebogenentwicklung....... 170

5.3.2 Bestimmung der Stichprobe und Auswahl der Auskunftspersonen180

5.3.3 Datenbasis und Stichprobenstruktur 186

5.4 Bewertung der Messmodelle........................................................... 193

5.4.1 Gütebeurteilung der Messmodelle der Facetten des Konstrukts Marktorientierung 194

5.4.2 Gütebeurteilung des Messmodells des Gesamtkonstrukts

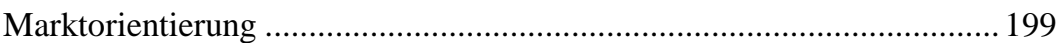

5.4.3 Gütebeurteilung der Messmodelle der Wirkungen .................... 207

$5.5 \quad$ Bewertung des Strukturmodells ........................................................ 211

5.5.1 Betrachtung der direkten Effekte und Erklärungsgehalt der

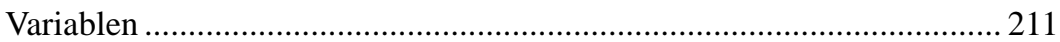

5.5.2 Bewertung der Mediationseffekte............................................. 213

5.6 Weitergehende Prüfungen der Modelle.......................................... 217

5.6.1 Modelltest auf disaggregierter Konstruktebene ......................... 217

5.6.2 Vergleich der MKTOR-Skala mit der neu entwickelten coMO-

Skala 221

5.6.3 Gruppenvergleich der Messmodelle .......................................... 224

5.6.4 Gruppenvergleiche im Strukturmodell .................................... 236

5.7 Zusammenfassung und Diskussion der Ergebnisse......................... 239

6 Fazit und Ausblick ............................................................... 246

6.1 Wissenschaftlicher Beitrag ............................................................ 246

6.2 Implikationen für die Praxis von Marketing und Vertrieb ............... 249

6.3 Limitationen der Untersuchung und Ansatzpunkte für weiterführende Forschung 251

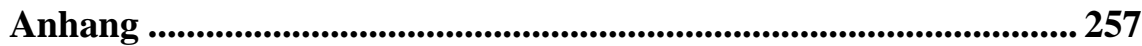

Literaturverzeichnis............................................................. 271 
VIII

\section{Abbildungsverzeichnis}

Abbildung 1: Push-, Pull- und kooperative mehrstufige Marketingstrategie ...... 29

Abbildung 2: Mehrstufiges Marketing. 36

Abbildung 3: Schematische Darstellung von reflektiven und formativen Messmodellen 71

Abbildung 4: Typen von Konstrukten zweiter Ordnung................................. 72

Abbildung 5: Stakeholder des Unternehmens. 104

Abbildung 6: Überschneidung von Markt- und Stakeholder-Orientierung....... 107

Abbildung 7: Strukturmodell der vorliegenden Studie ................................. 133

Abbildung 8: Schematisches Strukturgleichungsmodell............................... 154

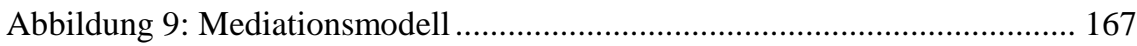

Abbildung 10: Ergebnisse der Hypothesenprüfungen ..................................... 216 


\section{Tabellenverzeichnis}

Tabelle 1: Ausgewählte Arbeiten zur verhaltensorientierten Sichtweise von Marktorientierung ......................................................................... 15

Tabelle 2: Ausgewählte Arbeiten zur kulturbasierten Sichtweise von Marktorientierung ........................................................................... 18

Tabelle 3: Unterschiedliche Messspezifikationen der MARKOR- und MKTOR-

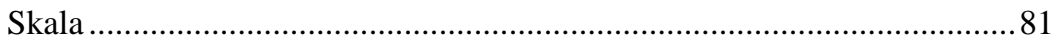

Tabelle 4: Items zur Messung der Kundenorientierung...................................138

Tabelle 5: Items zur Messung der Wettbewerbsorientierung ............................ 139

Tabelle 6: Items zur Messung der Drittparteienorientierung........................... 141

Tabelle 7: Items zur Messung der Marktstufenorientierung............................ 142

Tabelle 8: Items zur Messung der Abteilungsübergreifenden Koordination .....143

Tabelle 9: Items zur Messung der umfassenden Marktorientierung.................. 144

Tabelle 10: Items zur Messung der Unternehmenskollaboration ......................146

Tabelle 11: Items zur Messung der Innovativität ........................................... 147

Tabelle 12: Items zur Messung der Anpassung der Marketingaktivitäten..........148

Tabelle 13: Items zur Messung des Neuprodukterfolgs .................................... 150

Tabelle 14: Gütebeurteilung reflektiver Konstrukte....................................... 162

Tabelle 15: Gütebeurteilung formativer Messmodelle ..................................... 165

Tabelle 16: Gütebeurteilung von Strukturgleichungsmodellen .........................166

Tabelle 17: Gütekriterien von Mediationseffekten............................................168

Tabelle 18: Dimensionszuordnung relevanter Marktteilnehmer ......................173 
Tabelle 19: Struktur der Stichprobe nach Unternehmenscharakteristika 190

Tabelle 20: Struktur der Stichprobe nach Charakteristika der Auskunftspersonen 191

Tabelle 21: Güteprüfung der Dimension Kundenorientierung......................... 195

Tabelle 22: Güteprüfung der Dimension Wettbewerbsorientierung ................. 196

Tabelle 23: Güteprüfung der Dimension Drittparteienorientierung.................. 197

Tabelle 24: Güteprüfung der Dimension Marktstufenorientierung.................. 198

Tabelle 25: Güteprüfung der Dimension Abteilungsübergreifende Koordination 199

Tabelle 26: Ergebnisse der explorativen Faktoranalyse des Gesamtkonstrukts der Marktorientierung .............................................................................. 200

Tabelle 27: Ergebnisse des Tests auf Diskriminanzvalidität ........................... 201

Tabelle 28: Güteprüfung des Gesamtkonstrukts der umfassenden Marktorientierung 203

Tabelle 29: Ergebnisse der Güteprüfung des formativen Messmodells von Marktorientierung .................................................................................... 203

Tabelle 30: Ergebnisse der Kollinearitätsprüfung ........................................ 206

Tabelle 31: Ergebnisse der Prüfung auf Indikatorrelevanz für den faktorbasierten Ansatz 206

Tabelle 32: Gütebeurteilung des Konstrukts Unternehmenszusammenarbeit... 208

Tabelle 33: Gütebeurteilung des Konstrukts Innovativität 209

Tabelle 34: Gütebeurteilung des Konstrukts Anpassung der Marketingstrategie 209

Tabelle 35: Gütebeurteilung des Neuprodukterfolgs 210 
Tabelle 36: Ergebnisse der Schätzung des Strukturmodells .212

Tabelle 37: Bestimmtheitsmaße der endogenen Modellvariablen..... 212

Tabelle 38: Effektstärken und Prognoserelevanz des Teilstrukturmodells. 213

Tabelle 39: Ergebnisse der Prüfung auf Mediation .215

Tabelle 40: Ergebnisse der Prüfung auf Mediationsstärke .216

Tabelle 41: Vergleich der Bestimmtheitsmaße im aggregierten und disaggregierten Modell .................................................................... 218

Tabelle 42: Wirkbeziehungen mit disaggregiertem coMO-Modell 219

Tabelle 43: Vergleich der Bestimmtheitsmaße zwischen der MKTOR- und der coMO-Skala

Tabelle 44: Gruppenbildung auf Basis der jeweiligen Gruppenvariable. 228

Tabelle 45: Ergebnisse der Prüfung des coMO-Messmodells auf Unterschiede hinsichtlich der Voraussetzungen des Mehrstufigen Marketings 231

Tabelle 46: Ergebnisse der Prüfung des coMO-Messmodells auf Unterschiede hinsichtlich der Position in der Wertschöpfungskette

Tabelle 47: Ergebnisse der Prüfung des coMO-Messmodells auf Unterschiede hinsichtlich der Abteilungszugehörigkeit .235

Tabelle 48: Gruppenvergleich der Wirkbeziehung ALIGN $\rightarrow$ NPS 237

Tabelle 49: Ergebnisse der Prüfung auf Mediationsstärke mit ALIGN als Mediator bei Gruppenbetrachtung..... .239

Tabelle 50: Zusammenfassung der Hypothesen und Befunde .. 244 


\section{Abkürzungsverzeichnis}

\begin{tabular}{ll} 
Aufl. & Auflage \\
B2B & Business-to-Business \\
B2C & Business-to-Consumer \\
bspw. & beispielsweise \\
bzw. & beziehungsweise \\
CBV & Competence-Based View of the Firm \\
CRM & Customer Relationship Management \\
d. h. & das heißt \\
Ed(s) & Editor(s) \\
EFA & Explorative Faktoranalyse \\
et al. & et alii \\
etc. & et cetera \\
F\&E & Forschung und Entwicklung \\
f. & folgende \\
ff. & fortfolgende \\
Hervorh. d. Verf. & Hervorhebung durch den Verfasser \\
Hrsg. & Herausgeber \\
IT & Informationstechnik \\
KMO & Kaiser-Meyer-Olkin \\
MIMIC & Multiple-Indicators-Multiple-Causes \\
n & Fallzahl in einer Stichprobe \\
No. & Number \\
o.ä. & oder ähnlich \\
OEM & Original Equipment Manufacturer \\
PLS & Partial Least Squares \\
pp. & pages \\
PR & Public Relations \\
RBV & Resource-Based View of the Firm \\
RV & Relational View \\
\hline
\end{tabular}


S.

SGM

sog.

Sp.

SPSS

u. a.

vgl.

VIF

Vol.

z.B.
Seite

Strukturgleichungsmodell

sogenannte/r

Spalte

Statistical Package for the Social Sciences

und andere

vergleiche

Variance Inflation Factor

Volume

zum Beispiel 



\section{$1 \quad$ Einleitung}

\subsection{Marktorientierung: Relevanz und Problemstellung}

"The only way to get out of this mess is for us to become customer driven or market oriented. I'm not even sure what that means, but I'm damn sure that we want to be there. “

(Shapiro 1988)

In fast allen Märkten kommt einem zufriedenen Kunden eine entscheidende Rolle bei der Schaffung von Wettbewerbsvorteilen für ein Unternehmen zu. Lediglich in Märkten, die von Monopolisten oder Oligopolisten beherrscht werden und unzufriedenen Kunden somit keine Ausweichmöglichkeiten gegeben sind, muss ein Anbieter in deutlich geringerem Umfang auf die Schaffung von Kundenzufriedenheit achten. Die Orientierung hin zum Markt bzw. die Fokussierung des Unternehmens auf die Kunden soll ein Unternehmen in eine Position bringen, in der es Wettbewerbsvorteile realisieren kann. Wenngleich, wie das Zitat nach SHAPIRO zeigt, die genaue inhaltliche Interpretation dieser Marktorientierung oder Kundenfokussierung nicht immer bekannt ist, so zeigt es doch, dass schon vor langer Zeit die Bedeutung dieser Unternehmensausrichtung für eine Verbesserung der eigenen Wettbewerbsposition in der Marketing-Praxis erkannt wurde. Wettbewerbsvorteile sind essentiell für das langfristige Überleben eines Unternehmens am Markt und resultieren immer aus einem Anbieter- und einem Kundenvorteil. ${ }^{1}$ Um Wettbewerbsvorteile zu erzielen, sind Unternehmen einerseits bestrebt, ihre interne Effizienz zu steigern, um so Anbietervorteile zu generieren. Andererseits müssen Unternehmen auch immer ihren Kunden einen Wert bieten, der sich von dem der Konkurrenz abhebt und die Kunden zufrieden stellen kann. ${ }^{2}$

\footnotetext{
${ }^{1}$ Vgl. Plinke 2000b, S. 85.

2 Vgl. Narver/Slater 1990, S. 20 f.; Woodruff 1997, S. 142 ff.; Parasuraman 1997, S. 155.
} 
Der Zufriedenstellung der Kunden wird eine große Relevanz beigemessen, da sie in der Regel zu erhöhter Kundenloyalität und somit zu Folgekäufen führt. Dies wiederum zieht erhöhte Umsätze nach sich und trägt schließlich zum Erhalt der Geschäftsbeziehung bei. ${ }^{3}$ Die Fokussierung des Unternehmens auf die Kunden erscheint somit eine notwendige Voraussetzung für den Erfolg eines Anbieters zu sein. Insbesondere für Unternehmen, die auf Industriegütermärkten tätig sind, gilt, dass oftmals komplexe Leistungen kundenindividuell erstellt werden und dass in der Regel einzelne Kunden einen relativ großen Anteil am Umsatzvolumen des Anbieters ausmachen. ${ }^{4}$ Zudem ist die Anzahl der Kunden von Industriegüterunternehmen in der Regel geringer als bei solchen Unternehmen, die ihren Absatzmarkt im Konsumgüterbereich haben. Kundenzufriedenheit zu erreichen ist daher für Industriegüterunternehmen von höchster Wichtigkeit und kann auch als zentrale Zielgröße des Marketings auf Industriegütermärkten angesehen werden. ${ }^{5}$

Um aber Kunden zufrieden stellen und unternehmenssichernde Wettbewerbsvorteile generieren zu können, benötigen Unternehmen Informationen über ihre Märkte und müssen darauf aufbauend ihre Geschäftstätigkeit entsprechend gestalten. Die Ausrichtung der Unternehmensaktivitäten auf den Markt eines Unternehmens, insbesondere auf die Kunden und die relevanten Wettbewerber, wird als Marktorientierung bezeichnet. Marktorientierung ist kein neues Phänomen, sondern vielmehr eine Unternehmensphilosophie, die vor allem in gesättigten, von Nachfragern dominierten Märkten als Voraussetzung gilt, um unternehmerisch erfolgreich tätig zu sein. ${ }^{6}$ Heutzutage gilt Marktorientierung in vielen Unternehmen, die in entwickelten Märkten tätig sind, als Maxime des unternehmerischen Handelns. Ob in Werbeslogans oder als Unternehmensleitbild: Fast immer werden die Kunden, die Erfüllung ihrer Wünsche und die Schaffung von Werten für diese Kunden als eines der zentralen Leitbilder und Aufgaben des Unternehmens verstanden. Als altruistisches Motiv soll Marktorientierung aber nicht ver-

${ }^{3}$ Vgl. Heskett et al., 1994, S. $166 \mathrm{f}$.

${ }^{4}$ Vgl. Backhaus/Voeth 2009, S. 10, S. 493; Dahlke 2001, S. 73.

${ }^{5}$ Vgl. Simon/Homburg 1998, S. 5.

${ }^{6}$ Vgl. Kumar et al., 2011. 
standen werden. Denn der Fokus auf die Kunden und deren Wünsche und Bedürfnisse stellt einen wesentlichen Bestandteil einer Unternehmenskultur dar, die dafür sorgt, dass Unternehmen am Markt erfolgreich sind - eine im Kundensinne bessere Leistungserbringung, die im Umkehrschluss zu gesteigerter Zufriedenheit führen kann, ist dabei ein erwünschter Nebeneffekt. ${ }^{7}$ Der positive Zusammenhang zwischen Marktorientierung und Unternehmenserfolg wurde vielfach in der Wissenschaft bestätigt und gilt heute als unumstritten. ${ }^{8}$

Das Konzept der Marktorientierung mit dem Ziel der Zufriedenstellung von Kunden ist nicht neu. Schon vor über 60 Jahren postulierte DRUCKER, dass die Zufriedenstellung von Kunden die einzig wahre Grundlage für die Geschäftstätigkeit eins Unternehmens darstelle. ${ }^{9}$ Auch die Auffassung der Geschäftstätigkeit von LEVITT, die darauf beruht, den Kunden dazu zu bringen mit einem Unternehmen Geschäfte abschließen zu wollen, prägt bis heute das Verständnis der Marktorientierung. ${ }^{10}$ Trotz der Plausibilität und der weiten Akzeptanz von Marketingkonzepten, die den hohen Stellenwert von Kunden und deren Zufriedenheit betonen, werden diese Konzepte immer noch nicht vollständig von Praktikern im Bereich des Marketings angewendet. ${ }^{11}$ Zwar schätzen sich Unternehmen selbst so ein, dass sie bereits den Fokus der Unternehmenstätigkeit auf den Markt und insbesondere die Kunden legten. Häufig ist jedoch das Gegenteil der Fall. Somit kann konstatiert werden, dass eine „wahre“ Marktorientierung oftmals nicht oder nur oberflächlich vorhanden ist. ${ }^{12}$

Bisher wurde in der wissenschaftlichen Literatur die Konzeptualisierung von Marktorientierung vorrangig als Ausprägung von Anbieteraktivitäten oder einstellungen unternommen. Diese Aktivitäten beziehen sich fast ausschließlich auf die direkten Kunden und Wettbewerber des Anbieters. Bei dieser Art der Konzeptualisierung wurden jedoch Markteigenschaften vernachlässigt, die essentielle Bedeutung in Industriegütermärkten besitzen. In den meisten Industrien gibt

\footnotetext{
${ }^{7}$ Vgl. Lafferty/Hult 2001, S. 1 und die dort angegebene Literatur.

${ }^{8}$ Vgl. Kotler et al. 2007, S. 21. Vgl. für eine Übersicht z.B. Kirca et al. 2005; Ellis 2006.

${ }^{9}$ Vgl. Drucker 1954, S. 37.

${ }^{10}$ Vgl. Levitt 1960, S. 56.

${ }^{11}$ Vgl. Pulendran/Speed 1996.

12 Vgl. Steinman et al. 2000; Kumar et al. 2011.
} 
es eine Vielzahl von Markt- bzw. Produktionsstufen, die ein Produkt durchlaufen muss, bis es in der Regel in veränderter Form den Endverbraucher, den Konsumenten, erreicht. ${ }^{13}$ In Industriegütermärkten leitet sich nämlich die Nachfrage nach Gütern und Dienstleistungen von der nachfolgenden Marktstufe ab, da die nachfragenden Unternehmen wiederum in ihren jeweiligen Absatzmärkten als Anbieter tätig werden. Produkte und Dienstleistungen werden also nur deshalb von Unternehmen beschafft, da sie für die Erstellung oder den Vertrieb anderer Leistungen erforderlich sind. ${ }^{14}$ Marktstufen in Industriegütermärkten weisen somit die Besonderheit auf, dass die auf ihnen existierende Nachfrage ihren Ursprung in einer nachgelagerten Marktstufe hat. Letztlich werden somit alle nachgefragten Güter und Dienstleistungen von den Unternehmen beschafft, um die originäre Nachfrage des Endkonsumenten auf der letzten Marktstufe mittelbar oder unmittelbar zu befriedigen. Es handelt sich daher auf Industriegütermärkten fast ausnahmslos um eine abgeleitete Nachfrage. ${ }^{15}$ Für Industriegüterunternehmen erscheint es demnach sinnvoll, die Marktstufen, von denen sich die Nachfrage nach den eigenen Leistungen ableitet, ebenfalls bei der Ausrichtung der Unternehmensaktivitäten zu berücksichtigen. Ebenso kann vielfach beobachtet werden, dass der Absatz von Industriegütern durch zahlreiche andere Faktoren im Markt und Marktumfeld aktiv oder passiv beeinflusst wird. Das Konzept der Marktorientierung, wie es vielfach als Ausrichtung auf den unmittelbaren Markt verstanden wird, scheint vor diesem Hintergrund zu kurz zu greifen.

Vielmehr verdeutlicht die vorangegangene Argumentation, dass Unternehmen gerade in Industriegütermärkten, in denen letztlich über mehrere Marktstufen hinweg die Bedürfnisse von Endkonsumenten befriedigt werden müssen - Marktorientierung über alle Wertschöpfungsstufen hinweg umsetzen müssen.

\subsection{Zielsetzung und Aufbau der Arbeit}

Im Rahmen dieser Arbeit soll überprüft werden, inwiefern das Begriffsverständ-

${ }^{13}$ Vgl. Kleinaltenkamp/Rudolph 2002, S. 287.

${ }^{14}$ Vgl. Kleinaltenkamp/Rudolph 2002, S. 285.

15 Vgl. Günter 1997, S. 214. 
nis des Marktes in der bisherigen Marketingliteratur ausreicht, um Business-toBusiness-Märkte (B2B) zutreffend zu beschreiben. Da sich Märkte in der Realität nicht als homogene Gebilde darstellen, sondern sich aus unterschiedlichen Gruppen und Bestandteilen konstituieren, soll in dieser Arbeit untersucht werden, welche Bestandteile für das Geschäft von Anbietern im B2B-Sektor als relevant erachtet werden und inwiefern insbesondere die Berücksichtigung der abgeleiteten Nachfrage und des weiteren Marktumfelds das Verständnis des Marktes prägt. Die damit verbundene Forschungsfrage lautet:

1. Welche Gruppen und Bestandteile bilden den Markt in einem B2BKontext?

An eine adäquate Beschreibung des Marktes im B2B-Kontext schließt sich die Frage nach der Ausrichtung eines Unternehmens auf diesen Markt an. Daher soll insbesondere unter Berücksichtigung der abgeleiteten Nachfrage und etwaiger anderer identifizierter, separater Marktbestandteile überprüft werden, wie der Begriff der Marktorientierung im B2B-Kontext erweitert werden muss, um eine Unternehmensausrichtung auf den gesamten relevanten Markt erfassen zu könnnen. Ein Ziel dieser Arbeit ist es daher, das in zahlreichen theoretischen und empirischen Arbeiten erörterte Thema der Marktorientierung in den Kontext von mehrstufigen Industriegütermärkten zu bringen und ein Messinstrument zu entwickeln, das dieses Konzept innerhalb des B2B-Kontextes adäquat erfasst. Die entsprechende Forschungsfrage lautet demzufolge:

2. Wie kann das Konstrukt der Marktorientierung in einem B2B-Kontext sinnvoll konzeptualisiert und operationalisiert werden?

Unternehmen, die sich in mehrstufigen Märkten befinden, können ihre Unternehmensaktivitäten entsprechend ausgestalten und auch auf Marktstufen ausrichten, die der Stufe der eigenen Kunden nachgelagert sind, d.h. Absatzmärkte ihrer eigenen Kunden darstellen. Hierbei können in der Business-to-Business-Praxis 
Kooperationen mit Kunden zur Entwicklung von Produkten und Dienstleistungen für nachgelagerte Marktstufen eingegangen werden, um letztlich Leistungen zu erstellen, welche die Kunden auf unterschiedlichen Marktstufen zufrieden stellen können. In dieser Arbeit soll daher der Einfluss der Marktorientierung sowohl auf die unternehmensübergreifende als auch einzelbetriebliche Fähigkeit zur Produktentwicklung untersucht werden. Neben der kooperativen Produktentwicklung können Unternehmen im B2B-Bereich auch Marketingstrategien implementieren, welche die Abnehmer auf nachgelagerten Marktstufen und das weitere Marktumfeld berücksichtigen. Solche Marketingstrategien werden unter dem Begriff des Mehrstufigen Marketings zusammengefasst. ${ }^{16}$ Obwohl sich die Literatur insbesondere in den letzten Jahren verstärkt dem Mehrstufigen Marketing zuwendet, hat bisher noch keine ausreichende Verbindung des Konzepts der Marktorientierung und der Ausrichtung der Unternehmensaktivitäten, insbesondere der Marketingstrategie, auf mehrstufige Märkte stattgefunden. Studien, die Einflussfaktoren von Mehrstufigem Marketing und dessen Erfolgswirkungen untersuchen, wurden bisher nur vereinzelt durchgeführt. ${ }^{17}$ In dieser Arbeit soll Marktorientierung daher als Einflussfaktor des Mehrstufigen Marketings untersucht werden. Zudem soll als Konsequenz einer mehrstufig angelegten Unternehmensausrichtung die Wirkung auf die letztlich hervorgebrachten Unternehmensleistungen betrachtet werden. Die daraus abgeleitete Forschungsfrage lautet:

3. Welchen Einfluss übt Marktorientierung im B2B-Kontext auf die einzelbetriebliche und kollaborative Entwicklung von Leistungen, auf mehrstufige Marketingstrategien sowie auf den Erfolg des Unternehmens aus?

Zur Beantwortung der aufgestellten Forschungsfragen und den Gang der Untersuchungen ist diese Arbeit in sechs Hauptkapitel gegliedert, die nachstehend vorgestellt werden.

Der Einleitung (Kapitel 1) folgend werden in Kapitel 2 zunächst die für die Ar-

${ }^{16}$ Vgl. Kleinaltenkamp et al. 2012, S. 143.

${ }^{17}$ Vgl. Kleinaltenkamp et al. 2011; Schönhoff 2013; Fischer 2014; Homburg/Wilczek 2014. 
beit zentralen begrifflichen Grundlagen erläutert. Dazu werden die verschiedenen relevanten Auffassungsweisen von Marktorientierung vorgestellt (2.1) und anschließend Charakteristika mehrstufiger Industriegütermärkte sowie deren Bearbeitung in Form von Mehrstufigem Marketing aufgezeigt (2.2).

Zum besseren Verständnis des zentralen Konstrukts der Marktorientierung und zur begründeten Herleitung von Wirkzusammenhängen soll in Kapitel 3 die theoretische Fundierung dieser Arbeit entwickelt werden. Nach einer Argumentation der herangezogenen theoretischen Grundlagen werden diese zunächst vorgestellt (3.1) sowie anschließend auf ihre Eignung zur Erklärung des zentralen Konstrukts der Marktorientierung und der relevanten Wirkzusammenhänge untersucht (3.2).

In Kapitel 4 werden dann die einzelnen Konstrukte dieser Arbeit sowie deren Beziehungen zueinander dargestellt. Nach einigen allgemeinen (4.1) und speziell auf das zentrale Konstrukt der Marktorientierung bezogenen Vorbemerkungen (4.2) wird die theoriegeleitete Konzeptualisierung des Konstrukts der Marktorientierung vorgenommen (4.3). Anschließend werden die Konstrukte der Wirkungen von Marktorientierung konzeptualisiert (4.4). Nach der theoretischen Spezifizierung aller relevanten Konstrukte werden die Zusammenhänge der Konstrukte erläutert und zusammenfassend in Form eines Hypothesensystems dargestellt (4.5). Die zur Messung der einzelnen Konstrukte zur Anwendung kommenden Messvorschriften werden anhand der Konstruktoperationalisierungen erläutert (4.6).

Kapitel 5 dient schließlich der empirischen Prüfung des entwickelten Messmodells einer umfassenden Marktorientierung, der Prüfung der Messmodelle der Konsequenzen von Marktorientierung sowie der Schätzung des hergeleiteten Strukturmodells. Zunächst werden dazu die methodischen Grundlagen (5.1) sowie das gewählte Schätzverfahren mit relevanten Gütekriterien zur Modellbeurteilung erläutert (5.2). Im Anschluss daran wird die Datenerhebung und Datenbasis dargestellt (5.3), und die Bewertung der Messmodelle (5.4) und des Strukturmodells (5.5) durchgeführt. Kapitel 5 schließt mit den Ergebnissen weiterführender Prüfungen (5.6) und einer Zusammenfassung und Diskussion der erreichten 
Ergebnisse (5.7).

Die Arbeit endet mit einem Fazit und Ausblick in Kapitel 6. Darin wird der wissenschaftliche Beitrag und Erkenntnisfortschritt der Arbeit dargestellt (6.1) sowie Implikationen für die Marketing- und Vertriebspraxis vorgestellt (6.2). Die gesamte Arbeit schließt mit der Benennung von Limitationen im Rahmen des durchgeführten Forschungsprojekts sowie von Ansatzpunkten für weiterführende Forschungsvorhaben (6.3). 


\section{Grundlagen der Marktorientierung in mehrstufigen Märkten}

Um ein einheitliches, grundlegendes Verständnis von Marktorientierung, mehrstufigen Märkten und darauf ausgerichteten Unternehmensaktivitäten zu schaffen, sollen im folgenden Kapitel für die Abhandlung wichtige Grundbegriffe erläutert werden.

\subsection{Begriff und Merkmal der Marktorientierung}

Das Konzept der Marktorientierung hat seinen Ursprung in der Idee des Marketingkonzepts. Das Marketingkonzept rückt für Anbieterunternehmen nach der traditionellen Sichtweise die gleichzeitige Ausrichtung auf die Kunden und den Wettbewerb mit anderen Marktteilnehmern in den Mittelpunkt der Unternehmensaktivitäten. ${ }^{18}$ DRUCKER hebt den Kundenaspekt hervor und argumentiert sogar: "There is only one valid definition of business purpose: to create a customer”. ${ }^{19}$ Diese Philosophie ist bis heute das Grundgerüst der Marketingwissenschaft, nach der das ganze Unternehmen aus der Perspektive des Endresultats betrachtet wird, das heißt aus der Sicht der gewonnenen Kunden. ${ }^{20}$ Als Implementierung dieses Marketingkonzepts wird die Marktorientierung eines Unternehmens verstanden. ${ }^{21}$

Obwohl der Begriff „Marktorientierung“ seit Jahrzehnten Verwendung findet, besteht hinsichtlich seiner genauen Bedeutung weder in Praxis noch Wissenschaft genaue Einigkeit darüber, was ein marktorientiertes Unternehmen bzw. marktori-

\footnotetext{
${ }^{18}$ Vgl. Kotler et al., 2007.

${ }^{19}$ Drucker 1954, S. 39. DAY (1994, S. 37), RUEKERT (1992, S. 226) und PLINKE (1992, S. 830)

heben die Zufriedenstellung des Kunden hervor und ergänzen die Aussagen von DRUCKER dahingehend, dass der Mittelpunkt der Geschäftstätigkeit „creating a satisfied customer“ (Hervorh. d. Verf.) lauten müsste.

${ }^{20}$ Vgl. Van Raaij/Stoelhorst 2008, S. 1266.

${ }^{21}$ Vgl. Kohli/Jaworski, 1990, S. 1. Kleinaltenkanp/Dahlke, 1998, S. 32 bezeichnen Marktorientierung in diesem Zusammenhang als „Core Concept of Marketing“.
} 
entierte Mitarbeiter ausmacht. Auch umgekehrt werden für mutmaßliche Marktorientierung unterschiedliche Begrifflichkeiten synonym verwendet. ${ }^{22}$ So wird Marktorientierung in der Literatur mitunter als Kundenorientierung bezeichnet, obwohl nach dem heutigen Verständnis Kundenorientierung lediglich einen Teilbereich der Marktorientierung ausmacht. ${ }^{23}$ Andere Autoren bezeichnen marktorientierte Unternehmen als „market-driven“, d.h. dem Diktat der Märkte unterworfen. ${ }^{24}$ Dies würde Marktorientierung auf den Aspekt der Erfüllung von artikulierten Kundenwünschen beschränken und dem in dieser Arbeit vertretenen Verständnis ebenfalls nicht genügen. ${ }^{25}$ Einigkeit besteht in der Literatur lediglich darüber, dass Marktorientierung als Unternehmensausrichtung etwas grundsätzlich Verschiedenes von Marketingorientierung ist. Marketingorientierung wird als eine Ausrichtung verstanden, die die Marketingabteilung und die Marketingaktivitäten in den Vordergrund des unternehmerischen Handelns stellt. ${ }^{26}$ Wenngleich bei der Unternehmensausrichtung auf den Markt der Marketingabteilung eine wichtige Rolle zukommt, so stellt eine Marketingorientierung noch lange keine Garantie für zufriedene Kunden dar, was hingegen als Ziel von Marktorientierung verstanden werden kann.

Auch in der Praxis gelten bisweilen bestimmte Ausrichtungen und Verhaltensweisen eines Unternehmens als Marktorientierung, die jedoch in der Wissenschaft nicht als solche bezeichnet werden würden. Dies liegt wesentlich an uneinheitlichen Definitionen und zugrunde gelegten Bestandteilen von Marktorientierung, was es den Unternehmen schwierig - wenn nicht unmöglich - macht, eine zielgerichtete Ausrichtung und erfolgreiche Messung der eigenen marktorientierten Tätigkeiten durchzuführen. Diese unterschiedlichen Auffassungen, die hinsichtlich Marktorientierung bestehen, werden zudem durch kulturelle Einflüsse in den einzelnen Unternehmen noch weiter verstärkt. ${ }^{27}$

Obwohl sich die Wissenschaft schon seit längerem mit dem Thema Marktorien-

${ }^{22}$ Vgl. Harris 2001, S. 18.

${ }^{23}$ Vgl. Deshpandé et al. 1993 S. 33 f.; Kelley 1992.

${ }^{24}$ Vgl. Sheth/Sisodia 1999, S. 80.

${ }^{25}$ Vgl. Hamel/Prahalad 1994, S. 125; Narver/Slater 1998, S. 1004; Ketchen et al. 2007, S. 962 f.

${ }^{26}$ Vgl. Shapiro 1988, S. 119; Kohli/Jaworski, S. 3 f.; Avlonitis/Gounaris 1997.

${ }^{27}$ Vgl. Mason/Harris 2005. 
tierung auseinandersetzt, ist es bisher noch nicht gelungen, ein einheitliches Verständnis des Begriffs Marktorientierung zu erreichen. Auch die wesentlichen Inhalte der verschiedenen Konzeptualisierungen variieren so stark, dass bisher keine einheitliche Form der Kategorisierung bzw. Gruppierung von wissenschaftlichen Beiträgen existiert. Verschiedene Autoren schlagen vor, Marktorientierung anhand der Ebene ihrer Anwendung zu kategorisieren. ${ }^{28}$ Demnach kann Marktorientierung auf Unternehmens-, Projekt- bzw. Produktebene ${ }^{29}$ sowie auf Mitarbeiterebene $^{30}$ zum Einsatz kommen und unterschieden werden. Eine Aufteilung von wissenschaftlichen Arbeiten nach diesem Kategorisierungsansatz vermag aufgrund geringer Trennschärfe keine eindeutige Differenzierung der Marktorientierungsansätze hervorzubringen. Ebenso erweist sich diese Kategorisierung in der praktischen Anwendung als problematisch, da eher wenige Unternehmen Marktorientierung in Bezug auf Projekte oder Produkte unterschiedlich ausgestalten oder auffassen würden. Eine Betrachtung der marktorientierten Mitarbeitertätigkeiten kann ebenso nicht ohne weiteres losgelöst vom Verhalten der gesamten Organisation durchgeführt werden.

LAFFERTY/HULT schlagen eine Unterteilung der Literatur zu Marktorientierung in fünf Kategorien (decision-making process, market intelligence, culturally based behaviors, strategic marketing focus, customer orientation) vor. ${ }^{31}$ Diese Unterteilung ist jedoch zu feingliedrig, um alle Marktorientierungskonzepte nur einer einzigen Kategorie zuzuordnen. Vielfach würden Konzepte aufgrund ihrer weit gefassten inhaltlichen Reichweite mehreren Kategorien zugeordnet werden müssen. Andere Autoren bedienen sich daher nur zwei der Kategorien und gliedern auf diese Weise die Marktorientierungsliteratur. ${ }^{32}$ HOMBURG/PFLESSER

${ }^{28}$ Vgl. Steinhoff 2006, S. 55; Biemans 2003, S. 517.

${ }^{29}$ Vgl. Kok et al. 2003; Steinhoff, 2006.

30 Vgl. Utzig 1997; Saxe/Weitz 1982; Pelled et al. 2000; Kelley/Hoffman 1997. Tatsächlich beschreiben die Autoren kundenzentriertes Verhalten, was nur als ein Teil einer unternehmensweiten Marktorientierung zu sehen ist.

31 Vgl. Lafferty/Hult 2001, S. 95

32 GLEITSMANN (2007, S. 9) sieht bspw. eine Unterteilung der Literatur in eine kulturelle Perspektive und eine informationsorientierte Perspektive als sinnvoll an. Die Arbeiten der informationsorientierte Perspektive werden allerdings in der Regel auf den Aspekt der Informationsverarbeitung reduziert und können dementsprechend problemlos der verhaltendorientierten Perspektive zugerechnet werden. 
unterscheiden zwischen einer verhaltensorientierten und einer kulturellen Perspektive. ${ }^{33}$ Eine Erweiterung dieser Aufteilung umfasst noch eine „dritte“ Kategorie, die all jene konzeptionellen Beiträge beinhaltet, die keiner der beiden ersten Kategorien zugeordnet werden können. ${ }^{34}$ Auch diese Unterteilung birgt Probleme, da zwar einige Konzeptualisierungen kulturbezogen vorgenommen, das Konstrukt letztlich aber verhaltensorientiert gemessen wird. Eine strikte Aufteilung von wissenschaftlichen Arbeiten zum Thema Marktorientierung ist daher nach dieser Kategorisierung nur dann zweifelsfrei möglich, wenn entweder nur der konzeptionelle oder nur der empirische Aspekt der Arbeiten zur Einteilung herangezogen wird.

Wenngleich, wie gezeigt, verschiedene Kategorisierungen in der Marketingliteratur existieren, besteht die weiteste Verbreitung hinsichtlich der Einteilung in eine kulturbasierte sowie eine verhaltensorientierte Sichtweise. Zum Zwecke eines besseren Verständnisses von Marktorientierung soll dieser Gliederung gefolgt und in den nachstehenden Abschnitten diese beiden Sichtweisen erläutert werden. Vorgestellt werden ebenfalls die wichtigsten wissenschaftlichen Abhandlungen, welche die jeweilige Sichtweise vertreten, und auf die sich bis heute nachfolgende Arbeiten in ihren Konzeptualisierungen und auch Operationalisierungen von Marktorientierung beziehen. Auf einen umfassenden Literaturüberblick zum Thema Marktorientierung wird in dieser Arbeit jedoch bewusst verzichtet und stattdessen auf die metaanalytischen Arbeiten von RODRIGUEZ CANO ET AL., KIRCA ET AL., ELLIS und LIAO ET AL. verwiesen. ${ }^{35}$

\subsubsection{Verhaltensorientierte Sichtweise von Marktorientierung}

Marktorientierung wird innerhalb der verhaltensorientierten Sichtweise als eine Ansammlung von koordinierten Prozessen verschiedener Unternehmensabteilungen verstanden. Diese Prozesse beinhalten neben der unternehmerischen Entscheidungsfindung auch die tatsächliche Umsetzung der getroffenen Entschei-

\footnotetext{
${ }^{33}$ Vgl. Homburg/Pflesser 1999, S. 449.

${ }^{34}$ Vgl. Grozdanovic 2007, S. 16; Reichmann 2010, S. 14.

${ }^{35}$ Vgl. Rodriguez Cano et al. 2004; Kirca et al. 2005; Ellis 2006; Liao et al. 2011.
} 
dungen als gemeinsame Aktivitäten. ${ }^{36}$ Die Prozesse beinhalten im Wesentlichen, dem Unternehmen Marktinformationen zugänglich zu machen und zu nutzen. Als Hauptvertreter dieser Sichtweise unterscheiden KOHLI/JAWORSKI drei Elemente der Marktorientierung:

Als Ausgangspunkt von Marktorientierung sehen die Autoren die Gewinnung von Marktinformationen (intelligence generation) an. Die Marktinformationen umfassen dabei neben dem Verhalten und den Bedürfnissen der Kunden auch das Verhalten der Wettbewerber sowie Rahmenbedingungen und sonstige Einflüsse auf das Marktgeschehen. ${ }^{37}$ Laut Definition wurden zunächst allerdings bei der Gewinnung von Marktinformationen nur Kunden berücksichtigt. Später erweiterten die Autoren ihre Auffassung hinsichtlich der im Unternehmen zu erfassenden relevanten Marktinformationen. ${ }^{38}$ DENG/DART machen in diesem Zusammenhang direkt auf die Sammlung und Auswertung von Wettbewerberinformationen aufmerksam, die zur Ableitung von Handlungsoptionen notwendig sind. ${ }^{39}$ Die Sammlung von Marktinformation kann über verschiedene Wege erfolgen, wie etwa eine systematische Befragung von Kunden, informelle Kundengespräche sowie gezielte Marktforschungsprojekte. ${ }^{40}$

Die Sammlung von Marktinformationen stellt alleine noch keine Marktorientierung dar. Vielmehr müssen die gesammelten Informationen den sie benötigenden Abteilungen im Unternehmen zugänglich gemacht werden. Die Verbreitung von Marktinformationen (intelligence dissemination) stellt somit das zweite Element von Marktorientierung dar und ist nicht nur auf bestimmte Bereiche (wie etwa Marketing und Vertrieb) beschränkt, sondern durchzieht die gesamte Organisation. Die relevanten Informationen werden über formelle Wege oder durch informelle Gespräche zwischen Abteilungsmitgliedern weitergeleitet. ${ }^{41}$

Neben der Sammlung und Verteilung von marktrelevanten Informationen in einem Unternehmen ist auch eine Reaktion darauf (responsiveness) als drittes Ele-

\footnotetext{
${ }^{36}$ Vgl. Shapiro 1988, S. $120 \mathrm{ff}$.

${ }^{37}$ Vgl. Kohli/Jaworski 1990, S. 4.

${ }^{38}$ Vgl. Jaworski/Kohli 1996, S. 131

${ }^{39}$ Vgl. Deng/Dart 1994, S. 726.

${ }^{40}$ Vgl. Kohli et al. 1993, S. 476

${ }^{41}$ Vgl. Kohli/Jaworski 1990, S. 5.
} 
ment von Marktorientierung notwendig. Insbesondere RUEKERT sieht in der Ausarbeitung und Umsetzung der strategischen Marketingaspekte einen wesentlichen Bestandteil von Marktorientierung. ${ }^{42}$ Dazu gehören die Auswahl von Zielmärkten, eine auf die Kundenbedürfnisse abgestimmte Produktpolitik und alle Maßnahmen im Rahmen der Vermarktung dieser Produkte. ${ }^{43}$ JAWORSKI/KOHLI differenzieren hinsichtlich der Reaktionsfähigkeit des Unternehmens in späteren Arbeiten explizit zwischen der Entwicklung von (Marketing-)Plänen (response design) und der tatsächlichen Umsetzung dieser Pläne (response implementation), so dass noch einmal der Handlungsaspekt stärker in den Vordergrund gestellt wird. ${ }^{44}$

DAY hingegen sieht das Ziel der Informationssammlung, Informationsaufbereitung und anschließenden Verteilung des Wissens nicht unbedingt in der direkten Ausarbeitung und Umsetzung von Marketingstrategien. Der Autor betrachtet vielmehr alle Aktivitäten als Ziel von Marktorientierung, die im Rahmen des Kundenbeziehungsmanagements durchgeführt werden und zur Schaffung von Kundenzufriedenheit beitragen. ${ }^{45}$

Tabelle 1 gibt einen Überblick über die wichtigsten Arbeiten im Rahmen der verhaltensorientierten Sichtweise, auf die sich zahlreiche spätere Forschungsarbeiten hinsichtlich des Verständnisses von Marktorientierung beziehen.

\footnotetext{
${ }^{42}$ Vgl. Ruekert 1992.

${ }^{43}$ Vgl. Kohli/Jaworski 1990, S. 6.

${ }^{44}$ Vgl. Jaworski/Kohli 1993, S. 54.

${ }^{45}$ Vgl. Day 1994, S. 37.
} 
Tabelle 1: Ausgewählte Arbeiten zur verhaltensorientierten Sichtweise von Marktorientierung

\begin{tabular}{|c|c|c|c|c|c|}
\hline 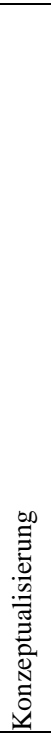 & 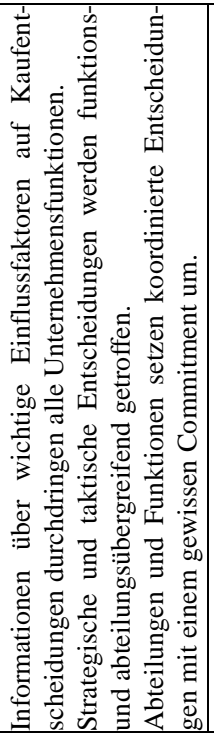 & 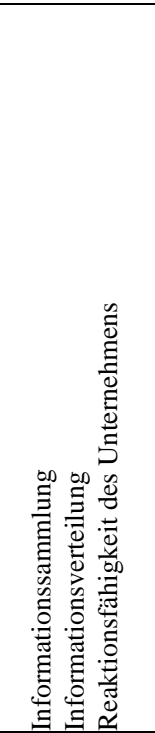 & 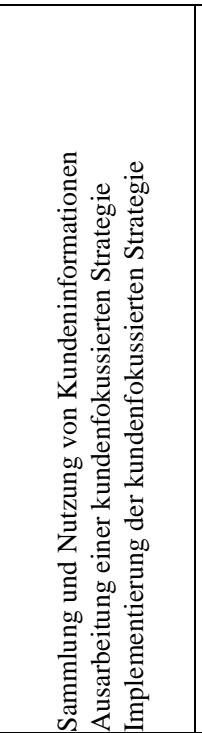 & 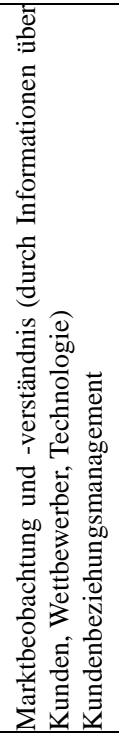 & 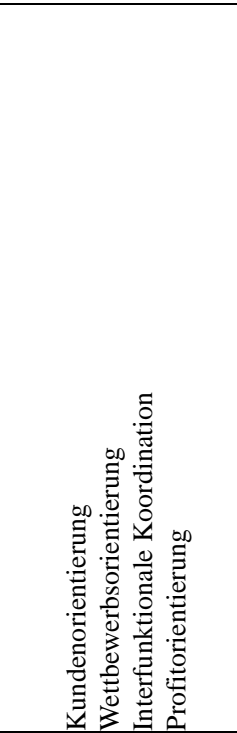 \\
\hline 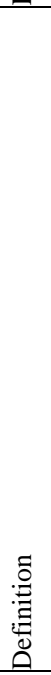 & 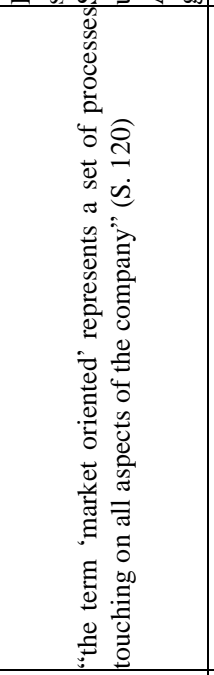 & 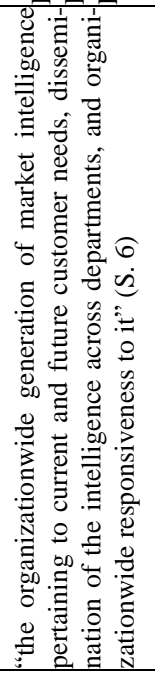 & 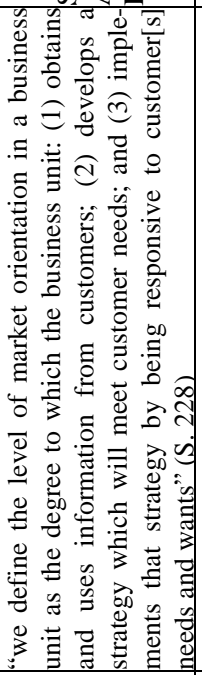 & 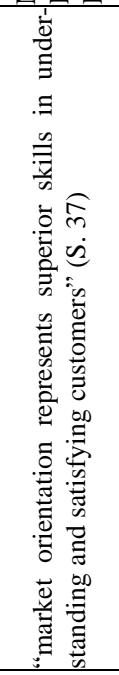 & 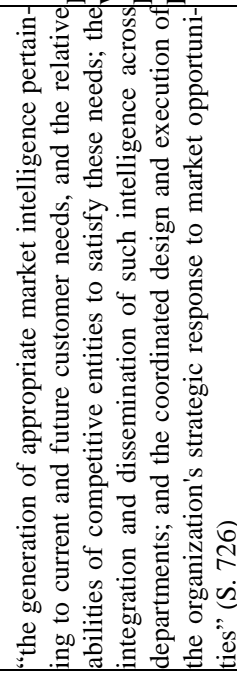 \\
\hline 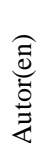 & 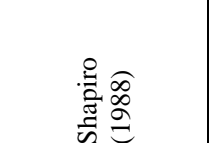 & 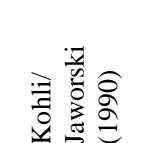 & 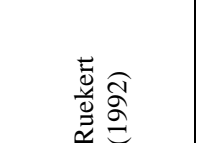 & $\overrightarrow{\stackrel{\Xi}{0}}$ & 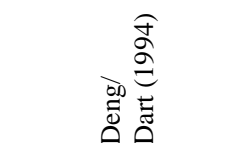 \\
\hline
\end{tabular}




\subsubsection{Kulturbasierte Sichtweise von Marktorientierung}

Die Arbeiten, die der kulturbasierten Sichtweise zugeordnet werden, verstehen Marktorientierung als Manifestation einer Unternehmenskultur. ${ }^{46}$ Diese Unternehmenskultur besteht aus Werten, Normen, Erwartungen und Überzeugungen und ruft ein bestimmtes auf den Markt gerichtetes Verhalten der Organisation und ihrer Mitglieder hervor. ${ }^{47}$ Innerhalb dieser Unternehmenskultur sieht sich das Unternehmen in der Pflicht, überlegene Werte für seine Kunden zu schaffen. ${ }^{48}$ Als einer der ersten Verfechter einer kulturbasierten Sichtweise zeigt PARASURAMAN, wie Anbieter durch eine kundenfokussierte Unternehmenskultur Wettbewerbsvorteile, insbesondere in hart umkämpften Märkten, generieren können. ${ }^{49}$ Dabei legt der Autor den Fokus auf Tätigkeiten, die aus den Überzeugungen der Unternehmung heraus entstehen und nicht durch bloße Arbeitsanweisungen abgedeckt werden können. Als eine der ersten empirischen Arbeiten, die Marktorientierung als mehr als nur eine Unternehmensfunktion betrachten, kann die Arbeit von HOOLEY ET AL. angesehen werden. Die Autoren zeigen, dass Marketing zwar lediglich als Unternehmensfunktion verstanden werden kann, erfolgreiche Unternehmen allerdings gleichzeitig Marktorientierung auch als unternehmensweite Implementierung des Marketingkonzepts und als Unternehmensphilosophie auffassen. ${ }^{50}$

Die heute bekannteste kulturbasierte Sichtweise auf Marktorientierung stammt von NARVER/SLATER. Die Autoren definieren Marktorientierung als „business culture that most effectively and efficiently creates superior value for customers““. ${ }^{51}$ Die Schaffung von Werten für den Kunden bedarf im Unternehmen demnach nicht mehr nur des Vergleichs zwischen Kosten und Nutzen der Leistungen des anbietenden Unternehmens. ${ }^{52}$ Vielmehr muss das Leistungsangebot eines

46 Vgl. Narver/Slater 1998a, S. 235.

47 Vgl. Parasuraman 1987, S. 40; Narver/Slater 1998a, S. 235; Harris 1998, S. 360.

${ }^{48}$ Vgl. Narver, Slater 1990; Deshpandé, Farley, Webster 1993.

${ }^{49}$ Vgl. Parasuranam 1997. Der Autor spricht hier allerdings nicht von Marktorientierung sondern von „customer-oriented corporate culture“ (S. 39).

${ }^{50}$ Vgl. Hooley et al. 1990, S. 11. Den Begriff Marktorientierung verwenden die Autoren allerdings explizit nur in Bezug auf die organisationale Struktur eines Unternehmens.

51 Narver/Slater 1990, S. 20.

52 Vgl. Plinke, 2000b, S. 85. 
Anbieters immer auch im Vergleich zur Mehrwertstiftung von Wettbewerbsleistungen gesehen werden. ${ }^{53}$ Ein wesentlicher Beitrag dieser Arbeit ist daher in der expliziten, gleichzeitigen Berücksichtigung von Kunden und Wettbewerbern zu sehen. Obwohl die Autoren Marktorientierung als Teil einer Unternehmenskultur betrachten, konzeptualisieren sie das Konstrukt anhand von drei marktorientierten Verhaltensweisen: customer orientation, competitor orientation und interfunctional coordination. ${ }^{54}$ In späteren Arbeiten betonen die Autoren, dass es sich bei Marktorientierung zwar um eine Unternehmenskultur handele, sich diese aber in den Einstellungen der Mitarbeiter widerspiegele und letztlich marktbezogene Aktivitäten begründe. ${ }^{55}$

DESHPANDÉ ET AL. entwickeln die kulturbasierte Definitionen von Marktorientierung hin zu einem kulturellen Selbstverständnis einer Organisation, das über die Kunden und Wettbewerber hinaus auch weitere Unternehmenszielgruppen (stakeholder) berücksichtigt. ${ }^{56}$ Bei der Messung des Konstrukts greifen die Autoren jedoch ebenfalls auf marktorientierte Verhaltensweisen zurück.

Eine tatsächlich auf kulturellen Elementen basierte Konzeptualisierung und Operationalisierung findet sich bei HOMBURG/PLESSER. Die Autoren nehmen allerdings auch hier zusätzlich Verhaltensaspekte auf und definieren Marktorientierung als eine Komposition von im Unternehmen geteilten Wertvorstellungen und auf den Markt gerichteten Unternehmensaktivitäten. ${ }^{57}$ Anzumerken ist hier, dass Marktorientierung teilweise als etwas der Organisation und teilweise den Mitarbeitern inhärentes angesehen wird. Gemeinsam ist allen Arbeiten jedoch, dass sie trotz der kulturellen Fokussierung nicht gänzlich ohne Bezug zum Verhalten der Mitarbeiter oder der Organisation in ihren Konzeptualisierungen oder Operationalisierungen auskommen.

Forschungsarbeiten, die heutzutage eine kulturbasierte Sichtweise von Marktorientierung annehmen, stützen sich im Wesentlichen auf die zuvor vorgestellten

\footnotetext{
${ }^{53}$ Vgl. Narver/Slater 1990, S. 21.

${ }^{54}$ Vgl. Narver/Slater 1990, S. 21. Andere Autoren rechnen die Arbeit von NARVER/SLATER (1990)

daher auch den verhaltensbasierten Ansätzen zu (vgl. Steinhoff 2006, S. 62).

55 Vgl. Slater/Narver 1995, S. 67; Narver et al. 1998, S. 241.

${ }^{56}$ Vgl. Deshpandé et al. 1993, S. 27.

${ }^{57}$ Homburg/Pflesser 2000, S. 450.
} 
Arbeiten, die noch einmal in Tabelle 2 zusammengefasst dargestellt werden.

Tabelle 2: Ausgewählte Arbeiten zur kulturbasierten Sichtweise von Marktorientierung

\begin{tabular}{|c|c|c|c|c|c|c|c|c|}
\hline 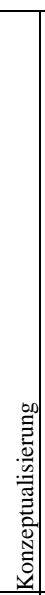 & 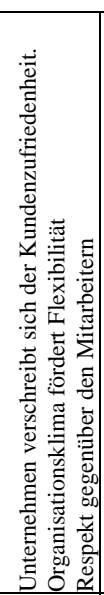 & 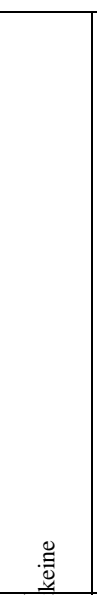 & 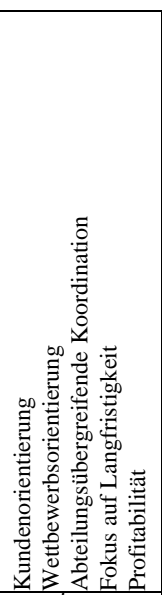 & 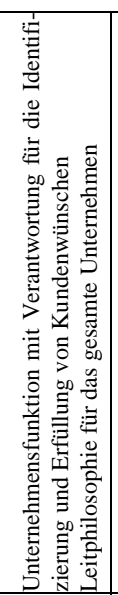 & 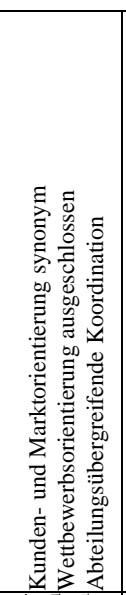 & & 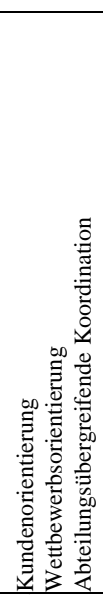 & 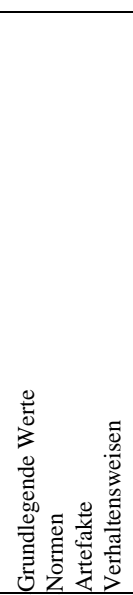 \\
\hline $\begin{array}{l}0 \\
:\end{array}$ & 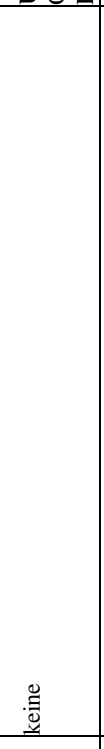 & 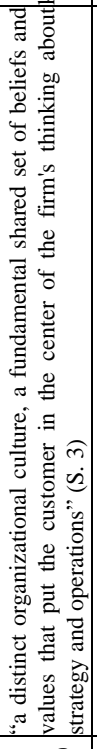 & 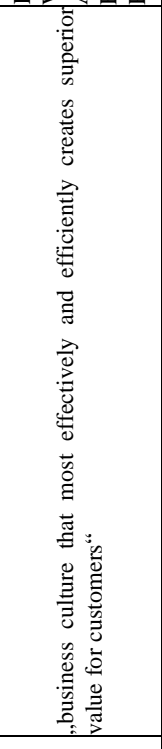 & 胥 & 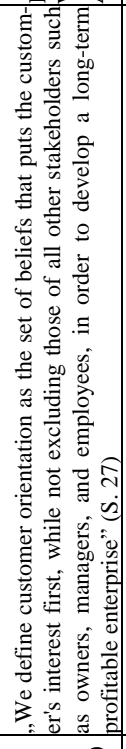 & 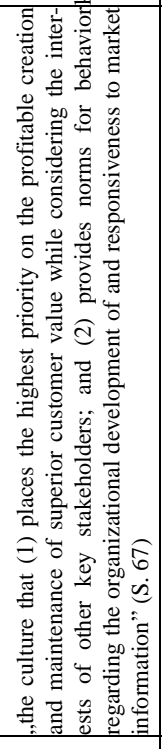 & 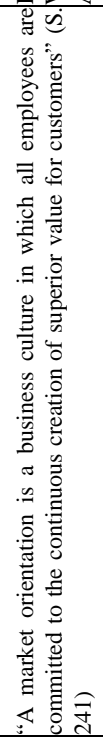 & 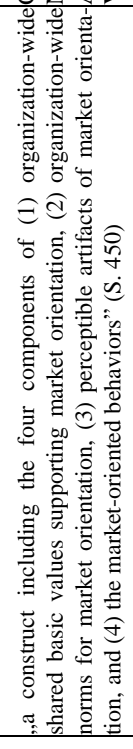 \\
\hline 侌 & 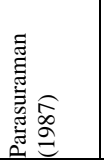 & 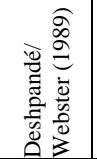 & 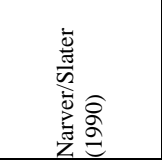 & 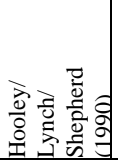 & 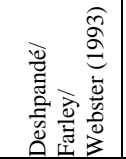 & 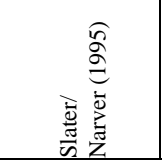 & 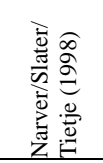 & 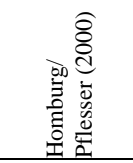 \\
\hline
\end{tabular}




\subsubsection{Gemeinsamkeiten der Sichtweisen}

Der wesentliche Unterschied zwischen der verhaltens- und der kulturbasierten Sichtweise auf Marktorientierung besteht darin, dass marktorientierte Aktivitäten als von der Unternehmenskultur losgelöst oder als Ausfluss einer marktorientierten Kultur angesehen werden. GEBHARDT ET AL. resümieren in diesem Zusammenhang „Researchers advancing a behavioral perspective suggest that the activities of a market orientation are separate from organizational culture [...], whereas others suggest that it is an organizational culture that encourages such behaviors". 58

Auch in neuerer Zeit geht die Diskussion darum weiter, ob Marktorientierung als Teil einer Unternehmenskultur oder als Verhalten betrachtet werden soll. Um die jeweiligen Vorteile beider Sichtweisen nutzen und bestimmte Phänomene im Unternehmenskontext erforschen zu können, wird Marktorientierung mitunter auch in eine Kultur- und eine Aktionskomponente aufgeteilt. ${ }^{59}$ Dabei wird eine kausale Beziehung zwischen einer marktorientierter Kultur und einem marktorientiertem Verhalten unterstellt.

Trotz der aufgeführten Unterschiede bestehen zwischen den verschiedenen Definitionen und Sichtweisen von Marktorientierung einige Übereinstimmungen. ${ }^{60}$ Diese übereinstimmenden Aspekte bilden den gemeinsamen Nenner hinsichtlich Marktorientierung in der Marketingwissenschaft:

- Ein wesentlicher Unternehmensfokus liegt auf den Kunden und ihren Bedürfnissen.

- Weitere Marktfaktoren (z.B. Wettbewerb, Technologie, gesetzliche Regulierungen, Einflussnehmer und weitere Stakeholder) finden Berücksichtigung in der Unternehmensausrichtung.

- Marktwissen wird im Unternehmen durch Sammeln und Verteilen von Informationen geschaffen.

\footnotetext{
${ }^{58}$ Gebhardt et al. 2006, S. 52.

${ }^{59} \mathrm{Vgl}$. Zhou et al. 2008; Hult et al. 2005.

${ }^{60}$ Vgl. Lafferty/Hult 2001, S. 100 ff.; Jaworski/Kohli 1996, S. 121; Day 1994, S. 37.
} 
- Auf Grundlage von Marktwissen werden Marketingaktivitäten geplant und koordiniert durchgeführt und letztlich Kundennutzen geschaffen.

Die in Abschnitt 2.1.2 genannte Arbeit von PARASURAMAN macht jedoch deutlich, dass alle beschrieben Gemeinsamkeiten, die sich als Aktivitäten eines Unternehmens darstellen, nicht den gleichen Erfolg beim Kunden hätten, wenn sie lediglich auf reinen Arbeitsanweisungen beruhen würden. Auch wäre Marktorientierung und daraus resultierende Kundenzufriedenheit in diesem Fall in jedem beliebigen Kontext in jedem Unternehmen implementierbar. ${ }^{61}$ Dies ist aber nicht der Fall, wie ein Blick in die Praxis nahe legt. Marktorientierung zum Zwecke der Steigerung von Kundenzufriedenheit kann somit nicht einzig und allein auf angewiesenen kundenfokussierten Tätigkeiten basieren. Vielmehr kann Kundenzufriedenheit nur aus echter Marktorientierung resultieren. Dafür muss eine innere Bereitschaft des Unternehmens und seiner Mitarbeiter vorliegen, kundenfokussiert zu handeln. Diese Bereitschaft wiederum leitet sich aus den Werten, Überzeugungen und Vorstellungen der Mitarbeiter ab, die in Summe die Organisationskultur bilden. In dieser Arbeit wird daher dem Ansatz gefolgt, dass es sich bei Marktorientierung um eine Unternehmenskultur handelt, die sich allerdings immer auch in Handlungen, d.h. in auf den Markt gerichteten Aktivitäten, niederschlagen muss. Marktorientierung als Unternehmenskultur beinhaltet also immer auch eine Handlungskomponente, die gegenüber den Kunden oder anderen Stakeholdern des Unternehmens Anwendung findet. ${ }^{62}$

Für das Verständnis von Marktorientierung in dieser Arbeit lässt sich also sagen, dass Marktorientierung als Verkörperung des Marketingkonzepts einerseits als Kultur der Unternehmung gesehen wird, die überlegene Werte für die Kunden und einen relativ hohen Erfolg für das Unternehmen schafft. ${ }^{63}$ Andererseits stellt Marktorientierung die Implementierung des Marketingkonzepts dar, d.h. jene gesamtunternehmerische Aktivitäten, die darauf ausgerichtet sind, die Kunden-

61 Vgl. Narver/Slater 1998a, S. 235.

62 Vgl. Barney 1986b, S. 659; Auch PLINKE (1992, S. 836 f.) beschreibt Marktorientierung als

Einstellungsmerkmal der Unternehmensmitarbeiter, die sich letztlich aber in Verhaltensweisen gegenüber Kunden äußern müsse.

${ }^{63}$ Vgl. Day/Wensley 1988, S. 43; Narver/Slater 1990, S. 20; Slater/Narver 1995, S. 63. 
wünsche besser als der Wettbewerb $\mathrm{zu}$ erfüllen. ${ }^{64}$ Zusammengefasst ist Marktorientierung ,a culture committed to the continuous creation of superior value for customers that manifests itself as a set of cross-functional processes and activities directed at creating and satisfying customers through continuous needs assessment”. 65

\subsection{Begriff und Merkmale von mehrstufigen Märkten und Mehrstufigem Marketing}

Da Marktorientierung als Kultur, wie erläutert, immer auch Aktivitäten nach sich zieht, die auf den Markt des Unternehmens gerichtet sind, soll im Folgenden gezeigt werden, was im Rahmen dieser Arbeit unter dem Markt eines Unternehmens verstanden wird. Die folgenden Abschnitte zielen dabei darauf ab, erste Antworten auf die Forschungsfrage 1 nach den Bestandteilen des Marktes in einem Business-to-Business-Kontext auf theoretischer Basis zu liefern. Im Vordergrund der Betrachtung stehen hierbei zunächst die Mehrstufigkeit des Absatzmarktes im B2B-Bereich und anschließend die Relevanz von Einflussnehmern im Marktumfeld. Vor dem Hintergrund dieser Stufenstruktur soll ferner betrachtet werden, wie ein Anbieter den Einsatz von Marketingaktivitäten auf Basis einer mehrstufigen Marktorientierung planen kann.

\subsubsection{Abgeleitete Nachfrage}

Leistungen im Business-to-Business-Bereich werden in der Regel von Produktions- oder Dienstleistungsunternehmen beschafft, um damit wiederum Leistungen zu erstellen, die den Bedarf anderer Unternehmen decken. ${ }^{66}$ Alternativ werden die Leistungen unverändert an den Handel zur weiteren Veräußerung an wieder andere Abnehmer geliefert. ${ }^{67}$ Nachfrager auf Business-to-Business-Märkten stellen somit in den meisten Fällen selbst Anbieter von Leistungen auf ihren jeweili-

\footnotetext{
${ }^{64}$ Vgl. Kohli/Jaworski 1990; Jaworski/Kohli 1993, S. 57; Wrenn 1997.

65 Vgl. Narver et al. 1998, S. 250.

66 Vgl. Kleinaltenkamp 2000, S. 173.

${ }^{67}$ Vgl. Engelhardt/Günter 1981, S. 24.
} 
gen Absatzmärkten dar. ${ }^{68}$ Die erstellten Leistungen durchlaufen mehrere Marktoder Handelsstufen, bevor sie in weiterverarbeiteter Form oder als Fertigprodukte letztlich den Endnutzer erreichen. ${ }^{69}$

Die Anzahl der Marktstufen, die zwischen dem Hersteller einer Leistung und der letztlichen Nutzung dieser Leistung liegen, hängt vom Grad der Arbeitsteilung in der betreffenden Wertschöpfungskette ab. ${ }^{70}$ Der Grad der Arbeitsteilung wird wiederum im Rahmen einer Make-or-Buy-Entscheidung dadurch bestimmt, ob es für ein Unternehmen günstiger ist, eine bestimmte Leistung selbst zu erstellen bzw. einen bestimmten Prozessschritt selbst vorzunehmen (Make) oder diese Leistungen am Markt von anderen Anbietern zu kaufen (Buy). ${ }^{71}$ Durch die zunehmende Arbeitsspezialisierung und die Konzentration auf Kernkompetenzen kann davon ausgegangen werden, dass in Zukunft ein Produkt eher mehr als weniger Marktstufen in einer Wertschöpfungskette bis zum Endkunden durchlaufen wird. ${ }^{72}$ KLEINALTENKAMP ET AL. zeigen in einer Studie, dass bereits jetzt in einigen Branchen über die Hälfte aller Produkte mehr als drei weiterverarbeitende Marktstufen durchlaufen, bevor sie den Endkunden erreichen. ${ }^{73}$ In der Wertschöpfungskette nehmen folglich nur das erste und das letzte Unternehmen in der Kette nicht die Doppelrolle als gleichzeitige Anbieter und Nachfrager ein. $^{74}$

Für einen Anbieter bedeutet die Stufenstruktur des Marktes, dass die Nachfrage seines direkten Kunden von der Nachfrage dessen Kunden, also von den indirekten Kunden des Anbieters und ferner von den Endkunden bestimmt wird. Die Nachfrage in der Wertschöpfungskette hängt somit von den Beschaffungsentscheidungen der nachfolgenden Marktstufen ab und leitet sich originär vom letztendlichen Nutzer dieser Leistung ab. In diesem Zusammenhang wird daher auch

${ }^{68}$ Vgl. Kleinaltenkamp/Rudolph 2002, S. 285

${ }^{69}$ Vgl. Kleinaltenkamp et al. 2012, S. 143.

${ }^{70}$ Vgl. Havenstein 2004, S. 57.

${ }^{71}$ Vgl. Besanko et al. 2010, S. $120 \mathrm{ff}$.

${ }^{72}$ Vgl. Plinke 2000c, S. 48 ff.

${ }^{73}$ Vgl. Kleinaltenkamp et al. 2010.

${ }^{74}$ Vgl. Flint 2004, S. 48 f. 
von abgeleiteter Nachfrage gesprochen. ${ }^{75}$ Der ursprüngliche Bedarf, für dessen Deckung eine Leistung in der Wertschöpfungskette erstellt wird, stammt somit vom letzten Glied der Kette, dem Endkunden. Dieser Bedarf wird sukzessive von den Unternehmen der dem Endkunden vorgelagerten Stufen des Marktes gedeckt. KLEINALTENKAMP ET AL. konstatieren in diesem Zusammenhang: „all business transactions in a downstream value chain are done in order to ultimately satisfy the final consumers' needs." 76

\subsubsection{Grundlagen des Mehrstufigen Marketings}

Obwohl das Wissen um die Wichtigkeit der abgeleiteten Nachfrage in Unternehmen verbreitet ist, werden die nachfolgenden Marktstufen nur selten bei der Planung von Marketingaktivitäten berücksichtigt. ${ }^{77}$ In Anlehnung an LEVITT's „Marketing Myopia"78 postulieren KLEINALTENKAMP ET AL., dass eine Betrachtung nur des direkten Kunden eine betriebswirtschaftliche Kurzsichtigkeit darstellt. Die Autoren sprechen in diesem Zusammenhang von "b2b marketing myopia”. ${ }^{79}$ Durch solch eine eingeschränkte Sicht ihres Marktes entgehen den Unternehmen Geschäftsmöglichkeiten und sie verzichten auf Unternehmenswachstum, denn alle Marktstufen bis hin zum Endnutzer oder Endkonsumenten einer Leistung bieten ein mögliches Ziel für Marketingmaßnahmen. ${ }^{80}$ Schließlich stellen die nachfolgenden Marktstufen genau wie die direkten Kunden auch die Geschäftsumgebung und somit ebenfalls einen relevanten Teil des Marktes eines Anbieters dar. Im Gegensatz zu einer einstufigen und eventuell kurzsichtigen Marktbetrachtung erscheint eine mehrstufige Marktbetrachtung bzw. eine auf die verschiedenen Marktstufen ausgerichtete Marketingstrategie im Business-toBusiness-Kontext zielführender. Durch eine mehrstufige Marketingstrategie wird es für einen Anbieter möglich, die verschiedenen nachgelagerten Marktstufen bis

\footnotetext{
${ }^{75}$ Vgl. Kleinaltenkamp/Rudolph 2002, S. 285.

${ }^{76}$ Kleinaltenkamp et al. 2009, S. 2.

77 Vgl. Kleinaltenkamp et al. 2011, S. 43.

${ }^{78}$ Vgl. Levitt 1960.

${ }^{79}$ Vgl. Kleinaltenkamp et al. 2009, S. 2.

${ }^{80}$ Vgl. Kleinaltenkamp et al. 2009, S. 2; Homburg/Wilczek 2014, S. 12 ff.; Kleinaltenkamp/Rudolph 2002, S. 287.
} 
hin zum Endkunden in ihren Kaufentscheidungen zu beeinflussen. ${ }^{81}$

Der Begriff des Mehrstufigen Marketings wird in der Literatur nicht einheitlich gebraucht. So finden sich zahlreiche Begriffe, die im Zusammenhang mit der Bearbeitung von mehrstufigen Märkten gebraucht werden und nur teilweise große inhaltliche Übereinstimmungen aufweisen. Es finden sich in der englischsprachigen Literatur Begriffe wie „vertical marketing systems“ ${ }^{\text {82 }}$, „vertical cooperati-

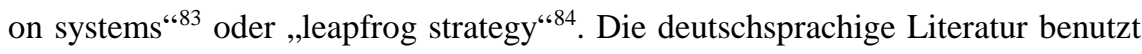
vielfach den inhaltlich verwandten Begriff „,vertikales Marketing“ ${ }^{85}$ Da die einzelnen Instrumentalbereiche einer mehrstufigen Marketingstrategie ebenfalls nicht eindeutig definiert sind, finden sich in der Literatur auch Begriffe, die, nicht zuletzt auch historisch bedingt, das heutige Verständnis von Marketingaktivitäten nicht vollumfänglich abbilden. So verwendet ENGELHARDT die Begriffe „mehrstufige Absatzstrategie“ und „mehrstufige Marktstrategie“ synonym. Zudem verweist der Autor im Zusammenhang mit der Bearbeitung mehrstufiger Märkte auf die ebenfalls häufig benutzten Begrifflichkeiten „Vertikalvertrieb“ und „integratives Absatzsystem“. ${ }^{86}$ Letztlich berühren die beschriebenen Tätigkeiten aber alle Instrumentalbereiche des Marketings und reichen somit weit über die suggerierte Beschränkung auf die Distributionspolitik eines Unternehmens hinaus.

Inhaltliche Unterscheidungen werden in der Literatur auch hinsichtlich der Bereitschaft zur Zusammenarbeit zwischen Anbieter und Kundenunternehmen getroffen. So versteht KUNKEL unter vertikalem Marketing eine „auf mehrere Marktstufen zielende absatzfördernde Strategie und deren taktische Ausgestaltung durch eine Unternehmung wie auch durch mehrere Unternehmungen“. ${ }^{87}$ Der Autor stellt dabei den kooperativen Aspekt der Strategie in den Vordergrund: Die Unternehmen der verschiedenen Marktstufen in der Wertschöpfungskette stim-

${ }^{81}$ Vgl. Kleinaltenkamp et al. 2009, S. 3.

${ }^{82}$ Vgl. McCammon, 1970, S. 43.

${ }^{83}$ Vgl. Ericsson, 1976, S. 29

${ }^{84}$ Vgl. Arnott, 1994, S. 79.

85 Vgl. Kunkel 1977; Homburg 2012, S. 872; Zentes/Swoboda 2001, S. 348.

${ }^{86}$ Vgl. Engelhardt 1976, S. 175.

${ }^{87}$ Kunkel 1977, S. 6 (Hervorh. d. Verf.). 
men ihre Strategien ab und richten sie auf die Endabnehmer aus. ${ }^{88}$

BAUMGARTH geht differenzierter vor und unterscheidet zwischen mehrstufigem Marketing und vertikalem Marketing. Der Autor definiert den Begriff des vertikalen Marketings im Gegensatz zu KUNKEL als „eine mehrere Stufen der Vertikalkette berücksichtigende Strategie und deren taktische Ausgestaltung durch eine Unternehmung, die versucht, ihr Marketingkonzept bis zum Endabnehmer durchzusetzen“. ${ }^{89}$ Mehrstufiges Marketing unterscheidet sich laut BAUMGARTH von vertikalem Marketing im Hinblick auf die konkrete Ausgestaltung der auf die Marktstufen gerichteten Marketingmaßnahmen. Beim mehrstufigen Marketing werden mehrere Marktstufen aktiv und direkt angesprochen, beim vertikalen Marketing müssen diese hingegen lediglich Berücksichtigung in der Marketingstrategie finden. ${ }^{90}$ Der Autor betrachtet das mehrstufige Marketing als eine einzelbetriebliche, unkooperative Strategie eines Anbieters. Eine Abstimmung der Marketingstrategien auf verschiedenen Marktstufen ist nicht erforderlich.

RUDOLPH hingegen bezeichnet schon Marketingstrategien, die nur eine oder auch mehrere Marktstufen ansprechen, als mehrstufiges Marketing, sofern es sich dabei nicht um die Stufe des direkten Kunden handelt. Der Autor definiert mehrstufiges Marketing wie folgt: „Mehrstufiges Marketing umfasst die Gesamtheit aller absatzpolitischen Maßnahmen, die auf eine (mehrere) den unmittelbaren Abnehmern nachfolgende Marktstufe(n) gerichtet sind. “91 SCHÖNHOFF ET AL. bauen auf dieser Definition auf und zeigen, dass es verschiedene Arten von Mehrstufigem Marketing gibt, die sowohl durch Einbeziehung des direkten Kunden, als auch durch dessen Umgehung charakterisiert werden können. In ihrer Definition von Mehrstufigem Marketing legen die Autoren den Fokus auf die Abstimmung innerhalb des Anbieterunternehmens zwischen jenen Marketingaktivitäten, die auf die direkten Kunden abzielen und solchen, die nachgelagerte

\footnotetext{
${ }^{88}$ Homburg (2012, S. 872) und Zentes/Swoboda (2001, S. 348 f.) stellen bei der Beschreibung des vertikalen Marketings ebenfalls den kooperativen Aspekt heraus.

89 Baumgarth 1998, S. 39 (Hervorh. d. Verf.)

${ }^{90}$ Vgl. Baumgarth 1998, S. 187.

91 Rudolph 1989, S. 34.
} 
Marktstufen ansprechen: „Multi-stage marketing involves all marketing and sales-related measures aiming at direct customers and subsequent market stages, i.e. customers of the customers, as well as the coordination of single-stage and overleaping marketing activities.“92

KLEINALTENKAMP ET AL. geben ebenfalls eine eigene Definition von Mehrstufigem Marketing und bauen dabei auch auf der Definition von RUDOLPH auf: "Multi-stage marketing involves all sales-related measures which are aimed at the subsequent market stages ("customers of the customer") which follow one or several primary customers in order to influence the buying behavior of these primary customers." ${ }^{93}$ Die Autoren rücken mit dieser Definition wieder die auf Industriegütermärkten existierende abgeleitete Nachfrage in den Fokus und verdeutlichen gleichzeitig das mit einer mehrstufigen Marketingstrategie verfolgte Ziel. Das Ziel besteht demnach darin, die direkten Kunden hinsichtlich ihrer Kaufentscheidungen zu beeinflussen. Als Adressaten einer mehrstufigen Marketingstrategie verweisen die Autoren zusätzlich zu den in der Definition genannten noch auf sog. Drittparteien, die das Kaufverhalten auf nachgelagerten Stufen beeinflussen können. ${ }^{94}$

Ein wesentlicher Unterschied in den Definitionen von SCHÖNHOFF ET AL. und KLEINALTENKAMP ET AL. resultiert aus den vom Mehrstufigen Marketing in diesen Ansichten umfassten Maßnahmen. KLEINALTENKAMP ET AL. nennen in ihrer Definition lediglich absatzpolitische Maßnahmen, die gemeinhin als Distributionspolitik verstanden werden können. ${ }^{95}$ SCHÖNHOFF ET AL. hingegen ergänzen diese Maßnahmen allgemein um Marketingaktivitäten und berücksichtigen somit laut ihrer Definition ein deutlich weiteres Spektrum an Unternehmensaktivitäten, das im Rahmen des Mehrstufigen Marketings eingesetzt werden kann.

Für den weiteren Verlauf der Arbeit sollen diese beiden Definitionen zusammengebracht werden, da sie gemeinsam das heute vorherrschende Verständnis von

${ }^{92}$ Vgl. Schönhoff et al. 2013, S. 2.

${ }^{93}$ Kleinaltenkamp et al. 2012, S. 143.

${ }^{94}$ Kleinaltenkamp et al. 2012, S. 149.

${ }^{95}$ Vgl. Meffert et al. 2012, S. 7; Gutenberg 1955, S. 13. 
Mehrstufigem Marketing prägen und die in dieser Arbeit beschriebenen Phänomene am treffendsten beschreiben können: Mehrstufiges Marketing umfasst (1) alle Marketingmaßnahmen, die auf die indirekten Kunden oder einflussnehmende Drittparteien gerichtet sind, sowie (2) die Koordination dieser Marketingaktivitäten mit den auf die direkten Kunden gerichteten Marketingaktivitäten mit dem Ziel, (3) das Kaufverhalten in der Wertschöpfungskette zu beeinflussen.

Dabei umfasst der Begriff der indirekten Kunden alle Abnehmer auf einer oder mehreren den direkten Kunden nachfolgenden Marktstufe(n) („Kunden des Kunden“). Eine unternehmensinterne Abstimmung der Marketingstrategien, die verschiedene Marktstufen zum Ziel haben, wird nach dieser Definition als notwendig erachtet. Eine Abstimmung zwischen den Unternehmen verschiedener Marktstufen kann ebenfalls erfolgen, ist aber nicht bei allen Ausgestaltungsformen des Mehrstufigen Marketings (z.B. beim unkooperativen Vorgehen) notwendig bzw. durchführbar. ${ }^{96}$ Die Definition zeigt zudem implizit die Wirkmechanismen des Mehrstufigen Marketings auf, die im Wesentlichen aus einer Kombination von Pull- und Push-Effekten bestehen.

\subsubsection{Wirkmechanismen des Mehrstufigen Marketings}

Ziel einer mehrstufigen Marketingstrategie ist es, Einfluss auf das Beschaffungsverhalten von Akteuren in einer Wertschöpfungskette zu nehmen. Dazu kann sowohl Einfluss auf die direkten Kunden, als auch auf die indirekten Kunden auf nachgelagerten Marktstufen ausgeübt werden. ${ }^{97}$

Eine reine Push-Strategie beinhaltet eine einstufige Marktbetrachtung und kann somit nur Einfluss auf die Kaufentscheidungen der direkten Kunden nehmen. Die Marketingmaßnahmen des Anbieters werden auf die direkten Abnehmer gerichtet und haben zum Ziel, die Anbieterleistungen sozusagen in den Markt zu „drücken“. ${ }^{98}$ Bei der Push-Strategie bietet der Anbieter den direkten Kunden Anreize, damit diese seine Produkte verstärkt nachfragen. Anreize können bspw. die Gewährung von besonderen Einkaufskonditionen oder die Unterstützung bei Marke-

\footnotetext{
${ }^{96}$ Vgl. Schönhoff et al. 2013, S. 3.

${ }^{97}$ Vgl. Kleinaltenkamp/Rudolph 2002, S. 291; Kleinaltenkamp et al. 2012, S. 143.

98 Vgl. Kleinaltenkamp/Rudolph 2002, S. 291.
} 
tingaktivitäten (z.B. durch Produktbroschüren, Schulungsmaßnahmen) des direkten Abnehmers sein. ${ }^{99}$ Häufige Anwendung findet die Push-Strategie vor allem im Handelsbereich. ${ }^{100}$ Im Bereich der weiterverarbeitenden Produktion und im Dienstleistungsbereich kommen Push-Strategien aber ebenfalls zum Einsatz. ${ }^{101}$ Im Gegensatz dazu findet bei Pull-Strategien eine mehrstufige Marktbetrachtung statt: Marketingaktivitäten werden hierbei auf nachgelagerte Marktstufen gerichtet und sollen dort eine höhere Anbieterakzeptanz und Präferenz für die Leistungen dieses Anbieters schaffen. ${ }^{102}$ Die Präferenzen der indirekten Kunden für die Anbieterleistungen lösen einen Nachfrage-Sog aus, der sich bis auf die Stufe der direkten Kunden überträgt und letztlich zur Kaufentscheidung durch die direkten Kunden führt. Der geschaffene Nachfrage-Sog wird auch als Pull-Effekt bezeichnet, weshalb die dahinterstehende Strategie auch Pull-Strategie genannt wird. ${ }^{103}$

Vereinfacht werden diese Strategien in Abbildung 1 dargestellt. In der Praxis können die Wertschöpfungsketten deutlich mehr Weiterverarbeitungs- und Handelsstufen beinhalten, was eine Marktbearbeitung entsprechend komplexer gestaltet. In der Praxis ist die Anwendung von nur einer der beschriebenen Strategien oftmals mit Problemen verbunden. So zeigen direkte Kunden, sofern sie im Rahmen einer Pull-Strategie umgangen bzw. in die „Zange genommen“ werden, mitunter starke Widerstände, die je nach Machtkonstellationen im Marktgefüge große Risiken für den Anbieter bergen können. ${ }^{104}$ Reine Push-Strategien bergen ebenfalls große Risiken, wenn z.B. der direkte Kunde wenig Produktwissen und nicht das erforderliche Marketing-Knowhow besitzt oder ihm gleichwertige Alternativen durch konkurrierende Anbieter zur Verfügung stehen. Daher werden sowohl im Bereich des Handels als auch im produzierenden Gewerbe oftmals Kombinationen dieser Strategien, z.B. in Form einer kooperativen mehrstufigen Marketingstrategie (vgl. Abbildung 1) angewandt. ${ }^{105}$

${ }^{99}$ Vgl. Kleinaltenkamp/Rudolph 2002, S. 292.

100 Vgl. Frazier 1999, S. 235.

101 Vgl. Kleinaltenkamp/Rudolph 2002, S. 292.

102 Vgl. Backhaus/Voeth 2010, S. 296; Rätsch/Bazing 2010, S. 664.

103 Vgl. Homburg 2012, S. 872.

${ }^{104}$ Vgl. Kleinaltenkamp/Rudolph 2002, S. 292; Kleinaltenkamp et al. 2011, S. 44.

105 Vgl. Nuneva/Jensen 2010, S. 307; Chiou et al. 2010, S. 431. 
Push-Strategie

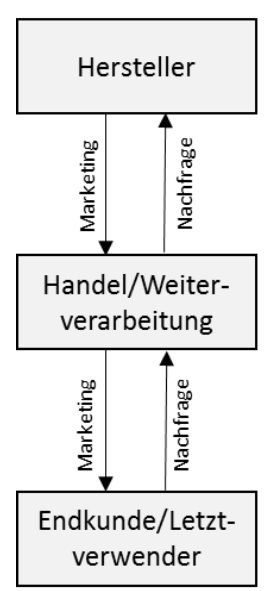

Pull-Strategie

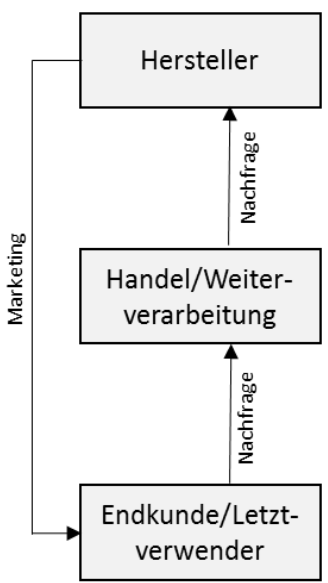

Kooperatives Mehrstufiges Marketing

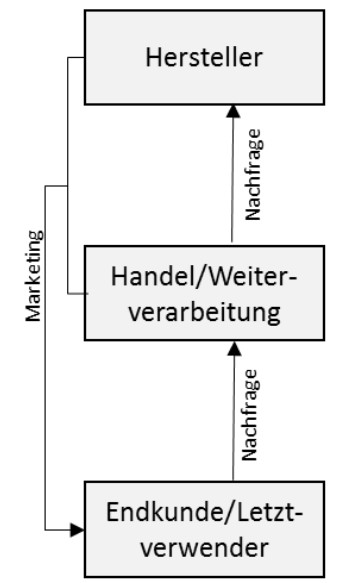

Abbildung 1: Push-, Pull- und kooperative mehrstufige Marketingstrategie (Quelle: in Anlehnung an Kleinaltenkamp et al. 2012, S. 148)

\subsubsection{Anwendungsvoraussetzungen des Mehrstufigen Marketings}

Auch wenn eine mehrstufige Marketingstrategie, die sowohl Push- als auch PullWirkungen integrieren würde, sinnvoll erscheint, kommt in der Praxis dennoch nicht immer Mehrstufiges Marketing zum Einsatz. Dies kann einerseits an den betreffenden Produkten, andererseits an den involvierten Unternehmen liegen, die jeweils gewisse Voraussetzungen für einen sinnvollen und erfolgreichen Einsatz erfüllen müssen. ${ }^{106}$

Zu den Leistungsvoraussetzungen zählt vor allem, dass die angebotenen Waren und Dienstleistungen sich von denen des Wettbewerbs abheben und den Verwendern der entsprechenden Leistungen einen Vorteil bieten müssen. Diese Vorteile können sich an die direkten Kunden oder aber auch an die nachfolgenden Marktstufen richten. Vorteile können beispielsweise durch relativ niedrige Preise, eine

106 Kleinaltenkamp et al. 2012, S. 156; Hillebrand/Biemans 2011, S. 74. 
leichtere (Weiter-) Verarbeitung oder durch qualitative Eigenschaften entstehen, die den Abnehmern in ihren Absatzmärkten wiederum Wettbewerbsvorteile verschaffen. Diese Vorteile müssen in den Augen der Abnehmerstufen signifikant sein und eine Relevanz für den Kauf der entsprechenden Leistung aufweisen. ${ }^{107}$ Zudem müssen diese Wettbewerbsvorteile auch in irgendeiner Form auf den nachfolgenden Marktstufen kommuniziert werden können. Herausforderungen können darin bestehen, die nachgelagerten Abnehmer davon zu überzeugen, dass der geschaffene Wettbewerbsvorteil auf die Eigenschaften des Vorprodukts des betreffenden Anbieters zurückzuführen ist, insbesondere dann, wenn das Produkt viele Weiterverarbeitungsstufen durchläuft, die vorteilhafte Wirkung sich aber erst beim Letztverwender zeigt. ${ }^{108}$ Erschwert wird dies zusätzlich dann noch dadurch, dass viele Vorprodukte nach der Weiterverarbeitung (z.B. in der Chemischen Industrie) überhaupt nicht mehr als Komponente identifizierbar sind und ihre vorteilhafte Wirkung nur noch schwer nachweisbar bzw. dieser bestimmten Komponente zurechenbar ist. Einfacher gestaltet es sich, auf die Vorteile des Vorprodukts hinzuweisen, wenn dieses durch Markennamen oder Markenzeichen des Anbieters auf nachfolgenden Marktstufen wiedererkannt werden kann. Im besten Fall gelingt es, ein Ingredient Branding zu implementieren und das Produkt des Kunden auch noch mit der Marke des Vorproduktes zu versehen. ${ }^{109}$ Die Kunden der Kunden können mit der Marke eine bestimmte Produktqualität assoziieren und Vertrauen zum Anbieter aufbauen. ${ }^{110}$ In einigen Fällen spezialisierter oder technologisch anspruchsvoller Produkte erscheint der Einsatz von Mehrstufigem Marketing unumgänglich. Insbesondere, wenn der direkte Kunde wenig Wissen über die technischen Eigenschaften der Vorprodukte besitzt, diese aber für die Weiterverarbeitung oder die Letztverwender von entscheidender Bedeutung sind, erscheint eine direkte Kontaktaufnahme mit den Kunden der Kunden bzw. den Letztverwendern der betreffenden Leistung erfolgsversprechend.

Zu den Unternehmensvoraussetzungen, die für den Einsatz von Mehrstufigem

${ }^{107}$ Kleinaltenkamp et al. 2012, S. 156; Hillebrand/Biemans 2011, S. 74.

108 Vgl. Kleinaltenkamp et al. 2012, S. 156; Hillebrand/Biemans 2011, S.74.

${ }^{109}$ Vgl. Kleinaltenkamp et al. 2012, S. 158.

${ }^{110}$ Vgl. Norris 1992, S22; Nufer/Kocher 2010, S. 7. 
Marketing erfüllt sein müssen, zählt insbesondere, dass der Anbieter ein ausreichendes Markt- und Marketing-Knowhow auf den nachgelagerten Marktstufen besitzen muss. ${ }^{111}$ Ein Anbieter, der versucht, seine Produkte mehrstufig zu vermarkten, muss wissen, wie er Informationen über nachgelagerte Abnehmer sammelt und diesen auch relevante Informationen über seine angebotenen Produkte zur Verfügung stellt. ${ }^{112}$ Möglichkeiten, sich fehlendes Wissen anzueignen, bestehen für Anbieter darin, im Rahmen von Kooperationen mit direkten Kunden auch Marktinformationen auszutauschen. Alternativ kann ein Anbieter auf nachgelagerten Märkten eigene Marktforschungsaktivitäten durchzuführen. ${ }^{113}$ Anbieter, die sich dazu entscheiden, ihre Marketingaktivitäten bis auf die Stufe der Endkonsumenten auszudehnen, benötigen entsprechendes Wissen über die dortigen „Gesetze des Marktes“ und über den Einsatz von Massenmedien, die im Business-to-Business-Marketing eher selten zum Einsatz kommen. ${ }^{114}$ Durch die Ansprache der Kunden des Kunden bzw. der Endkonsumenten kann es einem Anbieter gelingen, diese Marktstufen von den Vorzügen der eigenen Produkte zu überzeugen und mögliche Vorbehalte gegen deren Einsatz oder Konsum zu entkräften. ${ }^{115}$

Eine weitere Voraussetzung für den Einsatz von Mehrstufigem Marketing betrifft die Akzeptanz dieser Maßnahmen bei den direkten Kunden. ${ }^{116}$ Diese können Widerstände gegen den Einsatz der betreffenden Produkte oder sogar gegen die Anwendung von Mehrstufigem Marketing selbst entwickeln. Widerstände können aus einer möglichen Einschränkung der eigenen Marketingaktivitäten oder aus dem Fehlen von Anreizen, die betreffenden Produkte einzusetzen, resultieren. ${ }^{117}$ Durch die Wahl einer kooperativen Form des Mehrstufigen Marketings, also unter Einbeziehung der direkten Kunden, kann es allerdings gelingen, etwaige

\footnotetext{
111 Vgl. Kleinaltenkamp et al. 2012, S. 158 f.

112 Vgl. Hillebrand/Biemans 2011, S.75.

113 Vgl. Hillebrand/Biemans 2011, S.74 f.

${ }^{114}$ Vgl. Kleinaltenkamp et al. 2012, S. 159 f.

115 Vgl. Hillebrand/Biemans 2011, S.75.

${ }^{116}$ Vgl. Kleinaltenkamp et al. 2011, S. 44.

${ }^{117}$ Vgl. Kleinaltenkamp et al. 2012, S. 160.
} 
Widerstände auf dieser Marktstufe zu überwinden. ${ }^{118}$ Besteht auf Seiten der direkten Kunden allerdings kein Interesse, in eine mehrstufige Strategie eingebunden zu werden, so kann ein Anbieter dennoch unter Umständen versuchen, aufgrund seiner Machtposition ein mehrstufiges Marketingkonzept zu implementieren. ${ }^{119}$

\subsubsection{Möglichkeit der praktischen Ausgestaltung der einzelnen Marketinginstrumente}

Anbieter müssen entscheiden, ob und wie eine mehrstufige Marketingstrategie implementiert werden soll, um bei den indirekten Kunden eine Präferenz für die eigenen Produkte zu schaffen und so einen Nachfrage-Sog auszulösen.

Bei der Ausgestaltung einer mehrstufigen Marketingstrategie muss ein Anbieter entscheiden, ob er einzelbetrieblich oder mit dem unmittelbaren Kunden kooperativ die nachfolgenden Marktstufen ansprechen möchte. Nicht alle unmittelbaren Kunden sind dazu bereit. Entscheidend ist daher für den Anbieter, genügend Wissen über die nachgelagerten Märkte und entsprechendes MarketingKnowhow zu besitzen, um eine marktstufenübergreifende Marketingstrategie umsetzen zu können. In erster Linie kann dieses Wissen durch entsprechende Marktforschung und direkten Kontakt mit den indirekten Kunden erworben werden.

Neben dem Wissen über nachgelagerte Märkte gehört es auch zu einer mehrstufigen Marketingstrategie, dieses Wissen in marketingpolitischen Maßnahmen umzusetzen. Dazu stehen einem Unternehmen grundsätzlich alle Marketinginstrumente zur Verfügung, die auch im einstufigen Marketing zur Anwendung kommen. Diese Instrumente sind vornehmlich die Vertriebs-, Preis-, Kommunikations- und Produktpolitik.

Bei der Ausgestaltung von kooperativen mehrstufigen Marketingaktivitäten wird eine gewisse Abstimmung der Vertriebsaktivitäten als Voraussetzung angesehen. Eine indirekte Vertriebsstrategie, bei der die Leistungen zunächst an Händler

${ }^{118}$ Vgl. Homburg/Wilczek 2014, S. 15.

${ }^{119}$ Vgl. Kleinaltenkamp et al. 2012, S. 161. 
oder Weiterverarbeiter verkauft werden, bildet die Grundlage mehrstufiger Marketingaktivitäten. ${ }^{120}$ Preisliche Absprachen mit den unmittelbaren Kunden sind kartellrechtlich nur eingeschränkt möglich, so dass der Fokus auf der Produktund Kommunikationspolitik liegt.

In der Praxis lässt sich beobachten, dass Anbieter von Produkten bestimmte Spezifikationen direkt beim Endkunden bzw. Anwender des finalen Produkts erfragen, und so dazu beitragen, dass der direkte Kunde wiederum eine Leistung mit möglichst hohem Kundenvorteil anbieten kann. Grundsätzlich besteht die Aufgabe einer mehrstufigen Produktpolitik darin, die Bedürfnisse der Abnehmer auf allen relevanten Marktstufen zu berücksichtigen. Diese Ausrichtung kann dann auch über die Wünsche der unmittelbaren Kunden und Endkunden hinausgehen und Abnehmer auf Zwischenstufen einschließen. Wesentliche Bedeutung kommt im produzierenden Gewerbe neben dem Produkt selbst auch den produktbegleitenden Dienstleistungen zu. Insbesondere bei komplexen Produkten können sich nämlich Anbieter auch durch Beratungsleistungen hinsichtlich der Produktgestaltung oder Unterstützung bei Forschung und Entwicklung differenzieren. Bei essentiell wichtigen Komponenten z.B. in der Steuerungstechnik von Anlagen und Maschinen kann ein Anbieter etwa durch Kosten- und Wirtschaftlichkeitsanalysen oder Mitarbeiterschulungen beim Endkunden auf sich aufmerksam machen und seine Leistungen bewerben. Auch im After-Sales-Bereich stehen einem Anbieter produktpolitische Maßnahmen auf nachgelagerten Märkten wie etwa der technische Kundendienst oder Garantieleistungen zur Verfügung. Ähnliches gilt auch, wenn der direkte Abnehmer ein Händler ist. Hier können Anbieter und Händler durch ein abgestimmtes Sortiment und eine geographisch differenzierte Ansprache und den Austausch von Endkundeninformationen Werte beim Endkunden schaffen. ${ }^{121}$

Denkbare mehrstufige kommunikationspolitische Maßnahmen beinhalten zum Großteil eine Verknüpfung von Werbeinhalten und -botschaften über mehrere Marktstufen hinweg, so dass den Abnehmern auf den verschiedenen Marktstufen

${ }^{120}$ Vgl. Kleinaltenkamp et al. 2012, S. 165.

${ }^{121}$ Vgl. Simpson et al. 2001, S. $127 \mathrm{ff}$. 
in konsistenter Art und Weise die Leistungsvorteile kommuniziert werden können. ${ }^{122}$ Zudem können Anbieter ihren direkten Kunden Werbezuschüsse gewähren bzw. insbesondere im Handel Angebotsaktionen unterstützen. Eine Abstimmung zwischen den PR-Abteilungen von Anbieter und direktem Kunden kann ebenfalls bei relevanten Pressemitteilungen vorgenommen werden. Oftmals sind solche Maßnahmen im Automobilbereich zwischen OEMs und den Herstellern von wesentlichen Fahrzeugkomponenten wie etwa Getrieben zu beobachten.

Verkaufsförderung durch den Besuch von Messen, Schulungen der Verkäufer des direkten Kunden hinsichtlich der Eigenschaften der Anbieterleistungen oder direkte persönliche Beratungsgespräche auf nachgelagerten Marktstufen sind in der Praxis häufig anzutreffende mehrstufige kommunikationspolitische Maßnahmen. Die für den Endkunden jedoch am deutlichsten wahrnehmbare Form von abgestimmten mehrstufigen Kommunikationsmaßnamen ist das Ingredient Branding. Hierbei können nicht mehr beobachtbare Produkteigenschaften durch Markennamen oder Markenzeichen des Anbieters auf dem Endprodukt wieder kenntlich gemacht werden. Da dies allerdings einen starken Eingriff in die Gestaltungsfreiheit der einstufigen Marketingaktivitäten des direkten Kunden bedeutet und eher von langfristiger, strategischer Zusammenarbeit geprägt ist, sind solche Maßnahmen in der Praxis je nach Branche nur selten zu beobachten. ${ }^{123}$ Da in Unternehmen, die Mehrstufiges Marketing betreiben, in der Regel einstufige und mehrstufige Maßnahmen gleichzeitig zum Einsatz kommen, müssen diese Maßnahmen koordiniert werden, um einen möglichst hohen Nutzen zu generieren. Insgesamt gilt es, bei der Anwendung von Mehrstufigem Marketing die einzelnen Maßnahmen, die auf den indirekten Kunden abzielen, mit denen, die auf die direkten Kunden gerichtet sind, innerhalb des Anbieterunternehmens abzustimmen. Beim kooperativen Vorgehen ist zusätzlich eine Abstimmung zwischen Anbieter und direktem Kunden notwendig. ${ }^{124}$

122 Vgl. Kleinaltenkamp et al. 2012, S. 170.

123 Insbesondere in der chemischen Industrie wird häufig auf ein Ingredient-Branding verzichtet, da Zulieferer und Kunden in anderen Märkten als Konkurrenten auftreten. In der Konsumgüterindustrie hingegen ist diese Marketingmaßnahme häufiger zu beobachten. Die Konsumenten nehmen Ingredient-Branding oftmals als zusätzlichen Qualitätsindikator wahr (vgl. Desai/Keller 2001).

124 Vgl. Kleinaltenkamp et al. 2012, S. 170. 
Mehrstufiges Marketing muss sich aber nicht ausschließlich auf direkte und indirekte Kunden richten. In komplexeren Marktstrukturen können auch Drittparteien, die auf Produktgestaltung und Absatz einen wesentlichen Einfluss haben, Berücksichtigung finden. LIU/GUPTA zeigen, wie Medikamentenhersteller ihre Werbemaßnahmen auf Patienten bzw. Konsumenten ausrichten und dies zu insgesamt mehr Arztbesuchen führt. Weder Ärzte noch Patienten kaufen allerdings direkt die betreffenden Medikamente, sondern verschreiben und konsumieren diese lediglich. ${ }^{125}$ Den Krankenkassen kommt hierbei eine entscheidende Einflussnehmerrolle zu, da sie die Medikamente letztlich in den Leistungskatalog aufnehmen und auch bezahlen müssen. Krankenkassen werden daher auch von Herstellern medizinischer Analysegeräte (z.B. Wärmebildkameras zur Messung der Körpertemperatur) direkt angesprochen, da die Kaufentscheidung durch Praxen und Krankenhäuser stark davon abhängt, ob der geplante Einsatz der betreffenden Geräte auch über die Krankenkasse abgerechnet werden kann. Bei den beschriebenen oder anderen neuartigen Produkten bzw. neuen Einsatzgebieten bestehender Produkte sind in der Praxis auch Abstimmungen von verschiedenen Anbietern hinsichtlich ihrer Marketingmaßnahmen in der Praxis zu beobachten. Nicht selten werden bestimmte Produktkategorien, Verwendungs- oder Fertigungstechniken auf den verschieden Stufen der Wertschöpfungskette gemeinsam von verschiedenen Herstellern beworben. ${ }^{126}$ So eine horizontale kooperative Marketingstrategie soll aber in dieser Arbeit nicht näher beleuchtet werden. $\mathrm{Zu}$ dieser Art der Marketingkooperation sei auf die Arbeit von BUCKLIN/SENGUPTA verwiesen. ${ }^{127}$

Eine umfassende mehrstufige Marketingstrategie, die den Einsatz verschiedener Marketinginstrumente beinhaltet, zeigt Abbildung 2 graphisch. Hierbei sind sowohl direkte und indirekte Kunden als auch Drittparteien Ziel der Marketingmaßnahmen.

\footnotetext{
125 Vgl. Liu/Gupta 2011.

${ }^{126}$ Vgl. Kleinaltenkamp et al. 2012, S. 164.

${ }^{127}$ Vgl. Bucklin/Sengupta 1993.
} 


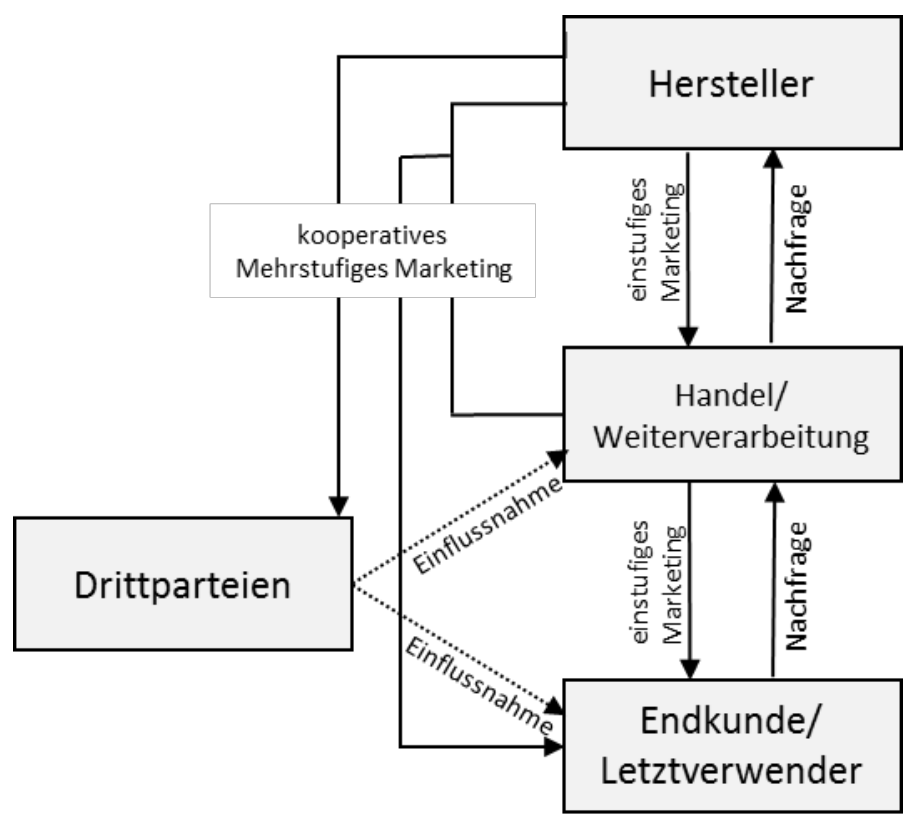

Abbildung 2: Mehrstufiges Marketing

(Quelle: in Anlehnung an Kleinaltenkamp et al. 2012, S. 150)

Die vorangegangenen Ausführungen haben gezeigt, dass der Markt eines Unternehmens im B2B-Sektor unter anderem nachgelagerte Marktstufen und einflussnehmende Drittparteien umfasst, die im Rahmen einer Marketingstrategie differenziert angesprochen werden sollten. Die auf konzeptionellen Arbeiten und einzelnen empirischen Beobachtungen fußenden Erkenntnisse geben erste Hinweise auf die verschiedenen Marktgruppen als separaten Bestandteile des Markts und sollen zur Beantwortung der Forschungsfrage 1 in Abschnitt 5.3.1 durch weitere empirische Ergebnisse ergänzt werden. Dabei sollen gegebenenfalls darüber hinaus noch weitere relevante Marktgruppen identifiziert werden, um ein vollständiges Bild über die Bestandteile des Markts im B2B-Bereich zu erhalten. 


\section{Diskussion der theoretischen Grundlage}

\subsection{Bestimmung der theoretischen Grundlage}

In diesem Kapitel wird die theoretische Grundlage vorgestellt, auf der diese Arbeit basiert und mit der das Konstrukt einer umfassenden für den Business-toBusiness-Bereich gültigen Marktorientierung und seine Auswirkungen theoretisch fundiert werden.

Dazu sind grundsätzlich verschiedene ökonomische oder auch verhaltenswissenschaftliche Ansätze möglich. Verschiedene theoretische Ansätze können nicht nur unabhängig voneinander zur Erklärung von Phänomenen genutzt werden. Es ist auch möglich, der Idee des theoretischen Pluralismus folgend, verschiedene Fragestellungen durch den Einsatz sich ergänzender und nicht widersprechender Theorien zu beantworten. ${ }^{128}$

Die vorliegende Arbeit bezieht sich zur Beantwortung der gesetzten Fragestellungen auf den ressourcenbasierten Ansatz (Resource-Based View), den kompetenzbasierten Ansatz (Competence-Based View) sowie den relationalen Ansatz (Relational View).

Die genannten Theorien sollen das theoretische Konstrukt der Marktorientierung fundieren und begründen, wie der Erfolg von Unternehmen einzelbetrieblich und kooperativ erklärt werden kann. Dabei dienen die ressourcen- und kompetenzbasierten Ansätze im Speziellen der Fundierung der Konzeptualisierung von Marktorientierung und begründet auch die Herleitung der Unternehmensaktivitäten und Erfolgsvariablen. Der relationale Ansatz wird dazu herangezogen, die Zusammenarbeit von Unternehmen und den daraus resultierenden Einfluss auf die Wettbewerbsvorteile eines Unternehmens zu erklären.

Zweck dieses Abschnitts ist dabei nicht, die Theorien in ihrer Vollständigkeit

${ }^{128}$ Vgl. Feyerabend 1965; zum theoretischen Pluralismus in der Marketingforschung vgl. Fritz 1995, S. 27. 
darzustellen. Vielmehr beschränkt sich die Darstellung auf jene Aspekte, die für die Zielsetzung dieser Arbeit von Relevanz sind.

\subsubsection{Resource-Based View und Competence-Based View}

Der ressourcenbasierte Ansatz, auch Ressource-Based View of the Firm - kurz Resource-Based View (RBV) genannt, entstammt der strategischen Managementlehre und hat zum Ziel zu erklären, wie Unternehmen aufgrund ihrer unterschiedlichen Ausstattung an Ressourcen unterschiedlich starke Wettbewerbspositionen erlangen, die letztlich für den Unternehmenserfolg entscheidend sind. ${ }^{129}$

Zunächst war der RBV nur als Ergänzung der industrieökonomischen Konzepte gedacht und stützte sich auf die Arbeiten von CAVES und PORTER, gilt allerdings heute als eigenständige Theorie. ${ }^{130}$ Der RBV unterscheidet sich von den industrieökonomischen Konzepten vor allem in der Hinsicht, dass er grundsätzlich von einer Unterschiedlichkeit der Unternehmen einer Branche ausgeht, die vor allem durch eine unternehmensspezifische Ausstattung an Ressourcen begründet wird. ${ }^{131}$ Laut RBV ist die unterschiedliche Ausstattung der Unternehmen mit Ressourcen dadurch bedingt, dass Ressourcen nur unzureichend mobil bzw. zwischen den Firmen nur eingeschränkt handelbar und innerhalb einer Branche heterogen verteilt sind. ${ }^{132}$ Durch die heterogene Verteilung der Ressourcen kann laut RBV ein Unternehmen durch seine firmenspezifischen, einzigartigen Ressourcen dauerhafte Wettbewerbsvorteile erlangen, die es von anderen Unternehmen innerhalb der Branchen absetzen und einen positiven Einfluss auf dessen Unternehmenserfolg ausüben. ${ }^{133}$

Damit unterscheidet sich der RBV deutlich von der bis dahin verbreiteten Theorie des Market-Based View, der die Leistungsunterschiede von Unternehmen mit äußeren Markt- und Umwelteinflüssen begründet und somit nur bedingt geeignet

\footnotetext{
${ }^{129}$ Vgl. Barney 1986a, S. 1239; Barney 1991.

130 Vgl. Caves 1980; Porter 1980.

131 Vgl. Connor 1991; Bresser 2010, S. 68 und die dort angegebene Literatur.

132 Vgl. Barney 1991, S. 101. Vgl. Capron/Hulland 1999 zur Immobilität von Ressourcen.

133 Vgl. Barney 1991; Mahoney/Pandian 1992, S. 365.
} 
ist, Unterschiede innerhalb derselben Branche zu erklären. ${ }^{134}$ Im Rahmen des RBV hingegen ermöglicht die spezifische Ausstattung mit Ressourcen dem Unternehmen, Wettbewerbsvorteile in zweierlei Hinsicht zu erreichen. Zum einen kann das Unternehmen seine Leistungen im Vergleich zu den Wettbewerbern kostengünstiger bzw. effizienter herstellen und anbieten. Diese Effizienzsteigerung kann auch als Anbietervorteil verstanden werden, da sich der Anbieter hinsichtlich des Einsatzes von Produktionsfaktoren und Produktionsprozessen einen Vorteil gegenüber seinen Konkurrenten verschafft. Ermöglicht werden diese Anbieter- oder Kostenvorteile bspw. durch bestimmte Prozesstechnologien, die Unternehmensgröße und den etwaigen Zugang zu kostengünstigen Produktionsfaktoren, so dass große Anbieter sog. Economies of Scale nutzen können. ${ }^{135}$ Zum anderen kann das Unternehmen seine Ressourcen so einsetzen, dass es in der Wahrnehmung der Kunden bessere Leistungen als der Wettbewerb anbietet und somit die Kundenwünsche möglichst effektiv erfüllen kann. Solche Kundenvorteile können durch den Einsatz von Ressourcen wie Forschungs- und Entwicklungskompetenz oder auch über die Reputation der Produkte oder des Unternehmens selbst entstehen.

Die obigen Ausführungen zeigen, wie weit der Begriff der Ressourcen gefasst und was alles darunter verstanden werden kann. Dies liegt nicht zuletzt daran, dass der Begriff der Unternehmensressource in der wissenschaftlichen Literatur uneinheitlich gebraucht wird. So werden Ressourcen zum Teil als Auflistung verschiedener Unternehmensattribute beschrieben, die von strategischer Bedeutung sind: „all assets, capabilities, organizational processes, firm attributes, information, knowledge, etc. controlled by a firm that enable the firm to conceive of and implement strategies that improve its efficiency and effectiveness. " ${ }^{136}$ Von anderen Autoren wird der Begriff der Ressourcen noch deutlich weiter verstanden und es werden darunter alle Stärken und Schwächen eines Unternehmens bzw.

\footnotetext{
${ }^{134}$ Vgl. Porter 1980; sowie zusammenfassend KUß ET AL. (2007, S. 60). Vgl. auch Amit/Shoemaker 1993 für einen Ansatz, der eine interne Ressourcenbetrachtung und eine externe Umweltanalyse miteinander verbindet.

${ }^{135}$ Vgl. Grant 1991, S. 118; Kuß et al. 2007, S. 58.

${ }^{136}$ Barney 1991.
} 
alle Vermögenswerte subsumiert, die dem Unternehmen semi-dauerhaft zur Verfügung stehen. Dabei wird lediglich zwischen materiellen und immateriellen Vermögenswerten unterschieden. ${ }^{137}$

Unter materiellen Ressourcen wird dabei alles Handhabbare eines Unternehmens verstanden, das buchhalterisch erfassbar und somit vom Unternehmen auch wieder veräußerbar ist. $\mathrm{Zu}$ den materiellen Ressourcen können unter anderem Grundstücke, Gebäude, Anlagen, Maschinen, Rohmaterialien sowie Halb- und Fertigerzeugnisse gezählt werden. Die immateriellen Ressourcen bestehen aus sehr abstrakten Werten wie Unternehmenskultur, Wissen und Fähigkeiten, aber auch aus etwas greifbareren Werten wie z.B. Reputation, Marken oder Patenten. ${ }^{138}$ Eine etwas feinere Untergliederung als lediglich in materielle und immaterielle Ressourcen nimmt BARNEY vor, indem er die Ressourcen eines Unternehmens in „financial, physical, human, and organizational assets“ aufteilt. ${ }^{139}$ Noch differenzierter unterscheidet HUNT, indem er Ressourcen noch in die zusätzlichen Kategorien der rechtlichen, informationellen und relationalen Ressourcen untergliedert. Der Autor hebt dabei hervor, dass Ressourcen, allen voran die Beziehungen zu Kunden, nicht von einem Unternehmen im engeren Sinne besessen werden. Vielmehr ist für ein Unternehmen bei solchen Ressourcen lediglich der Zugang und eine bestimmte Verfügungsgewalt entscheidend, um daraus einen strategischen Wettbewerbsvorteil ziehen zu können. ${ }^{140}$ GRANT unterscheidet bei der Klassifikation von Ressourcen zwischen den Effekten, die die Ressourcen auf das Unternehmen und dessen Umfeld ausüben. Die durch die Ressourcennutzung verursachten Markteintrittsbarrieren, Monopolstellungen, Verhandlungspositionen sowie Kosten- und Differenzierungsvorteile stehen dabei im Mittelpunkt. ${ }^{141}$ Einen gänzlich anderen Klassifikationsansatz wählt WERNERFELT, wenn er Ressourcen danach klassifiziert, inwiefern sie für das Unternehmen handelbar

${ }^{137}$ Vgl. Wernerfelt 1984), S. 172. Auch BARNEY (2001, S. 54) nahm später eine ähnliche Sichtweise ein und vereinfachte in einer Rückblende seines Artikels von 1991 seine eigene Definition von

Ressourcen: „resources are the tangible and intangible assets a firm uses to choose and implement its strategies."

138 Vgl. Hunt/Morgan 1995, S. 8.

${ }^{139}$ Barney 1995, S. 50.

${ }^{140}$ Vgl. Hunt 2000, S. 128. 
bzw. frei disponierbar sind. ${ }^{142}$ Eine dahingehende Unterscheidung treffen auch FREILING ET AL. Die Autoren betrachten jene Produktionsfaktoren als sog. Assets, die am Markt frei verfügbar sind und daher von jedem Unternehmen erworben werden können. Ressourcen entstehen den Autoren zufolge hingegen erst, wenn diese Produktionsfaktoren im Unternehmen selbst durch verschiedene Prozesse zu Firmenspezifika werden. Diese so aufgewerteten Ressourcen können dann nicht mehr frei am Markt gehandelt werden und besitzen zudem auch keinen oder zumindest einen deutlich geringeren Wert außerhalb des ursprünglichen Unternehmens. ${ }^{143}$

Die zahlreichen existierenden Klassifikationen insbesondere von nichtphysischen Ressourcen stellen allerdings nicht immer überschneidungsfreie Konzepte dar. VILLALONGA schlägt daher vor, der Einfachheit halber nur nach tangiblen und intangiblen Ressourcen zu unterscheiden. ${ }^{144}$ Zusammenfassend kann daher festgehalten werden, dass Ressourcen materielle oder immaterielle Attribute darstellen, über die ein Unternehmen frei verfügen kann.

Auch unter der Bedingung, dass ein Unternehmen seine Ressourcen zielgerichtet einsetzen kann, führt nicht der Einsatz jeder Ressource dazu, dass sich ein Unternehmen auch strategisch von seinen Wettbewerbern absetzen kann. Die Ressourcen, die einem Unternehmen ermöglichen, einen Wettbewerbsvorteil zu erlangen, müssen zusätzlich bestimmten Kriterien genügen, die sowohl BARNEY als auch PETERAF ausführlich beschreiben und die unter dem englischen Akronym VRIN (valuable, rare, in-imitable, non-substitutable) bzw. VRIO ${ }^{145}$ zusammengefasst werden können: ${ }^{146}$

- Ressourcen müssen insoweit wertvoll sein, als sie dem Unternehmen einen Wert stiften können hinsichtlich Effizienz- oder Effektivitätssteigerung. Zum

\footnotetext{
${ }^{141}$ Vgl. Grant 1991, S. 118.

142 Vgl. Wernerfelt 1989, S. 6 .

${ }^{143}$ Vgl. Freiling et al. 2008b, S. 1151.

${ }^{144}$ Vgl. Villalonga 2004, S. 206.

${ }^{145}$ Das VRIO-Konzept kann als Weiterentwicklung des VRIN-Konzepts betrachtet werden und betont eine Kompetenzperspektive. Durch den Organisationsaspekt wird die Bedeutung der unternehmerischen Fähigkeiten berücksichtigt, das Potenzial der Ressourcen voll auszuschöpfen (vgl. Gersch et al. 2014, S. 168).

${ }^{146}$ Vgl. Barney 1991; 1995; Peteraf 1993.
} 
Zwecke der Effizienzsteigerung werden hier insbesondere „low-cost manufacturing skills“ und zum Zwecke der Effektivitätssteigerung die Maßgabe „exploit market opportunities“ angeführt. ${ }^{147}$

- Ressourcen müssen einer gewissen Knappheit unterliegen und dürfen auch nicht unbeschränkt erweitert werden können, da andernfalls jeder Wettbewerber ebenfalls über diese Ressourcen verfügen könnte. Somit würden sie keinen Wettbewerbsvorteil für ein einzelnes Unternehmen erzeugen können.

- Ressourcen dürfen nicht in Gänze imitierbar sein. Ansonsten könnten auch die Wettbewerber sich diese Ressourcen ohne große Mühe beschaffen, und somit würde wiederum für kein Unternehmen einer Branche ein Wettbewerbsvorteil entstehen.

- Ressourcen dürfen nicht substituierbar sein. Das bedeutet, dass es für eine Ressource keine strategisch äquivalente Alternative geben darf, die selbst nicht einer gewissen Knappheit oder Nicht-Imitierbarkeit unterliegt. Andernfalls könnten fehlende oder schlecht ausgebildete Ressourcen leicht ersetzt werden, so dass die vermeintlichen wettbewerbsstrategischen Ressourcen keinem Unternehmen zum Vorteil gereichen würden.

- Zusätzlich zu diesen Eigenschaften sollte ein Unternehmen in der Lage sein, die Wettbewerbspotentiale seiner Ressourcen durch eine unterstützende Organisation voll auszuschöpfen.

Einige der strategisch relevanten Ressourcen können auf Märkten erworben werden, für andere hingegen besteht diese Möglichkeit nicht und sie können nur im Unternehmen selbst entwickelt werden. ${ }^{148}$ Insbesondere für buchhalterisch schwierig zu bestimmende Vermögenswerte wie Reputation, Händlerloyalität oder Kundenvertrauen existieren in der Regel keine Märkte. Ebenso verhält es sich mit Werten und Fähigkeiten, die von den Mitarbeitern erst durch „learning on the job“" verinnerlicht werden können. ${ }^{149}$ Auch das im Unternehmen verfügbare Wissen über Märkte und Unternehmensprozesse sowie dessen Kombination durch die Mitarbeiter des Unternehmens stellt eine erfolgskritische Ressource

${ }^{147}$ Barney 1991, S. 106.

148 Vgl. Helfat/Peteraf 2009, S. 93. 
dar. ${ }^{150}$ Nicht zuletzt stellt auch die Einstellung der Mitarbeiter in Form einer inneren Verbundenheit bzw. Commitment eine Ressource für dauerhaften Unternehmenserfolg dar. Solche mitarbeiterinhärenten Fähigkeiten und andere immaterielle Vermögenswerte stellen mitunter sogar die wichtigsten Ressourcen dar, da sie ohne größeren zeitlichen Verzug weder imitier- noch substituierbar sind. ${ }^{151}$ Oftmals sind an der Erstellung solch höchst intangibler Ressourcen mitunter zahlreiche Mitarbeitern und Abteilungen beteiligt, die ein Netz von sozialen Verflechtungen bilden. Die Erstellung selbst stellt zudem häufig einen langwierigen Prozess im Unternehmen dar, dessen Ergebnis als historisch gewachsen betrachtet werden kann. Somit bleibt der genaue Entstehungsprozess von intangiblen Ressourcen aufgrund der sozialen Komplexität und Historizität oftmals für Außenstehende teilweise oder gänzlich verborgen, was in einer schweren Imitierbarkeit resultiert. ${ }^{152}$ Physische Ressourcen werden hingegen als deutlich leichter imitierbar angesehen. Jedoch können sie oftmals nur durch die Kombination mit immateriellen Ressourcenbestandteilen wie bspw. sozialen Beziehungen und einer förderlichen Unternehmenskultur vollständig nutzbar gemacht werden. ${ }^{153}$ Es sind somit vornehmlich die intangiblen Ressourcen eines Unternehmens, die direkt oder indirekt in besonderem Maße zur Schaffung von Wettbewerbsvorteilen und Unternehmenserfolg beitragen. ${ }^{154}$

Der RBV ist vielfach als theoretische Grundlage zur Erklärung von realen Marktsituationen kritisiert worden. Darauf soll im Folgenden kurz eingegangen werden und aufgezeigt werden, wie die Heranziehung weiterer sich nicht widersprechender Theorien und eine sorgfältige Ausgestaltung von empirisch durchgeführten Studien in der Lage sind, die aufgeworfenen Kritikpunkte weitgehend zu berücksichtigen.

Als schwerwiegendsten Kritikpunkt am RBV führen PRIEM/BUTLER an, dass es sich bei dieser Theorie nach den von BARNEY aufgestellten Postulaten um

\footnotetext{
${ }^{149}$ Vgl. Dierickx/Cool 1989, S. 1505.

${ }^{150}$ Vgl. Kogut/Zander 1992; Grant 1996.

${ }^{151}$ Vgl. Grant 1991, S. 119. Dierickx/Cool 1989, S. 1507.

152 Vgl. Schreyögg/Conrad 2006, S. VII.

${ }^{153} \mathrm{Vgl}$. Hunt/Lambe 2000, S. 21.

${ }^{154}$ Vgl. Kaplan/Norton 2004; Villalonga 2004; Lopez 2006; Haanes/Fjeldstad 2000.
} 
eine tautologische Definition handele. ${ }^{155}$ Demnach führten wertstiftende und seltene Ressourcen zu Wettbewerbsvorteilen. Wenn aber Wertstiftung nur durch Effizienz- oder Effektivitätssteigerung erreichbar sei und ein Wettbewerbsvorteil sich nur in Form von erhöhter Effizienz oder Effektivität darstellen lasse, bleibe der aus dem RBV zu ziehende Erkenntnisgewinn fraglich. Um den Tautologievorwurf zu entkräften, schlagen LÜDEKE ET AL. vor, in empirischen Arbeiten bei der Operationalisierung darauf zu achten, dass die Indikatoren der einzelnen Konstrukte sorgfältig und vor allem unterschiedlich formuliert werden, um so eine eindeutige Unterscheidung von abhängigen und unabhängigen Variablen zu erreichen. SCHMIDT/KEIL hingegen schlagen vor, den Wert einer Ressource zu bestimmen, bevor durch diese Ressource Werte in Form von Marktleistungen geschaffen werden. Eine solche ex-ante Wertbestimmung resultiert demnach aus den Erwartungswerten unter Unsicherheit. ${ }^{156}$ Praktisch ist eine solche Bestimmung jedoch nur schwer durchzuführen und wird letztlich wesentlich von der Subjektivität der bewertenden Verantwortlichen im Unternehmen bestimmt. ${ }^{157}$ Auf diese Weise lässt sich zwar das Tautologieproblem theoretisch umgehen, es werden aber gleichzeitig praktische Probleme bei der ex ante Wertbestimmung der Ressource und der ex post generierten Output-Werte aufgeworfen.

Ein weiterer Kritikpunkt bezieht sich auf den statischen Charakter der Theorie. ${ }^{158}$ Der RBV dient der Erklärung von dauerhaften Wettbewerbsvorteilen, wobei aufgrund von dynamischen Umwelten und von sich kontinuierlich wandelndem Wettbewerb und Kundenerwartungen nicht damit zu rechnen ist, dass ein Unternehmen Vorteile im Wettbewerb dauerhaft erreichen kann, zumal auch in der Praxis die Imitation und Substitution von Ressourcen beobachtet werden kann. ${ }^{159}$ Die Nachahmung oder Substitution von strategischen Ressourcen ist, wenn auch nur mit einem gewissen zeitlichen Verzug, in fast allen Fällen möglich. Die obigen Ausführungen haben gezeigt, dass dies bei materiellen Ressourcen zudem

155 Vgl. Priem/Butler 2001.

156 Vgl. Schmidt/Keil 2013.

${ }^{157}$ Vgl. Foss et al. 2008; Kraaijenbrink et al. 2010.

${ }^{158}$ Vgl. Lockett/Thompson 2001; Priem/Butler 2001.

${ }^{159}$ Vgl. Bresser et al. 2000. 
noch leichter möglich ist als bei immateriellen. Kritisch zu hinterfragen ist ferner, inwiefern gerade die Imitation überhaupt ein Problem bei kurzen Produktlebenszyklen darstellt. Durch den Wegfall von Zugangsbarrieren und den schnellen Markteintritt von Wettbewerbern kann es sogar förderlich für ein Unternehmen sein, wenn dadurch Produkt- oder Marktstandards verbreitet werden. ${ }^{160}$ Um diese Kritikpunkte zu berücksichtigen, wäre es denkbar, die abhängige Variable nicht direkt als Wettbewerbsvorteil zu messen, sondern stattdessen den Erfolg eines bestimmten Produkts als Proxy von Wettbewerbsvorteilen zu ermitteln. Der Erfolg dieses Produkts ist dann nicht von dauerhafter Natur, sondern besteht maximal nur so lange, wie auch der Produktlebenszyklus dieses Produkts dauert. Zahlreiche empirische Studien, die auf dem RBV basieren, verwenden dieses Vorgehen und bedienen sich eines leichter messbaren Unternehmens- oder Produkterfolgs anstatt des eher abstrakt wirkenden Wettbewerbsvorteils als abhängiger Variable. ${ }^{161}$ Jedoch muss dabei beachtet werden, dass Erfolgsmaße und Wettbewerbsvorteile nicht immer identisch sind. ${ }^{162}$ Die auf diese Weise beobachteten Wettbewerbsvorteile sind somit nicht notwendiger Weise dauerhaft und können dadurch die Prämissen des RBV verletzen, der von einer dauerhaft überlegenen Stellung des Unternehmens im Wettbewerb ausgeht.

Ein weiterer Kritikpunkt am RBV ist eng mit der statischen Sicht der Theorie verbunden. In der wissenschaftlichen Literatur wird in Frage gestellt, dass der alleinige Besitz bzw. der Zugang zu Ressourcen Unterschiede im Unternehmenserfolg erklären kann. Vielmehr gehen zahlreiche Autoren davon aus, dass Ressourcen lediglich Potenziale darstellen, das Unternehmen aber darüber hinaus auch in der Lage sein muss, diese Ressourcen zielführend einzusetzen. Die Nutzung der Ressourcen ist wiederum nur durch bestimmte Fähigkeiten bzw. Kompetenzen möglich. Durch diese Kompetenzen kann das Unternehmen das Potenzial der Ressourcen ausschöpfen, indem es bewusst auf die Marktgegebenheiten eingeht. ${ }^{163}$ Aus diesen Überlegungen leitet sich der kompetenzbasierte Ansatz

\footnotetext{
${ }^{160}$ Vgl. Garud/Kumaraswamy 1993.

${ }^{161}$ Vgl. Newbert 2007, S. 126; Crook et a1. 2008, S. 1142.

162 Vgl. Coff 1999; Powell 2001, S. 875; Peteraf/Barney 2003, S. 313; Ketchen et al. 2007, S. 962.

${ }^{163}$ Vgl. Freiling 2004, S. 31.
} 
(Competence-Based View of the Firm - CBV) zur theoretischen Erklärung von Erfolgsunterschieden von Unternehmen ab.

Wie auch bei der Definition von Ressourcen gibt es allerding auch im CBV keine allgemeingültige Definition von Kompetenzen. Die dieser wissenschaftlichen Theorie zugehörigen Arbeiten setzen nämlich unterschiedliche Schwerpunkte bei der Erklärung von Kompetenzen, so dass das Verständnis von Kompetenzen bisweilen leicht variiert. ${ }^{164}$ Gemeinsamkeit besteht hingegen darin, dass der kompetenzbasierte Ansatz als Weiterführung des RBV angesehen werden kann. Der CBV sieht die Fähigkeiten eines Unternehmens als zentral an, die im Unternehmen verfügbaren Ressourcen auch zielgerichtet einzusetzen. Der Besitz bzw. der Zugang zu Ressourcen kann dieser Theorie zufolge zwar als Wurzel von Wettbewerbsvorteilen angesehen werden, vermag aber allein noch keine Wettbewerbsvorteile zu generieren. ${ }^{165}$ Kompetenzen sind jedoch nicht mit den Handlungen des Unternehmens gleichzusetzen, sondern befähigen lediglich das Unternehmen, auf die Kunden oder auf den Markt gerichtete Aktivitäten vorzunehmen. ${ }^{166}$ FREILING versteht darunter eine kreative Sichtweise auf neue Kundenlösungen und die Anpassung des eigenen Leistungsangebots an die Kundeanforderungen. Auch eine proaktive Berücksichtigung von noch nicht artikulierten Kundenwünschen mit dem Ziel, die eigenen Absatzmärkte frühzeitig zu formen, fällt darunter. ${ }^{167}$ Der CBV nimmt somit im Gegensatz zum RBV eine dynamische Sichtweise ein, nach der ein Unternehmen durch den Einsatz seiner Potenziale auf sich ändernde Marktgegebenheiten reagieren kann.

Ähnlich wie die Ressourcen innerhalb der Erklärungen des RBV unterliegen auch die Kompetenzen innerhalb des CBV einer starken Historizität und sozialen Komplexität. Demnach kann eine Kompetenz nur in einem bestimmten Kontext oder in einer bestimmten Situation als wettbewerbsstrategisch angesehen wer-

\footnotetext{
${ }^{164}$ Vgl. Pralahad/Hamel 1990 zum „core competence approach“; Langlois 1992 zum „capabilities approach”; Foss 1996 zur „competence perspective”; Teece et al. 1997 zum „dynamic capabilities approach”; Freiling 2004 zum „,competence-based view of the firm” bzw. zur „competence-based theory of the firm".

165 Vgl. Freiling 2004, S. 30.

166 Vgl. Duschek 2004, S. 613 f

${ }^{167}$ Vgl. Freiling 2004, S. 32.
} 
den. ${ }^{168}$ Bei der Generierung von Wettbewerbsvorteilen durch Kompetenzen wird der gemeinsame Einsatz verschiedener Ressourcen durch Koordination und Kooperation als entscheidend betrachtet, da eine Ressource in der Regel allein als dafür unzureichend angesehen wird. ${ }^{169}$ Große Bedeutung kommt dabei den einzelnen Mitarbeitern in unterschiedlichen Abteilungen und der Leitung des Unternehmens zu, die zur Schaffung von Wettbewerbsvorteilen ihre Aktivitäten untereinander abstimmen müssen. ${ }^{170}$

Auch PENROSE verdeutlichte die Notwendigkeit, Ressourcen zu kombinieren, um daraus Wettbewerbsvorteile ziehen zu können. Die Autorin formulierte in diesem Zusammenhang, dass es zwar die Ressourcen sind, die ein Unternehmen ausmachen und bezeichnete folglich die Unternehmung auch als Ressourcenbündel („Pool of Resources“), doch machte die Autorin auch deutlich, dass Ressourcen alleine lediglich ein Potential eines Unternehmens darstellten. ${ }^{171}$ Werte würden erst durch den Einsatz der Ressourcen bzw. der aus den Ressourcen stammenden „Services“ geschaffen werden. Dazu sei es auch notwendig, bestimmte Ressourcen mit anderen Ressourcen zu kombinieren, um einen möglichst großen Nutzen daraus zu ziehen. ${ }^{172}$

Der Idee des CBV nach entstehen Wettbewerbsvorteile gerade dann, wenn Unternehmen die Fähigkeiten besitzen, ihre Vermögenswerte oder andere Ressourcen durch organisationale Prozesse zielführend miteinander $\mathrm{zu}$ verbinden. ${ }^{173}$ Dabei sollten Ressourcen auch koordiniert in mehreren Anwendungsfeldern zum Einsatz kommen und zudem von den verschiedenen Bereichen innerhalb des Unternehmens gemeinsam genutzt werden. ${ }^{174}$ Ohne die Fähigkeit, Ressourcen sinnvoll einzusetzen oder verschiedene Ressourcen miteinander zu kombinieren, können Unternehmen folglich keinen Wettbewerbsvorteil erreichen. Die Fähigkeit der Ressourcenkombination wird im Ansatz der Dynamic Capabilities wei-

\footnotetext{
${ }^{168}$ Vgl. Freiling et al. 2008b, S. 1149.

169 Vgl. Grant 1991, S. 119.

${ }^{170}$ Vgl. Grant 1991, S. 122

${ }^{171}$ Vgl. Penrose 1959, S. 149.

172 Vgl. Penrose 1959, S. 25.

${ }^{173}$ Vgl. Day 1994, S. 38.

${ }^{174}$ Vgl. Prahalad/Hamel 1990, S. 81 f.
} 
terverfolgt. Nach diesem theoretischen Ansatz liegt der Fokus zur Erreichung von Wettbewerbsvorteilen auf der Ressourcenkombination. ${ }^{175}$ Die Fähigkeit, zielgerichtet und bewusst eine Ressourcenkombination zu schaffen, zu erweitern und anzupassen, wird darin als notwendige Vorstufe angesehen, um über Ressourcen, seien sie materieller oder immaterieller Art, einen Wettbewerbsvorteil zu erzielen. ${ }^{176}$

Wenngleich der CBV einige Kritikpunkte, die am RBV geäußert werden, insbesondere dessen mangelnde dynamische Sichtweise, kompensieren kann, so trifft das Argument der Tautologie auch auf den CBV zu. Schließlich geht auch der CBV davon aus, dass wertstiftende Kompetenzen zu Vorteilen im Wettbewerb im Sinne von Wertgenerierung über Effizienz- oder Effektivitätsvorteile führen. Um dieses definitorische Problem zu umgehen, führen FREILING ET AL. daher aus, dass weniger die erreichten Wettbewerbsvorteile als vielmehr die dauerhafte Wettbewerbsfähigkeit das vorrangige Erklärungsziel dieser Theorie bilden sollten. Darunter verstehen die Autoren die Fähigkeit, sich gegenüber Kunden, Wettbewerbern und Drittparteien im Markt zu behaupten, um den Fortbestand des Unternehmens zu sichern. ${ }^{177}$

\subsubsection{Relational View}

Um ihre Unternehmensziele zu erreichen, haben Unternehmen die Möglichkeit, Ressourcen, die ihnen nicht direkt zur Verfügung stehen, indirekt über Partnerschaften mit anderen Unternehmen zu nutzen. Oftmals ist es für Unternehmen essentiell wichtig, sich bei der Entwicklung und Vermarktung von Produkten auf neuen Märkten mit Partnerunternehmen verschiedener Marktstufen zusammenzuschließen. ${ }^{178}$ So kann es gelingen, die Ressourcen der Partnerunternehmen zu nutzen, daraus zu lernen und die eigenen Ressourcen ebenfalls möglichst effizient in eine Partnerschaft einzubringen.

Die gemeinsame Nutzung von Ressourcen und daraus erwachsende Wettbe-

\footnotetext{
175 Vgl. Teece et al. 1997; Eisenhardt/Martin 2000.

176 Vgl. Helfat et al. 2007, S. 4; Bresser 2010, S. 75.

177 Vgl. Freiling et al. 2008b, S. 1150.

178 Vgl. Varadarajan/Cunningham 1995, S. 285 f.; Roy/Sivakumar 2010.
} 
werbsvorteile mit dem Resource-Based View (RBV) zu erklären, wurde zwar in der Literatur unternommen. ${ }^{179}$ Dieses Vorgehen muss aber kritisiert werden, da der RBV klar darlegt, dass die Ressourcen, die zu Wettbewerbsvorteilen führen, vom Unternehmen selbst besessen oder zumindest vollständig kontrolliert werden müssen. ${ }^{180}$ Bei der Betrachtung von Netzwerken nach dem RBV versucht jedes Partnerunternehmen die Ressourcen der anderen Partner zu internalisieren, um letztlich aus der entstehenden Kooperationsrente einseitige Unternehmensvorteile zu ziehen. Dadurch scheint aber die langfristige Existenz von Kooperationen nicht gegeben. Der Blickwinkel des RBV richtet sich somit immer nur auf ein Unternehmen.

In der Praxis kann aber beobachtet werden, dass oftmals ein Unternehmen allein nicht in der Lage ist, in bestimmten Märkten Erfolge zu verzeichnen. Erst eine langfristige, partnerschaftliche Zusammenarbeit ermöglicht dann eine erfolgreiche Marktbearbeitung. LAVIE verweist in diesem Zusammenhang auf zahlreiche Studien, die zeigen, wie die Wettbewerbsvorteile eines Unternehmens von den Fähigkeiten und den Ressourcen von Partnerunternehmen abhängen können. Der Autor kommt zu dem Schluss, dass es schon für ein Unternehmen ausreichen kann, lediglich Zugang zu Ressourcen oder zumindest Zugang zum Nutzen der betreffenden Ressourcen zu haben, um Wettbewerbsvorteile zu generieren. ${ }^{181}$ So ein Zugang kann in verschiedenen Formen durch Unternehmenszusammenarbeit bzw. in Unternehmensnetzwerken erfolgen.

Der Relational View (RV) berücksichtigt diesen Zugang zu Ressourcen über Partnerunternehmen und kann als komplementäre Theorie zum RBV verstanden werden. Der RV setzt den Fokus weniger auf das einzelne Unternehmen als vielmehr auf die Netzwerkbeziehung von mindestens zwei rechtlich unabhängigen Organisationen. Im Gegensatz zum RBV, der die Quelle von Wettbewerbsvorteilen in der Ressourcenausstattung eines einzelnen Unternehmens sieht, geht der RV davon aus, dass Wettbewerbsvorteile aus der Zusammenarbeit von Unter-

\footnotetext{
${ }^{179}$ Vgl. Allred et al. 2011; Hult et al. 2008; Jap 1999; Varadarajan/Cunningham 1995.

${ }^{180}$ Vgl. Wernerfelt 1984, S. 172; Barney 1991, S. 101; Amit/Shoemaker 1993, S. 35.

${ }^{181}$ Vgl. Lavie 2006, S. 641.
} 
nehmen resultieren. ${ }^{182}$ Wettbewerbsvorteile entstehen dabei aus unternehmensübergreifenden Ressourcen, die der Unternehmensbeziehung immanent sind. Unternehmen erreichen nach dem RV eine der Konkurrenz überlegene Stellung insbesondere durch die Schaffung von beziehungsspezifischen Ressourcen, durch den Prozess des Wissensaustausches zwischen den Unternehmen, durch das Zusammenbringen von komplementären Ressourcen sowie durch eine effektive Netzwerksteuerung. ${ }^{183}$ Unter beziehungsspezifischen Ressourcen werden dabei alle Investitionen eines Unternehmens in die Beziehung zum Partnerunternehmen verstanden. Diese Investitionen können materieller und immaterieller Natur sein und beispielsweise aus der Schulung von Mitarbeitern oder der Anschaffung von Maschinen bestehen, die auf die Prozesse des Partnerunternehmens zugeschnitten sind. ${ }^{184}$ Auch ein Wissensaustausch erscheint für die Schaffung von Wettbewerbsvorteilen besonders wichtig, wenn über die verschiedenen Stufen der Wertschöpfungskette Wissen über Kundenwünsche, neue verfügbare Technologien und Arbeitsprozesse ausgetauscht wird und dies somit leichter zu innovativen Fertigungsprozessen oder Produkten führen kann. ${ }^{185}$ Die Kombination von komplementären Ressourcen bezieht sich auf Synergievorteile, die zwei Unternehmen erzielen können, wenn sie ihre jeweiligen Ressourcen zusammenbringen. Die Ressourcen können gemeinsam einen größeren Wettbewerbsvorteil erzielen, als dies für jedes Unternehmen individuell möglich gewesen wäre. ${ }^{186}$ Dies kann beispielsweise durch die Kombination von Reputation oder besonderem Marktwissen des einen Unternehmens und einer besonders effizienten Fertigungstechnik des anderen Unternehmens geschehen. ${ }^{187}$ Als weitere Quelle von Wettbewerbsvorteilen wird die effektive Netzwerksteuerung und -kontrolle angesehen. Da Wettbewerbsvorteile, die aus beziehungsspezifischen Investitionen resultieren, in der Regel nur solange für das einzelne Unternehmen und das Netzwerk Bestand haben, wie die Investitionen nicht durch Partnerunternehmen ausgenutzt

${ }^{182}$ Vgl. Dyer/Singh 1998, S. 661.

${ }^{183}$ Vgl. Dyer/Singh 1998, S. 662.

${ }^{184}$ Vgl. Dyer/Singh 1998, S. 662 f.

185 Vgl. Dyer/Singh 1998, S. 664 f.; Urban/von Hippel 1988, S. 569.

${ }^{186}$ Vgl. Dyer/Singh 1998, S. 666 f.

${ }^{187}$ Vgl. Oliver 1997, S. 707; Saxton 1997, S. 444 f. 
werden, müssen Mechanismen greifen, die ein opportunistisches Verhalten der Partner verhindern. Solche Mechanismen können in Form von Verträgen implementiert werden oder aus Vertrauen und direkten früheren Erfahrungen mit dem Partnerunternehmen resultieren. ${ }^{188}$

Da der von DYER/SINGH stark geprägte Relational View stark durch die Spezifika des damaligen Untersuchungsgegenstandes ${ }^{189}$ geprägt ist, haben sich insbesondere in neuerer Zeit Autoren mit den genauen Umständen beschäftigt, unter denen der RV als Theorie zur Erklärung von Unternehmenserfolg herangezogen werden kann. KOBAYASHI identifiziert in diesem Zusammenhang die geographische Dichte der kooperierenden Unternehmen als Erfolgsbedingung. Demnach müssen die Unternehmen geographisch dicht zusammen liegen, um die Kosten des Materialtransports möglichst niedrig zu halten und auf diese Weise einen Effizienzgewinn zu realisieren. ${ }^{190}$ Andere Autoren sehen die geographische Dichte bei der Entwicklung innovativer Produkte ebenfalls als förderlich an, da hierbei kurze Austauschwege für Informationen mit Unternehmen existieren, die ähnliche Ideen verfolgen. Hierbei rücken die Autoren im Besonderen regionale Technologie-Cluster in den Vordergrund ihrer Überlegungen. ${ }^{191}$ Um Effizienzvorteile zu sichern, müssen Unternehmen weiterhin die gesamten Kosten der Materialbeschaffung im Auge behalten, was nicht nur den letztlich gezahlten Preis betrifft, sondern die gesamten Kosten der Transaktion, d.h. von der Produktentwicklung beim Lieferanten bis hin zum fertigen Produkt des Unternehmens. Dabei kommt der beziehungsspezifischen Investition eine hohe Bedeutung zu, da hierdurch Prozesse leichter abgestimmt und schon in einem frühen Entwicklungsstadium Änderungen am Produkt oder Produktionsprozess zwischen den kooperierenden Unternehmen leicht durchgeführt werden können. ${ }^{192}$ Ferner stellt der intensive Austausch von Wissen zwischen den Mitarbeitern der Partner-

\footnotetext{
${ }^{188}$ Vgl. Dyer/Singh 1998, S. 669.

${ }^{189}$ Als empirische Basis der theoretischen Erläuterungen des RV diente der japanische Automobilhersteller Toyota mit seinem Netzwerk von Zulieferern. Durch den RV sollte insbesondere die bessere Wettbewerbsposition von Toyota im Vergleich zu den amerikanischen Automobilherstellern begründet werden.

${ }^{190}$ Vgl. Kobayashi 2014, S. 80.

${ }^{191}$ Vgl. Tracey et al. 2014, S. 11.
} 
unternehmen auf einer persönlichen Ebene als auch durch ein systematisches Austauschnetzwerk eine wichtige Bedingung dar, um Wettbewerbsvorteile zu erzielen. Hierbei stehen auch die Koordination von Entwicklungsschritten und die entsprechenden Verantwortlichkeiten im Vordergrund der Betrachtung. ${ }^{193}$ Beachtet werden sollte allerdings, dass zwar gerade größere Unternehmen einen Wissensaustausch aufgrund ihrer Machtstellung einfordern können, dass aber letztlich auch kleine Unternehmen von solch einem Wissensaustausch und transfer in Übereinstimmung mit dem RV profitieren können. ${ }^{194}$ Letztlich eignet sich der RV zur Erklärung von Unternehmenserfolg nur, wenn die betrachteten Kooperationen langfristig angelegt und die daraus entstehenden Produkte eher als komplex einzustufen sind. ${ }^{195}$ Technologisch einfache Produkte hingegen benötigen keine so weitreichende Abstimmung zwischen den Unternehmen. Dieser Punkt deckt sich mit der Bedingung für den erfolgreichen Einsatz von Mehrstufigem Marketing, nach der für eher technologisch anspruchsvolle Produkte eine gemeinsame Vermarktung zwischen Unternehmen und Kunde verstärkt in Betracht gezogen werden sollte (vgl. Abschnitt 2.2.2.2). Die obigen Ausführungen machen deutlich, dass der RV als theoretische Grundlage geeignet ist, den Erfolg von kooperierenden Unternehmen zu erklären, sofern die betrachteten Kooperationen die zuvor genannten Charakteristika aufweisen. Daneben existieren noch Bedingungen, die die zur Erklärung herangezogenen Ressourcen selbst betreffen. Wesentliches Merkmal des Relational Views ist nämlich, dass die in Unternehmenskollaborationen geschaffenen oder kombinierten Ressourcen genau wie beim RBV wertvoll, selten sowie schwer zu substituieren und imitieren sein müssen, um Wettbewerbsvorteile zu generieren. ${ }^{196}$ Insbesondere Ressourcen, die sich auf andere Mitglieder in einem Netzwerk beziehen, und zur Koordination notwendige Netzwerkfähigkeiten sind für Konkurrenten nur äußerst schwer zu imitieren. ${ }^{197}$ Solche Fähigkeiten verbinden zahlreiche Ressourcen unternehmens-

\footnotetext{
192 Vgl. Kobayashi 2014, S. 80 ff.

193 Vgl. Dyer/Nobeoka 2000.

${ }^{194}$ Vgl. Cai et al. 2013, S. 2065; Jones et al. 2014.

195 Vgl. Kobayashi 2014, S. 85.

${ }^{196}$ Vgl. Dyer/Singh 1998, S. 660.

197 Vgl. Hyvönen/Tuominen 2007, S. 424.
} 
übergreifend, die für das Gestalten von Kunden- und Händlerbeziehungen erfolgskritisch sind und beinhalten nicht zuletzt ein hohes Maß an immanentem Wissen, das sich nur über lange Zeitperioden entwickeln kann. ${ }^{198}$ Unternehmensübergreifende Fähigkeiten sind somit durch eine hohe Historizität und soziale Komplexität gekennzeichnet, die sie für ein Unternehmen strategisch bedeutsam werden lassen.

MESQUITA ET AL. zeigen anhand von Informationsaustauschprozessen in der Zuliefererindustrie im Maschinenbau, wie eng der RV mit dem RBV verflochten ist und wie mit dem jeweiligen theoretischen Ansatz bestimmte Arten von Wettbewerbsvorteilen erklärt werden können. ${ }^{199}$ Es zeigt sich, dass der Relational View zur Erklärung von Wettbewerbsvorteilen komplementär zum RBV herangezogen werden kann, wenn Ressourcen zur gemeinsamen Nutzung in eine Unternehmenspartnerschaft eingebracht oder dadurch erst generiert werden.

\subsection{Eignung der theoretischen Grundlagen zur Erklä- rung des Konstrukts der Marktorientierung und re- levanter Konsequenzen}

Das Phänomen einer kulturell begründeten Marktorientierung zeigt Eigenschaften, nach denen Marktorientierung sowohl als wettbewerbsstrategische Ressource als auch als Kompetenz eines Unternehmens betrachtet werden kann. Im Folgenden soll gezeigt werden, wie eine umfassende Marktorientierung, die neben Kunden und Wettbewerbern auch nachgelagerte Marktstufen und Drittparteien berücksichtigt, auf Basis des RBV, des CBV und des RV wettbewerbsstrategische Bedeutung für Unternehmen im B2B-Sektor besitzt.

Ressourcen und Fähigkeiten werden im Sinne des RBV dann als strategisch bedeutsam angesehen, wenn sie das Unternehmen befähigen, relativ zum Wettbewerb bessere Entscheidungen zu treffen. Diese strategischen Entscheidungen führen zu Wettbewerbsvorteilen, die wiederum zu einem höheren Unternehmens-

\footnotetext{
${ }^{198}$ Vgl. Day/Van den Bulte, 2002; Jarratt 2004.
}

${ }^{199}$ Vgl. Mesquita et al. 2008. 
erfolg beitragen. ${ }^{200}$ Inwiefern Marktorientierung wettbewerbsstrategische Wichtigkeit besitzt, kann anhand der Erfüllung der VRIN-Kriterien analysiert werden. Zunächst soll daher gezeigt werden, wie Marktorientierung zu einer verbesserten Wettbewerbsposition beitragen und somit als wertvoll bezeichnet werden kann. Anschließend soll die Seltenheit, Nicht-Imitierbarkeit und NichtSubstituierbarkeit betrachtet werden.

Dass Marktorientierung zu verbesserten Marktpositionen bzw. zu einem dauerhaften Wettbewerbsvorteil führt, wurde in zahlreichen Studien theoretisch erörtert und empirisch belegt. ${ }^{201}$ Die Fähigkeit, aus den gewonnenen und verarbeiteten Marktinformationen eine Strategie abzuleiten und somit auf Wettbewerber und Kundenverhalten zu reagieren, wird dabei besonders hervorgehoben. ${ }^{202}$ PORTER differenziert zwischen jenen Strategien, die Wettbewerbsvorteile in Form von Kostenvorteilen und solchen Strategien, die Wettbewerbsvorteile durch Differenzierungsvorteile generieren. ${ }^{203}$ Überlegenheit am Markt kann für ein Unternehmen somit nur daraus resultieren, dass es den Kunden seine Leistungen kostengünstiger anbietet oder Kundenwünsche besser bzw. effektiver erfüllt. In beiden Fällen bedeutet dies, dass ein Unternehmen in der Lage ist, für die Kunden einen größeren Wert als der Wettbewerb zu schaffen. HUNT/MORGAN erklären den Beitrag der Marktorientierung zum Unternehmenserfolg über den Effektivitätsvorteil folgendermaßen: „market orientation [...] would be a resource if it provided information that enabled a firm to produce [...] an offering well tailored to a market segment's specific tastes and preferences”. ${ }^{204}$ Durch relativ niedrigere Kosten kann ebenfalls eine überlegene Wettbewerbsposition erreicht werden. Dabei muss allerdings darauf geachtet werden, die Kosten, die der Analyse zugrunde gelegt werden, nicht nur auf die reinen Herstellungskosten zu beziehen, sondern zusätzlich Marketing- und After-Sales-Kosten einzubeziehen. ${ }^{205}$ Durch

\footnotetext{
200 Vgl. Ketchen et al. 2007, S. 962.

${ }^{201}$ Vgl. Kumar et al. 2011; Murray et al. 2011; Day 1994; Jaworski/Kohli 1993; Lado et al. 1992;

Kohli/Jaworski 1990; Day/Wensley 1988.

202 Vgl. Porter 2004; Ruekert 1992; Porter/Millar 1985.

203 Vgl. Porter 2004, S. 62; S. 119.

${ }^{204}$ Hunt/Morgan 1995, S. 11.

205 Vgl. Porter 2004, S. 62.
} 
das im Rahmen von Marktorientierung erworbene Wissen über Produkte, Preise und Strategien von Wettbewerbern sowie durch das Wissen über relevante Kundensegmente sollte es möglich sein, die Ausarbeitung von Kommunikationsstrategien und insbesondere Werbemaßnahmen kostengünstiger oder genau auf die Bedürfnisse der einzelnen Kundensegmente zuzuschneiden. ${ }^{206}$ Auch SLATER/NARVER sehen im Marktwissen den Schlüssel zu einem dauerhaften Wettbewerbsvorteil, indem dadurch einerseits Leistungen effektiver entwickelt, andererseits Menschen und Maschinen effizienter im Leistungserstellungsprozess $^{207}$ eingesetzt werden können. ${ }^{208}$

Oftmals werden in der Literatur aber nicht die Wettbewerbsvorteile im Sinne von Kunden- und Anbietervorteilen direkt gemessen. Es werden stattdessen hilfsweise Manifestationen von Effektivitäts- und Effizienzvorteilen anhand von interessierenden Messgrößen erhoben. JAWORSKI/KOHLI kommen bspw. in ihrer Studie zu dem Ergebnis, dass Marktorientierung eine wichtige Determinante des Unternehmenserfolgs ist. ${ }^{209}$ Unternehmenserfolg kann den Autoren zufolge als Ausdruck einer überlegenen Wettbewerbsposition verstanden werden. Als Ausbildung von Wettbewerbsvorteilen werden ebenfalls in der Literatur Produkt- und Servicequalität, Kostenkontrolle, Innovationskraft und Markteintrittsgeschwindigkeit genannt, die alle auf Marktorientierung zurückgeführt werden können. ${ }^{210}$ NARVER/SLATER stellen einen signifikanten Einfluss der Marktorientierung auf die Unternehmensprofitabilität fest. Die Autoren sehen dies ebenfalls als Indiz für eine überlegene Wettbewerbsposition aufgrund einer marktorientierten Unternehmensausrichtung. ${ }^{211}$ LUO ET AL. heben die Bedeutung von Marktorientierung für den Erfolg eines Unternehmens besonders hervor und bezeichnen

\footnotetext{
${ }^{206}$ Vgl. Hunt/Lambe 2000, S. 27; Glazer 1991, S. 11.

${ }^{207}$ Vgl. Kleinaltenkamp 2007, Sp. 1041-1042 zu den Dimensionen des Leistungserstellungsprozesses.

208 Vgl. Slater/Narver 2000, S. 122.

${ }^{209}$ Vgl. Jaworski/Kohli 1993, S. 64

${ }^{210}$ Vgl. Slater 1996, S. 82 ff.

${ }^{211}$ Vgl. Narver/Slater 1990, S. 32. Vgl. ebenfalls Hunt/Morgan 1995, S. 11 f. zu dieser Argumentationslogik.
} 
Marktorientierung daher auch als „key strategic resource“. ${ }^{212}$

Die dargestellten Möglichkeiten für Unternehmen, sich durch Marktorientierung Erfolge im Markt zu sichern, legen nahe, dass es sich auch für Wettbewerber lohnen würde, sich auf diese Art strategisch auszurichten, was entscheidende Implikationen für den Aspekt der Seltenheit von Marktorientierung als wettbewerbsstrategische Ressource hätte. Ein Blick in die Praxis zeigt zwar, dass Marktorientierung heutzutage in immer mehr Unternehmen bewusst praktiziert wird, dennoch gibt es weiterhin Unternehmen, die bisher nicht an der Maxime der Marketingkonzeption festhalten und ihre Unternehmensprozesse entsprechend nicht am Markt orientieren. ${ }^{213}$ Insgesamt ergibt sich somit kein eindeutiges Bild hinsichtlich der Seltenheit von Marktorientierung, sofern diese als Unternehmensausrichtung auf Kunden und Wettbewerber aufgefasst wird.

KUMAR ET AL führten vor diesem Hintergrund eine Langzeitstudie zur Marktorientierung durch und gingen der Frage nach, ob eine ausgeprägte Marktorientierung einem Unternehmen dauerhaft Wettbewerbsvorteile böte. Die Autoren wollten wissen, ob auch Konkurrenten über die Zeit ihre Unternehmensaktivitäten auf die Kunden ausrichten könnten und sich somit der Wettbewerbsvorteil verlöre. Marktorientierung würde dadurch an Seltenheit verlieren und wäre nicht mehr als knappe, strategische Ressource zu betrachten. Durch die zunehmende Verbreitung von Marktorientierung schlagen die Autoren daher vor, dass Firmen ihr Verständnis von Marktorientierung erweitern sollten, um wieder einzigartige Alleinstellungsmerkmale im Wettbewerb zu erreichen („companies must continuously identify new dimensions of this construct to distinguish themselves“). ${ }^{214}$ Als mögliche Erweiterung sehen die Autoren unter anderem die Berücksichtigung von multi-kulturellen Eigenschaften vor dem Hintergrund der zunehmenden wirtschaftlichen Globalisierung. Die Empfehlungen der Autoren berücksichtigen somit zwar nur in abstrakter Weise das weitere Marktumfeld eines Unternehmens, geben aber dennoch einen Hinweis auf die Wichtigkeit von sozio-kulturellen

212 Luo et al. 2005, S. 54. Die Autoren bewerten ebenfalls „entrepreneurial orientation“ und „innovative capability“ als strategische Ressourcen.

213 Vgl. Kumar et al. 2011.

214 Kumar et al. 2011, S. 28. 
Einflüssen und Einflussnehmern für den Erfolg eines Unternehmens. Die Empfehlungen der Autoren für eine Erweiterung der Marktorientierung beziehen sich jedoch nicht auf die Betrachtung nachgelagerter Marktstufen. KLEINALTENKAMP ET AL. hingegen empfehlen, das Verständnis von Marktorientierung genau dahingehend $\mathrm{zu}$ erweitern und zeigen, dass eine mehrstufige Marktorientierung in Unternehmen in der Geschäftspraxis bisher eher selten verbreitet ist. Die Autoren stellen fest, dass die Geschäftspraxis, die indirekten Kunden konkret mit in die Geschäftsplanung einzubeziehen, nicht weit verbreitet ist. Wenn ein Unternehmen allerdings eine solche mehrstufige Marktorientierung verfolgt, geschieht dies oftmals mit dem Ziel, eine strategisch günstige Wettbewerbsposition einzunehmen. ${ }^{215}$ Eine erweiterte, umfassende Marktorientierung, die das weitere Marktumfeld und nachgelagerte Marktstufen berücksichtigt, würde somit eine knappe Ressource darstellen und somit wettbewerbsstrategische Implikationen besitzen.

Vor Imitation durch Wettbewerber kann die Marktorientierung eines Unternehmens durch ihre Historizität und soziale Komplexität geschützt werden. Kompetenzen und Ressourcen gelten als nicht oder nur unter hohem finanziellen Aufwand zu imitieren, wenn sie innerhalb des Unternehmens historisch gewachsen und durch das Mitwirken der Mitarbeiter sozial höchst komplex gestaltet sind. Für Wettbewerber bleibt dadurch oftmals der genaue Zusammenhang zwischen den einzelnen Bestandteilen der Ressourcen und dem daraus resultierenden Wettbewerbsvorteil verborgen. ${ }^{216}$ Die Eigenschaften der Historizität und sozialen Komplexität treffen insbesondere auf immaterielle Ressourcen und Fähigkeiten zu, so dass sie als nicht vollständig imitierbar gelten und auch als Hauptgründe für die Erreichung von Wettbewerbsvorteilen gesehen werden können. ${ }^{217}$ Marktorientierung nimmt als solch eine immaterielle Ressource und Kompetenz somit einen besonderen Stellenwert bei der Erreichung von Wettbewerbsvorteilen ein. Dabei werden sowohl organisationale als auch kulturelle Aspekte der Markt-

\footnotetext{
${ }^{215}$ Vgl. Kleinaltenkamp et al. 2011, S. $43 \mathrm{f}$.

${ }^{216}$ Vgl. Barney 1991, S. 107; Barney 2001, S. 50; Srivastava et al. 1998, S. 6; Hunt/Morgan 1995, S. 13; Hunt 1997, S. 437.

${ }^{217}$ Vgl. Hult et al. 2008, S. 528.
} 
orientierung als hilfreich bei der Generierung von Wettbewerbsvorteilen angesehen. ${ }^{218}$ PARASURAMAN resümiert in diesem Zusammenhang, dass eine auf den Kunden ausgerichtete Unternehmenskultur von Wettbewerbern nicht kopiert werden könne, da es sich hierbei nicht zuletzt um die Persönlichkeit einer Firma handele. $^{219}$ Die Kompetenz, Marktwissen in verschiedenen Abteilungen und zwischen verschiedenen Unternehmen nutzbar zu machen sowie Beziehungen innerhalb des eigenen Unternehmens und mit externen Unternehmen aufzubauen, kann als sozial höchstkomplex angesehen werden. Da solche Beziehungen zu Vertriebspartnern und Kunden historisch wachsen, können sie nicht oder nur unter großen Anstrengungen von Konkurrenten imitiert werden. ${ }^{220}$

Als weiteres Kriterium darf eine Ressource, mit der ein dauerhafter Wettbewerbsvorteil generiert werden soll, nicht durch eine oder mehrere andere Ressourcen ersetzbar sein, mit denen dieselbe strategische Ausrichtung erreicht werden kann. ${ }^{221}$ Marktorientierung als Teil einer Unternehmenskultur wächst, wie erläutert, historisch und ist tief im Unternehmen verwurzelt, so dass sie auch nicht ohne weiteres durch andere Ressourcen ersetzt werden kann. ${ }^{222}$ Wenn ein Unternehmen im Rahmen seiner Marktorientierung in den Besitz von Kundeninformationen gelangt, die es ihm ermöglichen, auf die individuellen Bedürfnisse des Kunden zugeschnittene Leistungen anzubieten, wird es für den Wettbewerb sehr schwierig sein, eine andere Ressource nutzbar zu machen, die eine ähnliche Leistungsindividualisierung ermöglicht. ${ }^{223}$ Auch die Kompetenz, das Wissen über verschiedene Aspekte des Marktes zu kombinieren und in neue Angebote zu transferieren ist nur äußerst schwer zu ersetzen. Aufgrund der Schwierigkeiten, äquivalenten Ersatz für Marktorientierung zu finden, bleibt für Konkurrenten vielfach nur die Möglichkeit, entweder selbst eine entsprechende Marktorientierung langwierig aufzubauen oder das gesamte betreffende Unternehmen samt

${ }^{218}$ Zu den organisationalen Aspekten vgl. Day 1994; Hult et al. 2005, S. 1175; Morgan et al. 2003, S. 309. Zu den kulturellen Aspekten vgl. Barney 1995, S. 59; Hunt/Lambe 2000, S. 20.

${ }^{219}$ Vgl. Parasuraman 1987, S. 46.

${ }^{220}$ Vgl. Barney 1992, S. 45; Srivastava et al. 1998, S. 6 f.

${ }^{221}$ Vgl. Barney 1991, S. 111.

${ }^{222}$ Vgl. Barney 2001, S. 50.

${ }^{223}$ Vgl. Srivastava et al. 1998, S. 7; vgl hierzu auch Minculescu 2013, S. 41. 
seiner Ressourcen und Kompetenzen zu akquirieren. ${ }^{24}$ Dies wirft allerdings zahlreiche Problem auf, die damit verbunden einhergehen, eine zunächst fremde Unternehmenskultur zu übernehmen bzw. im übernommenen Unternehmen fremde kulturelle Elemente zu implementieren.

In den vorangegangenen Ausführungen wurde im Wesentlichen die Wertgenerierung von Marktorientierung aus der Perspektive des direkten Kunden betrachtet. Für die Bestimmung der wertstiftenden Eigenschaft einer Ressource oder Kompetenz ist allerdings nicht immer nur der direkte Kunde entscheidend. In Märkten, die durch eine abgeleitete Nachfrage charakterisiert sind, gilt vielmehr „the customers of the final product determine the value of the resources”. ${ }^{225}$ Ein umfassend marktorientiertes Unternehmen müsste demnach, um Werte zu schaffen, Leistungen anbieten, die in bestimmtem Maße auch auf die Bedürfnisse von nachfolgenden Marktstufen zugeschnitten sind. Vor dem Hintergrund der abgeleiteten Nachfrage in mehrstufigen Märkten erscheint eine Berücksichtigung von Kundenwünschen nachgelagerter Marktstufen als effektivitätssteigernd. Indem das Unternehmen die Bedürfnisse seiner indirekten Kunden effektiv befriedigt, könnte das Unternehmen eigene Wettbewerbsvorteile als Funktion von Kundenvorteilen auf nachgelagerten Märkten erzielen. ${ }^{226}$

Ebenso kann ein auch auf nachfolgende Marktstufen ausgerichtetes Unternehmen Effizienzvorteile erzielen, wenn es gelingt, Marketing- und Serviceaktivitäten kostengünstiger zu gestalten als der Wettbewerb. Eine Möglichkeit, dies zu erreichen, ist, diese Tätigkeiten mit der direkt nachfolgenden Marktstufe abzustimmen und die damit verbundenen Kosten aufzuteilen. Das Unternehmen könnte insbesondere bei einer kooperativ ausgestalteten Form des Mehrstufigen Marketings bspw. das nötige Markt- und Marketing-Knowhow durch den direkten Kunden beschaffen bzw. das in den jeweiligen Unternehmen schon vorhandene Wissen teilen. Das Potenzial des in der Wertschöpfungskette vorhandenen Wissens könnte insgesamt durch eine mehrstufig ausgerichtete Marktorientierung besser ausgeschöpft und Kundenbedürfnisse effektiver befriedigt sowie die Kosten der gesam-

\footnotetext{
${ }^{224}$ Vgl. Capron/Hulland 1999, S. 42; Hunt/Morgan 1995, S. 8.

225 Peteraf/Bergen 2003, S. 1038.
} 
ten Wertschöpfungskette zudem gesenkt werden. ${ }^{227}$

In Bezug auf die Imitationsmöglichkeiten einer mehrstufig ausgeprägten Marktorientierung kann festgehalten werden, dass diese im Vergleich zu einer einstufigen Marktorientierung noch deutlich sozial komplexer und somit nur unvollständig von Wettbewerbern zu imitieren ist. Eine mehrstufig ausgerichtete Marktorientierung macht nämlich zusätzlich zu einer Interaktion und sozialen Verbindung mit den direkten Kunden noch eine weitere Ausrichtung auf die nachgelagerten Kunden erforderlich. Gleiches gilt für eine Marktorientierung, die darüber hinaus auch solche Individuen und Organisationen einschließt, die den Absatz bei den direkten Kunden und in nachgelagerten Marktstufen beeinflussen können.

In einer mehrstufigen Marktkonstellation, in der auch Wettbewerber aus anderen Marktstufen als der eigenen im Stande sind, den indirekten Kunden Lösungen anzubieten, ist eine genaue Kenntnis der betreffenden Stufen und des Marktumfeldes von großer Bedeutung. Insbesondere in technologisch dynamischen bzw. innovativen Märkten, wo auch bisher unbekannte Wettbewerber durch den Einsatz von verschiedenen Ressourcen dieselben Kundenprobleme zu lösen vermögen, benötigt ein Anbieter eine sehr gute Marktkenntnis über existierende wie auch potentielle Wettbewerber. ${ }^{228}$ Marktorientierung als strategische Ressource scheint hier nicht durch andere Ressourcen ersetzbar zu sein, mit denen die gleichen Ziele erreicht werden können. Sollte dennoch ein Substitut gefunden werden, würde eine umfassende Marktorientierung als Ressource an Wert verlieren und keinen dauerhaften Wettbewerbsvorteil mehr generieren können. ${ }^{229}$ Im Sinne des CBV ist jedoch nicht unbedingt die Fähigkeit Wissen zu sammeln und aufzubereiten oder das Marktwissen selbst als Ressource für den Erfolg des Unternehmens entscheidend. Der Erfolg beruht vielmehr auf einer Ausschöpfung des damit verbundenen Potenzials durch die zielgerichtete Nutzung dieses Wissens und anderer Ressourcen. In Anlehnung an das VRIO-Konzept kommt der organisationalen Koordination von Ressourcen zur Potenzialausschöpfung eine

${ }^{226}$ Vgl. Günter 1997, S. 218 ff.

${ }^{227}$ Vgl. Cachon/Fisher 2000.

${ }^{228}$ Vgl. Srivastava et al. 2001, S. 794 f.

${ }^{229}$ Vgl. Peteraf/Bergen 2003, S. 1038. 
wesentliche Bedeutung zu. Marktorientierung kann als Bestandteil einer übergeordneten Unternehmenskultur als so ein koordinatives Element betrachtet werden, das die formalen und informalen Unternehmensstrukturen zur Weiterentwicklung von Ressourcen und zur letztlichen Potenzialnutzung unterstützt. ${ }^{230}$ MENGUC/AUH, die ebenfalls auf die gesamtunternehmerische, kulturelle Eigenschaft von Marktorientierung fokussieren, verbinden Marktorientierung mit wertschaffenden Unternehmensaktivitäten. Die Autoren konstatieren, dass im Unternehmen gesammeltes Marktwissen erst im Zusammenspiel mit Innovationsorientierung, also dem Willen und der Fähigkeit auch neue, die Kundenwünsche erfüllende Produkte zu entwickeln, sein volles Potential zur Schaffung von Kundenwerten und zur Steigerung des Unternehmenserfolgs entfalten kann. ${ }^{231}$ Für die strategische Bedeutung von Marktorientierung bleiben somit die Potenzialnutzung und insbesondere die Reaktionsfähigkeit auf Marktbedürfnisse entscheidend. Marktorientierung kommt aber nicht nur bei der Reaktion auf Marktbedürfnisse sondern gerade auch bei der Formung des Marktes große Bedeutung bei. Da der CBV davon ausgeht, dass Märkte nicht statisch sind oder als gegeben betrachtet werden können, sondern ein Unternehmen darin in einem gewissen Rahmen gestalterisch tätig werden kann, müssen die Fähigkeiten, die durch Marktorientierung koordiniert eingesetzt werden, auch eine solche proaktive Ausrichtung berücksichtigen. ${ }^{232}$ Diese Marktgestaltung kann sich sowohl auf die Abnehmer der eigenen Unternehmensleistung als auch auf Einflussnehmer im Markt beziehen. Bei der Berücksichtigung von Einflussnehmern spielt dann insbesondere die Ansprache und Beeinflussung der Politik und anderer „Standardsetzer“ eine wichtige Rolle, um die Eckpfeiler der eigenen Märkte so zu formen, dass die eigenen Potenziale im Wettbewerb möglichst gewinnbringend eingesetzt werden können. ${ }^{233}$

Neben der aktiven Marktprägung zur Verbesserung der Nutzungsmöglichkeiten

\footnotetext{
${ }^{230}$ Vgl. Freiling et al. 2008a, S. 7.

${ }^{231}$ Vgl. Menguc/Auh 2006; vgl. zur Verbindung von Markt- und Innovationsorientierung auch Deshpandé/Farley 1993; Reichmann 2010.

${ }^{232}$ Vgl. Freiling et al. 2008a, S. 6; Freiling 2004, S. 32.

${ }^{233}$ Vgl. Gersch et al. 2010, S. 2.
} 
des Unternehmenspotenzials kommt Marktorientierung weiterhin auch eine wichtige Rolle bei der reaktiven und proaktiven Wertgenerierung für Kunden. Die kulturbasierte Eigenschaft von Marktorientierung begünstigt die Nutzung des im Unternehmen vorhandenen Wissens und fördert die im Wettbewerb erforderliche Reaktionsfähigkeit des Unternehmens. ${ }^{234}$ Dabei spielen nicht zuletzt auch der Austausch und die Kombination von Marktinformationen und anderen Ressourcen als Teil einer kulturell im gesamten Unternehmen verankerten Marktorientierung eine entscheidende Rolle. HULT ET AL. vertreten in ihrer Arbeit hingegen den Standpunkt, dass es weniger die Kultur als vielmehr nur die Aktivitäten des Unternehmens sind, über die Werte für die Kunden generiert werden. Die Autoren schreiben: „customers do not purchase a firm's goods and services simply because the firm has a particular type of culture“. ${ }^{235}$ NARVER/SLATER bringen diese beiden Standpunkte zusammen und machen in diesem Zusammenhang klar, dass marktorientierte Unternehmensaktivitäten bei der Schaffung von Kundenwerten zwar eine wesentliche Rolle spielen, aber nicht von einer dahinterstehenden Unternehmenskultur losgelöst betrachtet werden dürfen. Wenn Marktorientierung als strategisch bedeutsam betrachtet werden soll, muss den Autoren zufolge immer auch eine kulturelle Komponente von Marktorientierung vorhanden sein: ,,[i]f a market orientation were simply a set of activities completely disassociated from the underlying belief system of an organization, then whatever an organization's culture, a market orientation could easily be implanted by the organization at any time. But such is not what one observes." 236

Als kulturelle Komponente durchzieht Marktorientierung somit das gesamte Unternehmen und sorgt dafür, dass das Wissen über Kundenbedürfnisse und Marktgegebenheiten sowie weitere im Unternehmen verfügbare Ressourcen zielgerichtet kombiniert und darauf aufbauend neue Produkte entwickelt und angeboten werden. Auf diese Weise sorgt Marktorientierung dafür, das im Unternehmen verfügbare Potenzial besser auszuschöpfen.

Die Fähigkeiten, mit denen das Wissen zielgerichtet gewonnen und anschließend

${ }^{234}$ Vgl. Dickson 1996, S. 104; Ngo/O’Cass 2012, S. 862 f.

${ }^{235}$ Hult et al. 2005, S. 1174. 
genutzt werden kann, sind vielfältig. Als strategisch bedeutsam und somit in engem Zusammenhang mit Wettbewerbsvorteilen stehend identifizieren VORHIES/MORGAN insbesondere Fähigkeiten zur Ressourcenkombination, die den verschiedenen Aufgabenfeldern des Marketings zugerechnet werden. ${ }^{237}$ Bei den genannten Aufgabenfeldern handelt es sich um solche Fähigkeiten, die das Unternehmen in die Lage versetzen, auf sich ändernde, geäußerte und latente Kundenwünsche dynamisch zu reagieren. ${ }^{238}$ Eines dieser Aufgabenfelder ist der klassische Marketing-Mix mit den Bestandteilen der Produktentwicklung, der Preis-, Distributions- bzw. Vertriebs- und Kommunikationspolitik. ${ }^{239}$ Weitere Aufgabenfelder stellen das Management von Marktinformationen sowie die Marketing-Planung dar. Diese beiden Aufgabenfelder beinhalten Fähigkeiten zur Sammlung, unternehmensinternen Weiterverarbeitung und Nutzung von Kunden-, Wettbewerbs- und Umweltdaten. Weiterhin wird darunter die Fähigkeit verstanden, Marketingstrategien so zu planen, dass die unternehmensspezifischen Ressourcen optimal die Bedingungen des Marktes erfüllen. ${ }^{240}$ Der letzte Aufgabenbereich betrifft die Marketingimplementierung. Hierunter werden Fähigkeiten verstanden, die beabsichtigten Marketingstrategien auch tatsächlich umzusetzen. $^{241}$

Diese genannten Aufgabenbereiche des Marketings werden in zahlreichen Studien mit dem Unternehmenserfolg in Verbindung gebracht. Sie stehen mit Marktorientierung in sehr engem Verhältnis bzw. bilden vielfach Bestandteile verschiedener Definitionen von Marktorientierung. ${ }^{242}$ Marktorientierung kann somit als die Kompetenz betrachtet werden, die das Unternehmen in die Lage versetzt, Marktwissen durch eine geeignete Koordination und Kombination von Fähigkeiten und anderen Ressourcen wettbewerbsstrategisch einzusetzen und dabei das Potenzial des gesamten Unternehmens bestmöglich auszuschöpfen.

\footnotetext{
236 Narver/Slater 1998a, S. 235

237 Vgl. Vorhies/Morgan 2005, S. 82.

${ }^{238}$ Vgl. Morgan et al. 2009, S. 910.

239 Vgl. Day 1994; 2011; Vorhies/Morgan 2003; Dutta et al. 1999; Dutta et al. 2003; Weitz/Jap 1995; Shapiro et al. 1997; McKee et al. 1992.

${ }^{240}$ Vgl. Morgan et al. 2003. Für eine ausführliche Behandlung des Themas vgl. Kuß et al. 2007.

241 Vgl. Noble/Mokwa 1999.
} 
Strategische Wettbewerbsvorteile entstehen oftmals aber nicht nur durch die einoder mehrstufige Marktausrichtung und das Handeln eines einzelnen Unternehmens. Im Einklang mit dem Relational View wird ein Anbieter vielfach erst in die Lage versetzt, Werte zu generieren, wenn er mit anderen Unternehmen eng zusammenarbeitet. ${ }^{243}$ Dabei nimmt der Informationsaustausch über den Markt und insbesondere über nachgelagerte Markstufen eine herausragende Stellung ein. ${ }^{244}$ Aber auch die gemeinsame Produktentwicklung kann von großer Bedeutung sein, wenn sie zu einem erhöhten Nutzen bei den Kunden oder nachgelagerten Kunden führt. ${ }^{245}$ In Bezug auf eine marktstufenübergreifende Generierung von Kundenwerten schreiben BAKER ET AL., dass Anbieter durch die enge Zusammenarbeit mit Unternehmen der unmittelbar folgenden Marktstufe, die ebenfalls marktorientiert agieren, zusammen größeren Kundennutzen für den Endnutzer stiften können, als dies für jedes Unternehmen alleine möglich wäre. Auf diese Weise kann es dem Anbieter gelingen, seinen Wettbewerbsvorteil weiter auszubauen. ${ }^{246}$ Auch POWELL ET AL. bestätigen in einer Studie, die in der Biotechnologieindustrie durchgeführt wurde: „when knowledge is broadly distributed and brings a competitive advantage, the locus of innovation is found in a network of interorganizational relationships.” ${ }^{247}$ Letztlich führt die Unternehmenszusammenarbeit zu größerem Kundennutzen beim Endnutzer, wenn Marketing- und Produktentwicklungsaktivitäten zwischen den verschiedenen Marktteilnehmern und Marktstufen koordiniert werden. Auf Anbieterseite können auf diese Weise ebenfalls Vorteile entstehen. So können beispielsweise marktstufenübergreifende Marktinformationen effizienter von mehreren Unternehmen gesammelt und die Kosten der Informationssammlung und Aufbereitung zwischen den kooperierenden Unternehmen geteilt werden. Dies würde nach PLINKE eine Effizienzsteigerung und somit einen Anbietervorteil darstellen, der sich letztlich wiederum in einer

242 Vgl. Vorhies/Morgan 2005, S. 90; Narver/Slater 1990; Kohli/Jaworski 1990; Rueckert 1992.

243 Vgl. Srivastava et al. 2001; Day/van den Bulte, 2002; Martin/Grbac, 2003, Hyvönen/Tuominen 2007; Allred et al. 2011.

244 Vgl. Dyer/Singh 1998, S. 665; Azuma 2005, S. 52.

245 Vgl. Ragatz et al. 1997, S. 191; Slater 1996, S. 84 f.

${ }^{246}$ Vgl. Baker et al. 1999, S. 54.

247 Powell et al. 1996, S. 119. 
verbesserten Wettbewerbsposition widerspiegelt. ${ }^{248}$ Zusätzlich bietet eine Kooperation den Vorteil, dass die kooperierenden Unternehmen jeweils ihre spezialisierten Fähigkeiten voll nutzen können. ${ }^{249}$ SLATER/NARVER bestätigen empirisch, dass die kooperative Form der Informationsbeschaffung im Gegensatz zu verschiedenen anderen Formen mittelbar die stärkste Verbindung zur Generierung eines Kundenvorteils aufweist. Gerade im Rahmen mehrstufiger Märkte erscheinen eine kooperative Marketingstrategie und eine abgestimmte Beschaffung von Marktinformationen sinnvoll. Für die praktische Ausgestaltung bedeutet dies in der Regel, dass dasjenige Unternehmen die in der gesamten Wertschöpfungskette benötigten Marktinformationen am effizientesten beschaffen kann, welches sich direkt vor der Marktstufe der Endkunden befindet. ${ }^{250}$ Analoges gilt, wenn eine mehrstufige Unternehmensausrichtung nicht auf die Endkunden sondern eine vor den Endkunden liegende nachgelagerte Marktstufe abzielt. Der RV bietet somit über die in Kooperation generierten Unternehmensrenten die theoretische Grundlage zur Erklärung von Wettbewerbsvorteilen, die aus der unternehmensübergreifenden Koordination und Verrichtung von Unternehmenstätigkeiten zur Erfüllung von Kundenwünschen entstehen.

Marktorientierung beinhaltet als Ressource, wie dargestellt, nicht nur die Sammlung und Aufbereitung von Marktwissen, sondern koordiniert auch als Kompetenz die Aktivitäten, welche dafür notwendig sind, um aus diesem Wissen neue Produkte zur Befriedigung von Kundenwünschen auf unterschiedlichen Marktstufen zu entwickeln. Marktorientierung ist daher mit der Innovativität des Unternehmens und der unternehmensübergreifenden Zusammenarbeit zur Entwicklung von Leistungen für nachgelagerte Marktstufen koordinativ eng verbunden. Marktorientierung bietet somit die Grundlage, für nachgelagerte Marktstufen durch die Nutzung und Kombination von Ressourcen und Fähigkeiten eines Anbieters und seines direkten Kunden Werte zu schaffen.

Die vorangegangenen Ausführungen haben gezeigt, dass die theoretischen Ansätze des ressourcenbasierten und des kompetenzbasierten Ansatzes sowie der rela-

\footnotetext{
${ }^{248}$ Vgl. Plinke 2000, S. 77.

${ }^{249}$ Vgl. Slater/Narver 2000.
} 
tionale Ansatz geeignet erscheinen, das Konstrukt einer umfassenden Marktorientierung im B2B-Kontext theoretisch zu durchdringen. Darüber hinaus liefern die gewählten Ansätze einen Beitrag zur theoretischen Begründung einzelbetrieblicher und kooperativer Konsequenzen von Marktorientierung. Diese einzelbetrieblichen und kooperativen Unternehmensaktivitäten sollen in Abschnitt 4.4 nach der Konzeptualisierung des zentralen Konstrukts der umfassenden Marktorientierung näher erläutert werden.

${ }^{250}$ Vgl. Glazer 1991, S. 9. 


\section{Bestimmung und Zusammenhang der un- tersuchten Konstrukte}

\subsection{Allgemeines zur Konzeptualisierung und Operationa- lisierung von Konstrukten}

Marktorientierung ist wie zahlreiche andere im Bereich der Sozialwissenschaften und besonders im Marketing verwendete theoretische Konstrukte inhaltlich nur schwer zu erfassen und entsprechend nur eingeschränkt direkt beobacht- und messbar. Nicht zuletzt resultiert dieser Umstand daraus, dass Marktorientierung sowohl als Kultur als auch als Bündel von Aktivitäten aufgefasst werden kann. Um das Konstrukt der Marktorientierung dennoch greifbar und letztlich messbar zu machen, soll im Folgenden kurz der Sinn und Zusammenhang zwischen der Konzeptualisierung und der Operationalisierung eines Konstrukts dargestellt werden.

Wenn Konstrukte in der Marketingforschung auf theoretischen Überlegungen beruhen, werden sie auch als „theoretische Konstrukte“, „hypothetische Konstrukte“, „latente Konstrukte“ oder „latente Variablen“ bezeichnet. ${ }^{251}$ Nach BAGOZZI/FORNELL wird ein theoretisches Konstrukt als „abstract entity which represents the 'true', unobservable state or nature of a phenomenon" beschrieben. ${ }^{252}$

Mit der Konzeptualisierung wird das Ziel verfolgt, ein Konstrukt möglichst genau zu beschreiben und von anderen Konstrukten abzugrenzen. Das nicht direkt beobachtbare Untersuchungsobjekt soll somit möglichst umfassend und theoretisch konkret beschrieben bzw. definiert werden. ROSSITER geht von drei Ebenen aus, die bei der Konzeptualisierung eines Forschungsgegenstandes berück-

\footnotetext{
${ }^{251}$ Vgl. Homburg/Giering 1996, S. 6; Weiber/Mühlhaus 2010, S. V.

252 Bagozzi/Fornell 1982, S. 24; vgl. auch Bagozzi/Phillips 1982, S. 465.
} 
sichtigt werden müssen. ${ }^{253}$

Auf der Subjektebene soll festgelegt werden, wer die Beurteilung des Konstrukts vornehmen soll. Anhand der Zielgruppe müssen die relevanten Aspekte des Konstrukts festgelegt und überprüft werden, ob das zu messende Konstrukt überhaupt von der anvisierten Zielgruppe beurteilt werden kann. Oftmals sind dies Mitarbeiter oder Führungskräfte eines Unternehmens. Aber auch Kunden können eine Beurteilung eines unternehmensbezogenen Konstrukts vornehmen. Auf der Objektebene soll festgelegt werden, auf welche Objekte sich das Konstrukt bezieht. So soll sichergestellt werden, dass die Zielgruppe ein einheitliches Verständnis des Untersuchungsgegenstandes hat. Der Abstraktionsgrad des Konstrukts wird auf der Attributebene festgelegt. Hier werden die einzelnen einzubeziehenden oder auszuschließenden Facetten des Konstrukts bestimmt. Durch die Herausarbeitung der einzelnen inhaltlichen Dimensionen eines Konstrukts soll ein besseres Verständnis des Forschungsgegenstandes erreicht werden. ${ }^{254}$ Theoretische Konstrukte können anhand der Anzahl ihrer Dimensionen bzw. ihrer inhaltlich unterschiedlichen Facetten unterschieden werden. Bei eindimensionalen Konstrukten können alle inhaltlichen Bereiche direkt der Ebene des Konstrukts zugeordnet werden. Bei mehrdimensionalen Konstrukten hingegen besteht das Konstrukt aus inhaltlich verwandten aber dennoch eigenständigen Facetten. ${ }^{255}$ Grundsätzlich ist es auch möglich, dass sich diese eigenständigen Facetten wiederum aus verschiedenen inhaltlichen Bereichen zusammensetzen, die dann mindestens zwei Ebenen unterhalb der Konstruktebene angesiedelt sind. Nachdem die Konzeptualisierung eines latenten Konstrukts durchgeführt wurde, kann schließlich die Definition des betrachteten Konstrukts erfolgen. ${ }^{256}$

Die Operationalisierung eines Konstrukts dient dazu, die theoretisch erläuterten Sachverhalte und einzelnen Facetten eines Konstrukts in beobachtbare und messbare Sachverhalte zu überführen. ${ }^{257}$ Hierdurch sollen die theoretischen Konstruk-

253 Vgl. hierzu und im Folgenden Rossiter 2002.

254 Vgl. Homburg/Giering 1996, S. 5.

255 Vgl. Homburg/Giering 1996, S. 5 f.

256 Vgl. Weiber/Mühlhaus 2010, S. 79.

257 Vgl. Weiber/Mühlhaus 2010, S. 86. 
te bzw. theoretischen Variablen, die sich einer direkten Messung entziehen, anhand von Korrespondenzregeln durch beobachtbare Sachverhalte dargestellt werden. ${ }^{258}$ Die Korrespondenzregeln spezifizieren, wie über Messvorschriften die latenten Konstrukte bzw. Dimensionen „greifbar“ gemacht werden. ${ }^{259}$ Hierzu müssen manifeste, direkt beobachtbare Variablen gefunden werden, die mit den theoretischen Sachverhalten in möglichst engem Zusammenhang stehen. ${ }^{260}$ Diese Indikatorvariablen bzw. Indikatoren lassen sich bei einem eindimensionalen Konstrukt direkt auf der Ebene des Konstrukt verdichten. Bei mehrdimensionalen Konstrukten findet die Verdichtung der manifesten Variablen auf der Ebene der einzelnen Dimensionen statt. ${ }^{261}$

Die Messung eines Konstrukts kann durch einen oder mehrere Indikatoren erfolgen. In der Literatur werden die für das jeweilige Forschungsvorhaben bedeutsamen Konstrukte in der Regel durch mehrere Indikatoren gemessen. Empfohlen wird dieses Vorgehen, da dadurch die Bedeutung eines Konstrukts genauer erfasst, nicht reliable Indikatoren ausgeglichen und eine feinere Abstufung der Konstruktausprägungen bei den Befragungseinheiten erreicht werden kann. ${ }^{262}$ Die Anweisungen, wie ein latentes Konstrukt über Messindikatoren abgebildet werden soll, werden auch als Messmodell bezeichnet. ${ }^{263}$ Die Messmodelle legen fest, ob ein Konstrukt oder eine Konstruktdimension über reflektive oder formative Indikatoren gemessen werden soll.

Um reflektive Messmodelle handelt es sich, wenn die Indikatoren von dem zugehörigen Konstrukt verursacht werden. ${ }^{264}$ Das bedeutet, dass die Ausprägung des latenten Konstrukts die Ausprägung der zugeordneten Indikatoren bestimmt. Das Messmodell legt somit fest, dass die Indikatoren die Wirkung des ursächlichen Konstrukts darstellen. Da die Indikatoren eine gemeinsame Ursache haben, soll-

\footnotetext{
258 Vgl. Fassott/Eggert 2005, S. 34 f.

259 Vgl. Homburg/Giering 1996, S. 6.

${ }^{260}$ Vgl. Bagozzi 1998.

${ }^{261}$ Vgl. Homburg/Giering 1996, S. 6.

262 Vgl. Churchill 1979, S. 66. Zu den Vorteilen der Messung über nur einen Indikator (Single-ItemMessung) vgl. Fuchs/Diamantopoulos 2009, S. 197 ff.; Bergkvist/Rossiter 2007; Diamantopoulos et al. 2012.

${ }^{263}$ Vgl. Weiber/Mühlhaus 2010, S. 35.

${ }^{264}$ Vgl. Edwards/Bagozzi 2000, S. 161; Bollen 1989, S. 65.
} 
ten sie auch entsprechend untereinander hoch korrelieren. Es ist auch möglich, einzelne Indikatoren auszutauschen oder aus dem Messmodell auszuschließen, ohne dass dies zu einer inhaltlichen Veränderung des betreffenden Konstrukts führt. ${ }^{265}$ Eine Veränderung der latenten Variable würde eine Veränderung aller entsprechenden Indikatoren nach sich ziehen, so dass von einem Gleichlauf zwischen den Indikatoren ausgegangen werden kann.

Die Berechnung eines reflektiven Konstrukts $\xi$ anhand seiner Indikatoren ergibt sich durch $\mathrm{x}_{\mathrm{i}}=\lambda_{\mathrm{i}} \xi+\delta_{\mathrm{i}}$, wobei $\mathrm{x}_{\mathrm{i}}$ für die Ausprägung des i-ten direkt beobachtbaren Indikators der latenten Variable $\xi$ steht. $\lambda_{\mathrm{i}}$ stellt die Faktorladung auf den jeweiligen Indikator dar und $\delta_{\mathrm{i}}$ repräsentiert die nicht durch die latente Variable erfasste Varianz des entsprechenden Indikators (vgl. Abbildung 3). ${ }^{266}$

Im Gegensatz dazu stellen bei formativen Messmodellen die Indikatoren die Ursache für das ihnen zugeordnete Konstrukt dar. Eine Veränderung eines Indikators verursacht somit auch eine Veränderung des betreffenden Konstrukts. Die Indikatoren müssen hierbei nicht miteinander korrelieren, da sie auch unterschiedliche Elemente eines Konstrukts bzw. einer Dimension erklären können. ${ }^{267}$ Daher sind auch positive und negative Einflüsse der einzelnen Indikatoren auf das betreffende Konstrukt möglich. Eine Eliminierung eines Indikators würde auch eine inhaltliche Veränderung des Konstrukts nach sich ziehen. ${ }^{268}$ Eine inhaltliche Änderung des Konstrukts setzt aber wiederum eine genaue Überprüfung oder Veränderung der Konzeptualisierung bzw. der Definition des Konstrukts voraus.

Die Berechnung eines formativen Konstrukts $\eta$ geschieht durch die Linearkombination der dem Konstrukt zugewiesenen Indikatoren und berechnet sich durch $\eta=\sum_{\mathrm{i}} \mathrm{x}_{\mathrm{i}} \mathrm{\gamma}_{\mathrm{i}}+\zeta$, mit $\eta$ als Ausprägung der latenten Variable, die durch die Indikatoren $\mathrm{x}_{\mathrm{i}}$ bestimmt wird. $\mathrm{y}_{\mathrm{i}}$ repräsentiert die Gewichtung, mit der der jeweilige Indikator bei der Berechnung von $\eta$ berücksichtigt wird. Die Residualgröße $\zeta$ bezeichnet die nicht erklärte Abweichung des tatsächlichen Konstruktwerts vom

${ }^{265}$ Vgl. Herrmann et al. 2006, S. 47.

${ }^{266}$ Vgl. Edwards/Bagozzi 2000, S. 161.

${ }^{267}$ Vgl. Fassott/Eggert 2005, S. 38.

${ }^{268}$ Vgl. Eberl 2006, S. 652. 
errechneten Wert (vgl. Abbildung 3). ${ }^{269}$
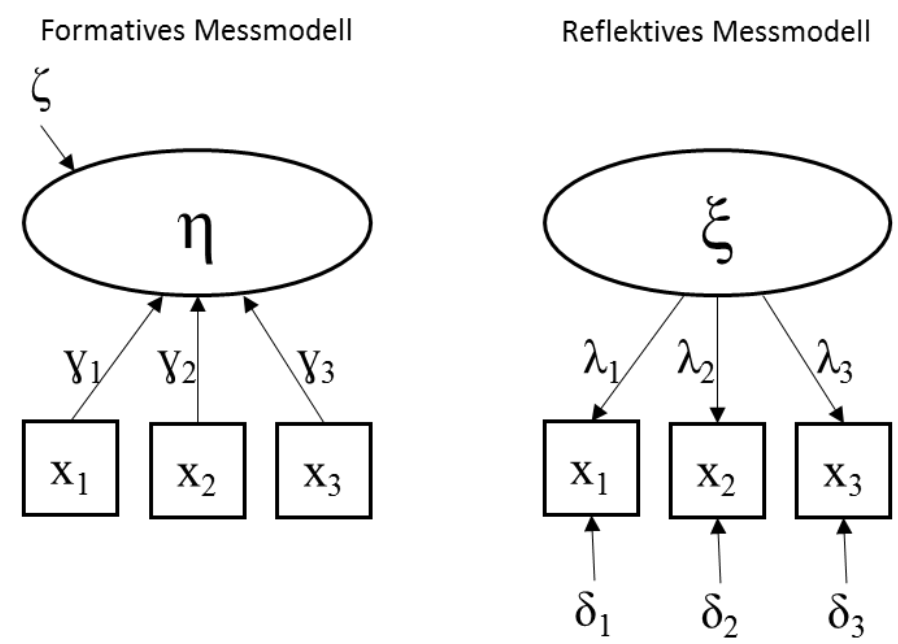

Abbildung 3: Schematische Darstellung von reflektiven und formativen Messmodellen (Quelle: in Anlehnung an Edwards/Bagozzi 2000, S. 161 f.)

Die grundsätzlichen Messeigenschaften der zuvor dargestellten eindimensionalen Konstrukte lassen sich auch auf mehrdimensionale Konstrukte übertragen. ${ }^{270}$ Oftmals werden Konstrukte nämlich auf einem Abstraktionsniveau konzeptualisiert, das ein Messmodell zweiter Ordnung erforderlich macht. ${ }^{271}$ Dabei werden die verschiedenen Dimensionen als Indikatoren des Konstrukts höherer Ordnung aufgefasst, so dass hierbei das Konstrukt wieder reflektiv oder formativ über die Dimensionen gemessen werden kann. ${ }^{272}$ Die einzelnen Dimensionen können dabei ebenfalls entweder reflektiv oder formativ gemessen werden, so dass sich insgesamt vier verschiedene Messmodelle für Konstrukte zweiter Ordnung ergeben. ${ }^{273}$ Alle Konstrukte zweiter Ordnung, die demnach spezifiziert werden kön-

${ }^{269}$ Vgl. Edwards/Bagozzi 2000, S. 162.

${ }^{270}$ Vgl. Giere et al. 2006, S. $680 \mathrm{ff}$.

${ }^{271}$ Vgl. Albers/Götz 2006, S. 672; Jarvis et al. 2003, S. 204. Grundsätzlich sind auch Messmodelle noch höherer Ordnung zur Messung von latenten Variablen möglich (vgl. z.B. Griese 2001, S. 191.).

${ }^{272}$ Vgl. Albers/Götz 2006, S. 672

${ }^{273}$ Vgl. Jarvis et al. 2003, S. 205. 
nen, sind in Abbildung 4 dargestellt.

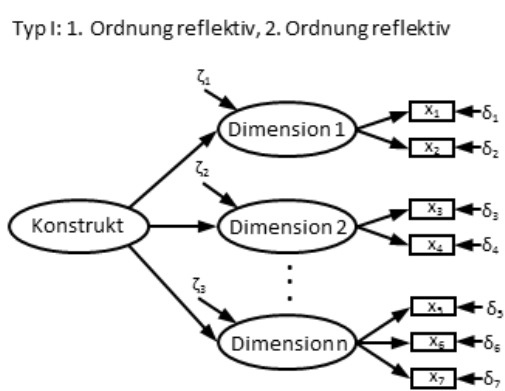

Typ III: 1. Ordnung formativ, 2. Ordnung reflektiv

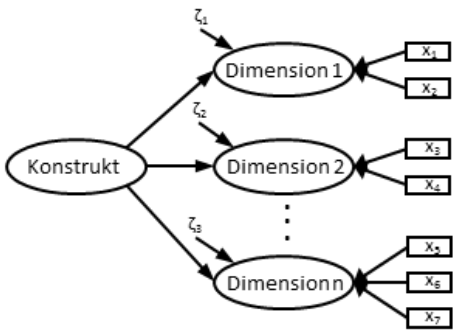

Typ II: 1. Ordnung reflektiv, 2. Ordnung formativ

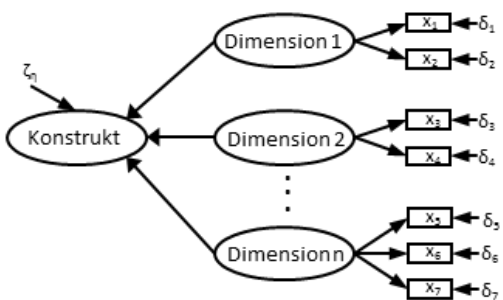

Typ III: 1. Ordnung formativ, 2. Ordnung reflektiv

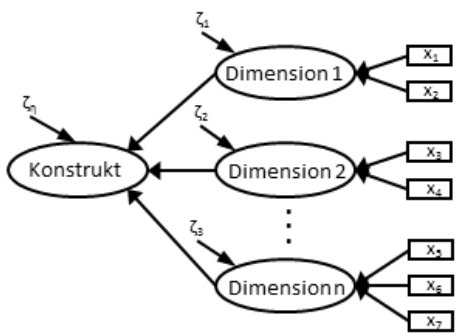

Abbildung 4: Typen von Konstrukten zweiter Ordnung (Quelle: in Anlehnung an Jarvis et al. 2003, S. 205; Albers/Götz 2006, S. 671)

ALBERS/GÖTZ untersuchten diese vier möglichen Messvorschriften latenter Konstrukte auf ihre Sinnhaftigkeit und argumentieren, dass durch die Verwendung von reflektiven Dimensionen eines Konstrukts diese eigentlich keine unterschiedlichen Facetten des Konstrukts repräsentieren können, da sie auch immer gleichzeitig austauschbare Indikatoren des Konstrukts darstellen müssen. Würde es sich um tatsächlich unterschiedliche Facetten desselben Konstrukts handeln, müssten diese formative Eigenschaften besitzen. Die Autoren sehen daher keine sinnvollen Anwendungsmöglichkeiten für die Modell-Typen I und III. ${ }^{274} \mathrm{Kon}$ strukte zweiter Ordnung sollten daher nur dann verwendet werden, wenn die Dimensionen tatsächlich verschiedene Facetten eines Konstrukts darstellen. Eine 
Entscheidung zwischen den Typen II und IV, also zwischen einer reflektiven und einer formativen Messung der Dimensionen sollte neben den theoretischen Erwägungen auch vom Ziel der Forschungsarbeit abhängig gemacht werden. Sollen bspw. konkrete Einzelmaßnahmen abgeleitet werden, um bestimmte Unternehmensziele zu erreichen, sollten die Dimensionen anhand von formativen Indikatoren gemessen werden. Interessieren jedoch die Facetten als Treiber des Konstrukts und ist eine Berücksichtigung von Messfehlern gewünscht, bietet sich das Modell Typ II an. ${ }^{275}$

Die Vielzahl der dargestellten Konzeptualisierungs- und Messmöglichkeiten hat auch in der Marketingforschung ihren Niederschlag gefunden. Insbesondere das Konstrukt der Marktorientierung wurde von verschiedenen Forschern unterschiedlich aufgefasst, konzeptualisiert und schließlich operationalisiert. In der Marketingliteratur ist daher eine Vielzahl von Konzeptualisierungen und Messskalen zu finden, wie der folgende Abschnitt zeigt.

\subsection{Zur Messung des Konstrukts Marktorientierung}

Mit der steigenden Anzahl von Studien zum Thema Marktorientierung wuchs auch die Anzahl der Konzeptualisierungen und Operationalisierungen dieses Konstrukts. Die in der Literatur zu findenden Messmodelle reichen von kleineren thematisch-inhaltlichen Anpassungen etablierter Skalen zu gänzlich neuen Konzeptualisierungen und Messvorschriften. Letztere wurden oftmals nur in einzelnen Studien verwendet und sind nicht als Replikationen in der einschlägigen Fachliteratur wiederzufinden. Der Vergleichbarkeit solcher nur einmal verwendeten bzw. neu entwickelten Konzeptualisierungen und Operationalisierungen sowie den damit gewonnenen empirischen Ergebnissen sind somit enge Grenzen gesetzt. GROVER fordert daher: „Lack of standardization of operationalization of constructs hampers the progress of any discipline. It is time we agreed upon terms and their conceptualizations.”276

\footnotetext{
${ }^{274}$ Vgl. Albers/Götz 2006, S. 672 f.

${ }^{275}$ Vgl. Albers/Götz 2006, S. 673.

${ }^{276}$ Vgl. Grover 1996, S. 115.
} 
Messskalen, die in der Forschung häufiger verwendet werden, zeichnen sich in der Regel durch ein breites Anwendungsspektrum und eine leichte Nachvollziehbarkeit der Konzeptualisierung sowie durch syntaktische Simplizität aus. ${ }^{277}$ Daneben existiert noch eine Fülle von Studien, in denen die Items verschiedener Skalen miteinander kombiniert werden. ${ }^{278}$ Oftmals wird ein solches Vorgehen verfolgt, um auf Basis von Gütekriterien einer empirischen Messung eine Verbesserung hinsichtlich der Messvalidität zu erreichen. Ein solches durch Gütekriterien getriebenes Vorgehen muss allerdings stark kritisiert werden, da hierbei die theoretische Fundierung der Konstruktkonzeptualisierung verloren zu gehen droht. Denn nach FARRELL ist eine Skalenkombination zwar schnell und einfach durchgeführt, doch bietet sie der Marketingwissenschaft nur oberflächlich durch eine hohe erreichte Güte einen Mehrwert. Bei einem solchen Vorgehen werden nämlich mitunter Items eliminiert, die eine große theoretische Bedeutung für die inhaltliche Erfassung eines Konstrukts besitzen. Ebenso kann eine Vermengung verschiedener Skalen $\mathrm{zu}$ theoretisch unplausiblen ItemZusammenstellungen führen. Es besteht somit die Gefahr, eine Operationalisierung abweichend von einer zuvor entwickelten Konzeptualisierung zu entwickeln. Ob eine solche Operationalisierung angesichts eines unklar definierten Untersuchungsgegenstandes neben reliablen auch valide Messergebnisse liefern kann, bleibt somit fraglich. Ein solches Vorgehen sollte daher, sofern keine ausreichende theoretische Grundlage vorhanden ist, gänzlich unterbleiben. ${ }^{279}$

Im Folgenden sollen die in der Literatur am häufigsten verwendeten Skalen zur Messung von Marktorientierung (MKTOR und MARKOR) und ihre unterschiedliche Handhabung in Bezug auf das Faktor- bzw. Dimensionsdesign betrachtet werden. Vor dem Hintergrund der unterschiedlichen Anwendung der Messskalen sollen in Abschnitt 4.3 und 4.6.1 die im Rahmen dieser Arbeit erarbeitete Kon-

${ }^{277}$ Vgl. Narver/Slater 1990; Rueckert 1992; Kohli et al. 1993; Deng/Dart 1994; Pelham/Wilson 1996; Lado et al. 1998.

${ }^{278}$ Werden die Daten zur Messung eines Konstrukts über einen Fragebogen erhoben, hat sich in der Marketingforschung der Begriff „Item“ für eine einzelne Frage etabliert. Ein Item stellt in der Regel einen manifesten Indikator dar, weshalb die Begriffe Item und Indikator in dieser Arbeit austauschbar verwendet werden.

${ }^{279}$ Vgl. Farrell 2002, S. 10. 
zeptualisierung und Operationalisierung begründet und näher erläutert werden. Die ursprüngliche MKTOR-Skala (MarKeT ORientation) wurde in den USA entwickelt und dort erstmals getestet. ${ }^{280}$ In zahlreichen internationalen Studien wurde die Skala ebenfalls benutzt und grundsätzlich als übertragbar eingestuft. ${ }^{281}$ Die Befragten beantworten darin in einer Zustimmungsskala von 1 (stimme voll zu) bis 7 (stimme gar nicht zu) Fragen zu den drei Bestandteilen von Marktorientierung nämlich customer orientation, competitor orientation und interfunctional coordination. Die Antwortwerte werden dann für jeden Bestandteil einzeln gemittelt. Anschließend werden die Mittelwerte der Dimensionen auf Konstruktniveau noch einmal gemittelt, um den endgültigen Wert für Marktorientierung zu erhalten. In einigen Replikationsstudien wird jedoch der Zwischenschritt der Mittelwertbildung auf Dimensionsebene umgangen. Bei dieser Messung werden alle Items auf Konstruktebene gemittelt, was der Konstruktfacette der Kundenorientierung aufgrund ihrer höheren Anzahl von Indikatoren einen größeren Einfluss verschafft. ${ }^{282}$ Die ursprünglich von NARVER/SLATER durchgeführte Mittelwertbildung über die Mittelwerte der einzelnen Dimensionen impliziert hingegen eine Gleichgewichtung der einzelnen Bestandteile.

Trotz der Inkonsistenz hinsichtlich der kulturbasierten Konzeptualisierung (vgl. Abschitt 2.1.2) und aktivitätsbasierten Messung besitzt der Messansatz dennoch bis heute einen großen Einfluss auf nachfolgende Forschungsarbeiten.

Die MARKOR-Skala (MARKet ORientation) wurde ebenfalls in den USA entwickelt und erstmals getestet. Auch bei dieser Skala und ihren Abwandlungen ist die Durchführung einer zweifachen Mittelwertbildung zur Bestimmung der Konstruktwerte bis heute gängige Praxis. ${ }^{283}$ Dabei werden zuerst Mittelwerte über die Indikatoren, dann über die Dimensionen intelligence generation, intelligence dissemination und responsiveness zur Messung des Gesamtkonstrukts gebildet. KOHLI ET AL. zeigen allerdings in ihrer Untersuchung, wie fragil die unterstell-

${ }^{280}$ Vgl. Narver/Slater 1990, S. 23.

281 Vgl. Rodriguez Cano et al. 2004, S. $180 \mathrm{f}$

282 Vgl. Greenley 1995; Deshpandé/Farley 1998. In der originalen MKTOR-Skala werden sechs Items verwendet, um Kundenorientierung zu messen, vier Items zur Messung von Wettbewerbsorientierung und fünf Items zur Messung von Abteilungsübergreifender Koordination.

${ }^{283}$ Vgl. Cadogan et al. 2009, S. 78. 
te drei-faktorielle Struktur von Marktorientierung ist, so dass in Replikationsstudien mithin je nach erreichten Gütekriterien drei oder vier Faktoren extrahiert und den Faktoren entsprechende Dimensionen zur Messung von Marktorientierung herangezogen werden. ${ }^{284}$ Ebenfalls gebräuchlich ist analog zur Verwendung der MKTOR-Skala ein ein-faktorielles Design, bei dem die Indikatoren direkt auf der Konstruktebene verdichtet werden. Die Verwendung eines unidimensionalen Designs muss trotz seiner einfachen Handhabung in der Wissenschaft abgelehnt werden. Faktoranalytische Untersuchungen zahlreicher Studien geben nämlich Hinweise darauf, dass es sich bei Marktorientierung um ein mehrdimensionales Konstrukt handelt. ${ }^{285}$ Diese Erkenntnisse haben auch dazu geführt, dass weniger der Einfluss des Gesamtkonstrukts auf verschiedene unternehmensrelevante Konsequenzen sondern vielmehr der individuelle Einfluss der einzelnen Komponenten im Fokus vieler Untersuchungen steht. ${ }^{286}$

Losgelöst von der Anzahl der Faktoren bzw. Dimensionen, die der Messung zugrunde gelegt werden, stellt sich die Frage nach der Kausalität der Facetten von Marktorientierung. Die Mehrheit der konzeptionell gering fundierten Arbeiten geht grundsätzlich von einem reflektiven Zusammenhang zwischen den Indikatoren und dem Konstrukt der Marktorientierung aus. ${ }^{287}$ Unabhängig davon, ob Marktorientierung kultur- oder verhaltensbasiert gesehen wird, messen die beiden betrachteten Skalen das Konstrukt über verschiedene Unternehmensmaßnahmen, die ein Anbieterunternehmen intern und nach außen gerichtet vollzieht. Konzeptualisiert wird Marktorientierung in beiden Fällen über die Summe verschiedener Aktivitäten, was ein formatives Modell impliziert. ${ }^{288}$

Teilweise wird dieser Umstand einer theoretisch begründeten formativen Messung von Marktorientierung in der Literatur erkannt, jedoch nicht immer berücksichtigt. WANG ET AL. versuchen, die MARKOR-Skala in einer formativen Weise zu verwenden, verwerfen diese Art der Operationalisierung allerdings, da

${ }^{284}$ Vgl. Kohli et al. 1993; Jaworski/Kohli 1993; Siguaw/Diamantopoulos 1995.

285 Vgl. Han et al. 1998; Siguaw/Diamantopoulos 1995, Menguc/Auh 2006; Hsieh et al. 2008.

${ }^{286}$ Vgl. Balakrishnan 1996; Han et al. 1998; Noble et al. 2002; Olson et al. 2005; Perry/Shao 2005;

Hsieh et al. 2008; Carbonell/Escudero 2010; He et al. 2011.

${ }^{287}$ Vgl. Coltman et al 2008, S. 1258.

${ }^{288}$ Vgl. Temme/Kreis 2005, S. 195; Coltman et al. 2008, S. 1251. 
sie bessere Gütekriterien des Messmodells mittels einer reflektiven Messung erreichen. Die Autoren entscheiden sich somit klar gegen die eigens dargelegte theoretische Fundierung der Konstrukts. ${ }^{289}$

BRETTEL ET AL. nehmen ebenfalls die MARKOR-Skala als Grundlage ihrer Messung von Marktorientierung und spezifizieren das Konstrukt als formatives Modell. Die Autoren betrachten die einzelnen von KOHLI ET AL. dargelegten Bestandteile von Marktorientierung als jeweils unabhängige Konstrukte, die nicht in irgendeiner Form auf der Konstruktebene von Marktorientierung verdichtet werden.

Auch die MKTOR-Skala wurde in der Literatur schon formativ behandelt, wenngleich bei Verwendung dieser Skala keine Messung auf der ersten Ebene Anwendung findet, sondern eine multi-dimensionale Konstruktstruktur bevorzugt wird. Bspw. bezeichnen AUH/MERLO in ihrer Studie die MKTOR-Skala als formativ und behandeln Marktorientierung als multi-dimensionales Konstrukt. Die auf der ersten Ebene formativ ermittelten Dimensionswerte bilden dann gemeinsam den Konstruktwert von Marktorientierung. Die Autoren gehen aber nicht weiter auf die genaue Operationalisierung oder eine Messvalidierung des so spezifizierten Konstrukts ein, da Marktorientierung nicht im Vordergrund ihrer Studie steht. ${ }^{290}$ An dieser Stelle soll die grundsätzliche Schwierigkeit erwähnt werden, Marktorientierung auf der ersten (Item-)Ebene formativ zu messen. Da die Items der MKTOR-Skala als auch der MARKOR-Skala ursprünglich in jeweils reflektiven Zusammenhängen entwickelt wurden, messen die einzelnen Items auch teilweise sehr ähnliche Sachverhalte. Bei einer formativen Verwendung können allerdings hoch korrelierende Items unter Umständen gravierende Probleme im Hinblick auf die Schätzbarkeit des Modells verursachen. ${ }^{291}$ Dieses Problem könnte durch die Eliminierung von Indikatoren umgangen werden, was allerdings wieder dem grundsätzlichen Verständnis einer formativen Messung widerspricht, nach der jeder Indikator einen bestimmten Teil eines Konstrukts abdeckt und nur aufgrund

\footnotetext{
${ }^{289}$ Vgl. Wang et al. 2013, S. $339 \mathrm{f}$.

290 Vgl. Auh/Merlo 2012, S. 867.

${ }^{291}$ Eine hohe Korrelation der Items ist bei reflektiven Messungen gewünscht, führt aber bei formativen Messungen zu Multikollinearität (vgl. Abschnitt 5.2.3).
} 
von theoretischen Überlegungen ausgeschlossen werden sollte. Bei einer formativen Interpretation und Anwendung der Skalen auf der ersten Ebene würde ein solches Vorgehen aber möglicherweise den Inhalt des Konstrukts Marktorientierung verändern und somit eine Neu-Konzeptualisierung erforderlich machen.

Einer anderen Argumentation, Marktorientierung nicht auf der ersten Ebene zu messen, folgt ROSSITER. Aufgrund des gegebenen hohen Abstraktionsniveaus von Marktorientierung beschreibt der Autor das Konstrukt als Konstrukt zweiter Ordnung. ${ }^{292}$ Das bedeutet, dass jede Dimension durch Indikatoren gemessen werden muss und schließlich jede Dimension selbst als Indikator des Gesamtkonstrukts fungiert.

In der neueren Literatur finden sich Autoren, die dieser Auffassung folgen und Marktorientierung als Konstrukt höherer Ordnung konzeptualisieren und messen. Dabei finden sich Modelle höherer Ordnung, die Marktorientierung sowohl auf der Dimensions- als auch auf der Konstruktebene reflektiv messen. Dies stellt nach ALBERS/GÖTZ ein Modell höherer Ordnung vom Typ I dar (vgl. Abschnitt 4.1). Die mit diesem Messmodell verbundene geringe Sinnhaftigkeit legen MURRAY ET AL. empirisch dar. Die Autoren messen die Marktorientierung von Exportunternehmen anhand einer Abwandlung der MARKOR-Skala. Bei einer komponentenweisen Vorgehensweise stellen die Autoren jedoch fest, dass die einzelnen Dimensionen von Marktorientierung unterschiedliche Effekte auf den Exporterfolg ausüben und somit nicht als austauschbar angesehen werden können. ${ }^{293}$ Die Austauschbarkeit der Indikatoren bzw. der Dimensionen ist aber Voraussetzung für das Vorliegen eines reflektiven Modells. Ähnliche mehrdimensionale Ansätze von Modellen des Typs I existieren auch für die MKTOR-Skala bzw. für Abwandlungen davon. ${ }^{294} \mathrm{Um}$ inhaltliche Probleme zu umgehen, die mit der Bildung von reflektiven Dimensionen verbunden sind, messen CADOGAN ET AL. die einzelnen Dimensionen über reflektive Indikatoren und bilden anschließend den Mittelwert davon auf der Konstruktebene. ${ }^{295}$ Dies wirft allerdings

292 Vgl. Rossiter 2002, S. 310.

293 Vgl. Murray et al. 2007, S. 55 f.

${ }^{294}$ Vgl. Gray et al. 1998; Carmen/Jose 2008; Coley et al. 2010.

${ }^{295}$ Vgl. Cadogan et al. 2009. 
möglicherweise wieder ein Problem mit der mathematisch bestimmten Gleichgewichtung jeder Dimension auf. Denkbar wäre nämlich, dass nicht alle Dimensionen gleichermaßen zum Konstrukt der Marktorientierung beitragen.

Daneben existieren Messmodelle höherer Ordnung, bei denen die Dimensionen über formative Items gebildet werden und sich der Konstruktwert aus einer Zusammenführung der einzelnen Dimensionswerte ergibt. Diese Messansätze kommen den Modellen des Typs IV nahe und werden von SANDVIK/SANDVIK zur Messung von Marktorientierung empfohlen. Die Autoren schlagen dabei die Besonderheit vor, die errechneten Dimensionswerte nicht wie oftmals üblich zu addieren sondern zu multiplizieren, um daraus eine finale Messung von Marktorientierung abzuleiten. ${ }^{296}$ Ob die etwa durch Multiplikation der Dimensionswerte erreichte Gewichtung der einzelnen Bestandteile auch der tatsächlichen Relevanz einer Dimension im Unternehmen entspricht, bleibt aber mehr als fraglich. Für den Wert des Gesamtkonstrukts jedenfalls bedeutet eine Multiplikation der Dimensionswerte, dass eine Vernachlässigung einer Dimension im Unternehmen in der Regel zu einer insgesamt gering ausgeprägten Marktorientierung führt.

Andere Arbeiten, die ebenfalls eine mathematisch festgelegte Gleichgewichtung der Dimensionen kritisch sehen, begegnen dem additiven und mittelwertbasierten Vorgehen dadurch, dass sie die Dimensionen bei der Konstruktmessung als formative Indikatoren über ihre jeweiligen Regressionsgewichte unterschiedlich stark zum Gesamtkonstrukt beitragen lassen. Die Dimensionen selbst werden hierbei hingegen durch reflektive Items spezifiziert. ${ }^{297}$ Ein solches Vorgehen entspricht den Modellen des Typs II. Wenngleich eine solche Konstruktspezifikation inhaltlich am meisten Sinn ergibt, werden bisher solche Ansätze allerdings nur auf Basis der MARKOR-Skala verfolgt. ${ }^{298}$ Konstrukte vom Typ III sind in der Marketingwissenschaft insgesamt eher selten zu finden. ${ }^{299}$ Das Konstrukt der Marktorientierung konnte daher in der Literatur bisher nicht als solch ein formatives Konstrukt erster Ordnung und reflektives Konstrukt zweiter Ordnung identi-

\footnotetext{
${ }^{296}$ Vgl. Sandvik/Sandvik 2003, S. 357.

297 Vgl. Navarro et al. 2010; Rodrigues-Pinto et al. 2011

${ }^{298}$ Vgl. Coltman et al. 2008; Rossiter 2002.

299 Vgl. Becker et al. 2012, S. 364.
} 
fiziert werden, obwohl keine messtechnischen Erwägungen dagegen sprechen. Mit der notwendigen konzeptionellen Fundierung könnten somit solche Messungen theoretisch zur Anwendung kommen.

Die kurze Darstellung obiger empirischer Arbeiten zeigt eine Vielfalt von Messmodellen auf Basis derselben zwei Messkalen. Daneben mögen noch andere Messansätze existieren, die hier allerdings aufgrund von mangelnden Informationen keine Erwähnung finden können. RINGLE ET AL. stellen im Hinblick auf Konstruktspezifikationen nämlich insgesamt fest, dass etwa ein Viertel aller Konstrukte höherer Ordnung in der relevanten Marketingliteratur nicht detailliert genug spezifiziert wird, um die Messung und die resultierenden Ergebnisse nachvollziehen zu können.300 Eine Interpretation und Vergleichbarkeit der erzielten Ergebnisse ist somit nicht in jedem Fall möglich. Tabelle 3 gibt einen Überblick über in der Literatur grundsätzlich nachvollziehbar dargestellte, unterschiedliche Messspezifikationen des Konstrukts Marktorientierung mittels der MARKORund MKTOR-Skala bzw. Derivaten der selbigen.

${ }^{300}$ Vgl. Ringle et al. 2012, S. xii. 
Tabelle 3: Unterschiedliche Messspezifikationen der MARKOR- und MKTOR-Skala

\begin{tabular}{|c|c|c|c|c|}
\hline Messung & $\begin{array}{l}\text { Unabhängige } \\
\text { Konstrukte }\end{array}$ & $\begin{array}{l}\text { Uni-dimensionales } \\
\text { Konstrukt }\end{array}$ & \multicolumn{2}{|l|}{$\begin{array}{l}\text { Multi-dimensionales } \\
\text { Konstrukt }\end{array}$} \\
\hline reflektiv & & & Тур I & Typ III \\
\hline & 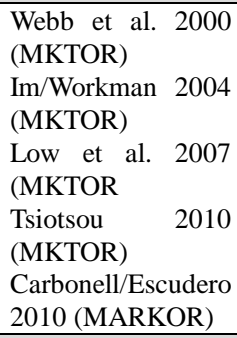 & $\begin{array}{l}\text { Greenley et al. } 1995 \\
\text { (MKTOR) } \\
\text { Deshpandé/Farley } \\
1998 \text { (MKTOR; } \\
\text { MARKOR) } \\
\text { Wang et al. } 2013 \\
\text { (MARKOR) }\end{array}$ & 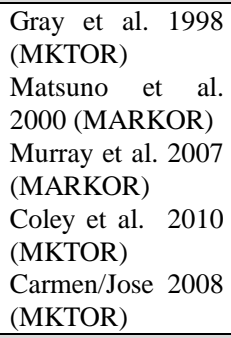 & \\
\hline formativ & & & Typ II & TYP IV \\
\hline & $\begin{array}{l}\text { Brettel et al. } 2008 \\
\text { (MARKOR) }\end{array}$ & & $\begin{array}{l}\text { Cadogan et al. } \\
2009 \\
\text { (MARKOR)* } \\
\text { Navarro et al. } \\
2010 \text { (MARKOR) } \\
\text { Rodriguez-Pinto et } \\
\text { al. } 2010 \\
\text { (MARKOR) }\end{array}$ & $\begin{array}{l}\text { Auh/Merlo } 2002 \\
\text { (MKTOR)* } \\
\text { Sandvik/Sandvik } \\
2003 \text { (MARKOR) }\end{array}$ \\
\hline
\end{tabular}

Eine Beurteilung der Ergebnisse, Vergleiche mit anderen Studien oder Messungen bzw. Replikationen können aufgrund der unterschiedlichen Messansätze somit nur schwer durchgeführt werden. Je nach veröffentlichten Gütekriterien und Ziel der Untersuchung kann zudem nicht immer überprüft werden, ob in den Studien tatsächlich eine empirische Bestätigung der theoretisch angenommenen Kausalrichtung der Dimensionen oder Items erreicht wurde. Zusammenfassend kann festgehalten werden, dass die etablierten MARKOR- und MKTOR-Skalen auf verschiedene Weise genutzt werden. Dabei werden zwischen Konstrukt, Dimension, und Items teilweise entgegengesetzte Wirkrichtungen unterstellt. ${ }^{301}$ Folglich können auch gravierende inhaltliche Interpretations- oder messtechnische Schwierigkeiten auftreten.

Grundsätzlich sollten die entwickelten Modelle zweiter oder höherer Ordnung

${ }^{301}$ Vgl. Wilcox et al. 2008, S. 1221. 
daher in erster Linie theoretisch-konzeptionellen Überlegungen folgen. Erst in zweiter Linie sollte darauf geachtet werden, dass die Modelle dergestalt aufgebaut sind, dass keine messtechnischen Schwierigkeiten auftreten. In den folgenden Abschnitten soll die Konzeptualisierung der in dieser Arbeit entwickelten umfassenden Marktorientierung theoretisch begründet dargelegt werden.

\subsection{Zur Konzeptualisierung des Konstrukts einer umfas- senden Marktorientierung}

Die folgenden Abschnitte sollen zur Beantwortung von Forschungsfrage 2 beitragen. Die Forschungsfrage 2 beschäftigt sich mit der sinnvollen Konzeptualisierung und Operationalisierung des zentralen Konstrukts der umfassenden Marktorientierung im Business-to-Business-Bereich.

\subsubsection{Theoretische Erwägungen}

Die Ausführungen in Abschnitt 3.2 zur theoretischen Basis der vorliegenden Untersuchung haben gezeigt, dass insbesondere der ressourcen- und der kompetenzbasierte Ansatz eine geeignete Grundlage für die theoretische Erläuterung des Konstrukts einer umfassenden Marktorientierung liefert. Der RBV liefert die Begründung dafür, wie Ressourcen dazu beitragen können, einen nachhaltigen Wettbewerbsvorteil am Markt zu erzielen. Insbesondere in mehrstufigen Märkten, in denen mehr als nur eine Ressource für den Unternehmenserfolg verantwortlich ist, ist es entscheidend geeignete Ressourcen so zu kombinieren, so dass das Unternehmen möglichst vollständig das vorhandene Potenzial ausschöpfen kann. Der kompetenzbasierte Ansatz bildet somit die theoretische Grundlage für eine erfolgsförderliche Ressourcen- und Fähigkeitskombination.

Marktorientierung sollte, um Wettbewerbsvorteile zu generieren, eine möglichst breite Auffassung des Marktes beinhalten. Ziel des Unternehmens muss es sein, ein breites Verständnis des Marktes zu erlangen und anschließend Leistungen anzubieten, die auf die Belange des Marktes zugeschnitten sind. Dazu muss das Wissen über möglichst viele relevante Aspekte des Marktes kombiniert und im Unternehmen an den entsprechenden Stellen zugänglich gemacht werden. Ent- 
scheidend ist hierbei, dass sowohl das Verhalten auf individueller Mitarbeiterebene als auch auf der Ebene der einzelnen Abteilungen dazu beiträgt, den Fokus der Unternehmenstätigkeit auf den entsprechenden Markt auszurichten. Zudem hat die Rolle der Führungskräfte, die selbst Aktivitäten der Wissenssammlung und -weitergabe übernehmen einen wesentlichen Einfluss. Die Führungskräfte tragen ebenfalls Verantwortung dafür, Ressourcen und Möglichkeiten im Unternehmen zu erkennen, zu fördern und so zu nutzen, dass möglichst effizient und effektiv Kundenwerte geschaffen werden können.

Die Fähigkeiten, Informationen zu sammeln, auszutauschen und darauf aufbauend Leistungen zu entwickeln und zu vermarkten, hängen in Industrienationen mit ausgebildeten Fachkräften weniger vom Können als vielmehr vom Wollen der einzelnen Mitarbeiter ab. Die Mitarbeiter müssen das Schaffen von Werten und die Befriedigung der Marktbedürfnisse verinnerlichen, so dass Marktorientierung wesentlich mehr ausmacht als nur das reine Sammeln und Auswerten von Daten. Andernfalls könnte Marktorientierung als simple Ansammlung von auf den Markt gerichteten Aktivitäten jederzeit mit entsprechend geschultem Personal von jedem Unternehmen problemlos weltweit implementiert werden. ${ }^{302}$ Dies würde allerdings der Annahme des RBV widersprechen, dass es sich bei der Marktorientierung um eine seltene und nur unzureichend kopierbare Ressource handelt. Das Bestreben, Marktwissen zur Schaffung von Werten zu nutzen, kann daher als Ausdruck eines stabilen Wertesystems bzw. als Manifestation einer Unternehmenskultur angesehen werden. ${ }^{303}$ Diesem Verständnis von Marktorientierung folgend, kann der Begriff der Marktorientierung in mehrstufigen Industriegütermärkten im Rahmen der vorliegenden Arbeit folgendermaßen definiert werden: Marktorientierung ist diejenige Unternehmenskultur, die eine differenzierte Fokussierung auf alle relevanten Marktteilnehmer ermöglicht und ein marktbezogenes Verhalten hervorruft, das notwendig ist, um überlegene Kundenwerte zu schaffen.

Nach dieser Definition ist das aus der Unternehmenskultur resultierende Verhal-

302 Vgl. Narver/Slater 1998a, S. 235.
303 Vgl. Narver/Slater 1998a, S. 235. 
ten sehr weit gefasst. Unter marktbezogenem Verhalten werden in dieser Arbeit die Sammlung, Nutzung und der unternehmensinterne Austausch von Markt- und Umfeldinformationen verstanden. DICKSON hebt die Wichtigkeit der Informationssammlung über die einzelnen relevanten Marktteilnehmer hervor und argumentiert: „Because the marketplace consists of consumers, competitors, distribution facilitators such as retailers and wholesalers, information agents such as consultants and trade associations, and regulatory institutions, changes in the behavior of all of those parties must be scanned and analyzed". ${ }^{304}$ Die Informationen beziehen sich somit auf Unternehmen im unmittelbaren Markt des Unternehmens wie etwa Kunden und Wettbewerber. Ferner beziehen sich die Informationen aber auch auf die Marktverhältnisse auf den nachgelagerten Marktstufen, was hauptsächlich die Kunden der Kunden und dortige Markttrends betrifft, sowie Drittparteien mit Einfluss auf Kaufprozesse. Das marktbezogene Verhalten betrifft sowohl individuelle Mitarbeitertätigkeiten als auch Aufgaben des Managements und Aktivitäten auf Abteilungsebene.

Um die Marktfokussierung des Unternehmens genauer betrachten zu können, soll der Markt differenziert betrachtet werden. Dadurch soll ermöglicht werden, die Einflüsse von Teilausrichtungen auf bestimmte Marktbestandteile bzw. Marktteilnehmer beobachten und bewerten zu können. ${ }^{305}$ Der Prozess von der Informationsverarbeitung bis zur anschließenden Nutzung des neu gewonnenen Wissens im Unternehmen steht dabei weniger im Fokus. Eine inhaltlich-konzeptionelle Anlehnung des in dieser Arbeit vorliegenden Verständnisses von Marktorientierung an das tätigkeitsfokussierte MARKOR-Konzept (intelligence generation, intelligence dissemination, responsiveness) ist somit nicht möglich. ${ }^{306}$ Das Verständnis von Marktorientierung in dieser Arbeit kann hingegen bei der Konzeptualisierung auf der Arbeit von NARVER/SLATER aufbauen. ${ }^{307}$ Neben der koordinierten Handlungsweise des Unternehmens wird hierbei zwischen einer Ausrichtung auf Kunden und auf Wettbewerber differenziert. Dass diese Teilausrichtun-

304 Dickson 1992, S. 75.

${ }^{305}$ Vgl. Coley et al. 2010, S. 143.

${ }^{306}$ Vgl. Kohli/Jaworski 1990; Kohli et al. 1993.

${ }^{307}$ Vgl. Narver/Slater 1990. 
gen unterschiedliche und eigenständige Facetten des Marktorientierungskonzepts darstellen, wurde in der Literatur hinlänglich untersucht und empirisch bestätigt. ${ }^{308}$ Die genannten Teilorientierungen erscheinen aber in einem B2B-Kontext nicht ausreichend, um den Markt in einer differenzierten Weise betrachten und bearbeiten zu können. Aus diesem Grund soll die Konzeptualisierung von NARVER/SLATER ergänzt werden.

Die einzelnen Facetten einer umfassenden, in dieser Arbeit untersuchten Marktorientierung sind die Kundenorientierung, die Wettbewerbsorientierung, die Marktstufenorientierung, die Drittparteienorientierung und die Abteilungsübergreifende Koordination. Diese Facetten bilden gemeinsam das Konstrukt der Marktorientierung und können nicht ausgetauscht oder eliminiert werden, da ansonsten das gesamte Marktverständnis einer inhaltlichen Veränderung unterworfen wäre.

Im Einklang mit den Theorien des RBV und der Dynamic Capabilities müssen die verschiedenen Dimensionen so kombiniert werden, dass sie gemeinsam die Unternehmensressource Marktorientierung abbilden können. Zudem müssen die Dimensionen, um als strategische Ressource zu gelten, alle ähnlich hoch ausgeprägt sein. Durch die Konzentration auf eine Dimension bzw. Vernachlässigung einer anderen Dimension können Unternehmen nach dieser Theorie nicht die vollen Wettbewerbsvorteile von Marktorientierung ausschöpfen und somit nicht ihr höchstmögliches Unternehmensergebnis erreichen. ${ }^{309}$ Eine niedrige Ausprägung einer Dimension kann dabei nicht etwa durch eine hohe Ausprägung einer anderen wettgemacht werden, was bedeutet, dass alle Teilausrichtungen und die Abteilungsübergreifende Koordination alle essentielle Bestandteile von Marktorientierung darstellen. ${ }^{310}$ Zwar würden unter rein messtechnischen Gesichtspunkten in einem formativen Modell auch Beiträge zum Konstrukt im nichtsignifikanten oder gar im negativen Bereich Sinn ergeben, doch würde dies dem RBV und dem CBV als theoretische Grundlagen in Bezug auf die Generierung

${ }^{308}$ Vgl. Siguaw/Diamantopoulos 1995; Farrell/Oczkowski 1997; Soehadi et al. 2001; Ward et al. 2006; Coley et al. 2010.

${ }^{309}$ Vgl. Greenley 1995; Slater/Narver 1994a.

${ }^{310}$ Vgl. Sandvik/Sandvik 2003, S. 356 f. 
und Kombination eines möglichst umfassenden Marktwissens widersprechen. Im nächsten Abschnitt soll näher auf die messtechnischen Gesichtspunkte eingegangen werden.

\subsubsection{Messtechnische Erwägungen}

Im Rahmen dieser Arbeit soll eine umfassende Marktorientierung nicht nur konzeptualisiert sondern auch valide gemessen werden. NARVER/SLATER definieren zwar mehrere Bestandteile (customer orientation, competitor orientation, interfunctional coordination) des Konstrukts und leiten diese theoretisch fundiert her. Allerdings ziehen die Autoren für ihre Untersuchungen eine eindimensionale Messung des Konstrukts heran, indem sie alle Bestandteile gleichgewichtet zum Gesamtmaß von Marktorientierung aggregieren. ${ }^{311}$ Zahlreiche Studien zeigen jedoch, dass es nicht nur aus theoretischer Sicht, sondern auch aus messtechnischer Sicht angebracht scheint, die einzelnen Bestandteile von Marktorientierung als eigenständige, unabhängige Dimensionen zu betrachten. ${ }^{312}$ Einige Studien zeigen zudem, dass die Komponenten anders als ursprünglich gedacht, nicht gleichermaßen zum Konstrukt der Marktorientierung beitragen. ${ }^{313}$ Eine unterschiedliche Gewichtung der Dimensionen sollte daher bei der Entwicklung einer neuen Skala für Marktorientierung in Industriegütermärkten berücksichtigt werden.

Die einzelnen Dimensionen der neu zu entwickelnden Messskala sollen als formative Indikatoren fungieren, die das Konstrukt gemeinsam bilden. Zwar sollen die Dimensionen in ähnlichem Umfang jeweils zum Konstrukt beitragen, doch soll eine a priori definierte Gleichgewichtung klar ausgeschossen werden. Der Einfluss der jeweiligen Indikatoren soll im Messmodell über die Regressionsgewichte frei schätzbar sein. Das Problem der hohen Indikatorkorrelationen bei der Verwendung der Dimensionen als formative Indikatoren ist in diesem Zusammenhang nicht relevant, da die Dimensionen zwar ein gemeinsames Konstrukt

\footnotetext{
311 Vgl. Narver/Slater 1990, S. 23.

312 Vgl. Dawes 2000; Langerak 2003; Im/Workman 2004; Hult et al. 2005; Ward et al. 2006; Zhou et al. 2008.

${ }^{313}$ Vgl. Day/Nedungadi 1994; Greenley 1995; Coley et al. 2010.
} 
bilden, aber dennoch grundverschiedene Aspekte desselben darstellen. Die erste Ebene des Konstrukts soll in reflektiver Weise gemessen werden. Ebenso wie in den etablierten Skalen sollen auch in der neuen Skala mehrere Items pro Dimension zur Messung herangezogen werden. Da diese Items als dahinterliegenden Faktor die Dimension selbst besitzen, ist hierbei mit hohen Korrelationen der Indikatoren untereinander zu rechnen. Eine hohe Korrelation ist hier allerdings erwünscht, da die Items reflektive Indikatoren erster Ordnung darstellen.

Zusammenfassend soll das Konstrukt einer umfassenden Marktorientierung die verschiedenen Marktbereiche eines Unternehmens abbilden und als theoretisches Konstrukt zweiter Ordnung definiert werden. Marktorientierung, wie sie in dieser Arbeit verstanden wird, soll auf der ersten Ebene reflektiv und auf der zweiten Ebene formativ gemessen werden.

\subsubsection{Berücksichtigung B2B-relevanter Aspekte der gängigen Mark- torientierungskonzepte}

Bevor eine detaillierte Konzeptualisierung der Dimensionen und Operationalisierung des in dieser Arbeit betrachteten Konstrukts einer umfassenden Marktorientierung vorgenommen wird, soll zunächst untersucht werden, inwiefern schon in den bestehenden Forschungsarbeiten sowohl auf theoretischer als auch auf empirischer Ebene B2B-relevante Aspekte von Märkten bzw. Marktorientierung berücksichtigt wurden. Dadurch sollen die Möglichkeiten einer Messstandardisierung ausgelotet werden.

Industriegütermärkte sind mehrstufige Märkte mit abgeleiteter Nachfrage als bestimmendem Charakteristikum. Dies bedeutet, dass auch das Konzept der Marktorientierung in diesem Anwendungskontext einen mehrstufigen Charakter aufweisen muss. ${ }^{314} \mathrm{Um}$ den definierenden Eigenschaften von Industriegütermärkten gerecht zu werden, muss auch das Konzept der Marktorientierung eine dahingehende Berücksichtigung aufweisen. Eine Berücksichtigung nachgelagerter Marktstufen müsste demnach zusätzlich zu den direkten Kunden und der direkten Abnehmerstufe auch die indirekten Kunden eines Anbieters und die auf diesen

${ }^{314}$ Vgl. Günter 1997, S. 214. 
Stufen herrschenden Marktdynamiken erfassen.

Ein zweites Marktcharakteristikum im Industriegüterbereich betrifft die einflussnehmenden Drittparteien. Daher müssten zusätzlich die Einflussnehmer entlang der Wertschöpfungskette, die das Kaufverhalten der direkten oder indirekten Kunden verändern und gestalten, Berücksichtigung in der Marktbetrachtung bzw. in einer umfassenden Marktorientierung finden. Dazu soll im folgenden Abschnitt analysiert werden, inwiefern die bestehenden und etablierten Konstruktkonzeptualisierungen und Operationalisierungen diese B2B-immanenten Charakteristika berücksichtigen.

\subsubsection{Berücksichtigung nachgelagerter Marktstufen}

Der Absatz eines Unternehmens im B2B-Sektor ist in erster Linie vom Kaufverhalten seiner direkten Kunden und vom Kaufverhalten der nachgelagerten bzw. indirekten Kunden abhängig. Die Bestimmung des Kunden und vor allem eine explizite Unterscheidung zwischen Kunden und den Kunden der Kunden hat in der Literatur zur Marktorientierungsforschung allerdings bisher wenig Widerhall gefunden. Allgemein werden unter dem Begriff des Kunden die Transaktionspartner eines anbietenden Unternehmens verstanden. Unternehmensaktivitäten, die sich ausschließlich an den direkten Transaktionspartnern ausrichten, erscheinen jedoch für die Marktorientierung eines Industriegüterunternehmens in mehrstufigen Märkten nicht ausreichend. Vielmehr muss ein Anbieter in mehrstufigen Märkten neben seinen direkten Kunden auch die weiteren Abnehmer auf nachfolgenden Marktstufen gegebenenfalls bis hin zum Letztverwender oder Konsumenten bei der Planung von Marketingaktivitäten betrachten. BÄUERLE propagiert eine mehrstufige Betrachtung der Nachfrager und definiert Kunden, also diejenigen Wirtschaftssubjekte, auf die eine Marktorientierung auszurichten ist, als „potenzielle und aktuelle Abnehmer einer unternehmerischen Leistung in unterschiedlichen Stufen der Wertschöpfungskette bis hin zum Endkunden (Konsument). “ ${ }^{315}$ Auch DAY/WENSLEY nehmen die Sichtweise ein, dass mehr als nur der direkte Kunde im Fokus der Marktorientierung eines Unternehmens stehen

${ }^{315}$ Bäuerle 2000, S. 14. 
sollte. Vielmehr seien auch die Position des Unternehmens in der Wertschöpfungskette und die Kostenstruktur der gesamten Wertschöpfungskette sowie die Koordination der Tätigkeiten zwischen Lieferanten, Herstellern und Unternehmen der nachfolgenden Marktstufe von großer Bedeutung. ${ }^{316}$

So zeigen auch empirische Studien, dass es insbesondere in Industriegütermärkten wichtig ist, die Unternehmensaktivitäten auf mehr als nur den direkten Kunden in der Wertschöpfungskette auszurichten. Vielmehr existieren in den Märkten Dynamiken und Abhängigkeiten, die es für ein Unternehmen notwendig erscheinen lassen, sich mit den nachfolgenden Stufen der Wertschöpfungskette zu befassen. SIGUAW ET AL. zeigen, dass marktorientiertes Handeln eines Unternehmens die Situation aller Unternehmen in der Wertschöpfungskette verbessern kann. Die Autoren untersuchen in diesem Zusammenhang, inwieweit die Marktorientierung eines Lieferanten die Marktorientierung des unmittelbar folgenden Händlers und weitere Faktoren innerhalb des Vertriebskanals beeinflussen kann. ${ }^{317}$ Die Autoren wählen hierzu allerdings eine dyadische Herangehensweise, so dass lediglich die Marktorientierung des Lieferanten gegenüber dem Händler und die Marktorientierung des Händlers gegenüber dem Konsumenten betrachtet werden. Eine Ausrichtung des Lieferanten auf weitere nachfolgende Marktstufen, z.B. die Endkunden oder Konsumenten, wird in dieser Studie nicht berücksichtigt. Andere Studien hingegen untersuchen die gemeinsame Marktorientierung von Unternehmen verschiedener Marktstufen gegenüber dem Endkunden. Im Vordergrund steht dabei die Ausrichtung der gesamten Wertschöpfungskette oder vertikaler bzw. horizontaler Allianzen auf den Endkunden. ${ }^{318}$ Dabei sehen die Autoren die Notwendigkeit, die verschiedenen Bereiche bei der Verarbeitung von Marktinformationen zu koordinieren und dieses möglichst effizient zwischen den Mitgliedern der Wertkette zu gestalten. ${ }^{319}$ Hierbei tritt allerdings die Ausrichtung des einzelnen Unternehmens auf das ihm in der Wertschöpfungskette unmittelbar

\footnotetext{
${ }^{316}$ Vgl. Day/Wensley 1988, S. 3; S. 17.

317 Vgl. Siguaw et al. 1998.

318 Vgl. Grunert et al. 2005; Carlson et al. 2011; Elg 2003.

${ }^{319}$ Die Studien stützen sich dabei auf die von KOHLIJAWORSKI (1990) beschriebenen Teilaufgaben der Marktorientierung: intelligence generation, intelligence dissemination und responsiveness.
} 
folgende Kundenunternehmen in den Hintergrund. Eine Unterscheidung zwischen der Marktorientierung gegenüber direkten Kunden und gegenüber Endkonsumenten wird daher ebenfalls nicht vorgenommen.

Eine der wichtigsten Arbeiten im Hinblick auf die Entwicklung einer Messskala für Marktorientierung stammt von KOHLI ET AL. ${ }^{320}$ In der Konzeptualisierung, die der Operationalisierung durch die Autoren vorausgeht, betonen KOHLI ET AL. die Bedeutung der Mehrstufigkeit von Märkten für die Marketingstrategie eines Unternehmens. KOHLI ET AL. beziehen sich u. a. auf DICKSON ${ }^{321}$ und schreiben, dass bei der Absatzplanung die Konsumenten, Wettbewerber, Absatzmittler wie etwa Händler aber auch die Regulierungsbehörden analysiert und in die Planung mit einbezogen werden müssen. So wird dann auch bei der Entwicklung der daraus abgeleiteten MARKOR-Skala ein Item aufgenommen, das die wahrgenommene Qualität der eigenen Produkte beim Endkunden bzw. Anwender messen soll. Eine scharfe Trennung zwischen einer Unternehmensausrichtung auf die direkte Abnehmerstufe und einer Ausrichtung auf die Endkunden oder andere dem unmittelbaren Abnehmer folgende Marktstufen kann aber aufgrund der tätigkeitsbasierten Dimensionsstruktur des Konstrukts nicht vorgenommen werden. Die Arbeit von NARVER/SLATER prägt bis heute in gleichem Maße die Konzeptualisierungen und Operationalisierungen von Marktorientierung in der Marketingliteratur. Die Autoren heben in ihrer Konzeptualisierung explizit die Wichtigkeit der abgeleiteten Nachfrage hervor. So proklamieren die Autoren, dass ein Anbieter nicht nur die Kosten- und Erlösstruktur seines direkten Abnehmers, sondern auch die Strukturen der Kunden seines direkten Abnehmers verstehen müsse, da von diesen letztlich die Nachfrage stamme, die der Anbieter zu befriedigen versuche. ${ }^{322}$ Anders als zu erwarten, wurde allerdings kein Item in die finale MKTOR-Skala aufgenommen, welches explizit die Mehrstufigkeit von Märkten berücksichtigt. In der Konstruktoperationalisierung wird letztlich keine Unterscheidung zwischen Abnehmern und Endkunden der vertriebenen Produkte vollzogen, obwohl diese je nach Produktart und insbesondere in Industriegüter-

${ }^{320} \mathrm{Vgl.} \mathrm{Kohli/Jaworski/Kumar} 1993$.

${ }^{321}$ Vgl. Dickson 1992. 
märkten völlig unterschiedlich sein können.

Eine weitere Arbeit, die hinsichtlich ihrer Konzeptualisierung und Operationalisierung große Anerkennung gefunden hat, ist die Arbeit von DENG/DART. Diese Arbeit fußt zu großen Teilen auf der von NARVER/SLATER. Auch hierin vertreten die Autoren die Auffassung, dass aufgrund der Mehrstufigkeit der Märkte insbesondere im Industriegüterbereich Marktorientierung weiter reichen muss als nur bis zur direkt nachfolgenden Marktstufe. Dies sei besonders dann erforderlich, wenn die direkt folgende Marktstufe eine reine Handelsstufe darstelle. In ihrer Operationalisierung des Marktorientierungskonstrukts entwickeln die Autoren zwei Items, die speziell zwischen den Händlern und Endkunden einer angebotenen Leistung unterscheiden. Aufgrund von Verbesserungsbemühungen hinsichtlich der Reliabilität der Messskala wurden diese Items jedoch nicht mehr von den Autoren in die endgültige Fassung der Operationalisierung überführt. ${ }^{323}$ Eine Berücksichtigung von mehrstufigen Märkten ist somit messtechnisch ausgeschlossen.

Aufgrund der Tatsache, dass bei den gängigsten Konstruktmessungen von Marktorientierung keine ausreichende Unterscheidung zwischen direkten und nachgelagerten Kunden getroffen wird, führen COLEY ET AL. aus, dass die bisherigen Modelle der Marktorientierung allesamt zu kurzsichtig und zu sehr auf den direkten Kunden fokussiert seien. ${ }^{324}$ Die Autoren schreiben, dass die gängigen Marktorientierungsmessmodelle den Konsumenten als Komponente des Konstrukts nicht explizit erwähnten oder stark vernachlässigten. Die Konsumenten spielen jedoch zweifelsfrei eine wichtige Rolle in vielen Industrien. COLEY ET AL. schreiben weiter, dass dem Konsumenten, der einen vom Kunden eindeutig verschiedenen Marktteilnehmer darstelle, in der Marktorientierung mehr Beachtung beigemessen werden müsse. ${ }^{325}$ Die Autoren erweitern deshalb die MKTOR-Skala und fügen als zusätzliche Dimension die Konsumentenorientierung hinzu. Generell ist die Erweiterung der Skala um die Konsumenten positiv zu sehen, da durch

\footnotetext{
${ }^{322}$ Vgl. Narver/Slater 1990, S. 21.

323 Vgl. Deng/Dart 1994, S. 741 f.

${ }^{324}$ Vgl. Coley et al. 2010, S. 143.

325 Vgl. Coley et al. 2010.
} 
diese Operationalisierung ein wesentlicher Marktbestandteil von Konsumgüterunternehmen berücksichtigt werden kann. Im Bereich des B2B-Marketings ist der Endkunde allerdings nicht immer von strategischer Bedeutung und teilweise sogar unbekannt. Die Skala bietet somit zwar wertvolle Hinweise auf die Existenz und Berücksichtigung von Letztverwendern, wird den Bedürfnissen von Industriegüterunternehmen aber nicht gerecht.

Die Analyse von Konsumenten und die strategische Ausrichtung an diesen sehen LADO ET AL. ebenfalls als wesentlichen Bestandteil einer umfassenderen Marktorientierung. Die Autoren nehmen in ihre Definition von Marktorientierung die Berücksichtigung verschiedener Stakeholder eines Unternehmens auf. Der Auffassung der Autoren nach muss ein marktorientiertes Unternehmen die Endkunden und Händler seiner Produkte sowie die Wettbewerber und das Marktumfeld analysieren und sollte darauf aufbauend seine Strategie ausrichten. Zudem bedürfe die Marktorientierung einer unternehmensinternen Koordination der Aktivitäten. ${ }^{326}$ LADO ET AL. berücksichtigen dabei konzeptionell die Mehrstufigkeit von Märkten durch die Einbeziehung einer Handelsstufe. Die Festlegung auf Händler als direkte Abnehmerstufe ist für ein im Industriegüterbereich vorherrschendes Marktverständnis jedoch nicht ausreichend, da hierbei jegliche Form der Weiterverarbeitung ausgeschlossen und somit die Anwendbarkeit der von den Autoren entwickelten Marktorientierungsskala eingeschränkt wird. ${ }^{327}$

Auch KIBBELING ET AL. unterscheiden konzeptionell zwischen Endkunden (end-user) und Kunden (direct customer) und heben in einer Studie die Bedeutung der Endkunden innerhalb von Wertschöpfungsketten hervor. Die Autoren entwickeln dazu das Konzept der Endkundenorientierung eines Lieferantenunternehmens und untersuchen insbesondere Effekte dieser Orientierung auf die Innovativität des Unternehmens und die Zufriedenheit der Endkunden. ${ }^{328}$ Per definitionem soll die Endkundenorientierung nur die Letztverwender der angebotenen

\footnotetext{
${ }^{326}$ Vgl. Lado et al. 1998, S. 34.

${ }^{327}$ Eine ähnliche Konzeptualisierung mit Berücksichtigung von mehrstufigen Märkten entwickelt auch Tomášková (2009). Durch die ausschließliche Berücksichtigung von Händlern als direkte Kunden schränkt die Autorin ebenfalls die Anwendbarkeit der entwickelten Skala stark ein. ${ }^{328}$ Vgl. Kibbeling et al. 2013.
} 
Leistungen berücksichtigen. In der finalen Skala beschäftigen sich allerdings zwei von fünf Items mit den Bedürfnissen der direkten Kunden. Aufgrund dieser Zusammenfassung von Items mit Bezug zu unterschiedlichen Marktakteuren kann diese Skala bestenfalls ansatzweise die Marktstufenstruktur im Rahmen einer Marktorientierung berücksichtigen.

Während COLEY ET AL. und KIBBELING ET AL. eine stärkere Berücksichtigung von Konsumenten gleichzeitig zur Berücksichtigung von direkten Kunden fordern, setzen andere Autoren die Marktakteure Kunden und Konsumenten gleich. Insbesondere im Konsumgütermarketing wird oftmals auch der Endkunde eines Produkts als Kunde eines Unternehmens aufgefasst. Diese Sichtweise ist aber ebenso kurzsichtig wie die zuvor dargestellte reine Fokussierung auf den direkten Kunden, da hierbei alle zwischengelagerten Marktstufen ausgeblendet werden. Die Haltung, dass in einer Marktbetrachtung sowohl die Konsumenten als auch die direkten Abnehmer Berücksichtigung finden sollten, nimmt DRUCKER schon früh ein. Der Autor zeigt anhand verschiedener Beispiele, dass nicht nur der Endkonsument als Kunde eines Unternehmens verstanden werden darf. Vielmehr steht das Unternehmen entlang der Wertschöpfungskette mit allen anderen Unternehmen in Verbindung, die ebenfalls als Kunden gesehen werden müssen. ${ }^{329}$ Auch RIEMER teilt diese Sichtweise und versteht den Begriff des Kunden als ein übergeordnetes Konstrukt, in dem nicht nur die Konsumenten, sondern auch direkte Kunden und alle nachgelagerten Wirtschaftssubjekte zusammengefasst werden. ${ }^{330}$

BIGNÉ ET AL. nehmen die Mehrstufigkeit am Beispiel der Keramikfliesenindustrie zum Anlass, den Begriff des Kunden genauer zu analysieren. In ihrer Konzeptualisierung von Marktorientierung verstehen die Autoren den Kunden eines Anbieters als den Endkunden oder Nutzer eines Produkts (final consumer). Die Autoren machen jedoch gleichzeitig darauf aufmerksam, dass eine Orientierung am Kunden auch alle Marktzwischenstufen beinhalten muss: „the company wishing to become more customer orientated has to adopt a broad concept of its

\footnotetext{
${ }^{329}$ Vgl. Drucker 1985, S. 80 ff.
}

${ }^{330}$ Vgl. Riemer 1986, S. 44. 
customers [...].“331 Da die Autoren aber alle auf die verschiedenen Marktteilnehmer ausgerichteten Aktivitäten in einer unidimensionalen Skala zusammenfassen, ist hier wiederum keine Unterscheidung bei der Unternehmensausrichtung auf verschiedene Marktakteure möglich. Eine explizite Messung der Berücksichtigung von nachgelagerten Marktstufen ist somit nicht möglich.

Die Schwierigkeiten, die mit einer ausgedehnten Sichtweise des Marktes und besonders mit der Ausrichtung auf nachgelagerte Marktstufen verbunden sein können, stellen KOHLI/JAWORSKI heraus. Aufgrund der Tatsache, dass der Markt eines Lieferanten nicht nur die direkten Kunden, sondern auch die Endnutzer umfasse sowie die dazwischen liegende Handelsstufe, müsse ein Lieferant die unterschiedlichen Bedürfnisse der Marktstufen und Einflussnehmer befriedigen. Dies könne mitunter sehr komplex werden. Schwierig werde es insbesondere dann, wenn Leistungen für einen Marktteilnehmer erstellt würden, dieser aber nicht derjenige sei, der den Lieferanten letztendlich bezahle. ${ }^{332}$ Eine umfassende Marktorientierung kann daher weder auf Konsumenten noch auf direkte Kunden beschränkt bleiben, sondern muss, wenn nötig, alle relevanten Marktstufen einschließen.

Aber nicht nur die Kunden und die Kunden der Kunden sind bei der Marktorientierung von Interesse. Auf den nachgelagerten Marktstufen befinden sich die Leistungen eines Anbieters ebenfalls im Wettbewerb mit Konkurrenzangeboten. Die Wettbewerber auf den nachgelagerten Marktstufen stellen daher ebenfalls Marktteilnehmer dar, die bei der Ausrichtung der Unternehmensaktivitäten Berücksichtigung finden müssen. HUNT/MORGAN beziehen daher in die Definition der Marktorientierung potenzielle Wettbewerber ein, die nicht notwendiger Weise auf derselben Marktstufe agieren müssen wie das Anbieterunternehmen selbst. Die Autoren sehen die Gefahr des Entstehens neuer Wettbewerber aufgrund von sich ändernder Technologie. ${ }^{333}$ Solch eine technologische Änderung könne zu weitreichenden Veränderungen in der Wertschöpfungskette führen, so dass Unternehmen in vor- oder nachgelagerten Marktstufen plötzlich zu Wettbe-

${ }^{331}$ Bigné et al. 2004, S. 176.

332 Vgl. Kohli/Jaworski 1990, S. 4. 
werbern des Anbieterunternehmens werden und so dessen Absatzchancen bis zum Endkunden bedrohen könnten. Eine Marktstufenbetrachtung sollte daher neben den dortigen Abnehmern immer auch alle potentiellen Wettbewerber berücksichtigen.

Zusammenfassend lässt sich sagen, dass in der Literatur die Mehrstufigkeit von B2B-Märkten und die damit verbundene Wichtigkeit von direkten und indirekten Kunden sowie die Gefahr durch Wettbewerb auf nachgelagerten Marktstufen durchaus erkannt wird. In der Marktorientierungsliteratur finden daher in konzeptioneller Hinsicht die Kunden der Kunden und die Gefahr durch nachgelagerte Substitutionsmöglichkeiten Berücksichtigung. Aufgrund von unterschiedlichen Prämissen bei der Skalenentwicklung und unterschiedlicher Schwerpunktsetzung des jeweiligen Marktorientierungskonstrukts gelingt es aber bisher keiner Arbeit in messtechnischer Hinsicht, den Aspekt der Mehrstufigkeit dieser Märkte adäquat abzubilden.

\subsubsection{Berücksichtigung von Drittparteien}

Der Absatz eines Anbieters wird in erster Linie vom Kaufverhalten seiner Kunden und deren Kunden bestimmt. Dieses Kaufverhalten kann aber von sogenannten Drittparteien beeinflusst werden, weshalb eine umfassende Marktorientierung insbesondere im Industriegüterbereich auch solche Einflussnehmer berücksichtigen sollte. In der Literatur zur Marktorientierung wird zwar ebenfalls die Wichtigkeit dieser Akteure im Marktumfeld eines Unternehmens erkannt, jedoch nur teilweise in ausreichendem Maße in Konzeptualisierungen und Operationalisierungen von Marktorientierung berücksichtigt, wie die folgenden Ausführungen zeigen.

BÄUERLE sieht eine sehr starke Verbindung zwischen dem Absatz eines Unternehmens und dem Einfluss Dritter auf das Kaufverhalten der Kunden, so dass er Kunden und Einflussnehmer zu einer Gruppe zusammenfasst „[Kunden] umfassen alle Personen, die den Kauf durchführen, sowie alle anderen an Auswahl und

\footnotetext{
${ }^{333}$ Vgl. Hunt/Morgan 1995, S. 11.
} 
Nutzung von Produkten und Dienstleistungen Beteiligten.“334 Wenngleich der Autor die immense Bedeutung von Einflussnehmern hervorhebt, so werden in dessen Ausführungen zwei höchst unterschiedliche Gruppen von Marktteilnehmern zusammengefasst, die von einem Unternehmen auch durchaus in unterschiedlicher Weise bei der Planung von Marketingaktivitäten berücksichtigt werden. Eine Trennung von Kunden und Einflussnehmern scheint daher in Wissenschaft und Unternehmenspraxis angeraten.

Andere Autoren, sehen ebenfalls eine sehr enge Beziehung zwischen Kunden und Einflussnehmern, erweitern aber nicht das Kundenverständnis, sondern betrachten einflussreiche Drittparteien als eigenständige Akteure im Marktumfeld eines Unternehmens.

Eine der in der Marketingforschung am häufigsten verwendeten Konzeptualisierungen von Marktorientierung ist die Arbeit von NARVER/SLATER. Die Autoren heben die Wichtigkeit des Marktumfeldes des Anbieterunternehmens für dessen Absatzchancen hervor. Insbesondere ökonomische und politische Einflüsse werden erwähnt. ${ }^{335}$ Konkret benennen die Autoren in einer späteren Veröffentlichung die Bedeutung von Regulierungsbehörden und Lieferanten, die einen signifikanten Einfluss auf den Erfolg des Anbieters haben können. ${ }^{336}$ Die Autoren führen aus, dass Marktorientierung weitergefasst auch die Berücksichtigung von allen Einflussnehmern beinhalten muss, die sich entweder positiv oder negativ auf den geschaffenen Kundennutzen und die Wettbewerbsposition des Anbieters auswirken können. ${ }^{337}$ In der häufig genutzten und von NARVER/SLATER entwickelten Messskala MKTOR werden allerdings explizit keine Daten hinsichtlich des weiteren Marktumfeldes gesammelt und analysiert, obwohl dieser Aspekt von den Autoren als wichtig hervorgehoben wird. Die finale Version der MKTORSkala beschäftigt sich nur sehr allgemein und implizit mit dem weiteren Umfeld des Anbieters. So wird lediglich untersucht, ob das Unternehmen die Bedürfnisse

${ }^{334}$ Bäuerle 2000, S. 14.

335 Vgl. Narver/Slater 1990, S. 21.

${ }^{336}$ Vgl. Slater, Narver 1994b, S. 22.

${ }^{337}$ Vgl. Slater/Narver 1995, S. 68. 
des Zielmarktes abdeckt. ${ }^{338}$ Eine genaue Definition des Zielmarktes geben die Autoren allerdings nicht, so dass den befragten Unternehmen viel Interpretationsspielraum bei der Beantwortung des entsprechenden Items gelassen wird. Auf Drittparteien, die Einfluss auf den Markt bzw. auf das Kaufverhalten der Kunden haben, wird nicht direkt eingegangen.

Die ebenfalls häufig verwendete Konzeptualisierung von KOHLI/JAWORSKI erwähnt explizit, dass besonderes jene exogenen Faktoren im Rahmen von Marktorientierung berücksichtigt werden müssten, die auf die Bedürfnisse und Präferenzen von Kunden einwirkten. ${ }^{339}$ Bezugnehmend auf die Konzeptualisierung von NARVER/SLATER führen KOHLI ET AL. aus, dass Marktorientierung oftmals einen zu engen Fokus besitze und damit eine Vernachlässigung von relevanten Umfeldentwicklungen einhergehe. ${ }^{340}$ Die Autoren möchten dieser Kurzsichtigkeit begegnen, indem sie drei Items entwickeln, die die Informationssammlung bezüglich verschiedener Einflussnehmer im Marktumfeld messen. In der finalen MARKOR-Skala, ist allerdings nur noch ein Item enthalten, das sich auf das weitere einflussnehmende Marktumfeld eines Unternehmens bezieht. ${ }^{341}$ Die übrigen zwei Items wurden aufgrund von Reliabilitätserwägungen aus der Skala ausgeschlossen. Aufgrund der Elimination kann der Bedeutung, die Drittparteien für den Absatz eines Unternehmens besitzen, nicht gerecht werden.

Da die beiden meist genutzten Konzeptualisierungen und Operationalisierungen letztlich nicht in vollem Umfang der Forderung nach einer breiteren Auffassung und Messung von Marktorientierung gerecht werden, ${ }^{342}$ entwickeln MATSUNO ET AL. auf Basis der MARKOR-Skala die sog. Extended Market OrientationSkala (EMO). ${ }^{343}$ Mit der EMO-Skala wird der Markt weiter gefasst, so dass neben den Kunden und Wettbewerbern auch Informationen über Lieferanten, soziokulturelle, ordnungspolitische und makroökonomische Faktoren gesammelt werden. Die Autoren erweitern hierzu die Item-Batterien der bestehenden Dimensio-

\footnotetext{
338 Vgl. Narver/Slater 1990; Deshpandè/Farley 1998, S. 226.

339 Vgl. Kohli/Jaworski 1990, S. 4.

340 Vgl. Kohli et al. 1993, S. 474.

${ }^{341}$ Vgl. Jaworski/Kohli 1993, S. 64; Kohli et al. 1993, S. 476.

${ }^{342}$ Vgl. Jaworski/Kohli, 1996; Kohli et al. 1993; Slater/Narver 1995.

343 Vgl. Matsuno et al. 2005; vgl. auch Matsuno et al. 2000.
} 
nen (intelligence generation, intelligence dissemination, responsiveness). Somit werden zwar die Schwerpunkte bei der Informationssammlung und -verarbeitung im Unternehmen verändert, eine differenzierte Analyse und Unternehmensausrichtung auf einzelne Gruppen von Marktteilnehmern bleibt allerdings weiterhin unmöglich.

Eine differenzierte Betrachtung von einzelnen Gruppen wird in der Arbeit von LADO ET AL. vorgenommen. Die Autoren berücksichtigen ebenfalls in ihrer Konzeptualisierung von Marktorientierung die Einflussnahme auf Kaufentscheidungen durch Dritte. Die Autoren erweitern das Konstruktverständnis dadurch, dass sie die Unternehmensausrichtung an Umweltfaktoren als eigenständige Dimension konzeptualisieren. ${ }^{344}$ Diese Dimension von Marktorientierung wird jedoch in der endgültigen Messskala stark vernachlässigt. Lediglich eines der insgesamt 36 Items behandelt die Informationsauswertung bezüglich der Marktumwelt. Akteure, die Kundenentscheidungen beeinflussen, werden zudem teilweise dem Bereich der Kunden zugerechnet, ${ }^{345}$ so dass eine strikte Trennung zwischen Kunden und einflussnehmenden Drittparteien nicht möglich ist. ${ }^{346}$ Gemeinsam ist all diesen Berücksichtigungen des Marktumfeldes, dass dabei nur solche Einflüsse bzw. Drittparteien betrachtet werden, die einen direkten Einfluss auf das Kaufverhalten der Kunden und nachgelagerten Kunden ausüben. HULT geht jedoch noch einen Schritt weiter und führt an, dass ein Unternehmen, um nachhaltig zu wirtschaften, auch „additional stakeholders“ im Rahmen von Marktorientierung mit betrachten muss. ${ }^{347}$ Darunter versteht der Autor alle, die in irgendeiner Weise vom Handeln eines Unternehmens betroffen sind. Insbesondere zielt der Autor dabei auf die natürliche Umwelt, soziale Gerechtigkeit und Gleichbehandlung ab und zeigt große Parallelen zur sog. Corporate Social Responsibility auf. Langfristig gesehen kann es für ein Unternehmen Sinn erge-

\footnotetext{
344 Vgl. Lado et al. 1998.

345 Vgl. dazu auch das Kundenverständnis von BÄUERLE (2000, S. 24).

346 Auch Tomášková (2009) berücksichtigt die Ausrichtung an der Unternehmensumwelt als eigenständige Dimension von Marktorientierung. Allerdings wird die Bedeutung dieser Einflussnehmergruppe nicht theoretisch hergeleitet und zielt auf die sozio-ökonomische Umwelt des Anbieters ab und kann daher nicht in ausreichendem Maße die Einflussnahme auf das Kaufverhalten der Kunden und nachgelagerten Kunden berücksichtigen.
} 
ben, die Marktorientierung dahingehend zu erweitern. Für die vorliegende Arbeit sollen jedoch nur solche Personen und Institutionen betrachtet werden, die sich im direkten Marktumfeld des Unternehmens und der Abnehmer befinden und dort auch einen Einfluss auf das Kaufverhalten der direkten und indirekten Kunden ausüben können. Die soziale Verantwortung, die ein Unternehmen trägt, wird in dieser Arbeit hingegen nicht als Teil einer umfassenden Marktorientierung betrachtet. ${ }^{348}$

Zusammenfassend kann gesagt werden, dass genau wie die Berücksichtigung von Marktstufenstrukturen ebenso die Berücksichtigung von Drittparteien in der Literatur zwar in einigen Arbeiten durchgeführt, dies aber der Bedeutung, die diese Marktbestandteile für den Erfolg eines Anbieters haben können, bisher nicht gerecht wird. In den überwiegenden Fällen der Konzeptualisierungen und Operationalisierungen ist es zudem nicht möglich, eine Unternehmensausrichtung differenziert auf die Gruppe der Drittparteien als eigenständigen Bestandteil einer Marktorientierung zu identifizieren und zu messen.

Marketingkonzeptionen im Business-to-Business-Bereich, die sich lediglich auf Markttransaktionen zwischen Verkäufer und Käufer konzentrieren und dabei den darauffolgenden Nutzer einer angebotenen Leistung oder die Kaufentscheidung beeinflussende Drittparteien außer Acht lassen, müssen sich somit eine stark eingeschränkte Sicht des Marktes und des Marktumfeldes nachsagen lassen. Um den Fokus dieser eingeengten Sichteise zu erweitern, soll in dieser Arbeit eine umfassendere Konzeptualisierung und darauf aufbauend eine Operationalisierung von Marktorientierung vorgenommen werden.

Im Folgenden werden die einzelnen Facetten einer umfassenden Marktorientierung, die für die erfolgreiche Bearbeitung von Industriegütermärkten erforderlich sind und die zuvor genannten Unzulänglichkeiten bestehender Skalen wettmachen, konzeptualisiert.

\footnotetext{
${ }^{347}$ Vgl. Hult 2011, S. 2.

348 Auch FRITZ (1998) sieht eine solche unternehmerische Grundhaltung als etwas von Marktorientierung verschiedenes an. Der Autor spricht in diesem Zusammenhang von Umwelt- und Gesellschaftsorientierung.
} 


\subsubsection{Kundenorientierung}

Marktorientierung bedeutet, seine Kunden zu verstehen und ihren Bedürfnissen entsprechend Leistungen anzubieten. Dies kann nur gelingen, wenn die Kunden im Mittelpunkt des unternehmerischen Handelns des Anbieters stehen. Ziel eines Unternehmens im B2B-Sektor sollte es somit sein, Kundenwünsche in Erfahrung zu bringen, diese effizient und effektiv zu erfüllen und dadurch Kundenzufriedenheit zu schaffen.

Um die Anforderungen und Bedürfnisse des Marktes zu kennen, müssen die Wünsche der Kunden kontinuierlich beobachtet werden. Nur durch ein permanentes Monitoring des Marktes ist es möglich, Änderungen der Kundenstruktur und der Kundenwünsche überhaupt festzustellen. Einmal in Erfahrung gebrachte Kundenwünsche stellen lediglich eine Momentaufnahme einer bestimmten, bekannten Kundengruppe dar. Wichtig ist es aber auch, die Bedürfnisse, gerade von noch nicht bedienten potentiellen Kunden, zu kennen und ebenfalls Anstrengung dahingehend zu unternehmen, zukünftige Bedürfnisse zu antizipieren. ${ }^{349}$

Die Schaffung und Sicherung von Kundenzufriedenheit wird in vielen Unternehmen durch die Unternehmensführung als ein strategisches Ziel deklariert und gegebenenfalls im Unternehmensleitbild, Mission Statement, Code of Conduct o.ä. festgeschrieben. Der Kundenfokus bleibt somit nicht nur für externe Interessenten sondern gerade auch für die Mitarbeiter des eigenen Unternehmens ständig präsent und trägt zur Förderung einer kundenfokussierten Unternehmenskultur bei.

Ob das Unternehmen auch tatsächlich seinen selbstgegebenen Leitlinien folgt, muss im Anschluss auf operativer Ebene mit geeigneten Instrumenten überprüft werden. Prinzipiell sind alle Mitarbeiter an der Erfüllung der Kundenwünsche beteiligt. Ein besonderer Kundenfokus besteht allerdings in den Abteilungen mit direktem Kundenkontakt, da sich hier die Mitarbeiter befinden, die auf die einfachste und schnellste Weise die Bedürfnisse der Kunden und die erzielte Kundenzufriedenheit in Erfahrung bringen können. Vornehmlich betrifft dies die Abteilungen Marketing und Vertrieb sowie After-Sales Services und Beschwer-

${ }^{349}$ Vgl. Narver/Slater 1998b, S. 1003; Narver et al. 2004. 
demanagement, durch die das Leistungsbündel ${ }^{350}$, d.h. die eigentliche Hauptleistung und entsprechende begleitende Dienstleistungen, angeboten werden.

Eine solche Ausrichtung des Unternehmens wird auch als Kundenorientierung bezeichnet und in dieser Arbeit wie folgt definiert: Kundenorientierung bedeutet, ein ausreichendes Verständnis seiner Kunden zu haben, um so in der Lage zu sein, für sie dauerhaft einen überlegenen Wert zu schaffen. ${ }^{351}$

\subsubsection{Wettbewerbsorientierung}

Die auf dem Markt angebotenen Leistungen stehen in der Regel mit Konkurrenzprodukten im Wettbewerb um die Kunden. Die Kunden werden daher eine Kaufentscheidung nach Abwägung ihrer Nutzenmaximierung zwischen den verschiedenen angebotenen Produkten treffen. Um im Markt bestehen zu können, ist es daher unerlässlich, über die Wettbewerber und deren Produkte informiert zu sein. Wettbewerbsorientierung bedeutet daher, dass die Mitarbeiter und das Management des Unternehmens ständig über Wettbewerberstrategien im Bilde sind. Hier sind wieder Aktivitäten auf Ebene der Mitarbeiter mit großem Kundenkontakt als auch der stark ausgeprägte Wille des Managements, besser zu sein als die Wettbewerber, notwendig. ${ }^{352}$ Dabei werden die Wettbewerber sowohl hinsichtlich langfristiger, strategischer Potenziale als auch hinsichtlich kurzfristiger, operativer Handlungen, wie bspw. Preisreduktionen oder Werbekampagnen analysiert und Szenarien entwickelt, wie auf Wettbewerberaktionen reagiert werden kann. ${ }^{353}$ Die Wettbewerbsaktionen müssen dabei immer vom generierten Kundennutzen aus beurteilt werden. ${ }^{354}$ Bedeutung erlangt hierbei die Rationalität, mit der dann folgende Reaktionen durchgeführt werden. Eine Überreaktion etwa in Form von Rabattschlachten, wäre nämlich auf Dauer für keinen der betroffenen Anbieter nützlich. ${ }^{355}$ Auch umgekehrt müssen die Reaktionen des Wettbewerbs auf die

\footnotetext{
${ }^{350}$ Vgl. Engelhardt et al. 1993.

351 Vgl. Narver/Slater 1990, S. 21.

352 Vgl. Bendle/Vandenbosch 2014, S. 782.

353 Vgl. Dawes 2000, S. 191.

${ }^{354}$ Vgl. Soehadi et al. 2001, S. 287.

355 Vgl. Brodie et al. 1996.
} 
eigenen Handlungen antizipiert werden, um erfolgreich im Markt zu bestehen. ${ }^{356}$ Letztlich muss das Unternehmen aus den gewonnenen Informationen Schlüsse für das eigene Leistungsprogramm ziehen, da die Kunden die Leistungen eines Unternehmens immer im Vergleich zu den angebotenen Leistungen der Wettbewerber sehen. Anpassungen des Leistungsprogramms sind über den Preis oder die Leistung selbst möglich. In der Praxis spielen daher Anpassungen im Hinblick auf den technischen Umfang oder die Qualität einer Leistung eine Rolle. Ferner besteht ebenso die Möglichkeit, die über den Wettbewerb gewonnenen Daten in der Form zu nutzen, dass neue Marktsegmente erschlossen werden oder Anstrengungen dort forciert werden, wo die Wettbewerber bisher keine zufriedenstellenden Leistungen anbieten. Weiterhin gilt es, neben den technisch ähnlichen Angeboten der Wettbewerber aus der eigenen Branche auch solche Wettbewerber zu identifizieren, die mit technisch neuen oder völlig anderen Lösungen dieselben Bedürfnisse der Kunden befriedigen können. ${ }^{357}$ Innovative und branchenfremde Lösungsmöglichkeiten werden daher ebenso beobachtet, um deren Marktpotenzial abzuschätzen. Wettbewerbsorientierung als Teil einer Marktorientierung muss daher auch ein breites Verständnis von Wettbewerb und Wettbewerbern besitzen. ${ }^{358}$

Zusammengefasst bedeutet Wettbewerbsorientierung, dass ein Anbieter die kurzfristigen Stärken und Schwächen sowie die langfristigen Potenziale und Strategien sowohl von gegenwärtigen als auch von potenziellen Wettbewerbern versteht. ${ }^{359}$

\subsubsection{Drittparteienorientierung}

Um am Markt erfolgreich zu sein, reicht es heutzutage oftmals nicht mehr aus, lediglich nach Kundenwünschen spezifizierte Leistungen anzubieten. GEBHARDT ET AL. zeigen anhand von Beispielen aus der Baubranche, wie neben den Bauherren auch Händler und Architekten Erwartungen an bestimmte

${ }^{356}$ Vgl. Dawes 2000, S. 191.

${ }^{357}$ Vgl. Levitt 1960.

${ }^{358}$ Vgl. Balakrishnan 1996, S. 259.

${ }^{359}$ Vgl. Narver/Slater 1990, S. 21 f. 
Produkte stellen, deren Erfüllung für den Erfolg des Anbieters von entscheidender Bedeutung sein kann. 360

Es zeigt sich, dass neben den Marktteilnehmern, mit denen das Unternehmen in direktem geschäftlichen Kontakt steht, auch zahlreiche Institutionen und Individuen existieren, die sich im Umfeld des Unternehmens befinden und einen Einfluss auf das Marktgeschehen haben können. ${ }^{361}$ Häufig wird im Zusammenhang mit solchen Einflussnehmern auch von Stakeholdern gesprochen. Da aber auch der Begriff des Stakeholders in der Literatur unterschiedlich gebraucht wird, soll für diese Arbeit zunächst eine Verständnisgrundlage hinsichtlich dieses Begriffs geschaffen werden:

DONALDSON/PRESTON zeigen ein sehr weit gefasstes Verständnis von Stakeholdern und zählen dazu alle Gruppen und Individuen, die ein Interesse am Unternehmen selbst und an der Unternehmenstätigkeit haben. ${ }^{362}$ FREEMAN sieht den Begriff enger und sieht nicht das Interesse, sondern die Möglichkeit der gegenseitigen Einflussnahme als entscheidendes Kriterium an. Der Autor definiert Stakeholder als ,any group or individual who can affect or is affected by the achievement of the organization's objectives“. ${ }^{363}$ Um diese Einflussnahme ausüben zu können, müssen zwischen dem Unternehmen und den Stakeholdern nicht notwendigerweise rechtsverbindliche Verträge geschlossen werden. Dennoch sind die Stakeholder oftmals mittelbar oder unmittelbar von der Geschäftstätigkeit betroffen, so dass für das Unternehmen zumindest eine moralische Verpflichtung gegenüber diesen Stakeholdern bestehen kann. ${ }^{364}$ In der Regel gehören zu dieser Gruppe der betroffenen Stakeholder die Menschen und politischen Vertreter in Gemeinden und Städten, in denen das Unternehmen seinen Sitz hat oder Produktionsstätten betreibt. Tätigkeiten des Unternehmens, durch die es seiner moralischen Verpflichtung nachkommt, werden auch als Social Corporate Responsibility bezeichnet. ${ }^{365}$ Dieser Stakeholder-Auffassung nach kann das Unternehmen

\footnotetext{
${ }^{360}$ Vgl. Gebhardt et al. 2006, S. 45.

361 Vgl. Kleinaltenkamp/Rudolph 2002, S. 287.

362 Vgl. Donaldson/Preston 1995, S. 67.

363 Freeman 1984, S. 4.

364 Vgl. Gotteland et al. 2007; Neuenburg 2010, S. 58.

365 Vgl. Maignan/Ferrell 2004, S. 4.
} 
seine Aktivitäten nicht mehr nur auf seine Kunden und andere Abnehmer ausrichten, sondern muss auch betroffene Stakeholder mit in seine Planung einbeziehen. ${ }^{366}$

In der Regel existieren zwischen den dargestellten Stakeholdern und einem Unternehmen ein gegenseitiges Interesse und eine gegenseitige Einflussnahme. ${ }^{367}$ Abbildung 5 stellt diesen Zusammenhang dar.

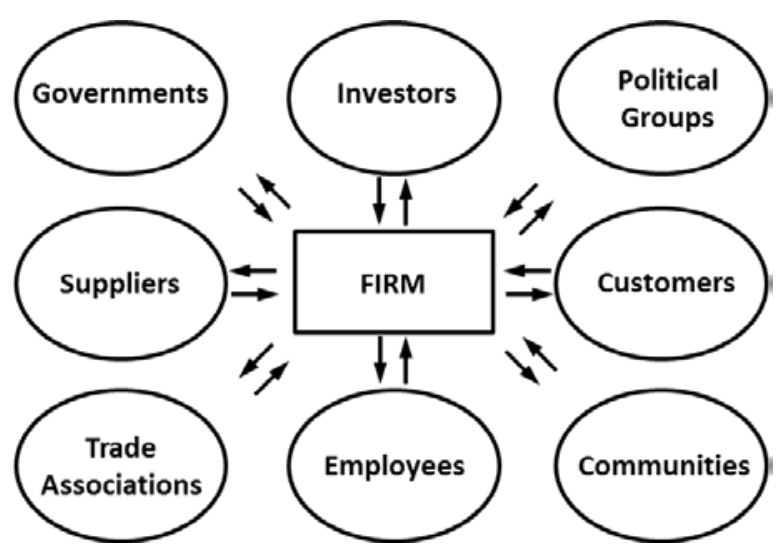

Abbildung 5: Stakeholder des Unternehmens (Quelle: Donaldson/Preston 1995, S. 69)

Es existieren jedoch auch Personen und Institutionen im Marktumfeld, die einseitig einen Einfluss auf das Unternehmen ausüben und an denen sich daher das Unternehmen in seinen Entscheidungen ausrichten muss. ${ }^{368}$ Zur Art dieser Einflussnahmen durch Stakeholder schreiben HENRIQUES/SADORSKY: „Stakeholders can [...] influence the practices of an organization via direct pressure or by conveying information". ${ }^{369}$ Die Autoren teilen die Gruppe der Stakeholder dabei in regulierende Stakeholder, organisationale Stakeholder, Stakeholder des Gemeinwesens und Medien ein. Regulierende Stakeholder sind vor allem im

${ }^{366}$ Vgl. Maignan/Ferrell 2004, S. 3.

367 Vgl. Freeman/Reed 1983, S. 91; Maignan/Ferrell 2004, S. 4; Gotteland et al. 2007, S. 51.

${ }^{368}$ Vgl. Jones 1995, S. 407; vgl. auch Savage et al. 1991, S. 61.

369 Henriques/Sadorsky 1999, S. 89. 
Bereich der staatlichen Verwaltung und Gesetzgebung zu finden und können den Rahmen setzten und ändern, innerhalb dessen ein Unternehmen Geschäfte abschließen darf. ${ }^{370}$ Einfluss auf das Unternehmen wird beispielsweise durch die Festsetzung von technischen Normen oder Umweltstandards ausgeübt. Solche Standards können auch in den Abnehmerbranchen des Anbieters existieren und so indirekt über das Kaufverhalten der Kunden den Absatz des Anbieters beeinflussen. Ebenfalls zu den regulierenden Stakeholdern gehören Verbände und Unternehmenszusammenschlüsse, die versuchen, Einfluss auf solche Regularien nehmen. ${ }^{371}$ Die organisationalen Stakeholder bestehen vor allem aus anderen Unternehmen, die mit dem betrachteten Unternehmen als Lieferanten oder Kunden in Verbindung stehen. Angestellte Mitarbeiter und Anteilseigner des Unternehmens werden ebenso zu dieser Gruppe von Stakeholdern gezählt. Die Stakeholder des Gemeinwesens beinhalten lokale Gemeindegruppen, Umweltaktivisten und andere Lobbygruppe und sind in der Lage, die öffentliche Meinung zu prägen und somit in positiver wie negativer Weise das Image eines Unternehmens zu beeinflussen. Der Stakeholdergruppe der Medien kommt eine besondere Bedeutung zu, da diese ähnlich wie die Stakeholder des Gemeinwesens auf die Meinung der Öffentlichkeit einwirken. Insbesondere durch Veröffentlichen oder Zurückhalten von Informationen über ein Unternehmen und dessen Geschäftstätigkeit können die Medien im Einzelfall einen starken Einfluss ausüben.

In der Literatur ergeben sich enge Parallelen und Überschneidungen zwischen dem Konzept der Stakeholder-Orientierung und dem Konzept der Marktorientierung. Dies geschieht aufgrund der Tatsache, dass oftmals sowohl Kunden als auch Wettbewerber zu den Stakeholdern eines Unternehmens gerechnet werden. Diese beiden Stakeholder-Gruppen werden aufgrund ihrer Wichtigkeit für das anbietende Unternehmen auch als primäre Stakeholder bezeichnet. ${ }^{372}$

In zahlreichen Konzeptualisierungen und Operationalisierungen von StakeholderOrientierung werden folglich explizit Kunden und Wettbewerber als Stakeholder

\footnotetext{
370 Vgl. Neuenburg 2010, S. 59 f.

${ }^{371}$ Vgl. hierzu und im Folgenden Henriques/Sadorsky 1999, S. 89.

372 Vgl. Ferrell et al. 2010, S. 95.
} 
bezeichnet. ${ }^{373}$ Aber auch umgekehrt wird in Konzeptualisierungen von Marktorientierung mitunter berücksichtigt, dass neben den primären Stakeholdern auch weitere Stakeholder als Einflussfaktoren in die Unternehmensausrichtung einbezogen werden müssen. Beispielsweise berücksichtigen MATSUNO ET AL. in ihrer Konzeptualisierung von Marktorientierung solche zusätzlichen Einflussfaktoren. Die Autoren sind dabei der Auffassung, dass ein Unternehmen die verschiedenen am Marktgeschehen direkt und indirekt Beteiligten berücksichtigen muss, um marktorientiert handeln zu können. Neben den Wettbewerbern und Kunden, die bekanntermaßen einen großen Einfluss auf das Handeln des Unternehmens ausüben können, zählen die Autoren hierzu zusätzlich Lieferanten, Regulierungsbehörden, soziale/kulturelle Trends und die makroökonomische Umwelt. ${ }^{374}$

Das Feld der Stakeholder schließt somit mögliche Einflussnehmer, vom Unternehmen Beeinflusste oder am Kaufprozess nicht unmittelbar beteiligte Drittparteien ein und findet teilweise schon Berücksichtigung in der Marktorientierungsliteratur (vgl. Abschnitt 4.3.3.2).

Im Rahmen der vorliegenden Untersuchung soll das Feld der Stakeholder, die sinnvoll im Rahmen von Marktorientierung berücksichtigt werden sollen, begrenzt werden. Dazu folgt die Arbeit im Folgenden dem Stakeholder-Verständnis von MAVONDO/FARRELL. Demnach werden nur solche Marktteilnehmer und Beeinflusser als relevante Drittparteien betrachtet, deren Handlungen einen direkten Einfluss auf die Erwartungen und Bedürfnisse der Kunden und letzten Endes auf deren Kaufentscheidungen ausüben. ${ }^{375}$ Diese Sichtweise ist auch konsistent mit der Auffassung von Marktorientierung von FERRELL ET AL., die den Blickwinkel der Marktorientierung über die Kunden und Konkurrenten eines Unternehmens hinaus nur auf diejenigen Stakeholder erweitern, deren Beziehung zum Unternehmen einen Einfluss auf das Kaufverhalten der aktuellen und potentiellen Unternehmenskunden ausübt (vgl. Abbildung 6). ${ }^{376}$ Das bedeutet auch,

${ }^{373}$ Vgl. Greenley/Foxall 1996; Yau et al. 2007; He et al. 2011.

${ }^{374}$ Vgl. Matsuno et al. 2005, S. 7.

${ }^{375}$ Vgl. Mavondo/Farrell 2000, S. 224.

${ }^{376}$ Vgl. Ferrell et al. 2010, S. 95. 
dass beispielsweise Anteilseigner und Beschäftigte des Unternehmens im Rahmen der Marktorientierung als Teil desselben und nicht etwa als einflussnehmende Drittpartei wie bei der Stakeholder-Orientierung betrachtet werden. Auch SHAPIRO ist der Ansicht, dass ein Unternehmen insbesondere solche Einflussnehmer beobachten muss, die, obwohl sie keine Geschäftsbeziehung mit dem anbietenden Unternehmen führen, das Kaufverhalten der Kunden beeinflussen und bei der Gestaltung der Produkte Einfluss nehmen auch wenn sie nicht einmal bei der Benutzung der betreffenden Produkte involviert sind. ${ }^{377}$

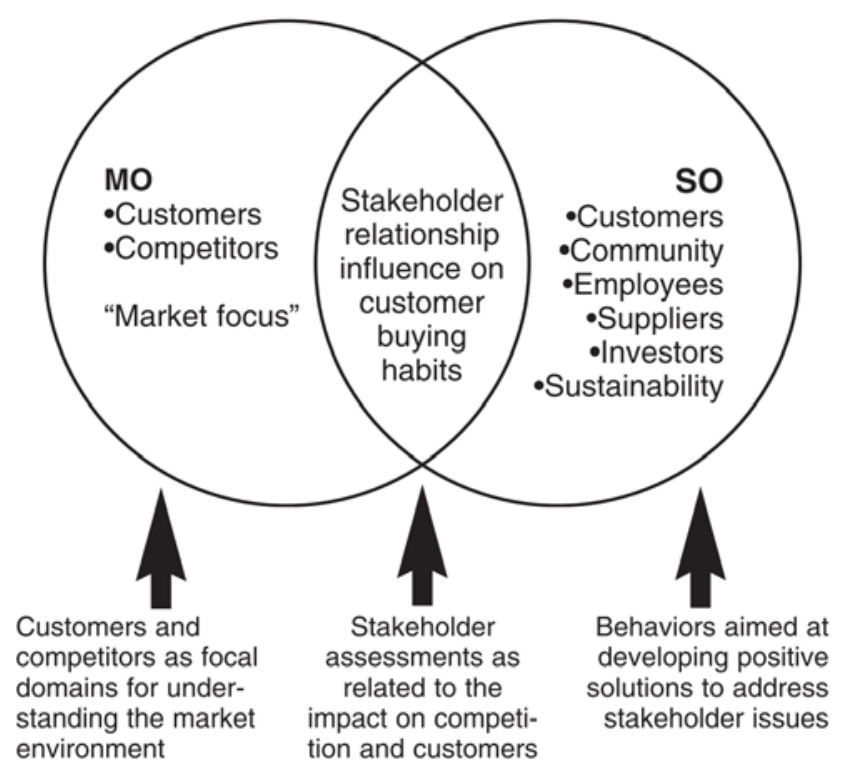

Abbildung 6: Überschneidung von Markt- und Stakeholder-Orientierung (Quelle: Ferrell et al. 2010, S. 95)

Trotz der in der Literatur erfolgten Vermengung von Kunden, Wettbewerbern und einflussnehmenden Drittparteien soll in dieser Arbeit eine strikte Trennung dieser Gruppen von direkten und indirekten Marktteilnehmern getroffen werden, um eine differenzierte Marktbetrachtung zu ermöglichen. Diese Arbeit folgt dabei

377 Vgl. Vgl. Shapiro 1988, S. 121. 
der Auffassung von GOTTELAND ET AL., die in diesem Zusammenhang konstatieren: „stakeholder orientation is considered as an extension of market orientation to include all agents affecting the processes and performance of organizations". 378

Letztlich kann die Einflussnahme auf das Kaufverhalten der Kunden durch Drittparteien bewusst oder unbewusst geschehen und sich über die Stufe der direkten Abnehmer hinaus auch auf alle nachfolgenden Marktstufen bis hin zum Endkunden erstrecken. Die Unterschiedlichkeit der Drittparteien spiegelt sich neben den diversen verfolgten Interessen auch in den jeweiligen Möglichkeiten der Einflussnahme wider. Für Unternehmen ist es daher nur schwer möglich, alle Drittparteien in ausreichendem Maße zu berücksichtigen. ${ }^{379}$ Aufgrund der begrenzten Ressourcen ist es daher für Unternehmen notwendig, diejenigen Drittparteien zu identifizieren, die den größten Einfluss auf die Wettbewerbsposition des Anbieters ausüben und eine Anpassung der Unternehmensstrategie erzwingen können. ${ }^{380}$ Eine Gleichgewichtung aller relevanten Drittparteien ist somit im Rahmen einer Drittparteienorientierung nicht zielführend. ${ }^{381}$ Vielmehr sollten Drittparteien nach ihrer Wichtigkeit klassifiziert werden. ${ }^{382}$ So können den Mitarbeitern des Anbieterunternehmens Anhaltspunkte für eine entsprechende Ausrichtung der Unternehmensaktivitäten gegeben werden.

Vor dem Hintergrund der großen und zahlreichen Möglichkeiten zur Einflussnahme durch Drittparteien auf die Absatzchancen eines Anbieters soll Drittparteienorientierung in dieser Arbeit als weitere eigenständige Dimension von Marktorientierung berücksichtigt werden. Drittparteienorientierung wird wie folgt definiert: Drittparteienorientierung bezeichnet die Berücksichtigung von Personen oder Organisationen, die sich nicht unmittelbar in der Wertschöpfungskette befinden, sondern im Umfeld der betreffenden Marktstufen agieren. Diese Drittparteien üben auf den jeweiligen Marktstufen einen Einfluss auf die Kaufentschei-

${ }^{378}$ Gotteland et al. 2007, S. 51.

${ }^{379} \mathrm{Vgl}$. Clarkson 1995.

${ }^{380}$ He et al. 2011, S. 70

${ }^{381}$ Zur Gleichgewichtung unterschiedlicher Unternehmenszielgruppen vgl. Donaldson/Preston 1995.

382 Vgl. Freeman 1994. 
dung der dortigen Kunden bzw. der Kunden der Kunden aus.

\subsubsection{Marktstufenorientierung}

Auf Industriemärkten durchlaufen die Produkte eines Lieferanten in der Regel mehrere Marktstufen, bevor sie ihrer Endverwendung zugeführt werden. ${ }^{383} \mathrm{Da}$ der Erfolg eines Anbieters aber vom Absatz seiner Produkte durch die gesamte Wertschöpfungskette bestimmt wird, ist es für den Anbieter von hoher Wichtigkeit, über diese Marktstufen informiert zu sein und gegebenenfalls seine Unternehmensaktivitäten darauf auszurichten. Wenn der Anbieter weiß, welchen Nutzen die Kunden der Kunden erwarten, wird es ihm möglich sein, seine direkten Kunden mit den entsprechenden wertstiftenden Komponenten zu beliefern. Vor dem Hintergrund der zu schaffenden Werte für die jeweils nachgelagerten Marktstufen schreibt FLINT: „firms must understand what their immediate downstream customers and their customers' customers value, ideally through to what end-use customers value." 384

Da alle Nachfrage ursprünglich von den Endkunden ausgeht, sollte diesen auch verstärkte Aufmerksamkeit gewidmet werden. ${ }^{385}$ Schließlich werden alle Rohstoffe, Komponenten, Teile etc. verarbeitet und zusammengesetzt, um mittelbar beim direkten und weiteren nachgelagerten Kunden Nutzen zu stiften.

Für einen Anbieter ist es daher essentiell, die einzelnen Stationen in der Wertschöpfungs- und Absatzkette seiner Produkte zu kennen und diese nachverfolgen zu können. Ein Lieferant in einem mehrstufigen Markt muss sich daher „zunächst Klarheit darüber [...] verschaffen, auf welchen Wegen seine Produkte in die nachfolgenden Marktstufen gelangen und was von dort aus weiter mit ihnen geschieht.“" ${ }^{386}$ Der Lieferant muss dazu die nachfolgenden Marktstufen beobachten und analysieren, auf welchen Marktstufen sein Produkt verändert, weiterverarbeitet oder einfach nur als fertige Komponente beispielsweise in eine Anlage eingesetzt wird. Auch reine Weiterveräußerungen durch Handelsunternehmen können

\footnotetext{
383 Vgl. Kleinaltenkamp/Rudolph 2002, S. 287.

${ }^{384}$ Flint 2004, S. 46.

385 Vgl. Coley et al. 2010, S. 143; Kibbeling et al. 2013.

${ }^{386}$ Kleinaltenkamp/Rudolph 2002, S. 297.
} 
für den Weg eines Produktes durch die verschiedenen Marktstufen von großer Bedeutung sein.

In der Regel treten Lieferanten von Komponenten nur selten in direkte Geschäftsbeziehungen mit den Endabnehmern von Maschinen und Anlagen ein. Dennoch kommt es vor, dass der Endabnehmer die Einhaltung bestimmter Spezifikationen direkt oder über die Marktzwischenstufen vom Komponentenhersteller einfordert. Hier treten die Endabnehmer dann teilweise in direkten Kontakt mit den Vorlieferanten, um ihre Bedürfnisse unmittelbar zu artikulieren. Unter bestimmten Umständen werden auch die Komponentenlieferanten vom Endabnehmer vorgeschrieben. ${ }^{387} \mathrm{Um}$ als gelisteter Komponentenlieferant berücksichtigt zu werden, wird ein Anbieter, der seinen Absatz durch die Wertschöpfungskette sichern möchte, durch ein sogenanntes Signaling ${ }^{388}$ auf seine Leistungen auf den nachfolgenden Marktstufen aufmerksam machen. Dies ist insbesondere in hoch spezialisierten und gering vertikal integrierten Industrien der Fall. ${ }^{389}$ Für Lieferanten wird es umso wichtiger, die nachfolgenden Marktstufen genau zu beobachten und die Unternehmenstätigkeit darauf auszurichten, je größer der Projektcharakter, je individualisierter die einzelnen Leistungen bzw. Vorleistungen sind und je wichtiger damit die Leistungsfähigkeit des Vorlieferanten wird. Eine Ausrichtung auf die nachfolgenden Marktstufen sollte neben der Analyse der Bedürfnisse der Kunden der Kunden auch eine aktive Ansprache im Sinne einer mehrstufigen Kommunikationsleistung beinhalten.

Neben der Kenntnis über die Marktstufen selbst und den dort am Produkt jeweils verrichteten Tätigkeiten, sollte ein Unternehmen ,analysieren, welche Gegebenheiten auf den betreffenden Marktstufen herrschen, d.h. vor allem, welche Substitutions- und Komplementaritätsbeziehungen zwischen den jeweiligen Produkten existieren und welche Wettbewerbsverhältnisse gegeben sind.“" ${ }^{390}$ Denn die Produkte des Anbieters stehen nicht nur mit den direkten branchenzugehörigen Konkurrenten im Wettbewerb. Zusätzlich konkurriert der Anbieter auch mit jedem

${ }^{387}$ Vgl. Trommen 2002, S. 26.

${ }^{388}$ Vgl. Kaas 1991.

${ }^{389}$ Vgl. Kleinaltenkamp et al. 2011, S. 43.

390 Kleinaltenkamp/Rudolph 2002, S. 297. 
Unternehmen, das in der Lage ist, die Bedürfnisse der Nachfrager auf einer beliebigen Stufe zwischen Anbieter und Endabnehmer zu befriedigen und lösungsäquivalente Werte zu schaffen. ${ }^{391}$ PETERAF/BERGEN sprechen in diesem Zusammenhang von funktionalen Substituten und entwickeln ein Muster zur Identifizierung von aktuellen und potentiellen Wettbewerbern. Die Autoren legen besondere Bedeutung auf die Substitutionsmöglichkeit durch Produkte bzw. Unternehmen, die mit scheinbar unähnlichen Lösungen dieselben Kundenbedürfnisse befriedigen. Die Autoren beschränken den Wettbewerb daher weniger auf die Unternehmen aus der unmittelbaren Branche, sondern sehen vielmehr Wettbewerb in allen funktional ähnlichen Produkten. ${ }^{392}$ Ein anschauliches Beispiel dazu liefert JONSSON, der für den Bereich der Holzindustrie aufzeigt, dass nicht nur Unternehmen der Holzbranche miteinander im Wettbewerb stehen. Vielmehr konkurrieren diese Unternehmen auch mit solchen der chemischen Industrie, wenn jene bei den Endkunden dieselben Anforderungen erfüllen. Dies kann beispielsweise im Bereich von Fußböden gegeben sein, wo Holzparkett mit verschiedenen Laminatprodukten um die Bedürfniserfüllung der Hausbesitzer konkurriert. ${ }^{393}$ Das bedeutet für einen Anbieter, der sich an den nachfolgenden Marktstufen ausrichtet, dass er dort auch solche Unternehmen berücksichtigen muss, die in der Lage sind, Bedürfnisse der nachgelagerten Kunden oder Endkunden zu befriedigen und dabei die Produkte des Anbieterunternehmens zu substituieren.

Um die Dynamiken des Marktes weitreichender zu erfassen und darauf angemessen reagieren zu können, schlagen KLEINALTENKAMP ET AL. eine „multistage market orientation” vor. ${ }^{394}$ Dem wird hier gefolgt. Solch eine Marktstufenorientierung wird im Rahmen dieser Arbeit als eine Dimension von Marktorientierung wie folgt definiert: Marktstufenorientierung ist die Ausrichtung eines Anbieterunternehmens auf Marktstufen in der Wertschöpfungskette, die der Marktstufe dieses Anbieters nicht unmittelbar folgen. In diesen nachgelagerten

\footnotetext{
${ }^{391}$ Vgl. Srivastava et al. 2001, S. 794 f.

392 Vgl. Peteraf/Bergen 2003.

${ }^{393}$ Vgl. Jonsson 2005.

394 Vgl. Kleinaltenkamp et al. 2009, S. 2.
} 
Marktstufen kann das Produkt des Anbieters weiterverarbeitet, weiterveräußert, letztlich verwendet oder aber auch substituiert werden.

\subsubsection{Abteilungsübergreifende Koordination}

Die verschiedenen Tätigkeiten in einem Unternehmen bauen aufeinander auf und befinden sich in gegenseitiger zeitlicher oder funktionaler Abhängigkeit. Mit der Ausrichtung an den Unternehmenszielen kann jede Unternehmensfunktion einen Beitrag zur Wertschöpfung leisten oder diese zumindest unterstützten. ${ }^{395}$ Somit leistet jeder Mitarbeiter, jede Abteilung und das Management des Unternehmens durch die Verrichtung von Tätigkeiten einen Beitrag zur Erfüllung der Kundenwünsche. NARVER/SLATER stellen in diesem Zusammenhang fest: „[a]ny point in the buyer's value chain affords an opportunity for a seller to create value for the buyer firm.““396 Damit dies gelingt, muss den Mitarbeitern der einzelnen Abteilungen bewusst sein, wer die Kunden des Unternehmens sind, welche Bedürfnisse sie haben und welche Konkurrenzprodukte auf dem Markt existieren. Ebenso müssen den Mitarbeitern relevante Informationen bezüglich der weiteren Verarbeitungs- und Handelsstufen der eigenen Produkte und bezüglich einflussreicher Drittparteien zur Verfügung gestellt werden. Nur so ist es möglich, dass auch Abteilungen ohne direkten Kundenkontakt ein Verständnis dafür entwickeln, wie sie an der Schaffung von Kundennutzen beteiligt sind. Da das Wissen über die Kunden und andere Aspekte des Marktes allerdings nicht immer dort erhoben und ausgewertet wird, wo es benötigt wird, müssen solche Informationen im Unternehmen verbreitet und ausgetauscht werden. Nur so kann sichergestellt werden, dass das im Unternehmen vorhandene Wissen auch effektiv zur Generierung von Kundenwerten genutzt wird. Dies kann auf offiziellem Wege z.B. durch vom Management bereitgestellte Marktberichte oder inoffiziell durch den sogenannten Flurfunk geschehen. Die Informationsverarbeitung und -bereitstellung im Unternehmen leistet somit einen besonderen Beitrag zu einer unternehmensweiten Marktorientierung. ${ }^{397}$

${ }^{395}$ Vgl. Porter/Millar 1985, S. 150.

${ }^{396}$ Narver/Slater 1990, S. 22.

${ }^{397}$ Vgl. Hult et al. 2005, S. 1178. 
In Wissenschaft und Praxis wird hervorgehoben, dass eine enge Abstimmung der Unternehmensaktivitäten zwischen den verschiedenen Abteilungen und eine optimale Ausnutzung der Unternehmensressourcen dazu beitragen, die Leistungserstellung weitgehend effizient zu gestalten und Synergien zu nutzen. ${ }^{398}$ Neben dem Wissen selbst nimmt die Fähigkeit der Mitarbeiter, dieses Wissen zielgerichtet zu kombinieren und zu verteilen einen hohen Stellenwert als Ressource ein. Können die verschiedenen Abteilungen gemeinsam auf diese Ressourcen zuzugreifen, vermag das Unternehmen effizienter zu arbeiten und dadurch den Kunden Vorteile gegenüber Wettbewerbern zu bieten.

Zusammenfassend lässt sich sagen: Abteilungsübergreifende Koordination versteht sich als die koordinierte Nutzung von Marktwissen und Unternehmensressourcen über Abteilungsgrenzen hinweg zur Schaffung von überlegenen Werten für die Zielkunden. ${ }^{399}$

Mit den vorangegangenen Abschnitten konnte die Forschungsfrage 2 nach der sinnvollen Konzeptualisierung und Operationalisierung einer umfassenden Marktorientierung im Business-to-Business-Kontext für den konzeptionellen Teil beantwortet werden. Die Teilfrage nach der Operationalisierung wird in Abschnitt 4.6.1 beantwortet.

\subsection{Konsequenzen einer umfassenden Marktorientierung und Hypothesenbildung}

Die marktorientierte Ausrichtung eines Anbieterunternehmens stellt freilich keinen Selbstzweck dar, sondern soll Unternehmen auf verschiedenen Wegen zu einer überlegenen Wettbewerbsposition verhelfen. Dabei spielen die von der Marktorientierung beeinflussten Fähigkeiten eine entscheidende Rolle, die auf die Schaffung und Vermarktung von Kundenwerten abzielen. Im Folgenden sollen daher einzelbetriebliche und kooperative, unternehmensübergreifende Unternehmenstätigkeiten dargestellt werden, durch die aus dem gewonnenen Marktwissen Werte für den Kunden generiert werden können. Die nachfolgenden Ab-

${ }^{398}$ Vgl. Narver/Slater 1990, S. 22; Grühn/Hein 2012, S. 14. 
schnitte sollen daher auf theoretischer Basis einen Beitrag zur Beantwortung der Forschungsfrage 3 liefern, die sich mit dem Einfluss von Marktorientierung auf Unternehmensaktivitäten und den letztlich erzielten Erfolg beschäftigt.

\subsubsection{Unternehmenskollaboration}

In der Literatur scheint unbestritten, dass sich die Absatzmärkte eines Unternehmens immer schneller wandeln, sei es durch Globalisierung, technologische Veränderungen oder immer kürzere Produktlebenszyklen in gesättigten Märkten. ${ }^{400}$ All dies ist keine neue Entwicklung der letzten Jahre. Unternehmen, die versuchen, im Wettbewerb erfolgreich zu sein, sehen jedoch eine Möglichkeit, diesen Marktentwicklungen zu begegnen, darin, verstärkt Kooperationen mit den Unternehmen der eigenen Wertschöpfungskette einzugehen. ${ }^{401}$ Solche Kooperationen können innerhalb der Wertschöpfungskette auf der gleichen Wertschöpfungsstufe zum Beispiel mit einem Wettbewerber horizontal oder mit einem Vertriebspartner oder weiterverarbeitenden Unternehmen vertikal erfolgen. ${ }^{402}$ Eine vertikale Kooperation bedeutet, dass sich die zusammenarbeitenden Unternehmen auf unterschiedlichen, nachfolgenden Stufen in der Wertschöpfungskette befinden. Oftmals werden vertikale Kooperationen eingegangen, um Produktinnovationen zu beschleunigen und somit durch die Zusammenarbeit von Anbieter und Nachfrager die Bedürfnisse des Endkunden schneller und besser zu befriedigen. ${ }^{403}$

In der heutigen Zeit erwarten Geschäftskunden, dass Lieferanten nicht nur effektiv die bestehenden Wünsche des Kunden befriedigen, sondern auch ein ausreichendes Verständnis für die Geschäftstätigkeit des Kunden besitzen, um so latente Wünsche erkennen und erfüllen zu können. ${ }^{404}$ Dies kann indes nur gelingen, wenn der Lieferant Informationen über das Geschäft seines Kunden besitzt. Diese

${ }^{399}$ Vgl. Narver/Slater 1990, S. 22.

400 Vgl. Schumpeter 1939, S. 86; Specht 1997, S. 402 f.; Weiber et al. 2006, S. 84 f.; Lisboa et al. 2011.

${ }^{401}$ Vgl. Hyvönen/Tuominen 2007; Martin/Grbac, 2003; Buzzell/Ortmeyer, 1995; Buchanan 1992.

402 Der Begriff der Unternehmenskollaboration wird in der Literatur nicht einheitlich gebraucht. Für dieselben Sachverhalte werden die Begriffe Collaboration, Cooperation, Alliance und Integration synonym verwendet (vgl. Singh 1997; Pinto/Pinto 1990).

${ }^{403}$ Vgl. Tracey et al. 2014, S. 3; Günter 2013, S. 390.

${ }^{404}$ Vgl. Blocker et al. 2011, S. 216. 
Informationen betreffen somit maßgeblich die Abnehmer des Kunden. Da Informationen jedoch zwischen Kunde und Lieferant asymmetrisch verteilt sind, müssen die beiden Unternehmen sich gegenseitig Informationen über den Markt der letztlich abzusetzenden Produkte bereitstellen sowie prozess-, entwicklungs- und produktionstechnisches Wissen miteinander austauschen. ${ }^{405}$ Im Sinne des Relational Views stellen der Austausch von Informationen und die Abstimmung bestimmter Prozesse bei der Produktentwicklung eine beziehungsspezifische Investition der Kooperationspartner dar. Die kooperierenden Unternehmen bringen dazu Teile ihrer Ressourcen und Fähigkeiten in die Beziehung ein, so dass auch das Partnerunternehmen darauf zurückgreifen kann.

Das Risiko für opportunistisches Verhalten eines Partners gegenüber dem anderen erscheint jedoch hoch. Ein Unternehmen könnte schließlich die Zusammenarbeit zur Aneignung technischer Expertise ausnutzen. Auch das Wissen über die Absatzprognosen des Kunden könnte z.B. primär zur Stärkung der Verhandlungsposition des Lieferanten oder zur Schwächung der Wettbewerbsposition des Kunden ausgenutzt werden. ${ }^{406}$ Damit nicht ein Unternehmen den Nutzen der in die Partnerschaft eingebrachten Ressourcen und Fähigkeiten übermäßig für sich in Anspruch nehmen kann, müssen innerhalb der Kooperation Mechanismen greifen, die einen Missbrauch verhindern. Unternehmen werden schließlich nur in Kooperationen einwilligen, wenn sie sich sicher sein können, dass bspw. das übertragene Wissen nur im Rahmen der Zusammenarbeit verwendet und nicht durch das Partnerunternehmen unberechtigterweise weitergegeben wird. Zudem werden die beteiligten Unternehmen darauf bedacht sein, die angestrebten Ergebnisse vorab exakt zu vereinbaren und sich auch über die Risikoverteilung zu einigen. Die Absicherung einer Unternehmenszusammenarbeit wird zudem stark von konkreten früheren Erfahrungen mit dem interessierenden Kooperationspartner oder von Kooperationserfahrungen im Allgemeinen geprägt. Durch vorangegangene Partnerschaften wird einem Anbieter zudem bewusst, welche Art von Ressourcen er selber einbringen kann und welche Fähigkeiten und Ressour-

\footnotetext{
${ }^{405}$ Vgl. Ragatz et al. 2002; Sinkovics/Roath 2004, S. 46; Eichentopf et al. 2011, S. 651.
}

${ }^{406}$ Vgl. Fang et al. 2008, S. 99; Li 2002, S. 1197; Xu/Beamon 2006. 
cen er vom Kooperationspartner einfordern muss, um eine möglichst hohe Kooperationsrente aus der Zusammenarbeit zu ziehen. Grundbedingung für eine Unternehmenszusammenarbeit von Lieferant und Kunde sind also für alle Beteiligten vorteilhafte Auswirkungen dieser Zusammenarbeit, bei denen die Synergieeffekte der eingebrachten Ressourcen genutzt werden können. ${ }^{407}$

Bei einer Unternehmenskollaboration zur Entwicklung von eher komplexen Produkten kann davon ausgegangen werden, dass auch die Zusammenarbeit eine gewisse zeitliche Spanne andauert. Während dieser Zeit werden nicht nur Ressourcen in die Beziehung eingebracht, es wachsen auch soziale Bindungen zwischen den Mitarbeitern des Anbieters und des Kunden. Eine Unternehmenszusammenarbeit stellt somit, sofern sie langfristig und partnerschaftlich angelegt ist, eine historisch gewachsene und sozial komplexe Fähigkeit dar, die es für ein Unternehmen wettbewerbsstrategisch zu nutzen gilt. Marktorientierung kommt im Zusammenhang der kooperativen Leistungsentwicklung eine wesentliche Koordinationsfunktion zu. Im Einklang mit dem kompetenzbasierten Ansatz sorgt Marktorientierung als Unternehmenskompetenz dafür, dass die Potenziale der von den Kooperationspartnern jeweils eingebrachten Ressourcen und Fähigkeiten möglichst vollständig und zielgerichtet ausgeschöpft werden.

Damit die Ziele einer Unternehmenszusammenarbeit dem Anbieter klar werden und durch die Zusammenarbeit erreicht werden können, muss der Anbieter über weitreichende Marktinformationen verfügen. Informationen über den eigenen Kunden zu besitzen, kann die Unternehmenszusammenarbeit insofern erleichtern und fördern, als dem Anbieter bereits zu Beginn der Kooperation bewusst wird, wo bspw. technologische und wirtschaftliche Schwächen oder Stärken des Kunden liegen und folglich eigene Ressourcen und Fähigkeiten für eine erfolgreiche Zusammenarbeit eingebracht werden müssen. Auch das Wissen über bestimmte Strukturen und Abläufe beim Kundenunternehmen sind für die Zusammenarbeit im Hinblick auf eine Prozessintegration und -abstimmung förderlich. Wenn ein Lieferant eine Kollaboration eingeht, ist es auch notwendig, über Wissen zu verfügen, das über den eigenen Absatzmarkt hinausgeht. Das Unternehmen muss

${ }^{407}$ Vgl. Jap 2001, S. 87; Barratt 2004, S. 36; Dyer/Singh 1998, S. 667. 
zusätzlich Wissen über die Abnehmer des eigenen Kunden und über die Dynamiken der nachgelagerten Marktstufen besitzen. Diese Dynamiken umfassen vor allem Wettbewerbsverhältnisse, Markttrends und das Verhalten von Personen und Organisationen, die die nachgelagerten Absatzmärkte beeinflussen können. Insbesondere vor dem Hintergrund des sogenannten Bullwhip Effects ist eine genaue Absatzplanung erforderlich, um kostspielige Produktions- und Lagerschwankungen zu vermeiden. ${ }^{408}$ Aufgrund von zeitlichen Verzögerungen und mangelndem Marktwissen kann sich nämlich eine fehlerhafte Absatzprognose z.B. in Folge von nicht innerhalb der Wertschöpfungskette abgesprochenen kurzfristigen Preisreduktionen im Konsumentenbereich durch die gesamte Wertschöpfungskette fortsetzen und verstärken. Auf diese Weise kann der Bullwhip Effect betriebliche Fehlplanungen auf den vorgelagerten Marktstufen verursachen. ${ }^{409} \mathrm{Je}$ ausgeprägter dieses Wissen über nachgelagerte Marktstufen ist, desto einfacher erscheint es, im Rahmen einer Kollaboration Verständnis für die Geschäftssituation des Kunden zu entwickeln, entsprechend auf die Belange des Kunden einzugehen und die Zusammenarbeit zu intensivieren.

Das im Unternehmen vorhandene Wissen muss zudem im eigenen Unternehmen verbreitet werden, so dass die verschiedenen Abteilungen und Unternehmensfunktionen die strategische Ausrichtung des eigenen Unternehmens und die Zusammenarbeit mit den Kunden besser verstehen. ${ }^{410}$ Die Ausrichtung am Wettbewerb hingegen hat keinen Einfluss auf die Ausgestaltung der Unternehmenszusammenarbeit. Zwar können Informationen über Wettbewerbsprodukte dazu führen, dass ein Anbieter gerade solche Produkte entwickelt, die sich vom Wettbewerb differenzieren, doch sollte die gemeinsame Produktentwicklung mit dem direkten Kunden für dessen Kunden dadurch nicht berührt werden.

Zusammenfassend lässt sich sagen, dass eine Unternehmenszusammenarbeit nur funktionieren kann, wenn der Lieferant eine Marktorientierung besitzt, die neben den eigenen Kunden auch auf nachgelagerte Abnehmer und weitere Einflussfaktoren ausgerichtet ist. Eine umfassende Marktorientierung ist demzufolge eine

${ }^{408} \mathrm{Vgl}$. Tabe et al. 1980.

${ }^{409}$ Lee et al. 2004. 
Voraussetzung, um im Rahmen einer Unternehmenskollaboration gemeinsam Produkte oder Dienstleistungen zu entwickeln, die der direkte Kunde im Prozess der Leistungserstellung für seine Kunden benötigt. Daraus lässt sich die folgende Hypothese ableiten:

H1: Je höher der Grad der Marktorientierung ist, desto stärker ist die Zusammenarbeit zwischen Lieferanten und Kunden ausgeprägt.

\subsubsection{Innovativität}

Im Unternehmen generiertes und verteiltes Wissen kann dazu dienen, Wettbewerbsvorteile zu erlangen. Wenn dieses Wissen Informationen über Kunden, Wettbewerber, Markt- und Technologieveränderungen beinhaltet, können damit Innovationen bzw. dem Wettbewerb überlegene Produkte geschaffen werden. ${ }^{411}$ Ein Unternehmen kann im Sinne des ressourcenbasierten Ansatzes Produkte anbieten, die den Kunden dadurch einen Mehrwert bieten, dass sie den Nutzen vergrößern oder die mit dem Kauf und der Nutzung des Produktes verbundenen Kosten senken. ${ }^{412}$ Unternehmen, die entsprechend Wissen über ihre Märke sammeln, können Möglichkeiten zur Veränderung und Verbesserung ihrer Produkte oder Prozesse ausfindig machen. ${ }^{413}$ Die Fähigkeit, neues und im Unternehmen bestehendes Wissen zu sammeln, aufbereiten und weiterentwickeln zu können, sehen GRIESE ET AL. als eine der zentralen Voraussetzungen für Innovativität an. Die Autoren erachten neben einer dafür ausreichenden Qualifikation der Mitarbeiter ebenfalls ein entsprechendes kulturelles Unternehmensumfeld als notwendig. ${ }^{414}$ Schließlich bedingt der Besitz von Wissen alleine noch keine Innovationen oder Wettbewerbsvorteile. ${ }^{415}$ Dies gilt vor allem, weil die Beschaffung von Marktwissen seit der Verbreitung und dem weitflächigen Einsatz von IT in Un-

\footnotetext{
410 Vgl. Day 1994, S. 45.

411 Vgl. Griese 2011, S. 158; Calantone 2002, S. 516. MENGUC/AUH (2006, S. 63) und VARADARAJAN/JAYACHANDRAN (1999, S. 129) betrachten Neuprodukterfolg als Maß für Innovationen.

412 Vgl. Narver/Slater 1990, S. 21.

413 Vgl. Hurley/Hult 1998, S. 44.

414 Vgl. Griese et al. 2012, S. 472.

415 Vgl. Griese 2011, S. 158.
} 
ternehmen deutlich leichter geworden ist. ${ }^{416}$ Wissen über Kunden und Wettbewerber alleine stellt nämlich für ein Unternehmen nicht mehr einen so starken Wettbewerbsvorteil dar wie etwa vor 20 Jahren noch. MOORMAN ET AL. verweisen daher auf die Nutzung des verfügbaren Wissens und resümieren in diesem Zusammenhang: „sustainable competitive advantage will depend less on who has information and increasingly on who is able to make the best use of that information”. ${ }^{417}$ Die Wissensnutzung bzw. die Bereitschaft dazu im Sinne von können und wollen kann Innovationen hervorbringen. Innovationen stellen also den primären Zweck der Wissensnutzung dar. Das Mittel zum Zweck ist die Innovativität eines Unternehmens. ${ }^{418}$ MENGUC/AUH schreiben dazu: „innovativeness, unlike innovation, is not an end but rather a means to an end”. ${ }^{419}$ Der Begriff der Innovativität ist in der Literatur jedoch nicht eindeutig beschrieben. ${ }^{420}$ Hauptsächlich finden sich kulturbezogene Definitionen wie „,...] the notion of openness to new ideas as an aspect of a firm's culture [...] the organization's orientation toward innovation” ${ }^{421}$ oder fähigkeitsbezogene Definitionen wie „the capacity to introduce [...] some new process, product, or idea in the organization”, ${ }^{422}$ Ähnlich wie bei der Marktorientierung ist somit auch bei der Innovativität eine enge Verbindung von Willen und Können zur erfolgreichen Entwicklung von Innovationen gegeben.

Die Innovativität eines Unternehmens wird zudem durch die Auswahl, Verknüpfung und Nutzung von strategischen Ressourcen und Fähigkeiten bestimmt, die zur Erstellung von Innovationen notwendig sind. ${ }^{423}$ Bei der Auswahl und Verknüpfung von Ressourcen und Fähigkeiten wird Marktorientierung und hierbei insbesondere der Aspekt der Kundenorientierung als wichtige Ausprägung einer

\footnotetext{
416 Vgl. Davis/Golicic 2010, S. 56 f.

417 Moorman et al. 1992, S. 314.

418 In der englischsprachigen Literatur werden zum Thema der Innovativität neben dem Begriff der innovativeness auch die Begriffe innovation orientation, innovation capability und innovation effort verwendet.

${ }^{419}$ Menguc/Auh 2006, S. 66.

${ }^{420}$ Für eine Übersicht der verschiedenen Definitionen von Innovativität vgl. Siguaw et al. 2006.

${ }^{421}$ Hurley/Hult 1998, S. 44. Vgl. auch Zhou et al. 2005, S. 1050; Derenthal 2009, S. 23; Amabile 1997, S. 52; Siguaw et al. 2006, S. 560.

422 Hult et al. 2004, S. 430. Vgl. auch Teichert/Bouncken 2011, S. 101.

${ }^{423}$ Vgl. Gersch et al. 2014, S. 170.
} 
Unternehmenskultur angesehen. ${ }^{424}$ Dahinter verbirgt sich die Implementierung des Marketingkonzepts im Unternehmen, welches den Interessen der Kunden höchste Priorität einräumt. ${ }^{425}$ Kundenorientierung bringt ein Unternehmen also dazu, kontinuierlich und proaktiv Wissen über die Bedürfnisse der Kunden zu sammeln, diese erfüllen zu wollen und dafür neue Produkte zu entwickeln. ${ }^{426}$ In der Literatur wird allerdings auch vor einer zu starken Ausrichtung auf die Kunden gewarnt. Die damit verbundene eingeengte Sichtweise des Marktes und Vernachlässigung der Bedürfnisse von potentiellen Kunden kann somit der Innovativität auch abträglich sein. Empirische Studien zeigen daher gemischte Resultate in Bezug auf diese Wirkbeziehung. ${ }^{427}$

Bei der Produktentwicklung erscheint eine alleinige Ausrichtung an Kundenbedürfnissen jedoch nicht zielführend, da hierbei die Reaktionen der Wettbewerber auf die neuen Produkte vernachlässigt werden. ${ }^{428}$ DAY/WENSLEY schlagen daher vor, auch Aktionen und Produkte der Konkurrenz zu berücksichtigen, um einen Wettbewerbsvorteil zu erreichen. ${ }^{429}$ Um mit Wettbewerbern Schritt zu halten bzw. diesen einen Schritt voraus zu sein, muss Wissen über Wettbewerber und insbesondere über deren technologischen Stand in die Entwicklung von neuen Produkten einfließen. ${ }^{430}$ Ein zu starker Fokus auf Wettbewerbsaktivitäten wird jedoch in der Literatur teilweise auch als innovationshemmend beschrieben, da ein Unternehmen riskiere, zu viele Ressourcen einzusetzen, um lediglich Wettbewerber zu kopieren, anstatt eigene innovative Produkte zu entwickeln. ${ }^{431}$ So zeigen sich auch hier wieder gemischte empirische Ergebnisse innerhalb der wissenschaftlichen Literatur. ${ }^{432}$ Trotz der diametralen Ergebnisse einiger Studien erscheint eine intensive Wettbewerbsanalyse dennoch notwendig, um solche Produkte zu entwickeln, die als innovativ angesehen werden und sich von Wett-

424 Vgl. Deshpandé et al. 1993; Narver/Slater 1990.

${ }^{425}$ Vgl. Han et al. 1998, S. 33; Deshpandé et al. 1993, S. 24.

${ }^{426}$ Vgl. Han et al. 1998, S. 33; Plinke 1992, S. 833.

${ }^{427}$ Vgl. Verhees/Meulenberg 2004; Im/Workman 2004, S. 124.

${ }^{428}$ Vgl. Narver/Slater 1990, S. 21 f.; Day/Wensley 1988; Armstrong/Colloppy 1996.

${ }^{429}$ Vgl. Day/Wensley 1988.

${ }^{430}$ Vgl. Han et al. 1998, S. 34.

${ }^{431}$ Vgl. Olsen et al. 2005, S. 51; Spanjol et al. 2011, S. 239.

${ }^{432}$ Vgl. Im/Workman 2004, S. 124; Derenthal 2009; S. 226; Han et al. 1998. 
bewerbsprodukten differenzieren.

Auch Wissen über Drittparteien und das weitere Marktumfeld im Sinne von weiteren Absatz- und Weiterverarbeitungsstufen kann der Entwicklung von neuen Produkten dienen. Zwar wird die Bedeutung der Drittparteien in verschiedenen Wertschöpfungsstufen stark unterschiedlich eingeschätzt; dennoch kann angenommen werden, dass auch das Wissen über Organisationen und Individuen, die Einfluss auf die Präferenzen der Kunden ausüben, den Produktentwicklungsprozess fördert. ${ }^{433}$ So kann die Beobachtung von Einflussnehmern Aufschluss darüber geben, welchen Trends die Kundenbedürfnisse möglicherweise unterliegen werden und wie darauf mit neuen Produkten entsprechend reagiert werden kann. Hervorzuheben ist hier, dass es sich bei der Wissenssammlung durch das Einbeziehen der nachfolgenden Marktstufen und durch die Berücksichtigung von einflussnehmenden Drittparteien um eine proaktive Form der Marktorientierung handelt. Auf Grundlage dieses Wissens kann ein Anbieter antizipieren, inwiefern die Bedürfnisse des direkten Kunden geformt und beeinflusst werden. Eine proaktive Marktorientierung steht im Gegensatz zu einer reaktiven Marktorientierung, die lediglich die artikulierten Bedürfnisse der aktuellen, direkten Kunden berücksichtigt. Solch eine reaktive Art der Marktorientierung kann die Innovationsfähigkeit im Unternehmen behindern und sich negativ auf die Generierung von neuen, erfolgreichen Produkten auswirken. ${ }^{434}$

Im Unternehmen wird Wissen oftmals an verschiedenen Stellen generiert und wiederum an anderer Stelle, meist in der Forschungs- und Entwicklungsabteilung, in physische Produkte umgesetzt. Eine Nutzbarmachung des im gesamten Unternehmen verteilten Wissens über den Markt und das Marktumfeld bedingt eine koordinierte Zusammenarbeit der verschiedenen Abteilungen. Indem verschiedene Unternehmensabteilungen zusammenarbeiten und ein gemeinsames Ziel verfolgen, können die Kapazitäten und Fähigkeiten der Mitarbeiter gesteigert werden. ${ }^{435}$ Wenn ein Unternehmen in der Lage ist, seine gesamten Ressourcen durch

\footnotetext{
433 Vgl. Fischer 2014, S. $160 \mathrm{ff}$.

434 Vgl. Berthon et al. 1999; Christensen/Bower 1996; Narver et al. 2004; Zhou et al. 2005.

435 Vgl. Han et al. 1998, S. 34f.; Xie et al. 2003. Vgl. auch Diller/Saatkamp 2002 zu Problemen im

Marketingprozess durch unzureichende Koordination und Zielsetzung.
} 
Abstimmung zwischen den Abteilungen effizient einzusetzen, können Wettbewerbsvorteile generiert werden. ${ }^{436}$ Insbesondere, um neue Produkte zu entwickeln, müssen sich Unternehmen auf die Zusammenarbeit der Marketing- sowie Forschungs- und Entwicklungsabteilung vom Beginn der Produktentwicklung bis zum fertigen Produkt verlassen können. ${ }^{437}$ Ist die Zusammenarbeit der Abteilungen und der Wille, Wissen im Unternehmen zu teilen, hingegen gering ausgeprägt, verringert sich auch die Innovativität des Unternehmens. ${ }^{438}$ Nur durch entsprechend ausgeprägte Abstimmungsprozesse zwischen Abteilungen kann das erworbene Marktwissen voll genutzt werden. ${ }^{439}$ CLERQ ET AL. fassen zusammen: „collaboration among functional departments provides a necessary platform for new product ideas". ${ }^{40}$

Innovativität stellt die Form des organisationalen Willens und die Fähigkeit dar, innovative, auf Marktwissen basierende Produkte den Kunden anzubieten. Eine unternehmensweite Marktorientierung mit dem Ziel, Kundenzufriedenheit zu erreichen, fördert somit die Innovationstätigkeit des Unternehmens. ${ }^{441}$ Dies führt zu folgender Hypothese:

H2: Je höher der Grad der Marktorientierung ist, desto stärker ist die Innovativität eines Unternehmens ausgeprägt.

\subsubsection{Anpassung der Marketingstrategien}

Da, wie in Abschnitt 2.2.1 gezeigt, die Nachfrage nach Leistungen im B2BBereich letztlich auf die Erfüllung der originären Endkundenbedürfnisse zurückgeht, müssen folglich alle Unternehmen einer Wertschöpfungskette direkt oder indirekt zur Erfüllung der abgeleiteten Nachfrage beitragen. Die gesamte Wertschöpfungskette steuert somit durch das Angebot von Komponenten oder Dienstleistungen zum finalen Produkt bei, das der Endkunde zur Befriedigung seiner Wünsche benötigt. Dazu dürfen Anbieter nicht mehr lediglich ihr Geschäft mit

${ }^{436}$ Vgl. Wooldridge/Minsky 2002.

${ }^{437}$ Vgl. Ernst et al. 2010.

${ }^{438}$ Vgl. Clerq et al. 2013, S. 60; Menon/Varadarajan 1992, S. 62.

${ }^{439}$ Vgl. Li/Clantone 1998, S. 15.

${ }^{440}$ Clerq et al. 2013, S. 56.

${ }^{441}$ Vgl. Han et al. 1998, S. 33; Nasution et al. 2011, S. 341; Jaworski/Kohli 1993, S. 56 f. 
dem unmittelbaren Kunden betrachten, sondern müssen eine marktstufenübergreifende Wertschöpfungsketten-Sichtweise annehmen. ${ }^{442}$ Nach dieser Sichtweise sind alle Unternehmen als Teil der Wertschöpfungskette daran beteiligt, dem Kunden bzw. Endkunden den größtmöglichen Nutzen zu liefern. Um den Unternehmen der nachgelagerten Marktstufen bzw. dem Endkunden entsprechend überlegene Werte zu bieten, müssen Lieferanten und Hersteller nach der Theorie des Relational Views enge Kollaborationen innerhalb der Wertschöpfungskette eingehen. In diese Partnerschaften müssen die beteiligten Unternehmen strategische Ressourcen und Fähigkeiten einbringen, um die Bedürfnisse der nachgelagerten Kunde adäquat erfüllen und selbst eine verbesserte Marktposition einnehmen zu können.

Bei der Befriedigung der Bedürfnisse auf nachgelagerten Marktstufen stehen sich folglich nicht mehr nur Unternehmen derselben Wertschöpfungsstufe als Wettbewerber gegenüber. Wettbewerb findet vor allem zwischen verschiedenen kollaborativen Netzwerken bzw. ganzen Wertschöpfungsketten im Sinne von „supply chain versus supply chain“ ${ }^{443}$ bzw. zwischen Supply-Systemen ${ }^{444}$ statt. Wettbewerbsvorteile können nach dieser Sichtweise für ein Unternehmen nicht mehr vorrangig durch geschickte Verhandlungen auf Kosten eines anderen Mitglieds derselben Wertschöpfungskette entstehen. ${ }^{445}$ Wettbewerbsvorteile entstehen stattdessen dadurch, dass die verschiedenen Unternehmen einer Wertschöpfungskette als Partner betrachtet, Schnittstellen zwischen den Unternehmen optimiert und Prozesse integriert werden. ${ }^{446}$ Ziel ist es, auf diese Weise dem Endkunden im Vergleich zu konkurrierenden Wertschöpfungsketten Leistungen effektiver oder effizienter anzubieten. Wenn nun aber Anstrengungen zur Kostenreduzierung bzw. Leistungsverbesserung einen Austausch von vertraulichen betrieblichen und finanziellen Informationen und eine Prozessintegration zwischen den Unternehmen in der Wertschöpfungskette bedingen, wäre es naheliegend, ebenfalls strate-

\footnotetext{
${ }^{442}$ Vgl. Chapman et al. 2003, S. 643.

${ }^{443}$ Ketchen/Giunipero 2004, S. 55; vgl. auch Hult et al. 2008.

${ }^{444}$ Vgl. Jarratt/Fayed 2001, S. 71.

445 Vgl. Barratt 2004, S. 36; Chapman et al. 2008, S. 644.

${ }^{446}$ Vgl. Chapman et al. 2008, S. 644.
} 
gische Marketingprozesse innerhalb der Wertschöpfungskette abzustimmen. ${ }^{447}$ Eine Kollaboration innerhalb der Wertschöpfungskette bringt daher nicht nur gemeinsame Forschungs- und Entwicklungsarbeit oder eine Integration der typischen Supply-Chain-Aktivitäten wie Einkauf und Logistik mit sich. ${ }^{448}$ Für vertikale Kooperationen zwischen Lieferant und Weiterverarbeiter kann es durchaus Sinn machen, die Folgemärkte durch abgestimmte Marketingaktivitäten gemeinsam zu beeinflussen und bspw. für in Kooperation entwickelte Produkte auch gemeinsam die entsprechenden Märkte zu erschließen. ${ }^{449}$ Dazu müssen die Marketingabteilungen und andere Unternehmensfunktionen von kooperierenden Unternehmen an der Markteinführung von Produkten beteiligt und untereinander abgestimmt werden. ${ }^{450}$ So ist es für beide Unternehmen möglich, den Abnehmern auf nachgelagerten Marktstufen bis hin zum Endkunden in einer konsistenten Strategie den gemeinsam geschaffenen Produktnutzen zu kommunizieren und zu liefern. ${ }^{451}$ Eine Abstimmung der Vertriebskanäle und Vertriebsaktivitäten als Aspekt der Marketingstrategie kann die Wertigkeit der angebotenen Leistungen gegenüber dem Endkunden unterstützen. Eine vertikale Preisabstimmung durch mehrere Marktstufen hinweg ist aufgrund kartellrechtlicher Bestimmungen jedoch nur eingeschränkt möglich. Im Bereich des Handels, wo also keine Weiterverarbeitung mehr stattfindet, darf der Hersteller sogar nur eine unverbindliche Preisempfehlung aussprechen. ${ }^{452}$ Gerade aber durch eine Koordination der Leistungsentgelte auf den verschiedenen Marktstufen könnte das Problem der mehrfachen Margenbildung umgangen und dem Endkunden die Leistung insgesamt effizienter bereitgestellt werden. ${ }^{453}$

Bei solch einer kollaborativen mehrstufigen Marketingstrategie müssen alle marketingrelevanten Prozesse zwischen den Unternehmen aufeinander abgestimmt werden, um für den Kunden des Kunden oder den Endkunden einen höheren

\footnotetext{
447 Vgl. Flint 2004, S. 48 f.

448 Vgl. Teichert/Bouncken 2011, S. 98; Barratt 2004.

449 Vgl. Günter 2013, S. 387.

${ }^{450}$ Vgl. Barratt 2004; Green et al. 2012.

451 Vgl. Kleinaltenkamp et al. 2012, S. 165 ff.

${ }^{452}$ Vgl. Berndt 2005, S. 14.

453 Vgl. Haucap/Klein 2012, S. 177; Schwalbe 2012, S. 159 f.
} 
Nutzen zu erzielen. ${ }^{454}$ Bei einer Abstimmung der Marketingstrategien für eher komplexe Produkte kann analog zur gemeinsamen Leistungsentwicklung (vgl. Abschnitt 4.4.1) davon ausgegangen werden, dass auch hier die Zusammenarbeit von Anbieter und Kunde eine gewisse zeitliche Spanne andauert. Während dieser Zeit werden nicht nur die jeweiligen Marketingfähigkeiten in die Beziehung eingebracht, es wachsen auch soziale Bindungen zwischen den Unternehmen, insbesondere zwischen den jeweiligen Marketing- und Vertriebsmitarbeitern des Anbieters und des Kunden. Eine Anpassung der Marketingstrategien zwischen Anbieter und direktem Kunden stellt somit, eine historisch gewachsene und sozial komplexe Fähigkeit dar, die einen wesentlichen Beitrag zur Erreichung von Wettbewerbsvorteilen leisten kann.

Marktorientierung kommt im Einklang mit dem kompetenzbasierten Ansatz große Bedeutung zu, das Potenzial des von den Kooperationspartnern jeweils eingebrachten Marktwissens und die damit verbundenen notwendigen Marketingfähigkeiten zielgerichtet auszuschöpfen. Für den Anbieter ist es daher erforderlich, Marktwissen zu besitzen, das nicht nur die Bedürfnisse der direkten Kunden abdeckt, sondern auch ein umfassendes Verständnis der Wertschöpfungskette insgesamt beinhaltet. Nur wenn ein Unternehmen auch über seine indirekten Kunden und deren Bedürfnisse Bescheid weiß, kann es diese durch eine Anpassung seiner Marketingstrategie bzw. durch Mehrstufiges Marketing adäquat berücksichtigen. Dazu gehört eine umfangreiche Analyse der nachgelagerten Marktstufen und der dort vorherrschenden Trends mit dem Ziel, sich das für die mehrstufige Vermarktung erforderliche Markt- und Marketing-Knowhow anzueignen.

Ebenso sind im Rahmen der Anpassung der Marketingstrategie die Einflussfaktoren der Marktumwelt auf diese interessierenden Marktstufen zu berücksichtigen. Eine gründliche Analyse von einflussreichen Drittparteien entlang der Wertschöpfungskette ist somit ebenfalls erforderlich. Die Ausrichtung des Unternehmens am Wettbewerb hingegen sollte keinen Einfluss auf die Anwendung von Mehrstufigem Marketing haben. Zwar sehen Unternehmen oftmals die Notwendigkeit,

${ }^{454}$ Vgl. Schönhoff 2013, S. 3; Fischer 2014, S. 88 ff. 
eine mehrstufige Marketingstrategie anzuwenden, wenn dies bereits Wettbewerber in der betreffenden Branche tun, doch scheint hierfür nicht die Wettbewerbsorientierung selbst, sondern eher ein etablierter Branchenprozess der Grund zu sein. 455

Durch den Austausch von Marktinformationen im eigenen Unternehmen kann ein besseres Verständnis über die Bedeutung der Wertschöpfungskette und die Notwendigkeit zur Abstimmung von Marketingmaßnahmen innerhalb der betreffenden Wertschöpfungskette realisiert werden. Wie bei einstufigen Marketingaktivitäten sollten daher bei der Planung von Mehrstufigem Marketing alle Abteilungen des Unternehmens ein Verständnis dafür haben, wie das Unternehmen in die Lage versetzt werden kann, Kundenwerte zu schaffen.

Die postulierten Zusammenhänge führen zu folgender Hypothese:

H3: Je höher der Grad der Marktorientierung ist, desto stärker kooperieren Kunden und Lieferanten bei der Ausgestaltung einer auf nachgelagerte Kunden gerichteten Marketingstrategie.

\subsubsection{Neuprodukterfolg}

Wie zuvor beschrieben, übt Marktorientierung einen positiven Einfluss auf die Unternehmenskollaboration, die Innovativität und die Anpassung der Marketingstrategie eines Unternehmens aus. Das Eingehen von Kooperationen mit Kunden zur Neuproduktentwicklung sowie das Entwickeln von abgestimmten Marketingstrategien oder die Schaffung von Innovativität im Unternehmen allein kann dem Unternehmen allerdings auf lange Sicht nicht das Überleben im Markt sichern. In einem marktwirtschaftlich organisierten Wirtschaftssystem funktioniert dies nur über die gewinnbringende Veräußerung von Dienstleistungen und Produkten. Auf lange Sicht und in ihrer Gesamtheit müssen daher die angebotenen Leistungen eines Unternehmens erfolgreich am Markt sein und die Kosten des Unternehmens decken. Die folgenden Erläuterungen sollen das Ziel des Ressourceneinsatzes, nämlich einen Wettbewerbsvorteil zu erreichen, im Einklang mit dem ressourcenbasierten, kompetenzbasierten und relationalen Ansatz dar-

${ }^{455}$ Vgl. Kleinaltenkamp et al. 2012, S. 151. 
stellen.

Wenn Unternehmen Kollaborationen eingehen, um neue Produkte zu entwickeln, so besteht die Möglichkeit, die gesamten Forschungs- und Entwicklungskosten, die bis zur Markteinführung der Endkundenprodukte entstehen, zwischen den Unternehmen zu teilen. ${ }^{456}$ Somit könnte ein einzelnes Unternehmen seine Kosten insgesamt senken und bei konstanter Marge ebenfalls seine Produktpreise senken. ALLRED ET AL. zeigen in einer empirischen Studie, dass eine enge Zusammenarbeit in der Wertschöpfungskette in den meisten Fällen die Kosten senkt und gleichzeitig sogar die Qualität der Produkte steigert. ${ }^{457}$ Weisen die Produkte dieses Herstellers eine ähnliche Qualität auf wie die seiner Wettbewerber, so kann er aufgrund der niedrigeren Preise einen Wettbewerbsvorteil erzielen, der sich im Erfolg seiner Neuprodukte niederschlägt. ${ }^{458}$ Da der Kunde bei gemeinsamer Produktentwicklungsarbeit das Ergebnis dieser Unternehmenszusammenarbeit im Sinne einer Kundenintegration ${ }^{459}$ maßgeblich mitgestalten kann und dafür auch eine Teilverantwortung trägt, wird er dafür Sorge tragen, dass das entwickelte Produkt seinen Vorstellungen entspricht. ${ }^{460}$ Die Produkte bzw. Komponenten, die der Lieferant beisteuert, werden in entsprechenden, eventuell schon vorab mit dem Partnerunternehmen vereinbarten Mengen abgenommen. Sofern keine strikte Exklusivität bei der Vermarktung vereinbart wird, kann der Lieferant die neu entwickelten Produkte oder Komponenten auch anderen Kunden anbieten. Hierbei sind die Kunden, mit denen kooperiert wird, von großer Bedeutung für das anbietende Unternehmen, da sie die Produkte als erste verwenden bzw. weiterverarbeiten und dem Anbieter so wertvolle Hinweise für die weitere Vermarktung der neu entwickelten Produkte liefern können. ${ }^{461}$ URBAN/VON HIPPEL zeigen, dass Produkte, die mithilfe von Kundeninput im Rahmen eines Lead-UserKonzepts entwickelt werden, auch bei anderen potenziellen Kunden eine deutlich

\footnotetext{
${ }^{456} \mathrm{Vgl}$. Banerjee/Lin 2001.

${ }^{457}$ Vgl. Allred et al. 2011, S. 148.

${ }^{458}$ Vgl. Zhou et al. 2008, S. 986.

${ }^{459}$ Vgl. Kleinaltenkamp, 2007; für eine Literaturzusammenfassung zu diesem Thema vgl. Eichentopf et al. 2011, S. $651 \mathrm{ff}$.

${ }^{460}$ Vgl. Eichentopf et al. 2011, S. 652

${ }^{461}$ Vgl. Geiger/Kleinaltenkamp 2011, S. 212.
} 
höhere Präferenz hervorrufen. ${ }^{462}$

Zahlreiche andere Studien stützen konzeptionell und empirisch den dargestellten positiven Zusammenhang, der zwischen einer Integration von Lieferanten und direkten Kunden bei der Neuproduktentwicklung besteht. ${ }^{463}$

Des Weiteren zeigt FANG empirisch, dass eine enge Prozessabstimmung zwischen Herstellern und den nachfolgenden Abnehmerstufen dazu führt, dass sich die entwickelten Produkte stärker vom Wettbewerb differenzieren. Jedoch können die gegenseitigen Prozessabhängigkeiten von Anbietern und direkten Kunden auch dazu führen, dass eine enge Prozessabstimmung notwendig wird, die wiederum die Entwicklungszeiten verlängert. ${ }^{464}$ Im Bereich der kollaborativen Produktentwicklung hebt GÜNTER daher das Simultaneous Engineering hervor. Bei zeitgleicher, abgestimmter Entwicklung von verschiedenen Komponenten kann es Zulieferern und Herstellern gelingen, die Innovationszyklen zu verkürzen, die Produktqualität zu erhöhen und somit die Markteinführung zu beschleunigen. Zudem können auch Vertrieb, Fertigung und Beschaffung synchronisiert werden. ${ }^{465}$ Ein enger Austausch von Marktinformationen zwischen dem Hersteller und den nachgelagerten Marktstufen beschleunigt ebenso die Markteinführung. Durch den Informationsaustausch kann auf Probleme, vor denen die Kunden auf ihren eigenen Absatzmärkten stehen, rechtzeitig durch die Lieferanten reagiert werden. ${ }^{466}$

Die Zusammenarbeit über die Grenzen des einzelnen Unternehmens hinaus stellt eine strategische Ressource im Sinne des Relational Views für das Unternehmen dar. Diese Ressource ist in sozialer Hinsicht ein äußerst komplexes Netz von Fähigkeiten, Investitionen, direkten und indirekten Verbindungen. ${ }^{467}$ Die Besonderheiten dieser Verbindungen zwischen den Unternehmen machen die Ressource für Wettbewerber weder vollständig beobachtbar und nachahmbar, noch am

462 Vgl. Urban/von Hippel 1988, S. 571 ff.

${ }^{463}$ Vgl. Chen/Paulraj 2004; Ragatz et al. 1997; Ragatz et al. 2002. Für eine umfassendere Auflistung weiterer Studien, die zu ähnlichen Ergebnissen kommen, vgl. Weiber/Jacob 2000, S. 602.

${ }^{464} \mathrm{Vgl}$. Fang 2008.

465 Vgl. Günter 2013, 418 ff.

${ }^{466}$ Vgl. Fang 2008, S. 99.

${ }^{467}$ Vgl. Jap 1999, S. 463 f. 
Markt akquirierbar. Für ein kollaborierendes Unternehmen kann daraus ein strategischer Wettbewerbsvorteil erwachsen. Somit lässt sich zusammenfassend sagen, dass eine gemeinsame Neuproduktentwicklung und der dazu notwendige Informationsaustausch zwischen Unternehmen unterschiedlicher Wertschöpfungsstufen dazu beitragen, dass die entwickelten Produkte schneller, preisgünstiger und qualitativ höherwertig am Markt verfügbar sein können. Dies verschafft dem Lieferanten einen Wettbewerbsvorteil und lässt den folgenden Schluss zu:

H4: Eine Unternehmenskollaboration zwischen Anbieter und Kunde übt einen positiven Einfluss auf den Neuprodukterfolg des Lieferanten aus.

BOWEN ET AL. betrachten Innovativität als strategische Ressource, die nach der Theorie des RBV dazu beitragen kann, einen Wettbewerbsvorteil zu generieren. ${ }^{468}$ Wettbewerbsvorteile durch Innovativität können erreicht werden, indem Ideen für Neuprodukte oder neue Prozesse innerhalb eines Unternehmens generiert werden. Dazu wird das Wissen über den Markt und andere relevante Bereiche eines Unternehmens im Rahmen von Innovativität genutzt, um Innovationen hervorzubringen. Als Maß für hervorgebrachte Innovationen betrachten MENGUC/AUH den Erfolg der Neuprodukte. ${ }^{469}$ Diejenigen Produkte, die sich in besonders innovativer Weise von den Wettbewerbsprodukten absetzen, lassen eine erfolgreiche Markteinführung erwarten. Anbieter, die willens und in der Lage sind, das im Unternehmen vorhandene Marktwissen in einer kreativen Art zu nutzen und dabei auch neue Prozesse anzuwenden, scheinen eher dafür prädestiniert, auch neue, innovative Leistungen erstellen zu können. Zahlreiche empirische Studien belegen einen positiven Zusammenhang zwischen der Innovativität eines Anbieters und der Schaffung von Innovationen bzw. dem Erfolg von neuen Produkten. ${ }^{470}$ Daraus lässt sich die folgende Hypothese ableiten:

H5: Die Innovativität eines Unternehmens übt einen positiven Einfluss auf den Neuprodukterfolg aus.

\footnotetext{
${ }^{468}$ Vgl. Bowen et al. 2010, S. 1080.

${ }^{469}$ Vgl. Meguc/Auh 2006, S. 63.

${ }^{470}$ Vgl. Hurley/Hult 1998; Vázquez et al. 2001; Derenthal 2009; Griese 2009; Ihinmoyan/Akinyele 2011.
} 
Ebenso kann davon ausgegangen werden, dass Unternehmen, die sich hinsichtlich ihrer Vermarktungsaktivitäten mit ihren direkten Kunden im Hinblick auf nachgelagerte Marktstufen abstimmen, gleichfalls Wettbewerbsvorteile realisieren können. SONG/THIEME zeigen nämlich empirisch, dass ein Informationsaustausch zwischen Lieferant und Hersteller hinsichtlich marketingrelevanter Informationen zu einem höheren Produkterfolg bestimmter Innovationen beim Hersteller führt. ${ }^{471}$ Es liegt die Vermutung nahe, dass auch die Komponenten bzw. Vorprodukte des Lieferanten am Markt erfolgreich sind. Schließlich ist es bei dauerhaft angelegten Kooperationen nicht möglich, dass lediglich ein Kooperationspartner Wettbewerbserfolge erzielt. Daraus kann folgende Hypothese abgeleitet werden:

H6: Die Anpassung der Marketingstrategien zwischen Anbieter und Kunde übt einen positiven Einfluss auf den Neuprodukterfolg aus.

Die drei hergeleiteten Hypothesen (H4, H5 und H6) postulieren einen Einfluss verschiedener Unternehmensaktivitäten auf den Neuprodukterfolg als Maß für den erreichten Wettbewerbsvorteil. Daneben wird Marktorientierung in zahlreichen Studien auch direkt mit dem Neuprodukterfolg in Verbindung gebracht. Marktorientierung zeigt sich in den zuvor dargestellten Hypothesen (H1, H2 und H3) allerdings auch als Einflussfaktor der drei dargestellten Unternehmensaktivitäten. Im Folgenden soll diese direkte und indirekte Verflechtung der verschiedenen Konstrukte näher erläutert werden.

Trotz der zahlreichen empirischen Studien, die einen direkten positiven Einfluss von Marktorientierung auf Unternehmens- oder Produkterfolg nachweisen, wird in der Literatur vielfach darauf hingewiesen, dass diese Beziehung meist nicht vollständig erklärt wird. ${ }^{42}$ Ansätze hingegen, bei denen weitere Variablen zwischen die Marktorientierung und ein bestimmtes Erfolgsmaß geschaltet werden, bieten einen höheren Erklärungsgehalt. Diese zwischengeschalteten Variablen mediieren dann die Wirkbeziehung von Marktorientierung auf den Erfolg des

${ }^{471}$ Vgl. Song/Thieme 2009, S. 51.

${ }^{472}$ Vgl. Zhou et al. 2008, S. 985; Hult et al. 2005, S. 1173; Varadarajan/Jayachandran 1999, S. 134. 
Unternehmens. KIRCA ET AL. weisen in ihrer Meta-Studie darauf hin, dass Innovativität einer der wichtigsten Mediatoren $^{473}$ zwischen Marktorientierung und Geschäftserfolg ist. ${ }^{474}$ Das Konstrukt der Innovativität oder andere mediierende Wege werden jedoch in vielen Modellen nicht berücksichtigt, um zu erklären, wie das gewonnene Marktwissen genutzt wird. HURLEY/HULT nehmen dies zum Anlass, um Innovativität als Bindeglied zwischen Marktorientierung und Wettbewerbsvorteilen sowie Unternehmenserfolg zu untersuchen. Die Autoren sehen Marktorientierung als Antezendenz von Innovativität und zeigen konzeptionell, dass Innovativität als Aspekt der Unternehmenskultur einen positiven Einfluss auf die Anzahl der im Unternehmen generierten und eingeführten Innovationen hat. ${ }^{475}$ Ebenfalls als Bindeglied zwischen Marktorientierung und Unternehmenserfolg betrachten SANDVIK/SANDVIK die Rolle der Innovativität. Die Autoren heben in ihrer Studie die mediierende Wirkung hervor, die Innovativität sowohl auf Effektivitäts- als auch auf Effizienzmaße besitzt. ${ }^{476} \mathrm{NGO} / \mathrm{O}^{` C A S S}$ bestätigen die Mediatorrolle von Innovativität zwischen Marktorientierung und Neuprodukterfolg auch empirisch. ${ }^{477}$

Neben der oft als Mediator berücksichtigten Innovativität werden selten die Zusammenarbeit innerhalb der Wertschöpfungskette und die Umsetzung der Marketingstrategie als Mediatoren in der Literatur betrachtet. ${ }^{478}$

In neuerer Zeit liegt der Fokus der Forschung und Praxis weiterhin weniger darauf, herauszufinden, ob Marktorientierung einen Einfluss auf den Erfolg eines Unternehmens hat. Dies wurde bereits hinlänglich gezeigt. Das Interesse liegt vielmehr darauf, herauszufinden, über welche Kanäle oder Mittel dieser Erfolg

\footnotetext{
473 Eine Variable gilt als Mediator, wenn diese als zwischengeschaltete Variable die Beziehung einer unabhängigen und einer abhängigen Variable mediiert. Das bedeutet, dass die mediierende Variable nach ihrer Einführung in einem Kausalmodell die direkte Beziehung zwischen den zwei anderen Variablen eliminiert (vollständige Mediation) oder dafür sorgt, dass die Beziehung sich signifikant verringert (partielle Mediation). Vgl. Baron/Kenny 1986, S. 1176.

${ }^{474}$ Vgl. Kirca et al. 2005, S. 38. Ebenfalls wichtige Mediatoren sind den Autoren zufolge Kundenloyalität und Qualität.

475 Vgl. Hurley/Hult 1998. Den Einfluss von Innovationen auf Wettbewerbsvorteile und Unternehmenserfolg erläutern die Autoren nur in theoretischem Rahmen unter Bezug auf Day 1994.

476 Vgl. Sandvik/Sandvik 2003, S. 361.

477 Vgl. Ngo/O’Cass 2012, S. 870

478 Vgl. Liao et al. 2011, S. 305.
} 
erreicht werden kann. ${ }^{479}$ In dieser Arbeit soll daher überprüft werden, ob die jeweils als direkte Wirkungen postulierten Zusammenhänge zwischen Marktorientierung und den Unternehmensaktivitäten sowie zwischen den Unternehmensaktivitäten und dem Neuprodukterfolg auch eine mediierte Wirkbeziehung darstellen. Es werden daher folgende Hypothesen getestet:

H7: Die Beziehung zwischen Marktorientierung und dem Neuprodukterfolg wird durch die markstufenübergreifende Unternehmenszusammenarbeit mediiert.

H8: Die Beziehung zwischen Marktorientierung und dem Neuprodukterfolg wird durch die Innovativität mediiert.

H9: Die Beziehung zwischen Marktorientierung und dem Neuprodukterfolg wird durch die markstufenübergreifende Anpassung der Marketingstrategien mediiert.

\subsection{Zusammenfassung der Hypothesen und das theoreti- sche Modell}

Die in den vorangegangenen Abschnitten hergeleiteten Hypothesen und das daraus begründete theoretische Modell sollen an dieser Stelle noch einmal in Abbildung 7 zusammenfassend dargestellt werden. Die Untersuchung der hypothetisierten Konsequenzen einer umfassenden Marktorientierung soll anhand des dargestellten Modells vorgenommen werden. Vor allem die Schätzung der Zusammenhänge zwischen dem Konstrukt der Marktorientierung und den resultierenden Wirkungen sind nicht zuletzt für die Relevanz von Marktorientierung für Unternehmen entscheidend.

${ }^{479}$ Vgl. Preacher/Hayes 2008, S. 879; Kirca et al. 2005, S. 30; Eggert at al. 2005, S. 103. 


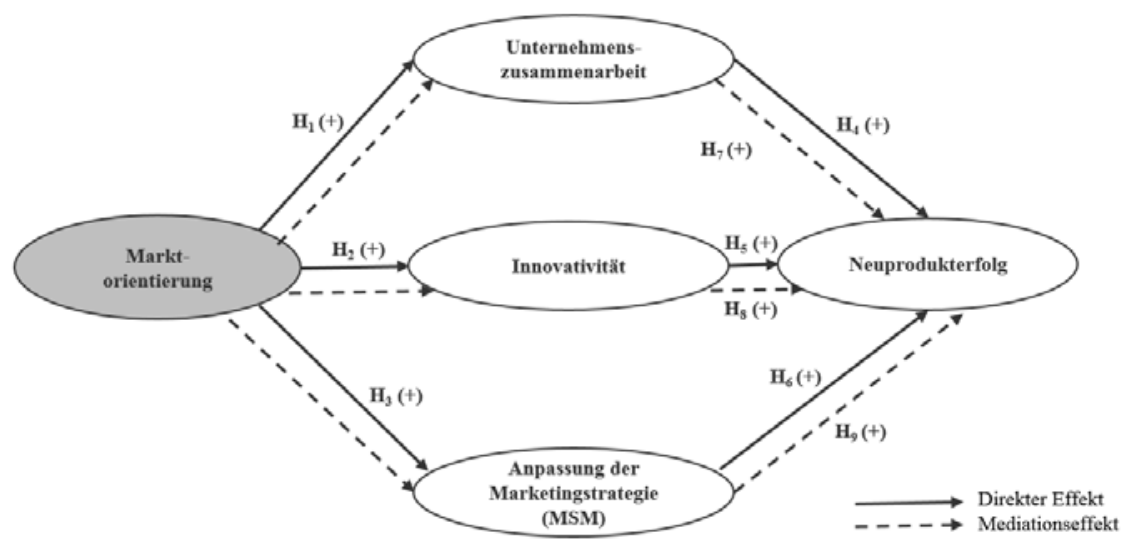

Abbildung 7: Strukturmodell der vorliegenden Studie

Die dargestellten Hypothesen sind zunächst theoretisch hergeleitet und in dieser Form noch keiner empirischen Überprüfung unterzogen worden. Verschiedene Forschungsansätze wurden in dieser Arbeit zusammengeführt, um erstens das Modell einer umfassenden Marktorientierung geeignet zu konzeptualisieren und zu messen, und zweitens relevante Konsequenzen der Marktorientierung entsprechend empirisch untersuchen zu können. Die zur Messung der Konstrukte notwendigen Messvorschriften sollen im folgenden Abschnitt 4.6 dargestellt werden. Die Untersuchung und Bewertung der hypothetischen Zusammenhänge wird in Kapitel 5.5 vorgenommen.

\subsection{Operationalisierung der Konstrukte}

In der Marketingliteratur kann beobachtet werden, dass manche Autoren mehr, manche weniger einer theoretischen Fundierung bei der Operationalisierung von Konstrukten folgen. Die Bandbreite der Sichtweisen, wie stark Messskalen theoretisch und sachlogisch verankert sein sollten, reicht von ROSSITER, der proklamiert, eine Skala allein auf theoretischen Überlegungen zu basieren und psychometrische Kennzahlen hierbei nicht weiter zu beachten, bis hin zu Autoren, die ihre hergeleiteten theoretischen Annahmen verwerfen, um bessere Güte- 
kriterien für ihre Messmodelle zu erreichen. ${ }^{480}$ Zwischen diesen beiden Extremen finden sich auch Autoren wie DIAMANTOPOULOS und FINN/KAYANDE, die den Standpunkt vertreten, dass eine sinnvolle Operationalisierung in vertretbarem Maße sowohl theoretischen Überlegungen als auch kritischen, psychometrischen Kennzahlen genügen müsse. ${ }^{481}$ In dieser Arbeit soll bei der Operationalisierung aller Konstrukte diesem vorgeschlagenen Mittelweg gefolgt werden.

\subsubsection{Zur Operationalisierung des Konstrukts Marktorientierung}

Um die Ergebnisse aus dieser Studie mit anderen Studien nach Möglichkeit vergleichbar zu machen, wurde bei der Konzeptualisierung und Operationalisierung versucht, auf bestehenden Arbeiten aufzubauen. Für die Entwicklung der Items wurden die von NARVER/SLATER auf Englisch veröffentlichten Items übersetzt und zu Grunde gelegt. Die übersetzten Items wurden im Rahmen einer Verständlichkeitsprüfung Vertriebs- und Marketingpraktikern vorgelegt und gegebenenfalls angepasst, ohne den ursprünglichen Inhalt und Sinn zu verfälschen. Damit folgt diese Arbeit nicht nur bei der Konzeptualisierung, sondern auch bei der Operationalisierung im Wesentlichen den Prämissen der Arbeit von NARVER/SLATER. Dieses Vorgehen war jedoch aufgrund der konzeptionellen Überschneidung nur bei den drei innerhalb der MKTOR-Skala begründeten Dimensionen Kundenorientierung, Wettbewerbsorientierung und Abteilungsübergreifende Koordination möglich.

Die Entscheidung, die bestehenden Items, wenn inhaltlich möglich, zu übernehmen, liegt in ihrer hohen Verständlichkeit begründet. HOOLEY ET AL. zeigen in einer Studie, dass die in der MKTOR-Skala verwendeten Items gut übersetzbar und für Praktiker in verschiedenen Branchen und Ländern bei Durchführung der Befragung zur Gewinnung von empirischen Daten inhaltlich sehr gut zu verstehen sind. ${ }^{482}$ Durch die relativ einfache Sprache ist die Skala geeignet, in Unternehmen eine schnelle Diagnose der eigenen Marktausrichtung vorzunehmen. ${ }^{483}$

480 Vgl. Rossiter 2002; Wang et al. 2013.

481 Vgl. Diamantopoulos 2005; Finn \& Kayande 2005.

${ }^{482}$ Vgl. Hooley et al. 2002.

${ }^{483}$ Vgl. Gauzente 1999, S. 78. 
Eine leichte Verständlichkeit der Fragebögen, sollte sich bei Unternehmensbefragungen in Form einer vollständigen Beantwortung der Fragebögen auswirken, weshalb auch in der vorliegenden Studie versucht wurde, wenn möglich auf die Items der MKTOR-Skala zurückzugreifen.

Zur Bewertung der Relevanz der den Dimensionen zugeordneten Items wurden Fokusgruppeninterviews durchgeführt. ${ }^{484}$ Die Fokusgruppenteilnehmer wurden gebeten, zu den einzelnen Dimensionen zunächst Ideen und dann im eigenen Unternehmen tatsächlich durchgeführte Aktivitäten zu benennen. Es zeigte sich, dass die Items der ursprünglichen MKTOR-Skala einen Großteil der genannten Aktivitäten abdecken. Bestand keine Überschneidung zwischen den genannten Aktivitäten und den Skalen-Items, wurden neue Items entwickelt und einem Pretest unterzogen.

Für die Dimensionen Drittparteienorientierung und Marktstufenorientierung liegen keine etablierten Skalen vor. In der Literatur wird, wenn die Übernahme von ganzen Skalen oder bestimmten Items nicht möglich ist, empfohlen, dem grundsätzlichen Vorgehen von CHURCHILL zur Skalenentwicklung zu folgen. Danach werden zunächst die einem Konstrukt zugeordneten Items durch umfangreiche Literatursichtung und Fokusgruppengespräche entwickelt. Die Items, welche die zwei neuen Dimensionen inhaltlich abdecken sollen, wurden allesamt aus den Fokusgruppendiskussionen und aus Literaturauswertungen entwickelt. Die Items wurden dann in einem Test auf ihre Reliabilität und Validität getestet und dabei solche Items ausgeschlossen, die bestimmte psychometrische Kennzahlen nicht erreichen. ${ }^{485}$

Die in dieser Arbeit entwickelte Skala für Marktorientierung soll als Messinstrument ein wesentlich umfassenderes Marktverständnis berücksichtigen. Die Ausrichtung eines Unternehmens auf die vier abgeleiteten Gruppen von Marktteil-

\footnotetext{
${ }^{484}$ Für Details zur Durchführung und zu den Teilnehmern der Interviews siehe Abschnitt 5.3.1. 485 Vgl. Churchill 1979. Für eine detaillierte Vorgehensweise vgl. Deng/Dart 1994; Chen/Quester 2005. Der Ansatz von Churchill ist nicht ohne Kritik geblieben. Insbesondere die auf mathematischstatistischen Methoden und Grenzwerten beruhende Exklusion von Items führt oftmals dazu, dass die Messung eines Konstrukts weitgehend von der ursprünglichen Definition abweicht. Vgl. hierzu Rossiter 2002 und 2005. Zur speziellen Kritik an der Entwicklung der häufig verwendeten Skalen von Marktorientierung vgl. Rossiter 2012.
} 
nehmern sowie die unternehmensinterne koordinierte Vorgehensweise sollen dementsprechend eine umfassende für Industriegütermärkte adäquate Marktorientierung messen können. Die daraus abgeleitete Messskala soll den Namen coMO als Referenz zur englischen Bezeichnung „comprehensive market orientation" tragen.

In dieser Arbeit soll das coMO-Konstrukt als Konstrukt zweiter Ordnung mit den einzelnen Dimensionen als konstituierende Elemente betrachtet werden. Das Konstrukt besteht aus formativen Dimensionen, die zusammen Marktorientierung bilden. Die einzelnen Dimensionen werden hingegen reflektiv gemessen. Das bedeutet, die einzelnen Items stellen Manifestationen ihrer jeweiligen Dimension dar und sind somit untereinander austauschbar, ohne den wesentlichen Inhalt der betreffenden Dimension zu verändern. Somit stellt Marktorientierung als coMOKonstrukt ein reflektives Konstrukt erster Ordnung und formatives Konstrukt zweiter Ordnung dar. Durch diese Art von Messmodell ist es möglich, der Forderung ALBERS‘ nachzukommen und anhand von Treibern das Entstehen und die Wirkung von Marktorientierung besser erklären zu können. ${ }^{486}$

Nachfolgend werden die reflektiven Operationalisierungen der fünf Dimensionen der umfassenden Marktorientierung sowie die zur Validierung notwendige zusätzliche globale Operationalisierung des Konstrukts dargestellt.

\subsubsection{Kundenorientierung}

Die Kundenorientierung soll inhaltlich die Aktivitäten des befragten Unternehmens, die sich auf den direkten Kunden beziehen, abbilden. Im Vordergrund stehen hierbei die Wahrnehmung der Kundenbedürfnisse, eine entsprechende Ausrichtung des Unternehmens sowie die tatsächliche, auch langfristige Verankerung der damit verbundenen Werte im Unternehmen.

In Tabelle 4 werden die für die Messung von Kundenorientierung (CUSTOR) verwendeten Items aufgeführt. Dabei werden nach Möglichkeit die von NARVER/SLATER entwickelten Items übernommen. ${ }^{487}$ Das Item zur systemati-

${ }^{486}$ Vgl. Albers 2010, S. 411.

${ }^{487}$ Narver/Slater 1990. 
schen und regelmäßigen Messung der Kundenzufriedenheit musste allerdings angepasst werden und stellt keine direkte Übersetzung dar, da sich im Rahmen der Gespräche mit den Fokusgruppen herausstellte, dass die Gruppe der angesprochenen Auskunftspersonen nur wenige Informationen über eine systematische Messung der Kundenzufriedenheit in ihrem jeweiligen Unternehmen besitzt. Dies kann zum einen daran liegen, dass viele Unternehmen gar keine systematische Messung durchführen, oder aber zum anderen, dass eine solche Messung vom Beschwerde- oder Claim-Management durchgeführt wird und das Wissen über Kundenzufrieden unsystematisch zwischen verschiedenen Abteilungen verteilt liegt. ${ }^{48}$ In Item custor5 soll die inhaltliche Anpassung Berücksichtigung finden und weniger die tatsächliche Messung der Kundenzufriedenheit, als vielmehr eine genuine Kundenwertschätzung im Vordergrund stehen.

Weiterhin gehen NARVER/SLATER davon aus, dass sich Kundenorientierung auch in After-Sales Aktivitäten nach dem Verkauf der Hauptleistung niederschlägt. Im Rahmen der vorliegenden Arbeit wurden allerdings so verschiedene Branchen befragt, dass nicht davon auszugehen war und auch in der Fokusgruppe bestätigt wurde, dass alle befragten Unternehmen einen bedeutsamen After-Sales Service betreiben. ${ }^{489}$ Auch im Pre-Test erreichte das Item nur eine geringe Reliabilität und wurde daher schließlich aus der Item-Batterie für die Dimension der Kundenorientierung entfernt.

\footnotetext{
${ }^{488}$ In anderen Studien, die sich auf die MKTOR-Skala stützen, erreicht dieses Item ebenfalls relativ niedrige Reliabilitätswerte und wird daher teilweise aus der jeweiligen Skala ausgeschlossen, was die unterschiedliche Auffassung und Durchführung von Kundenzufriedenheitsmessung in Unternehmen unterstreicht (vgl. Narver/Slater 1990; Ward et al. 2006; Coley et al. 2010; Farrell/Oczkowski 1997; Yau et al. 2010).

${ }^{489}$ Vgl. auch Greenley 1995, S. 58 zur unterschiedlichen Bedeutung des After-Sales Bereichs. Auch in der Studie von NARVER/SLATER weist das After-Sales-Item eine relativ geringe Korrelation mit der restlichen Skala von Kundenorientierung auf (vgl. Narver/Slater 1990, S. 24), was zudem den Ausschluss noch einmal bestärkt. Ebenso werden in anderen Studien, die die MKTOR-Skala verwenden, ähnlich problematische Tendenzen deutlich (vgl. Farrell/Oczkowski 1997; Morgan/Strong 1998; Ward et al. 2006; Gray et al. 1998).
} 
Tabelle 4: Items zur Messung der Kundenorientierung

\begin{tabular}{|c|c|c|c|}
\hline Konstrukt & Variablenlabel & Item & Quelle \\
\hline \multirow{5}{*}{ 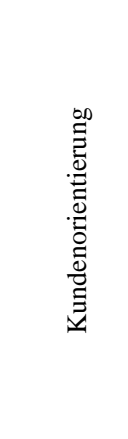 } & custor1 & $\begin{array}{l}\text { Unsere Geschäftsziele werden hauptsächlich durch } \\
\text { Kundenzufriedenheit bestimmt. }\end{array}$ & \multirow{4}{*}{$\begin{array}{c}\text { Narver/ } \\
\text { Slater } \\
1990\end{array}$} \\
\hline & custor2 & $\begin{array}{l}\text { Wir überprüfen ständig, wie sehr wir uns an den Kun- } \\
\text { denwünschen ausrichten. }\end{array}$ & \\
\hline & custor3 & $\begin{array}{l}\text { Die Aktivitäten unseres Unternehmens basieren auf } \\
\text { unserem Verständnis der Kundenbedürfnisse. }\end{array}$ & \\
\hline & custor4 & $\begin{array}{l}\text { Unsere Geschäftsstrategie wird von der Vorstellung } \\
\text { bestimmt, wie wir für unsere Kunden einen Mehrwert } \\
\text { schaffen können. }\end{array}$ & \\
\hline & custor5 & $\begin{array}{l}\text { Für unsere Kunden haben wir immer ein offenes Ohr und } \\
\text { nehmen ihre Bedürfnisse ernst. }\end{array}$ & $\begin{array}{l}\text { Eigenes } \\
\text { Item }\end{array}$ \\
\hline
\end{tabular}

\subsubsection{Wettbewerbsorientierung}

Da Kunden bei einer Kaufentscheidung die Leistungen eines Anbieters mit denen von konkurrierenden Anbietern vergleichen, stellt die Wettbewerbsorientierung des Anbieters einen wesentlichen Bestandteil von Marktorientierung dar. Es kann jedoch in zahlreichen Operationalisierungen beobachtet werden, dass gerade dieser Teilaspekt der Marktorientierung stark vernachlässigt wird. ${ }^{490}$ So misst beispielsweise in der finalen MARKOR-Skala kein Item die Sammlung von Informationen über Wettbewerber. ${ }^{491}$ In der Arbeit von FRITZ wird eine Orientierung am Wettbewerb ebenfalls als wesentlicher Bestandteil einer Marktorientierung angesehen, in der Messung des Konstrukts aber gänzlich vernachlässigt. 492 In der vorliegenden Arbeit soll Wettbewerbsorientierung entsprechend ihrer Wichtigkeit für eine umfassende Marktorientierung über reflektive Indikatoren explizit gemessen werden.

Die Items zur Wettbewerbsorientierung sollen diejenigen Aktivitäten des Unternehmens abbilden, die sich auf den Wettbewerb beziehen. Dabei sind vor allem direkte Wettbewerber derselben Branche zu berücksichtigen, aber auch auf bran-

490 Vgl. Vazquez et al. 2001, S. 77.

491 Vgl. Kohli et al. 1993; Jaworski/Kohli 1990.

492 Vgl. Fritz 1996, S. 61. 
chenfremde Anbieter und Produkte muss das Fokusunternehmen reagieren. Daher soll noch einmal deutlich gemacht werden, dass es sich bei der Messung nicht eine Wettbewerberorientierung ${ }^{493}$ sondern um Wettbewerbsorientierung ${ }^{494}$ handelt. Dabei stehen sowohl die Mitarbeiter des Unternehmens als auch die Aktivitäten des Unternehmens als Ganzes im Fokus. In

Tabelle 5 sind die Items zur Messung der Dimension Wettbewerbsorientierung (COMPOR) aufgelistet. Die Items konnten ebenfalls großenteils aus der MKTOR-Skala entlehnt werden. ${ }^{495}$ Ein weiteres Item wurde der Skala hinzugefügt, um auch Aktivitäten bezüglich branchenfremder Konkurrenz und Substitutionsmöglichkeiten zu erfassen.

Tabelle 5: Items zur Messung der Wettbewerbsorientierung

\begin{tabular}{|c|c|c|c|}
\hline Konstrukt & Variablenlabel & Item & Quelle \\
\hline \multirow{5}{*}{ 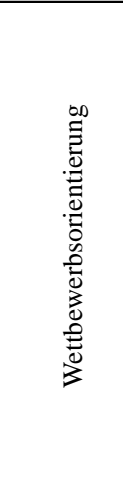 } & compor1 & $\begin{array}{l}\text { Unsere Vertriebsmitarbeiter tauschen sich regelmäßig } \\
\text { untereinander über die Strategien unserer Wettbewerber } \\
\text { aus. }\end{array}$ & \multirow{4}{*}{$\begin{array}{c}\text { Narver/ } \\
\text { Slater } \\
1990\end{array}$} \\
\hline & compor2 & $\begin{array}{l}\text { Wir reagieren schnell auf Aktionen von Wettbewerbern, } \\
\text { die uns bedrohen. }\end{array}$ & \\
\hline & compor3 & $\begin{array}{l}\text { Die Geschäftsleitung berät regelmäßig über die Stärken } \\
\text { und Strategien unserer Wettbewerber. }\end{array}$ & \\
\hline & compor4 & $\begin{array}{l}\text { Wir beobachten ständig unsere Konkurrenten und versu- } \\
\text { chen höherwertige Leistungen anzubieten oder ähnliche } \\
\text { Leistungen preisgünstiger. }\end{array}$ & \\
\hline & compor5 & $\begin{array}{l}\text { Wir analysieren systematisch die verschiedenen techno- } \\
\text { logischen Lösungsmöglichkeiten, mit denen unsere } \\
\text { Produkte in Konkurrenz stehen. }\end{array}$ & $\begin{array}{l}\text { Eigenes } \\
\text { Item }\end{array}$ \\
\hline
\end{tabular}

\subsubsection{Drittparteienorientierung}

Die Operationalisierung des Konstrukts der Drittparteienorientierung soll die Aktivitäten des Unternehmens abbilden, die im Zusammenhang mit der Informa-

${ }^{493}$ Vgl. z.B. Roth 2009; Gleitsmann 2007; Knack 2006. In der englischsprachigen Literatur ist hierzu von Competitor Orientation die Rede, vgl. z.B. Hult/Ketchen 2001; Fritz 1996; Deng/Dart 1994

${ }^{494}$ In der englischsprachigen Literatur ist hierzu von Competitive Orientation die Rede, vgl. z.B. He et al. 2011; Ward et al. 2006; Gatignon/Xuereb 1997; Balakrishnan 1996.

495 Vgl. Narver/Slater 1990. 
tionsgewinnung über solche Einflussnehmer stehen, die auf das Kaufverhalten der Kunden einwirken können, ohne mit dem Unternehmen in einer direkten Geschäfts- oder Konkurrenzbeziehung zu stehen. Damit werden Kunden und Wettbewerber ausgeklammert, die nach CLARKSON auch als „primary stakeholder" zu bezeichnen wären. Stattdessen werden bei der Operationalisierung nur „secondary stakeholder“ berücksichtigt, die zwar das Unternehmen als solches mittelbar beeinflussen aber nicht über den Fortbestand desselben direkt entscheiden können. ${ }^{496}$ Dies betrifft vor allem die Medien, die durch ihre Berichterstattung einen großen Einfluss auf die Kunden und Endkunden haben und die somit oftmals auch für die Prägung eines Firmenimages verantwortlich sein können. Ebenfalls werden solche Items entwickelt und mit aufgenommen, die die von SHAPIRO hervorgehobene Wichtigkeit jener Einflussnehmer widerspiegeln, die durch ihr Handeln auf die vom Unternehmen angebotenen Produkte einwirken. ${ }^{497}$ Die Items zur Messung dieses Konstrukts mussten großenteils neu entwickelt werden, da auf die einschlägigen Skalen von Stakeholder-Orientierung nicht zurückgegriffen werden kann. Dies liegt zum einen an der mangelnden Abgrenzung der Stakeholder-Orientierung von den dem Konstrukt der Marktorientierung inhärenten Stakeholdern wie Wettbewerbern und Kunden sowie zum anderen an der oftmals beschränkten Fokussierung auf weitere „primary Stakeholder“, allen voran Mitarbeiter, Lieferanten und Anteilseigner. ${ }^{498}$ Die entsprechenden Items zur Messung der Dimension Drittparteienorientierung (PARTOR) sind in Tabelle 6 aufgeführt.

496 Vgl. Clarkson 1995, S. 106 f.

${ }^{497}$ Vgl. Shapiro, 1988, S. 121.

498 Vgl. Greenley/Foxall 1996; Yau et al. 2007; He et al. 2011. 
Tabelle 6: Items zur Messung der Drittparteienorientierung

\begin{tabular}{|c|c|c|c|}
\hline Konstrukt & Variablenlabel & Item & Quelle \\
\hline \multirow{5}{*}{ 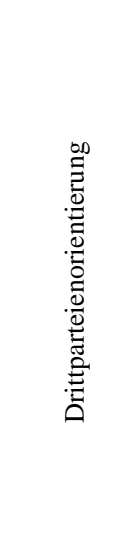 } & partor1 & $\begin{array}{l}\text { In unserem Unternehmen werten wir Informationen } \\
\text { über Institutionen aus, die allgemeine soziale Trends } \\
\text { (z.B. Umweltbewusstsein, Lebensstil) beeinflussen. }\end{array}$ & $\begin{array}{l}\text { in Anleh- } \\
\text { nung an } \\
\text { Matsuno } \\
\text { et al. } 2000\end{array}$ \\
\hline & partor2 & $\begin{array}{l}\text { Wir legen viel Wert auf die Außendarstellung unseres } \\
\text { Unternehmens. }\end{array}$ & \multirow{4}{*}{$\begin{array}{l}\text { Eigene } \\
\text { Items }\end{array}$} \\
\hline & partor3 & $\begin{array}{l}\text { Wir suchen regelmäßig Kontakt zu denjenigen Perso- } \\
\text { nen und Organisationen, die in unseren Absatzmärk- } \\
\text { ten bestimmte Produkte oder Standards empfehlen } \\
\text { bzw. vorschreiben. }\end{array}$ & \\
\hline & partor4 & $\begin{array}{l}\text { Wir beobachten regelmäßig, wie über unser Unter- } \\
\text { nehmen oder unsere Branche in den Medien (z.B. } \\
\text { Presse, Internetforen etc.) berichtet wird. }\end{array}$ & \\
\hline & partor5 & $\begin{array}{l}\text { Wir reagieren schnell auf ordnungspolitische Ände- } \\
\text { rungen (z.B. gesetzliche Regelungen, Verordnungen). }\end{array}$ & \\
\hline
\end{tabular}

\subsubsection{Marktstufenorientierung}

Bei der Operationalisierung von Marktstufenorientierung sollen die Aktivitäten des Unternehmens gemessen werden, die sich auf die Berücksichtigung von nachgelagerten Marktstufen beziehen. Hierbei geht es vor allem um die Informationssammlung über nachgelagerte Kunden und die Marktdynamiken, die auf diesen Markstufen vorherrschen und um die entsprechenden Reaktionen des Unternehmens darauf. Bei diesem Konstrukt konnte weder auf etablierte Skalen noch auf einzelne Items zurückgegriffen werden. KIBBELING ET AL. entwickeln zwar das konzeptionell verwandte Konstrukt der Endkunden-Orientierung eines Lieferanten, doch stellt sich bei der Operationalisierung heraus, dass die Autoren keine Marktdynamiken in den nachfolgenden Stufen berücksichtigen. Auch die übrigen Items setzen einen deutlichen Fokus auf den direkten Kunden und sehen eine Informationssammlung über die nachgelagerten Kunden eher als Nebenprodukt einer intensiven (direkten) Kundeorientierung. ${ }^{499}$ Die Skala erwies sich somit als ungeeignet für den Zweck der vorliegenden Arbeit. Dies hatte zur Folge, dass die Items zur Messung des Konstrukts der Marktstufenorientierung

499 Vgl. Kibbeling et al. 2013. 
(MULTOR) allesamt neu entwickelt werden mussten (Tabelle 7).

Tabelle 7: Items zur Messung der Marktstufenorientierung

\begin{tabular}{|c|c|c|c|}
\hline Konstrukt & Variablenlabel & Item & Quelle \\
\hline \multirow{5}{*}{ 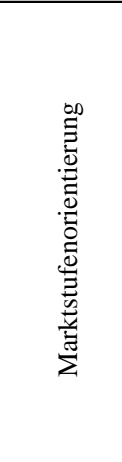 } & multor1 & $\begin{array}{l}\text { Wir verfolgen den Weg unserer Produkte durch die ver- } \\
\text { schiedenen Weiterverarbeitungsstufen bis zum Endkunden. }\end{array}$ & \multirow{5}{*}{$\begin{array}{l}\text { Eigene } \\
\text { Items }\end{array}$} \\
\hline & multor2 & $\begin{array}{l}\text { Wir sammeln Informationen, um die Präferenzen der Letzt- } \\
\text { verwender unserer Produkte einzuschätzen. }\end{array}$ & \\
\hline & multor3 & $\begin{array}{l}\text { Wir erklären den Abnehmern auf nachgelagerten Marktstu- } \\
\text { fen regelmäßig den Nutzen und die Qualität unserer Produk- } \\
\text { te (z.B. durch Produktbroschüren, Pressemitteilungen, } \\
\text { Beratung unseres Außendienstes etc.). }\end{array}$ & \\
\hline & multor4 & $\begin{array}{l}\text { Wir beobachten Trends und Marktverhältnisse auf den } \\
\text { nachfolgenden Marktstufen. }\end{array}$ & \\
\hline & multor5 & $\begin{array}{l}\text { Wir reagieren schnell, wenn der Absatz unserer Produkte in } \\
\text { nachgelagerten Marktstufen bedroht wird. }\end{array}$ & \\
\hline
\end{tabular}

\subsubsection{Abteilungsübergreifende Koordination}

Bei der Operationalisierung von Abteilungsübergreifender Koordination sollen die Aktivitäten des Unternehmens gemessen werden, die mit dem Austausch von Markt- und insbesondere Kundeninformationen in Verbindung stehen. Hierbei geht es vor allem darum, solche Aktivitäten zu messen, bei denen verschiedene Abteilungen desselben Unternehmens bzw. derselben Geschäftseinheit ihr Wissen und ihre Ressourcen mit dem Ziel einer effizienten Wertschöpfung teilen. Zur Messung des Konstrukts Abteilungsübergreifende Koordination (FUNCO) konnte ebenfalls auf die etablierte Messskala von NARVER/SLATER zurückgegriffen werden. ${ }^{500}$ Die entsprechenden übersetzten Items befinden sich in Tabelle 8.

${ }^{500}$ Vgl. Narver/Slater 1990. 
Tabelle 8: Items zur Messung der Abteilungsübergreifenden Koordination

\begin{tabular}{|c|c|c|c|}
\hline Konstrukt & Variablenlabel & Item & Quelle \\
\hline \multirow{5}{*}{ 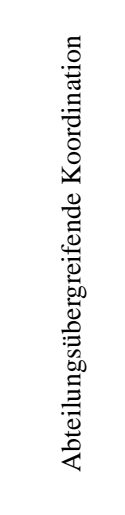 } & funco1 & $\begin{array}{l}\text { Bei uns findet ein reger Austausch zwischen den Abtei- } \\
\text { lungsleitern über potenzielle und aktuelle Kunden statt. }\end{array}$ & \multirow{5}{*}{$\begin{array}{c}\text { Narver/ } \\
\text { Slater } \\
1990\end{array}$} \\
\hline & funco2 & $\begin{array}{l}\text { Uns ist es wichtig, mit anderen Abteilungen über erfolgrei- } \\
\text { che und nicht erfolgreiche Erfahrungen mit Kunden zu } \\
\text { sprechen. }\end{array}$ & \\
\hline & funco3 & $\begin{array}{l}\text { Alle Abteilungen bei uns (z.B. Marketing/Vertrieb, Produk- } \\
\text { tion, F\&E, Finanzen etc.) sind an der Erfüllung der Kun- } \\
\text { denwünsche beteiligt. }\end{array}$ & \\
\hline & funco4 & $\begin{array}{l}\text { Unsere Abteilungsleiter verstehen, wie alle gemeinsam in } \\
\text { unserem Unternehmen dazu beitragen können, einen } \\
\text { Kundenwert zu schaffen. }\end{array}$ & \\
\hline & funco5 & $\begin{array}{l}\text { Alle Abteilungen teilen ihr Wissen und ihre Betriebsmittel } \\
\text { mit anderen Abteilungen, um sich gegenseitig in ihrer } \\
\text { Arbeit zu unterstützen. }\end{array}$ & \\
\hline
\end{tabular}

\subsubsection{Globale Konstruktoperationalisierung von Marktorientierung}

Neben der Operationalisierung der hergeleiteten fünf Dimensionen sollten zur Abschätzung der Validität des Konstrukts (vgl. Abschnitt 5.4.2) zudem globale Items entwickelt und im Rahmen der Untersuchung erhoben werden (vgl.

Tabelle 9). Die Items sollen die umfassende Marktorientierung direkt auf Konstruktebene messen. Eine Berücksichtigung von Konstruktdimensionen wird bei diesem Vorgehen bewusst umgangen. Die Items wurden als reflektives Messinstrument konzipiert und stellen somit Manifestationen des Konstrukts dar. Die Items orientieren sich dabei an den verschiedenen Definitionen von Marktorientierung, die in der Literatur große Beachtung gefunden haben und zielen auf den kulturellen, verhaltensbezogenen und strategischen bzw. Entscheidungscharakter von Marktorientierung ab. ${ }^{501}$ Ein viertes Item, das nach einer allgemeinen Einschätzung der Marktorientierung fragt, wurde ebenfalls in der Operationalisierung aufgenommen.

${ }^{501}$ Vgl. Narver/Slater 1990, S. 20; Kohli/Jaworski 1990, S. 6; Ruekert 1992, S. 228. 
Tabelle 9: Items zur Messung der umfassenden Marktorientierung

\begin{tabular}{|c|c|c|c|}
\hline Konstrukt & Variablenlabel & Item & Quelle \\
\hline \multirow{4}{*}{ 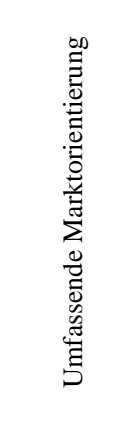 } & mo1 & $\begin{array}{l}\text { Verglichen mit anderen Unternehmen aus unserer Branche } \\
\text { gilt unsere Geschäftseinheit als marktorientiert. }\end{array}$ & \multirow{4}{*}{$\begin{array}{l}\text { Eigene } \\
\text { Items }\end{array}$} \\
\hline & mo2 & $\begin{array}{l}\text { Wie stark orientiert sich Ihre Geschäftseinheit in ihren } \\
\text { Entscheidungen an den Belangen der Kunden, an den Kun- } \\
\text { den der Kunden, an den Wettbewerbern und an den relevan- } \\
\text { ten Interessengruppen? }\end{array}$ & \\
\hline & mo3 & $\begin{array}{l}\text { In unserer Geschäftseinheit sammeln und nutzen wir syste- } \\
\text { matisch Informationen über unseren Markt und das Markt- } \\
\text { umfeld. }\end{array}$ & \\
\hline & mo4 & $\begin{array}{l}\text { In unserer Unternehmenskultur ist fest verankert, stets den } \\
\text { besten Wert für unsere Kunden zu liefern. }\end{array}$ & \\
\hline
\end{tabular}

Nach der Operationalisierung der fünf Dimensionen der umfassenden Marktorientierung werden in den folgenden Abschnitten die abhängigen Konstrukte des Hypothesensystems operationalisiert.

Mit den vorangegangenen Abschnitten kann die Forschungsfrage 2 zur sinnvollen Konzeptualisierung und Operationalisierung einer für den B2B-Bereich adäquaten Marktorientierung abgeschlossen werden. Die empirische Überprüfung der dargestellten Operationalisierung findet in den Abschnitten 5.4.1 und 5.4.2 statt.

\subsubsection{Operationalisierung der abhängigen Konstrukte}

Zur Operationalisierung der Wirkungen von Marktorientierung im Strukturgleichungsmodell wurde ebenfalls, wenn möglich, auf bestehende Skalen zurückgegriffen. Sofern keine adäquaten Items zur Verfügung standen, wurde dem Standardvorgehen gefolgt und anhand von theoretischen Überlegungen entsprechende Items abgeleitet. ${ }^{502}$ Diese Items wurden anschließend mehreren Pretests unterzogen, um eine reliable und valide Messung der abhängigen Konstrukte und letztlich eine hohe Interpretationsfähigkeit der Ergebnisse der Hypothesentests zu gewährleisten.

502 Vgl. zu diesem Vorgehen Ungruhe 2011; Griese 2011; Minculescu 2013. 


\subsubsection{Unternehmenskollaboration}

Anhand des Konstrukts Unternehmenskollaboration soll im Rahmen der Untersuchung erhoben werden, wie stark anbietende Unternehmen mit ihren Kunden bei gemeinsamen Projekten zusammenarbeiten. Das Verständnis und somit auch die Tätigkeiten und die Intensität einer Unternehmenskollaboration können sich bei der Zusammenarbeit von zwei Unternehmen stark unterscheiden. In dieser Arbeit wird darunter ein zielgerichtetes, geplantes Vorgehen verstanden, welches durch gegenseitiges Vertrauen geprägt ist. Die Operationalisierung lehnt sich daher inhaltlich stark an die Skala zur Messung einer externen Kollaboration von ALLRED ET AL. an. ${ }^{503}$ Darin wird die Zusammenarbeit zwischen Unternehmen verschiedener Stufen in der Wertschöpfungskette gemessen. Die Items der Skala wurden für die Zwecke dieser Arbeit angepasst und durch eigene Items ergänzt, um den Inhalt der im Rahmen der Kollaboration ausgetauschten Informationen näher zu spezifizieren. ${ }^{504}$ Zudem wurden Items ergänzt, die auf die Messung der routinierten und vertrauensvollen Eigenschaft der Zusammenarbeit abzielen. Insbesondere eine persönliche und regelmäßige Interaktion zwischen den Mitgliedern der verschiedenen Organisationen schafft eine Arbeitsroutine, eine gemeinsame Arbeitssprache und ein gemeinsames Problemverständnis, welches für eine effektive und effiziente Zusammenarbeit unabdingbar ist. ${ }^{505}$

Die Skala berücksichtigt aufgrund der Ergebnisse der Pre-Tests nicht die in der Literatur oftmals mit Unternehmenskollaborationen in Verbindung gebrachten gemeinsamen, strategischen Zielsetzungen. ${ }^{506}$ Items, die den Prozess einer strategischen Zielvereinbarung zwischen Anbieter und Kunde messen, wurden von der finalen Skala ausgeschlossen. Das Ziel der Unternehmenszusammenarbeit stellt somit gleichzeitig das Projektziel, nämlich die gemeinsame Produktentwicklung, dar. Die Items des Konstrukts sind in Tabelle $10 \mathrm{zu}$ finden.

\footnotetext{
503 Vgl. Allred et al. 2011, S. 140.

504 Vgl. Sammarra/Biggiero 2008, S. $810 \mathrm{ff}$.

505 Vgl. Dyer 1996, S. 273 f.

${ }^{506}$ Vgl. Allred et al. 2011; Campbell 1998; Wilson/Nielson 2001.
} 
Tabelle 10: Items zur Messung der Unternehmenskollaboration

\begin{tabular}{|c|l|l|c|}
\hline Konstrukt & Variablenlabel & Item & Quelle \\
\hline \multirow{2}{*}{} & collabo1 & $\begin{array}{l}\text { Wir arbeiten oft mit Kunden bei Neuproduktentwick- } \\
\text { lungen zusammen. }\end{array}$ & $\begin{array}{c}\text { Eigenes } \\
\text { Item }\end{array}$ \\
\cline { 2 - 4 } & collabo2 & $\begin{array}{l}\text { Wir teilen technische Expertise mit unseren Kunden } \\
\text { zur besseren Produktneuentwicklung. }\end{array}$ & $\begin{array}{c}\text { Allred et } \\
\text { al. } 2011\end{array}$ \\
\cline { 2 - 5 } & \multirow{2}{*}{ collabo3 } & $\begin{array}{l}\text { Wir tauschen Marktinformationen (z.B. Absatzprog- } \\
\text { nosen) mit unseren Kunden zur besseren Produktneu- } \\
\text { entwicklung aus. }\end{array}$ & $\begin{array}{c}\text { Eigenes } \\
\text { Item }\end{array}$ \\
\cline { 2 - 5 } & collabo4 & $\begin{array}{l}\text { Wir haben klare Richtlinien, wie wir die Zusammen- } \\
\text { arbeit mit Kunden bei gemeinsamen Projekten führen. }\end{array}$ & $\begin{array}{c}\text { Allred et } \\
\text { al. 2011 }\end{array}$ \\
\cline { 2 - 5 } \\
\cline { 2 - 4 }
\end{tabular}

\subsubsection{Innovativität}

Durch die Operationalisierung des Konstrukts Innovativität sollen die Handlungen der Untersuchungseinheit gemessen werden, die hinsichtlich der Umsetzung von Marktwissen in Innovationen bestehen. Messinstrumente, die eine zugrundeliegende kulturelle Verankerung des Innovativitätskonstrukts durch eine insgesamt positive Einstellung des Unternehmens hinsichtlich Neuerungen verschiedener Art berücksichtigen, erscheinen für die vorliegende Arbeit geeignet. Eine solche Messung geht implizit davon aus, dass das Unternehmen die Fähigkeit besitzt, Wissen in neue Produkte zu übertragen und dass eine Differenzierung im Markt eher durch die tatsächliche Bereitschaft dazu erreicht wird.

Nach CALANTONE ET AL. zeigt sich eine solche Innovativität in der Offenheit gegenüber Veränderungen im eigenen Unternehmen, die letztlich ein Schritt zur Einführung neuer Produkte am Markt sind. ${ }^{507}$ Dieses Verständnis grenzt Innovativität deutlich von Innovationen ab, die das Ergebnis der Innovativität in Form von modifizierten oder neuentwickelten Marktangeboten darstellen. Für die Messung von Innovativität in der vorliegenden Arbeit wurde daher die etablierte

${ }^{507}$ Vgl. Calantone et al. 2002, S. 517. 
Skala von CALANTONE ET AL. verwendet und an den Kontext dieser Studie angepasst (vgl. Tabelle 11). ${ }^{508}$ Die Messung des Konstrukts wurde diesen Autoren folgend über reflektive Indikatoren vorgenommen. Dies bedeutet, dass das Konstrukt der Innovativität die Ursache der beobachtbaren Werte der Messindikatoren darstellt.

Tabelle 11: Items zur Messung der Innovativität

\begin{tabular}{|c|c|c|c|}
\hline Konstrukt & Variablenlabel & Item & Quelle \\
\hline \multirow{4}{*}{ 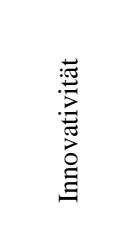 } & \multicolumn{2}{|c|}{ Unsere Geschäftseinheit (Business Unit) ... } & \multirow{4}{*}{$\begin{array}{l}\text { Calantone et } \\
\text { al. } 2002\end{array}$} \\
\hline & innova1 & ... probiert häufiger neue Ideen aus. & \\
\hline & innova2 & ... macht neue Vorgehensweisen ausfindig. & \\
\hline & innova3 & $\begin{array}{l}\text {... ist kreativ in ihren Arbeits- und Produktionsverfah- } \\
\text { ren. }\end{array}$ & \\
\hline
\end{tabular}

\subsubsection{Anpassung der Marketingstrategie}

Die Messung der Anpassung der Marketingaktivitäten soll anhand der zwischen Lieferanten und direkten Abnehmern abgestimmten Marketingaktivitäten erfolgen. Die gemessenen Marketingaktivitäten sollen einen Bezug zum Endkunden aufweisen und dabei alle wesentlichen Aktivitätsfelder des Marketings beinhalten. Dies betrifft die Kommunikationsstrategie, die alle Formen der zielgerichteten Verbreitung von Informationen beinhaltet, die das Leistungsangebot eines Unternehmens verdeutlichen. ${ }^{509}$ Ebenso ist die Vertriebsstrategie angesprochen. Die Preisstrategie wurde bei dieser Operationalisierung ausgeklammert, da sie aufgrund der kartellrechtlichen Einschränkungen nicht in allen Branchen ohne Weiteres möglich ist, so dass die angestrebte Allgemeingültigkeit der Messergebnisse diesbezüglich nicht erreicht werden könnte. Folglich stellt eine Abstimmung der Preisgestaltung zwischen Lieferant und Weiterverarbeiter in der Praxis eher die Ausnahme dar. ${ }^{510}$ Ein wesentlicher Aspekt bei der Abstimmung der Marke-

508 Vgl. Calantone et al. 2002, S. 520. Für die Übersetzung der Items wurde das getestete Messinstrument von GRIESE (2011, S. 202) übernommen.

509 Vgl. Plötner 2006, S. 498.

510 Vgl. Kleinaltenkamp et al. 2011, S. 44. 
tingaktivitäten liegt darin, für den Endkunden durch das Zusammenspiel der gesamten Wertschöpfungskette einen möglichst hohen Nutzen zu generieren. Daher sollte ebenfalls gemessen werden, inwiefern die Unternehmen auf unterschiedlichen Marktstufen ihre Schnittstellen optimieren, um möglichst effizient zu arbeiten. Zur Messung wurde die Skala von GREEN ET AL. herangezogen und an die zuvor genannten Aspekte angepasst. ${ }^{511}$ Ein weiteres Item wurde ergänzt, das noch einmal stärker auf den Inhalt der Kommunikationsmaßnahmen, also den gemeinsam generierten Kundennutzen abzielt. Die Produktpolitik wurde von der Messskala ausgeschlossen. Items, die eine Abstimmung zwischen Lieferanten und Kunden hinsichtlich der Produkte für nachgelagerte Marktstufen oder Endkunden messen, wurden aufgrund der weitgehenden inhaltlichen Abdeckung durch das Konstrukt der Unternehmenskollaboration nicht berücksichtigt. Den besagten Autoren folgend wurden die Items reflektiv konzipiert und stellen somit Konsequenzen der Anpassung der Marketingstrategien zwischen Lieferanten und direkten Abnehmern dar. Die verwendete Skala ist in

Tabelle 12 abgebildet.

Tabelle 12: Items zur Messung der Anpassung der Marketingaktivitäten

\begin{tabular}{|c|c|c|c|}
\hline Konstrukt & Variablenlabel & Item & Quelle \\
\hline 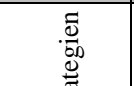 & \multicolumn{2}{|c|}{$\begin{array}{l}\text { Unsere Marketingverantwortlichen arbeiten mit unseren direkten Kunden } \\
\text { in der Wertschöpfungskette zusammen, um ... }\end{array}$} & \multirow{4}{*}{$\begin{array}{l}\text { Green et } \\
\text { al. } 2012\end{array}$} \\
\hline 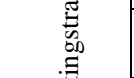 & align1 & $\begin{array}{l}\text {... eine Kommunikationsstrategie für die Endkunden- } \\
\text { Produkte zu erarbeiten. }\end{array}$ & \\
\hline 总 & align2 & $\begin{array}{l}\text {... eine Vertriebsstrategie für die Endkunden-Produkte zu } \\
\text { erarbeiten. }\end{array}$ & \\
\hline \multirow{2}{*}{ 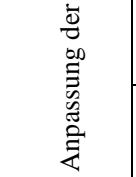 } & align3 & $\begin{array}{l}\text {... besser aufeinander abgestimmte Prozesse zu entwi- } \\
\text { ckeln, die den Endkunden einen Nutzen bringen. }\end{array}$ & \\
\hline & align4 & $\begin{array}{l}\text {... den Endkunden den für sie geschaffenen Nutzen zu } \\
\text { kommunizieren. }\end{array}$ & $\begin{array}{l}\text { Eigenes } \\
\text { Item }\end{array}$ \\
\hline
\end{tabular}

\subsubsection{Neuprodukterfolg}

Um den Neuprodukterfolg (NPS) als Manifestation eines Wettbewerbsvorteils zu

511 Vgl. Green et al. 2012, S. 1012. 
messen, sollten Effektivitäts- und Effizienzmaßzahlen verwendet werden. Aufgrund der Arbeitsteilung bei der Entwicklung von neuen Produkten sowie der gemeinsamen Ausarbeitung einer Marketingstrategie durch Anbieter und direkten Kunden kann angenommen werden, dass es einem Anbieter möglich ist, in derselben Zeit eine höhere Anzahl neuer Produkte zu entwickeln und am Markt einzuführen. Als Maßzahl für diese Effektivität soll daher die Anzahl der Neuproduktentwicklungen dienen. Werden Produkte entwickelt, für die auch tatsächlich ein Bedarf durch Nachfrager besteht, kann die Erfolgsquote der neuen Produkte am Markt als ein weiterer Maßstab für den Neuprodukterfolg herangezogen werden. Da die Entwicklung neuer Produkte im Unternehmen Ressourcen bindet oder verbraucht, sollten durch ein erfolgreiches Produkt möglichst schnell die Entwicklungskosten wieder amortisiert werden. Die Zeit, die eine Leistung von der Entwicklung bis zur Marktreife benötigt, möglichst kurz zu halten, erscheint daher als eine wesentliche Voraussetzung, um erfolgreich Produkte am Markt einzuführen. Als ein weiteres Maß des Neuprodukterfolgs sollte daher erfragt werden, wie oft das betreffende Unternehmen zuerst mit neuen Produkten am Markt ist. Durch die Zusammenarbeit bei der Entwicklung und Vermarktung von neuen Produkten kann davon ausgegangen werden, dass die Kosten geteilt und somit für das einzelne Unternehmen gesenkt werden können. Durch die Innovativität eines Anbieters werden zahlreiche Lösungsmöglichkeiten untersucht, die dazu führen können, dass neuartige Arbeits- und Produktionsverfahren angewendet werden, welche die Effizienz des Unternehmens fördern. Als viertes Maß für den Neuprodukterfolg sollten daher die Kosten für die Neuproduktentwicklung berücksichtigt werden.

In Übereinstimmung mit dem RBV, der einen dieser Arbeit zugrundeliegenden theoretischen Ansatz darstellt, sollte der Erfolg einer Unternehmung immer ins Verhältnis zu dem der Wettbewerber gesetzt werden. Auch Unternehmen derselben Branchen können jedoch nur schwer miteinander verglichen werden, da das Produktspektrum, die Absatzmärkte und somit auch die Wettbewerberstruktur gänzlich verschieden sein können. ${ }^{512}$ In dieser Untersuchung wurden daher aus-

512 Vgl. Slater/Narver 1994a, S. 51. 
schließlich Erfolgsmaßstäbe verwendet, die das befragte Unternehmen in ein Verhältnis zu seinen größten Wettbewerbern in einer bestimmten Produktkategorie setzen.

Die einzelnen Items des Konstrukts Neuprodukterfolg wurden in dieser Arbeit reflektiv gemessen und stellen somit Konsequenzen des Konstrukts dar. Die Items sind in Tabelle 13 zu finden.

Tabelle 13: Items zur Messung des Neuprodukterfolgs

\begin{tabular}{|c|c|c|c|}
\hline Konstrukt & \begin{tabular}{|l|} 
Variablenlabel \\
\end{tabular} & Item & Quelle \\
\hline \multirow{5}{*}{ 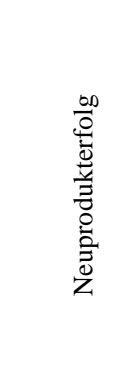 } & \multicolumn{3}{|c|}{$\begin{array}{l}\text { Wie bewerten Sie die folgenden Entwicklungen im Vergleich zum größten Wettbewer- } \\
\text { ber? }\end{array}$} \\
\hline & nps1 & $\begin{array}{l}\text { Die Anzahl von unseren neu eingeführten Produk- } \\
\text { ten am Markt. }\end{array}$ & $\begin{array}{l}\text { Baker/Sinkula } \\
1999\end{array}$ \\
\hline & nps2 & Die Erfolgsquote unserer neuen Produkte. & $\begin{array}{l}\text { Baker/Sinkula } \\
1999\end{array}$ \\
\hline & nps3 & $\begin{array}{l}\text { Wie oft ist Ihr Unternehmen als erstes mit neuen } \\
\text { Anwendungen am Markt? }\end{array}$ & $\begin{array}{l}\text { Baker/Sinkula } \\
1999\end{array}$ \\
\hline & nps4 & $\begin{array}{l}\text { Unsere Kosten für Produktentwicklun- } \\
\text { gen/Leistungsentwicklungen. }\end{array}$ & Eigenes Item \\
\hline
\end{tabular}




\section{Empirische Analyse des theoretischen Mo- dells}

\subsection{Methodische Grundlagen zu Strukturgleichungsmo- dellen}

Strukturgleichungsmodelle (SGM) werden eingesetzt, um zuvor aufgestellte theoretische Beziehungen von Konstrukten in ein lineares Gleichungssystem zu überführen und mathematisch-statistisch überprüfen zu können.

Die einzelnen a priori formulierten und theoretisch begründeten UrsacheWirkungszusammenhänge zwischen verschiedenen Variablen bzw. Konstrukten werden durch die SGM abgebildet. SGM erlauben eine kausale Interpretation der Zusammenhänge, indem sie zwischen unabhängigen und abhängigen Variablen je untersuchter Beziehung unterscheiden. ${ }^{513}$ Da mithilfe von Datensätzen Kausalitäten geprüft und gegebenenfalls bestätigt werden, werden die SGM auch den sogenannten Kausalanalysen zugerechnet und besitzen konfirmatorischen Charakter. ${ }^{514}$ Wesentlicher Vorteil der SGM gegenüber anderen Analyseverfahren ist, dass komplexe Beziehungen zwischen mehreren Variablen simultan geschätzt werden können. Vorteilhaft ist weiterhin, dass nicht nur direkte sondern auch mediierte oder moderierte Wirkbeziehungen geschätzt werden können. ${ }^{515}$ Die Variablen in einem SGM können latente, d.h. nicht direkt beobachtbare Variablen und beobachtbare bzw. manifeste Variablen sein. ${ }^{516}$ Dieselben Variablen können in einem SGM sowohl als unabhängige als auch als abhängige Variablen fungieren, so dass zwar innerhalb einer bestimmten Kausalität zwischen abhängigen und unabhängigen Variablen unterschieden werden kann, eine generelle Definiti-

\footnotetext{
${ }^{513}$ Vgl. Weiber/Mühlhaus 2010, S. 6; MacCallum/Austin 2000, S. 202 f.

${ }^{514}$ Vgl. Backhaus et al. 2011, S. 65.

${ }^{515}$ Vgl. Eggert et al. 2005, S. 102.

516 Vgl. Backhaus et al. 2011, S. 65.
} 
on innerhalb des SGM aber nicht erforderlich ist. ${ }^{517}$

Die zunehmende Verwendung von Strukturgleichungsmodellen in den Sozialwissenschaften ist nicht ohne Kritik geblieben. ${ }^{518}$ Kritisiert wird unter anderem, dass es aufgrund der relativ einfachen Implementierung in SGM zum häufigen Einsatz von neuartigen, bisher nicht widerspruchsfrei definierten latenten Konstrukten kommt, wenngleich bestimmte Zusammenhänge auch direkt manifest gemessen werden könnten. Oftmals werden auch spezifische Umweltbedingungen nicht ausreichend berücksichtigt. Zudem hängen die Ergebnisse in großem Maße von den Stichproben bzw. der Datenbasis ab, so dass eine Vergleichbarkeit der Daten und eine Allgemeingültigkeit der Ergebnisse nur schwer gewährleistet werden können. Zudem gehen die Modelle von linearen Wirkzusammenhängen zwischen den Variablen aus, die in der Realität allerdings nicht immer gegeben sind. ${ }^{519}$

Trotz der Kritik sollen die in dieser Arbeit interessierenden Konstrukte anhand eines SGM untersucht werden. Ein SGM wird für das in dieser Arbeit aufgestellte Hypothesensystem als geeignetes Analyseverfahren angesehen, da es sich insbesondere, wie in den vorangegangen Abschnitten erläutert, bei dem Konstrukt der umfassenden Marktorientierung um ein latentes Konstrukt handelt. Dieses Konstrukt muss hilfsweise über manifeste Indikatoren gemessen werden. Die Variablen Unternehmenszusammenarbeit, Anpassung der Marketingstrategie und Innovativität sind innerhalb des Hypothesensystems sowohl unabhängige als auch abhängige Variablen, so dass die einzelnen Kausalbeziehungen zwischen den verschiedenen Variablen simultan zu schätzen sind. Für die vorliegende Arbeit bietet sich daher die Verwendung eines Strukturgleichungssystems an. Der Kritik hinsichtlich der mangelnden Vergleich- und Generalisierbarkeit der Ergebnisse wird in den Abschnitten 5.3 (Datenerhebung und Datenbasis) und Abschnitt 5.6.3 (Gruppenvergleich der Messmodelle) begegnet. In den genannten Abschnitten soll deshalb dargelegt werden, inwiefern die Gruppe der Befragten als Stichprobe geeignet ist, um valide Auskunft über die in dieser Forschungsarbeit erhobenen Sachverhalte zu geben. Zudem soll überprüft werden, ob in unterschiedlichen

${ }^{517}$ Vgl. Weiber/Mühlhaus 2010, S. 18.

${ }^{518}$ Vgl. hierzu u. im Folgenden Diller 2006, S. 212 f.; MacCullum/Austin 2000, S. 211 f. 
Unternehmen unabhängig von den jeweiligen Marktgegebenheiten die interessierenden Sachverhalte gleich verstanden werden und die Ergebnisse der Messungen verallgemeinerbar sind.

Eine weitere Schwierigkeit im Zusammenhang mit kausalen Beziehungen in SGM ist die zeitliche Erfassung der Daten. Von einer Kausalität kann nur gesprochen werden, wenn die Ausprägung der unabhängigen Variable der Ausprägung der abhängigen zeitlich vorgelagert ist. ${ }^{520}$ Die Variablen im SGM beziehen sich also immer auf mindestens zwei Zeitpunkte. Da aber in den meisten Forschungsprojekten Daten aus ökonomischen Gründen nur zu einem Zeitpunkt erhoben werden und die Probanden kognitiv am einfachsten Auskunft zu einem IstZustand bzw. zu einem aktuellen Verhalten geben können, wird die Prämisse der zeitlichen Unterscheidung oftmals verletzt. Hilfsweise werden in einigen Forschungsarbeiten, bei denen die Daten nur zu einem Zeitpunkt erhoben werden, die Probanden nach Vergangenheitswerten oder Zukunftsprognosen gefragt. ${ }^{521}$ Auch in der vorliegenden Arbeit kann nicht zweifelsfrei die zeitliche Unterscheidung als gegeben angesehen werden. Jedoch handelt es sich bei dem unabhängigen Konstrukt der Marktorientierung um einen Teil einer Unternehmenskultur, die sich in der Regel in einem Unternehmen nur sehr langsam wandelt. Es kann also davon ausgegangen werden, dass die zeitgleiche Messung der Ausprägungen der Marktorientierung und der abhängigen Variablen kein Problem bei der Interpretation von Kausalitäten darstellt.

Der schematische Aufbau eines SGM ist in Abbildung 8 dargestellt. In einem vollständigen SGM werden graphisch die theoretischen Zusammenhänge im sog. Strukturmodell, die Messvorschriften der latenten Variablen im sog. Messmodell dargestellt. ${ }^{522}$ Die abhängigen latenten Variablen werden als endogene Variablen und die unabhängigen latenten Variablen als exogene Variablen bezeichnet. Die endogenen Variablen werden durch die exogenen Variablen innerhalb des SGM

\footnotetext{
${ }^{519}$ Vgl. Homburg/Klarmann 2006, S. 730; Scholderer et al. 2006, S. 643.

${ }^{520}$ Vgl. Weiber/Mühlhaus 2010, S. 13.

${ }^{521}$ Vgl. z.B. Baxter 2012; vgl. auch Kuß/Eisend 2010, S. 46 zum Problem der Zeitpunktbezogenheit bei Querschnittsuntersuchungen.

522 Gebräuchlich sind auch die Begriffe inneres Modell und äußeres Modell. Vgl. hierzu und im Folgenden Götz/Liehr-Gobbers 2004, S. 716; Backhaus et al. 2011, S. 65 ff.
} 
erklärt. Die Erklärung von endogenen Variablen kann in der Regel jedoch nie vollständig erfolgen, so dass jede endogene Variable eine durch das Modell nicht erklärbare Restvarianz $\zeta$ besitzt. Diese Restvarianz kann durch Messfehler oder durch nicht im Modell berücksichtigte Variablen begründet werden. ${ }^{523}$ Die exogenen Variablen werden nicht durch das Modell erklärt, sondern als von außen vorgegeben betrachtet. Messmodelle müssen für alle latenten Variablen aufgestellt werden. Dies betrifft sowohl latente exogene Variablen $\left(\xi_{1}\right)$ als auch latente endogene Variablen $\left(\eta_{1}, \eta_{2}\right)$. Bei den Messmodellen kann es sich um reflektive $(\lambda)$ oder formative Messvorschriften $(\pi)$ handeln. Jedes Konstrukt kann durch mehrere Indikatoren $\left(\mathrm{x}_{1}, \mathrm{x}_{2} ; \mathrm{y}_{1}, \mathrm{y}_{2}\right)$ gemessen werden. Die Richtung der Wirkungszusammenhänge zwischen den einzelnen Konstrukten im Strukturmodell wird mit Hilfe von Pfaden dargestellt.

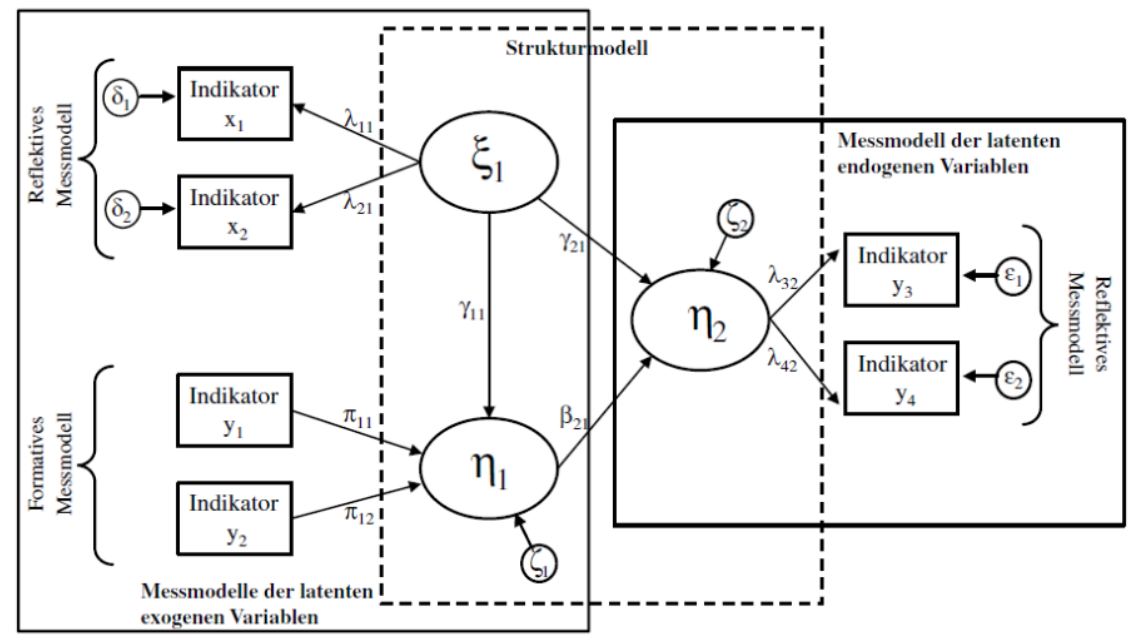

Abbildung 8: Schematisches Strukturgleichungsmodell (Quelle: Götz/Liehr-Gobbers 2004, S. 716)

${ }^{523}$ Vgl. Herrmann et al. 2006, S. 36. 


\subsection{Der Varianz-analytische Ansatz zur Schätzung des Strukturgleichungsmodells}

Das in dieser Arbeit aufgestellte Strukturgleichungsmodell soll mit Hilfe des Varianz-analytischen Verfahrens geschätzt werden. ${ }^{524}$ Hierbei werden die Fallwerte der Ausgangsdatenmatrix mit Hilfe der Kleinst-Quadrate-Schätzung sukzessive (Partialinformationen) möglichst genau reproduziert. ${ }^{525}$ Dieser Ansatz wird daher als Partial-Least-Squares (PLS)-Ansatz bezeichnet.

Nachfolgend werden die Gründe aufgeführt, die für eine Verwendung des PLSVerfahrens sprechen.

- Das zu erforschende Phänomen ist noch relativ neu und es liegen keine etablierten Konstrukttheorien oder Messvorschriften vor. ${ }^{526}$ Das Konstrukt der Marktorientierung ist zwar Gegenstand zahlreicher Untersuchungen in Strukturgleichungsmodellen, dennoch wurde in dieser Arbeit eine neue Konzeptualisierung und Messvorschrift entwickelt. Auf bewährte Messskalen wird zwar teilweise bei den übrigen latenten Konstrukten zurückgegriffen, insgesamt bleibt diese Untersuchung dennoch als überwiegend explorativ einzustufen.

- Die Anwendung von PLS bietet sich immer dann an, wenn das Strukturgleichungsmodell eine hohe Anzahl von Messvariablen besitzt und eher komplex ist. $^{527}$ Das vorliegende zentrale Messmodell von Marktorientierung wurde als zweistufiges Konstrukt mit insgesamt fünf Dimensionen und 24 Indikatoren operationalisiert. Das abgeleitete Strukturgleichungsmodell besteht aus fünf Konstrukten und insgesamt 20 manifesten Variablen. Wenngleich in der Literatur keine allgemeingültige Festlegung auf eine bestimmte Anzahl von

\footnotetext{
${ }^{524}$ Daneben existiert noch der Kovarianz-analytische Ansatz. Beide Ansätze unterschieden sich im Wesentlichen in der mathematischen Schätzmethode der Modellparameter. Eine ausführliche Unterscheidung der beiden Ansätze findet sich z.B. bei Fassott 2005, S. 24 ff.; Herrmann et al. 2006, S. 36 ff., Weiber/Mühlhaus 2010, S. 65 ff. Erwähnt sei hier lediglich, dass die beiden Verfahren nicht als substitutiv zu betrachten sind und ihre relative Überlegenheit und Anwendung sich immer nach der jeweiligen Forschungsfrage richten muss (vgl. Jöreskog/Wold 1982, S. 270; Hair et al. 2012, S. 415). 525 Vgl. Weiber/Mühlhaus 2010, S. 58.

${ }^{526}$ Vgl. Reinartz et al. 2009, S. 334; Weiber/Mühlhaus 2010, S. 253.

${ }^{527}$ Vgl. Henseler et al. 2009, S. 293 f.; Chin 2010, S. 660 f.
} 
Indikatoren, ab der ein Modell als komplex bezeichnet werden kann, existiert, spricht doch die insgesamt hohe Anzahl von Messvariablen für den Einsatz des PLS-Verfahrens. ${ }^{528}$

- Besteht der Fokus einer Untersuchung weniger in der Bestätigung einer Theorie sondern dient vielmehr der Prognose von Variablenausprägungen, so wird ebenfalls der Einsatz von PLS empfohlen. In der vorliegenden Arbeit dient der ressourcen- und kompetenzbasierte sowie der relationale Ansatz lediglich als theoretisches Gerüst und soll durch diese Arbeit nicht als Theorie getestet werden. In der vorliegenden Arbeit stehen vielmehr die Managementimplikationen im Vordergrund, auf welche Weise Unternehmen bestimmte Zielvariablen verändern können. ${ }^{529}$

- Ein weiterer Punkt betrifft die Stichprobengröße und -struktur. Anders als bei Kovarianz-analytischen Verfahren müssen die Daten beim PLS-Verfahren keiner multivariaten Normalverteilung entsprechen. Die PLS-Schätzungen basieren auf der Regressionsanalyse, für die keine Verteilungsannahme notwendig ist. ${ }^{530}$ REINARTZ ET AL. führen zudem an, dass die statistische Aussagekraft von PLS bei relativ kleinen Stichproben größer ist als die von Kovarianz-basierten Verfahren. ${ }^{531}$ Daher kann die Stichprobengröße deutlich geringer ausfallen, um ähnlich valide Ergebnisse zu erzielen wie bei Kovarianz-basierten Verfahren. ${ }^{532}$ In der wissenschaftlichen Praxis hat sich als quasi-Standard etabliert, dass bei der PLS-Analyse der Stichprobenumfang mindestens das Zehnfache der maximalen Anzahl an formativen Indikatoren, die einem Konstrukt zugeordnet sind oder das Zahnfache der maximalen Anzahl von Regressionspfaden auf eine der endogenen Variablen im Modell entsprechen soll. ${ }^{533}$ Aufgrund der eingeschränkten Erreichbarkeit der Auskunftspersonen gestaltet sich im Bereich der Business-to-Business-

${ }^{528}$ CHIN (2010, S. 661) und AKTER (2011, S. 1) sehen hingegen erst bei Modellen mit zehn oder mehr Konstrukten bzw. 50 Items den Einsatz von Varianz-basierten Verfahren als notwendig an.

${ }^{529} \mathrm{Vgl}$. Herrmann et al. 2006, S. 45.

${ }^{530}$ Vgl. Wold 1982; Weiber/Mühlhaus 2010, S. 63

${ }^{531}$ Vgl. Reinartz et al. 2009, S. $332 \mathrm{f}$.

532 Durch Resamplingmethoden (Bootstrapping) kann auch bei kleinen Stichproben die Güte des PLS-Modells beurteilt werden (vgl. Götz/Liehr-Gobbers 2004, S. 721). 
Forschung die Erhebung von Primärdaten im Vergleich zur Business-toConsumer-Forschung deutlich schwieriger. Wenngleich keine festen Vorschriften für den Mindeststichprobenumfang für Kovarianz-basierte Verfahren vorliegen, so zeigt sich dennoch, dass diese in der Regel deutlich über den geforderten Datensätzen des Varianz-analytischen Ansatzes angesiedelt sind. ${ }^{534}$ Dies kann unter Umständen für Analysen auf Basis des Gesamtdatensatzes, noch viel mehr aber auf Basis von Teilstichproben zu Problemen im Hinblick auf die Aussagefähigkeit des Modells führen. Eine vergleichende Mehrgruppenanalyse, bei der jeweils nur ein Teil des Gesamtdatensatzes zur Modellschätzung verwendet wird (vgl. Abschnitt 5.6), ist daher nur mit dem PLS-Verfahren möglich. ${ }^{535}$

- In einigen Studien wird der Einsatz von PLS als Analyseverfahren empfohlen, wenn das SGM Konstrukte mit formativen Messvorschriften enthält. ${ }^{536}$ Grundsätzlich ist die Einbeziehung von formativen Messmodellen in Kovarianz-analytischen Verfahren auch möglich, doch gestaltet sich die gemeinsame Verwendung von reflektiven und formativen Konstrukten, wie sie in dieser Arbeit untersucht werden sollen, wesentlich leichter in der PLSAnalyse. ${ }^{537}$ Zudem werden bei den Kovarianz-analytischen Verfahren die formativen Indikatoren in der Regel über Aufsummieren, Durchschnittsbildung oder andere Berechnungsvorschriften zu einer einzigen Variablen zu-

\footnotetext{
533 Vgl. Chin 1998b, S. 311.

${ }^{534}$ In der Literatur bestehen unterschiedliche Auffassungen über die Mindeststichprobengröße bei den Kovarianz-analytischen Verfahren. So werden feste Stichprobengrößen zwischen 100 und 400 vollständigen Datensätzen gefordert (vgl. Götz/Liehr-Gobbers 2004, S. 733; Ringle 2004; Herrmann et al. 2006, S. 44) oder es werden Faustregeln zu deren Bestimmung erstellt, die sich nach der Anzahl der zu schätzenden Parameter richten (vgl. z.B. Bagozzi/Yi 1988, S. 80; Scholderer/Balderjahn 2005, S. 97).

535 Theoretisch wäre im Rahmen von Kovarianz-analytischen Schätzverfahren auch mit einem relativ kleinen Datensatz ein Gruppenvergleich möglich. Dazu müsste allerdings die Modellkomplexität durch die Verwendung von Konstruktmittelwerten reduziert werden. Voraussetzung dafür ist aber das Vorliegen von Messinvarianz zwischen den Gruppen, die jedoch nicht bei allen Gruppenvergleichen erreicht wird.

536 Vgl. Diamantopoulos/Winklhofer 2001, S. 274; Fassott 2005, S. 24, Herrmann et al. 2006, S. 43; Reinartz et al. 2009, S. 333.

537 Vgl. Scholderer/Balderjahn 2005, S. 93; Fassott 2005, S. 24 f.; Weiber/Mühlhaus 2010, S. 67.
} 
sammengefasst, was einem Teilziel dieser Arbeit entgegenstünde. ${ }^{538}$ Unter anderem sollen in dieser Untersuchung die Treiber, also der Einfluss der einzelnen Indikatoren (Dimensionen) bei der Bildung des Konstrukts der umfassenden Marktorientierung, ermittelt werden.

Um die mit dem PLS-Ansatz ermittelten Schätzwerte der Modellparameter beurteilen zu können, sollen im Folgenden der Prozess und die Kriterien der Gütebeurteilung der Messmodelle und des Strukturmodells erläutert werden.

\subsubsection{Grundlagen der Gütebeurteilung}

Nachdem die Konzeptualisierung und Operationalisierung der interessierende Konstrukte, die Herleitung der Kausalbeziehungen zwischen diesen Konstrukten sowie die Auswahl des Schätzverfahrens erfolgt ist, soll nun die Güte der Modellschätzung im Mittelpunkt dieses Abschnitts stehen.

Voraussetzung für eine aussagekräftige Interpretation der Schätzwerte der Kausalbeziehungen ist eine möglichst fehlerfreie Messung der Konstrukte innerhalb des Strukturgleichungsmodells. Fehler in der Messung der Konstrukte führen zwangsläufig zu Fehlern in der Schätzung der Konstruktbeziehungen. ${ }^{539}$ Messfehler lassen sich in der Regel jedoch fast nie gänzlich ausschließen. In der Realität treten zwischen den tatsächlichen Werten und den gemessenen Werten einer Variable Diskrepanzen auf, die durch systematische oder zufällige Messverzerrungen hervorgerufen werden.

Die Gütemaße geben Aufschluss darüber, wie gut die Anpassung der einem latenten Konstrukt zugeordneten manifesten Variablen an den tatsächlichen Wert dieses Konstrukts gelingt. Insbesondere werden die Reliabilität und die Validität als Kriterien der Modellgüte herangezogen.

Die Gütebeurteilung der Reliabilität gibt Auskunft darüber, ob die Messung der latenten Variable durch die ihr zugeordneten Indikatoren intern konsistent ist. Eine reliable Messung liegt dann vor, wenn die Messung frei von zufälligen Einflüssen ist. ${ }^{540} \mathrm{Um}$ abschätzen zu können, welcher Anteil an der Ausprägungsvari-

538 Vgl. Fassott 2005, S. 25.

539 Vgl. Weiber/Mühlhaus 2010, S. 103.

${ }^{540}$ Vgl. Churchill 1979, S. 65. 
ation der Indikatoren von solchen Einflüssen bzw. Messfehlern verursacht wird, werden in der wissenschaftlichen Praxis meist Multi-Item-Skalen, d. h. mehrere Indikatoren, zur Messung eines Konstrukts empfohlen. ${ }^{541}$ Werden zur Messung einer latenten Variable mehrere Indikatoren verwendet, so bezeichnet CHURCHILL die Messung als reliabel, wenn “[...] independent but comparable measures of the same trait or construct of a given object agree.” ${ }^{42}$ Die Schätzung des Konstruktwerts kann folglich als zuverlässig angesehen werden, wenn die Ausprägungen der verschiedenen Messindikatoren desselben Konstrukts ähnliche Werte annehmen.

Die Gütebeurteilung der Validität zeigt auf, inwiefern die ausgewählten manifesten Indikatoren in der Lage sind, den wahren Wert des betreffenden latenten Konstrukts zu messen. Ein Messwert wird dann als valide angesehen, wenn er frei von systematischen und zufallsbedingten Fehlern ist. ${ }^{543}$ Bei der Validitätsprüfung wird ermittelt, ob eine Messskala auch genau das misst, was im Rahmen der Forschung a priori festgelegt wurde. Validität betrifft somit die konzeptionelle Richtigkeit des Messinstruments. ${ }^{544}$ Zusammengefasst lässt sich Validität folgendermaßen beschreiben: “A measure is valid when the differences in observed scores reflect true differences on the characteristic one is attempting to measure and nothing else”. 545

In der Literatur werden zahlreiche Gütekriterien aufgeführt, um die Reliabilität und Validität eines Messmodells zu bestimmen. Hierbei werden grundsätzlich unterschiedliche Kriterien angewendet, um die Güte von reflektiven und die Güte von formativen Messungen zu beurteilen. Diese Gütemaßzahlen werden in den nachstehenden Abschnitten 5.2.2 und 5.2.3 behandelt und folgen den von HENSELER ET AL. vorgeschlagenen Kriterien. ${ }^{546}$

\footnotetext{
${ }^{541}$ Vgl. Steenkamp/Baumgartner 2000, S. 198. Zu den Empfehlungen von Single-Item-Messungen vgl. Bergkvist/Rossiter 2007; Fuchs/Diamantopoulos 2009; Diamantopoulos et al. 2012.

542 Churchill 1979, S. 65.

${ }^{543}$ Vgl. Churchill 1979, S. 65.

544 Vgl. Weiber/Mühlhaus 2010, S. 103.

${ }^{545}$ Churchill 1979, S. 65

${ }^{546}$ Vgl. Henseler et al. 2009, S. 298 ff.
} 


\subsubsection{Gütekriterien reflektiver Messmodelle}

Bei reflektiven Messmodellen wird davon ausgegangen, dass die Ausprägungen der Indikatorvariablen von einem dahinterliegenden latenten Konstrukt bzw. Faktor verursacht werden. Änderungen der latenten Variable bedingen somit eine Änderung aller diesem Konstrukt zugeordneten Indikatoren, die als miteinander hoch korrelierend angesehen werden. Die hohe Korrelation bzw. Konsistenz der Indikatoren stellt die Basis der Gütebeurteilung reflektiver Messmodelle dar. Ziel der Güteprüfung ist es, Reliabilität und Validität der Messmodelle zu bestätigen.

Die Reliabilität der Messungen soll in dieser Arbeit einerseits durch die Bestimmung der Reliabilität der einzelnen Indikatoren und andererseits durch die Bestimmung der Reliabilität des jeweiligen Konstrukts insgesamt abgeschätzt werden.

Bei Vorliegen von Indikatorreliabilität kann ein wesentlicher Anteil der Indikatorvarianz durch den dahinterliegenden Faktor erklärt werden. Angestrebt wird, dass die Faktorladungen der Indikatoren hinreichend groß sind und weniger als die Hälfte der Varianz Messfehlern zugeschrieben wird. ${ }^{547}$ Reliabilität auf Faktorebene wird anhand der Konstruktreliablität ermittelt. Ein Konstrukt gilt dann als reliabel, wenn die ihm zugeordneten Indikatoren untereinander eine hohe Korrelation aufweisen. Gering korrelierende Indikatoren können zur Steigerung der Reliabilität eliminiert werden. 548

Für die Prüfung der Validität müssen Proxy-Kriterien verwendet werden, da der tatsächliche Wert einer Variablen durch den Einfluss von systematischen und zufälligen Fehlern unbekannt bleibt. Im Rahmen dieser Arbeit soll die Validität der Messungen über die Inhalts- und Diskriminanzvalidität bestimmt werden.

Inhaltsvalidität liegt dann vor, wenn die Messindikatoren dem inhaltlichsemantischen Bereich des betreffenden Konstrukts zugeordnet werden können und alle Bedeutungsinhalte dieses Konstrukts abbilden. ${ }^{549}$ Als Hinweis auf Inhaltsvalidität dient das Vorliegen einer unidimensionalen Faktorstruktur pro Konstrukt und die a priori intendierte Zuordnung der Indikatoren zu ihren jeweiligen

${ }^{547}$ Vgl. Homburg/Giering 1996, S. 16; Krafft et al. 2005, S. 73.

${ }^{548}$ Vgl. Bagozzi/Yi 1988, S. 82. 
Konstrukten mit Hilfe einer explorativen Faktoranalyse.

Diskriminanzvalidität überprüft die Unterschiedlichkeit von Konstrukten, die mit demselben Messinstrument erfasst wurden. Um valide Ergebnisse zu erzielen, muss sich zeigen, dass sich die Messungen von unterschiedlichen Konstrukten auch tatsächlich unterscheiden. ${ }^{550}$ Um dies zu erreichen, muss die gemeinsame Varianz zwischen einem Konstrukt und seinen Indikatoren größer sein als die Varianz zwischen diesem Konstrukt und anderen latenten Variablen. ${ }^{551}$

Nachfolgend werden die verschiedenen Gütemaße in Tabelle 14 aufgeführt, auf die die Konstrukte bzw. Indikatoren im Rahmen dieser Arbeit geprüft werden. Daneben existieren aber noch weitere Gütemaße, die in dieser Arbeit allerdings keine weitere Beachtung erfahren. ${ }^{552}$ Die Gütebeurteilung von Messmodellen erfolgt immer anhand einer Gesamtschau von verschiedenen Gütekriterien. Die in dieser Arbeit verwendeten Kriterien sind somit gemeinsam im Stande, eine fundierte Aussage über die Reliabilität und Validität der interessierenden Modelle zu treffen. Eine Bestimmung und spätere Diskussion weiterer Gütemaße wird daher als nicht weiter zielführend erachtet.

\footnotetext{
${ }^{549}$ Vgl. Weiber/Mühlhaus 2010, S. 128; Krafft et al. 2005, S. 73.

${ }^{550}$ Vgl. Bagozzi/Phillips 1982, S. 469.

551 Vgl. Hulland 1999, S. 199.

552 Vgl. Anhang 1 für die Angabe von zusätzlichen Gütekennzahlen (Kaiser-Meyer-Olkin-Kriterium und der Anteil der erklärten Varianz).
} 
Tabelle 14: Gütebeurteilung reflektiver Konstrukte

(Quelle: in Anlehnung an Krafft et al. 2005, S. 75)

\begin{tabular}{|c|c|c|c|}
\hline Gütekriterium & Definition & Methode/Kriterium & Schwellenwert \\
\hline $\begin{array}{l}\text { Indikatorrelia- } \\
\text { bilität }\end{array}$ & $\begin{array}{l}\text { Erklärungsgrad } \\
\text { der Indikato- } \\
\text { renvarianz } \\
\text { durch das } \\
\text { Konstrukt } \\
\end{array}$ & $\begin{array}{l}\text { Höhe der Faktorla- } \\
\text { dungen; Extrahierte } \\
\text { Kommunalitäten }\end{array}$ & $\begin{array}{l}\text { Faktorladungen }>0,4 \text {, besser }> \\
0,7^{553} ; \text { Kommunalitäten }>0,4^{554}\end{array}$ \\
\hline $\begin{array}{l}\text { Konstruktreli- } \\
\text { ablität }\end{array}$ & $\begin{array}{l}\text { Erklärungsgrad, } \\
\text { wie gut ein } \\
\text { Konstrukt } \\
\text { durch die ihm } \\
\text { zugeordneten } \\
\text { Indikatorvari- } \\
\text { ablen gemessen } \\
\text { wird } \\
\end{array}$ & $\begin{array}{l}\text { Cronbach-Alpha; } \\
\text { Item-Skala- } \\
\text { Korrelation; Com- } \\
\text { posite Reliability; } \\
\text { durchschnittlich } \\
\text { extrahierte Varianz } \\
\text { (DEV) }\end{array}$ & $\begin{array}{l}\text { Cronbach Alpha }>0,7^{555} \text {; Item- } \\
\text { total-Korrelation }>0,5^{556} \text {; Compo- } \\
\text { site Reliability }>0,6^{557} ; \text { DEV }> \\
0,5^{558}\end{array}$ \\
\hline Inhaltsvalidität & $\begin{array}{l}\text { Abbildungsgrad } \\
\text { der Konstrukt- } \\
\text { bedeutung } \\
\text { durch die } \\
\text { Indikatoren }\end{array}$ & $\begin{array}{l}\text { Expertengespräche; } \\
\text { Explorative Faktor- } \\
\text { analyse (EFA); } \\
\text { Anteil der erklärten } \\
\text { Varianz }\end{array}$ & $\begin{array}{l}\text { Abdeckung des Gesamtkonstrukts } \\
\text { durch Facetten; Unidimensionale } \\
\text { Faktorstruktur pro Konstrukt; } \\
\text { Bestätigung der a-priori- } \\
\text { Faktorstruktur }{ }^{559} \text {; Anteil der } \\
\text { erklärten Varianz >0,5 }\end{array}$ \\
\hline $\begin{array}{l}\text { Diskrimi- } \\
\text { nanzvalidität }\end{array}$ & $\begin{array}{l}\text { Unterschied- } \\
\text { lichkeitsgrad } \\
\text { der Messungen } \\
\text { verschiedener } \\
\text { Konstrukte mit } \\
\text { einem Messin- } \\
\text { strument }\end{array}$ & $\begin{array}{l}\text { Explorative Faktor- } \\
\text { analyse (EFA); } \\
\text { Fornell-Larcker- } \\
\text { Kriterium; Chi2- } \\
\text { Differenztest }\end{array}$ & $\begin{array}{l}\text { Starke Konstrukt-Indikator- } \\
\text { Beziehung und Kreuzladungen }< \\
0,4^{560} \text { bei gemeinsamer EFA aller } \\
\text { Indikatoren; DEV > quadrierte } \\
\text { Korrelationen zwischen den Fakto- } \\
\text { ren; keine signifikante Verbesse- } \\
\text { rung des Chi2-Wertes }\end{array}$ \\
\hline
\end{tabular}

\subsubsection{Gütekriterien formativer Messmodelle}

Anders als bei reflektiven Konstrukten stellen die Indikatoren bei formativen Konstrukten die Ursachen dieser Konstrukte dar. Dies bedeutet auch eine signifi-

553 Vgl. Homburg/Giering 1996, S. 12; Nach CHIN (1998b, S. 325) sind allerdings vereinzelt Werte zwischen 0,5 und 0,6 akzeptabel.

${ }^{554}$ Vgl. Yau et al. 2007; WEIBER/MÜHLHAUS (2010, S. 107) hingegen verlangen Werte von > 0,5. 555 Vgl. Nunnally 1978, S. 245; zur Kritik an diesem Wert vgl. Weiber/Mühlhaus 2010, S. 115 und die dort angegebene Literatur.

556 Vgl. Zaichkowsky 1985, S. 343.

${ }^{557}$ Vgl. Bagozzi/Yi 1988, S. 82.

${ }^{558}$ Vgl. Fornell/Larcker 1982, S.46.

${ }^{559}$ Vgl. Kaiser 1974, S. 31 ff.; Patil et al. 2008, S. 164.

${ }^{560}$ Vgl. Miocevic/Crnjak-Karanovic 2012, S. 119. 
kante Unterscheidung bei der Interpretation der Messmodelle, so dass hierbei auch nicht die Gütekriterien und Prüfverfahren der reflektiven Modelle Anwendung finden dürfen. ${ }^{562}$ Alle auf Indikatorkorrelation beruhenden Verfahren zur Güteprüfung verlieren an Aussagekraft, da bei formativen Konstrukten ja gerade die Unterschiedlichkeit der Indikatoren im Zentrum der Konstruktbildung steht. Im Zuge der Modellspezifikation von formativen Konstrukten wird das interessierende Konstrukt zunächst auf Indikatorrelevanz getestet. Einen ersten Anhaltspunkt über die Qualität eines Indikators liefert die Korrelation zwischen den einzelnen Indikatoren und dem Konstrukt selbst, was in diesem Fall z.B. über ein Außenkriterium gemessen werden kann. ${ }^{563}$ Die einzelnen Indikatoren werden auf ihren Beitrag zur Konstruktbildung hin untersucht. Die formative Messung von Konstrukten mit Hilfe des PLS-Ansatzes beruht auf der Regressionsanalyse. Für jeden Indikator wird daher ein Regressionsgewicht ermittelt. Da jeder Indikator auch einen wesentlichen inhaltlichen Bereich des latenten Konstrukts abbildet, wird gefordert, dass die Indikatoren einen nennenswerten, signifikanten Einfluss auf das Konstrukt haben. ${ }^{564}$ Die Gewichte geben dabei den Beitrag zur Erklärung des betreffenden Konstrukts an. ${ }^{565}$ Geringe absolute Gewichte dürfen dabei aber nicht als ein schlecht spezifiziertes Modell interpretiert werden. ${ }^{566}$ Vielmehr dienen die Gewichte als Vergleich zwischen den Indikatoren. ${ }^{567}$ Anders als bei reflektiven Modellen sind Indikatoren mit geringen Ladungen nicht zu eliminieren, da sonst das theoretische Konstrukt konzeptionell verändert werden würde. ${ }^{568}$

Eine hohe Korrelation von Indikatoren kann, anders als bei reflektiven Modellen, ein Problem darstellen. Da formative Modelle auf dem Prinzip der multiplen Regressionsanalyse beruhen, dürfen die Indikatoren nicht linear voneinander abhängig sein. Andernfalls würde es zu Verzerrungen der Parameterschätzwerte

\footnotetext{
${ }^{561}$ Vgl. Bagozzi/Yi 1988, S. 81.

562 Vgl. Krafft et al. baker 2005, S. 76; Fassott/Eggert 2005, S. 38 f.

563 Vgl. Diamantopoulos/Winklhofer 2001, S. 272.

${ }^{564}$ Vgl. Diamantopoulos et al. 2008, S. 1215; Chin 1998b, S. 307; Seltin/Keeves 1994, S. 4356.

565 Vgl. Chin 1998b, S. 307.

${ }^{566}$ Vgl. Götz/Liehr-Gobbers 2004, S. 729.

${ }^{567}$ Vgl. Sambamurthy/Chin 1994, S. 231f.
} 
oder gar zu einer mathematischen Nicht-Lösbarkeit der Parameterschätzung kommen. ${ }^{569}$ Ein Indikator mit hoher Multikollinearität wird dahingehend interpretiert, dass der Bedeutungsgehalt des Indikators redundant ist, keine Relevanz vorliegt und der Indikator somit eliminiert werden kann. ${ }^{570}$

Ein formatives Konstrukt kann auf externe Validität hin geprüft werden. Dabei wird überprüft, wie stark die einzelnen Indikatoren mit einem Außenkriterium korrelieren. Als Außenkriterium kann eine reflektive Operationalisierung desselben Konstrukts durch sog. Globalindikatoren dienen. ${ }^{571}$ Die Globalindikatoren sollten eine starke Verbindung zu den formativen Indikatoren aufweisen und das Konstrukt inhaltlich zusammengefasst abbilden. ${ }^{572}$ Durch dieses Vorgehen wird es möglich, Fehlerterme zu bestimmen und zu überprüfen, wie groß der Anteil der erklärten Varianz des Konstrukts durch die formativen Indikatoren ist. ${ }^{573}$

Die nachfolgend in Tabelle 15 aufgeführten Gütekriterien formativer Messmodelle werden in der Literatur bisweilen aufgrund der ähnlichen kausalen IndikatorKonstrukt-Beziehung auch zur Gütebeurteilung von Strukturmodellen herangezogen. Diesem Vorgehen wird auch in dieser Arbeit gefolgt, so dass teilweise dieselben Kriterien zur Abschätzung der Strukturmodellgüte Anwendung finden.

\footnotetext{
${ }^{568}$ Vgl. Bollen/Lennox 1991, S. 308; Coltman et al. 2008, S. 1253; Jarvis et al. 2003, S. 203.

569 Vgl. Wilcox et al. 2008, S. 1222; Albers/Hildebrandt 2006, S. 16.

570 Vgl. Krafft at al. 2005, S. 79; Götz/Liehr-Gobbers 2004, S. 729; Diamantopoulos et al. 2008, S. 1212.

${ }^{571}$ Vgl. Diamantopoulos/Winklhofer 2001, S. 272; Weiber/Mühlhaus 2010, S. 209. Dieses Vorgehen wird auch als Multiple-Indicators-Multiple-Causes (MIMIC) Modell bezeichnet.

572 Vgl. Diamantopoulos/Winklhofer 2001, S. 272.

${ }^{573}$ Vgl. Krafft et al. 2005, S. 80.
} 
Tabelle 15: Gütebeurteilung formativer Messmodelle

(Quelle: in Anlehnung an Krafft et al. 2005, S. 82)

\begin{tabular}{|c|c|c|c|}
\hline Gütekriterium & Definition & Methode/Kriterium & Schwellenwert \\
\hline Indikatorrelevanz & $\begin{array}{l}\text { Überprüfung der } \\
\text { Indikatoren auf } \\
\text { ihren Beitrag zur } \\
\text { Konstruktbildung }\end{array}$ & $\begin{array}{l}\text { Überprüfung der Regres- } \\
\text { sionskoeffizienten; Test } \\
\text { auf Multikollinearität; } \\
\text { Korrelation der Indikato- } \\
\text { ren mit einem Außenkri- } \\
\text { terium }\end{array}$ & $\begin{array}{l}\text { Signifikante t-Werte } \\
\text { der standardisierten } \\
\text { Regressionsgewichte; } \\
\text { Regressionsgewichte } \\
>0,1^{574} \text {; Variance } \\
\text { Inflation Factor (VIF) } \\
<5^{575} \text {; Signifikante } \\
\text { Item- } \\
\text { Außenkriteriums- } \\
\text { Korrelation }{ }^{576}\end{array}$ \\
\hline Externe Validität & $\begin{array}{l}\text { Evaluation der } \\
\text { Gültigkeit der } \\
\text { Konstruktmessung }\end{array}$ & $\begin{array}{l}\text { Korrelation mit Außen- } \\
\text { kriterium; MIMIC- } \\
\text { Modell } \\
\text { Mes77 (gleichzeitige } \\
\text { Indikatoren) }\end{array}$ & $\begin{array}{l}\text { Stärke, Richtung, } \\
\text { Signifikanz des Zu- } \\
\text { sammenhangs; R2 im } \\
\text { MIMIC-Modell } \geq \\
0,33^{578}\end{array}$ \\
\hline
\end{tabular}

\subsubsection{Gütekriterien des Strukturmodells}

Beim PLS-Schätzverfahren können keine inferenzstatistischen Tests zur Gütebeurteilung des Gesamtmodells durchgeführt werden. Da dem Varianz-analytischen Verfahren keine Verteilungsannahmen zu Grunde liegen, muss die Modellgüte über nicht parametrische Tests bestimmt werden. ${ }^{579}$ Als Gütekriterien werden hier die Kausalbeziehungen zwischen den Konstrukten herangezogen. Um die Güte der Beziehungen abzuschätzen, dienen die Höhe sowie die Signifikanzen der Parameter auf Basis von t-Werten mittels Boostrapping-Prozedur. ${ }^{580}$ Zudem wer-

\footnotetext{
574 Vgl. Seltin/Keeves 1994, S. 4356.

575 Vgl. Diamantopoulos/Riefler 2008, S. 1193; die Schwelle für einen kritischen VIF-Wert wird in der Fachliteratur unterschiedlich angesetzt. LUO ET AL. (2005, S. 60) sehen erst Werte von > 10 als bedenklich an, WEIBER/MÜHLHAUS (2010, S. 207 f.) empfehlen hingegen schon eine Prüfung bei Werten $>3$.

576 Vgl. Diamantopoulos/Winklhofer 2001, S. 272.

${ }^{577}$ Beim MIMIC-Verfahren (Multiple Indicators, Multiple Causes) findet eine gleichzeitige Konstruktmessung durch reflektive und formative Indikatoren statt.

578 Vgl. Chin 1998b, S. 323

${ }^{579}$ Vgl. Krafft et al. 2005, S. 83; Chin 1998b, S. 316.

580 Vgl. Herrmann et al. 2006, S. 59; Efron/Gong 1983, S. 37 ff. Zur Ermittlung der t-Werte wird im Rahmen dieser Arbeit durchgehend auf ein Boostrapping-Verfahren zurückgegriffen, das auf $500 \mathrm{zu}$ ziehenden Stichproben mit je 384 Fällen beruht.
} 
den der Anteil der erklärten Varianz der endogenen Variablen, der Erklärungsbeitrag der exogenen Variablen im Modell und die Prognoserelevanz jeweils als Gütekriterium angeführt. Tabelle 16 fasst die Kenngrößen zusammen.

Tabelle 16: Gütebeurteilung von Strukturgleichungsmodellen

(Quelle: in Anlehnung an Krafft et al. 2005, S. 85)

\begin{tabular}{|c|c|c|c|}
\hline Gütekriterium & Definition & Methode/Kriterium & Schwellenwert \\
\hline $\begin{array}{l}\text { Ausmaß und Signifi- } \\
\text { kanz der Pfadkoeffi- } \\
\text { zienten }\end{array}$ & $\begin{array}{l}\text { Stärke der Wirkbe- } \\
\text { ziehung zwischen } \\
\text { den Konstrukten }\end{array}$ & $\begin{array}{l}\text { Standardisierte ß- bzw. } \\
\gamma \text {-Koeffizienten }{ }^{581} \text {; t- } \\
\text { Werte nach der } \\
\text { Boostrapping- } \\
\text { Methode }^{582}\end{array}$ & $\begin{array}{l}\text { Pfadkoeffizienten }> \\
0,2^{583} \\
\text { t-Werte }>1,96\end{array}$ \\
\hline Bestimmtheitsmaß & $\begin{array}{l}\text { Anteil der erklärten } \\
\text { Varianz des Kon- } \\
\text { strukts }\end{array}$ & $\begin{array}{l}\mathrm{R}^{2} \text {-Werte (interpretierbar } \\
\text { wie bei multipler Re- } \\
\text { gression) }\end{array}$ & $\begin{array}{l}\mathrm{R}^{2} \approx 0,67 \text { (substan- } \\
\text { ziell) } \\
\mathrm{R}^{2} \approx 0,33 \text { (moderat) } \\
\mathrm{R}^{2} \approx 0,19 \\
(\text { schwach) } \\
\end{array}$ \\
\hline Erklärungsbeitrag & $\begin{array}{l}\text { Substanzieller } \\
\text { Einfluss der exoge- } \\
\text { nen Variablen auf } \\
\text { die endogenen } \\
\text { Variablen im Mo- } \\
\text { dell }\end{array}$ & $\mathrm{f}^{2}$ (Effektgröße) & $\begin{array}{l}\mathrm{f}^{2} \approx 0,02 \text { (gering) } \\
\mathrm{f}^{2} \approx 0,15 \text { (mittel) } \\
\mathrm{f}^{2} \approx 0,35(\text { stark })^{585}\end{array}$ \\
\hline Prognoserelevanz & $\begin{array}{l}\text { Anpassung des } \\
\text { Modells an die } \\
\text { empirischen Daten }\end{array}$ & $\begin{array}{l}\mathrm{Q}^{2} \text { (Stone-Geisser- } \\
\text { Kriterium); q² (pfadbe- } \\
\text { zogenes Stone-Geisser- } \\
\text { Kriterium) }\end{array}$ & $\begin{array}{l}\mathrm{Q}^{2}>0 \\
\mathrm{q}^{2}>0^{586}\end{array}$ \\
\hline
\end{tabular}

\subsubsection{Gütebeurteilung von Mediationseffekten}

Zusätzlich zu den in Abschnitt 5.2.4 vorgestellten Gütekriterien des Strukturmodells sollen in diesem Abschnitt die Gütekriterien für die in Abschnitt 4.4.4 hypothetisierten Mediationseffekte vorgestellt werden. Als Mediationseffekt wird der Einfluss einer intervenierenden Drittvariablen auf die Beziehung zwischen einer

581 Zur Benennung der Koeffizienten vgl. Abbildung 8.

582 Vgl. Herrmann et al. 2006, S. 59

${ }^{583}$ Vgl. Chin 1998a, S. xiii. CHIN sieht idealerweise erst Koeffizienten > 0,3 als bedeutungsvoll an.

584 Vgl. Chin 1998b, S. 323.

${ }^{585}$ Vgl. Chin 1998b, S. 317

${ }^{586}$ Vgl. Fornell/Bookstein 1982, S. 449; Chin 1998b, S. 318. 
endogenen und einer exogenen Variable bezeichnet. ${ }^{587}$ Ziel der Berücksichtigung von Mediatorvariablen ist es, die Wirkweise der unabhängigen Variable im Modell besser erklären zu können. ${ }^{588}$ In der vorliegenden Untersuchung wurde theoretisch hergeleitet, dass die Unternehmenszusammenarbeit, die Anpassung der Marketingstrategie und die Innovativität die Beziehung zwischen Marktorientierung (exogene Variable) und Neuprodukterfolg (endogene Variable) mediieren sollen. Bildlich ist die Beziehung der exogenen, endogenen und mediierenden Variablen in Abbildung 9 dargestellt.

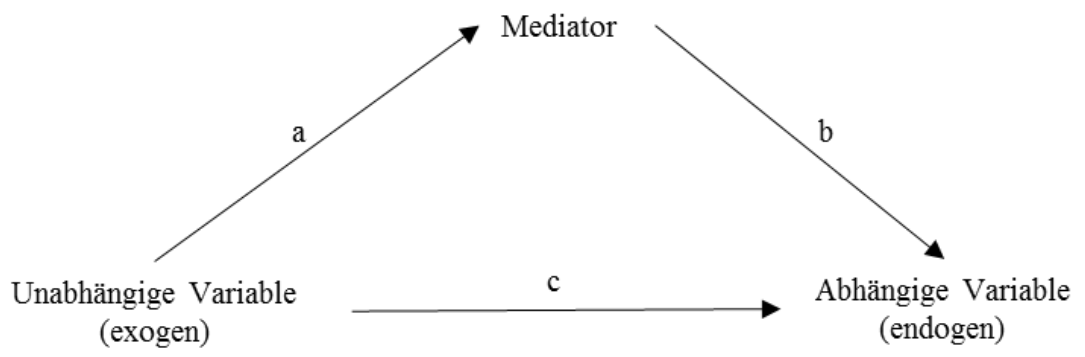

Abbildung 9: Mediationsmodell

(Quelle: in Anlehnung an Baron/Kenny 1986, S. 1176)

Eine Variable kann dann als Mediator bezeichnet werden, wenn die folgenden Voraussetzungen erfüllt sind: 589

- Eine Veränderung der unabhängigen Variable führt zu signifikanten Änderungen der zwischengeschalteten Variable (Pfad a).

- Änderungen der zwischengeschalteten Variable führen zu signifikanten Einflüssen auf die abhängige Variable (Pfad b).

- Die ursprünglich signifikante direkte Beziehung (Pfad c) wird nichtsignifikant (vollständige Mediation) bzw. bleibt signifikant, wird aber kleiner (partielle Mediation), wenn die Mediatorvariable im Modell eingeführt

\footnotetext{
${ }^{587}$ Vgl. Preacher/Hayes 2008, S. 879.

${ }^{588} \mathrm{Vgl}$. Baron/Kenny 1986, S. 1176.

${ }^{589}$ Vgl. Eggert et al. 2005, S. 105; Baron/Kenny 1986, S. 1176.
} 
wird. ${ }^{590}$ Eine sukzessive Prüfung von Teilmodellen (nur der direkte Effekt (Pfad c)); direkte und indirekte Effekte (Pfade a, b, c)) kann unter Zuhilfenahme des z-Tests nach SOBEL umgangen werden. ${ }^{591}$ Hierbei reicht der Nachweis der Signifikanz des indirekten Effekts (Pfad a und Pfad b) als Beleg für Mediation. ${ }^{592}$

Von Interesse ist bei der Interpretation von Mediationseffekten mehr als eine dichotome Einteilung in vollständige und partielle Mediation vornehmen zu können. Um die Stärke des Mediationseffekts genauer zu bestimmen, schlagen SHROUT/BOLGER vor, den mediierten Teil der Beziehung zwischen exogener und endogener Variable als Proportion $\mathrm{P}_{\mathrm{M}}$ zwischen indirektem und totalem Effekt anzugeben. ${ }^{593}$ Als totaler Effekt wird hierbei die Beziehung zwischen der exogenen und endogenen Variable verstanden, der sich aus dem direkten Effekt (Pfad c) und dem indirekten Effekt (Pfad a und Pfad b) zusammensetzt. Tabelle 17 gibt die Gütekriterien der Mediationseffekte wider.

Tabelle 17: Gütekriterien von Mediationseffekten

\begin{tabular}{|l|l|l|l|}
\hline Gütekriterium & Definition & Methode/Kriterium & Schwellenwert \\
\hline $\begin{array}{l}\text { Nachweis von } \\
\text { Mediation }\end{array}$ & $\begin{array}{l}\text { Indirekter Effekt der } \\
\text { exogenen Variable } \\
\text { über den Mediator } \\
\text { auf endogene Variab- } \\
\text { le }\end{array}$ & $\begin{array}{l}\text { Signifikanz des z-Werts } \\
\text { (Sobel-Test) }\end{array}$ & Z >1,96 \\
\hline $\begin{array}{l}\text { Stärke der Mediati- } \\
\text { on }\end{array}$ & $\begin{array}{l}\text { Betrachtung des } \\
\text { direkten Effekts }\end{array}$ & $\begin{array}{l}\text { Ausprägung und Signi- } \\
\text { fikanz der direkten } \\
\text { Effekte; PM-Wert }\end{array}$ & $\begin{array}{l}\text { Partielle Mediation: } \\
\text { signifikanter t-Wert } \\
\text { des direkten Effekts } \\
\text { (> 1,96), niedriger } \\
\text { PM-Wert; vollständi- } \\
\text { ge Mediation: nicht } \\
\text { signifikanter t-Wert } \\
\text { (<1,96), hoher PM- } \\
\text { Wert }\end{array}$ \\
\hline
\end{tabular}

\footnotetext{
${ }^{590}$ Für eine feinere Unterscheidung der Arten von Mediation sei auf ZHAO ET AL. (2010) verwiesen. Die Autoren kategorisieren Mediationen anhand der Ausprägungen und Signifikanzen der direkten und mediierten Variablenbeziehungen.

591 Vgl. Sobel 1982.

592 Vgl. Eggert et al. 2005, S. 105.

593 Vgl. Shrout/Bolger 2002, S. 434.
} 


\subsubsection{Gütebeurteilung von Messmodellen zweiter Ordnung}

Bei dem in dieser Arbeit konzeptualisierten Konstrukt der Marktorientierung handelt es sich um ein mehrdimensionales Konstrukt, das auf der ersten Ebene reflektiv und auf der zweiten Ebene formativ gemessen wird. Die Gütebeurteilungen von mehrdimensionalen Konstrukten werfen allerdings messtechnische Schwierigkeiten auf, weshalb sich in der wissenschaftlichen Praxis Hilfsverfahren zur Validierung solcher Messmodelle etabliert haben. ALBERS/GÖTZ empfehlen dazu, eine MIMIC (Multiple Indicators, Multiple Causes)Vorgehensweise. ${ }^{594}$ Das Konstrukt der umfassenden Marktorientierung wird hierzu zusätzlich durch reflektive Indikatoren erhoben, die auf globale Weise den Gesamtcharakter von Marktorientierung erfassen können. Sofern die Varianz des Konstrukts auf der zweiten Ebene nur in unzureichendem Maße durch die Dimensionen der ersten Ebene erklärt werden kann, deutet dies darauf hin, dass die konzeptualisierten Dimensionen das Konstrukt nicht in seiner Gesamtheit erfassen können.

Eine andere Möglichkeit der Gütebeurteilung besteht darin, das Konstrukt höherer Ordnung in ein Konstrukt erster Ordnung umzuwandeln. Dazu können die einzelnen Dimensionen durch ihre jeweiligen Faktorwerte ersetzt werden. ${ }^{595}$ Die Faktorwerte können dann als formative Indikatoren des Konstrukts der umfassenden Marktorientierung genutzt werden. Zur Gütebeurteilung können hierbei die Kriterien der formativen Messmodelle herangezogen werden.

Zur Prüfung der Validität der Konstruktmessung kann zusätzlich noch die Beziehung des Konstrukts zu anderen Konstrukten dienen. Zeigt sich bei der Einbettung des Konstrukts in ein nomologisches Netzwerk, dass die entsprechenden Pfade signifikant sind und der postulierten Richtung der Hypothesen folgen, kann dies als Vorliegen von nomologischer Validität aufgefasst werden. ${ }^{596}$ Als nomologisches Netzwerk können die Wirkrichtung der Indikatoren des Messmodells sowie das Strukturgleichungsmodell der vorliegenden Arbeit dienen.

In der Literatur wird auch die Möglichkeit aufgezeigt, Konstrukte höherer Ord-

${ }^{594}$ Vgl. hierzu und im Folgenden Albers/Götz 2006, S. 674; Becker et al. 2012, S. 365 ff.
${ }^{595}$ Vgl. Albers/Götz 2006, S. 674; Giere et al. 2006, S. 688; Becker et al. 2012, S. 366. 
nung durch einen einzigen aggregierten Indexwert handhabbar zu machen. ${ }^{597}$ Die Gütebeurteilung könnte dann ebenfalls im Rahmen eines nomologischen Netzwerks durchgeführt werden. Die Berechnung eines sog. Composite Scores erscheint in dieser Arbeit aber nicht als zielführend, da es ja gerade für die vorliegende Untersuchung von Interesse ist, die Treiber bzw. die einflussstärksten Dimensionen zu identifizieren. Insbesondere in der Erfolgsfaktorenforschung ist dieser Umstand hervorzuheben. Schließlich erscheint es durchaus plausibel, dass Marktorientierung einen Einfluss auf das Marktergebnis eines Unternehmens hat. Von größerem Interesse ist hingegen eher, welche der Dimensionen dazu am meisten beiträgt. ${ }^{598}$

\subsection{Datenerhebung und Datenbasis}

\subsubsection{Wahl der Erhebungsmethode und Fragebogenentwicklung}

Bei der Konzeptualisierung und Messung des Konstrukts einer umfassenden Marktorientierung sowie des aufgestellten Hypothesensystems kann nicht auf bestehende Daten aus früheren Forschungsvorhaben zurückgegriffen werden. Die Daten müssen daher als Primärinformationen neu erhoben werden, um Aussagen über Reliabilität und Validität treffen zu können.

Um ein besseres Verständnis des Konstrukts der Marktorientierung aus Sicht der Marketingpraxis zu erlangen wurden zunächst Interviews mit 13 Praktikern aus den Bereichen Vertrieb und Marketing geführt. Die Interviewpartner waren größtenteils bei großen und mittleren Unternehmen in leitender Funktion im Bereich Marketing und Vertrieb oder in der Unternehmensführung tätig. Drei der interviewten Personen waren in einfacher Position im Vertrieb oder als Applicationbzw. Produktmanager tätig. Die Interviewpartner gaben an, ihre Haupttätigkeitsfelder in den folgenden Märkten zu haben: Elektro-, Automatisierungs/Steuerungsbranche, Antriebs- und Motorenbau, Anlangenbau, Metallverarbei-

596 Vgl. Giere et al. 2006, S. 689; Diamantopoulos/Winklhofer 2001, S. 273.

597 Vgl. Giere et al. 2006, S. 689.

598 Vgl. Albers 2010, S. 415. 
tung und Chemische Industrie. Die Durchführung der Interviews war Leitfadenbasiert und fand auf der Hannover Messe statt. Aufgrund der Befragungssituation konnten die Interviews nicht aufgezeichnet, sondern lediglich schriftlich zusammengefasst dokumentiert werden. Die Interviewpartner können aufgrund ihres Überblicks über die zentralen inhaltlichen Aspekte des Interviews als Experten bezeichnet werden. ${ }^{599}$ Ziel der Interviews war es, tiefere Einsichten in das im Rahmen der Business-to-Business-Praxis vorherrschende Marktverständnis zu erlagen und eventuelle Unterschiede zum Verständnis des Marktes in der einschlägigen Marketing-Fachliteratur festzustellen. Die Interviewpartner wurden dazu gebeten, ihren Markt, ihre Wertschöpfungsketten sowie alle für ihr Geschäft relevanten Marktteilnehmer zu beschreiben. Anschließend wurden die Interviewpartner zu den Aktivitäten ihres eignen Unternehmens befragt, die auf die verschiedenen Marktteilnehmer und Marktstufen gerichtet sind. Abschließend wurde den Interviewten die Möglichkeit gegeben, noch weitere ihrer Meinung nach relevante Aspekte von Marketing und Vertrieb im B2B-Bereich zu benennen. Mehrfach wurden hier die Machtkonstellationen zwischen dem eigenen Unternehmen und dem direkten Kunden als Einflussfaktor auf die Ausgestaltung der eigenen Vertriebstätigkeiten genannt. Neben der Gewinnung von Erkenntnissen über das im B2B-Bereich vorherrschende Marktverständnis wurde die Auswertung der Experteninterviews zudem darüber hinaus genutzt, um die thematischen Schwerpunkte für die zwei folgenden Fokusgruppeninterviews abzuleiten. Im Rahmen der sich anschließenden Fokusgruppeninterviews wurden schwerpunktmäßig noch einmal die für Unternehmen relevanten Marktteilnehmer und auch verschiedenen marktbezogenen Aktivitäten diskutiert, die als Ausdruck marktorientierten Handelns in der Business-to-Business-Praxis verstanden werden. Die Teilnehmer der Fokusgruppeninterviews waren Studierende des weiterbildenden Studiengangs „Executive Master of Business Marketing“ an der Freien Universität Berlin. Die Studierenden zeichnen sich dadurch aus, dass sie für die Zulassung zum Studium eine einschlägige, mehrjährige Berufserfahrung im Bereich Marketing oder Vertrieb im B2B-Umfeld nachweisen müssen. Die Teilneh-

${ }^{599}$ Vgl. Hitzler 1994, S. 24. 
mer waren teilweise in leitender, teilweise in rein operativer Funktion im Unternehmen tätig. Es kann also bei den Befragten insgesamt auf eine gewisse Expertise zu den inhaltlichen Themen der Gruppeninterviews geschlossen werden. Aufgrund der ähnlichen funktionalen Verantwortlichkeiten in den jeweiligen Unternehmen kann zudem bei den Interviewteilnehmern auf eine relativ homogene Gruppe hinsichtlich ihres aktuellen beruflichen Hintergrundes geschlossen werden. Es kann aufgrund des damit gegebenen ähnlichen Verständnisses von Fachtermini und Geschäftsabläufen von einer hohen Validität und Qualität der Ergebnisse der Gruppendiskussionen ausgegangen werden. ${ }^{600}$ Durch die Tatsache, dass die Gruppenteilnehmer verschiedene Unternehmen aus gänzlich unterschiedlichen Branchen repräsentieren, die eine große Varianz im Leistungsspektrum besitzen, konnten unterschiedliche Ansichten des jeweils relevanten Marktes erfasst werden. Durch diese Unternehmensheterogenität und insbesondere durch die Unterschiedlichkeit der jeweiligen Wertschöpfungsketten war es möglich, eine breite Übersicht über Markteinflussfaktoren und Marktteilnehmer zu gewinnen. ${ }^{601}$

In den Fokusgruppeninterviews wurden die Teilnehmer gebeten, ihre jeweiligen Absatzmärkte und die Struktur der nachgelagerten Wertschöpfungskette zu beschreiben sowie Markteilnehmer und Einflussnehmer in ihren jeweiligen Branchen zu nennen. Die genannten Markteilnehmer und Einflussnehmer wurden den vier Marktorientierungsdimensionen zugeordnet, die die Teilausrichtung des Unternehmens auf eine bestimmte Marktteilnehmergruppe beschreiben. Um dabei der subjektiven Interpretation der Interviewergebnisse durch den Interviewer zu begegnen, wurden die Teilnehmer anschließend aufgefordert, die Zuordnung der genannten Markteilnehmer und Einflussnehmer kritisch zu überprüfen. ${ }^{602}$ So konnte gewährleistet werden, dass die genannten Marktteilnehmer den korrekten Dimensionen zugeordnet wurden und die Dimensionen in verschiedenen Branchen existent und relevant sowie inhaltlich abgegrenzt und weitgehend überschneidungsfrei sind. Von Bedeutung ist ebenfalls, dass keiner der genannten

${ }^{600}$ Vgl. Birn 2000, S. 272.

${ }^{601}$ Vgl. Callingham 2004, S. 104 
Marktteilnehmer oder Einflussnehmer nicht einer der vier Gruppen sinnvoll und nachvollziehbar zugeordnet werden konnte. Daraus konnte geschlussfolgert werden, dass auch in der Marketingpraxis die vier Marktbereiche (Kunden, Wettbewerber, Drittparteien, nachgelagerte Marktstufen) unabhängige Dimensionen als Teile einer Marktorientierung darstellen. Die vier für die befragten Marketingund Vertriebspraktiker relevanten Anspruchsgruppen, die gleichzeitig den Markt ausmachen, sind mit beispielhaften Nennungen durch die Fokusgruppen in

Tabelle 18 wiedergegeben. Die im Rahmen der Konzeptualisierung propagierten Dimensionen von Marktorientierung konnten dabei bestätigt werden.

Durch die Auflistung und Kategorisierung der relevanten Marktteilnehmer kann gleichzeitig Forschungsfrage 1 abschließend beantwortet werden. Forschungsfrage 1 beschäftigte sich mit der Zusammensetzung des Marktes im B2B-Bereich. Die identifizierten relevanten Bestandteile des Marktes sind somit: Kunden, Wettbewerber, Drittparteien und nachgelagerte Marktstufen.

Tabelle 18: Dimensionszuordnung relevanter Marktteilnehmer

\begin{tabular}{|l|l|l|l|}
\hline \multicolumn{2}{|l|}{ Gruppen relevanter Marktteilnehmer } & \multicolumn{2}{l|}{} \\
\hline Kunden & Wettbewerber & Drittparteien & $\begin{array}{l}\text { Nachgelagerte } \\
\text { Marktstufen }\end{array}$ \\
\hline - Kunden & - Wettbewerb & - Berater & - Endkunden \\
- Key-Accounts & - Konkurrenz & - Wirtschaftsverbände & - Endnutzer \\
- OEMs & - Branchenprimus & - Gutachter & - Lead-User \\
- Handel & - Innovationsführer & - Trendsetter & - Kunden der \\
- Käufer & - "Copy-Cats" und & - Politik & Kunden \\
- Projektpartner & "Follower" & - Branchenvertreter & - Indirekte \\
- Weiterverarbei- & & - Verbraucherschützer & Kunden \\
tungsbetriebe & & - Multiplikatoren & - Konsumen- \\
& & - Pressevertreter/Medien & ten \\
& & - Institute \& Universitäten & \\
& & - Umweltschutzorganisatio- & \\
& & - nen & \\
\end{tabular}

Aus den Experten- und Fokusgruppeninterviews konnten neben den relevanten Marktteilnehmern auch solche Unternehmenstätigkeiten abgeleitet werden, die

${ }^{602}$ Vgl. zu diesem Vorgehen Shao/Zhou 2006, S. 161; Birn 2000, S. 280. 
auf die zuvor genannten Marktteilnehmer ausgerichtet werden. Ein Großteil der abgeleiteten Items war inhaltlich deckungsgleich mit den im Rahmen der MKTOR-Skala entwickelten Items. Die abgeleiteten Tätigkeiten wiesen allerdings im Gegensatz zur ursprünglichen Skala eine deutliche Tendenz zur Nutzung von elektronischen Medien auf (z.B. Kommunikation mit dem Kunden per E-Mail, Nutzung elektronischer CRM-Systeme, Abruf und Weiterleitung von online verfügbaren Markt- und Absatzdaten). Für die Entwicklung der Skala waren allerdings weniger die genutzten Medien oder die genaue Form der Aktivitäten, sondern vielmehr der Inhalt und das angestrebte Ziel der Aktivitäten von Bedeutung. Daher wurden die Tätigkeiten weitgehend von ihren Medien abstrahiert, so dass zur Messung der in den Interviews genannten und mit der MKTORSkala inhaltlich identischen Tätigkeiten die Items der ursprünglichen MKTORSkala übersetzt und in die Item-Batterie aufgenommen wurden. Aus den in den Interviews genannten marktorientierten Tätigkeiten konnten insgesamt 43 Items abgeleitet werden, die potentiell zur Messung von Marktorientierung herangezogen werden können (vgl. Anhang 2).

Die Items wurden zur Überprüfung ihrer Verständlichkeit und Sinnhaftigkeit sowie für eine erste Einschätzung der Inhaltsvalidität 12 fachkundigen Wissenschaftlern der Freien Universität Berlin vorgelegt. Die Wissenschaftler wurden gebeten, die einzelnen in einem Fragebogen zufällig angeordneten Items denjenigen Dimensionen zuzuordnen, deren Inhalt sie mutmaßlich abbildeten. Items, die sich nicht eindeutig einer Dimension zuordnen ließen, wurden aus der ItemBatterie gelöscht oder nach Rücksprache mit Marketingpraktikern so umformuliert, dass die darin beschriebene Tätigkeit leichter einer Dimension eindeutig zugeordnet werden konnte. ${ }^{603}$

Die Items wurden anschließend einem ersten quantitativen Pretest unterzogen. ${ }^{604}$ Dazu wurde ein Fragebogen erstellt, der sich inhaltlich, semantisch und optisch weitgehend an den in der Fachliteratur zur Qualitätssicherung in der Umfragefor-

${ }^{603}$ Vgl. zu diesem Vorgehen Anderson/Gerbing 1991, S. 734.

${ }^{604}$ Vgl. Kaase 1999, S. 49. 
schung beschriebenen und etablierten Standards orientierte. ${ }^{605}$ Allerdings wurde abweichend von den etablierten Standards auf sogenannte Eisbrecherfragen verzichtet, da angenommen wurde, dass die Gruppe der befragten Personen eher wenig Zeit in die Beantwortung der Fragen investieren möchte. Zudem wurden die befragten Personen durch die persönliche Ansprache per E-Mail ausreichend mit dem Thema der Studie und der zugesicherten Datensicherheit vertraut gemacht, so dass der Umfang des Fragebogens selbst auf die wesentlichen interessierenden Sachverhalte beschränkt wurde. Die befragten Personen stammten überwiegend aus der Gruppe der ehemaligen Studenten des weiterbildenden Masterstudiengangs Executive Master of Business Marketing der Freien Universität Berlin. Insgesamt wurden 113 Personen mit einer persönlichen E-Mail oder über das soziale Netzwerk „Xing“ kontaktiert. Zudem wurde durch einen Einleitungstext der thematische Schwerpunkt der Umfrage erläutert und Anonymität zugesichert. Des Weiteren wurde im Fragebogen weitgehend auf Fragen zu sensiblen persönlichen oder geschäftlichen Inhalten verzichtet. Die Teilnehmer der Umfrage wurden dazu angehalten, möglichst objektiv zu antworten, um Probleme mit Datenverfälschungen z.B. aufgrund von sozial erwünschtem Antwortverhalten zu minimieren. ${ }^{606}$ Anschließend wurden die Items zum Konstrukt der Marktorientierung und zu den einzelnen Konstruktdimensionen präsentiert. Im zweiten Teil des Fragebogens sollten die Items zu den Konsequenzen der Marktorientierung und Items zu verschiedenen Moderatorvariablen beantwortet werden. Dazu wurden die Befragten gebeten, die Fragen vor dem Hintergrund der vom Unternehmen vertriebenen Hauptprodukte und bestimmter Kundenbeziehungen zu beantworten. Der letzte Teil des Fragebogens beinhaltete demographische Angaben zu den Befragten selbst und zu deren Unternehmen bzw. Business Units. Insgesamt konnten $n=79$ verwertbare Datensätze zwischen Januar und Juni 2013 generiert werden. Aufgrund von Filtereinstellungen im Fragebogen konnten die Gütekriterien der Dimension Marktstufenorientierung lediglich mit $n=51$ Datensätzen bestimmt werden.

${ }^{605} \mathrm{Vgl}$. Kuß/Eisend 2010, S. $107 \mathrm{ff}$.

${ }^{606}$ Vgl. Podsakoff et al. 2003, S. 881. 
Mit den gewonnenen Daten wurden die in Abschnitt 5.2.2 und 5.2.3 beschriebenen Gütetests durchgeführt. Items, die in diesem Pretest eine schlechte Reliabilität aufwiesen, jedoch aus sachlogischen oder theoretischen Überlegungen nicht ausgeschlossen werden konnten, da sie die in den Gruppeninterviews identifizierten Bereiche von Marktorientierung betrafen, wurden umformuliert. Items, die nicht eindeutig einem Konstrukt zugeordnet werden konnten oder hohe Kreuzladungen zu einem anderen als dem vorgesehenen Konstrukt aufwiesen, wurden ebenfalls umformuliert oder eliminiert. Die in diesem Pretest erzielten Ergebnisse waren aufgrund der relativ geringen Stichprobengröße nicht alle signifikant. Eine Auswahl der zu löschenden und der in der Item-Batterie zu behaltenden Items sollte daher im Rahmen eines Pretests immer sehr wohl überlegt sein. Einige Items, wurden aus diesem Grunde trotz geringfügiger Unterschreitung der geforderten Reliabilität für den nächsten Pretest beibehalten.

Die neue Item-Batterie wurde anschließend in einem zweiten quantitativen Pretest wieder in Fragebogenform getestet. Dazu wurden 1.500 Personen mit einer personalisierten Ansprache kontaktiert. Insgesamt konnten hierbei $n=145$ verwertbare Datensätze zwischen Juli und August 2013 generiert werden. Aufgrund von Filtereinstellungen im Fragebogen konnten die Gütekriterien der Dimension Marktstufenorientierung lediglich mit $\mathrm{n}=92$ Datensätzen bestimmt werden. Anhand der gewonnenen Daten waren nur wenige Ausschlüsse oder kleinere Anpassungen der Items notwendig, bevor die Items in die Hauptuntersuchung überführt werden konnten. Zudem wurden für die Hauptuntersuchung weitere reflektive Items zur globalen Messung von Marktorientierung hinzugefügt, um möglichst fehlerfrei die externe Validität mit Hilfe des MIMIC-Vorgehens (vgl. Abschnitt 5.2.2) bestimmen zu können. Zudem wurden für die Hauptuntersuchung die ItemReihenfolge und die Seitenreihenfolge innerhalb des Fragebogens randomisiert, um verzerrende Einflüsse, die aus dem Item-Kontext resultieren können, zu vermeiden. ${ }^{607}$

Der Gestaltung von Fragebögen kommt bei der Erhebung von Primärdaten, bei der während der einzelnen Erhebung selbst keine Änderungen vorgenommen

${ }^{607}$ Vgl. Podsakoff 2003, S. 887. 
werden können, große Bedeutung zu. Eine generelle Herausforderung von Umfragen liegt daher in der Schaffung eines einheitlichen Verständnisses der untersuchten Konstrukte. Durch branchen- oder unternehmensspezifische Eigenheiten können bestimmte Sachverhalte unterschiedlich aufgefasst und bewertet werden. Um ein einheitliches Verständnis der im Fragebogen erfassten Konstrukte zu gewährleiten, verdeutlichen DAY/NEDUNGADI, dass das Frage- und Antwortdesign des Fragebogens sorgfältig gestaltet sein sollte. Nur so ist es möglich, auch über verschiedene Branchen und Unternehmenspositionen hinweg verlässliche Daten zu erhalten. ${ }^{608}$

Ein Problem, das oftmals mit der Generierung von Primärdaten in Querschnittserhebungen verbunden ist, stellt der Common-Method-Bias dar. CommonMethod-Bias bezeichnet die substanzielle Verzerrung von Konstruktbeziehungen in Strukturgleichungssystemen. ${ }^{609}$ Der Common-Method-Bias resultiert aus der sog. Common-Method-Variance, einem Messfehler, der auf den Einfluss der verwendeten Messmethode hinsichtlich der Varianz der Messungen zurückgeführt werden kann. ${ }^{610}$ Ursachen für Common-Method-Bias bzw. CommonMethod-Variance werden in personen- und itemspezifischen Einflüssen sowie in bestimmten Item- und Erhebungskontexten gesehen. ${ }^{611} \mathrm{Um}$ diesen Ursachen entgegenzuwirken und die Common-Method-Variance zu minimieren, wurden das Design und der Aufbau des Fragebogens mit Sorgfalt konzipiert.

Die Wortwahl der Items wurde daher anhand der Ergebnisse der Pretests und nach Diskussionen mit Marketing-Wissenschaftlern und Marketing-Praktikern für den finalen Fragebogen mit dem Ziel angepasst, ein möglichst hohes und einheitliches Verständnis der Items und ein möglichst niedriges Niveau von CommonMethod-Variance zu erreichen. Insbesondere wurden dazu folgende Empfehlungen der Fachliteratur befolgt: ${ }^{612}$

- Den Umfrageteilnehmern wurde Anonymität zugesichert.

\footnotetext{
608 Vgl. Day/Nedungadi 1994, S. 34.

${ }^{609}$ Vgl. Temme et al. 2009, S. 124.

610 Synonym wird auch der Begriff „,method effect“ verwendet (vgl. Cote/Buckley 1987, S. 315).

${ }^{611}$ Vgl. Podsakoff et al. 2003, S. 881 ff.; Temme et al. 2009, S. 124.

612 Vgl. Podsakoff 2003, S. 887 f.; Conway/Lance 2010, S. 330.
} 
- Den Befragten wurde erläutert, dass sie die Fragen möglichst objektiv und nicht auf persönlichen Gefühlen aufbauend beantworten sollten.

- Die Reihenfolge der Dimensionen von Marktorientierung und die Items innerhalb der Dimensionen bzw. Konstrukte wurden randomisiert.

- Die Items wurden daraufhin geprüft, ob sie mehrdeutig waren und mehr als einen zu bewertenden Inhalt aufwiesen.

- Die Skalenanker wurden innerhalb des Fragebogens sofern möglich bzw. erforderlich variiert. Das Antwortformat der Erfolgsvariablen wurde abweichend von der sonst verwendeten 7er-Likert-Skala als Schieberegler zwischen zwei Extrempunkten konzipiert.

- Item-Syntax und verwendete Fachbegriffe wurden möglichst einfach gehalten und an eine verständliche „Praktikersprache“ bzw. Fachjargon angepasst.

- Vermeintlich vage Konzepte wie Letztverwender und Endkunden wurden erläutert.

Die Hauptuntersuchung wurde als großzahlige Befragung angelegt, um für die Güteprüfung des aufgestellten Strukturgleichungsmodells eine ausreichend hohe Anzahl von Datensätzen zu generieren. Generell stehen für die Durchführung von Umfragen verschiedene Medien zur Verfügung. Die am häufigsten genutzten sind dabei die persönliche, die schriftlich, die telefonische und die Online-Umfrage. ${ }^{613}$ Neben der Erreichbarkeit der Untersuchungszielgruppe sollten bei der Wahl des Umfrage-Mediums auch forschungsökonomische Erwägungen berücksichtigt werden. Da bei Umfragen im Business-to-Business-Bereich oftmals Berufstätige aus verschiedenen Unternehmen befragt werden sollen, die sich räumlich entfernt befinden, bietet sich hierbei insbesondere die Online-Umfrage an. Die Befragten haben so die Möglichkeit, weitgehend orts- und zeitunabhängig an der Umfrage teilzunehmen. Das Organisieren von persönlichen Treffen oder das Rücksenden von schriftlichen Fragebögen entfällt, senkt dadurch den mit der Umfrage verbundenen Aufwand und kann so die Antwortquote der angeschriebenen Personen erhöhen. ${ }^{614}$ Auch die Reisekosten und der Zeitaufwand für den Interviewer, die

${ }^{613}$ Vgl. Kuß/Eisend 2010, S. 114.

${ }^{614}$ Vgl. Bonometti/Tang, 2006, S. 99; Evans/Mathur 2005, S. 198. 
mit persönlichen oder telefonischen Interviews verbunden sind, können minimiert werden, da eine persönliche Anwesenheit des Interviewers bei den Auskunftspersonen nicht notwendig ist. ${ }^{615}$ Unter Berücksichtigung der Forschungsökonomie wurde eine Querschnittsuntersuchung durchgeführt. Die Auskunftspersonen wurden nur zu einem Zeitpunkt befragt, was die Zeitdauer der Datenerhebung und somit der gesamten Untersuchung reduziert. ${ }^{616}$

Für die Beantwortung des Fragebogens wurden geschlossene Fragen gewählt, um die Beantwortung zu erleichtern und somit die Antwortquote zu erhöhen. Zudem sollten für den Einsatz von Strukturgleichungsmodellen standardisierbare Werte verwendet werden, welche durch eine einfache Codierung der Variablenausprägung durch geschlossene Fragen leicht erreicht werden kann. ${ }^{617}$ Die unabhängigen Variablen des Strukturgleichungssystems (Kundenorientierung, Wettbewerbsorientierung, Drittparteienorientierung, Marktstufenorientierung und Abteilungsübergreifende Koordination sowie die globale Messung von Marktorientierung) wurden anhand einer unipolaren, numerischen 1-bis-7-Antwortskala erhoben. ${ }^{618}$ Die oftmals auch als Likert-Skala bezeichneten Antwortmöglichkeiten reichten von „stimme gar nicht zu (1)“ bis „stimme voll zu (7)“. Mit Hilfe derselben Skalenantworten wurden die abhängigen bzw. mediierenden Variablen „Innovativität“, „Abstimmung der Marketingaktivitäten“ und „Unternehmenszusammenarbeit“ gemessen. Die abhängigen Erfolgsvariablen wurden anhand eines Schiebereglers als quasi-kontinuierliche Variable gemessen. Durch diese Arten der Skalierung der Antwortmöglichkeiten wurde eine ausreichende Varianz in den Daten angestrebt, um sinnvolle Aussagen zu Kausalbeziehungen treffen zu können.

Um weiterhin die Reliabilität der Daten zu erhöhen, sollten sich die Fragen auf den gegenwärtigen Zustand des Unternehmens oder auf aktuelle Aktivitäten beziehen. Ziel eines solchen Vorgehens ist es, eine oftmals fehlerbehaftete gedank-

\footnotetext{
${ }^{615}$ Vgl. Kaas 1999, S. 46

${ }^{616}$ Vgl. Kuß/Eisend 2010, S. 47. Auf die damit einhergehenden Probleme bezüglich der festzustellenden Kausalitäten sei auf Abschnitt 5.1 verwiesen.

${ }^{617}$ Vgl. Kuß/Eisend 2010, S. 83.

${ }^{618}$ Vgl. Rossiter 2012, S. 111.
} 
liche Rekonstruktion der Vergangenheit zu vermeiden. ${ }^{619}$

\subsubsection{Bestimmung der Stichprobe und Auswahl der Auskunftsperso- nen}

In Strukturgleichungsmodellen kann die Stabilität der Schätzung von der Stichprobengröße abhängen. Die statistische Power eines Modells wird daher als Maß verwendet, um den für ein Modell zur Schätzung notwendigen Stichprobenumfang zu bestimmen. Die statistische Power gibt die Wahrscheinlichkeit an, signifikante Ergebnisse bei der Modellberechnung zu ermitteln, wenn auch tatsächlich signifikante Beziehungen vorliegen. ${ }^{620}$ Mit Hilfe der Software g*Power 3.1.9.2 ${ }^{621}$ wurde für das in dieser Arbeit aufgestellte Modell ein Mindeststichprobenumfang von $\mathrm{n}=81$ ermittelt. $^{622}$

Die Daten, die in dieser Untersuchung erhoben wurden, beziehen sich auf das Verhalten einzelner Organisationsmitglieder und Abteilungen sowie auf die Organisation als Ganzes. Eine häufig dazu verwendete Methode der Datensammlung ist die Nutzung von sog. Key-Informants als Auskunftspersonen. ${ }^{623} \mathrm{Key}-$ Informants besitzen eine bestimmte Qualifikation bzw. spezifisches Wissen oder haben eine bestimmte Position im Unternehmen, die es ihnen ermöglicht, qualifizierte Aussagen über bestimmte für die Untersuchung relevante Sachverhalte zu treffen. ${ }^{624}$ Die Datenerhebung ausschließlich auf Key-Informants zu stützen, ist in der Literatur nicht ohne Kritik geblieben. So wird bspw. in der Marketingforschung diskutiert, inwiefern Key-Informants überhaupt in der Lage sind, fehlerfrei Auskunft zu geben. Insbesondere, wenn es sich um unterschiedliche Sachverhalte in einer Organisation handelt, kann angezweifelt werden, dass eine Person alleine zu allen Aspekten valide Auskunft geben kann. In der vorliegenden Untersuchung stellen bspw. die verschiedenen Aktivitäten des Unternehmens und die

\footnotetext{
619 Vgl. March/Sutton 1997, S. 699; Homburg et al. 2012, S. 604 f.

${ }^{620}$ In der Literatur wird eine statistische Power von 0,8 als hoch angesehen, so dass auch in dieser Untersuchung der Wert verwendet wird (Cohen 1988, S. 56; Akter et al. 2011, S. 2 f.).

621 Vgl. Faul et al. 2009.

${ }^{622}$ Zur Berechnung des Stichprobenumfangs wurden folgende Input-Parameter verwendet: Effektgröße $=0,1 ; \alpha$-Fehlerwahrscheinlichkeit $=0,05$.

${ }^{623} \mathrm{Vgl}$. Seidel 1974, S. $818 \mathrm{ff}$.
} 
Erfolgskennzahlen verschiedene Untersuchungsgegenstände dar, die möglicherweise nicht ohne weiteres von einer einzigen Person beurteilt werden können. Einige Studien zeigen, dass aus diesem möglichen Problem resultierend ein beträchtlicher Anteil der Variablenvarianz erklärt werden kann. ${ }^{625}$ Die Ergebnisse von Studien können somit durch die Erhebungsmethode, d.h. durch die jeweilige Auskunftsperson beeinflusst werden.

Probleme, die möglicherweise durch die Key-Informant-Methode verursacht werden, können allerdings durch eine sinnvolle Auswahl der Auskunftspersonen minimiert werden. HOMBURG ET AL. zeigen, wie die Validität und Reliabilität der erhobenen Daten durch eine Auswahl von geeigneten Key-Informants erhöht werden kann. ${ }^{626}$ In der vorliegenden Arbeit wurde diesen Empfehlungen gefolgt, und es wurden nur solche Personen kontaktiert, die sich in einer gehobenen Position im Unternehmen befanden und zudem eine längere Verweildauer in relevanten Unternehmenspositionen aufwiesen. So konnte sichergestellt werden, dass die Auskunftspersonen Erfahrung mit den relevanten Unternehmensprozessen besaßen und die Einschätzung des Marktes und der Unternehmenskennzahlen reliabel und valide erfolgte. ${ }^{627}$ Die gehobene Position im Unternehmen sorgte dafür, dass die Auskunftspersonen Zugang zu relevanten Information und eine direkte Entscheidungsbefugnis hatten oder zumindest eine große Nähe zu den Trägern strategischer Unternehmensentscheidungen besaßen.

Ein weiteres in der Literatur diskutiertes Problem, das aus der Key-InformantMethode entstehen kann, ist das Problem der Selbsteinschätzung. Key-Informants können mitunter ihre Antworten systematisch über- oder unterschätzen. Dies könnte im Rahmen der vorliegenden Untersuchung zu systematischen Verzerrungen führen, da die Items des Fragebogens genau so angelegt sind, dass die KeyInformants Selbstauskünfte geben sollten. Eine Verzerrung der Antworten kann bspw. aus Voreingenommenheit des Befragten aufgrund seiner Unternehmenszugehörigkeit resultieren. Um eine solche Datenverfälschung zu vermeiden, schla-

\footnotetext{
${ }^{624}$ Bagozzi et al. 1991, S. 423.

${ }^{625}$ Vgl. March/Sutton 1997; Bagozzi et al. 1991, S. 424; Podsakoff et al. 2003, S. 881 f.

${ }^{626}$ Vgl. Homburg et al. 2012, S. 601.

${ }^{627}$ Vgl. auch Kumar et al. 1993, S. 1639.
} 
gen einige Autoren vor, mehrere Auskunftspersonen pro Untersuchungseinheit zu verwenden und so die Reliabilität der Daten zu erhöhen. ${ }^{628}$ Allerdings kann dies wieder neue Probleme aufwerfen, die aus einer geringen Übereinstimmung der Antworten verschiedener Auskunftspersonen sowie aus niedrigen Rücklaufquoten von vollständigen, mehrfachen Datensätzen entstehen. ${ }^{629}$ FOREMAN ET AL. sehen eine Möglichkeit, die Auskunft mehrerer Personen desselben Unternehmens zu nutzen, in der Auswertung der schriftlichen Unternehmenskommunikation. ${ }^{630}$ Die Autoren analysieren hierzu die an Investoren gerichteten Geschäftsberichte, um anhand von konkreten Maßnnahmen die Ausprägung der Kunden- und Wettbewerbsorientierung zu bestimmen. Da solche Berichte in der Regel von mehreren Mitarbeitern angefertigt und redigiert werden, gehen die Autoren davon aus, dass die subjektiven Einflüsse einzelner Mitarbeiter bei der Bewertung der eigenen Unternehmensaktivitäten nicht ins Gewicht fallen. Zur Bestimmung der abhängigen Erfolgsvariable betrachten die Autoren die Aktienrendite des betreffenden Unternehmens. Wenngleich zumindest die Messung des Erfolgs objektiven Kriterien folgt, kann auch hier wieder bei der Bewertung von Marktorientierung an der Unvoreingenommenheit der gesamten Mitarbeiter gezweifelt werden. ${ }^{631}$

Aufgrund der nicht ausräumbaren Zweifel hinsichtlich dieser Unvoreingenommenheit schlagen verschiedene Autoren vor, bestimmte Sachverhalte bzw. Konstrukte nicht von Personen desselben Unternehmens, sondern von Personen außerhalb des betreffenden Unternehmens bewerten zu lassen. ${ }^{632}$ Ein solches Vor-

\footnotetext{
${ }^{628}$ Vgl. Bowman/Ambrosini 1997; Phillips 1981; Oakley 2012, S. 1100; SLATER (1995, S. 265)

schlägt z.B. vor, drei Personen für eine Erhöhung der Messreliabilität zu befragen.

${ }^{629}$ Vgl. Gundlach/Cadotte 1994, S. 523; Hughes/Garrett 1990, S. 193; Slater 1995, S. 265.

630 Vgl. Foreman et al. 2014, S. 355 f. Homburg et al. 2012, S. 601.

${ }^{630}$ Vgl. auch Kumar et al. 1993, S. 1639.

630 Vgl. Bowman/Ambrosini 1997; Phillips 1981; Oakley 2012, S. 1100; SLATER (1995, S. 265)

schlägt z.B. vor, drei Personen für eine Erhöhung der Messreliabilität zu befragen.

${ }^{630}$ Vgl. Gundlach/Cadotte 1994, S. 523; Hughes/Garrett 1990, S. 193; Slater 1995, S. 265.

630 Vgl. Foreman et al. 2014, S. 355 f.

${ }^{631}$ Vgl. Higgins/Gulati 2003

632 TSAI ET AL. (2011) schlagen vor, die Wettbewerbsorientierung von einem Wettbewerber beurteilen zu lassen. Auch DESHPANDÉ ET AL. (1993) schlagen eine Evaluierung der Kundenorientierung eines Unternehmens durch die Kunden dieses Unternehmens vor.
} 
gehen würde zwar möglicherweise die Qualität der Daten erhöhen, doch würde es auch wieder andere Probleme aufwerfen. Die Auswahl geeigneter Personen zur Beurteilung der interessierenden Sachverhalte könnte sich nämlich problematisch gestalten oder gar unmöglich sein. Die Auskunftspersonen müssten demnach in der vorliegenden Untersuchung in der Lage sein, auch bestimmte unternehmensinterne Prozessschritte hinsichtlich der abteilungsübergreifenden Koordination zu beurteilen. Aufgrund mangelnder Einblicke für Unternehmensexterne in die genauen Geschäftsabläufe bietet diese Form der Datenerhebung keine Ansatzpunkte für die vorliegende Arbeit. ${ }^{633}$

Für das vorliegende Forschungsvorhaben erscheint aufgrund der dargelegten Einschätzungen die Key-Informant-Methode gegenüber den Multi-InformantMethoden und vor allem aufgrund von forschungsökonomischen Erwägungen als vorteilhaft. Im Rahmen dieser Arbeit wurde es daher als zielführend angesehen, die Datenerhebung anhand von einfachen Key-Informants pro Untersuchungseinheit mittels eines auf Selbsteinschätzungen beruhenden Fragebogens durchzuführen. ${ }^{634}$

Zur Kontaktierung von Auskunftspersonen wurden in dem sozialen Netzwerk „Xing“ Berufstätige in relevanten Unternehmenspositionen angeschrieben. Xing ist eine Online-Plattform, in der Mitglieder sich vor allem mit beruflichen Kontakten verknüpfen. Die Plattform ist für alle Branchen und Unternehmenspositionen offen. Das Netzwerk wurde zur Datengenerierung genutzt, da es im deutschsprachigen Raum mit über 7 Mio. Mitgliedern das meist genutzte soziale Business-Netzwerk ist. ${ }^{635}$

Im Zeitraum von August 2013 bis Februar 2014 wurden insgesamt 3.500 Personen persönlich angeschrieben und gebeten, an der Umfrage teilzunehmen. Im

633 Trotz der mit einem solchen Vorgehen verbundenen zweifelhaften Ergebnisvalidität finden sich in der Literatur dennoch Studien, die unternehmensinterne Prozesse durch Unternehmensexterne (Kunden) bewerten lassen (vgl. z.B. Webb et al. 2000).

${ }^{634}$ Vgl. Deshpandé/Farley 2004, S. 16 zu dieser Vorgehensweise und der Validität der so gewonnenen Daten.

635 Vgl. Pressebox 2014. Trotz der hohen Anzahl von Mitgliedern verschiedener Branchen kann nicht davon ausgegangen werden, dass die Mitgliederstruktur mit der tatsächlichen Branchenstruktur im deutschsprachigen Raum identisch ist. Eine Repräsentativität der Stichprobe für den Business-toBusiness-Bereich im gesamten deutschsprachigen Raum ist somit nicht gegeben. 
Anschreiben wurde den Personen Anonymität zugesichert, das grobe Ziel der Studie erläutert und als Dankeschön für die Teilnahme ein Ergebnisbericht der Studie versprochen. Zur eigentlichen Umfrage wurde per Hyperlink weitergeleitet. Nach ca. zwei bis drei Wochen erfolgte wiederum eine personalisierte Erinnerung, an der Umfrage teilzunehmen. Die Auswahl geeigneter Auskunftspersonen erfolgte mittels einer sog. Talent-Manager-Mitgliedschaft im Xing-Netzwerk. Diese Art der Mitgliedschaft dient hauptsächlich der Personalbeschaffung und wird vor allem von Human-Ressource-Abteilungen und Headhuntern genutzt, um passende Kandidaten für offene Stellen im Unternehmen zu finden. Durch diese Art der Mitgliedschaft werden die Profile der Mitglieder vollständig offen gelegt, so dass deren Berufserfahrung und Qualifikationen sichtbar werden. Durch Filtereinstellungen konnten innerhalb des Netzwerks geeignete Auskunftspersonen für die Umfrage identifiziert werden.

Da die Umfrage auf Key-Informants aufbaut, mussten solche Teilnehmer identifiziert und kontaktiert werden, die vor allem eine große Markt- und Kundennähe besitzen, um die auf Kunden gerichteten Aktivitäten des eigenen Unternehmens gut beurteilen zu können. Darüber hinaus sollten die Befragten eine umfassende Kenntnis des Wettbewerbs und der Wertschöpfungskette sowie des Marktumfeldes besitzen. Zudem mussten die Befragten in der Lage sein, Auskunft über firmeninterne Abstimmungsprozesse und den Erfolg des eigenen Unternehmens im Markt zu geben. Die hierarchische Position im Unternehmen sollte gehoben sein, um den Zugang zu wichtigem innerbetrieblichen Wissen und Informationen über das Marktgeschehen zu gewährleisten. Da von Unternehmen zu Unternehmen die genauen Positionsbezeichnungen variieren, mussten die Profile der potenziellen Auskunftspersonen zunächst daraufhin untersucht werden, ob eine ausreichende Qualifikation und Position innerhalb des Unternehmens bzw. in der Business Unit vorlag. ${ }^{636}$ Typische Unternehmenspositionen, die die letztlich angeschriebenen Auskunftspersonen inne hatten, waren etwa Marketingmanager, Senior Marketingmanager, Marketingleiter, Sales Director, Vice Sales Director, Vertriebslei-

${ }^{636}$ Vgl. Gök/Hacioglu 2010 für eine Auflistung unterschiedlicher Bezeichnungen für inhaltlich ähnliche funktionale Marketingpositionen in Unternehmen. 
ter, Sales Manager, Senior Sales Manager, Key Account Manager, Senior Key Account Manager, Technischer Vertrieb, Market Manager, Produktmanager und Business Development. ${ }^{637}$ Eher technik-orientierte Positionen wie Technischer Vertrieb und Produktmanager wurden nur angeschrieben, sofern im Lebenslauf der Befragten erkennbar war, dass eine frühere Position im Bereich des Marketings bekleidet wurde oder eine sonstige ausreichende Qualifikation vorlag. ${ }^{638}$ Ein Ziel der vorliegenden Untersuchung ist es, allgemeingültige Kausalzusammenhänge aufzustellen und ein branchenübergreifendes Messinstrument von Marktorientierung im Business-to-Business-Bereich zu entwickeln. Die Auskunftspersonen, die angeschrieben wurden, sollten daher auch in verschiedenen Branchen tätig sein. Von der Untersuchung ausgeschlossen wurden allerdings Unternehmen, die ihre Produkte direkt als Konsumgüter an den Einzelhandel vertreiben. Auch wenn zwischen Konsumguthersteller und Handel eine Businessto-Business-Beziehung vorliegt, so existiert eine Reihe von branchenspezifischen Charakteristika, die der Allgemeingültigkeit der angestrebten Ergebnisse entgegensteht. Diese branchenspezifischen Charakteristika wurden zudem aufgrund des B2B-Schwerpunkts nicht in den Fokusgruppendiskussionen erwähnt oder in den Beschreibungen der Teilnehmer ihrer relevanten Wertschöpfungsketten widergespiegelt.

Zur Untersuchung wurden auch nur solche Unternehmen herangezogen, bei denen sich zwischen dem Fokusunternehmen und dem Konsumenten noch mindestens eine Marktstufe befand. Auf diese Weise konnten Business-to-ConsumerUnternehmen ausgeschlossen werden, deren direkte Absatzmärkte keiner abgeleiteten Nachfrage unterliegen.

Zudem wurden nur rechtlich unabhängige Unternehmen oder deren eigenständig operierende Business Units angeschrieben. Damit wurden Beteiligungsgesellschaften und Joint Ventures ausgeschlossen, da hierbei nicht gewährleistet werden kann, dass marktbezogene Entscheidungen im Unternehmen selbst getroffen

${ }^{637}$ Vgl. Tull et al. 1991 für die Verteilung der Marketingaufgaben über verschiedene Abteilungen hinweg.

${ }^{638}$ Hier schienen insbesondere Personen geeignet zu sein, die an Weiterbildungsprogrammen mit Marketing- oder Vertriebsschwerpunkt teilgenommen hatten. 
werden.

Letztlich wurden keine Kleinstunternehmen mit weniger als zehn Mitarbeitern angeschrieben. ${ }^{639}$ In vielen kleinen Betrieben existiert oftmals keine klare Funktions- und Bereichstrennung, wie es sie etwa in größeren Unternehmen gibt. Daher kann es bei Unternehmen mit einer so geringen Anzahl von Mitarbeitern zu inhaltlichen Problemen bei der Beantwortung der Fragen hinsichtlich der abteilungsübergreifenden Koordination kommen.

\subsubsection{Datenbasis und Stichprobenstruktur}

Insgesamt erklärten sich 943 der angeschriebenen 3.500 Personen bereit, an der Umfrage teilzunehmen. Davon füllten 488 Personen den Fragebogen vollständig aus. Die vollständigen Datensätze wurden anschließend bereinigt. Aus den vollständigen Fragebögen wurden solche Datensätze ausgeschlossen, die auf ein inkonsistentes oder unmotiviertes Antwortverhalten der Auskunftspersonen schließen ließen oder deren Unternehmen die in Abschnitt 5.3.2 geforderten Kriterien nicht erfüllten.

Um die Verzerrung der Ergebnisse durch eine gehäufte Teilnahme von Mitgliedern desselben Unternehmens bzw. derselben Business Unit gering zu halten, wurde den Befragten die Möglichkeit gegeben, freiwillig den Unternehmensnamen bzw. ihre Business Unit anzugeben. Sollte eine Häufung von Datensätze aus derselben Untersuchungseinheit stammen, wären diese von der weiteren Analyse ausgeschlossen worden. Die Ergebnisse zeigen allerdings, dass nur in zwei Fällen jeweils zwei Datensätze sich auf Sachverhalte in derselben Untersuchungseinheit beziehen. Eine Bereinigung der Datensätze nach diesem Kriterium wurde daher als nicht notwendig erachtet. ${ }^{640}$

Nach der Datenbereinigung blieben noch 384 vollständige und verwertbare Datensätze übrig, die als Basis für die vorliegende Untersuchung dienen. Der Stichprobenumfang liegt somit deutlich über der mittels Power-Analyse ermittelten

639 Zur Größenklassifikation von Unternehmen vgl. Europäische Union 2003.

${ }^{640}$ Anzumerken ist, dass 156 Auskunftspersonen Angaben zu ihrer Geschäftseinheit machten. Häufungen von Datensätzen aus denselben Geschäftseinheiten, bei denen keine Angaben dazu gemacht wurden, können nicht ausgeschlossen werden. 
Mindeststichprobengröße von $n=81$. Die letztlich erreichte, bereinigte Antwortquote liegt bei $11 \%$, was im Rahmen von vergleichbaren Business-to-BusinessUntersuchungen zwar durchaus als akzeptabel angesehen werden kann, ${ }^{641}$ aber dennoch bedeutet, dass das Antwortverhalten von einem Großteil potentieller Auskunftspersonen nicht erfasst werden konnte. ${ }^{642}$ Gerade deshalb erscheint eine weitere Untersuchung der gewonnenen Daten vor der eigentlichen Schätzung der Mess- und Strukturmodelle als unverzichtbar. Nach Beendigung der Umfrage wurde daher analysiert, inwiefern die gewonnenen Daten durch einen sog. NonResponse-Bias verzerrt sein könnten. ${ }^{643}$ Es wird in der Wissenschaftspraxis der Auffassung gefolgt, dass das Antwortverhalten der Personen, die später an Umfragen teilnehmen und antworten, dem theoretischen Antwortverhalten der NichtAntwortenden recht ähnlich ist. ${ }^{644}$ Die Gruppe der Spät-Antwortenden steht somit stellvertretend für alle angeschriebenen Personen, die den Fragebogen nicht oder nicht vollständig ausgefüllt haben. Um mögliche Unterschiede im Antwortverhalten zu identifizieren, sollen die Variablenausprägungen der frühen und der späten Antworten verglichen werden. Dazu wurden die Antworten derjenigen, die auf die erste Nachricht im Xing-Netzwerk reagierten, mit denen verglichen, die erst auf die Erinnerungsnachricht reagierten. ${ }^{645}$ Für das Forschungsvorhaben relevante Variablen wurden mit Hilfe von t-Tests auf Unterschiede getestet. Es zeigte sich, dass sich die Gruppen der Früh-Antwortenden und der Spät-Antwortenden insgesamt sehr ähnlich sind. Lediglich hinsichtlich ihrer Drittparteienorientierung unterscheiden sich die Gruppen signifikant voneinander. Der Vergleich zeigt, dass diejenigen Auskunftspersonen, die früher antworteten, eine in allen Items höher ausgeprägte Drittparteienorientierung besitzen. Dieses Antwortverhalten

${ }^{641}$ Vgl. Ungruhe 2011, S. 143; Minculescu 2013, S. 200; Gleitsmann 2007, S. 144.

642 Vgl. Rogelberg/Stanton 2007, S. 198.

${ }^{643}$ Vgl. Armstrong/Overton 1977.

${ }^{644}$ Vgl. Armstrong/Overton 1977, S. 397.

${ }^{645}$ Der Erhebungszeitraum umfasste insgesamt sieben Monate und die Auskunftspersonen mussten aufgrund der Xing-Talentmanager Bestimmungen gestaffelt angeschrieben werden. Erst-Nachrichten und Erinnerungen wurden somit parallel an potentielle Teilnehmer verschickt. Um dennoch klar zwischen frühen und späten Antworten unterscheiden zu können, wurde in den Erinnerungsnachrichten ein von der ersten Nachricht marginal abweichender Hyperlink zur Online-Umfrage gesetzt. Frühe und späte Antworten wurden somit in unterschiedlichen, wenngleich inhaltlich identischen Umfragen erfasst. 
kann möglicherweise dadurch erklärt werden, dass die Drittparteienorientierung die Ausrichtung auf außerhalb des eigentlichen Absatzmarktes stehende Organisationen, wie etwa auch Institute und Universitäten, beinhaltet (vgl. Tabelle 18). Zudem umfasst Drittparteienorientierung auch, wie wichtig dem Unternehmen die eigene Außendarstellung, nicht zuletzt auf Online-Kanälen, ist. Die erfolgte Einladung zur Umfrageteilnahme wurde wie beschrieben online über das XingNetzwerk versendet und war schon im Einleitungstext eindeutig als Forschungsprojekt der Freien Universität Berlin beschrieben. Vor dem Hintergrund dieser Vorgehensweise bei der Kontaktierung und dem Inhalt der Drittparteienorientierung erscheint es durchaus plausibel, dass Nachrichten von universitären Forschungseinrichtungen in einem Online-Netzwerk spät oder gar nicht beantwortet werden.Tabelle 19 stellt anhand von Charakteristika der Auskunft gebenden Unternehmen überblicksartig die Struktur der zur weiteren Analyse verwendeten Stichprobe dar. Die Daten zeigen eine recht heterogene Struktur der Stichprobe, so dass keine Klassifikation oder Kategorie die Datenstruktur und die Ergebnisse entscheidend bestimmt.

Die Analyse der Branchenstruktur offenbart, dass die meisten Unternehmen dem Anlagen- und Maschinenbau (20,8 \%) zuzurechnen sind. Annähernd gleichverteilt vertreten sind die Elektroindustrie (13,5\%), die Industrielle Automatisierungstechnik (13,5\%), die Chemieindustrie (13,0 \%) und die Automobilindustrie (12,8 \%). Etwas geringer vertreten sind die Metallindustrie/-verarbeitung (7,3 \%), die Softwarebranche (6,8 \%) sowie Handel \& Logistik (3,9 \%). Die Ergebnisse der Untersuchung besitzen somit keinen speziellen Branchenfokus.

Die Daten zeigen zudem, dass eher mittlere und große Unternehmen an der Umfrage teilgenommen haben, was dem Ziel, auch abteilungsübergreifende Prozesse messen zu können, entgegen kommt. Lediglich 9,1 \% der Umfrageteilnehmer repräsentieren Kleinunternehmen mit weniger als 50 Mitarbeitern. Kleinstunternehmen mit weniger als zehn Mitarbeitern wurden von der weiteren Analyse wie in Abschnitt 5.3 .2 begründet ausgeschlossen.

Die Produktkategorien der angebotenen Leistungen werden von Komponenten (26,6 \%) und Fertigprodukten (25,8 \%) dominiert. Ebenfalls vertreten sind Ma- 
schinen (15,6 \%), Systemlösungen (10,4 \%) sowie Dienstleistungen (7,8 \%), so dass hinsichtlich der Kaufabsichten des direkten Kunden eine Vielzahl von Weiterverarbeitungs- und Einsatzmöglichkeiten der angebotenen Leistungen repräsentiert wird. Die Unterschiedlichkeit der Produktkategorien und die damit verbundenen Nutzungs- und Weiterverarbeitungsmöglichkeiten sind der angestrebten Gültigkeit der Studienergebnisse in einer Vielzahl von potentiellen Wertschöpfungsketten zuträglich.

Die Positionen der Unternehmen in der Wertschöpfungskette machen deutlich, dass sich bei fast der Hälfte (45,6 \%) der befragten Unternehmen zwei oder mehr Marktstufen zwischen dem Unternehmen und dem Letztverwender der angebotenen Leistungen befinden. Bei 36,5 \% der Unternehmen existiert nur eine Zwischenstufe bis zum Letztverwender der Leistung und 15,5 \% bezeichnen ihre direkten Kunden als Letztverwender. Die letztgenannte Kategorie betrifft vor allem Unternehmen, die Dienstleistungen, Software und Maschinen vertreiben. Diese Leistungen werden beim Kunden nicht mehr weiterverarbeitet, dienen aber als Produktionsfaktoren bei der Erstellung von Gütern für nachfolgende Marktstufen. 
Tabelle 19: Struktur der Stichprobe nach Unternehmenscharakteristika

\begin{tabular}{|c|c|c|c|c|c|}
\hline \multicolumn{3}{|l|}{ I. Branche } & \multicolumn{3}{|l|}{ II. Produktkategorie } \\
\hline & absolut & relativ & & absolut & relativ \\
\hline Anlagen-/ Maschinenbau & 80 & $20,8 \%$ & Komponenten & 102 & $26,6 \%$ \\
\hline Elektroindustrie & 52 & $13,5 \%$ & Fertigprodukte & 99 & $25,8 \%$ \\
\hline Industrielle Automatisierung & 52 & $13,5 \%$ & Maschinen/Anlagen & 60 & $15,6 \%$ \\
\hline Chemieindustrie & 50 & $13,0 \%$ & Systemlösungen & 40 & $10,4 \%$ \\
\hline Automobilindustrie & 49 & $12,8 \%$ & Dienstleistungen & 30 & $7,8 \%$ \\
\hline Metallindustrie/ -verarbeitung & 28 & $7,3 \%$ & Rohmaterialien & 27 & $7,0 \%$ \\
\hline Computersoftware & 26 & $6,8 \%$ & Software & 13 & $3,4 \%$ \\
\hline Handel/Logistik & 15 & $3,9 \%$ & Sonstige & 13 & $3,4 \%$ \\
\hline andere & 32 & $8,3 \%$ & & & \\
\hline \multicolumn{3}{|l|}{ III. Anzahl der Mitarbeiter } & \multicolumn{3}{|c|}{$\begin{array}{l}\text { IV. Marktzwischenstufen zum Letzt- } \\
\text { verwender }\end{array}$} \\
\hline & absolut & relativ & & absolut & relativ \\
\hline 10 bis 49 & 35 & $9,1 \%$ & 1 Stufe & 140 & $36,5 \%$ \\
\hline 50 bis 249 & 91 & $23,7 \%$ & 2 Stufen & 117 & $30,5 \%$ \\
\hline 250 bis 999 & 85 & $22,1 \%$ & mehr als 2 Stufen & 58 & $15,1 \%$ \\
\hline 1.000 bis 5.000 & 86 & $22,4 \%$ & $\begin{array}{l}\text { Keine Zwischenstufe } \\
\text { Marktstufen unbe- }\end{array}$ & 58 & $15,1 \%$ \\
\hline \multirow{2}{*}{ mehr als 5.000} & 87 & $22,7 \%$ & kannt & 2 & $0,5 \%$ \\
\hline & & & Keine Angabe & 9 & $2,3 \%$ \\
\hline
\end{tabular}

Die Analyse der demographischen Daten der Umfrageteilnehmer gibt Auskunft darüber, welche Positionen im Unternehmen die Befragten innehaben.

Tabelle 20 zeigt die Selbstauskunft der Befragten. Demnach ordnen sich 91,7 \% der Umfrageteilnehmer mindestens einem der marktnahen Bereiche, in denen die Mitarbeiter klassischerweise direkten Kontakt zu den Kunden haben, zu. ${ }^{646}$ Die Befragten erscheinen somit in der Lage, Auskunft über den Absatzmarkt sowie über Unternehmensaktivitäten geben zu können, die unter Berücksichtigung von anderen Marktteilnehmern auf die Kunden gerichtet sind.

Die Dauer der bekleideten Position im gegenwärtigen Unternehmen beträgt in allen Fällen mindestens ein Jahr. Über zwei Drittel der Befragten geben an, seit mehr als drei Jahren in ihrem Unternehmen die bekleidete Position auszuüben. Die Umfrageteilnehmer sind folglich lange genug in ihren jeweiligen Unternehmen aktiv, um Auskunft über innerbetriebliche Prozesse geben zu können. Fast $82 \%$ der Auskunftspersonen arbeiten zudem seit mehr als sieben Jahren im an- 
gegeben Tätigkeitsbereich unabhängig von ihrem gegenwärtigen Unternehmen. Die Dauer der Tätigkeit in den angegebenen Unternehmensbereichen deutet darauf hin, dass die Befragten ein hinreichendes Wissen über die in dieser Arbeit untersuchten Forschungsgegenstände besitzen und fundierte Auskünfte darüber geben können. Die Umfrageteilnehmer können somit als qualifizierte KeyInformants betrachtet werden.

Tabelle 20: Struktur der Stichprobe nach Charakteristika der Auskunftspersonen

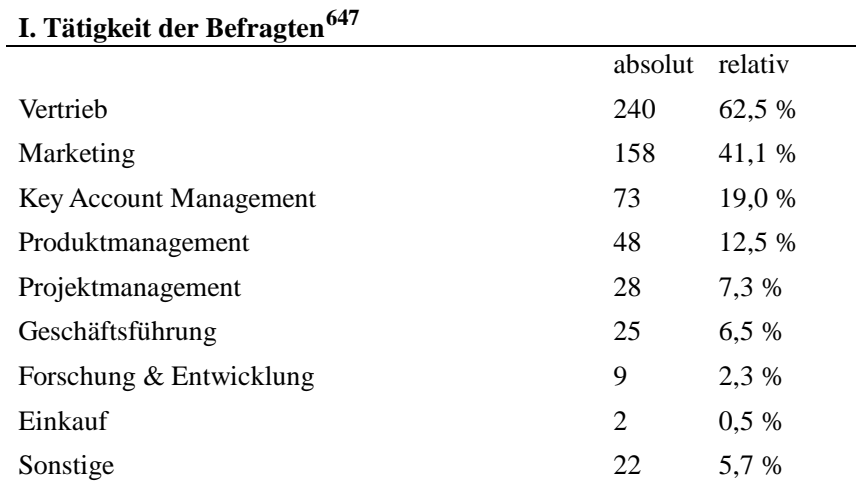

\section{Dauer der Tätigkeit im aktuellen Unternehmen}

\begin{tabular}{lll}
\hline & absolut & relativ \\
1-3 Jahre & 116 & $30,2 \%$ \\
3-7 Jahre & 102 & $26,6 \%$ \\
mehr als 7 Jahre & 166 & $43,2 \%$
\end{tabular}

\section{Dauer der Tätigkeit insgesamt (auch in anderen Unternehmen)}

\begin{tabular}{lll}
\hline & absolut & relativ \\
1-3 Jahre & 18 & $4,7 \%$ \\
3-7 Jahre & 38 & $9,9 \%$ \\
mehr als 7 Jahre & 314 & $81,8 \%$ \\
keine od. inkonsistente Angaben & 14 & $3,6 \%$
\end{tabular}

${ }^{646}$ Als kundennahe Bereiche werden in dieser Arbeit Marketing, Vertrieb, Key Account Management sowie der Bereich Service (in Tabelle 20 unter Sonstige aufgeführt) betrachtet.

${ }^{647}$ Eine Mehrfachnennung von Tätigkeitsbereichen war möglich. 
Wie in Abschnitt 5.3.1 beschrieben, wurde der Fragebogen derart gestaltet, dass Common-Method-Variance möglichst klein ausfällt oder gänzlich ausgeschlossen werden kann. Dennoch wurde post hoc eine Analyse durchgeführt, um durch die Auskunftspersonen oder durch den Fragebogen hervorgerufene starke systematische Verzerrungen der Daten zu identifizieren. Ein dazu häufig genutztes Verfahren ist der Harman's-One-Factor-Test. ${ }^{648}$ Hierzu wird über alle im Modell auftretenden Variablen eine explorative Faktoranalyse durchgeführt. Sollte nur ein Faktor extrahiert werden können oder ein einziger Faktor mehr als 50 \% der Gesamtvarianz erklären, kann auf das Vorliegen von Common-Method-Variance geschlossen werden. ${ }^{649}$ Anhand der vorliegenden Daten konnten insgesamt neun Faktoren nach dem Kaiser-Kriterium ${ }^{650}$ extrahiert werden, die gemeinsam 67,9 \% der Gesamtvarianz erklären. Der erste Faktor erklärt dabei 36,5 \% der Varianz. Nach dem Harman's-One-Factor-Test liegen demnach keine problematischen Verzerrungen vor. Da der Test allerdings nicht sehr sensitiv ist, können in der Regel nur äußerst starke Verzerrungen identifiziert werden. Die mittels Harman's-One-Factor-Test erzielten Ergebnisse sind somit nur bedingt geeignet, das Vorliegen von Common-Method-Variance auszuschließen.

Ein etwas aussagefähigerer Test beruht auf einem Vergleich von partiellen Korrelationen und geht auf LINDELL/WHITNEY zurück. ${ }^{651}$ Die Autoren schlagen vor, eine Markervariable in den Fragebogen zu integrieren. Die Markervariable sollte so beschaffen sein, dass sie nach theoretischer Überlegung mit einer oder mehreren Variablen der Studie unkorreliert ist und den gleichen Methodeneinflüssen unterliegen. ${ }^{652}$ Aus der empirisch gemessenen Korrelation zwischen der Markervariablen und einer anderen theoretisch unkorrelierten Variablen kann dann auf die aus der Messmethode stammende Common-Method-Variance geschlossen werden. Dazu wurde in der vorliegenden Arbeit die Markervariable im Fragebogen ebenfalls anhand einer 7er-Likert-Skala erhoben. Die Markervariable

\footnotetext{
648 Vgl. Harman 1967.

${ }^{649}$ Vgl. Xu et al. 2014, S. 395; Chengalur-Smith et al. 2010, S. 715.

650 Vgl. Kaiser 1974, S. $31 \mathrm{ff}$.

651 Vgl. Lindell/Whitney 2001.

652 Vgl. Temme et al. 2009, S. 131. Als Markervariable wurde in der vorliegenden Arbeit die Ausrichtung der Geschäftseiheit auf internationale Märkte erhoben.
} 
weist durchschnittlich eine Korrelation von $r=0,197$ mit den in der Studie erhobenen Konstrukten auf, was als sehr geringe Korrelation einzustufen ist und lediglich rund 4 \% gemeinsame Varianz darstellt. ${ }^{653}$ Indem die Höhe der Korrelation der Markervariable von allen anderen Korrelationen abgezogen wird, können die empirischen Daten um die Verzerrung bereinigt werden. Das Niveau der Korrelationen zwischen der Markervariablen und den übrigen Konstrukten liegt allerdings deutlich unterhalb der Höhe der Korrelationen der übrigen Variablen miteinander, so dass von einer Datenbereinigung abgesehen wurde. Letztlich kann zwar eine gewisse Verzerrung der Daten aufgrund von Common-MethodVariance nicht vollständig ausgeschlossen werden, Hinweise darauf sind aber eher schwach.

Beide Tests deuten darauf hin, dass die mögliche Existenz von Common-MethodVariance in dieser Arbeit als unkritisch angesehen werden kann. Die im Rahmen dieser Arbeit gemessenen Korrelationen zwischen den verschiedenen Konstrukten können somit unkritisch zur Bestätigung oder Verwerfung der aufgestellten Hypothesen herangezogen werden.

\subsection{Bewertung der Messmodelle}

Die in dieser Arbeit verwendeten reflektiven und formativen Messmodelle werden anhand der in den Abschnitten 5.2.2 und 5.2.3 vorgestellten Kriterien beurteilt. Die Berechnung der Werte des Cronbach-Alpha, der Faktorladungen, der Indikatoreliabilität, der Faktorreliabilität (Composite Reliability) sowie der durchschnittlich extrahierten Varianz (DEV) wurden mit Hilfe der Software SmartPLS 2.0.M3 ${ }^{654}$ durchgeführt. Die Werte der korrigierten Item-SkalaKorrelation und der Kommunalitäten sowie der $\mathrm{Chi}^{2}$-Differenztest wurden mittels

653 Vgl. Weiber/Mühlhaus 2010, S. 11. Anzumerken ist jedoch, dass die Markervariable zwar a priori entwickelt aber dennoch mit den übrigen Variablen nicht vollständig theoretisch unkorreliert erscheint. Eine sorgfältigere Wahl der Markervariable hätte tendenziell zu noch niedrigeren Korrelationen geführt.

${ }^{654}$ Ringle et al. 2005. Daneben existieren noch weitere Softwareprogramme, die zur Varianzbasierten Schätzung verwendet werden können (vgl. Temme/Kreis 2005). Die Programme gehen allesamt auf Algorithmen von WOLD (1982) zurück und unterscheiden sich im Wesentlichen in ihrer Benutzerfreundlichkeit 
IBM SPSS Amos 22.0.0.0 und Excel von Microsoft Office Professional Plus 2013 berechnet. Der Test auf Multikollinearität erfolgte mittels IBM SPSS Statistics 22.0.0.0.

Zunächst sollen zur Beurteilung der Messgüte die einzelnen Facetten des zentralen Konstrukts der umfassenden Marktorientierung im Fokus stehen. Die Validierung der Messmodelle erster Ordnung erfolgt somit für die Dimensionen Kundenorientierung, Wettbewerbsorientierung, Drittparteienorientierung, Marktstufenorientierung und Abteilungsübergreifende Koordination. Danach wird die Messvalidierung des Konstrukts zweiter Ordnung für das Gesamtkonstrukt der umfassenden Marktorientierung vorgenommen. Anschließend erfolgt die Gütebeurteilung der Konsequenzen der Marktorientierung.

\subsubsection{Gütebeurteilung der Messmodelle der Facetten des Konstrukts Marktorientierung}

Vor der Kennzahlen-basierten Abschätzung von Reliabilität und Validität der Messmodelle wurden die Skalen der einzelnen Dimensionen von umfassender Marktorientierung auf Inhaltsvalidität im Rahmen der durchgeführten Fokusgruppeninterviews geprüft. Dazu wurden die den jeweiligen Skalen zugehörigen Items berufstätigen Marketing- und Vertriebsexperten vorgelegt bzw. mit diesen entwickelt. ${ }^{655}$ In den Gruppendiskussionen mit den Experten konnte bestätigt werden, dass die aufgelisteten Items die Bereiche der verschiedenen Dimensionen von Marktorientierung inhaltlich weitgehend abdecken.

Die Ergebnisse der Kennzahlen-basierten Güteprüfung der Dimension Kundenorientierung sind Tabelle 21 zu entnehmen. Das Cronbach-Alpha zeigt einen Wert deutlich oberhalb des geforderten Mindestwerts auf. Gleichzeitig liegt der Wert aber nicht so hoch, dass auf redundante Items geschlossen werden kann. Erst Werte nahe 1 können auf redundante Items hinweisen, die die Messung nur auf den ersten Blick reliabel erscheinen lassen. ${ }^{656}$ Der Wert des Cronbach-Alpha ist also als völlig unkritisch anzusehen. Alle Indikatoren weisen eine Faktorla-

655 Vgl. Weiber/Mühlhaus 2010, S. 128.

656 Vgl. Rossiter 2002, S. 317. 
dung über den geforderten 0,7 auf und besitzen folglich auch eine Indikatorreliabilität, die über die geforderten 0,5 hinausgeht. Die Indikatoren korrelieren einzeln ausreichend hoch mit den übrigen Indikatoren der Skala, was sich in der korrigierten Item-Skala-Korrelation niederschlägt. Die Faktorvarianz (Kommunalitäten) zeigt, dass die Streuung jeder Messvariable zu mehr als der Hälfte von dem dahinterliegenden Faktor, nämlich der Dimension Kundenorientierung, erklärt werden kann. Weniger als die Hälfte der Streuung entfällt somit auf die im Modell nicht erklärbare Fehlervarianz. Die durchschnittlich extrahierte Varianz (DEV) liegt ebenfalls über dem geforderten Mindestwert von 0,5. ${ }^{657}$ Auch die Faktorreliabilität als Maß, inwiefern das Konstrukt als Ganzes durch die ihm zugeordneten Items reliabel gemessen werden kann, übersteigt deutlich den geforderten Mindestwert von 0,6.

Tabelle 21: Güteprüfung der Dimension Kundenorientierung

\begin{tabular}{|c|c|c|c|c|c|c|c|}
\hline & $\begin{array}{l}\text { Cron- } \\
\text { bach- } \\
\text { Alpha }\end{array}$ & $\begin{array}{c}\text { Faktorla- } \\
\text { dungen }\end{array}$ & $\begin{array}{l}\text { Indikator- } \\
\text { reliabilität }\end{array}$ & $\begin{array}{l}\text { Korrigierte } \\
\text { Item-Skala- } \\
\text { Korrelation }\end{array}$ & $\begin{array}{c}\text { Kommuna- } \\
\text { litäten }\end{array}$ & DEV & $\begin{array}{c}\text { Faktorreliabili- } \\
\text { tät (Composite } \\
\text { Reliability) }\end{array}$ \\
\hline custor1 & \multirow{5}{*}{0,876} & 0,782 & 0,611 & 0,670 & 0,518 & \multirow{5}{*}{0,669} & \multirow{5}{*}{0,910} \\
\hline custor2 & & 0,819 & 0,671 & 0,700 & 0,572 & & \\
\hline custor3 & & 0,835 & 0,697 & 0,730 & 0,636 & & \\
\hline custor4 & & 0,854 & 0,729 & 0,748 & 0,668 & & \\
\hline custor5 & & 0,797 & 0,635 & 0,678 & 0,541 & & \\
\hline
\end{tabular}

Für die Dimension Wettbewerbsorientierung ergeben sich ganz ähnliche Werte, die in Tabelle 22 dargestellt sind. Das Cronbach-Alpha, die Faktorladungen und die Indikatorreliabiltäten der einzelnen Items liegen über den kritischen Werten. Auch die Item-Skala-Korrelationen zeigen, dass das Messinstrument reliable Messergebnisse liefert. Die extrahierten Kommunalitäten aller Items erfüllen zwar die Mindestanforderungen von Werten $>0,4$, doch zeigt sich, dass nur etwas weniger als die Hälfte der Streuung der Items compor1 und compor3 durch

\footnotetext{
${ }^{657}$ Die in dieser und den nachfolgenden Tabellen dargestellte DEV weicht geringfügig vom Durchschnitt der dargestellten Item-Kommunalitäten ab. Grund hierfür sind die mathematisch unterschiedlichen Schätzverfahren der jeweils verwendeten Software.
} 
die Dimension Wettbewerbsorientierung erklärt werden kann. ${ }^{658}$ Die Messungen durch die beiden Items beinhalten somit eine vergleichsweise hohe Fehlervarianz. Die anderen Indikatoren weisen deutlich bessere Werte auf. In Summe allerdings erfüllen die Items ebenfalls den geforderten Wert für die durchschnittlich extrahierte Varianz (DEV). Eine Item-Elimination erscheint daher nicht erforderlich und es kann von einer reliablen Messung ausgegangen werden.

Tabelle 22: Güteprüfung der Dimension Wettbewerbsorientierung

\begin{tabular}{|c|c|c|c|c|c|c|c|}
\hline & $\begin{array}{l}\text { Cron- } \\
\text { bach- } \\
\text { Alpha }\end{array}$ & $\begin{array}{c}\text { Faktorla- } \\
\text { dungen }\end{array}$ & $\begin{array}{l}\text { Indikator- } \\
\text { reliabilität }\end{array}$ & $\begin{array}{l}\text { Korrigierte } \\
\text { Item-Skala- } \\
\text { Korrelation }\end{array}$ & $\begin{array}{l}\text { Kommu- } \\
\text { nalitäten }\end{array}$ & DEV & $\begin{array}{l}\text { Faktorreliabi- } \\
\text { lität (Compo- } \\
\text { site Reliabili- } \\
\text { ty) } \\
\end{array}$ \\
\hline compor1 & \multirow{5}{*}{0,860} & 0,758 & 0,575 & 0,620 & 0,454 & \multirow{5}{*}{0,643} & \multirow{5}{*}{0,900} \\
\hline compor2 & & 0,766 & 0,587 & 0,690 & 0,567 & & \\
\hline compor3 & & 0,812 & 0,660 & 0,636 & 0,484 & & \\
\hline compor4 & & 0,837 & 0,700 & 0,725 & 0,644 & & \\
\hline compor5 & & 0,831 & 0,691 & 0,718 & 0,628 & & \\
\hline
\end{tabular}

Die Dimension Drittparteienorientierung musste aufgrund unzureichender Gütemaße um den Indikator partor1 bereinigt werden (vgl. Tabelle 23). Der Indikator partor1 besitzt eine unzureichende Faktorladung und Indikatorreliabilität und korreliert zudem deutlich geringer mit den anderen Indikatoren als die übrigen Items untereinander. Zudem werden über zwei Drittel der Item-Streuung Messfehlern zugeschrieben. Der Indikator konnte aus sachlogischen Überlegungen entfernt werden, da ein Großteil seines Inhalts durch die verbleibenden Indikatoren abgedeckt werden kann. In der Literatur ist allerdings umstritten, wann ein Item genau aufgrund von relativ geringer Reliabilität aus einer Skala ausgeschlossen werden sollte. WEIBER/MÜHLHAUS plädieren dafür, dies immer dann zu tun, wenn dadurch das Cronbach-Alpha verbessert werden kann und dies gleichzeitig keinen inhaltlich sachlogischen Erwägungen entgegensteht. ${ }^{659}$ NETEMEYER ET AL. hingen halten eine Veränderung einer Itembatterie bei

${ }^{658}$ Auch in der Studie von YAU ET AL. (2007, S. 1314) weist das Item compor1 (Austausch der Vertriebler über Wettbewerberstrategien) eine geringe Item-Skala-Korrelation auf, so dass die generelle Eignung dieses Items noch einmal überdacht werden könnte.

659 Vgl. Weiber/Mühlhaus 2010, S. 112. 
Alphawerten über 0,8 zur Verbesserung der internen Konsistenz und Inhaltsvalidität einer Skala für nicht notwendig. ${ }^{660}$ Um jedoch eine „saubere“ Skala zu entwickeln, wurde das Item partor1 aufgrund seiner geringen Reliabilität ausgeschlossen. Das Cronbach-Alpha veränderte sich dadurch allerdings nur minimal. Nach der Eliminierung werden die geforderten Mindestanforderungen aller übrigen Gütekriterien ebenfalls erfüllt.

Die Kommunalitäten der Indikatoren partor2, partor3 und partor5 sind zwar als relativ niedrig anzusehen, geben aber keinen hinreichenden Anlass, die Items aus der Skala zu entfernen. In Summe erfüllen die Indikatoren den kritischen Wert der DEV und die Dimension als Ganze die Faktorreliabilität. Es kann also auch beim Konstrukt der Drittparteienorientierung von einer reliablen und validen Messung ausgegangen werden.

Tabelle 23: Güteprüfung der Dimension Drittparteienorientierung

\begin{tabular}{|c|c|c|c|c|c|c|c|}
\hline & $\begin{array}{l}\text { Cron- } \\
\text { bach- } \\
\text { Alpha }\end{array}$ & $\begin{array}{c}\text { Faktorla- } \\
\text { dungen }\end{array}$ & $\begin{array}{l}\text { Indikator- } \\
\text { reliabilität }\end{array}$ & $\begin{array}{l}\text { Korrigierte } \\
\text { Item-Skala- } \\
\text { Korrelation }\end{array}$ & $\begin{array}{l}\text { Kommu- } \\
\text { nalitäten }\end{array}$ & DEV & $\begin{array}{c}\text { Faktorreliabili- } \\
\text { tät (Composite } \\
\text { Reliability) }\end{array}$ \\
\hline partor1 & -- & 0,660 & 0,436 & 0,497 & 0,309 & -- & -- \\
\hline partor2 & \multirow{4}{*}{0,802} & 0,792 & 0,627 & 0,612 & 0,499 & \multirow{4}{*}{0,628} & \multirow{4}{*}{0,871} \\
\hline partor3 & & 0,780 & 0,608 & 0,593 & 0,459 & & \\
\hline partor4 & & 0,828 & 0,685 & 0,676 & 0,630 & & \\
\hline partor5 & & 0,768 & 0,590 & 0,584 & 0,437 & & \\
\hline
\end{tabular}

Die Ergebnisse der Güteprüfung des Konstrukts der Marktstufenorientierung sind in Tabelle 24 zu finden. Sämtliche Indikatoren und das Konstrukt als Ganzes erfüllen alle geforderten Gütekriterien. Das Cronbach-Alpha, die Faktorladungen, die Indikatorreliabilität und die Item-Skala-Korrelationen liegen deutlich über den kritischen Werten. Die Indikatoren weisen zudem eine relativ niedrige Fehlervarianz auf. Es kann somit von einer reliablen und validen Messskala ausgegangen werden. Alle Indikatoren können folglich beibehalten werden.

${ }^{660}$ Vgl. Netemeyer et al. 2003, S. 126. 
Tabelle 24: Güteprüfung der Dimension Marktstufenorientierung

\begin{tabular}{|c|c|c|c|c|c|c|c|}
\hline & $\begin{array}{l}\text { Cron- } \\
\text { bach- } \\
\text { Alpha }\end{array}$ & $\begin{array}{c}\text { Faktorla- } \\
\text { dungen }\end{array}$ & $\begin{array}{l}\text { Indikator- } \\
\text { reliabilität }\end{array}$ & $\begin{array}{l}\text { Korrigierte } \\
\text { Item-Skala- } \\
\text { Korrelation }\end{array}$ & $\begin{array}{l}\text { Kom- } \\
\text { munali- } \\
\text { täten }\end{array}$ & DEV & $\begin{array}{c}\text { Faktorrelia- } \\
\text { bilität } \\
\text { (Composite } \\
\text { Reliability) }\end{array}$ \\
\hline multor1 & \multirow{5}{*}{0,846} & 0,797 & 0,635 & 0,692 & 0,554 & \multirow{5}{*}{0,681} & \multirow{5}{*}{0,914} \\
\hline multor2 & & 0,864 & 0,746 & 0,780 & 0,717 & & \\
\hline multor3 & & 0,804 & 0,647 & 0,697 & 0,569 & & \\
\hline multor4 & & 0,845 & 0,715 & 0,748 & 0,652 & & \\
\hline multor5 & & 0,813 & 0,661 & 0,673 & 0,526 & & \\
\hline
\end{tabular}

Für die Dimension Abteilungsübergreifende Koordination ergeben sich ebenfalls Werte deutlich oberhalb der kritischen Grenzen. Der Wert des CronbachAlpha ist mit 0,884 als relativ hoch anzusehen, stellt aber dennoch kein Problem hinsichtlich redundanter Indikatoren dar. Die Items spiegeln jeweils unterschiedliche Aktivitäten und kognitive Fähigkeiten eines Unternehmens bzw. seiner Mitarbeiter wider. ${ }^{661}$ Etwas über die Hälfte der Varianz von Item funco3 muss Messfehlern zugeschrieben werden. Der kritische Wert für Kommunalitäten von 0,4 wird aber nicht unterschritten. Da das Item ansonsten sehr gute Werte hinsichtlich der Reliabilität aufweist, kann es ohne Probleme beibehalten werden. Die übrigen Items erfüllen ebenfalls allesamt die geforderten Kennzahlen.

Tabelle 25 zeigt zusammenfassend, dass für die Dimension Abteilungsübergreifende Koordination eine reliable und valide Messskala vorliegt und keine Indikatoren eliminiert werden müssen.

${ }^{661}$ Vgl. Rossiter 2002, S. 316 f. 
Tabelle 25: Güteprüfung der Dimension Abteilungsübergreifende Koordination

\begin{tabular}{|c|c|c|c|c|c|c|c|}
\hline & $\begin{array}{l}\text { Cron- } \\
\text { bach- } \\
\text { Alpha }\end{array}$ & $\begin{array}{c}\text { Faktorla- } \\
\text { dungen }\end{array}$ & $\begin{array}{l}\text { Indikator- } \\
\text { reliabilität }\end{array}$ & $\begin{array}{l}\text { Korrigierte } \\
\text { Item-Skala- } \\
\text { Korrelation }\end{array}$ & $\begin{array}{l}\text { Kommu- } \\
\text { nalitäten }\end{array}$ & DEV & $\begin{array}{l}\text { Faktorreliabi- } \\
\text { lität (Compo- } \\
\text { site Reliabili- } \\
\text { ty) }\end{array}$ \\
\hline funco1 & \multirow{5}{*}{0,884} & 0,845 & 0,714 & 0,742 & 0,649 & \multirow{5}{*}{0,684} & \multirow{5}{*}{0,915} \\
\hline funco2 & & 0,784 & 0,614 & 0,678 & 0,531 & & \\
\hline funco3 & & 0,788 & 0,621 & 0,654 & 0,491 & & \\
\hline funco4 & & 0,869 & 0,755 & 0,775 & 0,707 & & \\
\hline funco5 & & 0,847 & 0,718 & 0,756 & 0,664 & & \\
\hline
\end{tabular}

Nachdem für die Messvorschriften erster Ordnung die Messvalidierung erfolgreich durchgeführt wurde, soll im nächsten Abschnitt das Gesamtkonstrukt der Marktorientierung einer Güteprüfung unterzogen werden.

\subsubsection{Gütebeurteilung des Messmodells des Gesamtkonstrukts Marktorientierung}

Zur Prüfung des Gesamtkonstrukts soll zunächst die angenommene Dimensionsstruktur getestet werden. Dazu werden in einem ersten Schritt sämtliche Indikatorvariablen des Konstrukts einer explorativen Faktoranalyse unterzogen. ${ }^{662}$ Anhand der erhobenen Daten kann die fünf-dimensionale Konzeptualisierung und Operationalisierung von Marktorientierung anhand der fünf extrahierten Faktoren abgebildet werden (vgl. Tabelle 26). Dadurch wird auch quantitativ-statistisch die Einschätzung der berufstätigen Marketing- und Vertriebsexperten unterstützt. Die Praktiker hatten, wie beschrieben, im Rahmen von Fokusgruppeninterviews durch eine Zuordnung von Marktteilnehmern und mit diesen assoziierte Unternehmenstätigkeiten eindeutig einer der beschriebenen Dimensionen von Marktorientierung zugeordnet.

${ }_{662}$ Die Faktoren wurden anhand der Hauptachsenfaktorenanalyse nach dem Kaiser-Kriterium extrahiert und durch die Promax-Methode rotiert. 
Tabelle 26: Ergebnisse der explorativen Faktoranalyse des Gesamtkonstrukts der Marktorientierung

\begin{tabular}{|c|c|c|c|c|c|c|}
\hline Dimension & Indikator & Faktor1 & Faktor2 & Faktor3 & Faktor4 & Faktor5 \\
\hline \multirow{5}{*}{ 点 } & custor1 &,- 023 & ,008 & ,777 &,- 012 &,- 079 \\
\hline & custor2 &,- 005 & ,070 & 618 & ,093 &, 064 \\
\hline & custor3 &,- 028 &,- 044 & ,795 & 112 &,- 049 \\
\hline & custor4 & ,058 &,- 028 & ,864 &,- 105 & ,035 \\
\hline & custor5 & ,067 &, 070 & ,645 &,- 012 & ,017 \\
\hline \multirow{5}{*}{ 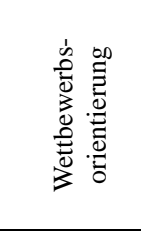 } & compor1 & ,118 & ,092 &,- 021 &, 547 &, 000 \\
\hline & compor2 &,- 059 & ,084 &,- 022 & ,696 & ,077 \\
\hline & compor3 &,- 054 &,- 022 & ,097 & ,722 &,- 036 \\
\hline & compor4 & ,055 &,- 048 &,- 020 & ,826 &,- 019 \\
\hline & compor5 & ,003 &,- 010 &,- 009 & ,786 & ,013 \\
\hline \multirow{4}{*}{ 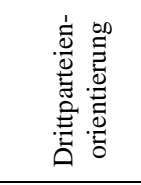 } & partor2 & ,041 & ,011 & ,040 &,- 082 & ,713 \\
\hline & partor3 & ,020 & ,060 & ,005 & ,147 & ,528 \\
\hline & partor4 & ,019 &,- 092 &,- 099 & ,010 & ,899 \\
\hline & partor5 &,- 012 & ,093 & ,049 & ,029 &, 566 \\
\hline \multirow{5}{*}{ 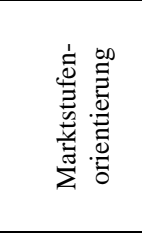 } & multor1 & ,746 & ,149 &,- 081 & ,002 &,- 054 \\
\hline & multor2 & ,826 &,- 036 & ,038 & ,024 & ,001 \\
\hline & multor3 & ,787 &,- 034 & ,027 &,- 093 & ,061 \\
\hline & multor4 & ,805 &,- 081 & ,012 & ,009 & ,054 \\
\hline & multor5 & ,559 &, 070 & ,076 & ,167 &,- 030 \\
\hline \multirow{5}{*}{ 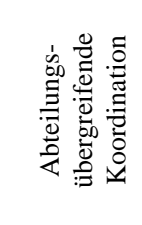 } & funco1 & ,081 & ,854 &,- 036 &,- 042 &,- 063 \\
\hline & funco2 &,- 006 & ,688 & ,007 & ,040 & ,017 \\
\hline & funco3 &,- 154 &, 563 & ,214 & ,040 & ,094 \\
\hline & funco4 & ,025 & ,816 & ,050 &,- 052 & ,027 \\
\hline & funco5 & ,003 & ,839 &,- 067 & ,054 &,- 036 \\
\hline
\end{tabular}

Die Faktoranalyse bestätigt nun, dass alle Indikatoren auf die intendierten Faktoren laden und nur geringe Kreuzladungen aufweisen, was einen ersten Hinweis auf Diskriminanzvalidität liefert. ${ }^{663}$ Kreuzladungen zwischen inhaltlich verwandten Konstrukten bzw. Faktoren sind allerdings keine Seltenheit, doch liegen alle in dieser Studie erreichten Kreuzladungen im akzeptablen Bereich und können

663 Vgl. Miocevic/Crnjak-Karanovic 2012, S. 119. 
als völlig unkritisch betrachtet werden. ${ }^{664}$

Um die Existenz von Diskriminanzvalidität, d.h. die weitgehende Unabhängigkeit der Dimensionen voneinander zu zeigen, soll das Fornell-Larcker-Kriterium herangezogen werden. ${ }^{665}$ Demzufolge muss die Varianz zwischen einem Konstrukt bzw. einem Faktor und seinen Indikatoren (DEV) größer sein als die Varianz, die dieser Faktor mit einem anderen Faktor gemeinsam hat. Tabelle 27 gibt die Ergebnisse des Tests auf Diskriminanzvalidität nach dem Fornell-LarckerKriterium wider. Die auf der Diagonale stehenden DEVs übertreffen bei weitem die quadrierten Korrelationen, die als gemeinsame Varianzen jeder Variablenpaarung interpretiert werden können. Das Fornell-Larcker-Kriterium kann somit als erfüllt betrachtet und folglich auf Diskriminanzvalidität geschlossen werden.

Tabelle 27: Ergebnisse des Tests auf Diskriminanzvalidität

\begin{tabular}{|l|l|l|l|l|l|}
\hline & 1 & 2 & 3 & 4 & 5 \\
\hline 1 CUSTOR & $\mathbf{0 , 6 6 9}$ & & & & \\
2 COMPOR & 0,348 & $\mathbf{0 , 6 4 3}$ & & & \\
3 FUNCO & 0,405 & 0,390 & $\mathbf{0 , 6 8 4}$ & & \\
4 PARTOR & 0,208 & 0,208 & 0,227 & $\mathbf{0 , 6 2 8}$ & \\
5 MULTOR & 0,273 & 0,333 & 0,233 & 0,285 & $\mathbf{0 , 6 8 1}$ \\
\hline
\end{tabular}

Ein weiterer Test auf Diskriminanzvalidität ist der Chi $^{2}$-Differenztest. Der Test basiert auf dem Kovarianz-analytischen Verfahren und vergleicht die $\mathrm{Chi}^{2}$-Werte als globale Gütemaße von unterschiedlichen Modellen miteinander. Es werden dabei ein unrestringiertes Modell, bei dem die Kovarianzen zwischen den verschiedenen Dimensionen des Konstrukts jeden Wert zwischen 0 und 1 annehmen können, mit einem restringierten Modell, bei dem die Kovarianz von jeweils zwei Dimensionen auf 1 festgelegt wird, miteinander verglichen. Die Modellrestriktion kann als Zusammenfassung von zwei Dimensionen zu einer Dimension interpretiert werden. Zeigt sich beim Test, dass die Werte der Chi²-Differenzen signifikant von Null verschieden sind, kann die Hypothese, dass das restringierte und

${ }^{664}$ Vgl. Kahn 2001, S. 318; Lin et al. 2008, S. 759.

${ }^{665}$ Vgl. Fornell/Larcker 1981, S. 46. 
das unrestringierte Modell dasselbe messen, verworfen werden. ${ }^{666}$ Wird die Hypothese verworfen, bedeutet dies gleichzeitig, dass die jeweils zwei fixierten Dimensionen nicht dasselbe messen und folglich unterschiedliche Inhalte zum Gegenstand haben. Anhand der erhobenen Daten zeigt der Test auf einem 5 \%Signifikanzniveau, dass keine zwei Dimensionen dieselben Inhalte des Marktorientierungskonstrukts erfassen (vgl. Anhang 3). Mittels Chi²-Differenztests kann ebenfalls auf Diskriminanzvalidität geschlossen werden.

Die drei durchgeführten Tests bestätigen, dass die verschiedenen theoretisch hergeleiteten und im Rahmen der Fokusgruppeninterviews identifizierten Bestandteile von Marktorientierung als eigenständige Dimensionen aufgefasst werden können. Für das vorliegende Messmodell kann auf Diskriminanzvalidität geschlossen werden. Es liegen zudem keine Anhaltspunkte vor, welche die formative Struktur zweiter Ordnung des Gesamtkonstrukts anzweifeln lassen.

Die externe Validität der formativen Messung von Marktorientierung soll mittels MIMIC-Modell eruiert werden. MIMIC-Modelle bieten den Vorteil, dass sie eine Abschätzung des Einflusses der einzelnen formativen Indikatoren erlauben. Neben den formativen Indikatoren benötigt ein vollständiges MIMIC-Modell auch eine reflektive globale Operationalisierung desselben Konstrukts. In der vorliegenden Arbeit wurde die umfassende Marktorientierung zusätzlich anhand von vier reflektiven Indikatoren als Konstrukt erster Ordnung operationalisiert. Die Ergebnisse der Güteprüfung des reflektiv gemessenen Gesamtkonstrukts gibt Tabelle 28 wider. Sowohl die einzelnen Items als auch das Konstrukt als Ganzes erfüllen die geforderten Mindestwerte der Gütebeurteilung. Es müssen somit keine Indikatoren eliminiert werden. Die reflektive Messung kann für das weitere Vorgehen im MIMIC-Modell zur Validierung der Indikatorrelevanz und externen Validität verwendet werden.

${ }^{666}$ Vgl. Weiber/Mühlhaus 2010, S. 135 f. 
Tabelle 28: Güteprüfung des Gesamtkonstrukts der umfassenden Marktorientierung

\begin{tabular}{|c|c|c|c|c|c|c|c|}
\hline & $\begin{array}{l}\text { Cron- } \\
\text { bach- } \\
\text { Alpha }\end{array}$ & $\begin{array}{c}\text { Faktorla- } \\
\text { dungen }\end{array}$ & $\begin{array}{l}\text { Indikator- } \\
\text { reliabilität }\end{array}$ & $\begin{array}{l}\text { Korrigierte } \\
\text { Item-Skala- } \\
\text { Korrelation }\end{array}$ & $\begin{array}{l}\text { Kom- } \\
\text { munali- } \\
\text { täten }\end{array}$ & DEV & $\begin{array}{l}\text { Faktorreliabili- } \\
\text { tät (Composite } \\
\text { Reliability) } \\
\end{array}$ \\
\hline mo1 & \multirow{4}{*}{0,846} & 0,864 & 0,746 & 0,739 & 0,703 & \multirow{4}{*}{0,685} & \multirow{4}{*}{0,897} \\
\hline mo2 & & 0,823 & 0,677 & 0,680 & 0,576 & & \\
\hline mo3 & & 0,812 & 0,659 & 0,655 & 0,525 & & \\
\hline mo4 & & 0,810 & 0,657 & 0,654 & 0,523 & & \\
\hline
\end{tabular}

Das MIMIC-Modell wird messtechnisch wie ein Strukturgleichungsmodell behandelt und geschätzt. Die fünf Dimensionen bilden dabei die unabhängigen Variablen, die globale Messung des Konstrukts die abhängige Variable. Als Gütekriterien werden daher auch die etablierten Kenngrößen aus der Strukturgleichungsbeurteilung herangezogen (vgl. Tabelle 16). Die Ergebnisse der Prüfung auf externe Validität anhand des Bestimmtheitsmaßes und auf Prognoserelevanz des gesamten Messmodells zeigt Tabelle 29. Ferner sind dort das Ausmaß und die Signifikanz der Pfadkoeffizienten sowie der Erklärungsbeitrag und die Prognoserelevanz jeder einzelnen Dimension berechnet.

Tabelle 29: Ergebnisse der Güteprüfung des formativen Messmodells von Marktorientierung

\begin{tabular}{|c|c|c|c|c|c|c|c|}
\hline Dimension & Pfadkoeffizient & $\begin{array}{c}\mathrm{t}-\text { Wert } \\
\text { (Signifikanz) }\end{array}$ & $\mathrm{R}^{2}$ & $\mathrm{R}^{2} \mathrm{exkl}$ & $\mathrm{f}^{2}$ & $\mathrm{Q}^{2}$ & $\mathrm{q}^{2}$ \\
\hline CUSTOR & 0,301 & $6,532(* * *)$ & \multirow{5}{*}{0,709} & 0,664 & 0,154 & 0,497 & 0,035 \\
\hline COMPOR & 0,085 & $2,013(* *)$ & & 0,706 & 0,010 & 0,460 & $-0,013$ \\
\hline PARTOR & 0,239 & $5,534(* * *)$ & & 0,678 & 0,105 & 0,376 & 0,026 \\
\hline MULTOR & 0,234 & $5,796(* * *)$ & & 0,675 & 0,117 & 0,514 & 0,043 \\
\hline FUNCO & 0,196 & $4,422(* * *)$ & & 0,690 & 0,065 & 0,520 & 0,001 \\
\hline
\end{tabular}

Die Ergebnisse zeigen, dass alle Dimension signifikant zum Konstrukt der Marktorientierung beitragen. Lediglich die Höhe des Pfadkoeffizienten der Dimension Wettbewerbsorientierung (COMPOR) und minimal die Höhe des Pfadkoeffizienten der abteilungsübergreifenden Koordination (FUNCO) liegen unterhalb des 
geforderten Werts von 0,2. ${ }^{667}$ Die Effektstärken der einzelnen Dimensionen liegen mit Ausnahme von COMPOR im Bereich einer mittleren Stärke. Gleiches gilt für die pfadbezogene Prognoserelevanz $\mathrm{q}^{2}$ der einzelnen Dimensionen. Mit Hilfe der Blindfolding-Prozedur ${ }^{668}$ kann somit gezeigt werden, dass das Messmodell in der Lage ist, für jede Dimension mit Ausnahme von COMPOR fehlende Daten genauer zu schätzen, als es durch das Einsetzen von Variablenmittelwerten möglich ist. Das Stone-Geisser-Kriterium $\mathrm{Q}^{2}$ liegt für alle endogenen Variablen im positiven Bereich, was für das Modell insgesamt eine Prognoserelevanz bedeutet. ${ }^{669}$ Das R $\mathrm{R}^{2}$ liegt mit 70,9 \% der über die Dimensionen erklärten Varianz in einem sehr hohen Bereich für explorative Studien. Die Dimensionen können somit einen substanziellen Teil der Varianz des Konstrukts erklären. ${ }^{670}$

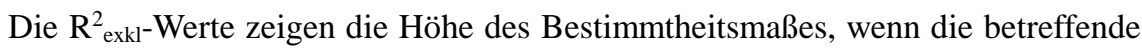
Dimension aus dem Messmodell entfernt wird.

Die ermittelten Kennzahlen zeigen insgesamt, dass die Konzeptualisierung von Marktorientierung als mehrdimensionales Konstrukt zweiter Ordnung nicht verworfen werden muss. Die Struktur der fünf Dimensionen wird somit als bestätigt angesehen.

Das Konstrukt der Marktorientierung soll in dieser Arbeit im Rahmen eines Strukturgleichungsmodells hinsichtlich seiner Einflüsse überprüft werden. Der PLS-Ansatz, mit dem das Strukturgleichungsmodell geschätzt werden soll, ist allerdings nicht ohne Einschränkungen in der Lage, Konstrukte höherer Ordnung zu schätzen. ${ }^{671}$ Um dennoch die zugrundeliegende formative Struktur von Marktorientierung beibehalten zu können, werden im Folgenden die einzelnen reflektiv gemessenen Dimensionen zu Faktorwerten aggregiert. ${ }^{672}$ Dadurch wird die erste Ebene von reflektiven Messmodellen eliminiert und das Gesamtmodell wieder für weitere Untersuchungen mittels PLS handhabbar gemacht (vgl. Abschnitt

\footnotetext{
667 Vgl. Chin 1998a S. XI.

${ }^{668}$ Vgl. Tenenhaus et al. 2005, S. $174 \mathrm{ff}$.

${ }^{669}$ Zur Bestimmung des Q2-Wertes wird die standardmäßig in SmartPLS ausgegebene Cross-

validated Redundancy verwendet (vgl. Chin 1998b, S. 318; Tenenhaus et al. 2005, S. 173 ff.)

${ }^{670}$ Vgl. Chin 1998b, S. 323.

${ }^{671}$ Vgl. Becker et al. 2012, S. 361.

672 Vgl. hierzu und im Folgenden Giere et al. 2006, S. 688.
} 
5.2.5). Die Faktorwerte der Dimensionen Kundenorientierung, Wettbewerbsorientierung, Drittparteienorientierung, Marktstufenorientierung und Abteilungsübergreifende Koordination werden nach diesem Ansatz als direkte formative Messindikatoren von Marktorientierung verwendet. Das so gemessene Konstrukt der Marktorientierung soll ebenfalls einer Güteprüfung unterzogen werden, bevor es zur Hypothesenbeurteilung im Strukturgleichungsmodell Anwendung findet. Zunächst sollen die Indikatoren des formativen Messmodells einem Test auf Multikollinearität unterzogen und anschließend auf ihre Relevanz getestet werden. Mittels Test auf Multikollinearität soll die lineare Abhängigkeit der Indikatoren und damit verbundene inhaltliche sowie messtechnische Schwierigkeiten ausgeschlossen und eine Indikatorelimination vermieden werden. Zur Überprüfung werden die fünf als theoretisch unabhängig betrachteten Indikatoren einer Regressionsanalyse unterzogen. Dabei werden abwechselnd je ein Indikator als abhängige Variable und die übrigen vier Indikatoren als unabhängige Variable festgesetzt. ${ }^{673}$ Die Ergebnisse des Tests auf Multikollinearität stellt Tabelle 30 dar. Es zeigt sich, dass jeder Indikator mit Ausnahme der Wettbewerbsorientierung zu weniger als 50 \% von den übrigen Indikatoren erklärt werden kann. Der Faktorwert der Wettbewerbsorientierung zeigt die höchsten VIF-Werte, die allerdings vollumfänglich in den geforderten Grenzen für Multikollinearität liegen.

${ }^{673}$ Vgl. Weiber/Mühlhaus 2010, S. 204; 214. 
Tabelle 30: Ergebnisse der Kollinearitätsprüfung

\begin{tabular}{|c|c|c|c|c|}
\hline $\begin{array}{c}\text { Faktorwerte } \\
\text { als abhängige Variable }\end{array}$ & $\mathrm{R}^{2}$ & $\begin{array}{c}\text { Faktorwerte } \\
\text { als unabhängige Variable }\end{array}$ & Toleranz & VIF \\
\hline \multirow{4}{*}{$\begin{array}{l}\text { Kundenorientierung } \\
\text { (CUSTOR) }\end{array}$} & \multirow{4}{*}{0,483} & COMPOR & ,559 & 1,790 \\
\hline & & PARTOR & ,610 & 1,641 \\
\hline & & MULTOR & (540 & 1,851 \\
\hline & & FUNCO & ,659 & 1,518 \\
\hline \multirow{4}{*}{$\begin{array}{l}\text { Wettbewerbsorientierung } \\
\text { (COMPOR) }\end{array}$} & \multirow{4}{*}{0,542} & CUSTOR & ,540 & 1,851 \\
\hline & & FUNCO & ,559 & 1,790 \\
\hline & & PARTOR & ,659 & 1,518 \\
\hline & & MULTOR & ,610 & 1,641 \\
\hline \multirow{4}{*}{$\begin{array}{l}\text { Abteilungsübergreifende } \\
\text { Koordination (FUNCO) }\end{array}$} & \multirow{4}{*}{0,495} & CUSTOR & 603 & 1,658 \\
\hline & & COMPOR & ,507 & 1,973 \\
\hline & & PARTOR & ,615 & 1,626 \\
\hline & & MULTOR & -580 & 1,725 \\
\hline \multirow{4}{*}{$\begin{array}{l}\text { Drittparteienorientierung } \\
\text { (PARTOR) }\end{array}$} & \multirow{4}{*}{0,390} & CUSTOR & ,519 & 1,929 \\
\hline & & COMPOR & ,495 & 2,022 \\
\hline & & FUNCO & (509 & 1,965 \\
\hline & & MULTOR & ,624 & 1,602 \\
\hline \multirow{4}{*}{$\begin{array}{l}\text { Marktstufenorientierung } \\
\text { (MULTOR) }\end{array}$} & \multirow{4}{*}{0,422} & CUSTOR & ,539 & 1,855 \\
\hline & & COMPOR & 483 & 2,070 \\
\hline & & FUNCO & ,506 & 1,975 \\
\hline & & PARTOR & ,659 & 1,517 \\
\hline
\end{tabular}

Eine lineare Abhängigkeit der Indikatoren ist somit als unkritisch einzustufen, so dass keine Indikatoren eliminiert werden müssen. Alle Indikatoren können folglich für die weitere Prüfung auf Indikatorrelevanz beibehalten werden. Tabelle 31 gibt die Ergebnisse der Prüfung auf Indikatorrelevanz für die faktorbasierten Dimensionswerte wider.

Tabelle 31: Ergebnisse der Prüfung auf Indikatorrelevanz für den faktorbasierten Ansatz

\begin{tabular}{|c|c|c|}
\multicolumn{1}{c|}{ Faktorwert der Dimension } & Regressionsgewichte & t-Werte (Signifikanz) \\
\hline CUSTOR & 0,376 & $7,258(* * *)$ \\
\hline COMPOR & 0,103 & $2,148(* *)$ \\
\hline PARTOR & 0,236 & $4,373(* * *)$ \\
\hline MULTOR & 0,291 & $6,129(* * *)$ \\
\hline FUNCO & 0,256 & $5,174\left(^{* * *}\right)$ \\
\hline Signifikanzniveau: ***: $\mathrm{p}<0,01 ; * *: \mathrm{p}<0,05 ; *$ : $<0,1 ;$ n.s.: nicht signifikant \\
\hline
\end{tabular}

Alle fünf Faktoren tragen substanziell und signifikant zum Konstrukt der umfas- 
senden Marktorientierung bei. Der geringste Einfluss auf das Konstrukt geht vom Indikator Wettbewerbsorientierung mit $\lambda=0,103$ aus. Der absolut gesehen niedrige Wert sollte allerdings nicht als Hinweis eines schlecht spezifizierten formativen Messmodells fehlinterpretiert werden. ${ }^{674}$ Der PLS-Ansatz schätzt schließlich die Gewichte der formativen Indikatoren so, dass ein möglichst hoher Anteil der Varianz der abhängigen Variable erklärt werden kann. ${ }^{675}$ Mit der Anzahl der zur Konstruktmessung herangezogenen formativen Indikatoren steigt zudem die Wahrscheinlichkeit, dass auch Indikatoren mit relativ niedrigen Ladungen darunter sind. Hier kommt es also darauf an, die einzelnen Indikatoren vor ihrem theoretischen Beitrag zum Konstrukt zu analysieren und die Signifikanzniveaus der Regressionsgewichte zu betrachten. ${ }^{676}$ Alle geschätzten Regressionspfade zeigen sich auf einem 5 \%-Niveau als signifikant und können aufgrund von theoretischen Erwägungen auch nicht ausgeschlossen werden.

Die Ergebnisse zeigen, dass für jeden Indikator Indikatorrelevanz angenommen werden kann. Der faktorbasierte Ansatz mit fünf formativen Indikatoren wird folglich im Strukturgleichungsmodell verwendet, um die Wirkungen der umfassenden Marktorientierung auf marktrelevante Unternehmensaktivitäten zu prüfen.

\subsubsection{Gütebeurteilung der Messmodelle der Wirkungen}

Nachfolgend wird die Validierung und Bewertung der jeweiligen Messmodelle der Wirkungen von Marktorientierung vorgenommen. Dabei kommen aufgrund ebenfalls reflektiver Messvorschriften dieselben Prüfverfahren und Gütekriterien zum Einsatz wie bei der Güteprüfung der Facetten von Marktorientierung (vgl. Abschnitt 5.4.1).

Die Ergebnisse der Güteprüfung für das Konstrukt der Unternehmenszusammenarbeit sind in Tabelle 32 abgebildet.

\footnotetext{
${ }^{674}$ Vgl. Götz/Liehr-Gobbers 2004, S. 728; Petter et al. 2007, S. 627.

${ }^{675}$ Vgl. Chin 1998b, S. 307.

${ }^{676}$ Vgl. Cenfetelli/Bassellier 2009, S. 694.
} 
Tabelle 32: Gütebeurteilung des Konstrukts Unternehmenszusammenarbeit

\begin{tabular}{|c|c|c|c|c|c|c|c|}
\hline & $\begin{array}{l}\text { Cron- } \\
\text { bach- } \\
\text { Alpha }\end{array}$ & $\begin{array}{c}\text { Faktorla- } \\
\text { dungen }\end{array}$ & $\begin{array}{l}\text { Indikator- } \\
\text { reliabilität }\end{array}$ & $\begin{array}{l}\text { Korrigierte } \\
\text { Item-Skala- } \\
\text { Korrelation }\end{array}$ & $\begin{array}{l}\text { Kom } \\
\text { muna- } \\
\text { litäten }\end{array}$ & DEV & $\begin{array}{c}\text { Faktorreliabili- } \\
\text { tät (Composite } \\
\text { Reliability) }\end{array}$ \\
\hline collabo1 & \multirow{5}{*}{0,846} & 0,828 & 0,685 & 0,705 & 0,622 & \multirow{5}{*}{0,621} & \multirow{5}{*}{0,891} \\
\hline collabo2 & & 0,851 & 0,724 & 0,756 & 0,726 & & \\
\hline collabo3 & & 0,737 & 0,543 & 0,590 & 0,416 & & \\
\hline collabo4 & & 0,746 & 0,557 & 0,566 & 0,379 & & \\
\hline collabo5 & & 0,771 & 0,594 & 0,644 & 0,526 & & \\
\hline
\end{tabular}

Das Konstrukt als Ganzes und die einzelnen Indikatoren weisen insgesamt zufriedenstellende Werte auf. Lediglich die Streuung des Indikators collabo4 (klare Richtlinien bei Zusammenarbeit) kann nur zu 37,9 \% über das Konstrukt erklärt werden. Zudem weist der Indikator die geringste Item-Skala-Korrelation auf. Da aber die übrigen Kennzahlen für die Beibehaltung dieses Indikators sprechen und anhand der a priori angestellten theoretischen Überlegungen die Inhaltsabdeckung dieses Items als wichtig angenommen wird, soll der Indikator dennoch beibehalten werden. Dennoch führen die Werte zu der Überlegung, ob eine Zusammenarbeit von Unternehmen, so wie sie von den Befragungsteilnehmern aufgefasst wird, immer auch durch explizite Regelwerke gelenkt werden muss. Vielmehr scheint eine enge Zusammenarbeit zwischen Anbieter und Kunde auch ohne klare Regeln und Formalien auszukommen. Ähnliches gilt für den Indikator collabo3 (Marktinformationen austauschen). Das Item kann durch den dahinterstehenden Faktor nur zu etwas weniger als der Hälfte erklärt werden. Die übrigen Kennzahlen und eine sachlogische Betrachtung sprechen allerdings für die weitere Beibehaltung dieses Items.

Das Konstrukt der Innovativität weist durchgehend sehr gute Werte auf, die in Tabelle 33 dargestellt werden. Die Skala kann unverändert für die weiteren Untersuchungen übernommen werden. 
Tabelle 33: Gütebeurteilung des Konstrukts Innovativität

\begin{tabular}{|c|c|c|c|c|c|c|c|}
\hline & $\begin{array}{l}\text { Cron- } \\
\text { bach- } \\
\text { Alpha }\end{array}$ & $\begin{array}{l}\text { Faktorla- } \\
\text { dungen }\end{array}$ & $\begin{array}{c}\text { Indikatorre- } \\
\text { liabilität }\end{array}$ & $\begin{array}{l}\text { Korrigierte } \\
\text { Item-Skala- } \\
\text { Korrelation }\end{array}$ & $\begin{array}{l}\text { Kommu- } \\
\text { nalitäten }\end{array}$ & DEV & $\begin{array}{c}\text { Faktorreliabili- } \\
\text { tät (Composite } \\
\text { Reliability) }\end{array}$ \\
\hline innova1 & \multirow{3}{*}{0,914} & 0,908 & 0,824 & 0,802 & 0,719 & \multirow{3}{*}{0,853} & \multirow{3}{*}{0,946} \\
\hline innova2 & & 0,941 & 0,885 & 0,864 & 0,882 & & \\
\hline innova3 & & 0,922 & 0,849 & 0,812 & 0,745 & & \\
\hline
\end{tabular}

Die Ergebnisse der Prüfung für das Konstrukt Anpassung der Marketingstrategie zeigen, dass das Konstrukt insgesamt als reliabel zu bezeichnen ist. Die Werte des Cronbach-Alphas und der Composite Reliability erreichen sehr gute Werte. Auch die einzelnen Indikatoren erreichen mit Ausnahme des Indikators align3 sehr gute Werte. Align3 bezieht sich auf die Prozessabstimmung zwischen Anbieter und direktem Kunden, um den nachfolgenden Marktstufen einen höheren Kundennutzen zu liefern. Da allerdings der geforderte Wert der Kommunalitäten noch knapp eingehalten wird und alle anderen Kriterien ebenso eingehalten werden, soll dieses Item für die weiteren Untersuchungen beibehalten werden. Tabelle 34 fasst die Ergebnisse der Güteprüfung zusammen.

Tabelle 34: Gütebeurteilung des Konstrukts Anpassung der Marketingstrategie

\begin{tabular}{|c|c|c|c|c|c|c|c|}
\hline & $\begin{array}{l}\text { Cron- } \\
\text { bach- } \\
\text { Alpha }\end{array}$ & $\begin{array}{c}\text { Faktorla- } \\
\text { dungen }\end{array}$ & $\begin{array}{l}\text { Indikator- } \\
\text { reliabilität }\end{array}$ & $\begin{array}{l}\text { Korrigierte } \\
\text { Item-Skala- } \\
\text { Korrelation }\end{array}$ & $\begin{array}{l}\text { Kommu- } \\
\text { nalitäten }\end{array}$ & DEV & $\begin{array}{c}\text { Faktorreliabili- } \\
\text { tät (Composite } \\
\text { Reliability) }\end{array}$ \\
\hline align1 & \multirow{4}{*}{0,870} & 0,869 & 0,755 & 0,795 & 0,788 & \multirow{4}{*}{0,717} & \multirow{4}{*}{0,910} \\
\hline align2 & & 0,838 & 0,702 & 0,756 & 0,708 & & \\
\hline align3 & & 0,806 & 0,649 & 0,606 & 0,411 & & \\
\hline align4 & & 0,873 & 0,762 & 0,742 & 0,641 & & \\
\hline
\end{tabular}

Als Erfolgskonstrukt wird der Neuprodukterfolg gemessen. Die Validierung dieses Konstrukts ergibt anhand einer explorativen Faktoranalyse, dass es sich nicht um ein unidimensionales Konstrukt handelt, da die zur Messung herangezogenen Indikatoren auf zwei Faktoren laden. Anders als die Items nps1 (Anzahl 
von Produkteinführungen), nps2 (Erfolgsquote) und nps3 (Schnelligkeit beim Markteintritt) bezieht sich das Item nps4 (Kosten der Leistungsentwicklung) nicht auf den absoluten oder relativen Erfolg bei der Einführung von Neuprodukten sondern auf den Aspekt der Kosten, die dem Unternehmen bis zur Marktreife entstehen. Folglich lädt dieses Item auch auf einen separaten Faktor. Eine ZweiFaktorlösung erscheint post-hoc in Anbetracht der gewonnenen Daten durchaus plausibel. Da jedoch die Reliabilität einer Single-Item-Messung für einen einzigen Faktor, der die Effizienz abbilden würde, nicht abgeschätzt werden kann, wird entschieden, das Konstrukt um den Effizienz-Indikator zu bereinigen. Durch die Elimination des Items nps4 kann die geforderte unidimensionale Faktorstruktur wieder erreicht werden. Anders als ursprünglich gedacht, bezieht sich das Konstrukt dann jedoch nur noch auf die Effektivität der Produkteinführung. Die Effizienz, mit der Neuprodukte eingeführt werden, musste in dieser Untersuchung als Maß für den Neuprodukterfolg aufgegeben werden. Die Ergebnisse der Güteprüfung, nachdem der Indikator nps4 entfernt wurde, zeigt Tabelle 35. Alle geforderten Gütemaße und insbesondere die Unidimensionalität werden nach der Elimination von nps4 eingehalten.

Tabelle 35: Gütebeurteilung des Neuprodukterfolgs

\begin{tabular}{|c|c|c|c|c|c|c|c|}
\hline & $\begin{array}{l}\text { Cronbach- } \\
\text { Alpha }\end{array}$ & $\begin{array}{c}\text { Faktorla- } \\
\text { dungen }\end{array}$ & $\begin{array}{l}\text { Indikator- } \\
\text { reliabilität }\end{array}$ & $\begin{array}{l}\text { Korrigierte } \\
\text { Item-Skala- } \\
\text { Korrelation }\end{array}$ & $\begin{array}{l}\text { Kommuna- } \\
\text { litäten }\end{array}$ & DEV & $\begin{array}{c}\text { Faktorreliabili- } \\
\text { tät (Composite } \\
\text { Reliability) }\end{array}$ \\
\hline nps1 & \multirow{3}{*}{0,799} & 0,862 & 0,743 & 0,692 & 0,709 & \multirow{3}{*}{0,713} & \multirow{3}{*}{0,882} \\
\hline nps2 & & 0,852 & 0,727 & 0,635 & 0,537 & & \\
\hline nps3 & & 0,819 & 0,670 & 0,611 & 0,482 & & \\
\hline nps4 & -- & 0,057 & 0,003 & 0,037 & 0,080 & -- & -- \\
\hline
\end{tabular}

Nach der Bereinigung des Konstrukts Neuprodukterfolg erfüllen die Wirkungskonstrukte alle zufriedenstellend die geforderten Gütekriterien. Die Messskalen der Konstrukte sind somit geeignet, um im Strukturgleichungsmodell für die Schätzung von Wirkbeziehungen herangezogen zu werden. 


\subsection{Bewertung des Strukturmodells}

Zur Schätzung des Strukturmodells wird auf die Varianz-analytische Methode (Partial Least Squares) zurückgegriffen und die Software SmartPLS 2.0.M3 ${ }^{677}$ benutzt. Zur Ermittlung der Signifikanzen von Wirkzusammenhängen wird auf das Boostrapping als Resamplingverfahren zurückgegriffen. Die Schätzungen basieren auf 500 aus den erhobenen Datensätzen gezogenen Stichproben mit je 384 Fällen.

Zunächst sollen die direkten Effekte im Modell und der Erklärungsgehalt der einzelnen Variablen betrachtet werden. Anschließend erfolgt eine Begutachtung der Mediatoreffekte im Modell.

\subsubsection{Betrachtung der direkten Effekte und Erklärungsgehalt der Variablen}

Die Betrachtung des Strukturgleichungsmodells und die Überprüfung der Hypothesen erfolgt anhand der Pfadkoeffizienten und der ermittelten t-Werte.

Tabelle 36 gibt die Ergebnisse wider. Die Ergebnisse zeigen, dass fünf von sechs Hypothesen bestätigt werden. Lediglich die Beziehung zwischen der Anpassung der Marketingstrategien und dem Neuprodukterfolg weist keine nennenswerten Effekte auf. Der Pfadkoeffizient ist zwar wie postuliert positiv, liegt aber nahe bei null. Hypothese H6 kann somit nicht unter Beachtung des angestrebten Signifikanzniveaus als bestätigt angesehen werden. Eine ausführliche Diskussion der Ergebnisse soll in Abschnitt 5.7 erfolgen.

${ }^{677}$ Ringle et al. 2005. 
Tabelle 36: Ergebnisse der Schätzung des Strukturmodells

\begin{tabular}{|c|c|c|c|c|}
\hline Hypothese & Beziehung & Pfadkoeffizient & t-Wert & Befund \\
\hline H1 & MO $\rightarrow$ COLLABO & $0,492^{* * *}$ & 10,880 & $\sqrt{ }$ \\
\hline H2 & MO $\rightarrow$ INNOVA & $0,605^{* * *}$ & 15,856 & $\sqrt{ }$ \\
\hline H3 & MO $\rightarrow$ ALLIGN & $0,519^{* * *}$ & 13,683 & $\sqrt{ }$ \\
\hline H4 & COLLABO $\rightarrow$ NPS & $0,185^{* * *}$ & 3,683 & $\sqrt{ }$ \\
\hline H5 & INNOVA $\rightarrow$ NPS & $0,404^{* * *}$ & 7,528 & $\sqrt{ }$ \\
\hline H6 & ALIGN $\rightarrow$ NPS & 0,057 n.s. & 1,422 & $(\sqrt{ })$ \\
\hline \multicolumn{2}{|c|}{ Signifikanzniveau: $* * *: p<0,01 ; * *: p<0,05 ; *: p<0,1 ;$ n.s.: nicht signifikant } \\
$\sqrt{ }=$ Hypothese empirisch gestützt; $(\sqrt{ })=$ Hypothese nicht durch signifikanten Pfad bestätigt \\
\hline
\end{tabular}

Neben der Stärke und Signifikanz der Wirkbeziehungen ist das Bestimmtheitsmaß $\mathrm{R}^{2}$ für die Güte eines Modells von Belang. Das $\mathrm{R}^{2}$ gibt an, wie groß der Varianzanteil einer abhängigen Variable ist, der durch die exogenen Variablen des Modells erklärt werden kann. Tabelle 37 zeigt die Bestimmtheitsmaße der endogenen Modellvariablen.

Tabelle 37: Bestimmtheitsmaße der endogenen Modellvariablen

\begin{tabular}{|c|c|c|c|c|}
\hline & COLLABO & INNOVA & ALIGN & NPS \\
\hline R2 & 0,242 & 0,366 & 0,269 & 0,293 \\
\hline
\end{tabular}

Die Konstrukte Unternehmenszusammenarbeit (COLLABO), Innovativität (INNOVA) und Anpassung der Marketingstrategie (ALIGN) werden zu je 24,2 \%, 36,6 \% und 26,9 \% erklärt. Dies sind überaus zufriedenstellende Werte, wenn man bedenkt, dass diese Konstrukte im vorliegenden Modell lediglich durch die Marktorientierung eines Unternehmens erklärt werden. Der Neuprodukterfolg kann im Modell zu 29,3 \% erklärt werden. Dieser Wert kann als moderat bezeichnet werden und zeigt gleichzeitig, dass neben den Variablen COLLABO, INNOVA, ALIGN noch weitere Einflussgrößen existieren müssen, die allerdings im Modell nicht berücksichtigt werden.

Des Weiteren kann die Effektstärke $\mathrm{f}^{2}$ Aufschluss darüber geben, in welchem 
Ausmaß die einzelnen unabhängigen Variablen zum Erklärungsgehalt einer abhängigen Variablen beitragen. Die Berechnung der Effektstärke $\mathrm{f}^{2}$ basiert auf dem veränderten Bestimmtheitsmaß $\mathrm{R}_{\text {exkl }}^{2}$ durch Ausschluss einer unabhängigen Variable. Im vorliegenden Modell kann dabei nur die Variable NPS sinnvollerweise als abhängige Variable fungieren. Die Variablen COLLABO, INNOVA, ALIGN dienen dabei als unabhängige Variable. Eine Berechnung der Effektstärke des Konstrukts der umfassenden Marktorientierung kann nur eingeschränkt interpretiert werden, da es sich hierbei um die einzige Einflussgröße auf die Variablen COLLABO, INNOVA, ALIGN handelt. Die Effektstärken-Analyse wird daher an einem Teilstrukturmodell ohne Marktorientierung vorgenommen.

Tabelle 38 zeigt die ermittelten Effektstärken der einzelnen unabhängigen Variablen auf den Neuprodukterfolg. Die Unternehmenszusammenarbeit zeigt dabei einen schwachen Einfluss und die Anpassung der Marketingstrategien einen sehr schwachen Einfluss auf den Neuprodukterfolg. Die Effektstärke der Innovativität kann hingegen als moderat bezeichnet werden.

Das Stone-Geisser-Kriterium Q² als Maß der Prognoserelevanz des betrachteten Teilstrukturmodells liegt für alle endogenen Variablen im positiven Bereich. Dies bedeutet, dass fehlende Daten anhand des Modells genauer geschätzt werden können, als dies durch das Einsetzen von Variablenmittelwerten zu erwarten wäre. Gleiches gilt für die Prognoserelevanz q2 der einzelnen Pfadbeziehungen. Die drei unabhängigen Konstrukte COLLABO, INNOVA, ALIGN weisen eine positive Prognoserelevanz auf (vgl.

Tabelle 38).

Tabelle 38: Effektstärken und Prognoserelevanz des Teilstrukturmodells

\begin{tabular}{|c|c|c|c|c|c|c|}
\hline $\begin{array}{c}\text { Abhängige } \\
\text { Variable }\end{array}$ & $\begin{array}{c}\text { Unabhängige } \\
\text { Variable }\end{array}$ & \multirow{2}{*}{$\mathrm{R}^{2}$} & $\mathrm{R}^{2}{ }_{\text {exkl }}$ & $\mathrm{f}^{2}$ & $\mathrm{Q}^{2}$ & $\mathrm{q}^{2}$ \\
\hline \multirow{3}{*}{ NPS } & COLLABO & & 0,268 & 0,036 & 0,140 & 0,023 \\
\cline { 2 - 6 } & INNOVA & \multirow{2}{*}{0,293} & 0,172 & 0,171 & 0,287 & 0,115 \\
\cline { 2 - 5 } & ALIGN & & 0,290 & 0,004 & 0,178 & 0,002 \\
\hline
\end{tabular}

\subsubsection{Bewertung der Mediationseffekte}

Zur Prüfung der Hypothesen H7, H8 und H9, die von einer mediierten Beziehung 
zwischen Marktorientierung und Neuprodukterfolg ausgehen, sollen die in Abschnitt 5.2.5 vorgestellten Gütekriterien Anwendung finden. Zunächst wird daher anhand des Sobel-Tests die Existenz und anhand des Verhältnisses zwischen direktem und indirektem Pfad zwischen unabhängiger und abhängiger Variable die Stärke der Mediation untersucht. Der aus dem Sobel-Test berechnete z-Wert gibt Auskunft darüber, mit welcher Wahrscheinlichkeit kein indirekter Effekt vorliegt, und eine entsprechende Nullhypothese zurückgewiesen werden muss. ${ }^{678}$ Die Ergebnisse in Tabelle 39 zeigen, dass hochsignifikante Mediationseffekte für die Variablen Unternehmenszusammenarbeit (COLLABO) und Innovativität (INNOVA) bestehen. Marktorientierung (MO) wirkt hier also nur zu einem Teil direkt auf das Konstrukt Neuprodukterfolg (NPS). Ein bedeutsamer Teil dieser Beziehung wird durch die mediierende Wirkung von Unternehmenszusammenarbeit und Innovativität erklärt. Die Hypothesen H7 und H8 werden somit angenommen. Hypothese H9, die die Anpassung der Marketingstrategie (ALIGN) als Mediator unterstellt, muss zurückgewiesen werden. Der z-Wert nimmt keinen Wert an, der auf das Vorliegen eines indirekten Effekts schließen lässt. Zudem erfüllt die Beziehung zwischen ALIGN und NPS nicht das Kriterium, nachdem beim direkten Effekt eine signifikante Beziehung vorliegen muss ( $\mathrm{t}$-Wert = $0,281) \cdot{ }^{679}$

${ }^{678} \mathrm{Vgl}$. Eggert et al. 2005, S. 106.

${ }^{679}$ Vgl. Eggert et al. 2005, S. 105; Baron/Kenny 1986, S. 1176. 
Tabelle 39: Ergebnisse der Prüfung auf Mediation

\begin{tabular}{|c|c|c|c|c|c|c|}
\hline 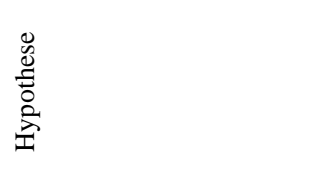 & 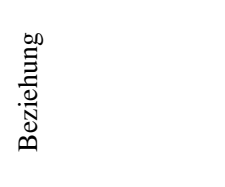 & 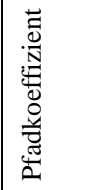 & 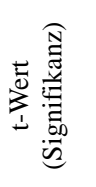 & 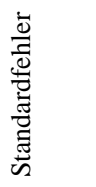 & 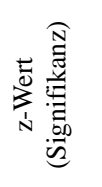 & 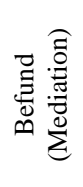 \\
\hline \multirow{2}{*}{$\begin{array}{c}\mathrm{H} 7 \\
(\mathrm{MO} \rightarrow \mathrm{COLLABO} \rightarrow \mathrm{NPS})\end{array}$} & $\begin{array}{c}\text { MO } \\
\rightarrow \text { COLLABO }\end{array}$ & 0,497 & $\begin{array}{c}11,214 \\
(* * *)\end{array}$ & 0,044 & \multirow{2}{*}{$\begin{array}{l}2,602 \\
(* * *)\end{array}$} & \multirow{2}{*}{$\sqrt{ }$} \\
\hline & COLLABO $\rightarrow$ NPS & 0,138 & $\begin{array}{l}2,675 \\
(* * *)\end{array}$ & 0,052 & & \\
\hline \multirow{2}{*}{$\begin{array}{c}\mathrm{H} 8 \\
(\mathrm{MO} \rightarrow \mathrm{INNOVA} \rightarrow \mathrm{NPS})\end{array}$} & $\mathrm{MO} \rightarrow$ INNOVA & 0,608 & $\begin{array}{c}14,858 \\
(* * *)\end{array}$ & 0,041 & \multirow{2}{*}{$\begin{array}{l}4,471 \\
(* * *)\end{array}$} & \multirow{2}{*}{$\sqrt{ }$} \\
\hline & INNOVA $\rightarrow$ NPS & 0,303 & $\begin{array}{l}4,688 \\
(* * *) \\
\end{array}$ & 0,065 & & \\
\hline \multirow{2}{*}{$\begin{array}{c}\text { H9 } \\
(\mathrm{MO} \rightarrow \text { ALIGN } \rightarrow \text { NPS })\end{array}$} & $\mathrm{MO} \rightarrow$ ALIGN & 0,506 & $\begin{array}{c}12,510 \\
(* * *)\end{array}$ & 0,040 & \multirow{2}{*}{$\begin{array}{c}-0,281 \\
\text { (n.s.) }\end{array}$} & \multirow{2}{*}{$X$} \\
\hline & ALIGN $\rightarrow$ NPS & $-0,015$ & $\begin{array}{l}0,281 \\
\text { (n.s.) }\end{array}$ & 0,052 & & \\
\hline
\end{tabular}

Aufgrund der Tatsache, dass bei Berücksichtigung der Mediatoren der direkte Pfad zwischen exogener und endogener Variable zwar jeweils kleiner wird aber signifikant bleibt, handelt es sich in allen Fällen um eine partielle Mediation. Die geschätzten direkten und indirekten Effekte sowie die Stärke der Mediation können Tabelle 40 entnommen werden. Der PM-Wert gibt das Verhältnis des indirekten Effekts zum Gesamteinfluss wider. Es zeigt sich, dass der Mediator COLLABO nur einen relativ kleinen Anteil von 12,4 \% und der Mediator INNOVA 27,5 \% der Wirkbeziehung zwischen MO und NPS erklärt. 
Tabelle 40: Ergebnisse der Prüfung auf Mediationsstärke

\begin{tabular}{|c|c|c|c|c|c|}
\hline Hypothese & Mediator & $\begin{array}{c}\text { direkter } \\
\text { Effekt }\end{array}$ & $\begin{array}{c}\text { t-Wert } \\
(\text { Signifikanz) }\end{array}$ & $\begin{array}{c}\text { indirekter } \\
\text { Effekt }\end{array}$ & PM-Wert \\
\hline $\begin{array}{c}\text { H7 } \\
(\mathrm{MO} \rightarrow \text { COLLABO } \rightarrow \text { NPS) }\end{array}$ & COLLABO & 0,487 & $\begin{array}{c}11,206 \\
(* * *)\end{array}$ & 0,069 & 0,124 \\
\hline $\begin{array}{c}\text { H8 } \\
(M O \rightarrow \text { INNOVA } \rightarrow \text { NPS) }\end{array}$ & INNOVA & 0,487 & $\begin{array}{c}11,206 \\
(* * *)\end{array}$ & 0,184 & 0,275 \\
\hline $\begin{array}{c}\text { H9 } \\
(M O \rightarrow \text { ALIGN } \rightarrow \text { NPS) }\end{array}$ & ALIGN & 0,487 & $\begin{array}{c}11,206 \\
(* * *)\end{array}$ & $-0,007$ & $-0,016$ \\
\hline \multicolumn{7}{|c|}{ Signifikanzniveau: $* * *: p<0,01 ; * *: p<0,05 ; *: p<0,1 ;$ n.s.: nicht signifikant } \\
\hline
\end{tabular}

Nach der Güteprüfung des Strukturmodells kann dem vorliegenden Modell in der Gesamtschau der zur Überprüfung herangezogenen Gütekriterien eine insgesamt gute Eignung bescheinigt werden. Die Betrachtung der Stärke und Signifikanz der direkten und indirekten Pfade, der Bestimmtheitsmaße, der Effektstärken und der Prognoserelevanz zeigt sich, dass das Modell, die a priori formulierten Hypothesen großenteils empirisch bestätigt. Die Ergebnisse der Hypothesenüberprüfung sind in Abbildung 10 zusammenfassend dargestellt.

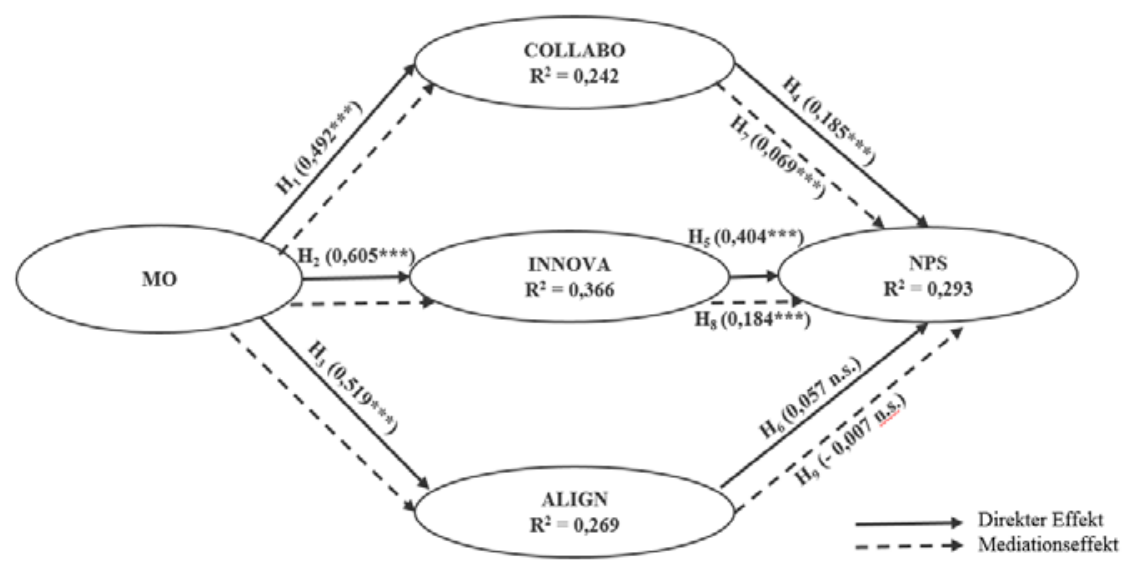

Abbildung 10: Ergebnisse der Hypothesenprüfungen

Mit der Hypothesenprüfung des Strukturgleichungsmodells wurde gleichzeitig

Forschungsfrage 3 beantwortet, die sich mit den Einflüssen der Marktorientie- 
rung hinsichtlich einzelbetrieblicher und kooperativer Unternehmenstätigkeiten sowie dem dadurch letztlich generierten Erfolg beschäftigte.

\subsection{Weitergehende Prüfungen der Modelle}

In einem weiteren Analyseschritt soll nun überprüft werden, welche Einflüsse auf die Modellgüte bei einer Modifizierung der Messskala von Marktorientierung zu erwarten wären. Die Untersuchungen zielen dabei auf den Informationsverlust aufgrund der faktorbasierten Dimensionsaggregation, auf die Allgemeingültigkeit des entwickelten Messinstruments sowie auf einen Vergleich zwischen der originalen MKTOR-Skala und der erweiterten formativen coMO-Skala ab.

Zudem soll noch einmal die Hypothese H6, die einen positiven Zusammenhang zwischen der Anpassung der Marketingstrategie (ALIGN) und dem Neuprodukterfolg (NPS) postuliert, im Lichte eines Gruppenvergleichs, der die Unternehmens- und Produktvoraussetzungen für das Mehrstufige Marketing berücksichtigt, durchgeführt werden.

\subsubsection{Modelltest auf disaggregierter Konstruktebene}

Im Rahmen dieser Arbeit wurde die Schätzung des Strukturmodells mittels einer faktorwertbasierten, formativen Messung von Marktorientierung vorgenommen. Der Schritt der Aggregation der einzelnen Dimensionen zu je einem formativen Indikator war aufgrund der Schätzmethodik der verwendeten Software notwendig. Da eine Aggregation von Daten in der Regel immer mit Informationsverlusten behaftet ist, soll in diesem Abschnitt überprüft werden, wie sich im Modell die Disaggregation des coMO-Messmodells auswirkt. Untersucht werden soll dabei, wie sich das Bestimmtheitsmaß ändert und welche besonderen Einflüsse hinsichtlich der Dimensionen auf die endogenen Variablen festgestellt werden können.

Mit dieser Untersuchung soll wissenschaftlichen Arbeiten Rechnung getragen werden, die Marktorientierung nicht als ein Konstrukt sehen. In der Literatur wird Marktorientierung vielfach als Zusammenstellung unabhängiger Konstrukte gesehen und daher eine Effektuntersuchung auf disaggregiertem Niveau vor- 
schlagen. ${ }^{680}$

Die Ergebnisse des Vergleichs zwischen aggregiertem und disaggregiertem Modell sind in Tabelle 41 dargestellt. Die Ergebnisse zeigen, dass die Aggregation zu geringfügig schlechteren $\mathrm{R}^{2}$-Werten bei den endogenen Variablen Unternehmenszusammenarbeit (COLLABO) und Innovativität (INNOVA) führt. Zudem kann sogar die Varianz des Neuprodukterfolgs (NPS) minimal besser im aggregierten Modell erklärt werden. Hervorzuheben ist, dass die Variable der Anpassung der Marketingstrategien (ALIGN) im disaggregierten Modell substanziell besser erklärt wird.

Tabelle 41: Vergleich der Bestimmtheitsmaße im aggregierten und disaggregierten Modell

\begin{tabular}{|c|c|c|c|c|}
\hline $\mathrm{R}^{2}$ & COLLABO & INNOVA & ALIGN & NPS \\
\hline Aggregiertes coMO-Modell & 0,242 & 0,366 & 0,269 & 0,293 \\
\hline Disaggregiertes coMO-Modell & 0,262 & 0,392 & 0,324 & 0,292 \\
\hline
\end{tabular}

Aus der in Tabelle 42 dargestellten Stärke und Signifikanz der Pfadkoeffizienten kann geschlossen werden, welche disaggregierten Dimensionen besonders stark auf die abhängigen Variablen wirken.

${ }^{680}$ Vgl. Tsiotsou 2010; Low et al. 2007; Ward et al. 2006; Im/Workman 2004; Lukas/Ferrell 2000; Webb et al. 2000; Siguaw/Diamantopoulos 1995. 
Tabelle 42: Wirkbeziehungen mit disaggregiertem coMO-Modell

\begin{tabular}{|c|c|c|c|c|}
\hline $\begin{array}{c}\text { Abhängige } \\
\text { Variable }\end{array}$ & $\begin{array}{c}\text { Unabhängige } \\
\text { Variable }\end{array}$ & Pfadkoeffizient & t-Wert & Signifikanz \\
\hline COLLABO & CUSTOR & 0,160867 & 2,534566 & $* *$ \\
\hline COLLABO & COMPOR & 0,075225 & 1,330499 & n.s. \\
\hline COLLABO & PARTOR & 0,151306 & 2,455326 & $* *$ \\
\hline COLLABO & MULTOR & 0,066037 & 1,329726 & n.s. \\
\hline COLLABO & FUNCO & 0,183057 & 2,814259 & $* * *$ \\
\hline INNOVA & CUSTOR & 0,071994 & 1,324556 & n.s. \\
\hline INNOVA & COMPOR & 0,108877 & 1,958192 & $* *$ \\
\hline INNOVA & PARTOR & 0,128778 & 2,374523 & $* *$ \\
\hline INNOVA & MULTOR & 0,132557 & 2,372434 & $* *$ \\
\hline INNOVA & FUNCO & 0,325401 & 5,371856 & $* * *$ \\
\hline ALIGN & CUSTOR & 0,019352 & 0,485113 & n.s. \\
\hline ALIGN & COMPOR & $-0,000797$ & 0,020323 & n.s. \\
\hline ALIGN & PARTOR & 0,113016 & 2,224818 & $* *$ \\
\hline ALIGN & MULTOR & 0,477153 & 8,260369 & $* * *$ \\
\hline ALIGN & FUNCO & 0,023408 & 0,546982 & n.s. \\
\hline NPS & COLLABO & 0,184424 & 3,812071 & $* * *$ \\
\hline NPS & INNOVA & 0,405565 & 7,395635 & $* * *$ \\
\hline NPS & ALIGN & 0,053749 & 1,239935 & n.s. \\
\hline Signifikanzniveau: $* * *: p$ & 0,$01 ; * *: p<0,05 ; *: p<0,1 ; n . s .:$ nicht & signifikant \\
\hline
\end{tabular}

Die Unternehmenszusammenarbeit wird dabei besonders stark durch die Kundenorientierung, Drittparteienorientierung und Abteilungsübergreifende Koordination signifikant beeinflusst. Vornehmlich eine hoch ausgeprägte innerbetriebliche Informationsteilung und ein starker Fokus auf den Kunden fördern die Zusammenarbeit mit Kunden.

Die Ergebnisse zeigen ferner, dass die Innovativität eines Unternehmens von der Ausrichtung auf Wettbewerber, Drittparteien und nachgelagerte Marktstufen abhängt. Die Ausrichtung an diesen drei Marktgruppen kann die Innovativität des Unternehmens fördern. Die größte Quelle von Innovativität liegt aber im Fokus- 
unternehmen selbst. Die Abteilungsübergreifende Koordination trägt hoch signifikant und mit einem Pfadkoeffizienten $\gamma=0,325$ am stärksten zur Innovativität bei. Interessanterweise kann eine Orientierung an den Kunden nicht als Quelle von Innovativität identifiziert werden. Diese Beobachtung stützt Autoren, die eine zu starke Kundenorientierung als erfolgshinderlich verstehen, da hierbei möglicherweise nur auf die Wünsche bestehender Kunden eingegangen wird und betriebliche Prozesse darauf abgestimmt werden. Eine geringe Unternehmensflexibilität, eine mangelnde Fähigkeit neue Produktideen zu entwickeln und Absatzmöglichkeiten zu erschließen, können die Folge sein. ${ }^{681}$

Die Anpassung der Marketingstrategie wird zu großen Teilen von der Ausrichtung eines Unternehmens auf nachgelagerte Marktstufen bestimmt. Nicht überraschend besteht ein sehr starker, hoch signifikanter Einfluss der Markstufenorientierung auf die verschiedenen mit dem direkten Kunden abgestimmten Marketingaktivitäten. Deutlich schwächeren aber dennoch signifikanten Einfluss hat die Drittparteienorientierung. Neben der Wettbewerbsorientierung und der innerbetrieblichen Zusammenarbeit besitzt interessanter Weise auch die Kundenorientierung keinen nennenswerten Einfluss auf abgestimmte mehrstufige Marketingaktivitäten. Eine entscheidende Determinante für Mehrstufiges Marketing stellt somit weniger die Ausrichtung auf den direkten Kunden als vielmehr auf den indirekten Kunden dar. Die Ergebnisse der Pfadkoeffizienten stehen im Einklang mit der substanziellen Verbesserung des Bestimmtheitsmaßes des Konstrukts ALIGN im disaggregierten coMO-Modell (vgl. Tabelle 41).

Angemerkt werden soll an dieser Stelle, dass die Wettbewerbsorientierung nur einen äußerst geringen positiven bzw. sogar einen leicht negativen Einfluss auf die Unternehmenskooperation und die Anpassung der Marketingstrategie besitzt. Diese Ergebnisse stimmen mit den Erkenntnissen verschiedener spieltheoretischer Experimente überein, nach denen eine Wettbewerbsorientierung, insbesondere wenn sie durch das Management in irrationaler Weise verfolgt wird, für die Zusammenarbeit von Unternehmen schädlich sein kann. ${ }^{62}$ Die dargestellten

${ }^{681}$ Vgl. Christensen/Bower 1996; Håkansson/Ford 2002, S. 135.

${ }^{682}$ Vgl. Bendle/Vandenbosch 2014, S. 789. 
Ergebnisse sind zwar nicht signifikant, doch geben sie Hinweise darauf, dass die Tendenz, „,andere Unternehmen schlagen zu wollen“, dazu führen kann, auch bei der Verteilung von Kooperationsrenten, die nach der Theorie des Relational Views anfallen, den Kooperationspartner möglicherweise übervorteilen zu wollen. Dies wäre der Kooperation insgesamt abträglich.

Die Wirkbeziehung zwischen den unabhängigen Variablen Unternehmenszusammenarbeit (COLLABO), Innovativität (INNOVA), Abstimmung der Marketingstrategien (ALIGN) und der abhängigen Variable Neuprodukterfolg (NPS) sind hinsichtlich der Stärke und Signifikanz weitgehend identisch. Dieses Ergebnis ist nicht weiter überraschend, da keine der Variablen dieses Teilmodells von der Disaggregation direkt betroffen ist.

Aus dem Vergleich der Ergebnisse des aggregierten und disaggregierten Modells lässt sich schließen, dass eine Aggregation für die Schätzung des Modells nur dann einen nennenswerten Einfluss hat, wenn wenige oder gar nur eine Dimension signifikant und hoch auf ein endogenes Konstrukt lädt. Dies ist im vorliegenden Modell beim Konstrukt der Anpassung der Marketingstrategien (ALIGN) der Fall. Dieses Konstrukt wird zum überwiegenden Teil von der Dimension Marktstufenorientierung beeinflusst. Die übrigen endogenen Konstrukte sind nicht von einer derartigen ein- oder zweidimensionalen Einflussnahme betroffen.

\subsubsection{Vergleich der MKTOR-Skala mit der neu entwickelten coMO- Skala}

Das im Rahmen dieser Arbeit theoretisch hergeleitete und entwickelte coMOMessinstrument fußt zu großen Teilen auf der von NARVER/SLATER im Jahre 1990 veröffentlichten MKTOR-Skala. Gegenstand dieses Abschnitts soll ein Vergleich der beiden Skalen sein. Anzumerken ist, dass ein direkter Vergleich mit der Original-MKTOR-Skala nicht möglich ist, da, wie Abschnitte 4.6.1.1, 0 und 0 hinsichtlich der Operationalisierung gezeigt haben, einige der ursprünglichen Items aus den jeweiligen Sub-Skalen entfernt bzw. verändert und neu entwickelte Items aufgenommen wurden. Auch durch die vorgenommenen Übersetzungen besteht die Möglichkeit einer nicht identischen Inhaltsabbildung der Items. Den- 
noch sollen die unterschiedlichen Messansätze miteinander verglichen werden wissentlich, dass nicht in allen Fällen die exakt gleichen Items zum Vergleich herangezogen werden können. Vor dem Vergleich seien an dieser Stelle noch einmal kurz die wesentlichen Unterschiede zwischen den beiden Messskalen umrissen.

NARVER/SLATER attestieren den drei von ihnen entwickelten Komponenten customer orientation, competitor orientation und interfunctional coordination „equal conceptual importance“ innerhalb der MKTOR-Skala. ${ }^{683}$ Die Ausprägung der Marktorientierung auf Konstruktebene wird folglich durch einfache Mittelwertbildung der Dimensionswerte erreicht.

Die im Rahmen dieser Arbeit entwickelte coMO-Skala geht von einem weiter zu fassenden Markt und folglich von einer umfassenderen Marktorientierung aus. Dieser Umstand schlägt sich in den zwei zusätzlichen Dimensionen Drittparteienorientierung und Marktstufenorientierung nieder. Zudem werden die insgesamt fünf Dimensionen als faktorwertbasierte, formative Indikatoren mit unterschiedlichen Regressionsgewichten zur Bildung von Marktorientierung herangezogen.

Die zum Vergleich heranzuziehenden Gütemaße beschränken sich auf die mit dem jeweiligen Messmodell erreichten Bestimmtheitsmaße. Untersucht werden sollen dabei der Erklärungsgehalt in einem MIMIC-Modell mit den vier aus den verschiedenen Definitionen von Marktorientierung abgeleiteten GlobalIndikatoren sowie der Erklärungsgehalt der exogenen Variablen des in dieser Arbeit aufgestellten Strukturgleichungssystems (vgl. Abschnitt 4.5). Tabelle 43 zeigt den Vergleich der Bestimmtheitsmaße in Abhängigkeit des Messmodells.

${ }^{683}$ Narver/Slater 1990, S. 26. 
Tabelle 43: Vergleich der Bestimmtheitsmaße zwischen der MKTOR- und der coMOSkala

\begin{tabular}{|c|c|c|c|c|c|}
\hline & $\begin{array}{c}\text { MIMIC- } \\
\text { Modell }\end{array}$ & \multicolumn{4}{|c|}{ Strukturgleichungsmodell } \\
\hline $\mathbf{R}^{2}$ & MO & COLLABO & INNOVA & ALIGN & NPS \\
\hline MKTOR-Skala & 0,612 & 0,237 & 0,354 & 0,157 & 0,293 \\
\hline coMO-Skala & 0,706 & 0,242 & 0,366 & 0,269 & 0,293 \\
\hline
\end{tabular}

Dass das fünf-dimensionale Modell eine höhere Erklärungskraft besitzt, ist vor dem Hintergrund der größeren Anzahl von erklärenden Indikatoren der neu entwickelten coMO-Skala und vor dem Hintergrund des höheren Aggregationsniveaus der MKTOR-Skala nicht überraschend. Innerhalb des MIMIC-Modells gelingt es dem coMO-Messinstrument über $9 \%$ mehr Varianz von Marktorientierung (MO) zu erklären. Unternehmenstätigkeiten, die einen Bezug zur Produktentwicklung aufweisen wie Innovativität (INNOVA) und Unternehmenszusammenarbeit (COLLABO) können etwas besser erklärt werden. Das Bestimmtheitsmaß der Variable Anpassung der Marketingstrategien (ALIGN) steigt substanziell im coMO-Messmodell.

Angemerkt werden soll an dieser Stelle die von NARVER/SLATER an der eigenen Skala geäußerte Kritik. Demnach stellen die Autoren die Gleichgewichtung der einzelnen Komponenten von Marktorientierung in Frage. ${ }^{684}$ Ein Vergleich der drei-dimensionalen MKTOR-Messskala mit frei geschätzten Regressionsgewichten pro Dimension und der von NARVER/SLATER ursprünglich vorgeschlagenen Mittelwertbildung, bei der von einer Gleichgewichtung der Dimensionen ausgegangen wird, zeigt eine (wenn auch nur geringe) Verbesserung des Bestimmtheitsmaßes von Marktorientierung. Im MIMIC-Modell steigt das Bestimmtheitsmaß von $\mathrm{R}^{2}=0,612$ auf $\mathrm{R}^{2}=0,620$ bei freier Schätzung der Regressionsgewichte. Der Unterschied der erklärten Varianz von Marktorientierung im MIMIC-Modell zwischen der coMO-Skala und der MKTOR-Skala stammt somit zum großen Teil aus der Berücksichtigung zusätzlicher Marktaspekte in Form der zwei hinzugefügten Dimensionen. Der Unterschied des Bestimmtheitsmaßes

${ }^{684}$ Vgl. Narver/Slater 1990, S. 33 f. 
resultiert folglich zu geringeren Teilen aus der freien Schätzung der Regressionsgewichte.

\subsubsection{Gruppenvergleich der Messmodelle}

Ein Teilziel dieser Arbeit ist es, ein allgemeingültiges, über verschiedene Unternehmen und Produktkategorien valides Messinstrument zu entwickeln. Dazu soll in diesem Abschnitt untersucht werden, ob die entwickelte coMO-Messskala von Marktorientierung bei unterschiedlichen Produkt-, Markt- und Umweltbedingungen, sowie in verschiedenen Abteilungen eines Unternehmens ohne Anpassungen verwendet werden kann. Zudem soll überprüft werden, ob die verschiedenen Dimensionen von Marktorientierung auch unter den genannten unterschiedlichen Bedingungen eine ähnliche Bedeutung bei der Bildung von Marktorientierung aufweisen.

Zur Überprüfung kann ein Mehrgruppenvergleich mithilfe des PLS-Ansatzes durchgeführt werden. Zunächst muss dazu überprüft werden, ob die Messmodelle der einzelnen Dimensionen auch dieselben Sachverhalte in den jeweiligen Gruppen messen. ${ }^{65}$ Die Gruppen werden anhand von relevanten Merkmalen unterschieden. Kann aufgrund der Ergebnisse des Messmodellvergleichs darauf geschlossen werden, dass in den jeweiligen Gruppen mithilfe der Messmodelle auch dieselben Sachverhalte gemessen werden, wird von Messäquivalenz gesprochen. Messäquivalenz stellt eine Voraussetzung dar, um die Strukturbeziehungen zwischen den einzelnen latenten Variablen vergleichen zu können. Durch die Messäquivalenz wird erreicht, dass Differenzen in den Pfadkoeffizienten der Strukturbeziehungen nicht auf Unterschiede der Messungen zwischen den Gruppen zurückzuführen sind. Im vorliegenden Modell sollen die Richtung und Stärke der Pfadkoeffizienten der einzelnen Dimensionen bzw. deren Einfluss auf das Gesamtkonstrukt der umfassenden Marktorientierung gruppenweise verglichen werden. Anhand von Gütekriterien können verschiedene Niveaus von Messäquivalenz unterschieden werden. ${ }^{686}$ Grundsätzlich lässt sich je nach erreichten Güte-

${ }^{685}$ Vgl. Weiber/Mühlhaus 2010, S. 226.

${ }^{686}$ Vgl. Steenkamp/Baumgartner 1998. 
kriterien zwischen vollständiger und partieller Messäquivalenz unterscheiden. Da aber in der Realität äußerst selten vollständige Messäquivalenz vorliegt, wird die Existenz von partieller Messäquivalenz als akzeptabel für weitergehende Gruppenvergleiche von Strukturbeziehungen angesehen. Wann partielle Messäquivalenz vorliegt, ist nicht eindeutig definiert und insbesondere im Rahmen des PLSAnsatzes, der keine globalen Gütemaße verwendet, äußert fraglich. Grundsätzlich lässt sich aber sagen, dass von partieller Messäquivalenz ausgegangen werden kann, wenn pro Konstrukt nur ein kleiner Teil der diesem Konstrukt zugeordneten Indikatoren nicht messäquivalent ist. ${ }^{677}$ Bei der Ermittlung der Messäquivalenzniveaus werden in der Literatur zumeist gesamthafte Gütekriterien verwendet, die auf Analyseverfahren des Kovarianz-analytischen Ansatzes basieren. Im Rahmen des Varianz-analytischen PLS-Ansatzes können diese Kriterien aber nicht zur Anwendung kommen. Stattdessen wird dem Ansatz von CHIN gefolgt, anhand einer t-Wert-Statistik signifikante Unterschiede der Faktorladungen der einzelnen Indikatoren und der Pfadkoeffizienten zwischen den Gruppen zu betrachten. ${ }^{68}$ Dieses Vorgehen wird als parametrischer Test bezeichnet und geht von einer Normalverteilung der einzelnen Variablenausprägungen aus.

In Anhang 4 wird ersichtlich, dass alle betrachteten Items die univariate Normalverteilungsannahme erfüllen. Die multivariate Normalverteilungsannahme wird hingegen verletzt. Um dennoch aussagefähige Gruppenvergleiche durchführen zu können, wird in der Literatur empfohlen, nicht-parametrische Tests anzuwenden, die auf keiner Verteilungsannahme beruhen. ${ }^{699}$ HENSELER schlägt dazu einen Test vor, der auf Daten der Bootstrapping-Prozedur basiert. ${ }^{690}$ Für jede Gruppe werden 500 Stichproben gezogen und die interessierenden Parameter geschätzt. Jeder der 500 für die erste Gruppe geschätzte Parameter wird anschließend mit

\footnotetext{
${ }^{687}$ Vgl. Temme/Hildebrandt 2009, S. 155. BYRNE ET Al. (1989) argumentieren, dass nur zwei Items pro Konstrukt bzw. Dimension messäquivalent sein müssen. Nach STEENKAMP/BAUMGARTNER (1998) sollte die Mehrheit der Indikatoren messäquivalent sein. ${ }^{688}$ Vgl. Chin 2000. In der Literatur wird zur Bestimmung der t-Werte oftmals auch die Methode von KEIL ET AL. (2000, S. 312 ff.) vorgeschlagen. Diese Methode beruht in ihrer ursprünglichen Form auf dem Jackknifing-Verfahren, einer speziellen Variante des Bootstrapping-Verfahrens, die allerdings nicht durch die im Rahmen dieser Arbeit verwendete Software unterstützt wird.

${ }^{689}$ Vgl. Qureshi/Compeau 2009, S. 198; Chin/Dibbern 2010.

690 Vgl. Henseler 2007. Vgl. hierzu auch Henseler et al. 2009, S. 309.
} 
jedem der 500 geschätzten Parameter der zweiten Gruppe verglichen. Die Anzahl der Vergleiche, bei denen ein Parameter aus der ersten Gruppe den Parameter der zweiten Gruppe übersteigt, spiegelt die Wahrscheinlichkeit wider, dass der Parameter der ersten Gruppe signifikant größer ist als der Parameter der zweiten Gruppe. Die Wahrscheinlichkeit, dass ein Schätzparameter der einen Gruppe tatsächlich größer ist als der der Vergleichsgruppe wird anhand des P-Wertes ermittelt. Der P-Wert berücksichtigt den $\alpha$-Fehler, einen Parameterunterschied fälschlicherweise festzustellen, und sollte daher im Bereich zwischen 0,05 und 0,95 liegen. ${ }^{691}$

QURESHI/COMPEAU zeigen anhand einer Simulationsstudie, dass parametrische und nicht-parametrische Testverfahren sehr ähnliche Ergebnisse liefern. ${ }^{692}$ Im Rahmen der vorliegenden Arbeit wird daher zur Überprüfung der Messäquivalenz lediglich auf den parametrischen Test zurückgegriffen. Zur Überprüfung der Wirkbeziehungen der einzelnen Dimensionen sollen jedoch sowohl der parametrische als auch der nicht-parametrische Test zum Einsatz kommen. So soll sichergestellt werden, dass mögliche Gruppeneinflüsse auf die Wirkbeziehungen der einzelnen Dimensionen nicht auf eine Verletzung der multivariaten Normalverteilungsannahme zurückzuführen sind.

Um Kategorien bzw. Gruppen bilden und die Datensätze entsprechend ihrer Variablenausprägung zuordnen zu können, wird einem in der Literatur üblichen Verfahren gefolgt. Hierbei werden metrisch skalierte Variablen zur Gruppeneinteilung in Kategorial-Variablen umcodiert. Dazu werden die Beobachtungen in drei möglichst gleichgroße Gruppen unterteilt. Die Gruppenvariable mit dem Drittel der höchsten Ausprägungen wird als Wertkategorie „hoch“, das Drittel der niedrigsten Ausprägungen als Wertkategorie „niedrig“ bezeichnet. Das mittlere Drittel wird von der weiteren Untersuchung ausgeschlossen. Durch die Exklusion des mittleren Drittels wird erreicht, dass deutlich unterschiedliche Gruppen miteinan-

${ }^{691}$ Vgl. Henseler et al. 2009, S. 309. Daneben existieren noch andere Methoden im Rahmen des PLS-Ansatzes, um Gruppenvergleiche durchzuführen (z.B. Tenenhaus et al. 2010; Chin/Dibbern 2010). Multi-Group-Analysen werden aber anders als bei Kovarianz-analytischer Software noch nicht durch die verfügbare PLS-Software unterstützt und daher im Rahmen dieser Arbeit auch nicht weiter betrachtet (vgl. Temme et al. 2010, S. 738).

692 Vgl. Qureshi/Compeau 2009, S. 206. 
der verglichen und mögliche Gruppenunterschiede besser sichtbar werden. Durch diese Art der Umcodierung von Gruppenvariablen werden dichotome Kategorialvariablen gebildet, die leicht miteinander verglichen werden können. ${ }^{693}$

Im Zuge der vorliegenden Arbeit werden zur Einschätzung der Generalisierbarkeit der entwickelten Messskala Gruppen aus dem Gesamtdatensatz gebildet und miteinander verglichen. Die Gruppen werden anhand verschiedener Eigenschaften der von den Unternehmen vertriebenen Leistungen, anhand der Konstellation der Marktmacht, anhand der Position des Unternehmens in der Wertschöpfungskette, sowie anhand der Abteilungszugehörigkeit gebildet. Die Konstellation der Marktmacht und die Leistungseigenschaften können als Voraussetzungen für den erfolgreichen Einsatz von Mehrstufigem Marketing verstanden werden (vgl. Abschnitt 2.2.2.2). Tabelle 44 gibt die gebildeten Gruppen anhand der Gruppenvariable und die zugehörige Gruppengröße wider.

${ }^{693}$ HENSELER/FASSOTT (2010, S. 720) kritisieren dieses Vorgehen aufgrund des damit einhergehenden Verlusts von Variablenvarianz, des Ausschlusses von einem Drittel der Daten und der eher willkürlichen Gruppenzuordnung von Beobachtungen. 
Tabelle 44: Gruppenbildung auf Basis der jeweiligen Gruppenvariable

\begin{tabular}{|c|c|c|c|}
\hline Gruppenvariable & Variablenname & Ausprägung & Gruppengröße \\
\hline \multirow{2}{*}{ Req1 } & \multirow{2}{*}{$\begin{array}{l}\text { Identifizierbarkeit der Leistung } \\
\text { für indirekte Kunden }\end{array}$} & Hoch (h) & $\mathrm{n}=118$ \\
\hline & & Niedrig (n) & $\mathrm{n}=168$ \\
\hline \multirow[b]{2}{*}{ Req2 } & \multirow{2}{*}{$\begin{array}{l}\text { Relevanz der Leistung beim } \\
\text { direkten Kunden }\end{array}$} & Hoch (h) & $\mathrm{n}=151$ \\
\hline & & Niedrig (n) & $\mathrm{n}=175$ \\
\hline \multirow[b]{2}{*}{ Req3 } & \multirow{2}{*}{$\begin{array}{l}\text { Technologisches Anspruchsni- } \\
\text { veau der Leistung }\end{array}$} & Hoch (h) & $\mathrm{n}=142$ \\
\hline & & & \\
\hline & & & \\
\hline \multirow[b]{2}{*}{ Req4 } & \multirow{2}{*}{$\begin{array}{l}\text { Alleinstellungsmerkmal der } \\
\text { Leistung }\end{array}$} & Hoch (h) & $\mathrm{n}=147$ \\
\hline & & Niedrig (n) & $\mathrm{n}=149$ \\
\hline \multirow[b]{2}{*}{ BuyPower } & \multirow[b]{2}{*}{ Marktmacht des Kunden } & Hoch (h) & $\mathrm{n}=174$ \\
\hline & & Niedrig (n) & $\mathrm{n}=138$ \\
\hline \multirow{3}{*}{ MaStage ${ }^{694}$} & \multirow{3}{*}{ Marktstufen zum Letztverwender } & 1 Zwischenstufe & $\mathrm{n}=140(58)$ \\
\hline & & $>2$ Zwischenstufen & $\mathrm{n}=58$ \\
\hline & & Keine Zwischenstufe & $\mathrm{n}=58$ \\
\hline \multirow{2}{*}{ Position } & \multirow{2}{*}{ Abteilung } & Vertrieb & $\mathrm{n}=94$ \\
\hline & & Marketing (u. a.) & $n=94$ \\
\hline
\end{tabular}

Die Ergebnisse des Messäquivalenz-Tests hinsichtlich der Gruppenunterteilung nach Produkteigenschaft sind in Anhang 5 aufgeführt. Es zeigt sich, dass für die globale Messung des Konstrukts Marktorientierung keine Messäquivalenz für den Gruppenvergleich zwischen hoher und niedriger Ausprägung der Variable Req1 (Identifizierbarkeit der Leistung für indirekte Kunden) vorliegt. Alle vier zur Messung herangezogenen Items unterscheiden sich zwischen den Gruppen signifikant. Ebenso erreicht nur ein Item der Dimension FUNCO bei diesem Gruppenvergleich Messäquivalenz, so dass die gesamte Dimension als nicht

${ }^{694}$ Voraussetzungen, um valide Ergebnisse bei Gruppenvergleichen zu erhalten, sind hinreichend große Stichproben und annähernd gleichgroße Gruppen. Dazu werden für die Vergleiche mit den Gruppen „Marktstufen zum Letztverwender“ mit der Ausprägung „> 2 Zwischenstufen“ und „Keine Zwischenstufe“ Zufallsstichproben von n = 58 aus der Grundgesamtheit der deutlich größeren anderen Gruppe („1 Zwischenstufe“) gezogen. Durch dieses Vorgehen werden für den Vergleich gleichgroße Stichproben gebildet. Anzumerken ist hierbei, dass die Power-Analyse ergeben hat, dass ein 
messäquivalent einzustufen ist. Als Folge sollten weitergehende Gruppenvergleiche bezüglich der Wirkbeziehungen der Dimensionen mit Vorsicht interpretiert werden. Die Übrigen Dimensionen CUSTOR, COMPOR, PARTOR und MULTOR erfüllen die Kriterien der Messäquivalenz.

Der Gruppenvergleich hinsichtlich einer hohen und niedrigen Ausprägung der Variable Req2 (Relevanz der Leistung beim direkten Kunden) zeigt wiederum, dass Messäquivalenz für die globale Konstruktmessung von Marktorientierung nur bedingt gegeben ist. Lediglich ein Indikator kann als messäquivalent bezeichnet werden. Gleiches gilt bei diesem Gruppenvergleich für die Dimension PARTOR. Hier ist ebenfalls nur ein Indikator messäquivalent. Die übrigen Dimensionen CUSTOR, COMPOR, FUNCO und MULTOR erfüllen die Kriterien der Messäquivalenz.

Messäquivalenz kann den Gruppen mit hoher und niedriger Ausprägung der Variable Req3 (Technologisches Anspruchsniveau der Leistung) bescheinigt werden. Alle Dimensionen und die globale Messung von Marktorientierung erfüllen die Kriterien der Messäquivalenz und können daher problemlos für weitergehende Gruppenvergleiche herangezogen werden.

Der Gruppenvergleich hinsichtlich der Variable Req4 (Alleinstellungsmerkmal der Leistung) zeigt, dass die Messung des globalen Konstrukts der Marktorientierung nur bedingt messäquivalent ist. Lediglich ein Indikator weist zwischen der Gruppe mit hoher und der Gruppe mit niedriger Ausprägung Messäquivalenz auf. Die fünf Subdimensionen des Konstrukts hingegen erfüllen alle die geforderten Kriterien der Messäquivalenz.

Den Gruppen mit hoher und niedriger Ausprägung der Variable BuyPower (Marktmacht der Kunden) kann Messäquivalenz bescheinigt werden. Alle Dimensionen und die globale Messung von Marktorientierung erfüllen die Kriterien der Messäquivalenz und können daher problemlos für weitergehende Gruppenvergleiche herangezogen werden.

Es bleibt festzuhalten, dass nur für die Gruppenvariablen Req3 und BuyPower in

Mindeststichprobenumfang von $n=81$ erforderlich ist (vgl. Abschnitt 5.3.2). Mit der kleinsten Stichprobengröße von $\mathrm{n}=58$ kann aber immer noch eine Power von 0,66 erreicht werden. 
allen Dimensionen und im Globalkonstrukt partielle Messäquivalenz bestätigt werden kann. Dennoch sollen im Weiteren auch die Gruppenvergleiche bezüglich der Variablen Req1, Req2 und Req4 hinsichtlich der Wirkbeziehungen der Dimensionen durchgeführt werden. Dieses Vorgehen wird als akzeptabel angesehen, da von den insgesamt sechs Sub-Skalen, die pro Vergleich herangezogen werden, lediglich zwei Skalen jeweils als nicht messäquivalent zu bezeichnen sind. In einem Fall (Req4) besteht sogar nur für eine Sub-Skala keine Messäquivalenz. Es sei aber darauf hingewiesen, dass für die Gruppenvergleiche, in denen nicht alle Sub-Skalen als messäquivalent einzustufen sind, die Ergebnisse der Wirkbeziehungen der Dimensionen nur bedingt aussagefähig sind.

Tabelle 45 zeigt die Ergebnisse der Gruppenvergleiche hinsichtlich der Wirkbeziehungen der einzelnen Dimensionen. Die Vergleiche wurden mittels parametrischem und nicht-parametrischem Test für die Gruppenvariablen der Leistungseigenschaften Req1, Req2, Req3 und Req4 sowie für die Gruppenvariable der Konstellation der Marktmacht BuyPower durchgeführt. 
Tabelle 45: Ergebnisse der Prüfung des coMO-Messmodells auf Unterschiede hinsichtlich der Voraussetzungen des Mehrstufigen Marketings

\begin{tabular}{|c|c|c|c|c|c|c|c|c|c|c|c|}
\hline \multirow[b]{2}{*}{$\begin{array}{c}\text { Struktur- } \\
\text { beziehung }\end{array}$} & \multirow{2}{*}{$\begin{array}{l}\begin{array}{c}\text { Gruppen- } \\
\text { variable }\end{array} \\
\text { Ausprägung }\end{array}$} & \multicolumn{2}{|c|}{ req1 } & \multicolumn{2}{|c|}{ req2 } & \multicolumn{2}{|c|}{ req3 } & \multicolumn{2}{|c|}{ req4 } & \multicolumn{2}{|c|}{ BuyPower } \\
\hline & & $\mathrm{n}$ & $\mathrm{h}$ & $\mathrm{n}$ & $\mathrm{h}$ & $\mathrm{n}$ & $\mathrm{h}$ & $\mathrm{n}$ & $\mathrm{h}$ & $\mathrm{n}$ & h \\
\hline \multirow{4}{*}{$\begin{array}{c}\text { CUSTOR } \\
\rightarrow \mathrm{MO}\end{array}$} & Pfadkoeffizient & 0,309 & 0,328 & 0,273 & 0,314 & 0,390 & 0,344 & 0,351 & 0,171 & 0,295 & 0,390 \\
\hline & t-Wert & \multicolumn{2}{|c|}{$-0,156$} & \multicolumn{2}{|c|}{$-0,403$} & \multicolumn{2}{|c|}{0,500} & \multicolumn{2}{|c|}{1,640} & \multicolumn{2}{|c|}{$-1,205$} \\
\hline & Sig.-Niveau & \multicolumn{2}{|c|}{ n.s. } & \multicolumn{2}{|c|}{ n.s. } & \multicolumn{2}{|c|}{ n.s. } & \multicolumn{2}{|c|}{ n.s. } & \multicolumn{2}{|c|}{ n.s. } \\
\hline & $\begin{array}{l}\text { P-Wert des } \\
\text { nicht- } \\
\text { parametrischen } \\
\text { Tests }\end{array}$ & \multicolumn{2}{|c|}{0,510} & \multicolumn{2}{|c|}{0,626} & \multicolumn{2}{|c|}{0,410} & \multicolumn{2}{|c|}{0,038} & \multicolumn{2}{|c|}{0,503} \\
\hline \multirow{4}{*}{$\begin{array}{c}\text { COMPOR } \\
\rightarrow \mathrm{MO}\end{array}$} & Pfadkoeffizient & 0,155 & 0,008 & 0,063 & 0,193 & 0,080 & 0,093 & 0,137 & 0,099 & 0,135 & $-0,02$ \\
\hline & t-Wert & \multicolumn{2}{|c|}{1,402} & \multicolumn{2}{|c|}{$-1,398$} & \multicolumn{2}{|c|}{$-0,134$} & \multicolumn{2}{|c|}{0,399} & \multicolumn{2}{|c|}{1,738} \\
\hline & Sig.-Niveau & \multicolumn{2}{|c|}{ n.s. } & \multicolumn{2}{|c|}{ n.s. } & \multicolumn{2}{|c|}{ n.s. } & & & & \\
\hline & $\begin{array}{l}\text { P-Wert des } \\
\text { nicht- } \\
\text { parametrischen } \\
\text { Tests }\end{array}$ & & & & & & & & & & \\
\hline & Pfadkoeffizient & 0,224 & 0,342 & 0,237 & 0,200 & 0,291 & 0,279 & 0,330 & 0,212 & 0,323 & 0,206 \\
\hline & t-Wert & & & & & & & & & & 95 \\
\hline PARTOR & Sig.-Niveau & & & & & & & & & & \\
\hline$\rightarrow \mathrm{MO}$ & $\begin{array}{l}\text { P-Wert des } \\
\text { nicht- } \\
\text { parametrischen } \\
\text { Tests }\end{array}$ & & & & & & & & & & \\
\hline & Pfadkoeffizient & 0,174 & 0,146 & 0,217 & 0,212 & 0,200 & 0,237 & 0,196 & 0,279 & 0,223 & 0,270 \\
\hline & t-Wert & & & & & & & & & & 194 \\
\hline MULTOR & Sig.-Niveau & & & & & & & & & & \\
\hline$\rightarrow \mathrm{MO}$ & $\begin{array}{l}\text { P-Wert des } \\
\text { nicht- } \\
\text { parametrischen } \\
\text { Tests }\end{array}$ & & 48 & & 83 & & 12 & & & & \\
\hline & Pfadkoeffizient & 0,173 & 0,254 & 0,252 & 0,148 & 0,133 & 0,117 & 0,093 & 0,237 & 0,103 & 0,222 \\
\hline & t-Wert & & 752 & & 39 & & 70 & & 414 & & \\
\hline FUNCO & Sig.-Niveau & & s. & & s. & & s. & & s. & & \\
\hline$\rightarrow \mathrm{MO}$ & $\begin{array}{l}\text { P-Wert des } \\
\text { nicht- } \\
\text { parametrischen } \\
\text { Tests }\end{array}$ & & 95 & & 54 & & 13 & & 08 & & 14 \\
\hline
\end{tabular}

Die Analyse mit Hilfe des parametrischen Tests zeigt lediglich für die Gruppenvariable BuyPower schwach signifikant unterschiedliche Einflüsse der Dimensi- 
on Wettbewerbsorientierung auf das Konstrukt der Marktorientierung. Dieser Unterschied der Pfadladungen wird auch durch den nicht-parametrischen Test bestätigt. Ferner deutet der nicht-parametrische Test für die Gruppenvariable Req1 darauf hin, dass die Wettbewerbsorientierung bei Unternehmen, die Produkte vertreiben, die weniger leicht für den indirekten Kunden identifizierbar sind, einen signifikant stärkeren Einfluss auf die Marktorientierung besitzt. Eine Interpretation dieses Ergebnisses bleibt vor dem Hintergrund der unzureichenden partiellen Messäquivalenz aus. Der nicht-parametrische Test zeigt zudem für die Gruppenvariable Req4, dass bei Unternehmen mit leicht zu substituierenden Produkten die Kundenorientierung signifikant stärker zum Konstrukt der Marktorientierung beiträgt. Dies ist insofern auch nicht verwunderlich, da gerade bei eher dem Commodity-Bereich zuordenbaren Produkten eine stärkere Kundenfokussierung erforderlich erscheint, um weiterhin als Lieferant bestehen zu bleiben. Ist hingegen das Produkt nur schwer zu ersetzen oder besteht gar eine monopolartige Stellung des Lieferanten, ist der Einfluss der Kundenorientierung signifikant geringer ausgeprägt.

Die Ergebnisse der Überprüfung auf Messäquivalenz für die Gruppen, die anhand ihrer Position in der Wertschöpfungskette gebildet werden, sind in Anhang 6 aufgeführt. Es zeigt sich, dass zwischen allen Gruppen für jede Dimension und für das Globalkonstrukt der Marktorientierung von partieller Messäquivalenz ausgegangen werden kann. Trotz der unterschiedlichen Position in der Wertschöpfungskette weisen die befragten Unternehmen ein weitgehend einheitliches Verständnis der Items auf. Die Ergebnisse sind als äußerst zufriedenstellend zu bewerten, so dass in einer weiteren Analyse der Einfluss der einzelnen Dimensionen auf das Konstrukt der Marktorientierung untersucht werden kann. Dafür werden alle Gruppen einem paarweisen Vergleich unterzogen, dessen Ergebnisse in Tabelle 46 dargestellt sind. 
Tabelle 46: Ergebnisse der Prüfung des coMO-Messmodells auf Unterschiede hinsichtlich der Position in der Wertschöpfungskette

\begin{tabular}{|c|c|c|c|c|c|c|c|}
\hline & $\begin{array}{l}\text { Variable der Grup- } \\
\text { penaufteilung }\end{array}$ & \multicolumn{2}{|c|}{ MaStage } & \multicolumn{2}{|c|}{ MaStage } & \multicolumn{2}{|c|}{ MaStage } \\
\hline 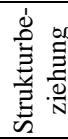 & Ausprägung & $\begin{array}{c}1 \text { Zwi- } \\
\text { schen- } \\
\text { stufe }\end{array}$ & $\begin{array}{c}>2 \\
\text { Zwi- } \\
\text { schenstu- } \\
\text { fen }\end{array}$ & $\begin{array}{c}\text { Keine } \\
\text { Zwi- } \\
\text { schenstu- } \\
\text { fe }\end{array}$ & $\begin{array}{c}1 \text { Zwi- } \\
\text { schenstu- } \\
\text { fe }\end{array}$ & $\begin{array}{l}\text { Keine } \\
\text { Zwischen- } \\
\text { stufe }\end{array}$ & $\begin{array}{c}>2 \text { Zwi- } \\
\text { schenstu- } \\
\text { fen }\end{array}$ \\
\hline \multirow{4}{*}{ 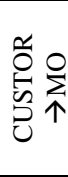 } & Pfadkoeffizient & 0,132 & 0,390 & 0,361 & 0,132 & 0,361 & 0,390 \\
\hline & t-Wert & \multicolumn{2}{|c|}{$-1,905$} & \multicolumn{2}{|c|}{1,575} & \multicolumn{2}{|c|}{$-0,217$} \\
\hline & Sig.-Niveau & \multicolumn{2}{|c|}{$*$} & \multicolumn{2}{|c|}{ n.s. } & \multicolumn{2}{|c|}{ n.s. } \\
\hline & $\begin{array}{c}\text { P-Wert des nicht- } \\
\text { parametrischen Tests }\end{array}$ & \multicolumn{2}{|c|}{0,935} & \multicolumn{2}{|c|}{0,091} & \multicolumn{2}{|c|}{0,555} \\
\hline \multirow{4}{*}{$\begin{array}{l}n_{0}^{\prime} \\
\sum_{0}^{\circ} \sum^{\circ}\end{array}$} & Pfadkoeffizient & $-0,014$ & 0,162 & 0,152 & $-0,014$ & 0,152 & 0,162 \\
\hline & t-Wert & \multicolumn{2}{|c|}{$-1,384$} & \multicolumn{2}{|c|}{1,265} & \multicolumn{2}{|c|}{$-0,079$} \\
\hline & Sig.-Niveau & \multicolumn{2}{|c|}{ n.s. } & \multicolumn{2}{|c|}{ n.s. } & \multicolumn{2}{|c|}{ n.s. } \\
\hline & $\begin{array}{c}\text { P-Wert des nicht- } \\
\text { parametrischen Tests }\end{array}$ & \multicolumn{2}{|c|}{0,976} & \multicolumn{2}{|c|}{0,031} & \multicolumn{2}{|c|}{0,539} \\
\hline \multirow{4}{*}{ 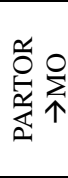 } & Pfadkoeffizient & 0,253 & 0,210 & 0,280 & 0,253 & 0,280 & 0,210 \\
\hline & t-Wert & \multicolumn{2}{|c|}{0,334} & \multicolumn{2}{|c|}{0,186} & \multicolumn{2}{|c|}{0,484} \\
\hline & Sig.-Niveau & \multicolumn{2}{|c|}{ n.s. } & \multicolumn{2}{|c|}{ n.s. } & \multicolumn{2}{|c|}{ n.s. } \\
\hline & $\begin{array}{c}\text { P-Wert des nicht- } \\
\text { parametrischen Tests }\end{array}$ & \multicolumn{2}{|c|}{0,367} & \multicolumn{2}{|c|}{0,444} & \multicolumn{2}{|c|}{0,311} \\
\hline \multirow{4}{*}{ 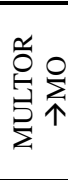 } & Pfadkoeffizient & 0,374 & 0,204 & 0,184 & 0,374 & 0,184 & 0,204 \\
\hline & t-Wert & & & & 304 & & \\
\hline & Sig.-Niveau & & & & & & \\
\hline & $\begin{array}{c}\text { P-Wert des nicht- } \\
\text { parametrischen Tests }\end{array}$ & & & & & & \\
\hline$\stackrel{2}{2}$ & Pfadkoeffizient & 0,335 & 0,116 & 0,178 & 0,335 & 0,178 & 0,116 \\
\hline 个 & $\mathrm{t}$-Wert & & & & 22 & & \\
\hline 0 & Sig.-Niveau & & & & & & \\
\hline 只 & $\begin{array}{c}\text { P-Wert des nicht- } \\
\text { parametrischen Tests }\end{array}$ & & & & & & \\
\hline & Signifikanzniveau: & & $; * *: \mathrm{p}$ & $5 . *$. & $1 \cdot n$ & & \\
\hline
\end{tabular}

Der Gruppenvergleich zeigt, dass lediglich der $\mathrm{t}$-Wert $(\mathrm{t}=\mathrm{-1,905)}$ der Dimension Kundenorientierung zwischen den Gruppen „1 Zwischenstufe,, und „>2 Zwischenstufen“ auf leicht signifikante Unterschiede der Pfadkoeffizienten hindeutet. Eine Überprüfung der parametrischen Testergebnisse mit Hilfe des nichtparametrischen Tests deutet hierbei allerdings auf keinen signifikanten Unterschied hin. Dagegen stellt der nicht-parametrische Test einen Unterschied der Pfadkoeffizienten dieser beiden Gruppen hinsichtlich der Dimension Wettbe- 
werbsorientierung fest. Ferner zeigt sich durch den nicht-parametrischen Test ein Unterschied der Pfadkoeffizienten der Dimension Wettbewerbsorientierung zwischen den Gruppen „Keine Zwischenstufe“ und "1 Zwischenstufe“. Dies führt zu dem Schluss, dass sich Unternehmen, in deren Wertschöpfungskette nur eine Zwischenstufe zum Letztverwender existiert, hinsichtlich ihrer Einschätzung der Wettbewerbsorientierung von den übrigen Gruppen unterscheiden. Unterschiede bei der Gewichtung der einzelnen Dimensionen zwischen den Gruppen „Keine Zwischenstufe“ und „>2 Zwischenstufen“ können weder durch den parametrischen noch den nicht-parametrischen Test festgestellt werden.

Ein weiterer Gruppenvergleich soll zwischen den Abteilungen Vertrieb und Marketing erfolgen. Ein Gruppenvergleich zwischen allen Probanden der vorliegenden Studie, die sich dem Vertrieb und allen, die sich dem Marketing zugehörig sehen, ist allerdings aufgrund der Möglichkeit der mehrfachen Abteilungsnennung nicht direkt möglich. Außerdem besteht zwischen den Gruppen ein deutlicher Größenunterschied. Für den Gruppenvergleich zwischen Vertriebs- und Marketingabteilung werden daher für die Gruppe der Marketingpraktiker auch solche Datensätze berücksichtigt, die neben der Nennung der Marketingabteilung noch eine zusätzliche Abteilung beinhalten. ${ }^{695}$ Aus der Gruppe der Vertriebler wird eine Zufallsstichprobe gezogen, so dass sich letztlich zwei gleichgroße Gruppen ergeben.

Die Ergebnisse der Überprüfung auf Messäquivalenz für die Gruppen, die anhand ihrer Abteilungszugehörigkeit im Unternehmen gebildet wurden, deuten auf Messäquivalenz in jeder Dimension und in der globalen Konstruktmessung hin. Die verwendeten Items scheinen somit von Vertrieblern und Marketingpraktikern überwiegend identisch aufgefasst zu werden. Anhang 7 zeigt das Ergebnis der Prüfung auf Messäquivalenz zwischen den beiden Gruppen.

Die Ergebnisse des Gruppenvergleichs hinsichtlich der Wirkbeziehungen der einzelnen Dimensionen zeigt Tabelle 47.

${ }^{695}$ Dies betrifft vor allem Mehrfachnennungen von Marketing und Produktmanagement. Die Mehrfachnennung von Marketing und Vertrieb wurde vom Gruppenvergleich ausgeschlossen. 
Tabelle 47: Ergebnisse der Prüfung des coMO-Messmodells auf Unterschiede hinsichtlich der Abteilungszugehörigkeit

\begin{tabular}{|c|c|c|c|}
\hline & $\begin{array}{l}\text { Variable der Gruppenauftei- } \\
\text { lung }\end{array}$ & \multicolumn{2}{|c|}{ Abteilung } \\
\hline Strukturbeziehung & Ausprägung & Vertrieb & Marketing \\
\hline \multirow{4}{*}{ CUSTOR $\rightarrow$ MO } & Pfadkoeffizient & 0,326 & 0,084 \\
\hline & t-Wert & \multicolumn{2}{|c|}{1,389} \\
\hline & Sig.-Niveau & \multicolumn{2}{|c|}{ n.s. } \\
\hline & $\begin{array}{c}\text { P-Wert des nicht- } \\
\text { parametrischen Tests }\end{array}$ & \multicolumn{2}{|c|}{0,042} \\
\hline \multirow{4}{*}{$\mathrm{COMPOR} \rightarrow \mathrm{MO}$} & Pfadkoeffizient & 0,058 & 0,303 \\
\hline & $\mathrm{t}$-Wert & \multicolumn{2}{|c|}{$-2,010$} \\
\hline & Sig.-Niveau & \multicolumn{2}{|c|}{$* *$} \\
\hline & $\begin{array}{l}\text { P-Wert des nicht- } \\
\text { parametrischen Tests }\end{array}$ & \multicolumn{2}{|c|}{0,984} \\
\hline \multirow{4}{*}{ PARTOR $\rightarrow$ MO } & Pfadkoeffizient & 0,261 & 0,353 \\
\hline & $\mathrm{t}$-Wert & \multicolumn{2}{|c|}{$-0,839$} \\
\hline & Sig.-Niveau & \multicolumn{2}{|c|}{ n.s. } \\
\hline & $\begin{array}{l}\text { P-Wert des nicht- } \\
\text { parametrischen Tests }\end{array}$ & \multicolumn{2}{|c|}{0,784} \\
\hline \multirow{4}{*}{ MULTOR $\rightarrow$ MO } & Pfadkoeffizient & 0,256 & 0,097 \\
\hline & t-Wert & \multicolumn{2}{|c|}{1,557} \\
\hline & Sig.-Niveau & \multicolumn{2}{|c|}{ n.s. } \\
\hline & $\begin{array}{c}\text { P-Wert des nicht- } \\
\text { parametrischen Tests }\end{array}$ & \multicolumn{2}{|c|}{0,155} \\
\hline \multirow{4}{*}{$\mathrm{FUNCO} \rightarrow \mathrm{MO}$} & Pfadkoeffizient & 0,197 & 0,101 \\
\hline & t-Wert & \multicolumn{2}{|c|}{0,765} \\
\hline & Sig.-Niveau & \multicolumn{2}{|c|}{ n.s. } \\
\hline & $\begin{array}{l}\text { P-Wert des nicht- } \\
\text { parametrischen Tests }\end{array}$ & \multicolumn{2}{|c|}{0,283} \\
\hline
\end{tabular}

Die Vergleiche wurden mittels parametrischem und nicht-parametrischem Test für die Gruppenvariable Abteilung durchgeführt. Für den Pfadkoeffizienten der Kundenorientierung ergibt sich dabei ein signifikanter Unterschied anhand des nichtparametrischen Tests. Demzufolge geben Vertriebsabteilungen der Kundenorientierung ein größeres Gewicht bei der Bildung des Konstrukts der umfassenden Marktorientierung. Die Wettbewerbsorientierung weist ebenfalls signifikante Unterschiede hinsichtlich ihrer Relevanz für das Marktorientierungskonstrukt auf. Beide Tests zeigen, dass Marketingpraktiker der Wettbewerbsorientierung 
eine größere Relevanz beimessen als Vertriebler. Für die übrigen Dimensionen können zwar keine signifikanten Unterschiede festgestellt werden, doch deuten die Ergebnisse darauf hin, dass auch die indirekten Kunden stärkere Beachtung in Vertriebsabteilungen finden. Der Fokus auf Drittparteien liegt in Marketingabteilungen höher.

Für weitergehende Forschungsarbeiten zeigt Anhang 10 die relative Häufigkeit, die ein Item in den insgesamt 9 Tests auf Messäquivalenz als nicht messäquivalent einzustufen war. Anhand der Tabelle kann abgelesen werden, welche Items sich besonders gut für Gruppenvergleiche eignen und welche - sollte es mit dem Inhalt der jeweiligen Dimension und dem Ziel der Untersuchung vereinbar sein aus der Skala gestrichen werden können. ${ }^{696}$

\subsubsection{Gruppenvergleiche im Strukturmodell}

Die im Modell aufgestellte Hypothese H6, nach der die Anpassung der Marketingstrategien (ALIGN) einen positiven Einfluss auf den Markterfolg neuer Produkte (NPS) haben soll, konnte anhand des vorliegenden Gesamtdatensatzes nicht bestätigt werden. Ebenfalls musste die Hypothese H9, die eine Mediation zwischen Marktorientierung und Neuprodukterfolg über den Mediator Anpassung der Marketingstrategien postuliert, verworfen werden. Die Anpassung der Marketingstrategien ist im Wesentlichen mit den Unternehmensaktivitäten gleichzusetzen, die dem Mehrstufigen Marketing zugerechnet werden. In diesem Abschnitt soll daher überprüft werden, inwiefern die Erfüllung der für ein erfolgreiches Mehrstufiges Marketing theoretisch notwendigen Voraussetzungen einen Einfluss auf diese ursprünglich postulierten Wirkbeziehungen hat.

Dazu soll im Folgenden ein Gruppenvergleich für die Gruppenvariablen Req1 (Identifizierbarkeit der Leistung für indirekte Kunden), Req2 (Relevanz der Leistung beim direkten Kunden), Req3 (Technologisches Anspruchsniveau der Leistung), Req4 (Alleinstellungsmerkmal der Leistung) sowie BuyPower (Marktmacht des Kunden) durchgeführt werden. Vor der Überprüfung der Wirkbezie-

${ }^{696}$ Vgl. zu diesem Vorgehen die Empfehlungen von Temme/Hildebrandt 2009, S. 155 ff.; Cheung/Rensvold 1999, S. 18. 
hung zwischen der Anpassung der Marketingstrategie und dem Neuprodukterfolg wird eine Überprüfung der Messäquivalenz der betreffenden Konstruktmessungen durchgeführt. Die Ergebnisse werden in Anhang 8 dargestellt und zeigen, dass bezüglich des Gruppenvergleichs von Req1 die Skala der Anpassung der Marketingstrategie und bezüglich des Gruppenvergleichs von Req2 die Skala des Neuprodukterfolgs nicht als messäquivalent bezeichnet werden kann. Es kann somit nicht vollständig ausgeschlossen werden, dass mögliche Unterschiede der Wirkbeziehungen zwischen den Gruppen auf ein uneinheitliches Verständnis der Skalen zurückzuführen sind. Bei den Gruppenvariablen Req3, Req4 und BuyPower kann auf partielle Messäquivalenz geschlossen werden.

Bei der Überprüfung der Wirkbeziehung stehen die Höhe und Signifikanz der Pfadkoeffizienten zwischen ALIGN und NPS im Fokus der Betrachtung. Die Ergebnisse der Gruppenvergleiche zeigen, dass sich die Wirkbeziehung zwischen der Anpassung der Marketingstrategien und dem Neuprodukterfolg hinsichtlich aller Gruppenvariablen positiv erhöht, sofern die Voraussetzungen des Mehrstufigen Marketings als erfüllt angesehen werden können. Im Detail sind die Ergebnisse der Gruppenvergleiche der Wirkbeziehung von ALIGN in Tabelle 48 dargestellt.

Tabelle 48: Gruppenvergleich der Wirkbeziehung ALIGN $\rightarrow$ NPS

\begin{tabular}{|c|c|c|c|c|c|}
\hline Gruppenvariable & Ausprägung & $\begin{array}{l}\text { Pfadkoeffizient } \\
\text { (Sig.-Niveau) }\end{array}$ & t-Wert & $\begin{array}{l}\text { Signifikanz- } \\
\quad \text { Niveau }\end{array}$ & $\begin{array}{c}\text { P-Wert des nicht- } \\
\text { parametrischen } \\
\text { Tests } \\
\end{array}$ \\
\hline \multirow{2}{*}{ Req1 } & $\mathrm{n}$ & $-0,044$ (n.s.) & \multirow{2}{*}{2,842} & \multirow{2}{*}{$* * *$} & \multirow{2}{*}{0,000} \\
\hline & $\mathrm{h}$ & $0,165(* * *)$ & & & \\
\hline \multirow{2}{*}{ Req2 } & $\mathrm{n}$ & 0,062 (n.s.) & \multirow{2}{*}{1,170} & \multirow{2}{*}{ n.s. } & \multirow{2}{*}{0,109} \\
\hline & $\mathrm{h}$ & $0,139(* * *)$ & & & \\
\hline \multirow{2}{*}{ Req3 } & $\mathrm{n}$ & $-0,058$ (n.s.) & \multirow{2}{*}{2,573} & \multirow{2}{*}{$* *$} & \multirow{2}{*}{0,000} \\
\hline & $\mathrm{h}$ & $0,128(* *)$ & & & \\
\hline \multirow{2}{*}{ Req4 } & $\mathrm{n}$ & 0,065 (n.s.) & \multirow{2}{*}{0,125} & \multirow{2}{*}{ n.s. } & \multirow{2}{*}{0,453} \\
\hline & $\mathrm{h}$ & 0,075 (n.s.) & & & \\
\hline \multirow{2}{*}{ BuyPower } & $\mathrm{n}$ & $0,108(* *)$ & \multirow{2}{*}{$-1,530$} & \multirow{2}{*}{ n.s. } & \multirow{2}{*}{0,969} \\
\hline & $\mathrm{h}$ & 0,001 (n.s.) & & & \\
\hline \multicolumn{6}{|c|}{ Signifikanzniveau: ${ }^{* * *}: \mathrm{p}<0,01 ; * *: \mathrm{p}<0,05 ; *$ : $\mathrm{p}<0,1 ;$ n.s.: nicht signifikant } \\
\hline
\end{tabular}

Bei hohen Ausprägungen der Variable Req1, wenn also die Leistungen beim 
indirekten Kunden identifizierbar bleiben, ändert der Pfadkoeffizient sein Vorzeichen von negativ auf positiv und wird auch hoch signifikant. Sowohl der parametrische als auch der nicht-parametrische Test bestätigen den Unterschied zwischen den Gruppen als signifikant.

Ähnliches gilt für die Variable Req2. Bei Leistungen, die für den direkten Kunden nicht zuletzt preislich bei der eigenen Leistungserstellung eine große Relevanz besitzen, zeigt sich ebenfalls eine deutliche Veränderung der Pfadkoeffizienten. Einen signifikanten Unterschied zwischen den Gruppen vermag der parametrische Test allerdings nicht festzustellen. Auch der nicht-parametrische Test liegt mit einem P-Wert von 0,109 etwas oberhalb des geforderten Wertes von 0,05.

Gruppenunterschiede auf signifikantem Niveau können auch zwischen Unternehmen mit hohen und niedrigen Ausprägungen der Variable Req3 festgestellt werden. So ändert sich die Wirkbeziehung von schwach negativ und nichtsignifikant nach signifikant positiv, wenn es sich um einen Anbieter von technologisch anspruchsvollen Leistungen handelt. Der parametrische und der nichtparametrische Test stützen die Ergebnisse dieses Gruppenunterschieds.

Signifikante Gruppenunterschiede für den Pfad der Anpassung der Marketingstrategie und Neuprodukterfolg können hinsichtlich der Gruppenvariable Req4 nicht festgestellt werden. Der Pfadkoeffizient erhöht sich zwar leicht, doch bleiben in beiden Gruppen die jeweiligen Pfade nicht-signifikant. Die Substituierbarkeit bzw. ein Alleinstellungsmerkmal einer Leistung scheinen somit keinen Einfluss auf den Erfolg einer mehrstufigen Marketingstrategie zu haben.

Letztlich macht der Gruppenvergleich noch deutlich, dass die Marktmacht des Kunden die Wirkung der Anpassung der Marketingstrategien beeinflussen kann. Liegt die Macht in einer Geschäftsbeziehung eher beim Kunden, so nimmt die Pfadbeziehung einen Wert nahe null an. Ist hingegen der Anbieter in einer mächtigeren Position, so wird der Pfadkoeffizient signifikant positiv. Der t-Wert des parametrischen Tests liegt knapp unterhalb des kritischen Signifikanzniveaus, um Gruppenunterschiede festzustellen. Die Ergebnisse des nicht-parametrischen Tests lassen jedoch auf existierende Unterschiede schließen.

Der in Abschnitt 5.5.2 vorgenommene Test auf Mediation ergibt für den Gesamt- 
datensatz, dass die Anpassung der Marketingstrategien keinen Mediator zwischen der Marktorientierung und dem Neuprodukterfolg darstellt. Eine Bedingung des Sobel-Tests auf Mediation ist das Vorliegen von signifikanten Konstruktbeziehungen. Da die betreffenden Pfade zwischen der Anpassung der Marketingstrategie und dem Neuprodukterfolg bei Erfüllung der Voraussetzungen des Mehrstufigen Marketings mit Ausnahme von Req4 signifikant werden (vgl. Tabelle 48), erscheint für die betreffenden Gruppen eine erneute Prüfung auf Mediation geboten. Der Test auf Mediation soll daher noch einmal für die Gruppen mit hoher Ausprägung der Variablen Req1, Req2 und Req3 sowie mit niedriger Ausprägung der Variable BuyPower durchgeführt werden. Die Mediationsprüfung zeigt, dass bei Vorliegen der Voraussetzungen für erfolgreiches Mehrstufiges Marketing ein Teil der Beziehung zwischen Marktorientierung und Neuprodukterfolg mediiert wird (Anhang 9). Für alle Gruppen liegt partielle Mediation vor. Der über den Mediator ALIGN erklärte Anteil des Gesamteffekts beläuft sich auf 13,1 \% bis 17,4 \%. Tabelle 49 zeigt die Ergebnisse des Tests auf Mediationsstärke.

Tabelle 49: Ergebnisse der Prüfung auf Mediationsstärke mit ALIGN als Mediator bei Gruppenbetrachtung

\begin{tabular}{|c|c|c|c|c|c|}
\hline $\begin{array}{c}\text { Gruppenvariable } \\
\text { (Ausprägung) }\end{array}$ & $\begin{array}{c}\text { Pfadkoeffizient } \\
\text { direkter Effekt) } \\
\text { MO } \rightarrow \text { NPS }\end{array}$ & t-Wert & Signifikanz & $\begin{array}{c}\text { indirekter } \\
\text { Effekt }\end{array}$ & PM-Wert \\
\hline Req1 (h) & 0,347 & 8,851 & $* * *$ & 0,070 & 0,167 \\
\hline Req2 (h) & 0,423 & 12,332 & $* * *$ & 0,067 & 0,137 \\
\hline Req3 (h) & 0,297 & 8,158 & $* * *$ & 0,063 & 0,174 \\
\hline BuyPower (n) & 0,405 & 11,624 & $* * *$ & 0,061 & 0,131 \\
\hline \multicolumn{6}{|r|}{ Signifikanzniveau: $* * *: p<0,01 ; * *: p<0,05 ; *: p<0,1 ;$ n.s.: nicht signifikant } \\
\hline
\end{tabular}

Hypothese H9, die eine mediierende Eigenschaft von ALIGN postuliert, kann somit unter den oben genannten Voraussetzungen beibehalten werden.

\subsection{Zusammenfassung und Diskussion der Ergebnisse}

In der vorliegenden Arbeit wurden für das zentrale Konstrukt der umfassenden Marktorientierung im B2B-Kontext in Anlehnung an die bestehende Konzeptua- 
lisierung und Operationalisierung von NARVER/SLATER aus dem Jahr 1990 fünf Dimensionen theoretisch hergeleitet und die daraus entwickelte coMOMessskala validiert. Die Bestandteile der Kunden-, Wettbewerbs-, Drittparteienund Marktstufenorientierung sowie die Abteilungsübergreifende Koordination können das latente Konstrukt der umfassenden Marktorientierung zu fast $71 \%$ $\left(\mathrm{R}^{2}=0,709\right)$ erklären. Die fünf Bestandteile stellen jeweils wesentliche und eigenständige Facetten des übergeordneten Gesamtkonstrukts dar.

Der größte Einfluss auf die Ausprägung der Marktorientierung geht von der Kundenorientierung aus. Dies zeigt, dass der Erfolg marktorientierter Unternehmen wesentlich durch die Ausrichtung an den Kundenbedürfnissen bestimmt wird. Ein Verständnis der Kunden und der Kundenwünsche sowie die Erfüllung der Kundenwünsche können als hauptsächlicher Treiber von Marktorientierung identifiziert werden.

Daneben stellen die Ausrichtung der Unternehmensaktivitäten auf die Drittparteien und die nachfolgenden Kunden ebenfalls bedeutsame Facetten des Gesamtkonstrukts dar. Dies ist besonders vor dem Hintergrund hervorzuheben, dass es sich bei diesen Bestandteilen um die Erweiterungen des etablierten Konzepts von NARVER/SLATER handelt. Es zeigt sich, dass eine „myopische“, rein kundenfokussierte Marktbetrachtung demnach das Konstrukt der Marktorientierung nicht vollständig zu erfassen scheint und in der Marketingpraxis dementsprechend weitergefasst wird. Die Ausprägung der Marktorientierung wird folglich signifikant vom Verständnis geprägt, welche weiteren Einflussfaktoren den Absatz der eigenen Leistungen bestimmen und welche Dynamiken in den Weiterverarbeitungs- und Absatzkanälen der eigenen Leistungen herrschen.

Bedeutsamen Anteil an der Bildung von Marktorientierung hat zudem die Facette der unternehmensinternen Prozessabstimmung. Abteilungsübergreifende Koordination im Sinne von Austausch von Marktwissen und anderen Ressourcen stellt somit eine wesentliche Voraussetzung von Marktorientierung dar. Einer bewussten Integration der verschiedenen Abteilungen in den Leistungserstellungsprozess kommt somit im Rahmen der Marktorientierung eine besondere Bedeutung zu. Den erhobenen Daten zufolge wird der Wettbewerbsorientierung bei der Imple- 
mentierung von Marktorientierung eine etwas geringere Bedeutung zugesprochen. Dennoch bildet die Ausrichtung der Unternehmensaktivitäten auf Wettbewerber einen nicht zu vernachlässigenden Bestandteil des Gesamtkonstrukts, was sich nicht zuletzt in einem signifikant positiven Pfadkoeffizient der Dimension niederschlägt. Bei der Schaffung von Kundenwerten müssen daher immer auch die für den Kunden verfügbaren Alternativen berücksichtigt werden.

Grundsätzlich lässt sich festhalten, dass jeder der fünf aufgestellten Dimensionen eine signifikante Bedeutung bei der Bildung von Marktorientierung zukommt.

Die Allgemeingültigkeit des entwickelten Messinstruments wurde anhand einer Mehrgruppenanalyse über verschiedene Produkt- und Markteigenschaften sowie Wertschöpfungsstufen und kundennahe Abteilungen getestet. Trotz der nicht durchgehend erreichten Messäquivalenz in allen Dimensionen bei allen Gruppenvergleichen, kann von insgesamt zufriedenstellender Gültigkeit und weiten Einsatzmöglichkeiten des Messinstruments ausgegangen werden. Ein Test der Relevanz der einzelnen Konstruktbestandteile zeigt, dass insbesondere der Einfluss der Wettbewerbsorientierung auf das Konstrukt der umfassenden Markorientierung variiert. Bei besonders schwer für den indirekten Kunden identifizierbaren Leistungen sowie in Marketingabteilungen sprechen die befragten Personen der Wettbewerbsorientierung eine signifikant höhere Bedeutung als in den jeweiligen Vergleichsgruppen zu. Bei Unternehmen, deren Leistungen nur eine Zwischenstufe bis zum Letztverwender durchlaufen, fällt die Relevanz der Wettbewerbsorientierung dagegen geringer als bei den Vergleichsgruppen aus.

Weiterhin zeigen die Gruppenvergleiche, dass Unternehmen mit leicht zu ersetzenden Leistungen der Kundenorientierung eine größere Bedeutung geben als solche Unternehmen mit schwer zu substituierenden Angeboten. Ebenfalls zeigt sich in Vertriebsabteilungen, dass auf signifikantem Niveau die Kundenorientierung und auf nicht signifikantem Niveau die Marktstufenorientierung stärker zum Konstrukt der Marktorientierung beiträgt, als es in Marketingabteilungen der Fall ist. Umgekehrtes gilt für die Relevanz der Wettbewerbsorientierung und mit Einschränkungen hinsichtlich der Signifikanz für die Drittparteienorientierung. Das Konstrukt der umfassenden Marktorientierung wurde ferner in einem Hypo- 
thesensystem hinsichtlich seiner Einflüsse auf die Wirkvariablen Unternehmenszusammenarbeit, Anpassung der Marketingstrategie und Innovativität geprüft.

Die Unternehmenszusammenarbeit mit dem direkten Kunden, die sich hauptsächlich der gemeinsamen Produktentwicklung widmet, kann im Modell über die Marktorientierung zu großen Teilen erklärt werden $\left(R^{2}=0,242\right)$. Hypothese H1, nach der eine Steigerung der Marktorientierung zu einer Steigerung der Zusammenarbeit führt, kann im Modell bestätigt werden. Unternehmen, die für die Produktentwicklung auf die Zusammenarbeit ihrer Kunden angewiesen sind, müssen demnach über eine gute Kenntnis ihres Marktes und ihres weiteren Marktumfeldes verfügen.

Die größte Wirkung hat Marktorientierung im Modell auf die Innovativität eines Unternehmens und kann diese als alleinige exogene Variable auch zu 36,6 \% erklären. Damit wird die Hypothese H2 bestätigt, die Marktorientierung als Treiber von Innovativität sieht. Die empirischen Ergebnisse zeigen, dass ein wesentlicher Teil der Einstellung und Fähigkeit eines Unternehmens zum Generieren und Ausprobieren von Produkt- und Prozessinnovationen aus dem Marktwissen und der Wissensverteilung im Unternehmen resultiert.

Die theoretisch hergeleitete Wirkbeziehung zwischen Marktorientierung und der Anpassung der Marketingstrategie und damit die Hypothese H3 konnte empirisch ebenfalls bestätigt werden. Das Modell zeigt, dass die Marketingaktivitäten, die mit dem direkten Kunden abgestimmt werden, zu einem großen Teil durch die Marktorientierung erklärt werden können $\left(R^{2}=26,9\right)$. Eine umfassende Marktorientierung eines Unternehmens ist somit als ein Ausgangspunkt von Mehrstufigem Marketing zu sehen, sofern die daraus abgeleiteten Aktivitäten mit dem Kunden kollaborativ implementiert werden.

Das Modell konnte zudem die Hypothesen H4 und H5, die einen positiven Wirkzusammenhang zwischen der Unternehmenszusammenarbeit bzw. der Innovativität und dem Neuprodukterfolg vermuten, als signifikant bestätigen. Mit einer Effektstärke von $\mathrm{f}^{2}=0,171$ und einem Pfadkoeffizienten von $\gamma=0,404$ hat Innovativität im Modell den stärksten Einfluss auf den Neuprodukterfolg. Eine positive Einstellung, Neues auszuprobieren, kann somit als eine Grundvoraussetzung 
für den Erfolg neuer Leistungsangebote gesehen werden. Darüber hinaus zeigt das Modell aber auch, dass die Zusammenarbeit mit Kundenunternehmen ebenfalls den Neuprodukterfolg - wenn auch in geringerem Maße - positiv beeinflusst. Das Modell konnte jedoch für den Gesamtdatensatz die aufgestellte Hypothese H6, die eine positive Wirkung der Anpassung der Marketingstrategien auf den Neuprodukterfolg postuliert, nicht bestätigen. Die Pfadbeziehung zeigt zwar einen leicht positiven aber dennoch nicht-signifikanten Zusammenhang zwischen den Variablen. Die Durchführung einer gruppenweisen Analyse dieses Zusammenhangs, zeigt allerdings, dass die in Hypothese H6 vermutete Wirkbeziehung signifikant positive Werte annimmt, wenn die Leistungen des Unternehmens und die Machtstellung des Anbieters die Voraussetzungen für einen erfolgreichen Einsatz von Mehrstufigem Marketing erfüllen. Technologisch anspruchsvolle, auf nachfolgenden Marktstufen identifizierbare und für den direkten Kunden relevante Leistungen sowie eine starke Position in der Geschäftsbeziehung gegenüber dem direkten Kunden wirken sich positiv moderierend auf die Beziehung zwischen der Anpassung der Marketingstrategien und dem Neuprodukterfolg aus.

Das aufgestellte Modell geht ferner von mediierten Beziehungen zwischen Marktorientierung und Neuprodukterfolg aus. Die Hypothesen H7 und H8, nach denen die Unternehmenszusammenarbeit und die Innovativität als Mediatoren fungieren, können bestätigt werden. Anhand der Daten zeigt sich allerdings, dass der Mediator Innovativität nur einen mittleren und der Mediator Unternehmenszusammenarbeit einen eher geringen Anteil der Wirkbeziehung zwischen Marktorientierung und Neuprodukterfolg erklären können. Die Hypothese H9, die von der mediierenden Wirkung der Variable Anpassung der Marketingstrategien ausgeht, kann im vorliegenden Modell anhand des Gesamtdatensatzes nicht bestätigt werden. Die Hypothese kann allerdings für die Gruppen bestätigt werden, bei denen die oben genannten Voraussetzungen für erfolgreiches Mehrstufiges Marketing als erfüllt angesehen werden können. Tabelle 50 stellt noch einmal zusammenfassend alle geprüften Hypothesen und die jeweiligen Befunde anhand der zugrunde gelegten Datenbasis dar. 
Tabelle 50: Zusammenfassung der Hypothesen und Befunde

\begin{tabular}{|c|c|c|c|c|c|}
\hline $\begin{array}{l}\text { Hypo- } \\
\text { these }\end{array}$ & Datengrundlage & Beziehung & $\begin{array}{l}\text { Pfadkoeffizient } \\
\text { (Signifikanz) }\end{array}$ & $\begin{array}{c}\text { Mediation: } \\
\text { z-Wert } \\
\text { (Signifikanz) } \\
\end{array}$ & Befund \\
\hline $\mathrm{H} 1$ & Gesamtdatensatz & $\mathrm{MO} \rightarrow \mathrm{COLLABO}$ & $0,492(* * *)$ & -- & $\sqrt{ }$ \\
\hline H2 & Gesamtdatensatz & $\mathrm{MO} \rightarrow \mathrm{INNOVA}$ & $0,605(* * *)$ & -- & $\sqrt{ }$ \\
\hline H3 & Gesamtdatensatz & $\mathrm{MO} \rightarrow$ ALLIGN & $0,519(* * *)$ & -- & $\sqrt{ }$ \\
\hline $\mathrm{H} 4$ & Gesamtdatensatz & COLLABO $\rightarrow$ NPS & $0,185(* * *)$ & -- & $\sqrt{ }$ \\
\hline $\mathrm{H} 5$ & Gesamtdatensatz & INNOVA $\rightarrow$ NPS & $0,404(* * *)$ & -- & $\sqrt{ }$ \\
\hline \multirow{6}{*}{ H6 } & Gesamtdatensatz & \multirow{6}{*}{ ALIGN $\rightarrow$ NPS } & 0,057 (n.s.) & -- & $(\sqrt{ })$ \\
\hline & Req1 (h) & & $0,165(* * *)$ & -- & $\sqrt{ }$ \\
\hline & Req2 (h) & & $0,139(* * *)$ & -- & $\sqrt{ }$ \\
\hline & Req3 (h) & & $0,128(* *)$ & -- & $\sqrt{ }$ \\
\hline & Req4 (h) & & 0,075 (n.s.) & -- & $(\sqrt{)}$ \\
\hline & BuyPower (n) & & $0,108(* *)$ & -- & V \\
\hline \multirow{3}{*}{ H7 } & \multirow{3}{*}{ Gesamtdatensatz } & $\mathrm{MO} \rightarrow$ COLLABO & $0,497(* * *)$ & \multirow{3}{*}{$2,602(* * *)$} & \multirow{3}{*}{$\sqrt{ }$} \\
\hline & & COLLABO $\rightarrow$ NPS & $0,138(* * *)$ & & \\
\hline & & $\mathrm{MO} \rightarrow \mathrm{NPS}$ & $0,487(* * *)$ & & \\
\hline \multirow{3}{*}{ H8 } & \multirow{3}{*}{ Gesamtdatensatz } & $\mathrm{MO} \rightarrow$ INNOVA & $0,608(* * *)$ & \multirow{3}{*}{ 4,471 (***) } & \multirow{3}{*}{$\sqrt{ }$} \\
\hline & & INNOVA $\rightarrow$ NPS & $0,303(* * *)$ & & \\
\hline & & $\mathrm{MO} \rightarrow \mathrm{NPS}$ & $0,487(* * *)$ & & \\
\hline \multirow{18}{*}{ H9 } & \multirow{3}{*}{ Gesamtdatensatz } & $\mathrm{MO} \rightarrow$ ALIGN & $0,506(* * *)$ & \multirow{3}{*}{$-0,281$ (n.s.) } & \multirow{3}{*}{$\mathrm{X}$} \\
\hline & & ALIGN $\rightarrow$ NPS & $-0,015$ (n.s..) & & \\
\hline & & $\mathrm{MO} \rightarrow \mathrm{NPS}$ & $0,487(* * *)$ & & \\
\hline & \multirow{3}{*}{$\begin{array}{c}\text { Req1 (h) } \\
\text { Identifizierbarkeit der } \\
\text { Leistung für indir. } \\
\text { Kunden } \\
\end{array}$} & $\mathrm{MO} \rightarrow$ ALIGN & $0,422(* * *)$ & \multirow{3}{*}{$2,764(* * *)$} & \multirow{3}{*}{$\sqrt{ }$} \\
\hline & & ALIGN $\rightarrow$ NPS & $0,165(* * *)$ & & \\
\hline & & $\mathrm{MO} \rightarrow \mathrm{NPS}$ & $0,347(* * *)$ & & \\
\hline & \multirow{3}{*}{$\begin{array}{l}\text { Req2 (h) } \\
\text { Relevanz der Leistung } \\
\text { beim direkten Kunden }\end{array}$} & $\mathrm{MO} \rightarrow$ ALIGN & $0,483(* * *)$ & \multirow{3}{*}{$3,156(* * *)$} & \multirow{3}{*}{$\sqrt{ }$} \\
\hline & & ALIGN $\rightarrow$ NPS & 0,139 (***) $^{* * 2}$ & & \\
\hline & & $\mathrm{MO} \rightarrow \mathrm{NPS}$ & $0,423(* * *)$ & & \\
\hline & \multirow{3}{*}{$\begin{array}{c}\text { Req3 (h) } \\
\text { Technologisches An- } \\
\text { spruchsniveau der } \\
\text { Leistung } \\
\end{array}$} & $\mathrm{MO} \rightarrow$ ALIGN & $0,489(* * *)$ & \multirow{3}{*}{$2,243(* *)$} & \multirow{3}{*}{$\sqrt{ }$} \\
\hline & & ALIGN $\rightarrow$ NPS & $0,128(* *)$ & & \\
\hline & & $\mathrm{MO} \rightarrow \mathrm{NPS}$ & $0,297(* * *)$ & & \\
\hline & \multirow{3}{*}{$\begin{array}{c}\text { Req4 (h) } \\
\text { Alleinstellungsmerkmal } \\
\text { der Leistung }\end{array}$} & $\mathrm{MO} \rightarrow$ ALIGN & $0,494(* * *)$ & \multirow{3}{*}{ 1,282 (n.s.) } & \multirow{3}{*}{$\mathrm{X}$} \\
\hline & & ALIGN $\rightarrow$ NPS & 0,075 (n.s.) & & \\
\hline & & $\mathrm{MO} \rightarrow \mathrm{NPS}$ & 0,299 (***) $^{* *}$ & & \\
\hline & \multirow{3}{*}{$\begin{array}{c}\text { BuyPower (n) } \\
\text { Marktmacht des Kunden }\end{array}$} & MO $\rightarrow$ ALIGN & $0,563(* * *)$ & \multirow{3}{*}{$2,113(* *)$} & \\
\hline & & ALIGN $\rightarrow$ NPS & $0,108(* *)$ & & $\sqrt{ }$ \\
\hline & & $\mathrm{MO} \rightarrow \mathrm{NPS}$ & $0,405(* * *)$ & & \\
\hline
\end{tabular}

Die durchgeführte Untersuchung des Strukturgleichungsmodells auf Ebene der Dimensionen des Gesamtkonstukts zeigt auf, welche Komponenten der umfas- 
senden Marktorientierung die drei abhängigen Unternehmensaktivitäten in besonderem Maße beeinflussen. Demzufolge erscheint es für die Unternehmenszusammenarbeit in annähernd gleichem Maße relevant, Kunden- und Drittparteieninformationen zu sammeln. Offensichtlich bedarf die Auswahl von geeigneten Kooperationspartner einer genauen Kenntnis derselben und weiterer Einflussnehmer im Marktumfeld. Zudem zeigt sich, dass auch die Fähigkeit, unternehmensintern Prozesse zu koordinieren und über Abteilungsgrenzen hinweg zusammenzuarbeiten, der Zusammenarbeit mit Kundenunternehmen zuträglich ist. Für die Innovativität zeigt das Modell, dass ein breites Marktwissen zwar förderlich ist, der größte Einfluss jedoch aus der Ressourcenkoordination und Verteilung dieses Wissens an relevante Abteilungen resultiert. Marketingaktivitäten, die sich auf indirekte Kunden beziehen, werden fast ausschließlich durch die Ausrichtung eines Unternehmens auf diese nachgelagerten Markstufen bestimmt. Eine wesentliche Voraussetzung für die Implementierung einer mehrstufigen Marketingstrategie ist somit eine genaue Kenntnis der Marktverhältnisse in nachgelagerten Wertschöpfungsstufen. 


\section{Fazit und Ausblick}

\subsection{Wissenschaftlicher Beitrag}

Antrieb für die vorliegende Forschungsarbeit war nicht zuletzt die Diskrepanz zwischen den konzeptionellen Überlegungen zahlreicher wissenschaftlicher Arbeiten hinsichtlich der für einen Anbieter im B2B-Sektor relevanten Aspekte des Marktes und der letztendlich darauf basierenden Operationalisierung von Marktorientierung. Zur Bestimmung des relevanten Marktes eines Anbieters im B2BBereich wurde zunächst Forschungsfrage 1 beantwortet. Dabei wurden sowohl konzeptionell als auch empirisch vier relevante Gruppen von Marktteilnehmern identifiziert, aus denen sich der Markt zusammensetzt.

Als Ziel dieser Arbeit wurde ferner die theoretisch fundierte Herleitung einer Konzeptualisierung und Operationalisierung von Marktorientierung im Businessto-Business-Kontext als Beantwortung von Forschungsfrage 2 ausgegeben. Ausgehend von dem in Forschungsfrage 1 identifizierten facettenreichen Markt zeigt die vorliegende Arbeit somit, dass ein herkömmliches Verständnis von Marktorientierung, welches im Wesentlichen nur Kunden und Wettbewerber berücksichtigt, kaum noch Ansatzpunkte für die Generierung von Wettbewerbsvorteilen zu liefern vermag. Marktorientiertes Handeln wird heutzutage vielfach als unternehmerische Selbstverständlichkeit eingeschätzt, so dass sich ein Anbieter darüber kaum noch differenzieren kann.

Als Ausgangspunkt einer diese Herausforderungen berücksichtigende Unternehmensausrichtung wurde Marktorientierung basierend auf der MKTOR-Skala von NARVER/SLATER als fünf-dimensionales Konstrukt konzeptualisiert, wobei die zwei vollständig neu entwickelte Dimensionen explizit die Gegebenheiten auf Industriegütermärkten berücksichtigen. Durch die erweiterte Marktauffassung bringt die vorliegende Arbeit die konzeptionellen Ausführungen zahlreicher Wissenschaftler mit den tatsächlichen Gegebenheiten und Notwendigkeiten der Busi- 
ness-to-Business-Praxis in messtheoretischen Einklang.

Ein Vergleich der neu entwickelten coMO-Messskala mit der ursprünglichen MKTOR-Skala zeigt, dass durch das neue Messinstrument ein wesentlicher Teil des relevanten Marktes mehr berücksichtigt und zusätzliche Varianz im Konstrukt der Marktorientierung erklärt werden kann. Die formative Beziehung der hergeleiteten Dimensionen zum Gesamtkonstrukt der Marktorientierung nimmt Überlegungen auf, nach denen sich Marktorientierung aus verschiedenen Unternehmensausrichtungen zusammensetzt, die gemeinsam das Konstrukt formen. Durch diese formative Sichtweise wird es möglich, den Einfluss einzelner Dimensionen auf beliebige Wirkgrößen partiell zu betrachten und deren Einfluss zu messen. Das zur empirischen Analyse verwendete PLS-Schätzverfahren untermauert die hohe Güte des entwickelten Messmodells. Die fünf-dimensionale Struktur kann als bestätigt angenommen und die für jede Dimension abgeleiteten Indikatoren als insgesamt reliabel bezeichnet werden.

Daran anschließend sollte das zentrale Konstrukt der Marktorientierung in ein Strukturgleichungsmodell eigebettet werden, um Wirkbeziehungen und die Schaffung von Wettbewerbsvorteilen empirisch überprüfen zu können (Forschungsfrage 3).

Die Wirkungen von Marktorientierung zeigen sich im Rahmen des aufgestellten Strukturgleichungsmodells. Hierbei wird ersichtlich, wie die auf mehrere Marktstufen und das weitere Marktumfeld ausgerichtete Orientierung eines Unternehmens insbesondere einen Einfluss auf Unternehmensaktivitäten ausübt, die einen kooperativen und unternehmensübergreifenden Charakter besitzen.

Die nach dem ressourcenbasierten, kompetenzbasierten und relationalen Ansatz zu erwartenden Wettbewerbsvorteile konnten empirisch über die Messung von Effektivitätsvorteilen im Rahmen des Neuprodukterfolgs bestätigt werden.

Auf der besagten theoretischen Grundlage konnte Marktorientierung als Teil einer Unternehmenskultur identifiziert werden, die es einem Unternehmen ermöglicht, weitreichende Marktinformationen zu sammeln und diese wertgenerierend intern und in Abstimmung und Zusammenarbeit mit seinen direkten Kunden einzusetzen. Marktorientierung stellt demnach gleichzeitig eine wertvolle Res- 
source und Kompetenz dar, die für einen Anbieter einen Wettbewerbsvorteil zu generieren vermag. Der Wert der Marktorientierung steigt, je mehr ein Unternehmen in der Lage ist, die für sein Geschäft relevanten Marktteilnehmer zu identifizieren und in seinen Marktaktivitäten zu berücksichtigen. Dies betrifft Informationen über Kunden und Wettbewerber in gleichem Maße wie Informationen über Einflussnehmer und nachgelagerte Marktstufen. Marktorientierung wird in dieser Arbeit daher nicht wie bisher weitgehend üblich als einstufiges Phänomen gesehen, sondern als ein Konstrukt, das die real existierende multiple Stufenstruktur von Industriegütermärkten berücksichtigt.

Einen Beitrag zur Theorie des relationalen Ansatzes liefert diese Arbeit insoweit als, der Wert, der mittels der relationalen Ressourcen generiert wird, in Bezug zum Endkunden bzw. nachgelagerten Kunden gesehen wird. Traditionell wird innerhalb des RV der generierte Wert in Netzwerken und Kooperationen nur durch einen Kooperationspartner bestimmt. ${ }^{677}$ Eine Berücksichtigung der Wertschätzung durch Kunden von Netzwerken findet im Rahmen des RV allenfalls implizit statt. Dass allerdings der Wert, der dem Endkunden, als Ausgangspunkt der abgeleiteten Nachfrage, entsteht, entscheidend für die Bewertung von Ressourcen ist, kann, wie in dieser Arbeit geschehen, durch die Einbeziehung des Mehrstufigen Marketings berücksichtigt werden. ${ }^{698}$ Zur bestmöglichen Ausschöpfung von Ressourcen und der damit verbunden Generierung von Werten bedarf es somit eines eng abgestimmten und auf den Endkunden gerichteten Einsatzes der in eine Partnerschaft eingebrachten Ressourcen. ${ }^{699}$ Der RV kann somit zusätzlich zu der sonst üblichen dyadischen Sichtweise problemlos im Kontext mehrstufiger Märkte zur Erklärung von Wettbewerbsvorteilen eines Kooperationspartners oder des gesamten Netzwerks herangezogen werden.

697 Vgl. Dyer/Singh 1998, S. 667.

${ }^{698}$ Vgl. Peteraf/Bergen 2003, S. 1038; Frels et al. 2003.

${ }^{699}$ Vgl. Möller 2006, S. 917. 


\subsection{Implikationen für die Praxis von Marketing und Ver- trieb}

Mit der vorliegenden Arbeit konnte gezeigt werden, dass eine umfassende Marktorientierung eine erfolgsversprechende strategische Option für Anbieter darstellt. Für Unternehmen ist das entwickelte Messinstrument hilfreich, die eigene Marktorientierung zu messen und gezielt zu fördern. Die signifikanten Beiträge aller entwickelten Dimensionen zum Gesamtkonstrukt der Marktorientierung bedeutet für die Marketingpraxis, dass alle Bestandteile einer umfassenden Marktorientierung für B2B-Unternehmen relevant sind. Durch eine eingeschränkte Fokussierung auf Kunden und Wettbewerber kann ein Unternehmen nicht sein volles Potenzial ausschöpfen, eine derartige Unternehmensausrichtung erscheint somit erfolgshinderlich.

Je nachdem welche Unternehmensziele verfolgt werden, können anhand der formativen Messweise von Marktorientierung geeignete Treiber bzw. Stellschrauben identifiziert werden. Die Arbeit zeigt, dass die Verbesserung der innerbetrieblichen Prozessabstimmung und Kooperation der verschiedenen Abteilungen einen wichtigen Ansatzpunkt zur Förderung der Innovativität eines Unternehmens bietet. Hingegen kommen andere Dimensionen für ein derartiges Ziel nicht in Frage. Die Ausrichtung am Kunden beispielsweise, insbesondere, wenn eine gewisse Routine in der Geschäftsbeziehung eingesetzt hat, birgt wenige Möglichkeiten, um Innovationen hervorzubringen.

Zur Förderung einer kooperativen Marktbearbeitung muss ein Unternehmen über eine gute, umfassende Kenntnis des eigenen Marktes verfügen. So können leichter potentielle Kooperationspartner identifiziert und die Zusammenarbeit erfolgreich gestaltet werden. Den einzelnen Mitarbeitern, insbesondere in marktnahen Positionen, sollte daher bewusst sein, welche Kundenwünsche im Markt existieren und wie das eigene Unternehmen in der Lage ist, diese unter Beachtung von Wettbewerbseinflüssen zu befriedigen. Vor dem Hintergrund einer multiplen Stufenstruktur in Industriegütermärkten sollten Unternehmensmitarbeiter zudem in die Lage versetzt werden, den weiteren Verarbeitungs- und Absatzweg der eigenen Produkte zu kennen und der Präsenz von einflussnehmenden Drittpartei- 
en gewahr werden.

Bei der Förderung bestimmter Dimensionen bzw. Handlungsweisen im Sinne von Marktorientierung müssen Unternehmen sich vergegenwärtigen, dass die kundennahen Abteilungen Marketing und Vertrieb den verschiedenen Bestandteilen von Marktorientierung teilweise eine andere Relevanz beimessen. Im Allgemeinen kommt im Vertrieb den direkten und indirekten Kunden eine größere Bedeutung im Rahmen von Marktorientierung zu. Marketingabteilungen hingegen sehen eine größere Wichtigkeit im weiteren Marktumfeld, d.h. bei Wettbewerbern und einflussnehmenden Drittparteien. Da die Ressourcen in Unternehmen zur Förderung der Marktorientierung nicht unerschöpflich sind, ist es möglicherweise schon erfolgsversprechend je nach Zielsetzung nur ausgewählte Abteilungen zu fördern.

Grundsätzlich lässt sich für ein Unternehmen als Ganzes festhalten, dass eine breite Basis an Marktwissen und der unternehmensinterne Austausch dieses Wissens beim Aufbau von Marktorientierung und letztlich bei der Erstellung von wertgenerierenden Leistungen für Kunden hilfreich sind. Inwiefern allerdings die Marktorientierung durch die reine Informationssammlung und -verteilung verändert werden kann, bleibt fraglich. Wenn Marktorientierung als Teil einer Unternehmenskultur betrachtet wird, muss eine Förderung derartiger Mitarbeiter-, und Abteilungstätigkeiten bzw. eine Implementierung neuer Arbeitsanweisungen folglich immer auch im Einklang mit den Unternehmenswerten und -leitlinien stehen.

Resultierend aus einer ausgeprägten Marktorientierung bietet sich für Unternehmen die strategische Handlungsoption, Mehrstufiges Marketing für einzelne Leistungen, einzelne Märkte oder für das gesamte Unternehmensangebot zu implementieren. Unternehmen, die sich für eine kooperative mehrstufige Marketingstrategie entscheiden, sollten sich dabei bewusst sein, dass diese Strategie nur erfolgsversprechend ist, wenn die mehrstufig zu vermarktenden Leistungen bestimmte Voraussetzungen erfüllen. Besonders erfolgsversprechend sind hierbei solche Leistungen, die auf nachfolgenden Marktstufen klar identifizierbar bleiben, d.h. die Leistungen des Anbieters wiedererkannt werden können. Gleiches 
gilt für Leistungen, die für den direkten Kunden preislich von großer Relevanz und insgesamt technologisch anspruchsvoll sind. Commodities und technologisch eher simple Produkte eignen sich hingegen deutlich weniger für eine mehrstufige Marketingstrategie. Daneben spielt die Machtposition des Unternehmens eine wichtige Rolle. Befindet sich der Anbieter in der eher mächtigeren Position einer Geschäftsbeziehung, so liegt es nahe, dass er die mehrstufigen Marketingpraktiken stärker nach seinen Vorstellungen und eher zu seinen Gunsten beeinflussen kann. Der letztlich daraus entstehende Erfolg von Neuprodukten übertrifft daher den Erfolg von mehrstufig agierenden Unternehmen in Geschäftsbeziehungen mit starken direkten Kunden.

\subsection{Limitationen der Untersuchung und Ansatzpunkte für weiterführende Forschung}

Im Zuge der Arbeit wurde die coMO-Messskala entwickelt und das Kostrukt der umfassenden Marktorientierung auf seine Wirkungen in Industriegütermärken hin untersucht. Bei der Entwicklung des Messinstruments wurde das Konstrukt der umfassenden Marktorientierung zwar einer gründlichen Untersuchung unterzogen, doch vermag die vorliegende Arbeit nicht, alle forschungsrelevanten inhaltlichen und methodischen Aspekte zu behandeln oder zufriedenstellend zu klären. Die Arbeit bietet daher neben den gewonnenen Erkenntnissen zahlreiche Anknüpfungspunkte für zukünftige Forschungsvorhaben, von denen die prägnantesten aus Sicht von Wissenschaft und Praxis im Folgenden ausgeführt werden.

Bei der Messvalidierung des Konstrukts der umfassenden Marktorientierung konnte insgesamt eine hohe Güte des Messmodells erreicht werden, wobei die Güte der Dimension Drittparteienorientierung (PARTOR) hintansteht. Im Rahmen der Identifizierung von relevanten Drittparteien wurde zwar der Idee gefolgt, nur Key-Stakeholder für den Absatzerfolg eines Anbieters zu berücksichtigen, doch wirft die entwickelte PARTOR-Sub-Skala, die Frage auf, ob alle wesentlichen Drittparteien und die jeweilige Form der Einflussnahme identifiziert werden konnten. Durch das Ziel, die Skala in möglichst vielen, verschiedenen Branchen und Marktumfeldern einsetzen zu können, wurde eine möglichst breite Auffas- 
sung von Drittparteien berücksichtigt. Scheinbar enthält die daraus entwickelte Skala aber Einflussnehmer, die in den Märkten der Befragungsteilnehmer keine durchgehende Relevanz besitzen. Insgesamt bleiben die Kennzahlen der PARTOR-Messung hinter denen der übrigen Dimensionen zurück. Möglicherweise ergeben sich für jede Industrie unterschiedliche Drittparteien, deren Wichtigkeit von Branche zu Branche oder von Unternehmen zu Unternehmen variiert. Hierbei kann der Reifegrad einer Industrie eine entscheidende Rolle spielen. Möglicherweise können bessere Ergebnisse erzielt werden, wenn die Drittparteien weiter abstrahiert in Kategorien nach ihrer Wichtigkeit oder ihrer Macht eingruppiert werden. ${ }^{700}$ Eine Item-Anpassung zum Zwecke einer höheren allgemeinen Validität der betreffenden Sub-Skala wäre für weitere Forschungsvorhaben überlegenswert.

Im Rahmen der vorliegenden Arbeit wurden zur Einschätzung der Allgemeingültigkeit der Skala zahlreiche Gruppenvergleiche durchgeführt. Nicht in jedem Fall konnte ein zufriedenstellendes Maß an Messäquivalenz innerhalb jeder Dimension erreicht werden. Es liegt daher die Vermutung nahe, dass bestimmte, häufig als nicht messäquivalent bezeichnete Indikatoren je nach Untersuchungskontext unterschiedlich aufgefasst werden können. Auch hier könnte eine ItemAnpassung der Indikatoren mit der geringsten Messäquivalenz Abhilfe schaffen. In der Literatur wird mitunter auch gefordert, solche Indikatoren von der Messung auszuschließen.

Einen Überblick der erreichten Messäquivalenz der einzelnen Indikatoren über die getätigten Gruppenvergleiche hinweg liefert Anhang 10. Für weitere Forschungsvorhaben könnte sich die Messung der reflektiven Konstruktbestandteile auf wenige dafür messäquivalente Indikatoren stützen. Einen wesentlichen Beitrag zur Allgemeingültigkeit der Skala könnte zudem ein Gruppenvergleich in einem anderen kulturellen Umfeld darstellen. Im Rahmen der Arbeit wurden zwar Daten aus Deutschland, Österreich und der Schweiz erhoben, da den Teilnehmern allerdings Anonymität zugesichert wurde, ist ein Gruppenvergleich nach Nationalitäten nicht ohne Weiteres möglich. Zudem wurden diese drei Länder bewusst

${ }^{700}$ Vgl. He et al. 2011, S. 68 f.; Mitchell et al. 1997, S. 854. 
aufgrund ihrer sprachlichen Übereinstimmung und kulturellen Ähnlichkeit für die Befragung ausgewählt, so dass anhand der befragten Personen keine Gruppenunterschiede zu erwarten wären.

Die vorliegende Skala wurde explizit für B2B-Unternehmen entwickelt. Vor dem Hintergrund, dass die gängigen Skalen von Marktorientierung für Industrie- und Konsumgüter in der Literatur als völlig austauschbar behandelt werden, wäre es auch im Falle der neu entwickelten coMO-Skala von Interesse zu sehen, inwiefern die Skala Anwendung im B2C-Bereich finden könnte. Denkbar wäre hierfür eine Elimination der Marktstufenorientierung (MULTOR). Da aber ein Großteil der Konsumgüter über den Handel vertrieben wird, wäre auch eine Abwandlung dieser Dimension im Sinne einer Handelsorientierung denkbar. Ob eine Anpassung der Drittparteienorientierung überhaupt erforderlich wäre, bleibt fraglich. Schließlich unterliegt auch der Absatz von Konsumgütern bisweilen starken Einflüssen von Drittparteien.

Die Ausführungen zur Datenerhebung und insbesondere zu den Auskunftspersonen in Abschnitt 5.3.2 machen deutlich, dass das gewählte Single-InformantDesign mit alleiniger Befragung im Anbieterunternehmen nicht in jedem Falle unreflektiert übernommen werden sollte. So wäre es grundsätzlich denkbar, jede Dimension der Skala von einer anderen betroffenen Stakeholder-Gruppe beantworten zu lassen, wie es verschiedene Autoren vorschlagen. ${ }^{701}$ Die Kundenorientierung eines Unternehmens könnte folglich von den Kunden selbst, die Wettbewerbsorientierung entsprechend von den Wettbewerbern des Anbieters eingeschätzt werden. Wenngleich damit validere Daten verbunden sein mögen, so wirft eine solche Vorgehensweise wieder neue Probleme hinsichtlich der Messung der Drittparteienorientierung oder Marktstufenorientierung auf. Mitunter ist nachgelagerten Kunden nicht einmal bekannt oder für sie überhaupt von Bedeutung, dass ein Anbieter den Weg seiner Produkte bis zum Endkunden verfolgt. Bei der Drittparteienorientierung stellt sich die Frage, welche der zahlreichen und grundverschiedenen Einflussnehmer zu befragen wären. Unter forschungsökonomischen Gesichtspunkten wäre ein solches Vorgehen daher nur im Rahmen von

${ }^{701}$ Vgl. Harris 2002, S. 240; Chen/Quester 2005, S. 780. 
kleinzahligen Erhebungen oder Fallstudienanalysen denkbar.

Die im Rahmen der Untersuchung befragten Unternehmen stammen aus zahlreichen Branchen, die vertriebenen Leistungen beinhalten immaterielle und materielle Leistungen und reichen von Rohstoffen über verschiedene Verarbeitungsstufen bis hin zu fertigen Maschinen und Anlagen. Auf diese Weise sollte ein möglichst breites Spektrum an Unternehmen berücksichtigt und die Generalisierbarkeit der Skala sichergestellt werden. Jedoch überwiegt der Anteil derjenigen Unternehmen, die physische Produkte anbieten gegenüber Dienstleistungsanbietern. Die Gruppe der Dienstleistungsanbieter ist mit $n=30$ relativ klein, so dass Gruppenvergleiche zwischen materiellen und immateriellen Produkten nicht sinnvoll durchgeführt werden können. Ansatzpunkt für weitergehende Forschung könnte die gezielte Befragung von Dienstleistern sein, um die Gültigkeit des Messinstruments für diese Gruppe zu validieren und gegebenenfalls neue Erkenntnisse für die mehrstufige Vermarktung von Dienstleistungen zu erlangen.

Ansatzpunkte für weitere Untersuchung finden sich zudem im nomologischen Netzwerk, in das das Konstrukt der umfassenden Marktorientierung eingebettet wurde.

Im Rahmen dieser Arbeit wurde Marktorientierung als Teil einer Unternehmenskultur identifiziert, die die Fähigkeiten zum unternehmensinternen und unternehmensübergreifenden Wissensaustausch fördert. Weitergehende Studien, die Antezendenzen von Marktorientierung wie etwa Markt-basierte Vergütungssysteme oder Trainings von marktorientierten Fähigkeiten betrachten, sind in der Literatur eher selten zu finden. Gerade aber die Untersuchung dieser Antezendenzen könnte Aufschluss über die Steigerung von mit Marktorientierung einhergehenden Fähigkeiten und dem damit verbundenen Erfolg geben. ${ }^{702}$

Nicht nur eine Untersuchung der Antezendenzen sondern auch eine Betrachtung weiterer Konsequenzen von Marktorientierung wäre denkbar. In der vorliegenden Arbeit wurde als abhängige Erfolgsgröße der Neuprodukterfolg als Effektivitätsmaß untersucht. In der Literatur wird allerdings immer wieder hervorgehoben,

702 Vgl. Kirca et al. 2005, S. 28; vgl. auch Griese 2011, S. 271 zum Einsatz von Trainings und Anreizsystemen zur Förderung von marktwissensrelevanten Fähigkeiten. 
dass gerade auch nicht direkt monetär messbare Größen durch eine erweiterte Marktorientierung beeinflusst werden können bzw. das Ziel von Mehrstufigem Marketing darstellen. Aufgrund der kooperativen Verhaltensweisen eines Anbieters im Rahmen von Mehrstufigem Marketing würden sich daher gerade solche Messgrößen eignen, die eher weichere Kenngrößen wie etwa das Vertrauen zwischen den Kooperationspartnern oder die Stabilität der Geschäftsbeziehung messen. ${ }^{703}$

Die in dieser Arbeit dargestellte Form des Mehrstufigen Marketings basiert auf einer kollaborativen Anpassung der Marketingstrategie von Anbieter und direktem Kunden. In der Marketing-Praxis sind allerdings auch solche Ausgestaltungsformen zu finden, die als marktstufenübergreifende Alleingänge eines Anbieters gesehen werden können. ${ }^{704}$ Solch ein nicht-kollaboratives Mehrstufiges Marketing wurde im Rahmen dieser Arbeit ausgeklammert, könnte aber sowohl als Konsequenz einer umfassenden Marktorientierung als auch als Antezendenz verschiedener Erfolgsgrößen weitere Einblicke in die Wirkmechanismen mehrstufiger Industriegütermärkte geben.

Die Generierung von Marktwissen wurde bei der Betrachtung einer umfassenden Marktorientierung weitgehend als die Aufgabe eines einzelnen Unternehmens betrachtet. Gerade aber vor dem Hintergrund kooperativer Geschäftsbeziehungen wäre eine stärkere Berücksichtigung von Wissensgenerierung über Netzwerke denkbar. Diese Überlegungen würden auch im Einklang mit dem relationalen Ansatz stehen, nach dem Wissen als strategische Ressource unter anderem durch ihre Zusammenarbeit von zwei oder mehr Unternehmen entstehen und genutzt werden kann.

Letztlich kann auch die gewählte Messmethode kritisch betrachtet werden. Aufgrund des eher exploratorischen Charakters der Studie wurde der PLS-Ansatz als Analyseverfahren gewählt. Aufgrund der fehlenden Verteilungsannahmen verzichtet dieser Ansatz auf globale Gütemaße und verlässt sich auf die Gesamtschau zahlreicher partieller Gütemaße, für die in der Literatur mehr oder weniger

\footnotetext{
${ }^{703}$ Vgl. Kleinaltenkamp et al. 2011, S. 44; Hillebrand/Biemans 2011, S. 73; Homburg et al. 2014;

${ }^{704}$ Vgl. Schönhoff et al. 2013, S. 2; Fischer 2014, S. 104 ff.; Homburg et al. 2014.
} 
strenge kritische Werte zu finden sind. Die letztliche Gütebeurteilung bleibt aber der Interpretation des Forschers vor dem Hintergrund des a priori definierten Untersuchungsziels überlassen. Deutlich normativere Gütebeurteilungen sind hingegen im Rahmen der Kovarianz-basierten Analyseverfahren zu finden. Um eine globale Vergleichbarkeit zumindest der reflektiven Bestandteile des entwickelten Messmodells und des Strukturgleichungsmodells zu erhalten, wäre es denkbar, sich im Rahmen einer Replikationsstudie auf das Kovarianz-basierte Schätzverfahren zu stützen.

Obwohl diese Arbeit nicht alle Aspekte der Konzeptualisierung und Operationalisierung abschließend klären kann und diesbezüglich neue Fragen und Probleme aufgezeigt werden, liefert die Arbeit doch einen wesentlichen Beitrag, Marktorientierung in Business-to-Business-Märkten adäquat zu messen. Die Untersuchung der aus der Marktorientierung resultierenden marktbezogenen Unternehmenstätigkeiten in einem Strukturmodell können nur erste Einblicke in die komplexen mehrstufigen Unternehmensbeziehungen in Industriegüterunternehmen geben. Die betrachteten Wirkbeziehungen sollen daher als wichtige Ansatzpunkte für zukünftige Forschungsvorhaben und Erkenntnisgewinne für die Marketingwissenschaft gesehen werden. 


\section{Anhang}

Anhang 1: Ergebnisse weiterer Güteprüfungen reflektiver Messungen

\begin{tabular}{|c|c|c|c|c|c|c|}
\hline $\begin{array}{l}\text { Konstrukt/ } \\
\text { Dimension }\end{array}$ & MULTOR & PARTOR & FUNCO & COMPOR & CUSTOR & MO \\
\hline KMO & 0,859 & 0,760 & 0,874 & 0,855 & 0,872 & 0,816 \\
\hline $\begin{array}{l}\text { \% der erklär- } \\
\text { ten Varianz }\end{array}$ & 60,351 & 50,615 & 60,821 & 55,527 & 58,704 & 58,192 \\
\hline Konstrukt & COLLABO & INNOVA & ALIGN & NPS & & \\
\hline KMO & 0,849 & 0,745 & 0,815 & 0,698 & & \\
\hline $\begin{array}{l}\text { \% der erklär- } \\
\text { ten Varianz }\end{array}$ & 53,388 & 78,183 & 57,086 & 57,606 & & \\
\hline
\end{tabular}

Anhang 2: Aus Interviews abgeleitete Items

\begin{tabular}{|l|l|}
\hline \multirow{3}{*}{} & $\begin{array}{l}\text { *Insgesamt wissen wir genau, welche Produkte unsere Kunden wollen und welche Anforderungen sie } \\
\text { daran stellen. }\end{array}$ \\
\cline { 2 - 3 } & Unsere Geschäftsziele werden hauptsächlich durch Kundenzufriedenheit bestimmt. \\
\cline { 2 - 3 } & Wir überprüfen ständig, wie sehr wir unseren Kunden gegenüber verpflichtet sind und uns an ihren Kun- \\
denwünschen ausrichten.
\end{tabular}


Fortsetzung Anhang 2: Aus Interviews abgeleitete Items

\begin{tabular}{|c|c|}
\hline \multirow{8}{*}{ 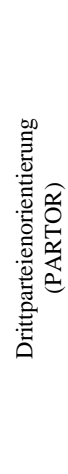 } & $\begin{array}{l}\text { In unserem Unternehmen sammeln und werten wir Informationen über Institutionen aus, die allgemeine } \\
\text { soziale Trends (z.B. Umweltbewusstsein, Lebensstil) beeinflussen und sich so auf unser Geschäft auswir- } \\
\text { ken könnten. }\end{array}$ \\
\hline & $\begin{array}{l}\text { *Alles in allem sind uns Personen und Organisationen im Umfeld der Marktstufen bekannt, an die unsere } \\
\text { Produkte direkt oder weiterverarbeitet geliefert werden. }\end{array}$ \\
\hline & $\begin{array}{l}\text { Wir brauchen lange Zeit, um auf ordnungspolitische Änderungen (z.B. gesetzliche Regelungen, Verordnun- } \\
\text { gen) zu reagieren. }\end{array}$ \\
\hline & $\begin{array}{l}\text { Wir suchen regelmäßig Kontakt mit denjenigen Personen und Organisationen, die Einfluss in den Marktstu- } \\
\text { fen haben, wo unsere Produkte weiterverarbeitet oder verwendet werden. }\end{array}$ \\
\hline & $\begin{array}{l}\text { *In unserer Geschäftseinheit pflegen wir Kontakte mit Vertretern aus Politik und Verwaltung, um sachdien- } \\
\text { liche Informationen zu sammeln und auszuwerten. }\end{array}$ \\
\hline & $\begin{array}{l}\text { *Grundsätzlich wissen wir, wer Einfluss auf die Kaufentscheidungen in den verschiedenen Marktstufen der } \\
\text { Wertschöpfungskette ausübt. }\end{array}$ \\
\hline & $\begin{array}{l}\text { *In unserer Geschäftseinheit werten wir systematisch Veröffentlichungen und Stellungnahmen von ver- } \\
\text { schiedenen Industrieverbänden (z.B. IHK, VDI) aus. }\end{array}$ \\
\hline & *Wir beobachten aufmerksam die Verbandsarbeit in den Branchen unserer Abnehmer. \\
\hline \multirow{11}{*}{ 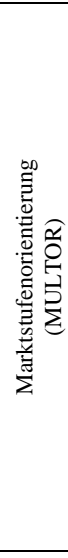 } & $\begin{array}{l}\text { *Der Weg unseres Produkts durch die nachgelagerten Vertriebs- und Weiterverarbeitungsstufen ist uns } \\
\text { bekannt. }\end{array}$ \\
\hline & $\begin{array}{l}\text { *Die Kunden unserer Kunden sind uns bekannt und werden bei der Entwicklung unserer Produkte berück- } \\
\text { sichtigt. }\end{array}$ \\
\hline & *Wir analysieren systematisch, wer die Kunden unserer Kunden sind. \\
\hline & $\begin{array}{l}\text { *Bei der Planung unserer Geschäftsstrategie berücksichtigen wir, wie wir einen Mehrwert für die Kunden } \\
\text { unserer Kunden auf nachgelagerten Marktstufen schaffen können. }\end{array}$ \\
\hline & $\begin{array}{l}\text { Wir erklären den Abnehmern auf nachgelagerten Marktstufen regelmäßig den Mehrwert unserer Produkte } \\
\text { (z.B. durch Produktbroschüren, Pressemitteilungen etc.). }\end{array}$ \\
\hline & Wir sammeln Informationen, um die Präferenzen der Letztverwender unserer Produkte einzuschätzen. \\
\hline & $\begin{array}{l}\text { Wir verfolgen den Weg unserer Produkte durch die verschiedenen Weiterverarbeitungsstufen bis zum } \\
\text { Endkunden. }\end{array}$ \\
\hline & $\begin{array}{l}\text { Wir reagieren schnell auf Aktionen von Firmen, die unsere Absatzchancen in nachgelagerten Marktstufen } \\
\text { bedrohen. }\end{array}$ \\
\hline & $\begin{array}{l}\text { *Die Geschäftsleitung berät regelmäßig über die Stärken und Strategien von Unternehmen, die beim } \\
\text { Endkunden dieselben Bedürfnisse/Funktionen erfüllen können wie unsere Produkte. }\end{array}$ \\
\hline & Wir beobachten, mit wem unsere Kunden im Wettbewerb stehen. \\
\hline & Wir beobachten, wo unsere Produkte letztlich zum Einsatz kommen. \\
\hline \multirow{8}{*}{ 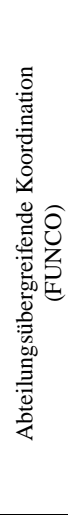 } & $\begin{array}{l}\text { *In unserer Geschäftseinheit findet ein reger Austausch von Marktinformationen zwischen verschiedenen } \\
\text { Abteilungen statt. }\end{array}$ \\
\hline & $\begin{array}{l}\text { *Alles in allem kooperieren die verschiedenen Abteilungen in unserer Geschäftseinheit eng miteinander, } \\
\text { wenn es um unsere Kunden geht. }\end{array}$ \\
\hline & $\begin{array}{l}\text { Bei uns findet ein reger Austausch zwischen den Abteilungsleitern über potenzielle und aktuelle Kunden } \\
\text { statt. }\end{array}$ \\
\hline & $\begin{array}{l}\text { Uns ist es wichtig, mit anderen Abteilungen über erfolgreiche und nicht erfolgreiche Erfahrungen mit } \\
\text { Kunden zu sprechen. }\end{array}$ \\
\hline & $\begin{array}{l}\text { Alle Abteilungen bei uns (z.B. Marketing/Vertrieb, Produktion, F\&E, Finanzen/Buchhaltung, etc.) sind an } \\
\text { der Erfüllung der Kundenwünsche auf unseren Zielmärkten beteiligt. }\end{array}$ \\
\hline & $\begin{array}{l}\text { Unsere Abteilungsleiter verstehen, wie alle gemeinsam in unserem Unternehmen dazu beitragen können, } \\
\text { einen Kundenwert zu schaffen. }\end{array}$ \\
\hline & $\begin{array}{l}\text { Alle Abteilungen teilen ihr Wissen und ihre Betriebsmittel mit anderen Abteilungen, um sich gegenseitig in } \\
\text { ihrer Arbeit zu unterstützen. }\end{array}$ \\
\hline & $\begin{array}{l}\text { *Wir teilen Marktinformationen mit allen relevanten Abteilungen (z.B. Marketing/Vertrieb, Produktion, } \\
\text { F\&E, Finanzen/Buchhaltung, etc.). }\end{array}$ \\
\hline & *Item, \\
\hline
\end{tabular}


Anhang 3: Ergebnisse des Chi-Quadrat-Differenz-Tests

\begin{tabular}{|c|c|c|c|}
\hline & \multicolumn{2}{|c|}{$\chi^{2}-M_{u}=450,36$} & \multirow{2}{*}{$\begin{array}{c}5 \text { \% Signifikanz-Niveau } \\
3,841\end{array}$} \\
\hline & $\chi^{2}-\mathrm{M}_{\mathrm{r}}$ & $\chi^{2}$-Differenz & \\
\hline $\operatorname{cov}($ Custor, Compor) $=1$ & 753,66 & 288,99 & ok \\
\hline $\operatorname{cov}($ Custor, Funco) = 1 & 750,59 & 285,92 & ok \\
\hline $\operatorname{cov}($ Custor, Partor $)=1$ & 724,58 & 259,91 & ok \\
\hline $\operatorname{cov}($ Custor, Multor $)=1$ & 919,99 & 455,32 & ok \\
\hline $\operatorname{cov}($ Compor, Funco $)=1$ & 732,40 & 267,73 & ok \\
\hline cov (Compor, Partor) = 1 & 624,17 & 159,49 & ok \\
\hline $\operatorname{cov}($ Compor, Multor $)=1$ & 795,48 & 330,81 & ok \\
\hline $\operatorname{cov}($ Funco, Multor $)=1$ & 1023,33 & 558,65 & ok \\
\hline $\operatorname{cov}($ Funco, Partor $)=1$ & 719,60 & 254,93 & ok \\
\hline $\operatorname{cov}($ Partor, Multor $)=1$ & 673,04 & 208,36 & ok \\
\hline
\end{tabular}


Anhang 4: Ergebnisse des Tests auf Normalverteilung

\begin{tabular}{|c|c|c|c|c|}
\hline Variable & Schiefe & $\begin{array}{l}\text { Kritisches } \\
\text { Verhältnis }\end{array}$ & Wölbung & $\begin{array}{l}\text { Kritisches } \\
\text { Verhältnis }\end{array}$ \\
\hline custor1 & $-0,374$ & $-2,994$ & $-0,311$ & $-1,243$ \\
\hline custor2 & $-0,295$ & $-2,360$ & $-0,760$ & $-3,042$ \\
\hline custor3 & $-0,798$ & $-6,383$ & 0,585 & 2,338 \\
\hline custor4 & $-0,849$ & $-6,794$ & 0,155 & 0,622 \\
\hline custor5 & $-1,330$ & $-10,643$ & 2,347 & 9,389 \\
\hline compor1 & $-0,367$ & $-2,939$ & $-0,651$ & $-2,603$ \\
\hline compor2 & 0,054 & 0,434 & $-0,759$ & $-3,034$ \\
\hline compor3 & $-0,168$ & $-1,343$ & $-1,045$ & $-4,180$ \\
\hline compor4 & $-0,464$ & $-3,709$ & $-0,657$ & $-2,627$ \\
\hline compor5 & $-0,485$ & $-3,880$ & $-0,631$ & $-2,524$ \\
\hline partor2 & $-0,916$ & $-7,326$ & 0,036 & 0,142 \\
\hline partor3 & $-0,520$ & $-4,160$ & $-0,584$ & $-2,336$ \\
\hline partor4 & $-0,840$ & $-6,721$ & $-0,217$ & $-0,868$ \\
\hline partor5 & $-0,876$ & $-7,006$ & 0,095 & 0,379 \\
\hline multor1 & $-0,669$ & $-5,355$ & $-0,430$ & $-1,719$ \\
\hline multor2 & $-0,712$ & $-5,695$ & $-0,507$ & $-2,028$ \\
\hline multor3 & $-0,893$ & $-7,142$ & $-0,371$ & $-1,483$ \\
\hline multor4 & $-0,809$ & $-6,471$ & $-0,090$ & $-0,359$ \\
\hline multor5 & $-0,244$ & $-1,950$ & $-0,887$ & $-3,546$ \\
\hline funco1 & $-0,163$ & $-1,301$ & $-0,895$ & $-3,579$ \\
\hline funco2 & $-0,374$ & $-2,994$ & $-0,717$ & $-2,867$ \\
\hline funco3 & $-0,447$ & $-3,577$ & $-0,677$ & $-2,708$ \\
\hline funco4 & $-0,286$ & $-2,291$ & $-0,739$ & $-2,955$ \\
\hline funco5 & $-0,076$ & $-0,606$ & $-0,751$ & $-3,002$ \\
\hline mo1 & $-0,691$ & $-5,526$ & 0,158 & 0,633 \\
\hline mo2 & $-0,570$ & $-4,558$ & $-0,005$ & $-0,021$ \\
\hline mo3 & $-0,745$ & $-5,958$ & $-0,006$ & $-0,023$ \\
\hline mo4 & $-0,724$ & $-5,790$ & 0,000 & $-0,001$ \\
\hline Multivariate & & & 149,429 & 35,720 \\
\hline
\end{tabular}


Fortsetzung Anhang 4: Ergebnisse des Tests auf Normalverteilung

Als substanzielle Verletzung einer univariaten Normalverteilung werden Schiefe- und Wölbungsmaße angesehen, die Beträge $|>2|$ bzw. $|>7|$ übersteigen. ${ }^{705}$ Das kritische Verhältnis, spiegelt das Verhältnis zwischen Wölbungs- bzw. Schiefemaßen und den jeweiligen Standardfehlern wider und sollte für eine strenge Auslegung auf einem Signifikanzniveau von $5 \%$ den Betrag $|1,96|$ nicht überschreiten. Die Prüfung der multivariaten Variablengesamtheit erfolgt anhand von Mardia's Koeffizient der multivariaten Wölbung. ${ }^{706}$ Werte $|>1,96|$ lassen auf eine Verletzung der Annahme einer multivariaten Normalverteilung schließen. ${ }^{707}$

705 Vgl. West et al. 1995, S. 74; TEMME/HILDEBRANDT (2009, S. 166) sehen bereits Werte $>1 \mid$ für Schiefe und Wölbung als Verletzung der Normalverteilung an. Diese strengeren Kriterien werden bei 26 der insgesamt 28 Indikatoren eingehalten.

${ }^{706}$ Vgl. Mardia 1970, S. $524 \mathrm{ff}$.

${ }^{707}$ Vgl. Weiber/Mühlhaus 2010, S. 147 f. 
Anhang 5: Ergebnisse der Prüfung der coMO-Skala auf Messäquivalenz in Abhängigkeit der Voraussetzungen für das Mehrstufige Marketing

\begin{tabular}{|c|c|c|c|c|c|c|c|c|c|c|c|c|}
\hline & & Gruppenvariable & \multicolumn{2}{|c|}{ req1 } & \multicolumn{2}{|c|}{ req2 } & \multicolumn{2}{|c|}{ req3 } & \multicolumn{2}{|c|}{ req4 } & \multicolumn{2}{|c|}{ BuyPower } \\
\hline Konstrukt & Item & Ausprägung & $\mathrm{n}$ & $\mathrm{h}$ & $\mathrm{n}$ & $\mathrm{h}$ & $\mathrm{n}$ & $\mathrm{h}$ & $\mathrm{n}$ & $\mathrm{h}$ & $\mathrm{n}$ & $\mathrm{h}$ \\
\hline \multirow{15}{*}{ 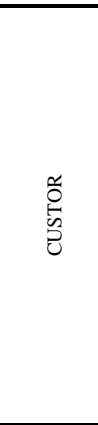 } & \multirow{3}{*}{ custor1 } & Faktorladung & 0,780 & 0,775 & 0,750 & 0,802 & 0,725 & 0,806 & 0,784 & 0,766 & 0,747 & 0,793 \\
\hline & & $\mathrm{t}$-Wert & \multicolumn{2}{|c|}{0,170} & \multicolumn{2}{|c|}{$-1,939$} & \multicolumn{2}{|c|}{$-3,094$} & \multicolumn{2}{|c|}{0,586} & \multicolumn{2}{|c|}{$-1,868$} \\
\hline & & Sig.-Niveau & \multicolumn{2}{|c|}{ n.s. } & \multicolumn{2}{|c|}{ * } & \multicolumn{2}{|c|}{$* * *$} & \multicolumn{2}{|c|}{ n.s. } & \multicolumn{2}{|c|}{ * } \\
\hline & \multirow{3}{*}{ custor2 } & Faktorladung & 0,843 & 0,771 & 0,819 & 0,776 & 0,808 & 0,825 & \begin{tabular}{l|l|}
0,817 \\
\end{tabular} & 0,802 & 0,827 & 0,813 \\
\hline & & $t$-Wert & \multicolumn{2}{|c|}{1,941} & \multicolumn{2}{|c|}{1,464} & \multirow{2}{*}{\multicolumn{2}{|c|}{$\frac{-0,671}{\text { n.s. }}$}} & 0,5 & 47 & & 524 \\
\hline & & Sig.-Niveau & & & & & & & $\mathrm{n}$. & & & s. \\
\hline & & Faktorladung & 0,853 & 0,791 & 0,861 & 0,831 & 0,857 & 0,792 & 0,845 & 0,834 & 0,844 & 0,845 \\
\hline & custor3 & $\mathrm{t}$-Wert & & & & & 2,0 & & 0,3 & & & 061 \\
\hline & & Sig.-Niveau & & & & & * & & $\mathrm{n}$. & & & s. \\
\hline & & Faktorladung & 0,873 & 0,813 & 0,877 & 0,814 & 0,857 & 0,839 & 0,848 & 0,869 & 0,856 & 0,842 \\
\hline & custor4 & $\mathrm{t}$-Wert & & & & & 0,6 & & $-0,7$ & 31 & & 598 \\
\hline & & Sig.-Niveau & & & & & $\mathrm{n}$. & & $\mathrm{n}$. & & & s. \\
\hline & & Faktorladung & 0,797 & 0,787 & 0,797 & 0,794 & 0,807 & 0,717 & 0,781 & 0,827 & 0,800 & 0,826 \\
\hline & custor5 & $\mathrm{t}$-Wert & & & & & 2,9 & & $-1,4$ & 175 & & 996 \\
\hline & & Sig.-Niveau & & & & & *** & & $\mathrm{n}$. & & & s. \\
\hline & & Faktorladung & 0,800 & 0,729 & 0,793 & 0,657 & 0,758 & 0,781 & 0,767 & 0,705 & 0,753 & \begin{tabular}{|l}
0,759 \\
\end{tabular} \\
\hline & compor1 & $\mathrm{t}$-Wert & & & & & -0, & & 1,7 & & & 183 \\
\hline & & Sig.-Niveau & & & & & $\mathrm{n}$. & & * & & & s. \\
\hline & & Faktorladung & 0,792 & 0,767 & 0,763 & 0,744 & 0,773 & 0,796 & 0,743 & 0,787 & 0,781 & 0,704 \\
\hline & compor2 & $\mathrm{t}$-Wert & & & & & $-0,8$ & & $-1,6$ & 06 & & 407 \\
\hline & & Sig.-Niveau & & & & & $\mathrm{n}$. & & $\mathrm{n}$. & & & $*$ \\
\hline ชี & & Faktorladung & 0,811 & 0,792 & 0,796 & 0,820 & 0,815 & 0,845 & 0,824 & 0,801 & 0,807 & 0,806 \\
\hline$\sum_{i}^{n}$ & compor3 & $\mathrm{t}$-Wert & & & & & -0, & & 0,7 & & & 31 \\
\hline 8 & & Sig.-Niveau & & & & & n. & & $\mathrm{n}$ & & & s. \\
\hline & & Faktorladung & 0,873 & 0,759 & 0,833 & 0,820 & 0,865 & 0,821 & 0,844 & 0,813 & 0,844 & 0,825 \\
\hline & compor4 & $\mathrm{t}$-Wert & & & & & 1,5 & & 1,0 & & & 662 \\
\hline & & Sig.-Niveau & & & & & $\mathrm{n}$. & & $\mathrm{n}$. & & & s. \\
\hline & & Faktorladung & 0,866 & 0,720 & 0,832 & \begin{tabular}{|l|l|}
0,817 \\
\end{tabular} & 0,847 & 0,816 & 0,821 & 0,845 & 0,843 & 0,813 \\
\hline & compor5 & $\mathrm{t}$-Wert & & & & & 1,0 & & $-0,7$ & & & 84 \\
\hline & & Sig.-Niveau & & & & & $\mathrm{n}$. & & $\mathrm{n}$. & & & s. \\
\hline & & Faktorladung & 0,805 & 0,741 & 0,824 & 0,682 & 0,801 & 0,823 & 0,791 & 0,792 & 0,774 & \begin{tabular}{|l}
0,807 \\
\end{tabular} \\
\hline & partor2 & $\mathrm{t}$-Wert & & & & & -0 & & $-0,0$ & & & 709 \\
\hline & & Sig.-Niveau & & & & & $\mathrm{n}$. & & $\mathrm{n}$ & & & s. \\
\hline & & Faktorladung & 0,766 & 0,803 & 0,821 & 0,743 & 0,812 & 0,715 & 0,789 & 0,780 & 0,702 & 0,810 \\
\hline & partor3 & $\mathrm{t}$-Wert & & & & & 2,0 & & 0,2 & & & 333 \\
\hline 요 & & Sig.-Niveau & & & & & * & & $\mathrm{n}$. & & & * \\
\hline$\frac{2}{4}$ & & Faktorladung & 0,863 & 0,721 & 0,875 & 0,765 & 0,829 & 0,825 & 0,846 & 0,835 & 0,835 & 0,790 \\
\hline$a^{2}$ & partor4 & t-Wert & & & & & 0,1 & & 0,2 & & & 211 \\
\hline & & Sig.-Niveau & & & & & $\mathrm{n}$. & & $\mathrm{n}$. & & & s. \\
\hline & & Faktorladung & 0,780 & 0,720 & 0,805 & 0,687 & 0,841 & 0,663 & 0,801 & 0,739 & 0,762 & \begin{tabular}{|l}
0,749 \\
\end{tabular} \\
\hline & partor5 & $\mathrm{t}$-Wert & & & & & 3,9 & & 1,6 & & & 269 \\
\hline & & Sig.-Niveau & & & & & *** & & $\mathrm{n}$. & & & s. \\
\hline
\end{tabular}


Fortsetzung Anhang 5: Ergebnisse der Prüfung der coMO-Skala auf Messäquivalenz in Abhängigkeit der Voraussetzungen für das Mehrstufige Marketing

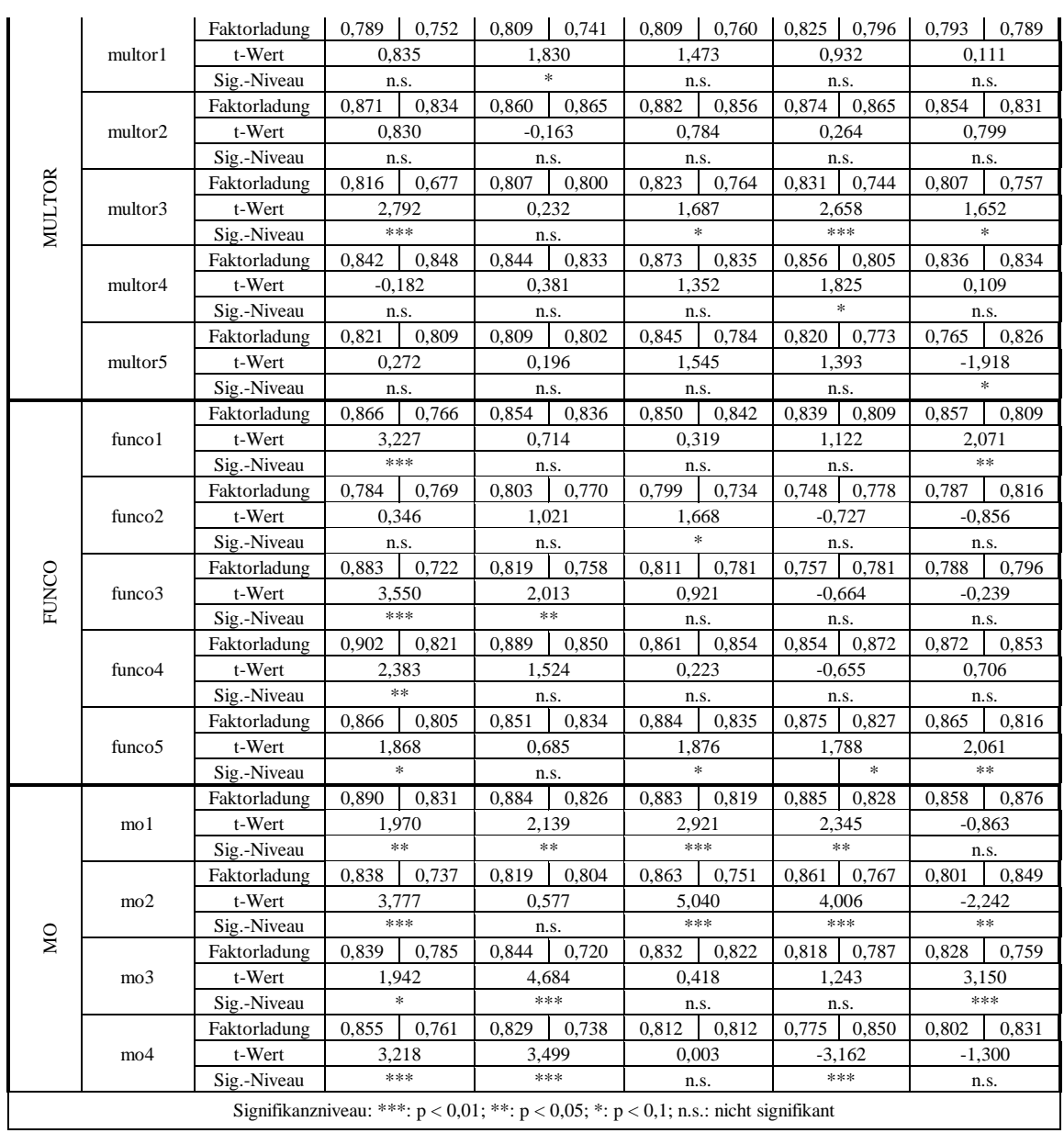


Anhang 6: Ergebnisse der Prüfung der coMO-Skala auf Messäquivalenz in Abhängigkeit der Position in der Wertschöpfungskette

\begin{tabular}{|c|c|c|c|c|c|c|c|c|}
\hline & & Gruppenvariable & MaStage & & MaStage & & MaStage & \\
\hline Konstrukt & Item & Ausprägung & $\begin{array}{l}1 \mathrm{Zwi}- \\
\text { schen- } \\
\text { stufe }\end{array}$ & $\begin{array}{l}>2 \text { Zwi- } \\
\text { schen- } \\
\text { stufen }\end{array}$ & $\begin{array}{l}\text { Keine } \\
\text { Zwi- } \\
\text { schen- } \\
\text { stufe }\end{array}$ & $\begin{array}{l}1 \text { Zwi- } \\
\text { schen- } \\
\text { stufe }\end{array}$ & $\begin{array}{l}\text { Keine } \\
\text { Zwi- } \\
\text { schen- } \\
\text { stufe }\end{array}$ & $\begin{array}{l}>2 \text { Zwi- } \\
\text { schen- } \\
\text { stufen }\end{array}$ \\
\hline \multirow{15}{*}{ 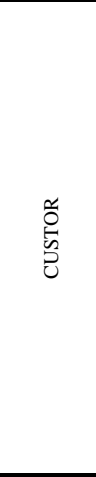 } & \multirow{3}{*}{ custor1 } & Faktorladung & 0,75 & 0,764 & 0,807 & 0,75 & 0,807 & 0,764 \\
\hline & & $\mathrm{t}$-Wert & & $-0,34$ & & 1,145 & & 0,987 \\
\hline & & Sig.-Niveau & n.s. & & n.s. & & n.s. & \\
\hline & \multirow{3}{*}{ custor2 } & Faktorladung & 0,855 & 0,88 & 0,836 & 0,855 & 0,836 & 0,88 \\
\hline & & t-Wert & & $-0,646$ & & $-0,408$ & & $-0,954$ \\
\hline & & Sig.-Niveau & n.s. & & n.s. & & n.s. & \\
\hline & \multirow{3}{*}{ custor3 } & Faktorladung & 0,871 & 0,835 & 0,798 & 0,871 & 0,798 & 0,835 \\
\hline & & t-Wert & & 1,048 & & $-1,666$ & & $-0,888$ \\
\hline & & Sig.-Niveau & n.s. & & $*$ & & n.s. & \\
\hline & \multirow{3}{*}{ custor4 } & Faktorladung & 0,832 & 0,891 & 0,857 & 0,832 & 0,857 & 0,891 \\
\hline & & t-Wert & & $-1,514$ & & 0,631 & & $-0,958$ \\
\hline & & Sig.-Niveau & n.s. & & n.s. & & n.s. & \\
\hline & \multirow{3}{*}{ custor5 } & Faktorladung & 0,855 & 0,864 & 0,783 & 0,855 & 0,783 & 0,864 \\
\hline & & $\mathrm{t}$-Wert & & $-0,248$ & & $-1,3$ & & $-1,637$ \\
\hline & & Sig.-Niveau & n.s. & & n.s. & & n.s. & \\
\hline \multirow{15}{*}{ 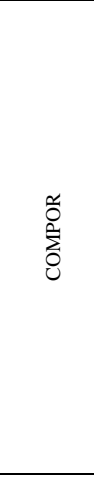 } & \multirow{3}{*}{ compor1 } & Faktorladung & 0,797 & 0,57 & 0,701 & 0,797 & 0,701 & 0,57 \\
\hline & & $\mathrm{t}$-Wert & & 2,797 & & $-1,155$ & & 1,836 \\
\hline & & Sig.-Niveau & $* * *$ & & n.s. & & $*$ & \\
\hline & \multirow{3}{*}{ compor2 } & Faktorladung & 0,727 & 0,806 & 0,751 & 0,727 & 0,751 & 0,806 \\
\hline & & $\mathrm{t}$-Wert & & $-1,434$ & & 0,38 & & $-1,15$ \\
\hline & & Sig.-Niveau & n.s. & & n.s. & & n.s. & \\
\hline & \multirow{3}{*}{ compor3 } & Faktorladung & 0,783 & 0,814 & 0,796 & 0,783 & 0,796 & 0,814 \\
\hline & & $\mathrm{t}$-Wert & & $-0,553$ & & 0,24 & & $-0,356$ \\
\hline & & Sig.-Niveau & n.s. & & n.s. & & n.s. & \\
\hline & \multirow{3}{*}{ compor4 } & Faktorladung & 0,831 & 0,849 & 0,828 & 0,831 & 0,828 & 0,849 \\
\hline & & $\mathrm{t}$-Wert & & $-0,291$ & & $-0,058$ & & $-0,364$ \\
\hline & & Sig.-Niveau & n.s. & & n.s. & & n.s. & \\
\hline & \multirow{3}{*}{ compor5 } & Faktorladung & 0,786 & 0,811 & 0,75 & 0,786 & 0,75 & 0,811 \\
\hline & & $\mathrm{t}$-Wert & & $-0,445$ & & $-0,587$ & & $-1,15$ \\
\hline & & Sig.-Niveau & n.s. & & n.s. & & n.s. & \\
\hline \multirow{12}{*}{ 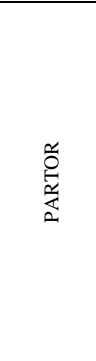 } & \multirow{3}{*}{ partor2 } & Faktorladung & 0,835 & 0,73 & 0,783 & 0,835 & 0,783 & 0,73 \\
\hline & & $\mathrm{t}$-Wert & & 1,358 & & $-0,834$ & & 0,642 \\
\hline & & Sig.-Niveau & n.s. & & n.s. & & n.s. & \\
\hline & \multirow{3}{*}{ partor3 } & Faktorladung & 0,782 & 0,749 & 0,773 & 0,782 & 0,773 & 0,749 \\
\hline & & $\mathrm{t}$-Wert & & 0,422 & & $-0,134$ & & 0,297 \\
\hline & & Sig.-Niveau & n.s. & & n.s. & & n.s. & \\
\hline & \multirow{3}{*}{ partor4 } & Faktorladung & 0,843 & 0,785 & 0,85 & 0,843 & 0,85 & 0,785 \\
\hline & & $\mathrm{t}$-Wert & & 0,914 & & 0,13 & & 1,131 \\
\hline & & Sig.-Niveau & n.s. & & n.s. & & n.s. & \\
\hline & \multirow{3}{*}{ partor5 } & Faktorladung & 0,626 & 0,815 & 0,781 & 0,626 & 0,781 & 0,815 \\
\hline & & $\mathrm{t}$-Wert & & $-2,509$ & & 2,058 & & $-0,419$ \\
\hline & & Sig.-Niveau & $* *$ & & *** & & n.s. & \\
\hline
\end{tabular}


Fortsetzung Anhang 6: Ergebnisse der Prüfung der coMO-Skala aufMessäquivalenz in Abhängigkeit der Position in der Wertschöpfungskette

\begin{tabular}{|c|c|c|c|c|c|c|c|c|}
\hline \multirow{15}{*}{ 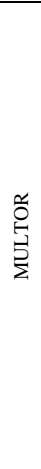 } & \multirow{3}{*}{ multor1 } & Fa ktorladung & 0,868 & 0,812 & 0,716 & 0,868 & 0,716 & 0,812 \\
\hline & & t-Wert & \multicolumn{2}{|c|}{1,591} & \multicolumn{2}{|c|}{$-1,778$} & \multicolumn{2}{|c|}{$-1,104$} \\
\hline & & Sig.-Niveau & \multicolumn{2}{|c|}{ n.s. } & \multicolumn{2}{|c|}{ * } & \multicolumn{2}{|c|}{ n.s. } \\
\hline & \multirow{3}{*}{ multor2 } & Faktorladung & 0,927 & 0,883 & 0,866 & 0,927 & 0,866 & 0,883 \\
\hline & & t-Wert & \multicolumn{2}{|c|}{1,833} & \multicolumn{2}{|c|}{$-0,724$} & \multicolumn{2}{|c|}{$-0,193$} \\
\hline & & Sig.-Niveau & \multicolumn{2}{|c|}{ * } & \multicolumn{2}{|c|}{ n.s. } & \multicolumn{2}{|c|}{ n.s. } \\
\hline & \multirow{3}{*}{ multor3 } & Faktorladung & 0,834 & 0,83 & 0,602 & 0,834 & 0,602 & 0,83 \\
\hline & & t-Wert & \multicolumn{2}{|c|}{0,114} & \multicolumn{2}{|c|}{$-2,099$} & \multicolumn{2}{|c|}{$-2,043$} \\
\hline & & Sig.-Niveau & \multicolumn{2}{|c|}{ n.s. } & \multicolumn{2}{|c|}{ ** } & \multicolumn{2}{|c|}{ *** } \\
\hline & \multirow{3}{*}{ multor4 } & Faktorladung & 0,886 & 0,867 & 0,808 & 0,886 & 0,808 & 0,867 \\
\hline & & t-Wert & \multicolumn{2}{|c|}{$\begin{array}{c}1 \\
0,555 \\
\end{array}$} & & & & \\
\hline & & Sig.-Niveau & & & & & & \\
\hline & & Faktorladung & 0,808 & 0,824 & 0,829 & 0,808 & 0,829 & 0,824 \\
\hline & multor5 & $\mathrm{t}$-Wert & & & & & & \\
\hline & & Sig.-Niveau & & & & & & \\
\hline & & Faktorladung & 0,885 & 0,866 & 0,787 & 0,885 & 0,787 & 0,866 \\
\hline & funco1 & $\mathrm{t}$-Wert & & & & & & \\
\hline & & Sig.-Niveau & & & & & & \\
\hline & & Faktorladung & 0,771 & 0,744 & 0,822 & 0,771 & 0,822 & 0,744 \\
\hline & funco2 & $\mathrm{t}$-Wert & & & & & & \\
\hline & & Sig.-Niveau & & & & & & \\
\hline & & Faktorladung & 0,834 & 0,768 & 0,78 & 0,834 & 0,78 & 0,768 \\
\hline z & funco3 & $\mathrm{t}$-Wert & & & & & & \\
\hline & & Sig.-Niveau & & & & & & \\
\hline & & Faktorladung & 0,921 & 0,869 & 0,83 & 0,921 & 0,83 & 0,869 \\
\hline & funco4 & $\mathrm{t}$-Wert & & & & & & \\
\hline & & Sig.-Niveau & & & & & & \\
\hline & & Faktorladung & 0,89 & 0,783 & 0,825 & 0,89 & 0,825 & 0,783 \\
\hline & funco5 & t-Wert & & & & & & \\
\hline & & Sig.-Niveau & & & & & & \\
\hline & & Faktorladung & 0,865 & 0,865 & 0,846 & 0,865 & 0,846 & 0,865 \\
\hline & mo1 & $\mathrm{t}$-Wert & & & & & & \\
\hline & & Sig.-Niveau & & & & & & \\
\hline & & Faktorladung & 0,749 & 0,863 & 0,743 & 0,749 & 0,743 & 0,863 \\
\hline & mo2 & t-Wert & & & & & & \\
\hline$\varrho$ & & Sig.-Niveau & & & & & & \\
\hline$\Sigma$ & & Faktorladung & 0,858 & 0,809 & 0,773 & 0,858 & 0,773 & 0,809 \\
\hline & mo3 & t-Wert & & & & & & \\
\hline & & Sig.-Niveau & & & & & & \\
\hline & & Faktorladung & 0,815 & 0,828 & 0,789 & 0,815 & 0,789 & 0,828 \\
\hline & mo4 & $\mathrm{t}$-Wert & & & & & & \\
\hline & & Sig.-Niveau & & & & & & \\
\hline
\end{tabular}


Anhang 7: Ergebnisse der Prüfung der coMO-Skala auf Messäquivalenz in Abhängigkeit der Abteilungszugehörigkeit

\begin{tabular}{|c|c|c|c|c|}
\hline & & Variable der Gruppenaufteilung & Abteilung & \\
\hline Konstrukt & Item & Ausprägung & Vertrieb & Marketing \\
\hline \multirow{15}{*}{ 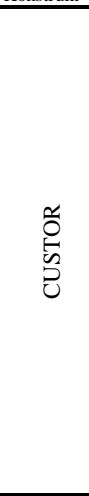 } & \multirow{3}{*}{ custor1 } & Faktorladung & 0,775 & 0,76 \\
\hline & & t-Wert & \multicolumn{2}{|c|}{0,353} \\
\hline & & Sig.-Niveau & \multicolumn{2}{|c|}{ n.s. } \\
\hline & \multirow{3}{*}{ custor2 } & Faktorladung & 0,813 & 0,746 \\
\hline & & $\mathrm{t}$-Wert & \multicolumn{2}{|c|}{1,526} \\
\hline & & Sig.-Niveau & \multicolumn{2}{|c|}{ n.s. } \\
\hline & \multirow{3}{*}{ custor3 } & Faktorladung & 0,85 & 0,79 \\
\hline & & t-Wert & \multicolumn{2}{|c|}{1,41} \\
\hline & & Sig.-Niveau & \multicolumn{2}{|c|}{ n.s. } \\
\hline & \multirow{3}{*}{ custor4 } & Faktorladung & 0,837 & 0,835 \\
\hline & & $\mathrm{t}$-Wert & \multicolumn{2}{|c|}{0,045} \\
\hline & & Sig.-Niveau & \multicolumn{2}{|c|}{ n.s. } \\
\hline & \multirow{3}{*}{ custor5 } & Faktorladung & 0,795 & 0,827 \\
\hline & & t-Wert & \multicolumn{2}{|c|}{$-0,889$} \\
\hline & & Sig.-Niveau & \multicolumn{2}{|c|}{ n.s. } \\
\hline \multirow{15}{*}{ 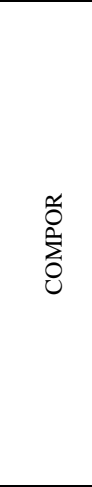 } & \multirow{3}{*}{ compor1 } & Faktorladung & 0,807 & 0,772 \\
\hline & & $\mathrm{t}$-Wert & \multicolumn{2}{|c|}{0,823} \\
\hline & & Sig.-Niveau & \multicolumn{2}{|c|}{ n.s. } \\
\hline & \multirow{3}{*}{ compor2 } & Faktorladung & 0,738 & 0,743 \\
\hline & & t-Wert & \multicolumn{2}{|c|}{$-0,122$} \\
\hline & & Sig.-Niveau & \multicolumn{2}{|c|}{ n.s. } \\
\hline & \multirow{3}{*}{ compor3 } & Faktorladung & 0,845 & 0,814 \\
\hline & & $\mathrm{t}$-Wert & \multicolumn{2}{|c|}{0,798} \\
\hline & & Sig.-Niveau & & 1.S. \\
\hline & & Faktorladung & 0,836 & 0,836 \\
\hline & compor4 & $\mathrm{t}$-Wert & & 0 \\
\hline & & Sig.-Niveau & & 1.s. \\
\hline & & Faktorladung & 0,792 & 0,868 \\
\hline & compor5 & $\mathrm{t}$-Wert & & 151 \\
\hline & & Sig.-Niveau & & ** \\
\hline & & Faktorladung & 0,693 & 0,81 \\
\hline & partor2 & t-Wert & & 036 \\
\hline & & Sig.-Niveau & & $* *$ \\
\hline & & Faktorladung & 0,742 & 0,797 \\
\hline & partor3 & $\mathrm{t}$-Wert & & 852 \\
\hline$\stackrel{0}{\ominus}$ & & Sig.-Niveau & & i.s. \\
\hline 产 & & Faktorladung & 0,796 & 0,827 \\
\hline 2 & partor4 & $\mathrm{t}$-Wert & & 632 \\
\hline & & Sig.-Niveau & & i.s. \\
\hline & & Faktorladung & 0,817 & 0,736 \\
\hline & partor5 & $\mathrm{t}$-Wert & & 365 \\
\hline & & Sig.-Niveau & & n.s. \\
\hline
\end{tabular}


Fortsetzung Anhang 7: Ergebnisse der Prüfung der coMO-Skala auf Messäquivalenz in Abhängigkeit der Abteilungszugehörigkeit

\begin{tabular}{|c|c|c|c|c|}
\hline \multirow{15}{*}{ 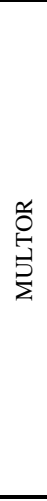 } & \multirow{3}{*}{ multor1 } & Faktorladung & 0,805 & 0,723 \\
\hline & & t-Wert & \multicolumn{2}{|c|}{1,725} \\
\hline & & Sig.-Niveau & \multicolumn{2}{|c|}{$*$} \\
\hline & \multirow{3}{*}{ multor2 } & Faktorladung & 0,887 & 0,815 \\
\hline & & t-Wert & \multicolumn{2}{|c|}{1,838} \\
\hline & & Sig.-Niveau & \multicolumn{2}{|c|}{$*$} \\
\hline & \multirow{3}{*}{ multor3 } & Faktorladung & 0,861 & 0,712 \\
\hline & & t-Wert & \multicolumn{2}{|c|}{3,859} \\
\hline & & Sig.-Niveau & \multicolumn{2}{|c|}{$* * *$} \\
\hline & \multirow{3}{*}{ multor4 } & Faktorladung & 0,879 & 0,843 \\
\hline & & t-Wert & \multicolumn{2}{|c|}{1,028} \\
\hline & & Sig.-Niveau & \multicolumn{2}{|c|}{ n.s. } \\
\hline & \multirow{3}{*}{ multor5 } & Faktorladung & 0,806 & 0,831 \\
\hline & & $\mathrm{t}$-Wert & \multicolumn{2}{|c|}{$-0,491$} \\
\hline & & Sig.-Niveau & \multicolumn{2}{|c|}{ n.s. } \\
\hline \multirow{15}{*}{ 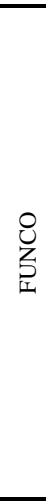 } & \multirow{3}{*}{ funco1 } & Faktorladung & 0,768 & 0,819 \\
\hline & & t-Wert & \multicolumn{2}{|c|}{$-1,397$} \\
\hline & & Sig.-Niveau & \multicolumn{2}{|c|}{ n.s. } \\
\hline & \multirow{3}{*}{ funco2 } & Faktorladung & 0,773 & 0,846 \\
\hline & & t-Wert & \multicolumn{2}{|c|}{$-1,827$} \\
\hline & & Sig.-Niveau & \multicolumn{2}{|c|}{$*$} \\
\hline & \multirow{3}{*}{ funco3 } & Faktorladung & 0,716 & 0,802 \\
\hline & & t-Wert & \multicolumn{2}{|c|}{$\frac{1}{-1,695}$} \\
\hline & & Sig.-Niveau & \multicolumn{2}{|c|}{$*$} \\
\hline & & Faktorladung & 0,849 & 0,867 \\
\hline & funco4 & $\mathrm{t}$-Wert & & \\
\hline & & Sig.-Niveau & & \\
\hline & & Faktorladung & 0,875 & 0,843 \\
\hline & funco5 & t-Wert & & \\
\hline & & Sig.-Niveau & & \\
\hline & & Faktorladung & 0,877 & 0,843 \\
\hline & mo1 & $\mathrm{t}$-Wert & & \\
\hline & & Sig.-Niveau & & \\
\hline & & Faktorladung & 0,859 & 0,826 \\
\hline & mo2 & t-Wert & & \\
\hline$\underline{0}$ & & Sig.-Niveau & & \\
\hline$\Sigma$ & & Faktorladung & 0,835 & 0,753 \\
\hline & mo3 & $\mathrm{t}$-Wert & & \\
\hline & & Sig.-Niveau & & \\
\hline & & Faktorladung & 0,772 & 0,813 \\
\hline & mo4 & t-Wert & & \\
\hline & & Sig.-Niveau & & \\
\hline
\end{tabular}


Anhang 8: Ergebnisse der Prüfung der Konstrukte ALIGN und NPS auf Messäquivalenz in Abhängigkeit der Voraussetzungen für das Mehrstufige Marketing

\begin{tabular}{|c|c|c|c|c|c|c|c|c|c|c|c|c|}
\hline \multirow[b]{2}{*}{ Konstrukt } & \multirow[b]{2}{*}{ Item } & \multirow[b]{2}{*}{ Ausprägung } & \multicolumn{2}{|c|}{ req1 } & \multicolumn{2}{|c|}{ req2 } & \multicolumn{2}{|c|}{ req3 } & \multicolumn{2}{|c|}{ req 4} & \multicolumn{2}{|c|}{ BuyPower } \\
\hline & & & $\mathrm{n}$ & $\mathrm{h}$ & $\mathrm{n}$ & $\mathrm{h}$ & $\mathrm{n}$ & $\mathrm{h}$ & $\mathrm{n}$ & $\mathrm{h}$ & $\mathrm{n}$ & $\mathrm{h}$ \\
\hline \multirow{12}{*}{$\frac{Z}{Z}$} & \multirow{3}{*}{ align1 } & Faktorladung & 0,890 & 0,828 & 0,824 & 0,868 & 0,856 & 0,869 & 0,895 & 0,813 & 0,864 & 0,857 \\
\hline & & $\mathrm{t}$-Wert & \multicolumn{2}{|c|}{$-2,389$} & \multicolumn{2}{|c|}{1,619} & \multicolumn{2}{|c|}{0,531} & \multicolumn{2}{|c|}{$-2,958$} & \multicolumn{2}{|c|}{$-0,296$} \\
\hline & & Sig.-Niveau & \multicolumn{2}{|c|}{$* *$} & \multicolumn{2}{|c|}{ n.s. } & \multicolumn{2}{|c|}{ n.s. } & \multicolumn{2}{|c|}{$* * *$} & \multicolumn{2}{|c|}{ n.s. } \\
\hline & \multirow{3}{*}{ align2 } & Faktorladung & 0,856 & 0,782 & 0,803 & 0,834 & 0,816 & 0,842 & 0,885 & 0,754 & 0,828 & 0,820 \\
\hline & & t-Wert & \multicolumn{2}{|c|}{$-2,191$} & \multicolumn{2}{|c|}{1,001} & \multicolumn{2}{|c|}{0,905} & \multicolumn{2}{|c|}{$-3,852$} & \multicolumn{2}{|c|}{$-0,241$} \\
\hline & & Sig.-Niveau & \multicolumn{2}{|c|}{$* *$} & \multicolumn{2}{|c|}{ n.s. } & \multicolumn{2}{|c|}{ n.s. } & \multicolumn{2}{|c|}{$* * *$} & \multicolumn{2}{|c|}{ n.s. } \\
\hline & & Faktorladung & 0,764 & 0,851 & 0,781 & 0,841 & 0,822 & 0,775 & 0,774 & 0,822 & 0,793 & 0,827 \\
\hline & align3 & t-Wert & & & 1, & & -1 , & & & & & 80 \\
\hline & & Sig.-Niveau & & & $n$ & & & & & & & \\
\hline & & Faktorladung & 0,856 & 0,844 & 0,861 & 0,860 & 0,823 & 0,878 & 0,900 & 0,886 & 0,876 & 0,837 \\
\hline & align4 & $\mathrm{t}$-Wert & & & -0 , & & & & & & & 555 \\
\hline & & Sig.-Niveau & & & $\mathrm{n}$ & & & & & & & \\
\hline & & Faktorladung & 0,859 & 0,865 & 0,856 & 0,870 & 0,817 & 0,842 & 0,829 & 0,840 & 0,863 & 0,846 \\
\hline & nps1 & $\mathrm{t}$-Wert & & & 0, & & & & & & & 499 \\
\hline & & Sig.-Niveau & & & $n$ & & & & & & & \\
\hline & & Faktorladung & 0,904 & 0,792 & 0,896 & 0,767 & 0,827 & 0,854 & 0,829 & 0,858 & 0,832 & 0,872 \\
\hline 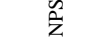 & nps2 & t-Wert & & & -4 , & & 0 , & & & & & \\
\hline & & Sig.-Niveau & & & * & & & & & & & \\
\hline & & Faktorladung & 0,850 & 0,809 & 0,861 & 0,776 & 0,789 & 0,826 & 0,755 & 0,803 & 0,803 & 0,825 \\
\hline & nps3 & t-Wert & & & -2 , & & & & & & & \\
\hline & & Sig.-Niveau & & & * & & & & $\mathrm{n}$ & & & \\
\hline & & Signifikanz & au: & $<0$, & $\mathrm{p}<$ & $5 ; *: \mathrm{p}$ &, $1 ; n$ & nich & fikant & & & \\
\hline
\end{tabular}


Anhang 9: Ergebnisse der Prüfung auf Mediation bei Gruppenbetrachtung

\begin{tabular}{|c|c|c|c|c|c|c|c|c|}
\hline $\begin{array}{c}\text { Gruppenvariable } \\
\text { (Ausprägung) }\end{array}$ & Beziehung & Pfadkoeffizient & $\mathrm{t}$-Wert & Signifikanz & $\begin{array}{c}\text { Standard- } \\
\text { fehler }\end{array}$ & z-Wert & Signifikanz & $\begin{array}{c}\text { Befund } \\
\text { (Mediation) }\end{array}$ \\
\hline \multirow{2}{*}{ Req1 (h) } & MO $\rightarrow$ ALIGN & 0,422 & 9,224 & $* * *$ & 0,046 & \multirow{2}{*}{2,764} & \multirow{2}{*}{$* * *$} & \multirow{2}{*}{$\sqrt{ }$} \\
\hline & ALIGN $\rightarrow$ NPS & 0,165 & 2,898 & $* * *$ & 0,057 & & & \\
\hline \multirow{2}{*}{ Req2 (h) } & MO $\rightarrow$ ALIGN & 0,483 & 14,155 & $* * *$ & 0,034 & \multirow{2}{*}{3,156} & \multirow{2}{*}{$* * *$} & \multirow{2}{*}{$\sqrt{ }$} \\
\hline & ALIGN $\rightarrow$ NPS & 0,139 & 3,237 & $* * *$ & 0,043 & & & \\
\hline \multirow{2}{*}{ Req3 (h) } & $\mathrm{MO} \rightarrow$ ALIGN & 0,489 & 12,368 & $* * *$ & 0,040 & \multirow{2}{*}{2,243} & \multirow{2}{*}{$* *$} & \multirow{2}{*}{$\sqrt{ }$} \\
\hline & ALIGN $\rightarrow$ NPS & 0,128 & 2,281 & $* *$ & 0,056 & & & \\
\hline \multirow{2}{*}{ Req4 (h) } & MO $\rightarrow$ ALIGN & 0,494 & 14,419 & $* * *$ & 0,034 & \multirow{2}{*}{1,282} & \multirow{2}{*}{ n.s. } & \multirow{2}{*}{$\mathrm{X}$} \\
\hline & ALIGN $\rightarrow$ NPS & 0,075 & 1,287 & n.s. & 0,058 & & & \\
\hline \multirow{2}{*}{ BuyPower (n) } & $\mathrm{MO} \rightarrow$ ALIGN & 0,563 & 16,270 & $* * *$ & 0,035 & \multirow{2}{*}{2,113} & \multirow{2}{*}{ ** } & \multirow{2}{*}{$\sqrt{ }$} \\
\hline & ALIGN $\rightarrow$ NPS & 0,108 & 2,131 & $* *$ & 0,051 & & & \\
\hline$\sqrt{ }=\mathrm{Hy}$ & $\begin{array}{r}\text { Signifika } \\
\text { empirisch ge }\end{array}$ & $\begin{array}{l}\text { iveau: ***: p } \\
\text { t; }(\sqrt{ })=\text { Hyp }\end{array}$ & $1 ; * *$ & $\begin{array}{l}0,05 ; *: p \\
\text { rch signifil }\end{array}$ & $\begin{array}{l}\text {, i n.s.: } \\
\text { en Pfad t }\end{array}$ & $\begin{array}{l}\text { ignifi } \\
\text { gt; } X\end{array}$ & . & ert \\
\hline
\end{tabular}


Anhang 10: Zusammenfassung der Indikatormessäquivalenz

\begin{tabular}{|c|c|c|c|}
\hline Konstrukt & Item & $\begin{array}{l}\text { Durchschnittlicher Betrag } \\
\text { der t-Werte }\end{array}$ & $\begin{array}{l}\text { Anzahl signifikanter Unter- } \\
\text { schiede (5 \%-Sig. Niveau) in } \\
\text { allen } 9 \text { Gruppenvergleichen }\end{array}$ \\
\hline \multirow{5}{*}{ 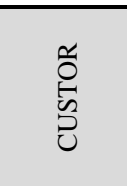 } & custor1 & 1,165 & 1 \\
\hline & custor2 & 0,976 & 0 \\
\hline & custor3 & 1,175 & 1 \\
\hline & custor4 & 1,028 & 1 \\
\hline & custor5 & 1,104 & 1 \\
\hline \multirow{5}{*}{$\sum_{0}^{\infty}$} & compor1 & 1,599 & 2 \\
\hline & compor2 & 1,012 & 1 \\
\hline & compor3 & 0,556 & 0 \\
\hline & compor4 & 0,798 & 1 \\
\hline & compor5 & 1,225 & 2 \\
\hline \multirow{4}{*}{ 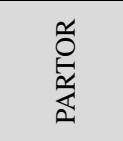 } & partor2 & 1,113 & 2 \\
\hline & partor3 & 0,893 & 2 \\
\hline & partor4 & 1,012 & 2 \\
\hline & partor5 & 1,744 & 4 \\
\hline \multirow{5}{*}{ 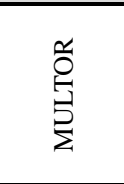 } & multor1 & 1,264 & 0 \\
\hline & multor2 & 0,825 & 0 \\
\hline & multor3 & 1,904 & 5 \\
\hline & multor4 & 0,854 & 0 \\
\hline & multor5 & 0,725 & 0 \\
\hline \multirow{5}{*}{$\begin{array}{l}0 \\
\text { Z } \\
S_{1}\end{array}$} & funco1 & 1,629 & 4 \\
\hline & funco2 & 1,083 & 0 \\
\hline & funco3 & 1,318 & 2 \\
\hline & funco 4 & 1,279 & 1 \\
\hline & funco5 & 1,629 & 2 \\
\hline \multirow{4}{*}{$\stackrel{O}{\Sigma}$} & mo1 & 1,376 & 4 \\
\hline & mo2 & 2,538 & 6 \\
\hline & mo3 & 2,067 & 4 \\
\hline & mo4 & 1,580 & 3 \\
\hline
\end{tabular}




\section{Literaturverzeichnis}

\section{A}

Akter, S., D'Ambra, J. \& Ray, P. (2011). An evaluation of PLS based complex models: the roles of power analysis, predictive relevance and GoF index. Proceedings of the 17th Americas Conference on Information Systems (AMCIS2011) (pp. 1-7). Detroit, USA: Association for Information Systems.

Albers, S. (2010). PLS and Success Factor Studies in Marketing. In V. E. Vinzi, W. W. Chin, J. Henseler, \& H. Wang (Eds.), Handbook of Partial Least Squares. Concepts, Methods and Applications, Berlin: Springer, S. 409-425.

Albers, S., \& Hildebrandt, L. (2006). Methodische Probleme bei der Erfolgsfaktorenforschung- Messfehler, formative versus reflektive Indikatoren und die Wahl des Strukturgleichungs-Modells. Schmalenbachs Zeitschrift Für Betriebswirtschaftliche Forschung, 58, 2-33.

Allred, C. R., Fawcett, S. E., Wallin, C., Magnan, G. M. (2011). A dynamic collaboration capability as a source of competitive advantage. Decision Sciences Journal, 42(1), 129-161.

Amabile, T. M. (1997). Motivating creativity in organizations: On doing what you love and loving what you do. California management review, 40(I), 3959.

Amit, R., Shoemaker, P.J.H. (1993). Strategic Assets and Organizational Rents. Strategic Management Journal, 14 (1), 33-46.

Armstrong, J., Collopy, F. (1996). Competitor Orientation: Effects of Objectives and Information on Managerial Decisions and Profitability. Journal of Marketing Research, 33(2), 188ᄀ199.

Arnott, N., (1994). Inside Intel's Marketing Coup. Sales \& Marketing Management, 146 (2), 78-81.

Auh, S., \& Merlo, O. (2012). The power of marketing within the firm: Its contribution to business performance and the effect of power asymmetry. Industrial Marketing Management, 41(5), 861-873. 
Avlonitis, G., Gounaris, S. (1997). Marketing orientation and company performance: industrial vs. consumer goods companies. Industrial Marketing Management, 26, 385-402.

Azuma, N. (2005). The paradox of competition in the world of volatility. Journal of Global Marketing, 18(1-2), 45-72.

B

Backhaus, K., Erichson, B., Weiber, R. (2011). Fortgeschrittene Multivariate Analysemethoden. Eine anwendungsorientierte Einführung, Berlin u.a.

Backhaus, K., \& Voeth, M. (2009). Industriegütermarketing, 9. Aufl., München: Vahlen.

Bagozzi, R. P. (1998). A Prospectus for Theory Construction in Marketing: Revisited and Revised. In: L. Hildebrandt, C. Homburg (Hrsg.): Die Kausalanalyse: Instrument der empirischen betriebswirtschaftlichen Forschung, Stuttgart, S. 45-81.

Bagozzi, R. P., Fornell, C. (1982). Theoretical Concepts, Measurements, and Meaning. In: Fornell, C. (Ed.): A Second Generation of Multivariate Analysis, Vol. 2, New York: Praeger Frederick, S. 24-38.

Bagozzi, R. P., \& Phillips, L. W. (1982). Representing and Testing Organizational Theories: A Holistic Construal. Administrative Science Quarterly, 27(3), 459-489.

Bagozzi, R. P., \& Yi, Y. (1988). On the evaluation of structural equation models. Journal of the Academy of Marketing Science, 16(1), 74-94.

Bagozzi, R., Yi, Y., \& Phillips, L. (1991). Assessing construct validity in organizational research. Administrative Science Quarterly, 36(1991), 421-458.

Baker, T. L., Simpson, P. M., Siguaw, J. A. (1999). The impact of suppliers' perceptions of reseller market orientation on key relationship constructs. Journal of the Academy of Marketing Science, 27(1), 50-57.

Baker, W. E., \& Sinkula, J. M. (1999). The Synergistic Effect of Market Orientation and Learning Orientation on Organizational Performance. Journal of the Academy of Marketing Science, 27(4), 411-427. 
Balakrishnan, S. (1996). Benefits of customer and competitive orientations in industrial markets. Industrial Marketing Management, 25(4), 257-269.

Banerjee, S., Lin, P. (2001). Vertical research joint ventures. International Journal of Industrial Organization, 19(1-2), 285-302.

Barney, J. B. (1986a). Strategic Factor Markets: Expectations, Luck, and Business Strategy. Management Science, 32(10), 1231-1241.

Barney, J. B. (1986b). Organizational Culture: Can It Be a Source of Sustained Competitive Advantage? The Academy of Management Review, 11(3), pp. 656-665.

Barney, J. B. (1991). Firm Resources and Sustained Competitive Advantage. Journal of Management, 17(1), 99-120.

Barney, J. B. (1992). Integrating organizational behavior and strategy formulation research: A resource-based analysis. In: P. Shrivastava, A. S. Huff, J. E. Dutton (Hrsg.): Advances in strategic management Greenwich (CT), USA, (pp. 39-61).

Barney, J. B. (1995). Looking inside for competitive advantage. Academy of Management Executive, 9(4), 49-61.

Barney, J. B. (2001). IS THE RESOURCE-BASED PERSPECTIVE MANAGEMENT “VIEW” A USEFUL FOR STRATEGIC RESEARCH? YES. The Academy of Management Review, 26(1), 41-56.

Baron, R. M., Kenny, D. A. (1986): The Moderator-Mediator Variable Distinction in Social Psychological Research: Conceptual, Strategic, and Statistical Considerations. Journal of Personality and Social Psychology, 51, 11731182.

Barratt, M. (2004). Understanding the meaning of collaboration in the supply chain. Supply Chain Management: An International Journal, 9(1), 30-42.

Baumgarth, C. (1998). Vertikale Marketingstrategien im Investitionsgüterbereich: dargestellt am Beispiel von Einsatzstoffen, Frankfurt am Main et al.

Bäuerle, T. (2000): Customer Focus Assessment, Wiesbaden.

Baxter, R. (2012). Chapter 5 Availability of Resources through Buyer-Seller Relationships. In Mark S. Glynn \& A. G. W. (Hrsg.), Business-to-Business 
Marketing Management: Strategies, Cases, and Solutions (Advances in Business Marketing and Purchasing, Volume 18) (pp. 119-140). Bingley (UK).

Becker,J.-M., Klein, K., Wetzels, M. (2012). Hierarchical Latent Variable Models in PLS-SEM: Guidelines for Using Reflective-Formative Type Models, Long Range Planning, 45(5-6), 359-394.

Bendle, N., \& Vandenbosch, M. (2014). Competitor Orientation and the Evolution of Business Markets. Marketing Science, 33(6), 781-795.

Bergkvist, L., \& Rossiter, J. R. (2007). The Predictive Validity of Multiple-Item Versus Single-Item Measures of the Same Constructs. Journal of Marketing Research (JMR), 44(2), 175-184.

Berndt, R. (2005). Marketingstrategie und Marketingpolitik. 4. Aufl., Berlin, Heidelberg.

Berthon, P., Hulbert, J. M., \& Pitt, L. F. (1999). To Serve or Create? STRATEGIC ORIENTATIONS TOWARD CUSTOMERS AND INNOVATION. California Management Review, 42(1), 37-58.

Besanko, D., Dranove, D., Schaefer, S., Shanley, M. (2010) Economics of Strategy. 5. Aufl., Hoboken.

Bigné, J. E., Blesa, A., Küster, I., \& Andreu, L. (2004). Market orientation: an antecedent to the industrial manufacturer's power. European Journal of Marketing, 38(1/2), 175-193.

Birn, R. J. (2000). The International Handbook of Market Research Techniques, 2. Aufl., London.

Bollen, K. A. (1989). Structural Equations with Latent Variables, New York.

Bonometti, R., \& Tang, J. (2006). A dynamic technique for conducting online survey-based research. Competitiveness Review, 16(2), 97-105.

Bowen, F. E., Rostami, M., Steel, P. (2010). Timing is everything: A metaanalysis of the relationships between organizational performance and innovation. Journal of Business Research, 63(11), 1179-1185.

Bresser, R. (2010). Strategische Managementtheorie. 2. Aufl., Stuttgart.

Bresser, R., Heuskel, D., \& Nixon, R. (2000). The Deconstruction of Integrated Value Chains: Practieal and Conceptual Challenges. In: Bresser, R., Hitt, M., 
Nixon R., Heuskel, D. (Eds.), Winning Strategies in a Deconstructing World, John Wi1ey: Chiehester-NewYork, pp. 1-21.

Brettel, M., Engelen, A., Heinemann, F., \& Vadhanasindhu, P. (2008). Antecedents of Market Orientation: A Cross-Cultural Comparison. Journal of International Marketing, 16(2), 84-119.

Brodie, R. J., Bonfrer, A., \& Cutler, J. (1996). Do managers ovęrreact to each others' promotional activity? Further empirical evidence. International Journal of Research in Marketing, 13(4), 379-387.

Buchanan, L. (1992). Vertical trade relationships: the role of dependence and symmetry in attaining organizational goals. Journal of Marketing Research, 29(February), 65-75.

Bucklin, L., \& Sengupta, S. (1993). Organizing successful co-marketing alliances. The Journal of Marketing, 57(April), 32-46.

Buzzell, R. D., Ortmeyer, G. (1995). Channel partnerships streamline distribution. Sloan Management Review, 36(3), 85-96.

\section{C}

Cachon, G. P., Fisher, M. (2000). Supply Chain Inventory Management and the Value of Shared Information. Management Science, 46(8), 1032-1048.

Cadogan, J. W., Diamantopoulos, A., \& Mortanges, C. P. de. (1999). A measure of export market orientation: scale development and cross-cultural validation. Journal of International Business Studies, 30(4), 689-707.

Cadogan, J., Kuivalainen, O., \& Sundqvist, S. (2009). Export Market-Oriented Behavior and Export Performance: Quadratic and Moderating Effects Under Differing Degrees of Market Dynamism and Internationalization. Journal Of International Marketing, 17(4), 71-89

Cai, S., Goh, M., de Souza, R., \& Li, G. (2013). Knowledge sharing in collaborative supply chains: twin effects of trust and power. International Journal of Production Research, 51(7), 2060-2076.

Calantone, R. J., Cavusgil, S. T., Zhao, Y. (2002). Learning orientation, firm innovation capability, and firm performance. Industrial Marketing Manage- 
ment, 31(6), 515-524.

Callingham, M (2004). Market Intelligence - How and Why Organizations use Market Research, London.

Cano, C. R., Carrillat, F. A., Jaramillo, F. (2004). A meta-analysis of the relationship between market orientation and business performance: evidence from five continents. International Journal of Research in Marketing, 21(2), 179200.

Capron, L., Hulland, J. (1999). Redeployment of Brands, Sales Forces, and General Marketing Management Expertise Following Horizontal Acquisitions: A Resource-Based View. Journal of Marketing, 63(2), 41-54.

Carbonell, P., Escudero, A. I. R. (2010). The effect of market orientation on innovation speed and new product performance. Journal of Business \& Industrial Marketing, 25(7), 501-513.

Carmen, C., Jose, G. (2008). The role of technological and organizational innovation in the relation between market orientation and performance in cultural organizations. European Journal of Innovation Management, 11(3), 413-434.

Caves, R. E. (1980). Industrial organization, corporate strategy and structure. Journal of Economic Literature, 58, 64-92.

Cenfetelli, R. T., \& Bassellier, G. (2009). Interpretation of formative measurement in IS research. MIS Quarterly, 33(4), 689-707.

Chapman, R. L., Soosay, C., Kandampully, J. (2003). Innovation in logistic services and the new business model: A conceptual framework. International Journal of Physical Distribution \& Logistics Management, 33(7), 630-650.

Chen, I. J., \& Paulraj, A. (2004). Towards a theory of supply chain management: the constructs and measurements. Journal of Operations Management, 22(2), 119-150.

Chengalur-Smith, I., Nevo, S., \& Pindaro Demertzoglou. (2010). An empirical analysis of the business value of open source infrastructure technologies. Journal of the Association for Information Systems Association for Information Systems, 11(11/12), 708-729.

Chin, W. W. (1998a). Issues and Opinion on Structural Equation Modeling. MIS 
Quarterly, 22(1), vii-xvi.

Chin, W. W. (1998b). The partial least squares approach to structural equation modeling. In: G. A. Marcoulides (Ed.), Modern methods for business research. Mahwah et al., S. 295-336.

Chin, W. W., (2000). Frequently Asked Questions - Partial Least Squares \& PLSGraph. [Online], http://disc-nt.cba.uh.edu/chin/plsfaq.htm [30.09.2014].

Chin, W. W. (2010). How toWrite Up and Report PLS Analyses. In: Vinzi, V.E., Chin W. W., Henseler, J., \& Wang, H. (Eds.): Handbook of Partial Least Squares. Concepts, Methods and Applications, Berlin: Springer, S. 655-690.

Chin, W. W., Dibbern, J. (2010). An Introduction to a Permutation Based Procedure for Multi-Group PLS Analysis: Results of Tests of Differences on Simulated Data and a Cross Cultural Analysis of the Sourcing of Information System Services Between Germany and the USA. In: Vinzi, V.E., Chin W. W., Henseler, J., \& Wang, H. (Eds.): Handbook of Partial Least Squares. Concepts, Methods and Applications, Berlin: Springer, S. 171-193.

Chiou, J., Wu, L., \& Chuang, M. (2010). Antecedents of retailer loyalty: Simultaneously investigating channel push and consumer pull effects. Journal of Business Research, 63(4), 431-438.

Christensen, C., \& Bower, J. (1996). Customer power, strategic investment, and the failure of leading firms. Strategic Management Journal, 17(3), 197-218.

Churchill Jr., G. A. (1979). A Paradigm for Developing Better Measures of Marketing Constructs. Journal of Marketing Research, 16(1), 64-73.

Clarkson, M. (1995). A stakeholder framework for analyzing and evaluating corporate social performance. Academy of Management Review, 20(1), 92117.

Coff, R. W. (1999). When Competitive Advantage Doesn't Lead and to Performance: The View Stakeholder Bargaining. Organization Science, 10(2), 119133.

Cohen, J. (1988). Statistical Power Analysis for the Behavioral Sciences, Hillside, NJ.

Coley, L. S., Mentzer, J. T., Cooper, M. C. (2010). IS “CONSUMER 
ORIENTATION” A DIMENSION OF MARKET ORIENTATION IN CONSUMER MARKETS? Journal of Marketing Theory \& Practice, 18(2), 141-154.

Coltman, T., Devinney, T., Midgley, D., \& Venaik, S. (2008). Formative versus reflective measurement models: Two applications of formative measurement. Journal of Business Research, 61(12), 1250-1262.

Conway, J. M., \& Lance, C. E. (2010). What Reviewers Should Expect from Authors Regarding Common Method Bias in Organizational Research. Journal of Business and Psychology, 25(3), 325-334.

Cote, J. A., \& Buckley, M. (1987). Estimating Trait, Method, and Error Variance: Generalizing Across 70 Construct Validation Studies. Journal Of Marketing Research (JMR), 24(3), 315-318.

Crook, T., Ketchen, D., Combs, J. G., \& Todd, S. Y. (2008). Strategic resources and performance: a meta-analysis. Strategic Management Journal, 29, 11411154.

\section{D}

Dahlke, B. (2001). Einzelkundenorientierung im Business-to-Business-Bereich. Konzeptualisierung und Operationalisierung, Wiesbaden.

Davis, D. F., Golicic, S. L. (2009). Gaining comparative advantage in supply chain relationships: the mediating role of market-oriented IT competence. Journal of the Academy of Marketing Science, 38(1), 56-70.

Dawes, J. (2000). Market Orientation and Company Profitability: Further Evidence Incorporating Longitudinal Data. Australian Journal of Management (University Of New South Wales), 25(2), 173.

Day, G. S. (1994). The Capabilities of Market-Driven Organizations. Journal of Marketing, 58(4), 37-52.

Day, G. S. (2011). Closing the Marketing Capabilities. Journal of Marketing, 75(July), 183-195.

Day, G. S., Nedungadi, P. (1994). Managerial representations of competitive advantage. Journal of Marketing, 58(2), 31-44. 
Day, G. S., \& Van Den Bulte, C. (2002). Superiority in customer relationship management: consequences for competitive advantage and performance (No. 02-123). Marketing Science Insitute.

Day, G. S., Wensley, R. (1988). Assessing Advantage: A Framework for Diagnosing Competitive Superiority. Journal of Marketing, 52(2), 1-20.

Deng, S., Dart, J. (1994). Measuring Market Orientation: A Multi-factor, Multiitem Approach. Journal of Marketing Management, 10(8), 725-742.

Derenthal. K. (2009). Innovationsorientierung von Unternehmen. Messung, Determinanten und Erfolgswirkungen, Wiesbaden.

Desai, K., \& Keller, K. (2002). The Effects of Ingredient Branding Strategies on Host Brand Extendibility. Journal Of Marketing, 66(1), 73-93.

Deshpandé, R., Farley, J. U. (1998). Measuring market orientation: generalization and synthesis. Journal of Market-Focused Management, 2(3), 213-232.

Deshpandé, R., \& Farley, J. U. (2004). Organizational culture, market orientation, innovativeness, and firm performance: an international research odyssey. International Journal of Research in Marketing, 21(1), 3-22.

Deshpandé, R., Farley, J. U., Webster, F. E. (1993). Corporate Culture, Customer Orientation, and Innovativeness in Japanese Firms: A Quadrad Analysis. Journal of Marketing, 57(1), 23-37.

Diamantopoulos, A. (2005). The C-OAR-SE procedure for scale development in marketing: a comment. International Journal of Research in Marketing, 22(1), 1-9.

Diamantopoulos, A., \& Riefler, P. (2008). Formative Indikatoren: Einige Anmerkungen zu ihrer Art, Validität und Multikollinarität. In: Zeitschrift für Betriebswirtschaft, 78(11), S. 1183-1196.

Diamantopoulos, A., \& Winklhofer, H. M. (2001). Index construction with formative indicators: An alternative to scale development. Journal of Marketing Research, 38, 269-277.

Diamantopoulos, A., Sarstedt, M., Fuchs, C., Wilczynski, P., \& Kaiser, S. (2012). Guidelines for choosing between multi-item and single-item scales for construct measurement: a predictive validity perspective. Journal Of The Acad- 
emy Of Marketing Science, 40(3), 434-449.

Dickson, P. R. (1992). Toward a General Theory of Competitive Rationality. Journal of Marketing, 56(1), 69-83.

Dickson, P. R. (1996). The Static and Dynamic Mechanics of Competition: A Comment on Hunt and Morgan's Comparative Advantage Theory. Journal of Marketing, 60(4), 102.

Dierickx, I., Cool, K. (1989). Asset Stock Accumulation and Sustainability of Competitive Advantage. Management Science, 35(12), 1504-1511.

Diller, H. (2006). Probleme der Handhabung von Strukturgleichungsmodellen in der betriebs- wirtschaftlichen Forschung. DBW - Die Betriebswirtschaft, 66(6), 611-617.

Diller, H., Saatkamp, J. (2002). Schwachstellen in Marketingprozessen. Ergebnisse einer explorativen Reengineering-Metananalyse. Marketing-ZfP, 24, S. 239-252.

Donaldson, T., Preston, L. E. (1995). The Stakeholder Theory of the Corporation: Concepts, Evidence, and Implications. The Academy of Management Review, 20(1), 65-91.

Drucker, P. F. (1954). The practice of management. New York: Harper and Row.

Drucker, P. F. (1985). Management: Tasks, Responsibilities, Practices. New York: Harper and Row.

Duschek, S. (2004). Kompetenzen, organisationale. In: Schreyögg, G., v. Werder, A. (Hrsg.): Handwörterbuch der Unternehmensführung und Organisation, 4. Aufl., Stuttgart: Schäffer-Poeschel, S. 612-618.

Dutta, S., Narasimhan, O., Rajiv, S. (1999). Success in High-Technology Markets: Is Marketing Capability Critical? Marketing Science, 18(4), 547-568.

Dutta, S., Zbaracki, M. J., Bergen, M. (2003). PRICING PROCESS AS A CAPABILITY: A RESOURCE-BASED PERSPECTIVE. Strategic Management Journal, 24(7), 615-630.

Dyer, J. H. (1996). SPECIALIZED SUPPLIER NETWORKS AS A SOURCE OF COMPETITIVE ADVANTAGE: EVIDENCE FROM THE AUTO INDUSTRY. Strategic Management Journal, 17(4), 271-291. 
Dyer, J. H., \& Nobeoka, K. (2000). Creating and managing a high-performance knowledge-sharing network: the Toyota case. Strategic Management Journal, 21(3), 345-367.

Dyer, J. H., \& Singh, H. (1998). The relational view: Cooperative strategy and sources of interorganizational competitive advantage. Academy of Management Review, 23(4), 660-679.

\section{E}

Edwards, J., \& Bagozzi, R. (2000). On the nature and direction of relationships between constructs and measures. Psychological Methods, 5(2), 155-174.

Efron, B., \& Gong, G. (1983), A Leisurely Look at the Bootstrap, Jackknife, and Cross-Validation. The American Statistician, 37(1), 36-48.

Eggert, A., Fassott, G., \& Helm, S. (2005). Identifizierung und Quantifizierung mediierender und moderierender Effekte in komplexen Kausalstrukturen. In: F. Bliemel, A. Eggert, G. Fassott, J. Henseler (Eds.): Handbuch PLSPfadmodellierung. Methode, Anwendung und Praxisbeispiele, Stuttgart: Schäffer/Poeschel, S. 101-116.

Eichentopf, T., Kleinaltenkamp, M., Van Stiphout, J. (2011). Modelling customer process activities in interactive value creation. Journal of Service Management, 22(5), 650-663.

Eisenhardt, K. M., Martin, J. A. (2000). Dynamic capabilities: what are they? Strategic Management Journal, 21(10-11), 1105-1121.

Engelhardt, W. H. (1976). Mehrstufige Absatzstrategien. Schmalenbachs Zeitschrift Für Betriebswirtschaftliche Forschung - Kontaktstudium, 28, S. 175182.

Engelhardt, W. H., Günter, B. (1981). Investitionsgüter-Marketing. Stuttgart et al.

Engelhardt, W. H., Kleinaltenkamp, M., Reckenfelderbäumer, M. (1993). Leistungsbündel als Absatzobjekte. Ein Ansatz zur Überwindung der Dichotomie von Sach- und Dienstleistungen. Zeitschrift für betriebswirtschaftliche Forschung, 45(5), S. 395-426.

Ericsson, D. (1976). Vertical Marketing Systems: Design and Development, 
Göteborg.

Ernst, H., Hoyer, W. D., Rübsaamen, C. (2010). Sales, Marketing, and Researchand-Development Cooperation Across New Product Development Stages: Implications for Success. Journal of Marketing, 74(September), 80-92.

Europäische Union (2003). EMPFEHLUNG DER KOMMISSION vom 6. Mai 2003 betreffend die Definition der Kleinstunternehmen sowie der kleinen und mittleren Unternehmen, L 124/36.

Evans, J., \& Mathur, A. (2005). The value of online surveys. Internet Research, 15(2), 195-219.

\section{F}

Fang, E. (2008). Customer Participation and the Trade-Off Between New Product Innovativeness and Speed to Market. Journal Of Marketing, 72(4), 90-104.

Fang, F., Guo, Z., Whinston, A. B. (2008). Collective Outsourcing to Market (COM): A Market-Based Framework for Information Supply Chain Outsourcing. Journal of the Association for Information Systems, 9(3/4), 98118.

Farrell, M. (2002). A critique of the development of alternative measures of market orientation. Marketing Bulletin, 13(Article 3), 1-13.

Farrell, M. A., \& Oczkowski, E. (1997). An Analysis of the MKTOR and MARKOR Measures of Market Orientation: An Australian Perspective. Marketing Bulletin, 8, 30-40.

Fassott, G. (2005). Die PLS-Pfadmodellierung: Entwicklungsrichtungen, Möglichkeiten und Grenzen. In: F. Bliemel, A. Eggert, G. Fassott, J. Henseler (Eds.): Handbuch PLS-Pfadmodellierung. Methode, Anwendung und Praxisbeispiele, Stuttgart: Schäffer/Poeschel, S. 19-29.

Fassott, G., Eggert, A. (2005). Zur Verwendung formativer und reflektiver Indikatoren in Strukturgleichungsmodellen: Bestandsaufnahme und Anwendungsempfehlungen. In: F. Bliemel, A. Eggert, G. Fassott, J. Henseler (Eds.): Handbuch PLS-Pfadmodellierung. Methode, Anwendung und Praxisbeispiele, Stuttgart: Schäffer/Poeschel, S. 31-47. 
Faul, F., Erdfelder, E., Buchner, A., \& Lang, A.-G. (2009). Statistical power analyses using $\mathrm{G}^{*}$ Power 3.1: Tests for correlation and regression analyses. Behavior Research Methods, 41, 1149-1160.

Ferrell, O. C., Gonzalez-Padron, T. L., Hult, G. T. M., Maignan, I. (2010). From Market Orientation to Stakeholder Orientation. Journal of Public Policy \& Marketing, 29(1), 93-96.

Feyerabend, P. K. (1965): Problems of Empiricism. In: Colodny, R. G. (Ed.): Beyond the Edge of Certainty, Englewood Cliffs N.J.: Prentice-Hall, pp. 145260.

Finn, A., \& Kayande, U. (2005). How fine is C-OAR-SE? A generalizability theory perspective on Rossiter's procedure. International Journal of Research in Marketing, 22(1), 11-21.

Fischer, A. (2014). Mehrstufiges Marketing in Wertschöpfungsketten: Einfluss und Auswirkungen in der Verpackungsindustrie. [Online], http://www.diss.fuberlin.de/diss/receive/FUDISS_thesis_000000097169, [17.10.2014]

Flint, D. J. (2004). Strategic marketing in global supply chains: Four challenges. Industrial Marketing Management, 33(1), 45-50.

Foreman, J., Donthu, N., Henson, S., \& Poddar, A. (2014). The Performance Implications of Planning, Implementation, and Evolution of Firms' Customer and Competitor Orientations. Journal Of Marketing Theory \& Practice, 22(4), 349-366.

Fornell, C., \& Bookstein, F. (1982). Two structural equation models: LISREL and PLS applied to consumer exit-voice theory. Journal of Marketing Research, 19(4), 440-453.

Fornell, C., \& Larcker, D. F. (1981). Evaluating Structural Equation Models with Unobservable Variables and Measurement Error. Journal of Marketing Research (JMR), 18(1), 39-50.

Foss, N. J. (1996). The Emerging Competence Perspective. In: Foss, N. J., Knudsen, C. (Eds.): Towards a Competence Theory of the Firm, London, pp. 1-12.

Foss, N., Klein, P., Kor, Y. Y., \& Mahoney, J. T. (2008). Entrepreneurship, subjectivism, and the resource-based view: toward a new synthesis. Strategic En- 
treupreneurship Journal, 2, 73-94.

Freeman, R. E. (1984). Strategic management: A stakeholder approach, Boston.

Freeman, R. E., Reed, D. L. (1983). Stockholders and Stakeholders: A New

Perspective on Corporate Governance. California Management Review, 25(3), 88-106.

Freiling, J. (2004). A Competence-based Theory of the Firm. Management Revue, $15(1), 27-52$.

Freiling, J., Gersch, M., \& Goeke, C. (2008a). Die kompetenztheoretische Erklärung von Unternehmungen anhand des Organisationalen Ambientes (Abstract). In A. Eisenkopf, C. Opitz, \& H. Proff (Hrsg.), Strategisches Kompetenz-Management in der Betriebswirtschaftslehre (S. 4-12). Wiesbaden: Gabler.

Freiling, J., Gersch, M., \& Goeke, C. (2008b). On the Path towards a Competence-based Theory of the Firm. Organization Studies, 29(8-9), 1143-1164.

Frels, J., Shervani, T., \& Srivastava, R. (2003). The integrated networks model: Explaining resource allocations in network markets. Journal of Marketing, 67(1), 29-45.

Fritz, W. (1995). Marketing-Management und Unternehmenserfolg, 2. Aufl., Stuttgart: Schäffer-Poeschel.

Fritz, W. (1996). Market orientation and corporate success: findings from Germany. European Journal of Marketing, 30(8), 59-74.

Fritz, W. (1998). Die Marktorientierung als Grunddimension und Erfolgsfaktor der Unternehmensführung. In: Hildebrandt, L./Homburg, C. (Hrsg.): Die Kausalanalyse. Ein Instrument der empirischen betriebswirtschaftlichen Forschung, Stuttgart, S. 183-208.

\section{G}

Garud, R., \& Kumaraswamy, A. (1993). CHANGING COMPETITIVE DYNAMICS IN NETWORK INDUSTRIES: AN EXPLORATION OF SUN MICROSYSTEMS' OPEN SYSTEMS STRATEGY. Strategic Management Journal, 14(5), 351-369. 
Gatignon, H., \& Xuereb, J.-M. (1997). Strategic Orientation of the Firm and New Product Performance. Journal of Marketing Research, 34(1), 77-90.

Gauzente, C. (1999). Comparing Market Orientation Scales: A Content Analysis. Marketing Bulletin, 10, 76-82.

Gebhardt, G. F., Carpenter, G. S., \& Sherry Jr., J. F. (2006). Creating a market orientation: a longitudinal, multifirm, grounded analysis of cultural transformation. Journal of Marketing, 70(4), 37-55.

Gersch, M., Goeke, C., \& Freiling, J. (2010). Lobbying: strategies to make a firm's competences generate value. In R. Sanchez \& A. Heene (Eds.), Enhancing Competences for Competitive Advantage (Advances in Applied Business Strategy, Volume 12), pp. 1-22, Emerald Group Publishing Limited.

Gersch, M., Adler, H., Dreher, C., \& Biedermann, A. (2014). Innovationen aus einer ressourcen- und kompetenzorientierten Perspektive. In: Burr, W. (Hrsg.): Innovationen - Theorien, Konzepte und Methoden der Innovationsforschung, Stuttgart, S. 163-219.

Geiger, I., Kleinaltenkamp, M. (2011). Instrumente des Geschäftsbeziehungsmanagements. In: M. Kleinaltenkamp, W. Plinke, I. Geiger, F. Jacob, A. Söllner (Hrsg.): Geschäftsbeziehungsmanagement - Konzepte, Methoden, Instrumente, Wiesbaden, S. 195-253.

Giere, J., Wirtz, B. W., Schilke, O. (2006). Mehrdimensionale Konstrukte. Konzeptionelle Grundlagen und Möglichkeiten ihrer Analyse mithilfe von Strukturgleichungsmodellen. Die Betriebswirtschaft, 66(6), 678-695.

Glazer, R. (1991). Marketing in an Information-Intensive Environment: Strategic Implications of Knowledge as an Asset. Journal of Marketing, 55(4), 1-19.

Gleitsmann, B. M. (2007). Internes Marketing, Unternehmenskultur und marktorientiertes Verhalten. Direkte, indirekte und moderierende Effekte, Wiesbaden.

Götz, O., \& Liehr-Gobbers, K. (2004). Analyse von Strukturgleichungsmodellen mit Hilfe der Partial-Least-Squares ( PLS ) -Methode. DBW - Die Betriebswirtschaft, 64(6), 714-738. 
Gotteland, D., Haon, C., Gauthier, C. (2007). Market orientation: synthesis and new theoretical directions. Recherche Et Applications En Marketing (English Edition), 22(1), 45-59.

Grant, R. (1991). The Resource-Based Theory of Competitive Advantage: Implications for Strategy Formulation. California Management Review, 33(3), $114-135$

Grant, R. M. (1996). Toward a Knowledge-based Theory of the Firm. Strategic Management Journal, 17, 109-122.

Gray, B., Matear, S., Boshoff, C., \& Matheson, P. (1998). Developing a better measure of market orientation. European Journal of Marketing, 32(9/10), 884-903.

Greenley, G. E. (1995). FORMS OF MARKET ORIENTATION IN UK COMPANIES. Journal Of Management Studies, 32(1), 47-66.

Greenley, G. E., \& Foxall, G. R. (1996). Consumer and nonconsumer stakeholder orientation in U.K. companies. Journal of Business Research, 35(2), 105116.

Griese, I. (2011). Wissensentwicklungskompetenz im Business-to-BusinessBereich. Der einzelne Kunde als Wissensquelle, Wiesbaden.

Griese, I., Pick, D., \& Kleinaltenkamp, M. (2012). Antecedents of knowledge generation competence and its impact on innovativeness. Journal of Business \& Industrial Marketing, 27(6), 468-485.

Grover, R. (1996). Market-focused: Some Fundamental Issues. Journal of Market-Focused Management, 1(2), 115-117.

Grozdanovic, M. (2007). Wettbewerbsorientierung von Unternehmen. Konzeption, Einflussfaktoren und Erfolgsauswirkungen, Wiesbaden.

Grühn, O., \& Hein, H.-H. (2012). Marketing Procurement 2012. Perspektiven der Kooperation zwischen Marketing und Einkauf. (pp. 1-28). St. Gallen, Schweiz.

Grunert, K. G., Jeppesen, L. F., Jespersen, K. R., Sonne, A.-M., Hansen, K., Trondsen, T., \& Young, J. A. (2005). Market orientation of value chains: A conceptual framework based on four case studies from the food industry. Eu- 
ropean Journal of Marketing, 39(5/6), 428-455.

Gundlach, G., \& Cadotte, E. (1994). Exchange interdependence and interfirm interaction: research in a simulated channel setting. Journal of Marketing Research, 31(4), 516-532.

Günter, B. (1997). Wettbewerbsvorteile, mehrstufige Kundenanalyse und Kundenfeedback im Business-to-Business-Marketing. In: K. Backhaus, B. Günter, M. Kleinaltenkamp, W. Plinke, H. Raffée (Hrsg.): Marktleistung und Wettbewerb, Wiesbaden, S. 213-231.

Günter, B. (2013). Projektkooperationen. In: Kleinaltenkamp et al. (Hrsg.): Auftrags- und Projektmanagement, Wiesbaden, S. 383 $\neg-425$.

Gutenberg, E. (1955). Grundlagen der Betriebswirtschaftslehre. Zweiter Band. Der Absatz. Würzburg.

\section{H}

Haanes, K., Fjeldstad, Ø. (2000). Linking intangible resources and competition. European Management Journal, 18(1), 52-62.

Hair, J., Sarstedt, M., Ringle, C., \& Mena, J. (2012). An assessment of the use of partial least squares structural equation modeling in marketing research. Journal of the Academy of Marketing Science, 40, 414-433.

Håkansson, H., \& Ford, D. (2002). How should companies interact in business networks? Journal of Business Research, 55(2), 133-139.

Hamel, G., Prahalad, C. K. (1994). Competing for the Future. Harvard Business Review, 72(4), 122.

Han, J., Kim, N., Srivastava, R. (1998). Market orientation and organizational performance: is innovation a missing link? The Journal of marketing, 62(October), 30-45.

Harman, H. H. 1967. Modern Factor Analysis, Chicago.

Harris, L. C. (2001). Market orientation and performance: objective and subjective empirical evidence from UK companies. Journal of Management studies, 38(1), 17-43.

Harris, L. C. (2002). Measuring market orientation: exploring a market oriented 
approach. Journal of Market-Focused Management, 5(1990), 239-270.

Harris, L. C. (1998). Cultural domination: the key to market-oriented culture? European Journal of Marketing, 32(3/4), 354.

Haucap, J., Klein, G. (2012). Einschränkungen der Preisgestaltung im Einzelhandel aus wettbewerbsökonomischer Perspektive. In: Ahlert, D. et al. (Hrsg.): Vertikale Preis- und Markenpflege im Kreuzfeuer des Kartellrechts, Wiesbaden, S. 169-186.

Havenstein, M. (2004). Ingredient Branding: Die Wirkung der Markierung von Produktbestandteilen bei konsumtiven Gebrauchsgütern, Wiesbaden.

Hawker, C., Nelson, J., \& Terry, S. (2004). Consumer-Driven Supply Chain Networks: Building the Supply Chain of the Future. Armonk, NY: IBM Business Consulting Services, IBM Corporation.

He, X., Zhang, X., Li, X., Piesse, J. (2011). Stakeholder Orientation and Organizational Performance in an Emerging Market. Journal of General Management, 36(3), 67-92.

Helfat, C., Finkelstein, S., Mitchell, W., Peteraf, M., Singh, H., Teece, D., \& Winter, S. (2007). Dynamic Capabilities: Understanding Strategic Change in Organizations. Malden, MA.

Helfat, C. E., Peteraf, M. A. (2009). Understanding dynamic capabilities: progress along a developmental path. Strategic Organization, 7(1), 91-102.

Henriques, I., Sadorsky, P. (1999). the Relationship Between Environmental Commitment and Managerial Perceptions of Stakeholder Importance. Academy of Management Journal, 42(1), 87-99.

Henseler, J. (2007). A New and Simple Approach to Multi-Group Analysis in Partial Least Squares Path Modeling. Proceedings of the 5th International Symposium on PLS and Related Methods, Ås, Norway, pp. 104-107.

Henseler, J., Ringle, C. M., Sinkovics, R. (2009). The use of partial least squares path modeling in international marketing. In: Advances in International Marketing, Bingley: Emerald, pp. 277-319.

Herrmann, A., Huber, F., Kressmann, F. (2006). Varianz- und kovarianzbasierte Strukturgleichungsmodelle. Ein Leitfaden zu deren Spezifikation, Schätzung 
und Beurteilung. Zeitschrift für betriebswirtschaftliche Forschung, 58(2), 3466.

Heskett, J. L., Jones, T. O., Loveman, G. W., Sasser, W. E., \& Schlesinger, L. (1994). Putting the Service-Profit Chain to Work Putting the Service-Profit Chain to Work. Harvard Business Review, 72(2), 164-174.

Higgins, M. C., \& Gulati, R. (2003). Getting Off to a Good Start: The Effects of Upper Echelon Affiliations on Underwriter Prestige. Organization Science, 14(3), 244-263.

Homburg, C. (2012). Marketingmanagement. Strategie - Instrumente - Umsetzung - Unternehmensführung, 4., überarb. u. erw. Aufl., Wiesbaden.

Homburg, C., \& Giering, A. (1996). Konzeptualisierung und Operationalisierung komplexer Konstrukte - Ein Leitfaden für die Marketingforschung. Marketing - Zeitschrift für Forschung und Praxis, 18(1), 5-24.

Homburg, C., \& Klarmann, M. (2006). Die Kausalanalyse in der empirischen betriebswirtschaftlichen Forschung-Problemfelder und Anwendungsempfehlungen. DBW - Die Betriebswirtschaft, 66(2006), 727-748.

Homburg, C., Klarmann, M., Reimann, M., \& Schilke, O. (2012). What Drives Key Informant Accuracy? Journal of Marketing Research (JMR), XLIX(August), 594-608.

Homburg, C., Pflesser, C. (2000). A Multiple-Layer Model of Market-Oriented Organizational Culture: Measurement Issues and Performance Outcomes. Journal of Marketing Research, 37(4), 449-462.

Homburg, C., Wilczek, H. (2014). Mehrstufiges Marketing: Erfolgswirkungen und Erfolgsfaktoren. [Online], http://imu2.bwl.unimannheim.de/fileadmin/files/imu/files/ ap/ri/RI_021.pdf [12.04.2014].

Homburg, C., Wilczek, H., \& Hahn, A. (2014). Looking Beyond the Horizon: How to Approach the Customers' Customers in Business-to-Business Markets. Journal of Marketing, 78(49), 58-77.

Hooley, G., Cox, T., Fahy, J., \& Shipley, D. (2000). Market Orientation in the Transition Economies of Central Europe: Tests of the Narver and Slater Market Orientation Scales. Journal of Business Research, 50, 273-285. 
Hooley, G. J., Lynch, J. E., \& Shepherd, J. (1990). The Marketing Concept: Putting the Theory into Practice. European Journal of Marketing, 24(9), 7-24.

Hsieh, Y.-C., Chiu, H.-C., Hsu, Y.-C. (2008). Supplier market orientation and accommodation of the customer in different relationship phases. Industrial Marketing Management, 37(4), 380-393.

Hulland, J. (1999). USE OF PARTIAL LEAST SQUARES (PLS) IN STRATEGIC MANAGEMENT RESEARCH: A REVIEW OF FOUR RECENT STUDIES. Strategic Management Journal, 20(2), 195-204.

Hult, G. T. M. (2011). Market-focused sustainability: market orientation plus! Journal of the Academy of Marketing Science, 39(1), 1-6.

Hult, G. T. M., Hurley, R. F., Knight, G. a. (2004). Innovativeness: Its antecedents and impact on business performance. Industrial Marketing Management, 33(5), 429-438.

Hult, G. T. M., Ketchen, D. J., Adams, G. L., Mena, J. A. (2008). Supply Chain Orientation and Balanced Scorecard Performance. Journal of Managerial Issues, 20(4), 526-544.

Hult, G. T. M., Ketchen, D. J., Slater, S. F. (2005). Market orientation and performance: an integration of disparate approaches. Strategic Management Journal, 26(12), 1173-1181.

Hunt, S. D. (1997). Competing through relationships: Grounding relationship marketing in resource-advantage theory. Journal of Marketing Management, 13(5), 431-445.

Hunt, S. D. (2000). A general theory of competition: resources, competences, productivity, economic growth. Thousand Oaks (CA), USA, u.a.

Hunt, S. D., Lambe, C. J. (2000). Marketing's contribution to business strategy: market orientation, relationship marketing and resource-advantage theory. International Journal of Management Reviews, 2(1), 17-43.

Hunt, S. D., Morgan, R. M. (1995). The comparative advantage theory of competition. Journal of Marketing, 59(2), 1-15.

Hurley, R. F., Hult, G. T. M. (1998). Organizational Learning: An integration and Empirical Examination, 62(July), 42-54. 
Hyvönen, S., Tuominen, M. (2007). Channel Collaboration, Market Orientation and Performance Advantages: Discovering Developed and Emerging Markets. The International Review of Retail, Distribution and Consumer Research, 17(5), 423-445.

Ihinmoyan, T., Akinyele, S. (2011). Relationship Between Market Orientation, Firm Innovativeness and Innovative Performance. The Journal Contemporary Management Research, 5(2), 42-54.

Im, S., Workman, J. P. jr. (2004). Market orientation, creativity, and new product performance in high-technology firms. Journal of Marketing, 68(2), 114132.

\section{$\mathbf{J}$}

Jap, S. D. (1999). Pie-Expansion Efforts: Collaboration Processes in BuyerSupplier Relationships. Journal of Marketing Research, 36(4), 461-475.

Jap, S. D. (2001). "Pie Sharing" in Complex Collaboration Contexts. Journal of Marketing Research (JMR), 38(1), 86-99.

Jarratt, D. (2004). Conceptualizing a relationship management capability. Marketing Theory, 4(4), 287-309.

Jarratt, D., \& Fayed, R. (2001). The impact of market and organisational challenges on marketing strategy decision-making: a qualitative investigation of the business-to-business sector. Journal of Business Research, 51(1), 61-72.

Jaworski, B. J., Kohli, A. K. (1993). Market orientation: Antecedents and consequences. Journal of Marketing, 57(3), 53-70.

Jaworski, B. J., Kohli, A. K. (1996). Market orientation: Review, refinement, and roadmap. Journal of Market-Focused Management, 1(2), 119-135.

Jöreskog, K. G., Wold, H. (1982). The ML and PLS Technique for Modelling with Latent Variables - Historical and Comparative Aspects. In: Jöreskog, K. G./Wold, H. (Hrsg.): Systems under Indirect Observation. Causality, Structure, Prediction. Amsterdam et al., S. 263 - 270. 
Jones, S. L., Fawcett, S. E., Wallin, C., Fawcett, A. M., \& Brewer, B. L. (2014). Can small firms gain relational advantage? Exploring strategic choice and trustworthiness signals in supply chain relationships. International Journal of Production Research, 52(18), 5451-5466.

Jones, T. M. (1995). Instrumental Stakeholder Theory: A Synthesis of Ethics and Economics. The Academy of Management Review, 20(2), 404-437.

Jonsson, R. (2005). The end consumer's choice of floorcovering in the Netherlands and the United Kingdom: a comparative pilot study of substitute competition. Journal of Wood Science, 51(2), 154-160.

\section{$\mathbf{K}$}

Kaas, K. P. (1991). Marktinformationen: Screening und Signaling unter Partnern und Rivalen. Zeitschrift für Betriebswirtschaft, 61(3), 357-370.

Kaase, M. (1999). Qualitätskriterien der Umfrageforschung, Berlin.

Kahn, K. B. (2001). Market orientation, interdepartmental integration, and product development performance. Journal of Product Innovation Management, 18(5), 314-323.

Kaiser, H. (1974). An index of factorial simplicity. Psychometrika, 39(1), 31-36.

Kaplan, R. S., Norton, D. P. (2004). Measuring the strategic readiness of intangible assets. Harvard business review, 82(4), 52-63.

Keil, M., Tan, B.C.Y., Wei, K.-K., Saarinen, T., Tuunainen, V., Wassenaar, A. (2000). A cross-cultural study on escalation of commitment behavior in software projects. MIS Quarterly 24 (2), 299-325.

Kelley, S. W., \& Douglas Hoffman, K. K. (1997). An Investigation of Positive Affect, Prosocial Behaviors and Service Quality. Journal of Retailing, 73(3), 407-427.

Ketchen, D. J., Hult, G. T. M., Slater, S. F. (2007). Toward greater understanding of market orientation and the resource-based view. Strategic Management Journal, 28(9), 961-964.

Kibbeling, M., Bij, H., Weele, A. (2013). Market Orientation and Innovativeness in Supply Chains: Supplier's Impact on Customer Satisfaction. Journal of 
Product Innovation Management, 30(3), 500-515.

Kleinaltenkamp, M. (2000). Einführung in das Business-to-Business-Marketing. In: M. Kleinaltenkamp, W. Plinke (Hrsg.), Technischer Vertrieb. Grundlagen des Business-to-Business-Marketing, 2. Auflage, S. 171-247, Berlin et al.

Kleinaltenkamp, M. (2007). Kundenintegration. In: R. Köhler (Hrsg.): Handwörterbuch der Betriebswirtschaft, 6. Aufl., Stuttgart, Sp. 1037-1048.

Kleinaltenkamp, M., Classen, M., Fischer, A. (2009). Multi-Stage Marketing Overcoming Marketing Myopia in B2B Markets. Proceedings of the 2009 ANZMAC Conference, Melbourne, Australia.

Kleinaltenkamp, M., Classen, M., Fischer, A. (2010). Multi-Stage Marketing: Overcoming Uncertainties in B2B Markets. Proceedings of the 2010 Global Marketing Conference of the KAMS, Tokyo, Japan.

Kleinaltenkamp, M., Classen, M., Fischer, A., Fabel, M., Pohl, A. (2011). Die Kunden der Kunden im Visier. Harvard Business Manager, 33(2), 43-47.

Kleinaltenkamp, M., Dahlke, B. (1998). Market orientation and customer orientation of industrial companies. Thexis, 4, 32-37.

Kleinaltenkamp, M., Rudolph, M. (2002). Mehrstufiges Marketing. In: M. Kleinaltenkamp, W. Plinke (Hrsg.), Strategisches Business-to-BusinessMarketing, 2. Aufl., Berlin et al., S. 283-319.

Kleinaltenkamp, M., Rudolph, M., Classen, M. (2012). Chapter 6 Multistage Marketing. In: M. S. Glynn, A. G. Woodside (Hrsg.), Business-to-Business Marketing Management: Strategies, Cases, and Solutions (Advances in Business Marketing and Purchasing, Volume 18), S. 141-174, Bingley (UK).

Knack, R. (2006). Wettbewerb und Kooperation: Wettbewerberorientierung in Projekten radikaler Innovation, Wiesbaden.

Kobayashi, M. (2014). Relational View: Four Prerequisites of Competitive Advantage. Annals of Business Administrative Science, 13, 77-90.

Kogut, B., Zander, U. (1992). KNOWLEDGE OF THE FIRM, COMBINATIVE CAPABILITIES, AND THE REPLICATION OF TECHNOLOGY. Organization Science, 3(3), 383-397.

Kohli, A. K., Jaworski, B. J. (1990). Market Orientation: The Construct, Re- 
search Propositions, and Managerial Implications. Journal of Marketing, 54(2), 1-18.

Kohli, A. K., Jaworski, B. J., Kumar, A. (1993). MARKOR: A Measure of Market Orientation. Journal of Marketing Research (JMR), 30(4), 467-477.

Kok, R. W., Hillebrand, B., \& Biemans, W. G. (2003). What Makes Product Development Market Oriented? Towards a Conceptual Framework. International Journal Of Innovation Management, 7(2), 137-162.

Kotler, P., Keller, K., Bliemel, F. (2007). Marketing-Management. Strategien für wertschaffendes Handeln. 12., aktual. Aufl., München.

Kraaijenbrink, J., Spender, J.-C., \& Groen, a. J. (2010). The Resource-Based View: A Review and Assessment of Its Critiques. Journal of Management, 36(1), 349-372.

Kumar, V., Jones, E., Venkatesan, R., Leone, R. P. (2011). Is Market Orientation a Source of Sustainable Competitive Advantage or Simply the Cost of Competing? Journal of Marketing, 75(1), 16-30.

Kunkel, R. (1977), Vertikales Marketing im Herstellerbereich. Bestimmungsfaktoren und Gestaltungselemente stufenübergreifender MarketingKonzeptionen, München.

Kuß, A., \& Eisend, M. (2010). Marktforschung. Grundlagen der Datenerhebung und Datenanalyse, 3., überarb. und erw. Aufl., Wiesbaden.

Kuß, A., Tomczak, T., Reinecke, S. (2007). Marketingplanung. Einführung in die marktorientierte Unternehmens- und Geschäftsfeldplanung. 5. Aufl., Wiesbaden.

$\mathbf{L}$

Lado, A. A., Boyd, N. G., Wright, P. (1992). A Competency-Based Model of Sustainable Competitive Advantage: Toward a Conceptual Integration. Journal of Management, 18(1), 77-91.

Lado, N., Maydeu-Olivares, A., \& Rivera, J. (1998). Measuring market orientation in several populations: A structural equations model. European Journal of Marketing, 32(1/2), 23-39. 
Lafferty, B. A., \& Hult, G. T. M. (2001). A synthesis of contemporary market orientation perspectives. European Journal of Marketing, 35(1/2), 92-109.

Langerak, F. (2003). The effect of market orientation on positional advantage and organizational performance. Journal of Strategic Marketing, 11(2), 93-115.

Lavie, D. (2006). The competitive advantage of interconnected firms: An extension of the resource-based view. Academy of Management Review, 31(3), 638-658.

Lee, H. L., Padmanabhan, V., Whang, S. (2004). Information Distortion in a Supply Chain: The Bullwhip Effect. Management Science, 50(12 Supplement), 1875-1886.

Levitt, T. (1960). Marketing myopia. Harvard Business Review, 38(4), 45-56.

Li, L. (2002). Information Sharing in a Supply Chain with Horizontal Competition. Management Science, 48(9), 1196-1212.

Liao, S.-H., Chang, W.-J., Wu, C.-C., Katrichis, J. M. (2011). A survey of market orientation research (1995-2008). Industrial Marketing Management, 40(2), 301-310.

Lin, C.-H., Peng, C.-H., \& Kao, D. T. (2008). The innovativeness effect of market orientation and learning orientation on business performance. International Journal of Manpower, 29(8), 752-772.

Lindell, M. K., \& Whitney, D. J. (2001). Accounting for common method variance in cross-sectional research designs. Journal of Applied Psychology, 86(1), 114-121.

Lisboa, A., Skarmeas, D., \& Lages, C. (2011). Entrepreneurial orientation, exploitative and explorative capabilities, and performance outcomes in export markets: A resource-based approach. Industrial Marketing Management, 40(8), 1274-1284.

Liu, Q., \& Gupta, S. (2011). The impact of direct-to-consumer advertising of prescription drugs on physician visits and drug requests: Empirical findings and public policy implications. International Journal of Research in Marketing, 28(3), 205-217.

Lockett, A., \& Thompson, S. (2001). The resource-based view and economics. 
Journal of Management, 27, 723-754.

López, V. A. (2006). An alternative methodology for testing a resource-based view linking intangible resources and long-term performance. Irish Journal of Management, 27(2), 49-66.

Low, D. R., Chapman, R. L., \& Sloan, T. R. (2007). Inter-relationships between innovation and market orientation in SMEs. Management Research News, 30(12), 878-891.

Lukas, B. A., \& Ferrell, O. C. (2000). The effect of market orientation on product innovation. Journal of the Academy of Marketing Science, 28(2), 239-247.

Luo, X., Sivakumar, K., Liu, S. S. (2005). Globalization, Marketing Resources, and Performance: Evidence From China. Journal of the Academy of Marketing Science, 33(1), 50-65.

\section{M}

MacCallum, R., \& Austin, J. (2000). Applications of structural equation modeling in psychological research. Annual Review of Psychology, 51, 201-226.

Mahoney, J., Pandian, J. (1992). The Resource-Based View within the Conversation of Strategic Management. Strategic Management Journal, 13(5), 363380.

Maignan, I., Ferrell, O. C. (2004). Corporate Social Responsibility and Marketing: An Integrative Framework. Journal of the Academy of Marketing Science, 32(1), 3-19.

March, J. G., \& Sutton, R. I. (1997). Organizational Performance as a Dependent Variable. Organization Science, 8(6), 698-706.

Mardia, K. V. (1970). Measures of multivariate skewness and kurtosis with applications. Biometrika, 57(3), 519-530.

Martin, J. H., Grbac, B. (2003). Using supply chain management to leverage a firm's market orientation. Industrial Marketing Management, 32(1), 25-38.

Mason, K., Harris, L. C. (2005). Pitfalls in Evaluating Market Orientation: An Exploration of Executives' Interpretations. Long Range Planning, 38(4), 373-391. 
Matsuno, K., Mentzer, J., \& Rentz, J. (2000). A refinement and validation of the MARKOR scale. Journal of the Academy of Marketing Science, 28(4), 527539.

Matsuno, K., Mentzer, J., \& Rentz, J. (2005). A conceptual and empirical comparison of three market orientation scales. Journal of Business Research, 58(1), 1-8.

Mavondo, F. T., Farrell, M. A. (2000). Measuring market orientation: are there differences between business marketers and consumer marketers? Australian Journal of Management, 25(2), 223-244.

McCammon, B. C. (1970). Perspectives for Distribution Programming. In: Bucklin, L. (Hrsg.): Vertical Marketing Systems, S. 32-51, Glenview.

McKee, D. O., Conant, J. S., Varadarajan, P. R., Mokwa, M. P. (1992). Successproducer and failure-preventer marketing skills: A social learning theory interpretation. Journal of the Academy of Marketing Science, 20(1), 17-26.

Meffert, H., Burmann, C., Kirchgeorg, M. (2012). Marketing. Grundlagen marktorientierter Unternehmensführung. Konzepte - Instrumente - Praxisbeispiele, 11., überarb. u. erweit. Aufl., Wiesbaden.

Menguc, B., Auh, S. (2006). Creating a Firm-Level Dynamic Capability through Capitalizing on Market Orientation and Innovativeness. Journal of the Academy of Marketing Science, 34(1), 63-73.

Menon, A., Varadarajan, P. R. (1992). A model of marketing knowledge use within firms. Journal of Marketing, 56(4), 53-71.

Mesquita, L. F., Anand, J., \& Brush, T. H. (2008). Comparing the ResourceBased and Relational Views: Knowledge Transfer and Spillover in Vertical Alliances. Strategic Management Journal, 29(9), 913-941.

Minculescu, I. (2013). Leistungsindividualisierung im B-to-B-Bereich. Die Einzigartigkeit im Rahmen der Dienstleistungsgestaltung, Wiesbaden.

Mitchell, R. K., Agle, B. R., \& Wood, D. J. (1997). Toward a Theory of Stakeholder Identification and Salience: Defining the Principle of Who and What Really Counts. Academy Of Management Review, 22(4), 853-886.

Möller, K. (2006). Role of competences in creating customer value: A value- 
creation logic approach. Industrial Marketing Management, 35(8), 913-924. Moorman, C., Zaltman, G., Deshpandé, R. (1992). Relationships between providers and users of market research: the dynamics of trust within and between organizations. Journal of Marketing Research, XXIX(August), 314-328.

Morgan, N. A., Vorhies, D. W., Mason, C. H. (2009). MARKET ORIENTATION, MARKETING CAPABILITIES, AND FIRM PERFORMANCE. Strategic Management Journal, 920(January), 909-920.

Morgan, R. E., Strong, C. A. (1998). Market orientation and dimensions of strategic orientation. European Journal of Marketing, 32(11/12), 1051-1073.

Murray, J., Gao, G., Kotabe, M. (2011). Market orientation and performance of export ventures: the process through marketing capabilities and competitive advantages. Journal of the Academy of Marketing Science, 39(2), 252-269.

Murray, J., Gao, G., Kotabe, M., \& Zhou, N. (2007). Assessing Measurement Invariance of Export Market Orientation: A Study of Chinese and NonChinese Firms in China. Journal of International Marketing, 15(4), 41-62.

\section{$\mathbf{N}$}

Narver, J. C., Slater, S. F. (1990). The effect of a market orientation on business profitability. Journal of Marketing, 54(4), 20-35.

Narver, J. C., Slater, S. F. (1998a). Additional Thoughts on the Measurement of Market Orientation: A Comment on Deshpande and Farley. Journal of Market-Focused Management, 2(3), 233-236.

Narver, J. C., Slater, S. F. (1998b). Customer-Led and Market-Oriented: Let's Not Confuse the Two. Strategic Management Journal, 19(10), 1001-1006.

Narver, J. C., Slater, S. F., MacLachlan, D. L. (2004). Responsive and Proactive Market Orientation and New-Product Success. Journal of Product Innovation Management, 21(5), 334-347.

Narver, J. C., Slater, S. F., \& Tietje, B. (1998). Creating a Market Orientation. Journal of Market-Focused Management, 2(3), 241-255.

Navarro, A., Acedo, F., Robson, M., Ruzo, E., \& Losada, F. (2010). Antecedents and consequences of firms' export commitment: An empirical study. Journal 
of International Marketing, 18(3), 41-61.

Netemeyer, R. G., Bearden, W. O., \& Sharma, S. (2003). Scaling procedures. Issues and applications. Thousand Oaks: Sage.

Neuenburg, J. (2010). Market-Driving Behavior in Emerging Firms, Wiesbaden.

Newbert, S. L. (2007). EMPIRICAL RESEARCH ON THE RESOURCEBASED VIEW OF THE FIRM: AN ASSESSMENT AND SUGGESTIONS FOR FUTURE RESEARCH. Strategic Management Journal, 28(September 2005), 121-146.

Ngo, L. V., O’Cass, A. (2012). In Search of Innovation and Customer-related Performance Superiority: The Role of Market Orientation, Marketing Capability, and Innovation Capability Interactions. Journal of Product Innovation Management, 29(5), 861-877.

Noble, C. H., \& Mokwa, M. P. (1999). Implementing Marketing Strategies: Developing and Testing a Managerial Theory. Journal of Marketing, 63(4), 57.

Noble, C. H., Sinha, R. K., Kumar, A. (2002). Market Orientation and Alternative Strategic Orientations: A Longitudinal Assessment of Performance Implications. Journal Of Marketing, 66(4), 25-39.

Nuneva, A. M., Jensen, S. (2010). Weltweite Markenführung am Beispiel der Heidelberger Druckmaschinen AG. In: C. Baumgarth (Hrsg.): B-to-BMarkenführung. Grundlagen -Konzepte -Best-Practive, Wiesbaden, S. 289316.

Nunnally, J. C. (1978). Psychometric Theory, 2. Aufl., New York.

\section{O}

Oakley, J. (2012). Bridging the gap between employees and customers. Journal of Marketing Management, 28(August), 1094-1113.

Oliver, C. (1997). Sustainable competitive advantage: Combining institutional and resource-based views. Strategic Management Journal, 18(November 1996), 697-713.

Olson E., Slater S., Hult G.T.M. (2005). The Performance Implications of Fit Among Business Strategy, Marketing Organization Structure, and Strategic 
Behavior. Journal Of Marketing, 69(3), 49-65.

$\mathbf{P}$

Parasuraman, A. (1987). Customer-oriented corporate cultures are crucial to services marketing success. Journal of Services Marketing, 1(1), 39-46.

Parasuraman, A. (1997). Reflections on gaining competitive advantage through customer value. Journal of the Academy of Marketing Science, 25(2), 154161.

Patil, V., Singh, S., Mishra, S., \& Donavan, T. (2008). Efficient theory development and factor retention criteria: Abandon the "eigenvalue greater than one”criterion. Journal of Business Research, 61(2), 162-170.

Pelham, A. M., \& Wilson, D. T. (1996). A Longitudinal Study of the Impact of Market Structure, Firm Structure, Strategy, and Market Orientation Culture on Dimensions of Small-Firm Performance. Journal of the Academy of Marketing Science, 24(1), 27.

Pelled, L. H., Cummings, T. G., \& Kizilos, M. A. (2000). The influence of organizational demography on customer-oriented prosocial behavior: An exploratory investigation. Journal of Business Research, 47(98), 209-216.

Penrose, E. T. (1959). The Theory of the Growth of the Firm. London.

Perry, M. L., Shao, A. T. (2005). Incumbents in a dynamic Internet related services market: Does customer and competitive orientation hinder or help performance?. Industrial Marketing Management, 34(6), 590-601.

Peteraf, M. A. (1993). The cornerstones of competitive advantage: a resourcebased view. Strategic Management Journal, 14(3), 179-191.

Peteraf, M. A., Bergen, M. E. (2003). Scanning dynamic competitive landscapes: a market-based and resource-based framework. Strategic Management Journal, 24(10), 1027-1041.

Petter, S., Straub, D., \& Rai, A. (2007). Specifying formative constructs in information systems research. MIS Quarterly, 31(4), 623-656.

Pinto, M., \& Pinto, J. (1990). Project team communication and cross-functional cooperation in new program development. Journal of Product Innovation 
Management, 7(3), 200-212.

Plinke, W. (1992). Ausprägungen der Marktorientierung im InvestitionsgüterMarketing. Zeitschrift Für Betriebswirtschaftliche Forschung, 44(9), 830 846.

Plinke, W. (2000a): Grundkonzeption des industriellen Marketing-Managements. In: Kleinaltenkamp, M., Plinke, W. (Hrsg.): Technischer Vertrieb. Grundlagen des Business-to-Business Marketing, 2. Aufl., Berlin et al, S. 101-168.

Plinke, W. (2000b). Grundlagen des Marktprozesses. In: Kleinaltenkamp, M., Plinke, W. (Hrsg.): Technischer Vertrieb. Grundlagen des Business-toBusiness Marketing, 2. Aufl., Berlin et al., S. 3-99., W. (2000c). Unternehmensstrategie. In: Kleinaltenkamp, M., Plinke, W. (Hrsg.): Strategisches Business-to-Business-Marketing, 2. Aufl., Berlin et al. S. 1-55.

Plötner, O. (2006): Grundlagen der Gestaltung der Kommunikationsleistung. In: Kleinaltenkamp, M., Plinke, W., Jacob, F., Söllner, A. (Hrsg.): Markt- und Produktmanagement. Die Instrumente des Die Instrumente des Business-toBusiness Marketing, 2. Aufl., Wiesbaden, S. 497-547.

Podsakoff, P. M., MacKenzie, S. B., Lee, J.-Y., \& Podsakoff, N. P. (2003). Common method biases in behavioral research: a critical review of the literature and recommended remedies. The Journal of Applied Psychology, 88(5), 879-903.

Porter, M. E. (2004). Competitive Advantage. Creating and Sustaining Superior Performance. New York (NY), USA.

Porter, M. E., Millar, V. E. (1985). How information gives you competitive advantage. Harvard Business Review, 63(4), 149-160.

Powell, T. C. (2001). COMPETITIVE ADVANTAGE: LOGICAL AND PHILOSOPHICAL CONSIDERATIONS. Strategic Management Journal, 22(9), 875-888.

Powell, W. W., Koput, K. W., Smith-Doerr, L. (1996). Interorganizational Collaboration and the Locus of Innovation: Networks of Learning in Biotechnology. Administrative Science Quarterly, 41(1), 116-145.

Prahalad, C. K., Hamel, G. (1990). The core competence of the corporation. 
Harvard Business Review, 79-91.

Pressebox (2014). XING im ersten Halbjahr 2014 weiter voll auf Wachstumskurs. [Online], http://www.pressebox.de/pressemitteilung/xing-ag/XING-imersten-Halbjahr-2014-weiter-voll-auf-Wachstumskurs/boxid/694413.

[29.08.2014]

Priem, R. L., \& Butler, J. E. (2001). TAUTOLOGY IN THE RESOURCEBASED VIEW AND THE IMPLICATIONS OF EXTERNALLY DETERMINED RESOURCE VALUE: FURTHER COMMENTS. Academy Of Management Review, 26(1), 57-66.

Pulendran, S., \& Speed, R. (1996). Planning and Doing: The Relationship between Marketing Planning Styles and Market Orientation. Journal of Marketing Management, 12(1-3), 53-68.

\section{Q}

Qureshi, I., \& Compeau, D. (2009). Assessing between-group differences in information systems research: A comparision of covariance-and componentbased SEM. MIS Quarterly, 33(1), 197-214.

\section{$\mathbf{R}$}

Ragatz, G. L., Handfield, R. B., Petersen, K. J. (2002). Benefits associated with supplier integration into new product development under conditions of technology uncertainty. Journal of Business Research, 55(5), 389-400.

Ragatz, G. L., Handfield, R. B., Scannell, T. V. (1997). Success factors for integrating suppliers into new product development. Journal of Product Innovation Management, 14, 190-202.

Rätsch, C. C., Bazing, E. (2010). Systematisches Marketing-Controlling am Beispiel der IT-Branche. In: C. Baumgarth (Hrsg.): B-to-B-Markenführung. Grundlagen -Konzepte -Best-Practive, Wiesbaden, S. 661-684.

Reichmann, R. (2010). Marktorientierung und Innovationsorientierung. Konzeption, Erfolgsauswirkungen und Typologisierung. [Online], http://deposit.dnb.de/cgi- 
bin/dokserv?idn=1009950843\&dok_var=d1\&dok_ext=pdf\&filename=10099 50843.pdf, [23.08.2011].

Riemer, M. (1986). Beschwerdemanagement, Frankfurt a.M. et al.

Ringle, C. M., Sarstedt, M., Straub, D. W. (2012). Editor's comments: a critical look at the use of PLS-SEM in MIS quarterly. MIS Quarterly 36 (1), iii-xiv.

Ringle, C. M., Wende, S., and Will, A. (2005). SmartPLS 2. Hamburg: SmartPLS, http://www.smartpls.com.

Rodríguez-Pinto, J., Carbonell, P., \& Rodríguez-escudero, A. I. (2011). Speed or quality? How the order of market entry influences the relationship between market orientation and new product performance. International Journal of Research in Marketing, 28(2), 145-154.

Rogelberg, S., \& Stanton, J. (2007). Introduction understanding and dealing with organizational survey nonresponse. Organizational Research Methods, 10(2), 195-209.

Rossiter, J. R. (2002). The C-OAR-SE procedure for scale development in marketing. International Journal of Research in Marketing, 19(4), 305-335.

Rossiter, J. R. (2005). Reminder: a horse is a horse. International Journal of Research in Marketing, 22, 23-25.

Rossiter, J. R. (2012). Further comment on “market orientation.” Australasian Marketing Journal (AMJ), 20(1), 108-112.

Roth, S. (2009). Strategisches Preismanagement für Dienstleistungen - Ergebnisse einer Studie in Neuseeland. In: B. Stauss (Hrsg.): Aktuelle Forschungsfragen im Dienstleistungsmarketing, Wiesbaden, S. $1 \neg-23$.

Roy, S., \& Sivakumar, K. (2010). Innovation generation in upstream and downstream business relationships. Journal of Business Research, 63(12), 13561363.

Rudolph, M. (1989), Mehrstufiges Marketing für Einsatzstoffe. Anwendungsvoraussetzungen und Strategietypen, Frankfurt am Main.

Ruekert, R. W. (1992). Developing a market orientation: An organizational strategy perspective. International Journal of Research in Marketing, 9(3), 225245. 
S

Sambamurthy, V., \& Chin, W. (1994). The Effects of Group Attitudes Toward Alternative GDSS Designs on the Decision-making Performance of Computer-Supported Groups. Decision Sciences, 25(2), 215-241.

Sammarra, A., \& Biggiero, L. (2008). Heterogeneity and specificity of Inter-Firm knowledge flows in innovation networks. Journal of Management Studies, 45(4), 800-829.

Sandvik, I. L., Sandvik, K. (2003). The impact of market orientation on product innovativeness and business performance. International Journal of Research in Marketing, 20(4), 355-376.

Savage, G. T., Nix, T. W., Whitehead, C. J., Blair, J. D. (1991). Strategies for assessing and managing organizational stakeholders. Academy of Management Executive, 5(2), 61-75.

Saxton, T. (1997). The Effects of Partner and Relationship Characteristics on Alliance Outcomes. Academy of Management Journal, 40(2), 443-461.

Schmidt, J., \& Keil, T. (2013). What Makes a Resource Valuable? Identifying the Drivers of Firm-Idiosyncratic Resource Value. Academy of Management Review, 38(2), 206-228.

Scholderer, J., Balderjahn, I. (2005). PLS versus LISREL: Ein Methodenvergleich. In: F. Bliemel, A. Eggert, G. Fassott, J. Henseler (Eds.), Stuttgart: Schäffer/Poeschel, S. 87-98.

Scholderer, J., Balderjahn, I., \& Paulssen, M. (2006). Kausalität, Linearität, Reliabilität: Drei Dinge, die Sie nie über Strukturgleichungsmodelle wissen wollten. DBW - Die Betriebswirtschaft, 66(6), 640-650.

Schönhoff, A., Geiger, I., \& Kleinaltenkamp, M. (2013). Does multi-stage marketing pay? Proceedings of the 2013 European Marketing Conference, Istanbul, Turkey.

Schreyögg, G., \& Conrad, P. (2006). Management von Kompetenz. Managementforschung 16, Wiesbaden: Gabler.

Schumpeter, J. A. (1939). Business Cycles, A Theoretical, Historical, and Statis- 
tical Analysis of the Capitalist Process, Band 1, New York u.a.: McGrawHill.

Schwalbe, U. (2012). Wettbewerbsökonomische Aspekte der Preisbindung in Wertschöpfungsketten. In: Ahlert, D. et al. (Hrsg.): Vertikale Preis- und Markenpflege im Kreuzfeuer des Kartellrechts, Wiesbaden, S. 155-168.

Seltin, N., \& Keeves, J. P. (1994). Path Analysis with latent Variables. In: T. Husen, T. Postlethwaite (Hrsg.): The International Encyclopedia of Education, 2. Aufl., Oxford, S. 4352-4359.

Shao, A. T., \& Zhou, K. (2006). Marketing Research: An Aid to Decision Making, 3. Aufl., Cincinnati.

Shapiro, B. P. (1988). What the Hell Is “Market Oriented”? Harvard Business Review, 66, 119-125.

Shapiro, B. P., Slywotzky, A. J., Doyle, S. X. (1997). Strategic Sales Management: A Boardroom Issue. Strategy and Business, 8(1), 29-36.

Sheth, J. N., Sisodia, R. S. (1999). Revisiting marketing's lawlike generalizations. Journal of the Academy of Marketing Science, 27(1), 71-87.

Sheth, J. N., Uslay, C. (2007). Implications of the Revised Definition of Marketing: From Exchange to Value Creation. Journal of Public Policy \& Marketing, 26(2), 302-307.

Siguaw, J. A., Diamantopoulos, A. (1995). Measuring market orientation: some evidence on Narver and Slater's three-component scale. Journal of Strategic Marketing, 3, 77-88.

Siguaw, J. A., Simpson, P. M., Baker, T. L. (1998). Effects of Supplier Market Orientation on Distributor Market Orientation and the Channel Relationship: The Distributor Perspective. Journal of Marketing, 62(3), 99-111.

Siguaw, J. A., Simpson, P. M., \& Enz, C. A. (2006). Conceptualizing Innovation Orientation: A Framework for Study and Integration of Innovation Research. Journal of Product Innovation Management, 23(6), 556-574.

Simon, H., Homburg, C. (1998). Kundenzufriedenheit: Konzepte, Methoden, Erfahrungen, 3. Aufl., Wiesbaden.

Simpson, P. M., Siguaw, J. A., \& Baker, T. L. (2001). A Model of Value Creation: 
Supplier Behaviors and Their Impact on Reseller-Perceived Value. Industrial Marketing Management, 30(2), 119-134.

Singh, K. (1997). The Impact of Technological Complexity and Interfirm Cooperation on Business Survival. Academy of Management Journal, 40(2), 339367.

Sinkovics, R. R., Roath, A. S. (2004). STRATEGIC ORIENTATION, CAPABILITIES, AND PERFORMANCE IN MANUFACTURER - 3PL RELATIONSHIPS. Journal of Business Logistics, 25(2), 43-64.

Slater, S. F. (1995). Issues in conducting marketing strategy research. Journal of Strategic Marketing, 3(4), 257-270.

Slater, S. F. (1996). The challenge of sustaining competitive advantage. Industrial Marketing Management, 25(1), 79-86.

Slater, S. F., Narver, J. C. (1994a). Does competitive environment moderate the market orientation-performance relationship? The Journal of Marketing, 58(January), 46-55.

Slater, S. F., Narver, J. C. (1994b). Market orientation, customer value, and superior performance. Business Horizons, 37(2), 22-28.

Slater, S. F., Narver, J. C. (1995). Market orientation and the learning organization. The Journal of Marketing, 59(3), 63-74.

Slater, S., Narver, J. (2000). Intelligence generation and superior customer value. Journal of the Academy of Marketing Science, 28(1), 120-127.

Sobel, M. (1982). Asymptotic confidence intervals for indirect effects in structural equation models. Sociological Methodology, 13(1982), 290-312.

Soehadi, A. W., Hart, S., \& Tagg, S. (2001). Measuring market orientation in the Indonesian retail context. Journal of Strategic Marketing, 9(4), 285-299.

Song, M., Thieme, J. (2009). The Role of Suppliers in Market Intelligence Gathering for Radical and Incremental Innovation. Journal of Product Innovation Management, 26(1), 43-57.

Specht, G. (1997). Markt- und technologiegetriebene Vorentwicklung im Produkt- und Systemgeschäft. In: K. Backhaus, B. Günter, M. Kleinaltenkamp, W. Plinke, H. Raffée (Hrsg.): Marktleistung und Wettbewerb, Wiesbaden, S. 
401-421.

Srivastava, R. K., Fahey, L., Christensen, H. K. (2001). The resource-based view and marketing: The role of market-based assets in gaining competitive advantage. Journal of Management, 27, 777-802.

Srivastava, R. K., Shervani, T. A., Fahey, L. (1998). Market-based assets and shareholder value: A framework for analysis. The Journal of Marketing, 62(January), pp. 2-18.

Steenkamp, J., \& Baumgartner, H. (1998). Assessing measurement invariance in cross-national consumer research. Journal of Consumer Research, 25(1), 78107.

Steenkamp, J., \& Baumgartner, H. (2000). On the use of structural equation models for marketing modeling. International Journal of Research in Marketing, 17, 195-202.

Steinhoff, F. (2006). Kundenorientierung bei hochgradigen Innovationen. Konzeptualisierung, empirische Bestandsaufnahme und Erfolgsbetrachtung, Wiesbaden.

Steinman, C., Deshpandé, R., \& Farley, J. U. (2000). Beyond Market Orientation: When Customers and Suppliers Disagree. Journal of the Academy of Marketing Science, 28(1), 109.

T

Tabe, T., Muramatsu, R., Tanaka, Y. (1980). Analysis of production ordering quantities and inventory variations in a multi-stage production ordering system. International Journal Of Production Research, 18(2), 245-257.

Teece, D. J., Pisano, G., \& Shuen, A. (1997). DYNAMIC CAPABILITIES AND STRATEGIC MANAGEMENT. Strategic Management Journal, 18(7), 509533.

Teichert, T., Bouncken, R. B. (2011). Rigidities Considered: Supplier Strategies for Integrated Innovation. International Journal of Innovation Management, 15(01), 95-119.

Temme, D., \& Hildebrandt, L. (2009). Gruppenvergleiche bei hypothetischen 
Konstrukten - Die Prüfung der Übereinstimmung von Messmodellen mit der Strukturgleichungsmethodik. Zfbf, 61(2),138-185.

Temme, D., Kreis, H. (2005). Der PLS-Ansatz zur Schätzung von Strukturgleichungsmodellen mit latenten Variablen: Ein Softwareüberblick. In: F. Bliemel, A. Eggert, G. Fassott, J. Henseler (Eds.): Handbuch PLSPfadmodellierung. Methode, Anwendung und Praxisbeispiele, Stuttgart: Schäffer/Poeschel, S.193-210.

Temme, D., Kreis, H., \& Hildebrandt, L. (2010). A Comparison of Current PLS Path Modeling Software: Features, Ease-of-Use, and Performance. In: V. E. Vinzi, W. W. Chin, J. Henseler, \& H. Wang (Eds.), Handbook of Partial Least Squares. Concepts, Methods and Applications, Berlin: Springer, S. 737-756.

Temme, D., Paulssen, M., \& Hildebrandt, L. (2009). Common Method Variance. Ursachen, Auswirkungen und Kontrollmöglichkeiten. DBW - Die Betriebswirtschaft, 69(2), 123-146.

Tenenhaus, M., Mauger, E., Gruinot, C. (2010). Use of ULS-SEM and PLS-SEM to Measure a Group Effect in a Regression Model Relating Two Blocks of Binary Variables. In: V. E. Vinzi, W. W. Chin, J. Henseler, \& H. Wang (Eds.), Handbook of Partial Least Squares. Concepts, Methods and Applications, Berlin: Springer, S. 125-140.

Tenenhaus, M., Vinzi, V. E., Chatelin, Y.-M., \& Lauro, C. (2005). PLS path modeling. Computational Statistics \& Data Analysis, 48(1), 159-205.

Tomášková, E. (2009). The Current Methods of Measurement of Market Orientation. European Research Studies, XII(3), 135-150.

Tracey, P., Heide, J. B., \& Bell, S. J. (2014). Bringing "Place" Back In: Regional Clusters, Project Governance, and New Product Outcomes. Journal Of Marketing, 78(6), 1-16.

Trommen, A. (2002): Mehrstufige Kundenintegration in Wertschöpfungssystemen, Wiesbaden.

Tsai, W., Su, K.-H., \& Chen, M. (2011). SEEING THROUGH THE EYES OF A RIVAL: COMPETITOR ACUMEN BASED ON RIVAL-CENTRIC PERCEPTIONS. Academy of Management Journal, 54(4), 761-778. 
Tsiotsou, R. H. (2010). Delineating the effect of market orientation on services performance: a component-wise approach. The Service Industries Journal, 30(3), 375-403.

$\mathbf{U}$

Ungruhe, M. (2011). Bezugsobjektspezifische Investitionen im Business- toBusiness-Marketing. [Online], http://www.diss.fuberlin.de/diss/receive/FUDISS_thesis_000000022080 [14.12.2014]

Urban, G. L., Von Hippel, E. (1988). Lead User Analyses for the Development of New Industrial Products. Management Science, 34(5), 569-582.

V

Van Raaij, E. M., Stoelhorst, J. W. (2008). The Implementation of a Market Orientation: A Review and Integration of the Contributions to Date. European Journal of Marketing, 42, 1265-1293.

Varadarajan, P., Jayachandran, S. (1999), Marketing Strategy: An Assessment of the State of the Field and Outlook. Journal Of The Academy Of Marketing Science, 27(2), 120-143.

Vázquez, R., Santos, M. L., Álvarez, L. I. (2001). Market orientation, innovation and competitive strategies in industrial firms. Journal of Strategic Marketing, 9(1), 69-90.

Verhees, F. J. H. M., \& Meulenberg, M. T. G. (2004). Market Orientation, Innovativeness, Product Innovation, and Performance in Small Firms. Journal of Small Business Management, 42(2), 134-154.

Villalonga, B. (2004). Intangible resources, Tobin's q, and sustainability of performance differences. Journal of Economic Behavior \& Organization, 54(2), 205-230.

Vorhies, D. W., Morgan, N. A. (2003). A Configuration Theory Assessment of Marketing Organization Fit with Business Strategy and Its Relationship with Marketing Performance. Journal of Marketing, 67(1), 100-115.

Vorhies, D. W., Morgan, N. A. (2005). Benchmarking marketing capabilities for 
sustainable competitive advantage. Journal of Marketing, 69(1), 80-94.

\section{W}

Wang, E. T. G., Hu, H., \& Hu, P. J.-H. (2013). Examining the role of information technology in cultivating firms’ dynamic marketing capabilities. Information \& Management, 50(6), 336-343.

Ward, S., Girardi, A., Lewandowska, A. (2006). A Cross-National Validation of the Narver and Slater Market Orientation Scale. The Journal of Marketing Theory and Practice, 14(2), 155-167.

Webb, D., Webster, C., \& Krepapa, A. (2000). An exploration of the meaning and outcomes of a customer-defined market orientation. Journal of Business Research, 48(2), 101-112.

Weiber, R., Jacob, F. (2000). Kundenbezogene Informationsgewinnung. In: Kleinaltenkamp, M., Plinke, W. (Hrsg.): Technischer Vertrieb. Grundlagen des Business-to-Business Marketing, 2. Aufl., Berlin et al, S. 523-612.

Weiber, R., Kollmann, T., Pohl, A. (2006). Das Management technologischer Innovationen. In: Kleinaltenkamp, M., Plinke, W., Jacob, F., Söllner, A. (Hrsg.): Markt- und Produktmanagement. Die Instrumente des Die Instrumente des Business-to-Business Marketing, 2. Aufl., Wiesbaden, S. 83-207.

Weiber, R., \& Mühlhaus, D. (2010). Strukturgleichungsmodellierung. Eine anwendungs- orientierte Einführung in die Kausalanalyse mit Hilfe von AMOS, SmartPLS und SPSS, Berlin et al.

Weitz, B. A., Jap, S. D. (1995). Relationship Marketing and Distribution Channels. Journal of the Academy of Marketing Science, 23(4), 305-320.

Wernerfelt, B. (1984). A Resource-based View of the Firm. Strategic Management Journal, 5, 171-180.

Wernerfelt, B. (1989). From critical resources to corporate strategy. Journal of General management, 14(3), 4-12.

West, S. G., Finch, J. F., Curran, P. J. (1995). Structural equation models with nonnormal variables: Problems and remedies. In: Hoyle, Rick H. (Ed.): Structural equation modeling: Concepts, issues, and applications, Thousand 
Oaks: Sage Publications, pp. 56-75.

Wilcox, J. B., Howell, R. D., Breivik, E. (2008). Questions about formative measurement. Journal of Business Research, 61(12), 1219-1228.

Wold, H. (1982): Soft Modeling. The Basic Design and Some Intentions. In: K. Jöreskog, H. Wold, H. (Eds): Systems under Indirect Observations. Causality, Structure, Prediction (Part II), Amsterdam, pp. 1-54.

Woodruff, R. (1997). Customer value: The next source for competitive advantage. Journal of the Academy of Marketing Science, 25(2), 139-153.

Wooldridge, B., \& Minsky, B. (2002). The role of climate and socialization in developing interfunctional coordination. The Learning Organization, 9(1), 29-38.

\section{X}

Xie, J., Song, M., Stringfellow, A. (2003). Antecedents and Consequences of Goal Incongruity on New Product Development in Five Countries: A Marketing View. Journal Of Product Innovation Management, 20(3), 233-250.

$\mathrm{Xu}$, J. D., Benbasat, I., \& Cenfetelli, R. T. (2014). THE NATURE AND CONSEQUENCES OF TRADE-OFF TRANSPARENCY IN THE CONTEXT OF RECOMMENDATION AGENTS. MIS Quarterly, 38(2), 379-406.

Xu, L., Beamon, B. (2006). Supply Chain Coordination and Cooperation Mechanisms: An Attribute-Based Approach. Journal of Supply Chain Management, 42(1), 4-12.

Y

Yau, O., Chow, R., Sin, L., Tse, A., Luk, C., Lee, S. (2007). Developing a scale for stakeholder orientation. European Journal of Marketing, 41(11/12), 1306-1327.

\section{Z}

Zaichkowsky, J. (1985). Measuring the involvement construct. Journal of Con- 
sumer Research, 12(December), 341-352.

Zentes, J., Swoboda, B. (2001). Grundbegriffe des Marketing: marktorientiertes globales Management-Wissen, 5. Aufl, Stuttgart: Schäffer-Poeschel.

Zhou, K. Z., Gao, G. Y., Yang, Z., Zhou, N. (2005). Developing strategic orientation in China: antecedents and consequences of market and innovation orientations. Journal of Business Research, 58(8), 1049-1058.

Zhou, K. Z., Kin, C., Tse, D. K. (2005). The effects of strategic orientations on technology-and market-based breakthrough innovations. Journal of Marketing, 69(2), 42-60.

Zhou, K. Z., Li, J. J., Zhou, N., Su, C. (2008). Market orientation, job satisfaction, product quality, and firm performance: evidence from China. Strategic Management Journal, 29(9), 985-1000. 